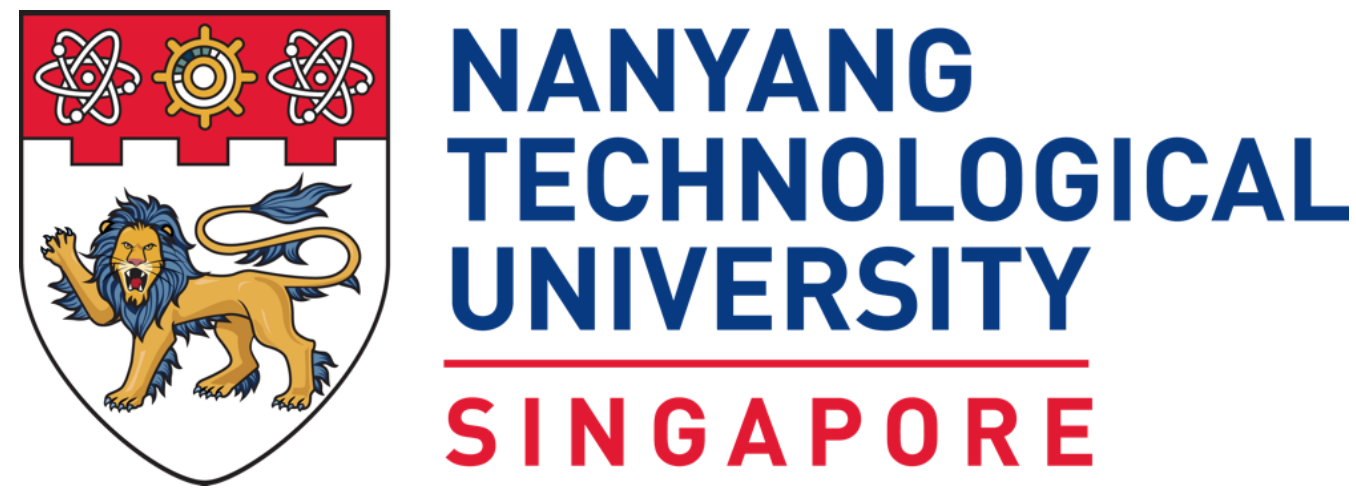

CHARACTERIZATION OF REGULATORY CENTRAL STALK EPITOPES OF MYCOBACTERIAL- AND ACETOGEN F-ATP SYNTHASES

\author{
BOGDANOVIĆ NEBOJŠA \\ SCHOOL OF BIOLOGICAL SCIENCES
}

2019 


\section{CHARACTERIZATION OF REGULATORY CENTRAL STALK EPITOPES OF MYCOBACTERIAL- AND ACETOGEN F-ATP SYNTHASES}

\section{BOGDANOVIĆ NEBOJŠA}

School of Biological Sciences

A thesis submitted to the Nanyang Technological University in partial fulfillment of the requirement for the degree of Doctor of Philosophy

2019 


\section{Statement of Originality}

I hereby certify that the work embodied in this thesis is the result of original research done by me except where otherwise stated in this thesis. The thesis work has not been submitted for a degree or professional qualification to any other university or institution. I declare that this thesis is written by myself and is free of plagiarism and of sufficient grammatical clarity to be examined. I confirm that the investigations were conducted in accord with the ethics policies and integrity standards of Nanyang Technological University and that the research data are presented honestly and without prejudice.

$24 / 01 / 2019$

Date

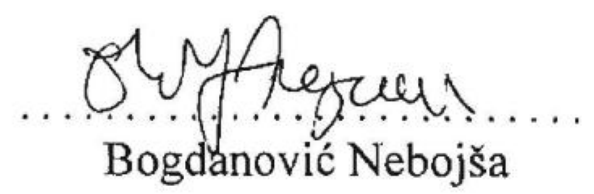




\section{Supervisor Declaration Statement}

I have reviewed the content and presentation style of this thesis and declare it of sufficient grammatical clarity to be examined. To the best of my knowledge, the thesis is free of plagiarism and the research and writing are those of the candidate's except as acknowledged in the Author Attribution Statement. I confirm that the investigations were conducted in accord with the ethics policies and integrity standards of Nanyang Technological University and that the research data are presented honestly and without prejudice.

$24 / 01 / 2019$

Date

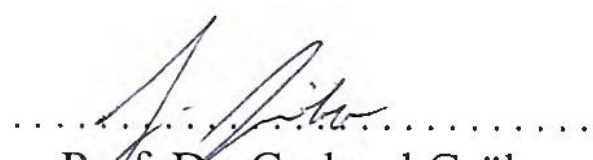

Pyff. Dr. Gerhard Grüber 


\section{Authorship Attribution Statement}

This thesis contains material from two papers published in the Journal of structural biology and The FEBS journal, as well as the third manuscript under revision where I was the first and/or co-author.

Chapters 3.1, 3.2 and 3.3 (excluding section 3.2.4) are published as Bogdanović, N., Sundararaman, L., Kamariah, N., Tyagi, A., Bhushan, S., Ragunathan, P., Shin, J., Dick, T., and Grüber, G. (2018) Structure and function of Mycobacterium-specific components of FATP synthase subunits alpha and epsilon. Journal of structural biology 204, 420-434

and

Joon, S., Ragunathan, P., Sundararaman, L., Nartey, W., Kundu, S., Manimekalai, M. S. S., Bogdanović, N., Dick, T., and Grüber, G. (2018) The NMR solution structure of Mycobacterium tuberculosis F-ATP synthase subunit epsilon provides new insight into energy coupling inside the rotary engine. The FEBS journal 285, 1111-1128

The contributions of the co-authors are as follows:

- Prof. Gerhard Grüber provided the initial project direction and the direction of the manuscript.

- I, together with Prof. Gerhard Grüber and Dr. Neelagandan Kamariah, wrote and edited the manuscript draft. The manuscript was revised by Prof. Shashi Bhushan and Dr. Priya Ragunathan.

- All cloning of the mutant $M t \varepsilon$ constructs, isolation of the proteins, reconstitution, crosslinking experiments, and activity measurements were conducted by me. The $\alpha^{\text {chi }}{ }_{3}: \beta_{3}: \gamma$ mutants S400C and V525C were generated together with Dr. Dhirendra Singh.

- The grids for the negative stain electron microscopy were prepared, and data collection was carried out by Prof. Shashi Bhushan and Dr. Anu Tyagi, while I have provided the purified, reconstituted proteins and processed the data.

- The ${ }^{15} \mathrm{~N}-\mathrm{HSQC}$ spectrum data collection for the mutant MtcE87A was performed by Dr. Shin Joon on the purified ${ }^{15} \mathrm{~N}$-labeled protein that was 
prepared by me. The comparative assignment of the ${ }^{15} \mathrm{~N}-\mathrm{HSQC}$ spectrum of $M t \varepsilon E 87$ A with $M t \varepsilon$ was performed by me under the guidance of Dr. Shin Joon, who has assigned and solved the Mte NMR solution structure.

- The fluorescent labeling of the proteins was carried out by me for the purpose of smFRET studies conducted by Prof. Michael Böersch at Jena University Hospital, Germany.

The contributions to the unpublished chapter 3.2.4 are as follows:

- The experiments were designed by Prof. Gerhard Grüber and Prof. Thomas Dick.

- The group of Prof. Thomas Dick kindly provided all the required plasmids and M. smegmatis WT strain for the recombineering experiments.

- All the experiments involving M. smegmatis mutant strain atp $C^{\mathrm{E} 87 \mathrm{~A}}$, including its generation, were carried out by me under the guidance of Dr. Carolyn Mulu Wu from Prof. Thomas Dick's group at MD6, NUS, Singapore.

Chapter 3.4 is included in the following manuscript:

Bogdanović, N., Trifunović, D., Sielaff, H., Westphal, L., Bhushan, S., Müller, V., and Grüber, G. (2019) The structural features of Acetobacterium woodii F-ATP synthase reveal the importance of the unique subunit gamma-loop in $\mathrm{Na}(+)$ translocation and ATP synthesis. The FEBS journal 286, 1894-1907

and its authors' contribution is as follows:

- Prof. Gerhard Grüber provided the initial project direction and the direction of the manuscript.

- I, together with Prof. Gerhard Grüber and Prof. Volker Müller wrote the manuscript, while it was revised by Dr. Hendrik Sielaf and Prof. Shashi Bhushan. 
- The A. woodii construct (vector with WT F-ATP synthase operon) was kindly provided by Prof. Volker Müller, Johann Wolfgang Goethe Universität, Frankfurt am Main, Germany.

- The generation of deletion mutant $\gamma_{\Delta 195-211}$ was carried out by me as well as the purification of WT and the mutant forms of F-ATP synthase of A. woodii.

- The grids for the negative stain electron microscopy were prepared and data collection was carried out by Prof. Shashi Bhushan, while I have provided the purified the protein and processed the data.

- The biochemical characterization including the ATP synthesis/hydrolysis and $\mathrm{Na}^{+}$translocation measurements of the proteoliposome-embedded enzyme was entirely carried out by Mr. Dragan Trifunović from Prof. Volker Müller's group who also provided the respective final figures.

$24 / 01 / 2019$

Date

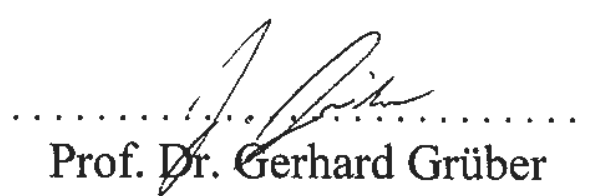




\section{Acknowledgments}

First and foremost, I would like to express my sincere gratitude to my Ph.D. supervisor, Professor Dr. Gerhard Grüber, for his countless hours of discussions and opinions that guided me through my past four years. His generous support provided me with a great learning opportunity that has influenced my scientific and professional development and enabled me to see the fabric of reality in a different light. Writing a Ph.D. thesis is never an easy task, but it has become a memorable experience because of his support and constructive suggestions and for that, I am grateful to have been a part of the Prof. Grüber's group.

Furthermore, I am sincerely grateful to Dr. Arvind Kumar for his keen interest in science that he shared with me and his constructive suggestions that lead to exciting interpretations and fruitful discussions, always challenging the current views. Many thanks go to Dr. Dhirendra Singh, who trained me initially in molecular biology and protein purification techniques and Dr. Hendrik Sielaff who, together with Dr. Singh, was always present to discuss scientific problems and experimental challenges with wit and enthusiasm, giving it a new dimension. My sincere appreciation goes to Dr. Balakrishna Asha, who selflessly shared her knowledge with care and genuine interest for the problem at hand. Dr. Yew Kwong's understanding of molecular biology provided me with an alternate approach to gene manipulations, and for that, I am expressing my honest gratitude. I am also thankful to Dr. Wilson Nartey with whom I spent numerous hours in discussions of science and life that sparked new ideas and concepts and Dr. Amaravadi Harikishore who has always been a great support and a helping hand in scientific endeavors, teaching me essential concepts in bioinformatics. Special appreciation goes to Dr. Carlyn Mulu $\mathrm{Wu}$, who has, with her immense patience and scientific prowess, guided me through the waters of microbiology and thought me a great deal about recombineering.

Moreover, I must give my heartfelt thanks to Dr. Ardina Grüber, for friendly teaching environment, her excellent work ethics and open conversations that made the working atmosphere pleasant. Dr. Malathy Sony Subramanian Manimekalai, Dr. Neelagandan Kamariah, Dr. Rishikesan, and Dr. Priya Ragunathan, Dr. Lavanya Sundararaman, Dr. Saw Wuan Geok, Dr. Shin Joon and Dr. Ankita Pan have contributed 
significantly to the professional working environment; therefore I express my sincere appreciation.

I would also like to express special gratitude for bright, young minds, my great juniors, Bharti, Mun, Chui Fann, Sherilyn, Jack and Robin who made the lab environment lively by discussing science and life, teaching me a great deal in return. I owe special thanks to my numerous friends from NTU and NUS for their support, interest, valuable hints, and shared experiences.

In addition, I gratefully acknowledge all the scientific support provided by our collaborators; Prof. Thomas Dick for providing all the plasmids for mycobacterial recombineering, Prof. Shashi Bhushan for all electron microscopy-related contributions, Prof. Volker Müller for kindly providing the WT construct and support for A. woodii project and Prof. Michael Böersch who conducted the smFRET experiment and analyzed the data.

I gratefully acknowledge the financial support rendered by School of Biological Sciences by providing me the research scholarship. This research was supported by the National Research Foundation (NRF) Singapore, NRF Competitive Research Programme (CRP), Grant Award Number NRF-CRP18-2017-01; Lead-PI G.G.). I am also grateful to the academic and technical staff at the School of Biological Sciences, who has helped me in one way or the other in my research work. I would also wish to acknowledge the funds of Prof. Dr. G. Grüber for the research support granted.

My hearty thanks go to my family members whose wit and quick thinking helped me achieve my academic success and my life goals. My parents Branko Bogdanović and Dr. Olivera Bogdanović, my grandmother Branka Marinković, my sister Mina and my close relatives Sanja, Zorana, Pavle, Vasilije, Petar and Danilo all provided endless support and inspiration to make further progress and excel in scientific research. Last but not least, my eternal gratitude goes to my late grandfather, Prof. Vidosav Marinković, whose persona and devotion inspired me and prepared for the challenges ahead. 
Dedicated to My parents and My sister 


\section{Table of Contents}

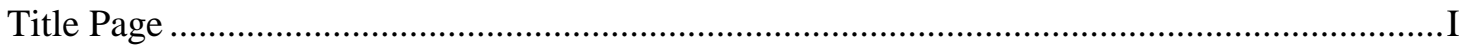

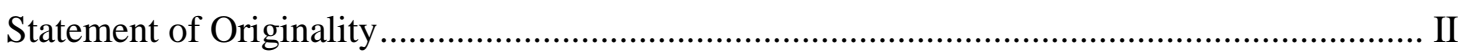

Supervisor Declaration Statement .................................................................................. III

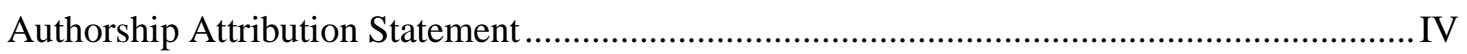

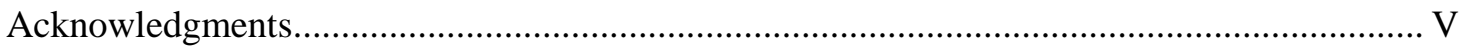

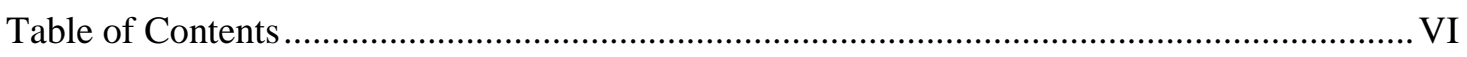

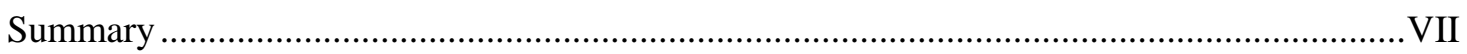

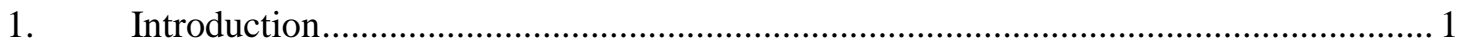

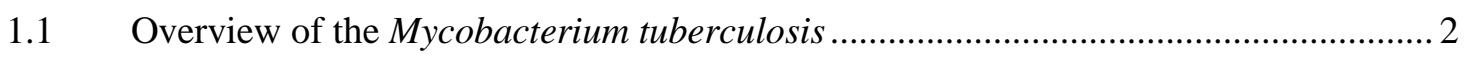

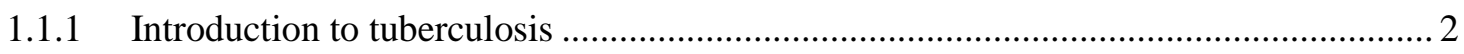

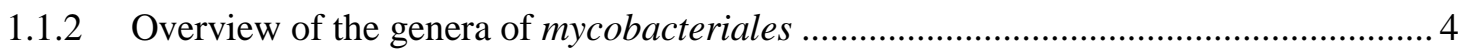

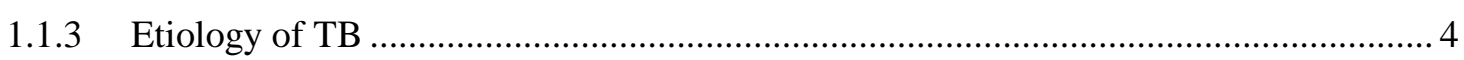

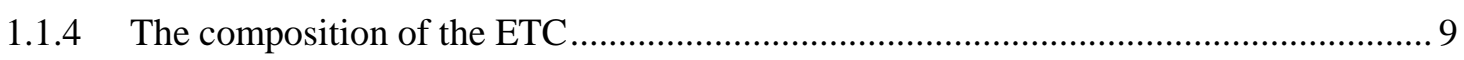

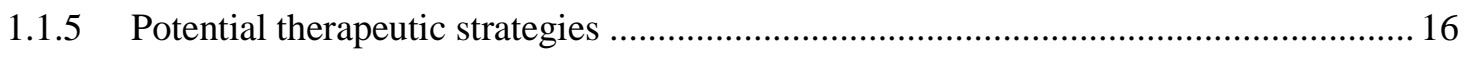

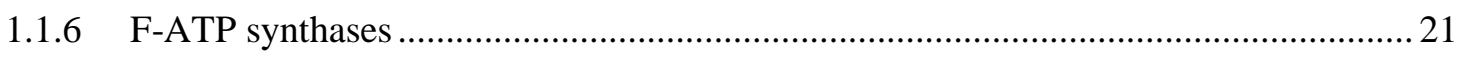

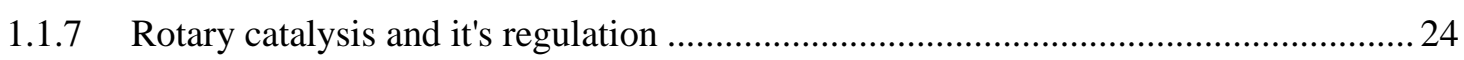

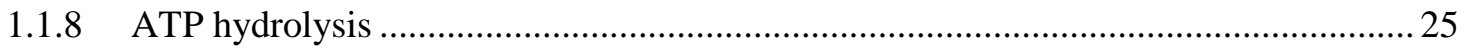

1.1.9 Structural features of bacterial subunit $\varepsilon$ and its regulatory capacity ........................2 27

1.1.10 Structural and functional features of mycobacterial F-ATP synthase .......................... 33

1.1.11 Catalytic features of $\alpha^{c h i}: \beta_{3}: \gamma$ complex and modeling of the $\alpha^{c h i}-\varepsilon$

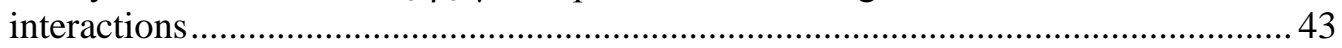

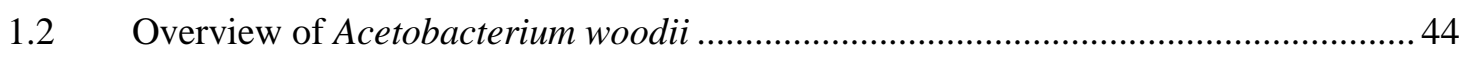

1.2.1 Metabolic pathways of carbon assimilation and energy generation ............................ 44

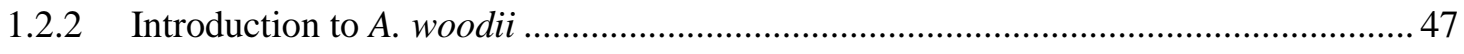

1.2.3 Carbon fixation and Wood-Ljungdahl pathway....................................................... 48

1.2.4 Structural and functional components of the $\mathrm{Na}^{+}$-translocating F-ATP

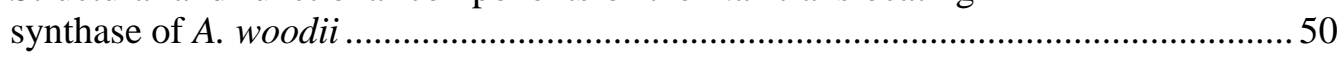

1.2.5 Structural and functional characterization of the $A$. woodii $c$-ring ...............................5 52

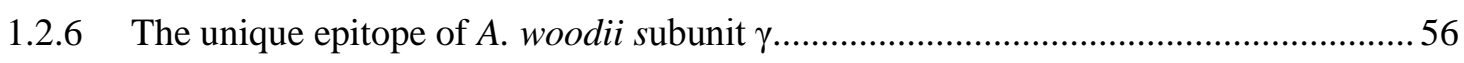

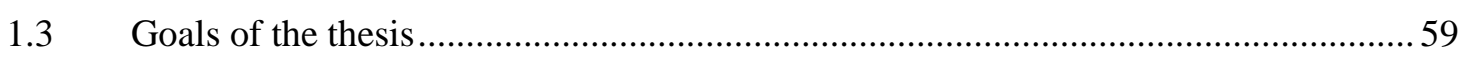

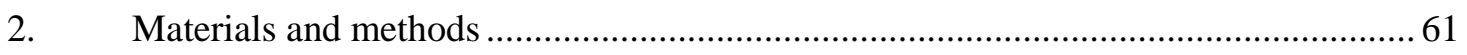

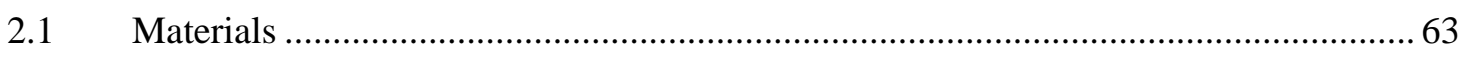

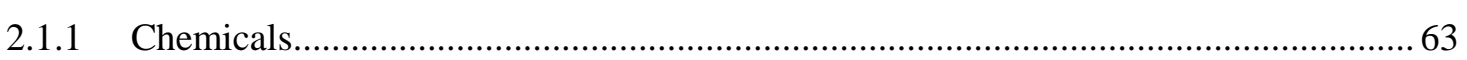

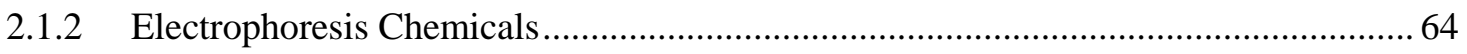

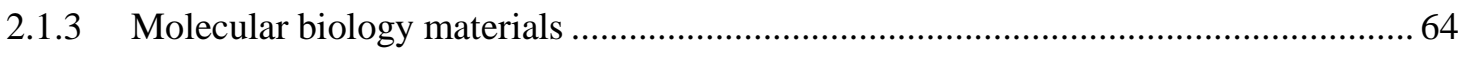

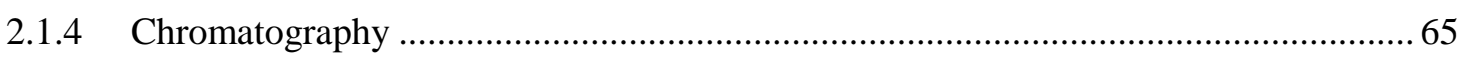




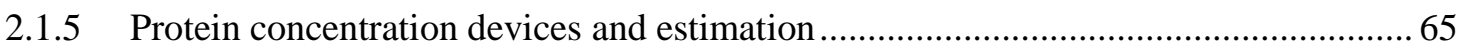

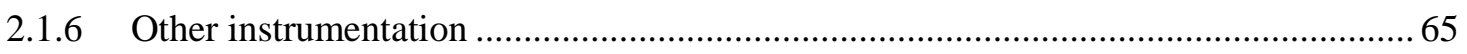

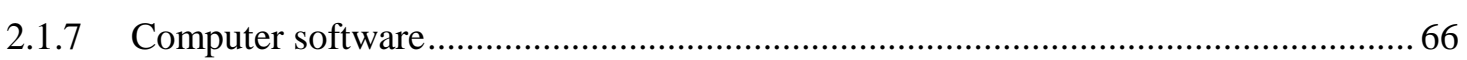

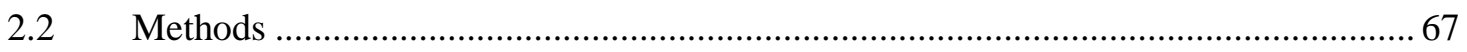

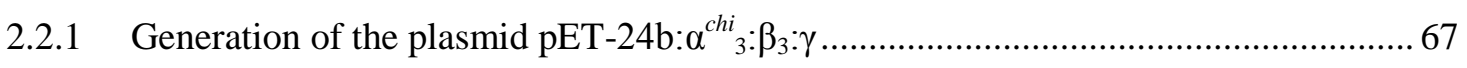

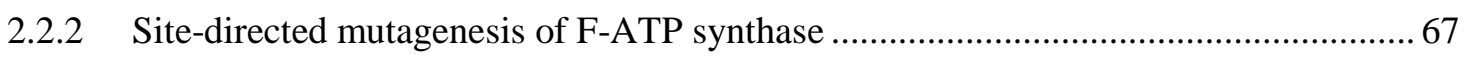

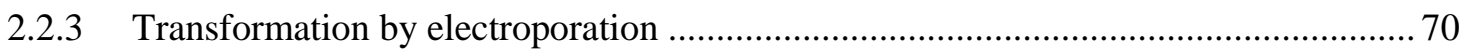

2.2.4 Cultivation conditions and expression of complex $\alpha_{3}^{\text {chi }}: \beta_{3}: \gamma$ F-ATP

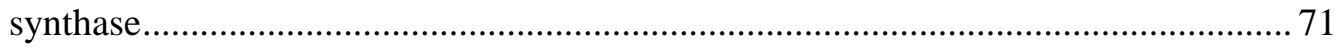

2.2.5 Purification of complex $\alpha^{\text {chi }}{ }_{3}: \beta_{3}: \gamma$ F-ATP from $E$. coli DK8 cells.................................. 71

2.2.6 Optimization of the purification of complex $\alpha_{3}^{\text {chi }}: \beta_{3}: \gamma$ F-ATP ..................................... 72

2.2.7 Cloning of various constructs of subunit $\varepsilon$ of F-ATP synthase from

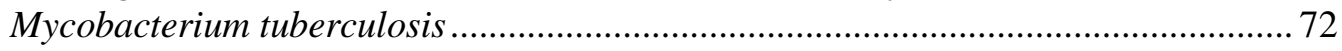

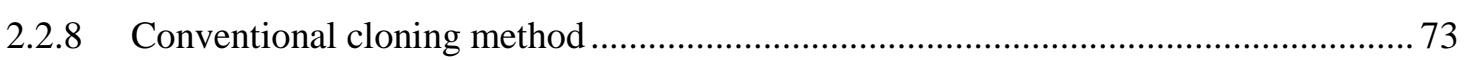

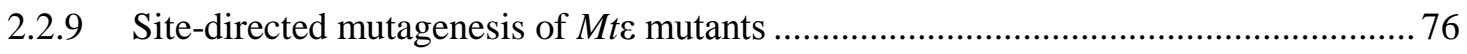

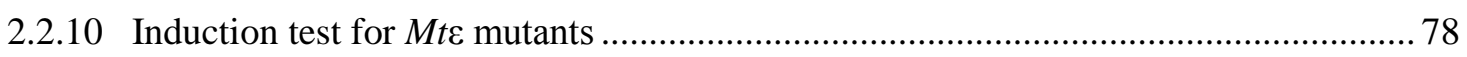

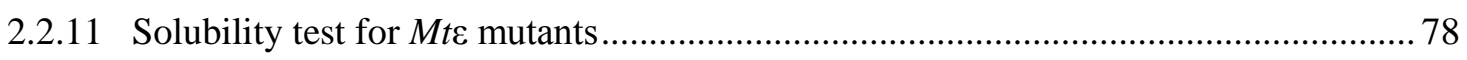

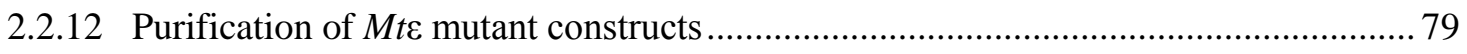

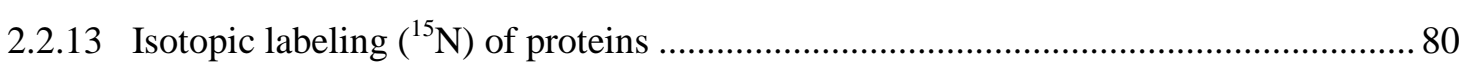

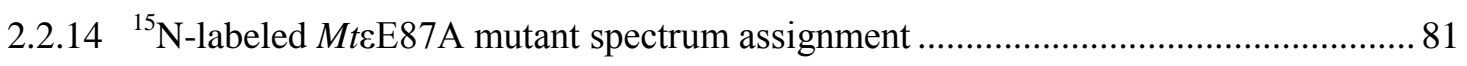

2.2.15 NADH coupled continuous assay for ATP hydrolytic activity

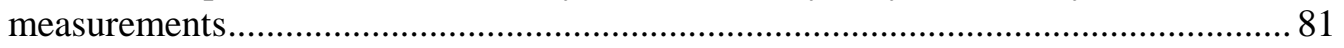

2.2.16 Reconstitution of the $\alpha^{c h i}: \beta_{3}: \gamma$ complex with $M t \varepsilon$ mutants......................................... 82

2.2.17 Negative stain electron microscopy (NS-EM) and 2D image analysis of the $\alpha^{c h i}{ }_{3}: \beta_{3}: \gamma$ - and $\alpha^{c h i}: \beta_{3}: \gamma: M t \varepsilon$ complexes of $\mathrm{F}_{1}$-ATP synthase ............................. 82

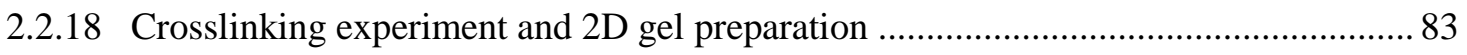

2.2.19 Densitometric analysis of reconstituted $\alpha^{\text {chi }}: \beta_{3}: \gamma: M t \varepsilon$ complexes ............................... 84

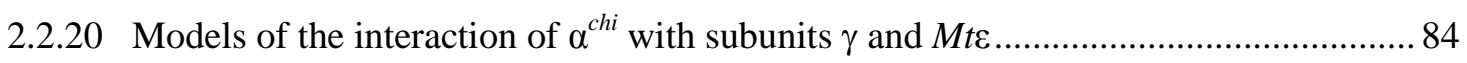

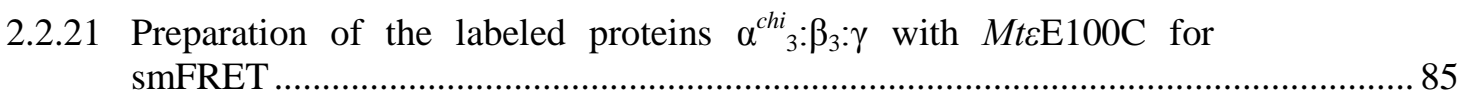

2.2.22 Preparation of electrocompetent cells of M. smegmatis mc $155^{2}$................................ 85

2.2.23 Transformation and preparation of the M. smegmatis mc $155^{2} \mathrm{pJV} 62$

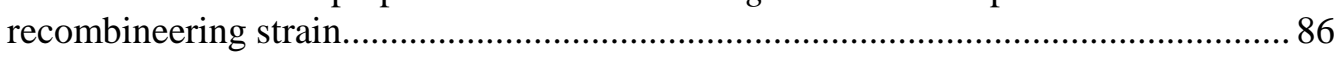

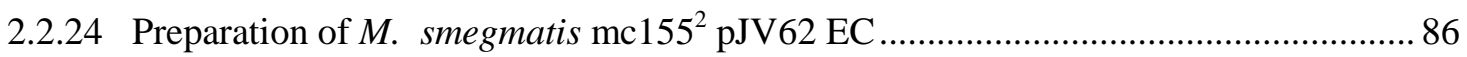

2.2.25 Design, transformation of the ssDNA M. smegmatis atp $C^{\mathrm{E} 87 \mathrm{~A}}$ oligo into the recombineering strain $M$. smegmatis mc $155^{2}$ pJV62 .................................. 87

2.2.26 PCR screening of E87A mutation in M. smegmatis mc $155^{2}$ pJV62 .......................... 87

2.2.27 Isolation of inverted membrane vesicle (IMV) isolation from $M$. smegmatis $m c 155^{2}$ (WT) and mutant atp $C^{\mathrm{E} 87 \mathrm{~A}}$...... 
2.2.28 ATP synthesis assay for screening the activities of $M$. smegmatis $m c 155^{2}(\mathrm{WT})$, mutant $a t p C^{\mathrm{E} 87 \mathrm{~A}}$ and mutant $a t p C^{\mathrm{E} 87 \mathrm{~A}}$ complemented strains IMVs

2.2.29 ATP hydrolysis assay for M. smegmatis mc155 (WT), mutant

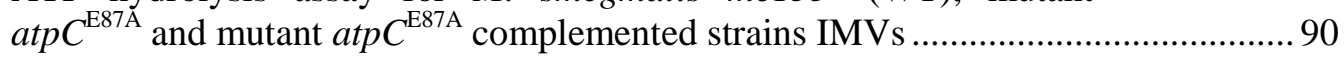

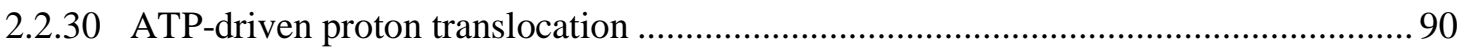

2.2.31 Determination of the intracellular ATP level of M. smegmatis $m c 155^{2}$ and mutant $\operatorname{atp} C^{\mathrm{E} 87 \mathrm{~A}}$

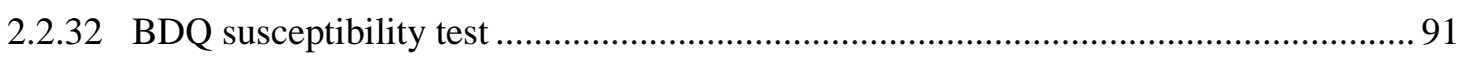

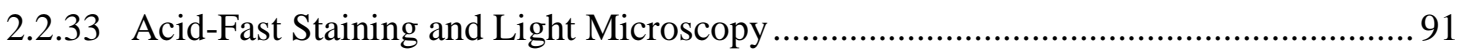

2.2.34 Quantification of proteins using bicinchoninic acid (BCA) assay and nanodrop

2.2.35 Structural model of the A. woodii F-ATP synthase and interactions of

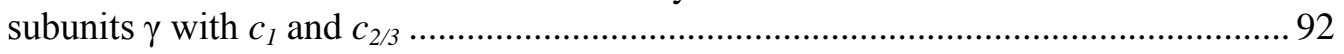

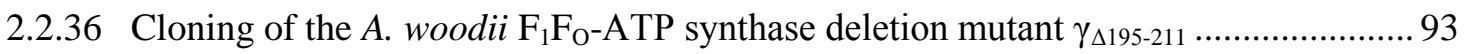

2.2.37 Purification of the A. woodii $\mathrm{F}_{1} \mathrm{~F}_{\mathrm{O}}$-ATP synthase WT and mutant $\gamma_{\Delta 195-211} \ldots \ldots \ldots \ldots \ldots . . . . . . .93$

2.2.38 Electron microscopy and 2D image analysis of A. woodii F-ATP

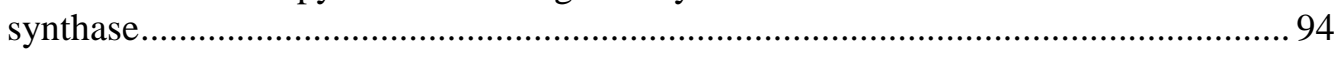

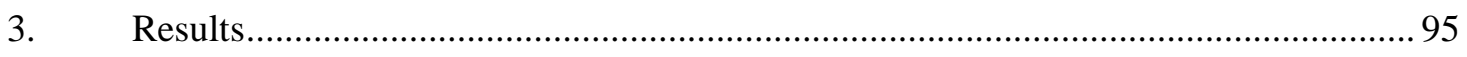

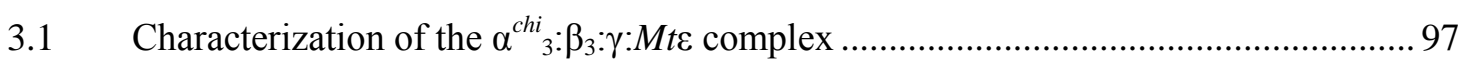

3.1.1 Optimized production, purification of the $\alpha^{c h i}: \beta_{3}: \gamma$ sub-complex and

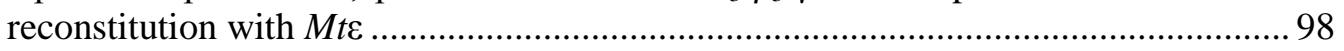

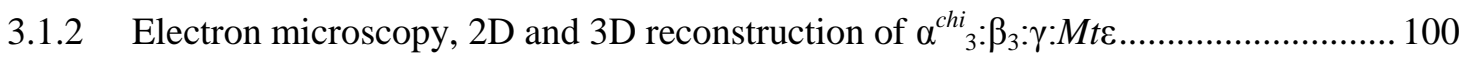

3.1.3 Kinetic properties of the $\alpha^{c h i}: \beta_{3}: \gamma-$ and $\alpha^{c h i}{ }_{3}: \beta_{3}: \gamma: M t \varepsilon$ complexes.................................. 104

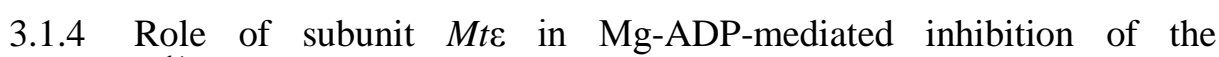

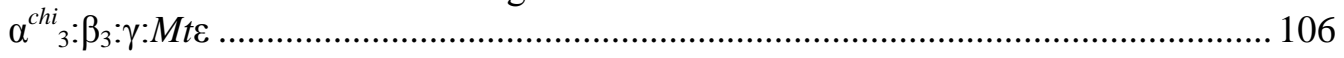

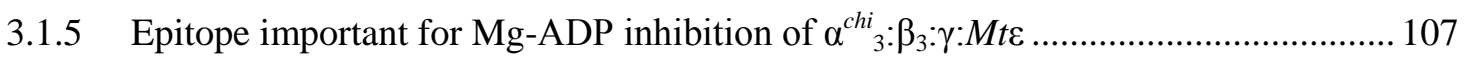

3.2 Characterization of epitopes of $M t \varepsilon$ important for catalytic activity and assembly.....

3.2.1 Characterization of epitopes of $M t \varepsilon$ important for catalytic activity and assembly......

3.2.2 Generating the mutant $M t \varepsilon E 87 \mathrm{~A}$ and its reconstitution with $\alpha^{c h i}{ }_{3}: \beta_{3}: \gamma$ complex........

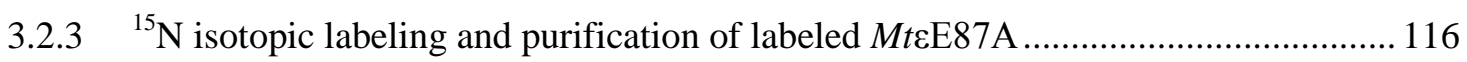

3.2.4 Effects of he $a t p C^{\mathrm{E} 87 \mathrm{~A}}$ on $M$. smegmatis bioenergetics ........................................... 120

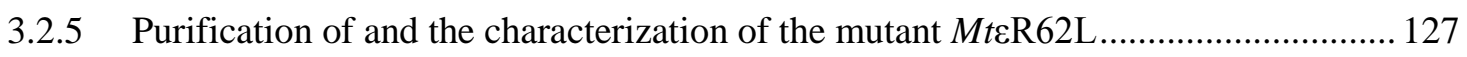

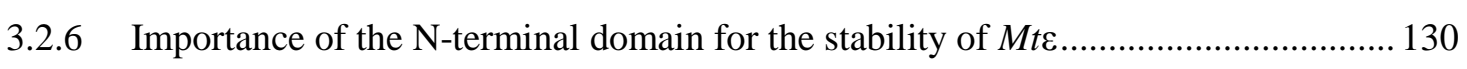

3.3 Interactions of mycobacteria-specific epitopes within mutant $\alpha^{c h i}: \beta_{3}: \gamma: M t \varepsilon$ complexes

3.3.1 Cloning, production, and purification of ( $\left.\alpha^{c h i}-\mathrm{S} 400 \mathrm{C}\right)-$ and ( $\alpha^{c h i}$ $\mathrm{V} 525 \mathrm{C})_{3}: \beta_{3}: \gamma$ complexes

3.3.2 Cloning, production, and purification of $M t \varepsilon$ mutants R113C, G118C, and D121C 
3.3.3 Reconstitution of $\left(\alpha^{c h i}-\mathrm{S} 400 \mathrm{C}\right)_{3}: \beta_{3}: \gamma$ and $M t \varepsilon \mathrm{D} 121 \mathrm{C}$

3.3.4 Reconstitution of ( $\left.\alpha^{c h i}-\mathrm{V} 525 \mathrm{C}\right)_{3}: \beta_{3}: \gamma$ and MtE mutants D121C, R113C and $\mathrm{G} 118 \mathrm{C}$

3.3.5 Cloning, production, and purification of the recombinant $M t \varepsilon \mathrm{E} 100 \mathrm{C}$ mutant for smFRET

3.3.6 Fluorescent labeling of the $\alpha^{c h i}{ }_{3}: \beta_{3}: \gamma: M t \varepsilon E 100 \mathrm{C}$ complex for the smFRET experiment.

3.4 Purification and characterization of $A$. woodii $\mathrm{F}_{1} \mathrm{~F}_{\mathrm{O}}$-ATP synthase using NS-EM and establishing a role of the unique subunit $\gamma$ unique loop.

3.4.1 Purification and ATPase activity of $A$. woodii $\mathrm{F}_{1} \mathrm{~F}_{\mathrm{O}}$-ATP synthase............................. 155

3.4.2 Negative stain electron microscopy of $A$. woodii $\mathrm{F}_{1} \mathrm{~F}_{\mathrm{O}}$-ATP synthase ....................... 157

3.4.3 Purification and ATPase activity of $A$. woodii $\gamma_{\Delta 195-211}$ mutant $\mathrm{F}_{1} \mathrm{~F}_{\mathrm{O}^{-}}$ ATP synthase

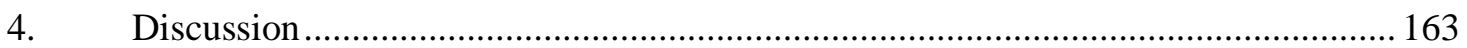

4.1 Structural and functional characterization of complexes $\alpha^{c h i}: \beta_{3}: \gamma$ and $\alpha^{c h i}: \beta_{3}: \gamma: M t \varepsilon$

4.2 Epitopes of mycobacterial subunit $\varepsilon$ involved in the regulation of ATPase activity...

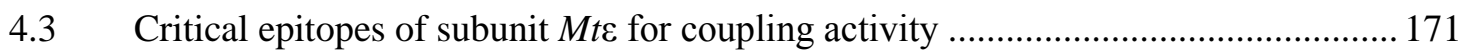

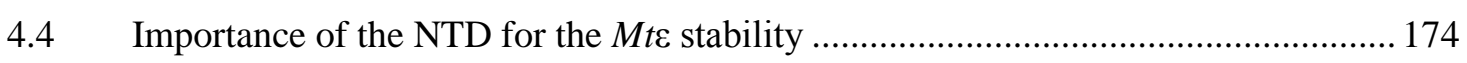

4.5 Residue E87 of $M t \varepsilon$ is important for $M$. smegmatis bioenergetics.

4.6 Spatial orientation of epitopes $M t \alpha_{514-549}$ and $M t \varepsilon C T D$ within

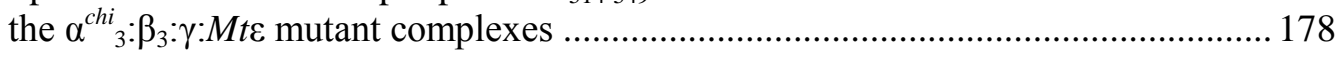

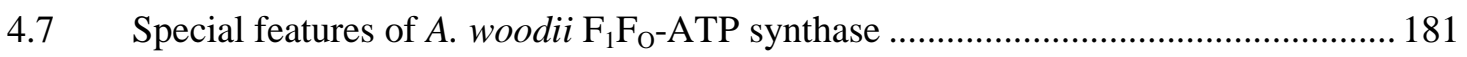

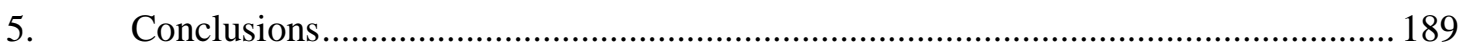

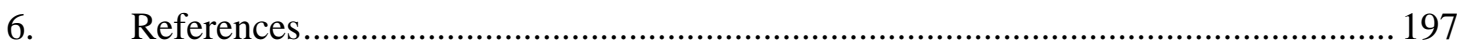

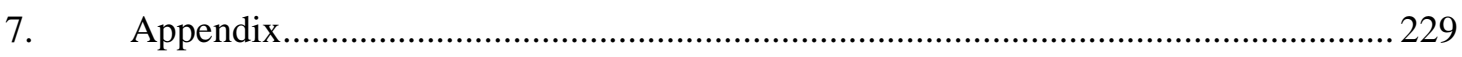




\section{List of Figures}

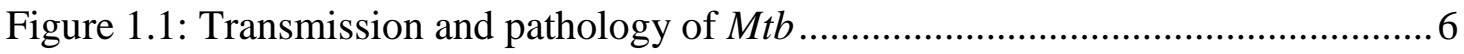

Figure 1.2: Organisation of the ETC from $M t b$ in aerobic conditions........................ 10

Figure 1.3: Organisation of the ETC from $M t b$ in hypoxic conditions........................ 11

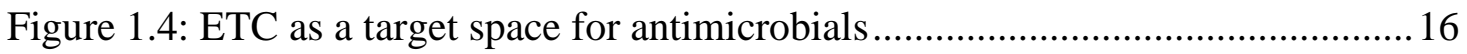

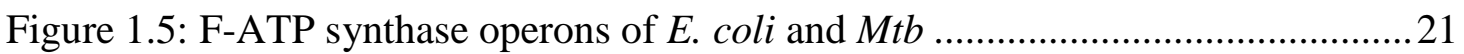

Figure 1.6: Structure of the E. coli F-ATP synthase solved using the cryo-EM ..........22

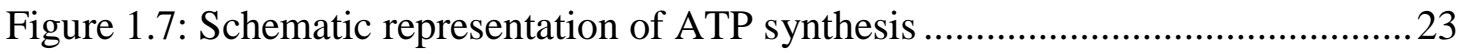

Figure 1.8: The conformations of subunit $\beta$ during catalysis ....................................26

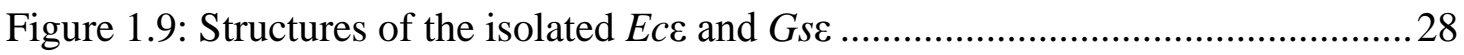

Figure 1.10: Sequence alignment of $G s \varepsilon$ and $E c \varepsilon$ showing the ATP binding pockets 29

Figure 1.11: Overview of the $E c \varepsilon$ in the extended conformation ...............................30

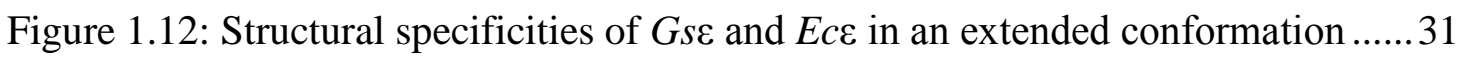

Figure 1.13: The structural alterations of the BDQ binging pocket in the $c$-ring ..........35

Figure 1.14: Sequence alignment and comparison of $\gamma$ and its unique features ............37

Figure 1.15: Sequence alignment and $M t b$ specific structural features of subunit $\alpha$....38

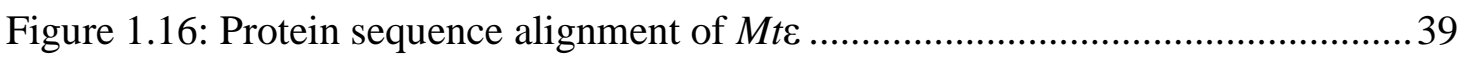

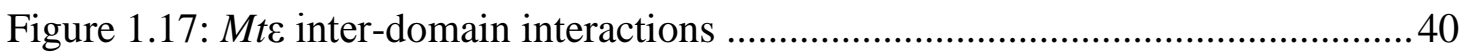

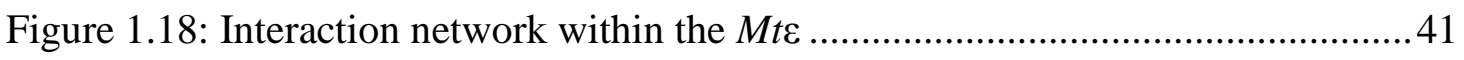

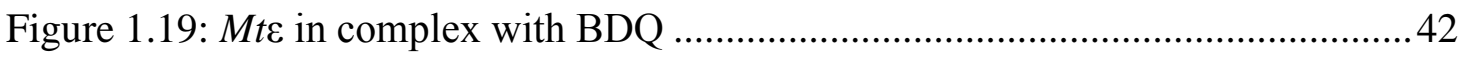

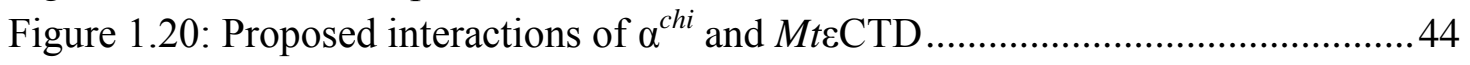

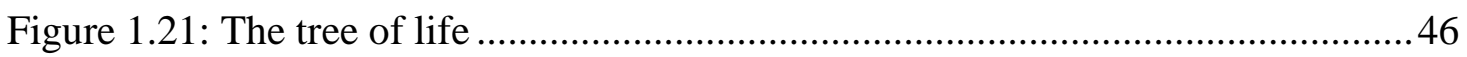

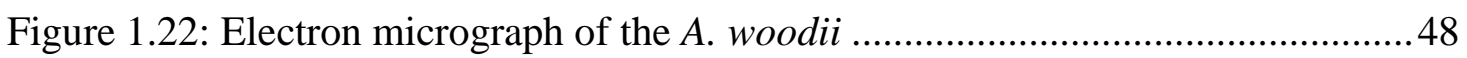

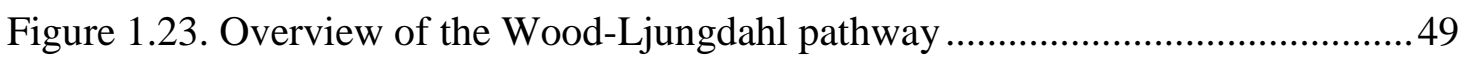

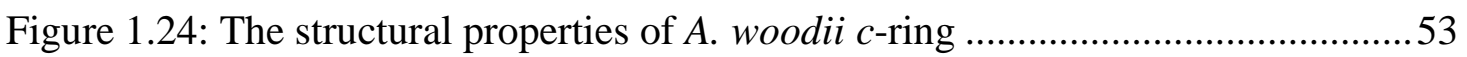

Figure 1.25. Protein sequence alignments of the $\gamma$ subunit of F-ATP synthases..........56

Figure 1.26: The unique A. woodii subunit $\gamma$-loop ${ }_{195}$ TSGKVKITEETKEEKSK $_{211} \ldots 57$

Figure 1.27: The phylogenetic tree of the selected bacterial and archaeal species.......58

Figure 2.1: Bar diagram mapping the mutations in $\alpha^{c h i}{ }_{3}: \beta_{3}: \gamma$ protein complex ............67

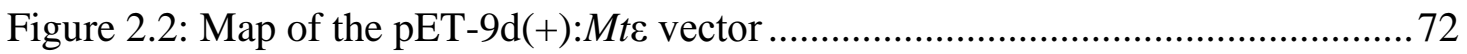

Figure 2.3: Scheme for the reaction of NADH coupled continuous assay. .................... 82

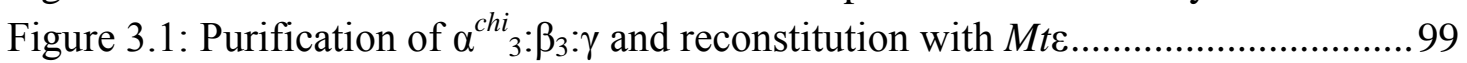

Figure 3.2: Densitogram and ATP hydrolysis of $\alpha^{c h i}: \beta_{3}: \gamma$ and $\alpha^{c h i}{ }_{3}: \beta_{3}: \gamma: M t \varepsilon$ complexes

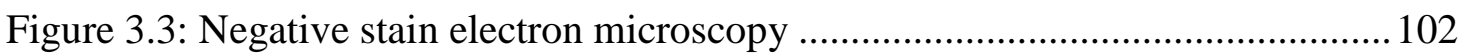

Figure 3.4: Surface representation of 3D reconstruction of $\alpha^{c h i}{ }_{3}: \beta_{3}: \gamma: M t \varepsilon$ complex... 103

Figure 3.5: FSC graph for negatively-stained reconstituted $\alpha^{c h i}{ }_{3} \beta_{3} \gamma: M t \varepsilon$ complex .... 103

Figure 3.6: ATPase activity of $\alpha^{c h i}{ }_{3}: \beta_{3}: \gamma \alpha^{c h i}{ }_{3}: \beta_{3}: \gamma: M t \varepsilon$ at variable Mg-ATP .............. 104

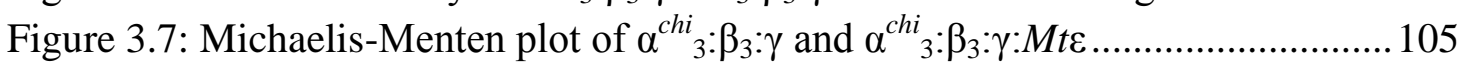

Figure 3.8: Effect of Mg-ADP on the inhibition of ATPase activity .......................... 106

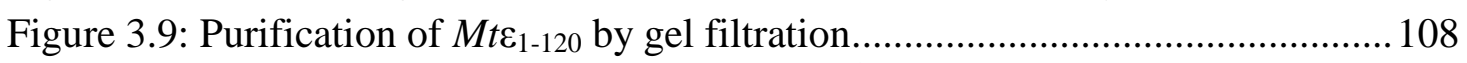

Figure 3.10: Reconstitution and densitometry of $\alpha^{c h i}{ }_{3}: \beta_{3}: \gamma: M t \varepsilon_{1-120}$ complex ............. 108

Figure 3.11: ATPase activity of the $\alpha^{c h i}: \beta_{3}: \gamma: M t \varepsilon_{1-120}$ complex................................. 109 
Figure 3.12: Inhibitory of Mg-ADP on the ATPase activity of the $\alpha^{c h i}: \beta_{3}: \gamma: M t \varepsilon_{1-120}$ complex.

Figure 3.13: The $M t \varepsilon$ intramolecular network of interactions around residue E87 ....112

Figure 3.14: Cloning of the substitution mutants MtEE87A.................................... 113

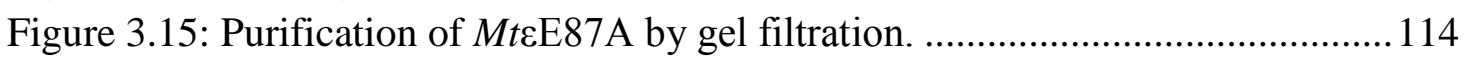

Figure 3.16: Reconstitution of $\alpha^{c h i}: \beta_{3}: \gamma$ and subunit MtEE87A mutant ..................... 115

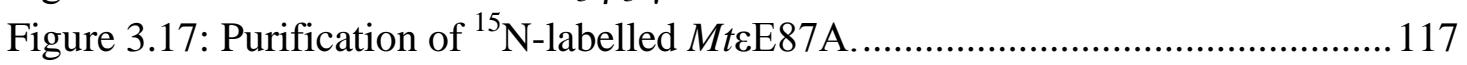

Figure 3.18. Effect of the E87A mutation $M t \varepsilon$ revealed by NMR .............................. 119

Figure 3.19: Generation of the M. smegmatis $a t p C^{\mathrm{E} 87 \mathrm{~A}}$ using recombineering .......... 121

Figure 3.20: Screening for the M. smegmatis atp $C^{\mathrm{E} 87 \mathrm{~A}}$ mutant strain ......................... 122

Figure 3.21: Growth of $M$. smegmatis $m c 155^{2}$ and $a t p C^{\mathrm{E} 87 \mathrm{~A}}$ and its susceptibility to BDQ ................................................................. 123

Figure 3.22: ATPase activity and proton pumping of the M. smegmatis IMVs .........125

Figure 3.23: ATP synthesis of the M. smegmatis IMVs........................................... 126

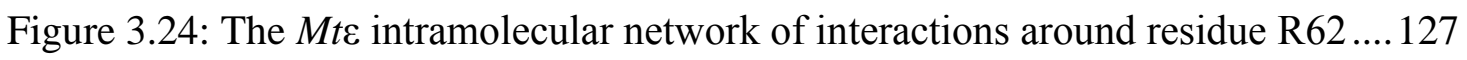

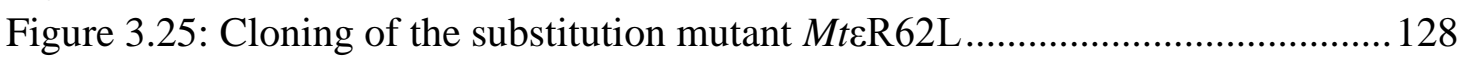

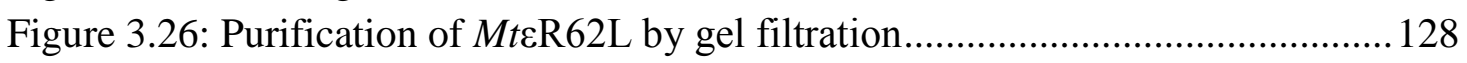

Figure 3.27: Reconstitution of $\alpha^{c h i}{ }_{3}: \beta_{3}: \gamma$ and subunit Mt\&R62L mutant ...................... 129

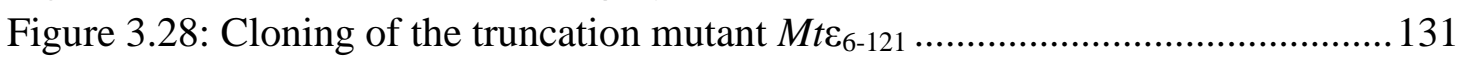

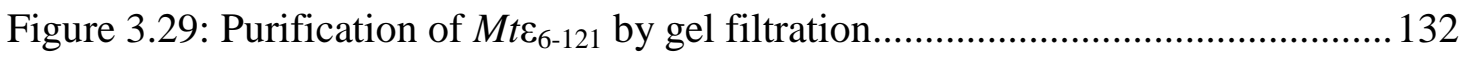

Figure 3.30: Reconstitution of $\alpha c^{h i}{ }_{3}: \beta_{3}: \gamma$ and subunit $M t \varepsilon_{6-121}$ mutant......................... 133

Figure 3.31: The model of the interacting residues in $\alpha^{c h i}{ }_{3}: \beta_{3}: \gamma: M t \varepsilon$ complex ........... 135

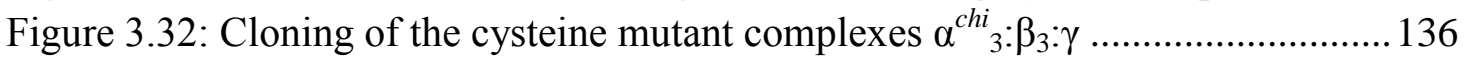

Figure 3.33: Purification of $\left(\alpha^{c h i}-\mathrm{S} 400 \mathrm{C}\right)_{3}: \beta_{3}: \gamma$ and $\left(\alpha^{c h i}-\mathrm{V} 525 \mathrm{C}\right)_{3}: \beta_{3}: \gamma \ldots \ldots \ldots \ldots \ldots \ldots . . . . . . . . .137$

Figure 3.34: Purification of $\left(\alpha^{\text {chi }}-\mathrm{S} 400 \mathrm{C}\right)_{3}: \beta_{3}: \gamma$ and $\left(\alpha^{\text {chi }}-\mathrm{V} 525 \mathrm{C}\right)_{3}: \beta_{3}: \gamma \ldots \ldots \ldots \ldots \ldots \ldots . . . . . . . .138$

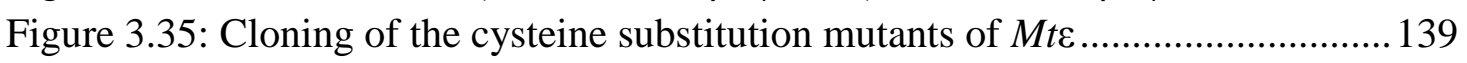

Figure 3.36: Purification of Mte mutants R113C, G118C, and D121C by gel filtration.

Figure 3.37: Redox-mediated formation of intramolecular disulfide

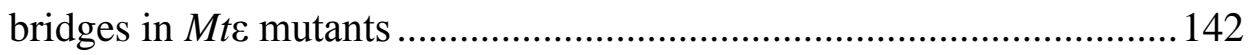

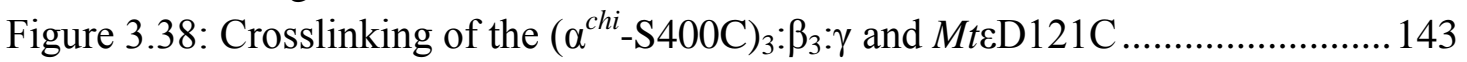

Figure 3.39: Crosslinking of the $\left(\alpha^{c h i}-\mathrm{V} 525 \mathrm{C}\right)_{3}: \beta_{3}: \gamma$ with $M t \varepsilon$ mutants

D121C, G118C and R113C

Figure 3.40: Determination of the constituent proteins of the crosslinking products. 145

Figure 3.41. Model of the $\alpha^{c h i}{ }_{3}: \beta_{3}: \gamma: M t \varepsilon E 100 \mathrm{C}$ complex used in SmFRET ................ 147

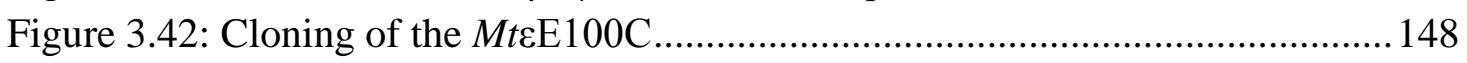

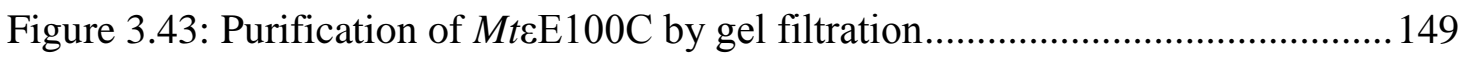

Figure 3.44: Reconstitution of $\alpha^{c h i}{ }_{3}: \beta_{3}: \gamma: M t \varepsilon E 100 C$, ATPase activity and crosslink formation

Figure 3.45: Reconstitution of the labeled $\alpha^{c h i}: \beta_{3}: \gamma$ and subunit

$M t \varepsilon E 100 \mathrm{C}$ mutant for smFRET

Figure 3.46: FRET efficiency histogram of the $\alpha^{c h i}{ }_{3}: \beta_{3}: \gamma: M t \varepsilon E 100 \mathrm{C}$ complex ............ 153

Figure 3.47: Purification of recombinant $A$. woodii $\mathrm{F}_{1} \mathrm{~F}_{\mathrm{O}}$-ATP synthase ..................... 155 
Figure 3.48: ATPase activity of the A. woodii F-ATP synthase 160

Figure 3.49: Negative stained EM of the recombinant A. woodii $\mathrm{F}_{1} \mathrm{~F}_{\mathrm{O}^{-}}$ ATP synthase 158

Figure 3.50: Restriction digestion and sequence verification of $A$. woodii $\gamma_{\Delta 195-211}$ mutant

Figure 3.51: Purification of recombinant $A$. woodii $\gamma_{\Delta 195-211}$ mutant $\mathrm{F}_{1} \mathrm{~F}_{\mathrm{O}}$-ATP synthase

Figure 3.52: ATPase activity of the $A$. woodii $\mathrm{F}_{1} \mathrm{~F}_{\mathrm{O}}$-ATP synthase 161

Figure 3.53: Effect of the $\gamma \Delta 195-211$ deletion on ATP synthesis and $\mathrm{Na}+$ transport

Figure 4.1: Surface representation of 3D reconstruction of $\alpha^{c h i}: \beta_{3}: \gamma: M t \varepsilon$ complex... 167

Figure 4.2: Synergistic effect of Mg-ADP and subunit $M t \varepsilon$ on ATP hydrolysis........ 168

Figure 4.3: Regulatory epitopes of bacterial F-ATP synthases ................................ 170

Figure 4.4: Communication of the rotational movement of $c$-ring through $M t \varepsilon \ldots . . . . .173$

Figure 4.5: Effect of W16A and E87A mutations on BDQ affinity and

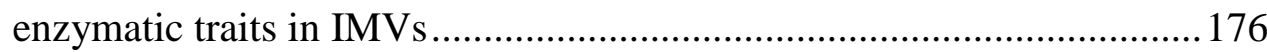

Figure 4.6: The interactions of the subunits $E c \varepsilon, G s \varepsilon$, and $M t \varepsilon$ within $\mathrm{F}_{1}$ domain.....179

Figure 4.7: 3D homology model fitting the 2D projections of NS $A$. woodii F-ATP synthase

Figure 4.8: Assembly model of $A$. woodii $\mathrm{F}_{1} \mathrm{~F}_{\mathrm{O}}$-ATP synthase showing the interactions

Figure 4.9: Interactions of the inner side of the $c$-ring in A. woodii FATP synthase

Figure 4.10: The position of the unique regulatory loops in subunit $\gamma$ 186 


\section{List of Tables}

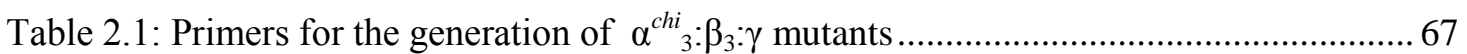

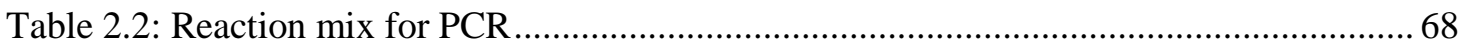

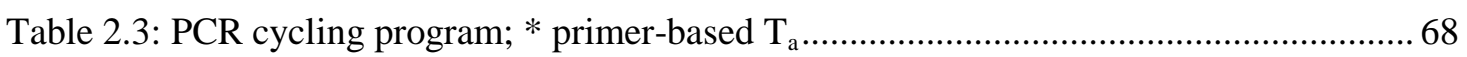

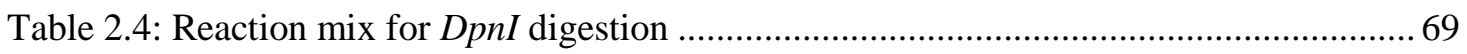

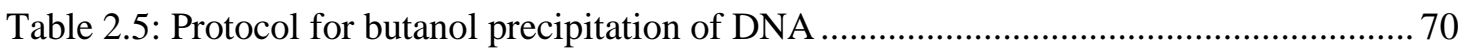

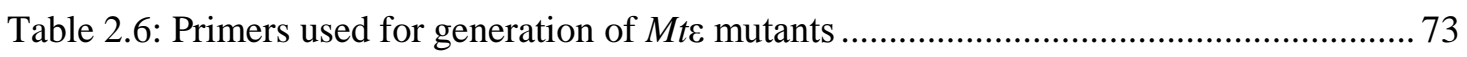

Table 2.7: Reaction mix for PCR for the generation of Mt\& mutants ...................................... 73

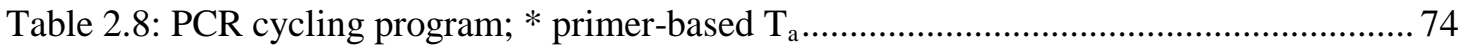

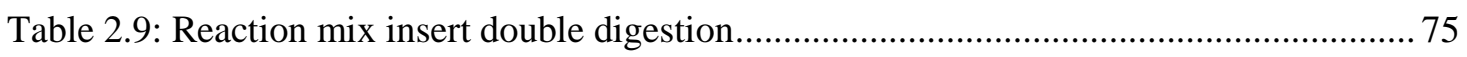

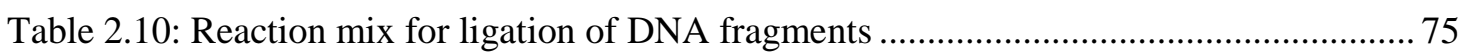

Table 2.11: Primers for the amplification of $M t \varepsilon$ mutant constructs........................................... 76

Table 2.12: Reaction mix for PCR for the generation of the $M t \varepsilon$ mutants................................ 76

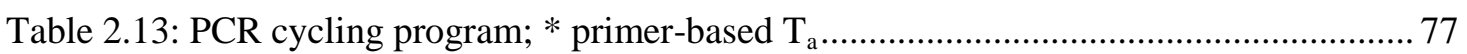

Table 2.14: Reaction mix for ligation of the MtcD121C mutant pDNA................................... 77

Table 2.15: Single strand DNA E87A oligonucleotide for recombineering ............................... 87

Table 2.16: Primers for the colony PCR screening of atp $C^{\mathrm{E} 87 \mathrm{~A}} M$. smegmatis

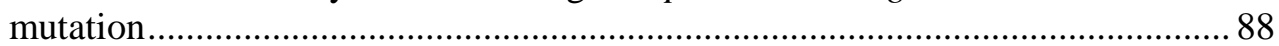

Table 2.17: Reaction mix for screening PCR of $a t p C^{\mathrm{E} 87 \mathrm{~A}}$ mutants ............................................ 88

Table 2.18: PCR program for screening PCR of $a t p C^{\mathrm{E} 87 \mathrm{~A}}$ mutants; ......................................... 88

Table 3.1: Enzymatic activities of the $\alpha^{c h i}: \beta_{3}: \gamma$ and $\alpha^{c h i}{ }_{3}: \beta_{3}: \gamma: M t \varepsilon$ complexes.......................... 105

Table 4.1: The prediction of the phosphorylation sites in subunit $\gamma$..................................... 187

Supplementary Table S1: The composition of the subunits in F-ATP synthases ....................230

Supplementary Table S2A: MALDI-TOF data of the $\alpha 1$ band of lane 2 in Figure 3.47C

Supplementary Table S2B: MALDI TOF-data of the $\alpha 2$ band of lane 2 in

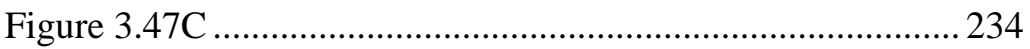

Supplementary Table S2C: MALDI-TOF data of the $\alpha 3$ band of lane 2 in

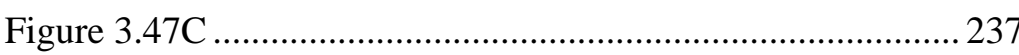

Supplementary Table S3A: MALDI-TOF data of the $110 \mathrm{kDa}$ band in Figure 3.47D ......

Supplementary Table S3B: MALDI-TOF data of the $35 \mathrm{kDa}$ band in Figure 3.47D .

Supplementary Table S3C: MALDI-TOF data of the $35 \mathrm{kDa}$ band in Figure 


\section{Summary}

F-ATP synthases are multimeric membrane-embedded proteins which can synthesize/hydrolyze ATP depending on the energy requirements of the cell. The canonical composition includes $F_{1}$ part comprised of $\alpha_{3}: \beta_{3}: \gamma: \delta: \varepsilon$ and $F_{O}$ part composed of subunits $a$ and $c$-ring attached to the dimeric peripheral stalk $b_{2}$ (1). In mycobacterial cells, ATP synthesis occurs by utilizing the proton-motive force (PMF) to drive the catalytic events in the F-ATP synthase and form ATP from ADP and $\mathrm{P}_{i}$, similarly to the mechanism found in other bacterial F-ATP synthases. In contrast, ATPase activity of $M t b$ enzyme is latent due to the action of a specific regulatory mechanism, allowing it to conserve ATP and thrive in the hypoxic environment (2). This trait gave rise to $M t b$ population establishment in energetically unfavorable conditions found in human lungs contributing to the latent TB infections (LTBI) and thus prompting a need for further structural and functional characterization of $M t \mathrm{~F}$ ATP synthase.

Previously it was established that the genetically-fused extended C-terminal domain of mycobacterial $\alpha(M t \alpha)$ reduced the ATPase activity of the complex $G$. stearothermophilus $\alpha_{3}: \beta_{3}: \gamma$ indicating its involvement in the inhibition of the ATPase activity (3). In the present study, the synergistic effect of mycobacteria-specific epitopes (MtaCTD and $M t \varepsilon)$ on inhibition of ATPase activity was revealed via the hybrid complex $\alpha^{c h i}{ }_{3}: \beta_{3}: \gamma: M t \varepsilon$ used as a model system. The complex $\alpha^{\text {chi }}{ }_{3}: \beta_{3}: \gamma: M t \varepsilon$ was visualized for the first time, using the negative-stain electron microscopy, revealing the binding of the heterologous $M t \varepsilon$ as well as the position of the $M t \alpha$-specific Cterminal epitope within the $3 \mathrm{D}$ reconstructed map (4). The interaction of these mycobacterial epitopes was described using mutagenesis and cysteine crosslinking experiments. The kinetic study of the ATPase activity advocates a strong dependence of the mycobacterial F-ATP synthase on Mte for coupling and establishment of the fully-functional complex.

The mutations in MtE were designed to dissect the role of its C-terminal domain, hinge domain and $\mathrm{N}$-terminal domain in the catalysis and stability of the reconstituted $\alpha_{3}^{c h i}: \beta_{3}: \gamma: M t \varepsilon_{(\mathrm{x})}$ complexes. The mutations $M t \varepsilon E 87 \mathrm{~A}, M t \varepsilon \mathrm{R} 62 \mathrm{~L}$, and $M t \varepsilon_{1-120}$ described the coupling and the entrapment of the inhibitory Mg-ADP, while 
the mutant $M t \varepsilon_{6-121}$ emphasized the role of $M t \varepsilon N T D$ for the protein stability in comparison to the other bacterial counterparts $(4,5)$.

In summary, the structural data obtained from NS-EM, together with the mutational and functional studies describe the importance of mycobacteria-specific

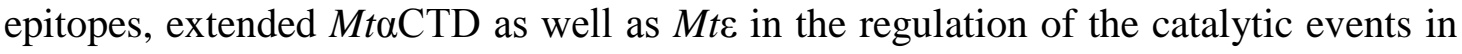
mycobacterial F-ATP synthases.

On the other side of evolution, away from the Kingdom of Bacteriae, an archaeal species Acetobacterium woodii displays unique features of its F-ATP synthase, developed in response to the environmental energy availability. The evolutionary pressure to adapt had driven the specialization of this F-ATP synthase to accommodate several structural and regulatory mechanisms specific for a particular niche. As halophilic, thermophilic, acetogenic species, A. woodii was facing hypersalinity, oxygen-deprivation, high temperatures, and high content of organic acids which all created an environment whereby energy conservation is paramount for survival.

To utilize the hypersalinity for the energy generation, A. woodii has invented a hybrid $\mathrm{Na}^{+}$-translocating $c$-ring that contains the V-ATPase-like subunit $c_{1}$ and F-ATP synthase $c_{2 / 3}$, arranged in the $c_{10}$ ring of the $c_{1}: c_{2 / 3}$ stoichiometry $1: 9(6,7)$. The $\mathrm{Na}^{+}-$ motive force operates on a similar principle found in PMF-dependant F-ATP synthases. By transversing through the $c$-ring down the chemical gradient, $\mathrm{Na}^{+}$ions affect its rotation, causing the rotation of the central stalk subunits $\gamma-\varepsilon$, which in turn imposes the structural changes in the nucleotide-binding $\alpha_{3} \beta_{3}$ catalytic hexamer and drives the ATP synthesis.

In this thesis, the entire $A$. woodii $\mathrm{F}_{1} \mathrm{~F}_{\mathrm{O}}$ ATP synthase was visualized for the first time, using NS-EM and the 2D projections were obtained pointing towards canonical subunit composition with a stoichiometry $\alpha_{3}: \beta_{3}: \gamma: \delta: \varepsilon: a: b_{2}:\left(c_{2 / 3}\right)_{9}: c_{1}$. A unique loop (195 TSGKEEKTEETKSK ${ }_{211}$ ) in the A. woodii subunit $\gamma$ was unraveled, and its role was examined by generating the deletion mutant $\gamma_{\Delta 195-211}$ whose ATP synthesis activity was entirely abolished coupled with diminished $\mathrm{Na}^{+}$-translocation. $\mathrm{T}$, the present thesis has established the structural basis of regulation of $\mathrm{Na}^{+}$-translocation, ATP synthesis, and revealed the regulatory role of the $A$. woodii unique $\gamma$-loop. 


\section{Introduction}




\subsection{Overview of the Mycobacterium tuberculosis}

\subsubsection{Introduction to tuberculosis}

Tuberculosis (TB) is described in the medical dictionary as a respiratory disease caused by pathogenic bacteria Mycobacterium tuberculosis complex, further referred to as $M t b$ (8). At its first stage, infected individuals do not exhibit any symptoms, classifying this stage as the latent tuberculosis infection (LTBI) (9). After the disease progresses to active tuberculosis, symptoms such as fever, chronic coughing, sputum blood, weight loss intensify, all caused by the increased bacterial load in the lungs. As the lungs are the primary compromised tissue, the pathogen can spread through aerosol caused by speaking, sneezing, and even breathing. High bacterial load in lungs is characteristic in the final stage, and half of the infected individuals with active tuberculosis do not survive. These facts make tuberculosis, apart from HIV, one of the most devastating diseases of humankind, causing around 9 million new infections per annum and 2 million deaths (10). Current statistics estimate 2.3 billion individuals with LTBI that act as a reservoir of this pathogen $(9,11)$.

Until today, many TB cases have emerged predominantly in India, Indonesia, China, Myanmar and as recently reported Ethiopia (12), and according to the statistics, these countries contribute to $72 \%$ of the global TB burden. The estimates from 2014 showcase a staggering number of 4.1 million unreported cases globally, of which these countries represent $79 \%$. These figures also include poorly treated and non-compliant patients that cause the relapse of $\mathrm{TB}$ and the emergence of the antibiotic resistance. The circumstances leading to such progression are predominantly economic and educational and are often rooted in poor diagnostics/treatment due to the lack of adequate medical facilities.

As a consequence, the strains resistant to rifampicin and isoniazid emerge, causing the occurrence of multi-drug resistant TB (MDR-TB), that effectively presents the root of the problem for future treatments $(13,14)$. Due to the patient noncompliance and unregulated/inadequate care, the MDR-TB strains develop further resistance, and their treatment requires the usage of the fluoroquinolones and aminoglycosides, which are in general less specific and less cost-effective (15). The effective MDR-TB treatment can take up to two years in comparison to the six-monthlong treatment of non-resistant TB with the first-line regiment antibiotics. Reports also emphasize that the improper regiments of these drugs increase the incidence of gene mutations whose product is the target of these antibiotics (15). As a result, new drugs 
such as injectable kapreomycin, kanamycin, and amikacin are included in the MDRTB treatment. The further development of the resistance to these drugs leads to the emergence of the extremely resistant strains (XDR-TB).

Many successful attempts to eradicate the actively-replicating TB have been reported and well documented, however, till this day, tuberculosis was avoiding the eradication through the "escape" into the persistence that manifests as a latent TB infection (LTBI). Most available antibiotics known until today, target the activelyreplicating bacteria, while the persister cells are less susceptible to the same agent due to the metabolic and morphological adaptations $(16,17)$. The first-line antibiotics such as isoniazid, ethambutol, together with the MDR-TB agent, ethionamide, target and arrest the biosynthesis of the cell wall during the division by interfering with the synthesis of the mycolic acids and arabinogalactan. This treatment is often used in combination with rifampicin (15), a potent inhibitor of RNA synthesis in cases of nonresistant TB. Different strategies include DNA synthesis inhibitors (fluoroquinolones) and clofazimine with its pleiotropic effect on the growth and potassium channels (15). The pathogen protein expression is usually targeted via ribosome interference using streptomycin, kapreomycin, amikacin, kanamycin, and drugs like linezolid in MDRTB cases where the resistance to rifampicin and isoniazid has occurred. All these drugs target cellular functions utilized during the active growth phase and replication, while the persisters are virtually unaffected with this treatment and present a reservoir of reemergence of the active TB upon the occurrence of immunodeficiency.

Recently discovered bedaquiline (BDQ), has shown high sterilizing activity in both actively-replicating and non-replicating persister cells (18-20). These properties have prompted a further investigation into its mechanism of action, resulting in the discovery of its target, a mycobacterial F-ATP synthase, the enzyme which synthesizes the bulk of the cellular ATP and provides the necessary energy to maintain the homeostasis during the infection. The potency of BDQ increases when used in combination with pyrazinamide (21), and even so, after the prolonged use, certain strains acquired the resistance (22). These outcomes emphasized the need to look deeper into the mycobacterial adaptive capacity and mode of drug actions in order to find the endogenous mechanism that will selectively incapacitate the $M t b$ upon a new, potential drug administration. In this context, the oxidative phosphorylation (OxPhos) pathway, which was long considered unsuitable due to its conserved nature between pro- and eukaryotes, has now been re-validated as a suitable target space to deliver 
antimicrobials (23). However, very few components of the mycobacterial electron transport chain (ETC) have been fully characterized, which presents a challenge that needs full attention. In order to understand the extent of the drug action, it is a prerequisite to look into the mechanism of infection and host-pathogen interaction, identify the critical points in its pathogenicity and immune response-mediated metabolic shift that contributes to the emergence of resistance to antimicrobials.

\subsubsection{Overview of the genera of mycobacteriales}

The prokaryotes have been described in the early literature as simple organisms owing it to their unicellular organization and lack of complex behaviors. In the $19^{\text {th }}$ and early $20^{\text {th }}$ century, the identification of the genera and species within was based mostly on the morphological properties of the colonies of the isolated microorganism (24). Their physical properties such as color, shape, and size of colonies, the speed of growth and nutritional requirements were recorded, which gave rise to a classification system that encompassed what we today know as saprophytes. The sophistication of the microscopy and chemical diagnostic tools like selective staining enabled more reliable classification and characterization of pathogens such as $M t b$, which was sorted in the Order of Actinobacteria. Mycobacterial species adopt a range of niches; some mycobacteria live in open environments adapting to the exclusive saprophytic lifestyle like $M$. smegmatis, while a predominantly saprophytic $M$. marinum (25) resorts to opportunistic infections displaying a tendency to infect humans. The obligate human pathogen $M t b$ displays behavior solely dependent on the human host and a lifestyle inseparable from the invasion of human lungs. Apart from $M t b$, there are other pathogenic species like $M$. bovis, a vector of tuberculosis capable of infecting bovine species (26,27), M. leprae, a causative agent of leprosy (28) and M. ulcerans, a causative agent of Buruli ulcer (29-31). M. bovis and M. leprae have been proven valuable for the research on pulmonary tuberculosis as their contagious capacity is lower than the one of $M t b$.

\subsubsection{Etiology of TB}

Numerous studies have shown that $M t b$ has a complicated relationship with the host (32-34). The healthy individuals come in contact with the patients that have an active TB form able to multiply and spread via aerosols. Upon the infection through the airways, it stations itself in the lungs parenchyma where it is actively replicating 
until it is being engaged by the components of host immune defense, predominantly macrophages (Figure 1.1) (32,33). The presence of pathogen promotes the activation of the macrophage to search and engulf the bacilli internalizing them via the phagocytosis and limiting their further spreading. Upon phagocytosis, the macrophage surmounts a physiological response; the phagosome compartment undergoes significant acidification reflected in the $\mathrm{pH}$ drop, typically down to 4.5-5, that activates the proteolytic and lipolytic enzymes. Lipolytic enzymes disrupt the pathogen cell wall/membrane while the proteolysis of the pathogen proteins provides the epitopes for presentation on the surface of the antigen-presenting cells (APC) such as macrophages and dendritic cells, which establishes the adaptive immune response (3436). However, $M t b$ is capable of attenuating the acidification through the secretion of urease that produces neutralizing ammonia from urea $(37,38)$ and buffers the entire compartment incapacitating the host enzymes to an extent.

Furthermore, macrophages surmount the "oxidative burst" (39) within the phagosome by secreting the high amounts of reactive oxygen species (ROS) such as superoxide anion radical $\left(\mathrm{O}_{2}{ }^{-}\right)$and hydrogen peroxide $\left(\mathrm{H}_{2} \mathrm{O}_{2}\right)$ as well as peroxy-nitrate (ONOO), a reactive nitrogen species (RNS) that can break down to $\mathrm{O}_{2}{ }^{--}$and nitric oxide (NO) (40-42), a potent signalling molecule that mediates a plethora of physiological roles (43-48). During infection, ROS and RNS can damage pathogen DNA, oxidize its' the lipids and nitrosylate the tyrosines of the proteins, affecting the homeostasis of the pathogen cell (49). Mtb copes with the oxidative burst by utilizing its' various anti-oxidative enzymatic systems to neutralize these reactive species $(50,51)$. Namely, $M t b$ expresses the catalase, a product of the gene $c a t G$ decomposing $\mathrm{H}_{2} \mathrm{O}_{2}$ to $\mathrm{H}_{2} \mathrm{O}$ and $\mathrm{O}_{2}$, as well as the genes of thioredoxin cluster (thioredoxin reductase, $\operatorname{tr} x B 2$ and thioredoxin, $\operatorname{thi} X, \operatorname{tr} x B 1$, and $\operatorname{tr} x C$ ) mitigating the response to $\mathrm{H}_{2} \mathrm{O}_{2}$ and NO (50). 


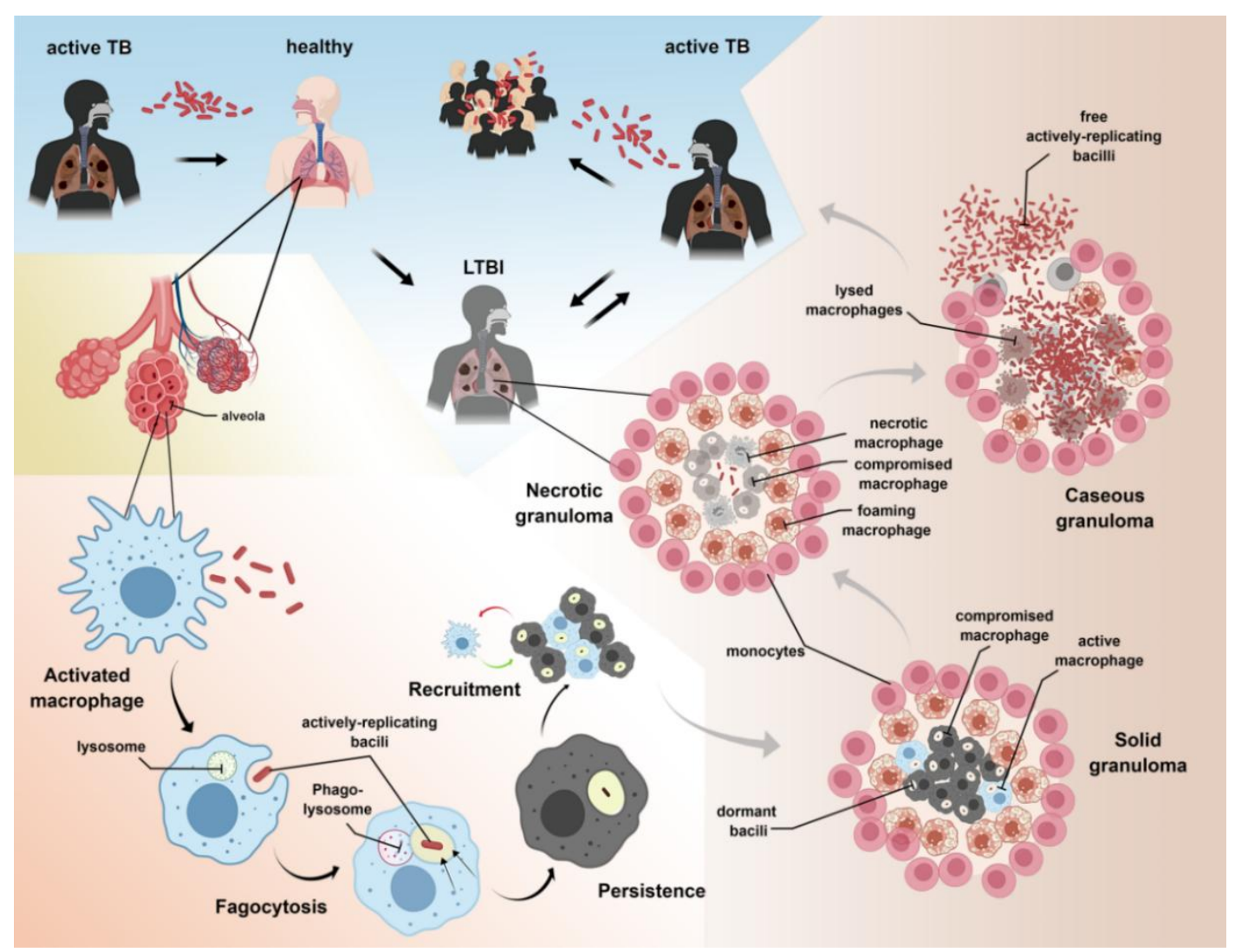

Figure 1.1: Transmission and pathology of $\boldsymbol{M t b}$. Inhalation of the pathogen-bearing aerosol causes the primary infection in healthy individuals. The infection induces the pro-inflammatory response of lung macrophages; activated macrophages internalize the bacilli via phagocytosis into their phagosomal compartment. Inside, upon the fusion of lysosome and early phagosome, the $\mathrm{pH}$ drops and the intra-phago-lysosomal concentration of ROS and RNS is increased. These environmental factors induce a physiological adaptation of $M t b$ from actively replicating to dormant cells, exhibiting a non-replicating small cell morphotype (persister phenotype). The compromised macrophages, unable to eliminate the bacilli, recruit additional activated macrophages as a line of defence and a solid granuloma is formed to contain the pathogen. The hallmark of mycobacterial infection is the presence of foamy macrophages that harbor lipid droplets in the cytoplasm to provide a substrate for the synthesis of leukotrienes and prostaglandins, immuno-modulators. Due to prolonged persistence, pathogen utilizes the macrophage-derived lipids, induces the necrotic macrophage death that resuscitates bacilli to initiate the active division (necrotic granuloma). Lysis of the incapacitated macrophage population precedes the formation of a caseous granuloma; the bacilli exit the granuloma causing the onset of active TB. At this stage, TB is transmitted from one individual to another, spreading further through the human population.

These mycobacterial defences cause the progression of TB that includes failure of the macrophage to clear the pathogen from the phagosome, which in turn starts to exhaust the macrophagal capacity for immune response and gives rise to persistence. This stage of the TB is tightly associated with the metabolic shift of $M t b$ and its escape to the dormant stage (52-54). The escape of mycobacteria into the dormancy forces a macrophage to surmount an enhanced immune response, via complex signaling 
pathways, by recruiting the $\mathrm{T}$ - and B-cells, other macrophages to the site of infection, creating a containment known as granuloma $(55,56)$.

The first stage solid granulomas are well-organized on the cellular level with a large number of the healthy macrophages still containing the pathogen within its phagosome without killing it. The initial immune response induces the fusion of a certain number of macrophages to form a foamy macrophage phenotype (57) that accumulates lipids in the lipid droplets maintained in the cytoplasm. Alternatively, this phenomenon is mediated via Mtb lipids that prevent the trafficking of the various forms of low-density lipoproteins (LDL) (55,58-60). During the infection, the lipid droplets act as a locally available substrate for production of prostaglandin $E_{2}$ and leukotrienes, paracrine hormones required for the maintenance of the immune response via macrophage recruitment (61-64). Prolonged exposure to the phagosomal environment induces the $M t b$ dormancy, a small cell morphotype (SCM) characterized by a series of metabolic and morphological adaptations.

The dormant $M t b$ cells (SCM) undergo a multi-phase process of septation of their cytoplasm upon nutrient starvation that occurs in the granuloma. In the first phase, the septated cells appear to have a multi-nuclear organization that finally progresses to a single mono-nuclear cell of a significantly smaller size (65). This phenomenon allows $M t b$ to control the cell wall thickening process (66-68) that plays a protective role and contributes to the extreme tolerance for the detrimental effects of the granuloma environment.

$M t b$ maintains its energy homeostasis by tuning the expression of the electrontransport chain (ETC) components that provide the proton-motive force and support ATP production (69). The central carbon assimilation pathway is shifted from the utilization of carbohydrates towards the use of the host-derived lipids, abundantly found in cytoplasmic lipid droplets of the foamy macrophages. The cessation of the anabolic reactions decreases the demand for the ATP and tolerates the establishment of lower proton-motive force required for survival of $M t b$ and persistence during the hypoxic conditions (70). All these adaptations come at the expense of the active $M t b$ cell growth and multiplication as a strategy to increase single-cell survivability and are beneficial for the pathogen from the aspect of its bioenergetic requirements to sustain life in limiting nutrient and oxygen conditions typical for the solid granuloma (70).

Furthermore, Mtb manages to evade the phagosome-mediated killing in the compromised macrophage inducing the necrotic macrophage cell death $(71,72)$. On the 
tissue scale, this is observed as an advanced stage of granuloma referred to as necrotic granuloma. The necrotic granuloma is a well-organized structure, which progressively fills up with solid cell debris originating from necrotic macrophages. Due to its relatively intact outer layer, the necrotic granuloma is still able to maintain hypoxic conditions and successfully limit the spread of the bacilli from its center to the other parts of the lungs.

However, further progression of the macrophage necrosis causes liquefaction of the center of granuloma, preceding to the restoration of the normal oxygen level. Together with the readily available nutrition from the necrotic macrophages, the normoxia rescues $M t b$ from its persister phenotype to metabolically fully active form $(73,74)$. The pathogen is now able to establish an actively-multiplying population, further damaging the granuloma until its integrity is entirely compromised. This final stage is known as caseous granuloma, and its further destructuring leads to the spread of the infection to other parts of the lungs, other tissues, and other individuals as well (Figure 1.1).

In many active TB cases, all stages of granuloma are observed simultaneously pointing out that the pathogen has a complex relationship with the host and can persist in a range of suboptimal and detrimental conditions for its growth. This persistencereactivation cycle enabled the $M t b$ to spread through the population and infect roughly $1 / 3$ of the world's population (11). This immense potential to spread uncontrollably, acquiring the resistance to the first- and second-line antibiotics has expedited the research to discover the new class of therapeutic agents that could cope with the Mtb's unmatched metabolic plasticity and emerging resistance.

As pointed out earlier, the discovery of the potent antibiotic, BDQ shifted the attention from targeting the DNA replication, protein and cell wall synthesis of Mtb towards targeting of its respirasome (ETC) that provides the energy in both life cycle stages, actively-dividing and dormant (persister) cells.

The canonical bacteriostatic and bactericidal agents such as isoniazid and rifampicin have already been in use for many years, in various administration regiments, causing the emergence of the MDR and XDR-TB. This unusually high microbial antibiotic resistance has warranted the investigation into the alternate druggable metabolic space, ultimately leading to the discovery and approval of BDQ by USA Food and Drug Agency (FDA) for use in the treatment of TB in humans (75). $\mathrm{BDQ}$, as a diarylquinoline drug surprisingly targets specifically mycobacterial F-ATP 
synthase $(18,76)$. Even though it has long been a theme of the scientific debates whether the mycobacterial ETC could be selectively targeted due to the high number of homologous complexes between the human mitochondria and $M t b$, the discovery of BDQ has reinforced the search for even more drugs that could selectively incapacitate the Mtb ETC. However, the difficulties faced on this frontline are bound to the fact that the mycobacterial ETC is far from understood in its entirety owing to the striking fact that $M t b$ thrives as an actively-replicating bacillus as well as metabolically downshifted persister under various immune-physiological stresses. The system that provides this advantage is a highly adaptive ETC, which will be considered in the following chapter.

\subsubsection{The composition of the ETC}

In $M t b, \mathrm{NADH}$ produced in the glycolysis and Krebs cycle is oxidized at the NADH-dehydrogenase of the ETC and through a series of redox events, the electrons are conveyed by the menaquinol/menaquinone pool to the terminal acceptor, molecular oxygen $\left(\mathrm{O}_{2}\right)$, thus forming the ETC. Simultaneously, the protons are being translocated across the plasma membrane, generating the proton gradient also known as a proton-motive force (PMF) which is being utilized by the F-ATP synthase, to produce ATP, the energy currency of the cell. $M t b$ exhibits various adaptations of the ETC in response to its environmental stimuli, and the organization of the ETC is highly variable in aerobic and hypoxic conditions.

During its' aerobic growth, NADH is oxidized by the ETC complex I, NADH dehydrogenase (Figure 1.2). The $M t b$ genome encodes two forms of complex I, namely NADH dehydrogenase 1 (NDH-1) and NDH-2. NDH-1 is a multi-subunit membrane-embedded respiratory complex with a function of oxidizing the NADH to $\mathrm{NAD}^{+}$, harvesting electrons in the process and providing $\mathrm{NAD}^{+}$for the next rounds of glycolysis and Krebs cycle. Furthermore, it represents the entry point of the electrons into the menaquinol/menaquinone pool (77) which are subsequently transferred to the ETC complex II, an $\mathrm{FADH}_{2}$-dependant succinate dehydrogenase (SDH). In $M t b$, deletion of the entire NDH-I operon (nuoABCDEFGHIJKLMN) does not seem to affect the redox homeostasis, thus rendering the NDH-1 dispensable for growth (70). Mutational studies involving the deletion of the nuoG, a subunit of the NDH-1 have shown that it plays a role in the inhibition of the macrophage apoptosis while it does not affect $M t b$ growth in vitro. Additionally, the M. smegmatis genome also codes for 
the nuоA-N, but the enzymatic assays did not detect activity advocating that the NDH1 is not possibly constitutively expressed (78).

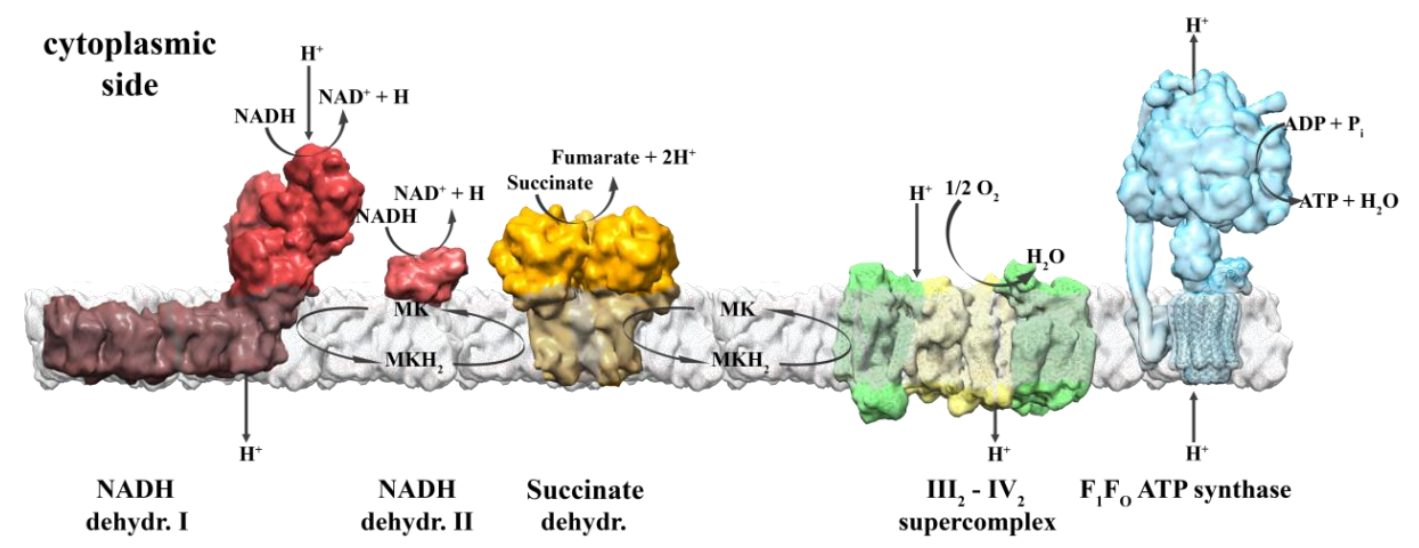

Figure 1.2: Organisation of the ETC from $M t b$ in aerobic conditions. Schematic diagram outlining the ETC components and F-ATP synthase of $M t b$ growing under aerobic conditions.

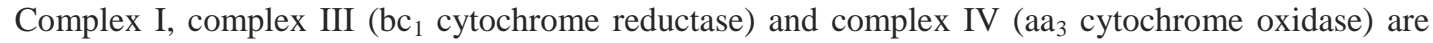
upregulated. These complexes translocate protons, conserving the energy of the oxidized NADH. Complex II (succinate dehydrogenase) does not translocate protons, while it reduces succinate to fumarate. Proton non-translocating NADH dehydrogenase II is contributing to the NADH/NAD ${ }^{+}$ homeostasis. The proton gradient generated by the ETC is utilized by F-ATP synthase to synthesize ATP. $\mathrm{MK}=$ menaquinone, $\mathrm{MKH}_{2}=$ menaquinol. The figure made in reflection to (79).

The presented evidence suggests that $M t b$ utilizes an additional NADH: oxidoreductase as a primary enzyme to oxidize NADH and to provide the flux of electrons to the ETC. The genes $n d h$ and $n d h A$ are found to be coding for two copies of a single-subunit monotopic protein, NDH-2 which does not conserve the energy by translocating the protons across the membrane. NDH-2 can directly transfer the harvested electrons onto the menaquinone/menaquinol pool supporting the growth in both, aerobic and hypoxic conditions (Figure 1.3). The growing body of evidence points out that the deletion of the $n d h A$ gene does not cause any phenotype distinguishable from the WT, while the inability to generate a $n d h$ deletion strain unequivocally advocates its essentiality in the survival of $M t b(37,80)$. Further experiments, including the transcriptional screening, indicate that $n d h$ undergoes different regulatory patterns during the aerobic growth and infection model, underpinning the importance of NDH-2 in adaptation to hypoxic conditions (81).

The next in line of the respiratory complexes is the succinate dehydrogenase (SDH), an $\mathrm{FADH}_{2}$-dependent multi-subunit (82) membrane-anchored enzyme, homologous to the mitochondrial ETC complex II. The SDH mediates the reduction of the succinate to fumarate obtaining an electron that is subsequently fuelled into the menaquinone/menaquinol $\left(\mathrm{MQ} / \mathrm{MQH}_{2}\right)$ pool without translocating the proton. 


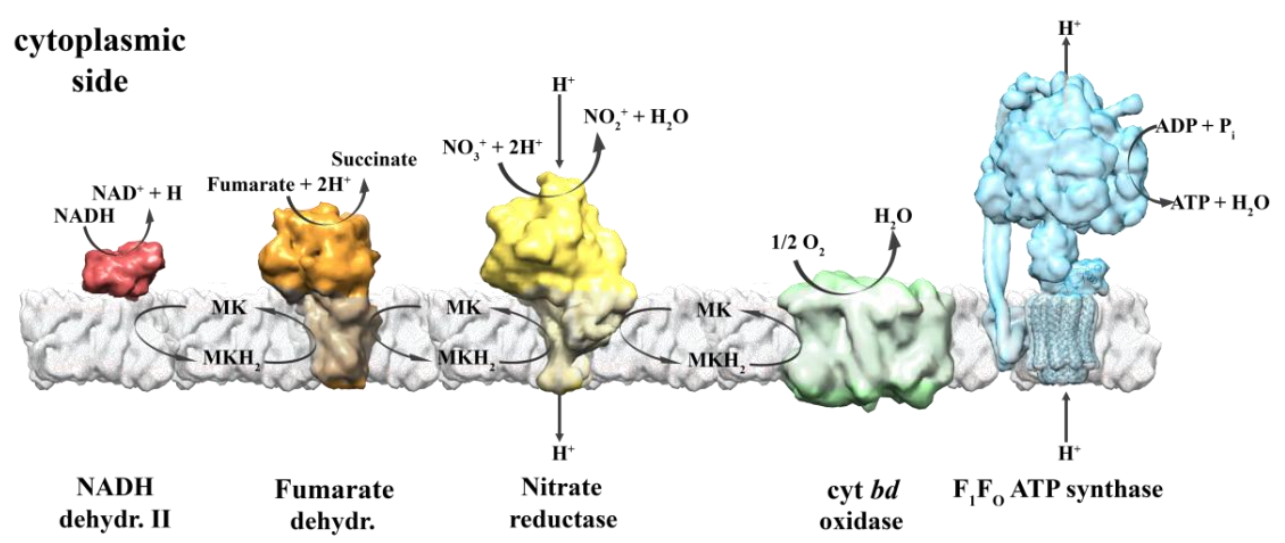

Figure 1.3: Organisation of the ETC from $M t b$ in hypoxic conditions. Schematic diagram outlining the ETC components and F-ATP synthase of Mtb growing under hypoxic conditions. The

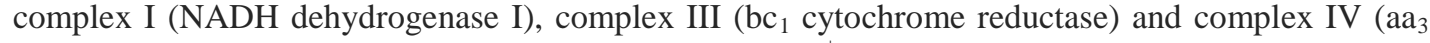
cytochrome oxidase) are downregulated. NADH/NAD ${ }^{+}$homeostasis is maintained by NADH dehydrogenase II. The $\mathrm{bc}_{1}$ cytochrome reductase and $\mathrm{aa}_{3}$ cytochrome oxidase are replaced by proton non-translocating cytochrome bd oxidase whose affinity for $\mathrm{O}_{2}$ is higher. Complex II (succinate dehydrogenase) operates in reverse, as a fumarate reductase. Upregulation of nitrate reductase is a hallmark of the growth in the hypoxic conditions. Proton gradient generated by the ETC is utilized by $\mathrm{F}$ - ATP synthase to synthesize ATP. $\mathrm{MK}=$ menaquinone, $\mathrm{MKH}_{2}=$ menaquinol. The figure made in reflection to (79).

The mycobacterial genes $S d h 1$ and $S d h 2$ encode two or three SDH isoforms $(83,84)$ with variable substrate affinity and reductive potential. In principle, the expression of the multiple enzyme forms with overlapping/similar roles could prove to be energetically inefficient and detrimental for the cell facing the nutrient/oxygen starvation, however, in mycobacteria, SDH seems to have a pivotal role in adaptation to hypoxic conditions by promoting the persistence. The mutational studies including the transposon mutagenesis confirmed the essentiality of the SDH for the energy homeostasis in $M t b$ which undoubtedly stems from the fact that it plays a role in the central carbon metabolism (Krebs cycle) as well as an electron donor to the ETC. The transposon-mediated deletion of $S d h 1(\Delta s d h 1)$ in $M$. smegmatis did not cause a growth phenotype when cultivated on a range of concentrations of succinate/fumarate due to the induced overexpression of the $s d h 2$ genes. The overexpression of $S d h 2$ acts as a compensatory mechanism indicating that $S d h l$ is non-essential for survival under both normoxic and hypoxic conditions. To the contrary of this observation, the $\Delta s d h 2$ mutant was not obtained when a single copy of the $s h d 2$ operon was present in the genome. However, the introduction of the additional shd2 operon allowed a recombineering event to occur and delete the $s d h 2$ operon from its native position pointing out the fact that a copy of the $s d h 2$ is essential for survival (83). 
Furthermore, the investigations also showed that in fact, the SDH variants respond differently to the environmental queues and that their expression pattern varies in response to the nutrient/oxygen availability (85). The upregulation of $S d h 2$ during the hypoxic conditions brings several advantages to the mycobacterial cell; it allows the flux of metabolites from the TCA eliminating the need for usage of NDH-1 (nuoABCDEFGHILMN) which is the primary source of ROS and saturates the $\mathrm{MQH}_{2}$ pool which in turn fuels the electrons in the $c y t b c_{1}-a a_{3}$ super complex. Moreover, the recently acquired evidence also suggests that in virtually anoxic conditions, succinate can be secreted from the Mtb to maintain the PMF allowing the cells to persist despite the lack of the terminal electron acceptor, oxygen, further strengthening the role of $\mathrm{SDH}$ in the maintenance of the PMF and redox homeostasis during adaptation to hypoxia.

Molecular oxygen $\left(\mathrm{O}_{2}\right)$ represents the undisputed molecule that acts as a terminal recipient of the electrons coming from the $\mathrm{MQ} / \mathrm{MQH}_{2}$ pool enabling the continuous flux of metabolites through the coupled reactions within the ETC as well as the generation of PMF. In mycobacteria, genetic screening identified two significant branches with this function. The primarily identified was cytochrome $c$ super complex that consists of two coupled enzymes, namely cytochrome c oxidoreductase, $\left(c y t-b c_{1}\right.$ complex) and cytochrome c oxidase $\left(c y t-a a_{3}\right)(86)$, while alternate electron route is characterized as a cytochrome $b d$.

The $c y t-b c_{1} a a_{3}$ super complex, a functional equivalent to the mitochondrial complexes III and IV, is a membrane-embedded multisubunit enzyme system encoded by the $q \operatorname{cr} C A B$ and $\operatorname{ctaBCDE}$ operons, respectively. Under typical aerobic conditions, the mycobacterial $c y t-b c_{1}$ accepts the electrons from the $\mathrm{MQH}_{2}$ and transfers it to the $c y t-a a_{3}$, which in turn reduces the molecular oxygen to $\mathrm{H}_{2} \mathrm{O}$. This event is coupled to the translocation of the protons, generating the PMF. Multiple attempts have been made to genetically disable the $c y t-b c_{1:} a a_{3}$ supercomplex in $M t b$ by deleting the qcrCAB but have yielded no viable strains, pointing out towards its absolute essentiality in cellular respiration under low oxygen tensions which $M t b$ encounters during the infection and persistence in macrophages. Even though annotated as indispensable in $M t b$, the $q c r C A B$ operon could, however, be recombined out from $M$. smegmatis genome leading to the severe growth impairments and the upregulation of $c y t-b d$ as a part of the compensatory mechanism (86). The observed differences between $M t b$ and $M$. smegmatis essentiality of the super complex III-IV, apart from its 
high bioenergetic efficacy, could be rooted in the recently discovered association with the superoxide dismutase (SOD). Mycobacterial SOD is closely associated with super complex III-IV, performing the canonical role of scavenging the ROS produced at its active centers (87) during the aerobic growth. The structural data obtained by cryoelectron microscopy (cryo-EM) revealed unprecedented details of the architectural organization of the super complex which forms a dimer of complex $\mathrm{III}_{2}$ flanked by two complexes IV and the SOD bound tightly to the $q \operatorname{crC}$ subunit of complex III (88).

In the hypoxic conditions, like the one specific for the macrophages during the persistence, $M t b$ strongly downregulates the genes of $c y t-b c_{1:} a a_{3}$ super complex due to the lowered requirements for the PMF and ATP generation (89). As an alternative electron sink, $c y t$-bd menaquinone oxidase genes are being upregulated to enable the unobstructed membrane electron flux by transferring the electrons from menaquinone/ol pool to $\mathrm{O}_{2}$ without coupling this transfer to the proton translocation through the membrane (90). Until today its role in mycobacterial bioenergetics remains unclear. The studies performed on the $M$. smegmatis show that the response to hypoxia involves upregulation of the $c y d A B D C$ genes encoding the $c y t$-bd oxidase to match the $\mathrm{O}_{2}$ supply (91).

Due to the lack of the structural and biochemical data, the mycobacterial $c y t-b d$ is compared to well-studied E. coli homolog (92). In E. coli genome, cyt-bd is encoded by two genomically distantly separated operons, $c y d A B$, and $c y d D C$, each with its distinct role (93). The $c y d A B$ encodes haem-containing $c y t$-bd oxidase (94) which facilitates respiratory electron flow during hypoxic conditions. The $c y d D C$ operon encodes heterodimeric membrane complex that functions as an ABC-type transporter involved in translocation of the haems required for assembly of all cytochromes (9496) as well as mediates the export of redox-active glutathione and cysteine which play a role in infection stage (97). Mutational disruption of any of these operons generated overlapping but distinct phenotypes (93).

In contrast to $E$. coli, in the $M t b$ genome, $c y d A B$ and $c y d D C$ are clustered into two adjacent operons, indicating their tighter functional correlation (98). Genetic deletion of $c y d A B$ ( $\triangle c y d A$ and $\triangle c y d B$ ) caused hypersensitivity of $M t b$ to Q203, a potent imidazopyridine that blocks electron transport via $c y t-b c_{1}$ (99) indicating the necessity of $c y t A B$ for electron transport which is also reported during $M t b$ hypoxia challenge. This phenotype was indistinguishable from the $\triangle c y d D C$ deletion 
demarcating the fact that the $\mathrm{ABC}$-transporter encoded by $c y d D C$ genes is required for assembly and function of the entire $c y t-b d$ oxidase.

These potent in vitro effects are also reflected in the persistence of bacilli reported in the studies on the in vivo mouse infection model (99). A 4-week-long Q203 treatment of the $\triangle c y d A B$ mutant caused a drop of bacterial counts below the limit of detection, while the $\triangle c y d A B c o m p l$ strain has elicited just a minor disadvantage in comparison to the WT under the hypoxic conditions (99). These facts point out to the essentiality of $c y t-b d$ as a terminal oxidase during the Q203 chemical inhibition of $c y t$ $b c_{1}: a a_{3}$ and its hypoxia-induced downregulation which both mimic the physiological state of persister $M t b$ cells found in microaerophilic conditions of the infected mouse lungs.

Interestingly, an additional study demonstrated a strong dependence of the persister impairment with the mutation introduced in the cydC gene. Namely, the terminal position of $c y d C$ in the $c y d A B D C$ (cyt- $b d$ ) operon allowed the transposonmediated insertive mutagenesis that disrupted the expression/structure of the subunit of $\mathrm{ABC}$ transporter involved in the cyt- $b d$ assembly. While the WT and the mutant displayed similar growth behavior in the untreated mice lungs, the treatment of mice with INH was able to significantly reduce bacterial counts of the $c y d C$ mutant strain in comparison to that of the WT (100). This persistence failure of the mutant strain could be attributed to the inactivation of the $\mathrm{ABC}$ transporter that in turn caused aberrant INH export, as well as it could be established due to the polar effect of the mutation on cyt- $b d$ oxidase assembly/function. Whichever is the case, it was not revealed during this investigation.

Another hampering factor is the histopathological organization of the tissue; $\triangle c y d A B$ mutant hypersensitivity to $\mathrm{Q} 203$ and hypoxia, and $c y d C$ mutant failure to persist during the INH treatment are established in mice. Nonetheless, this cannot be directly extrapolated to humans due to the essential differences between mouse and human lungs in the immune response to the $M t b$ infection. The mouse lung macrophages do not form typical granulomas, which in case of human lungs, exert an adverse oxidative and nitrosative pressure on the $c y t$-bd (101), and thus all the effects described here must be considered with reserve. The evidence advocating for the importance of $c y t-b d$ are numerous; the adaptation to hypoxic conditions undisputedly requires upregulation of $(c y d A B D C)$ cyt- $b d$, but the exact mechanism of its contribution and its impact on the $M t b$ physiology is not entirely understood. 
In addition to the $\mathrm{O}_{2}$ as a terminal electron receptor from the ETC in low oxygen tensions, there are ongoing investigations to address a question of an alternate electron acceptor during hypoxia. As these conditions could often arise in the advanced granulomas, it is not excluded that $M t b$ can utilize other readily available molecules as a respiratory substrate. Namely, human macrophages display an adverse oxidative burst due to $M t b$ persistence, secreting high concentrations of ROS and RNS, predominantly peroxy-nitrate (ONOO) (102). Peroxy-nitrate is formed by the generation of equimolar amounts of superoxide anion radical produced by macrophagal NADPH oxidase, NOX and nitric oxide, NO produced by macrophagal inducible nitric oxide synthase, iNOS. Even though NO exhibits beneficial effects acting as a potent modulator of numerous physiological parameters, it also serves as a part of the macrophage arsenal to eradicate the infection. Strikingly, Mtb has evolved a versatile system to, not only combat the oxidative burst but to utilize the nitrates obtained from decomposition of peroxy-nitrate as a respiratory substrate (103).

During the persistence, $M t b$ utilizes the truncated form of the hemoglobin as a secretory nitric oxide dioxygenase $(\mathrm{HbN})(104)$ to convert macrophage-derived NO to nitrate $\left(\mathrm{NO}_{3}{ }^{-}\right)$. The $\mathrm{NO}_{3}{ }^{-}$ions are being actively transported into the $M t b$ cell via the transporter demarcated as narK2 to be reduced to nitrite $\left(\mathrm{NO}_{2}{ }^{-}\right)$by the membranebound nitrate reductase (narGHIJ) as a part of ETC. The NADH-dependent nitrite reductase demarcated as $\operatorname{nir} D B$ further detoxifies nitrite by converting it to ammonia $\left(\mathrm{NH}_{3}\right)$. The hallmark of the persistence of $M t b$ is not, however, the upregulation of the reductases (narGHJI and nirDB), but the narK2 supporting the transport of the RNS into the $M t b$ cells which provides an alternate respiratory electron acceptor under hypoxia (105-107).

Other hypoxia-induced alterations that have been reported include the upregulation of the enzymes of the reductive branch of the Krebs cycle including fumarate reductase $(f r d A B C)$ that catalyzes a reaction of reduction of fumarate to succinate (81). As Mtb harbors the genes encoding Sdhl and Shd2 enzymes that catalyze the reverse reaction, the specific role of the fumarate reductase is not yet wellunderstood. However, it has been speculated that it might mediate increased flux of the oxidized equivalents $\left(\mathrm{NAD}^{+}\right.$and $\left.\mathrm{FAD}^{+}\right)$, changing the redox homeostasis and lowering the PMF to drive ATP synthesis at a lower rate than the one found in the aerobic conditions. These facts go well in line with the measured membrane potential of only $113 \mathrm{mV}$ (69), suggesting the existence of the mechanism that lowers the proton 
translocation rate in order reduce the energization of the membrane in the persister cells found in the hypoxic conditions.

As mentioned here, there are many ETC/metabolic adaptations and alternate electron routes utilized under the low oxygen tension to maintain the lowered membrane energization as well as support the cellular respiration. All these routes, in normoxic as well as hypoxic conditions, of which some contribute directly to the PMF generation by proton translocation, have a common ending point; a multi-subunit membrane-bound enzyme that acts as proton sink and synthesizes ATP in the process. In general consideration, the ATP synthases are also referred to as an ETC complex V. Considering the vast body of evidence that describes this group biochemically and structurally, mycobacterial F-ATP synthases will be described in the following chapters, and the importance of Mtb F-ATP synthase in virulence, as well as drug targeting, will be introduced.

\subsubsection{Potential therapeutic strategies}

Various classes of drugs have been screened against the activity of the respirasome, and some of them have high specificity coupled with strong sterilizing effects (Figure 1.4).

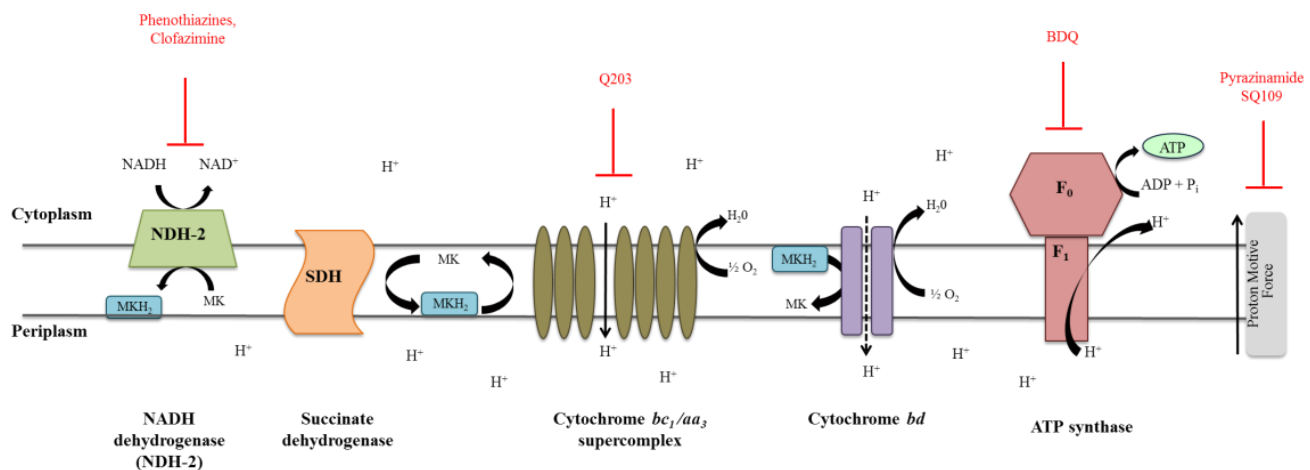

Figure 1.4: ETC as a target space for antimicrobials. The compounds like phenothiazines and clofazimine target the NDH2 dissipating the electron flow towards the terminal acceptor, $\mathrm{O}_{2}$. The novel compound Q203 targets the cytochrome $b c_{1}$ complex disturbing the transfer of electrons to molecular $\mathrm{O}_{2}$ during the aerobic respiration (99) affecting the synthesis of ATP. BDQ, a novel ATP synthase inhibitor, targets the subunit $\varepsilon$ (108) and $c$-ring blocking the proton conductance (109). Pyrazinamide, as well as novel SQ109, dissipate the PMF via multiple mechanisms.

The repurposed drug that was used as a neuroleptic $(110,111)$, that selectively incapacitates the NDH-2, is thioridazine. As phenothiazine derivative, thioridazine inhibits the transfer of the electrons from $\mathrm{NADH}$ to the $\mathrm{MKH}_{2} / \mathrm{MK}$ pool which 
prevents the entry of the electrons into the ETC (112). In turn, this affects the electron flux necessary for the cellular respiration and generation of ATP. The MDR- and XDR-TB strains often owe their resistance to the increased expression of the multidrug efflux pumps (113). The intense sterilizing action of thioridazine stems from the downregulation of the efflux pumps, which in turn reverses the resistance and the susceptibility to the first line antibiotics (114). The thioridazine, even though it is a highly potent inhibitor, due to its pleiotropic effects on the neuromuscular system (115), it is deemed as non-suitable for the prolonged treatments and is currently under consideration to be administered in cases of the isoniazid (INH) resistance in MDRTB (116-118).

Similarly to thioridazine, clofazimine (CFZ) acts on the NDH-2 inhibiting the NADH reduction and depleting the electron pool (119). It was initially developed as a treatment regimen for leprosy (Mycobacterium leprae), and due to its high sterilizing capacity, it entered the drug investigations as an anti-TB drug (120). The clofazimine shortens the first-line treatment for the MDR-TB, but the pleiotropic effects it causes, present a significant obstacle. Upon the administration, to the MDR-TB patients, it has been shown that CFZ causes skin de-pigmentation, similar to the hallmark of the leprosy treatment. It was reported that, in some patients, it causes serious complications such as atrial fibrillation and even cardiac arrest (121). The additional issues of the clinical use of CFZ are its pharmacokinetic drug-drug interactions and emergence of the resistance through the mutation in the transcription factor that regulates the expression of the multi-substrate efflux pump, MmpL5 (122,123). The upregulated pump in the CFZ-resistant mutants is reported to export BDQ as well, diminishing its effect on the cell and contributing to the cross-resistance.

Another newly discovered antimicrobial agent that entered the phase I of the clinical trials as the anti-TB drug is a derivative of phenothiazines, Q203 (124). The routine screenings of the commercially available libraries lead to the discovery (99) of this potent cytochrome $b c_{1}$ inhibitor that effectively reduces the formation of lung granulomas in mice. The Q203, when administered, eliminates actively-dividing bacilli via the inhibition of $c y t-b c_{1}$, which in turn affects the PMF generation. The studies performed on the mycobacterial inverted membrane vesicles (IMVs) further the understanding that Q203 significantly suppresses the capacity of IMVs for ATP synthesis, which most likely occurs due to the lowered PMF resulting from its action on $c y t-b c_{1}$ complex (125). In the development of any drug, it is of prime importance to 
consider its selectivity and toxicity to the host cells. Q203 performed exceptionally well, inhibiting the $50 \%$ growth of $M t b$ broth culture at $2.7 \mathrm{nM}$ while having no cytotoxic effect on any human cell lines tested at $10 \mu \mathrm{M}$, giving it a selectivity index $>3,700$ (99). It rapidly eliminated the actively-replicating bacilli while persisters were not affected, leaving the recalcitrant population intact. The facts were pointing toward $c y t-b d$, an alternately employed oxidase, upregulated during the persistence or hypoxia. Namely, upon the chemical inhibition of cyt- $b c_{1}: a a_{3}$ complex with Q203, cyt- $b d$ was able to provide sufficient electron flux towards $\mathrm{O}_{2}$ to fulfill the bioenergetics needs of the cell. The final verification of the cyt-bd implication in this phenomenon came from the mutational studies whereby the cyt- $b d$ was disabled via transposon mutagenesis to generate $\triangle c y t A B D C$ strain. The striking fact was that the Q203 was bactericidal for the cyt-bd deletion ( $\triangle c y t A B D C)$ strain, ultimately inhibiting the respiration in the presence of oxygen and drastically reducing the ATP intracellular pool, while its effect on the parental WT strain was only bacteriostatic, affecting ATP homeostasis to the lesser extent (126). Considering such high potency in $M t b$, it was of great concern whether the Q203 had a potential to inhibit mitochondrial complexes III and IV, but the toxicity reports indicated that it had no toxic effect on the cardiomyocytes (99).

Furthermore, another factual issue is also a possibility of the emergence of Q203 resistant strains. The routine screening in the original Q203 study identified strains that harbor mutations in $q c r B$, a subunit of the cyt- $b c_{1}$ (99) which could potentially hinder further development of the Q203 derivatives. However, when Q203 was tested on the $M t b$ clinical isolates, there were no reports of triggering any resistance to Q203, allowing the consideration of the combinatorial therapeutic approach for the MDR- and XDR-TB. Considering the mechanism of action and the effect on the ETC and Mtb bioenergetics, Q203 would be an ideal candidate in combination with a potential cyt-bd inhibitor. However, due to the lack of the facts regarding the structural and biochemical properties of mycobacterial cyt- $b d$, this strategy will have to be complemented with another anti-TB drug that targets the bioenergetic space other than cyt- $b d$.

In last forty years, the only drug that was approved by FDA (127) for the treatment of the MDR-TB is BDQ, a member of the drug class of diarylquinolines (DARQ) identified for its anti-mycobacterial activity in in vivo studies performed on Mtb, M. smegmatis and non-tuberculous mycobacteria (NTM) (18). As the lead 
DARQ compound with the highest potency, BDQ was particularly successful at inhibiting the drug-susceptible strains as well as clinical isolates resistant to first and second line anti-TB drugs (rifampicin, isoniazid, streptomycin, pyrazinamide, and moxifloxacin). The BDQ minimum inhibitory concentration (MIC) for drugsusceptible strains was found to be in the range of $0.03-0.120 \mu \mathrm{g} / \mathrm{ml}$, roughly five times lower than the MIC of rifampicin $(0.500 \mu \mathrm{g} / \mathrm{ml})(18,19)$. These properties provide a valuable treatment opportunity since TB requires lengthy regiments and prolonged exposure to the antimicrobial agents could convey toxicity for the host. Remarkably, upon oral administration into the infected mice over the eight days, BDQ had a pronounced bactericidal effect, eliminating the bacterial load with no counterindications to the host (18).

Furthermore, the clinical perspective directed the search towards the drug target, the mechanism of its action, and its specificity. The in vitro culturing of Mtb and $M$. smegmatis in the presence of BDQ concentrations ranging from 4-5 times higher than the minimal inhibitory concentration (MIC) lead to the development of the resistance to BDQ. The whole genome sequencing excluded the mutations in DNA gyrase $(g y r A)$ and identified the point mutations (A63P for $M t b$ and D32V for $M$. smegmatis) in the atpE, the gene that encodes the $c$-ring subunit of F-ATP synthase (18). Further experiments including the whole genome analyses carried out on the NTMs showed that the M. xenopi, M. novocastrense, and M. shimoidei have natural resistance towards BDQ reflected in the failure to inhibit their growth in vitro and rooted in the amino acid polymorphism $\left(a t p E^{\mathrm{A} 63 \mathrm{M}}\right)(128)$.

Also, the identification of the F-ATP synthase as a drug target raised the concerns of the selectivity of BDQ, since F-ATP synthase is present in the other prokaryotes (human gut microbiota) as well as host mitochondria. However, in vitro studies showed that to achieve the bactericidal effect of BDQ on pathogen Helicobacter pylori, closely related Corynebacterium glutamicum, gram-positive (Nocardia, Enterococcus feacalis, Streptococcus pneaumoniae, Streptococcus aureus) and gram-negative (Haemophilus influenzae, E. coli), it was required to administer much higher concentrations (MIC between 16 and $32 \mu \mathrm{g} / \mathrm{ml}$ ) putting BDQ in the group of highly specific anti-mycobacterial compounds (18). Apart from that, another important fact is that the host mitochondrial ATP synthase toxicity was not reported, propelling BDQ straight to the top one drug for MDR-TB treatment (76). 
The majority of these studies have validated mycobacterial F-ATP synthase as the drug target in the aerobic conditions of growth, but it was of prime importance to eliminate the bacilli that persisted in macrophages due to the hypoxia-induced metabolic and respiratory shift. The energy requirements for ATP synthesis are significantly lower in the hypoxia-grown bacilli, and thus, the ATP synthase was downregulated followed by the lowering of the ATP content of the cell (80). Considering that the BDQ targets ATP synthase which in actively-replicating bacilli produces high amounts of ATP, it was a matter of the debate whether it would have a potent sterilizing capacity on the dormant bacilli considering their lowered energy requirements, downregulated ATP synthase operon and decreased cellular ATP content. Nevertheless, it was shown that BDQ exhibits a strong bactericidal effect on the dormant (20) as well as on actively-replicating bacilli, advocating strongly the fact that the mycobacterial ATP synthase, is essential for ATP production. Coupling of the energy to cellular respiration in both life cycle stages and inhibition thereof lead to a complete loss of viability that ultimately to cell death.

The entire body of facts encompasses an immense amount of evidence pertaining to the importance of energy generation in mycobacteria and thus, considering the emergence of the resistance to BDQ in clinical isolates $(129,130)$, it is of uttermost importance to find an alternative way to target mycobacterial ATP synthase without causing the resistance in the long run. To that end, the mycobacterial ATP synthase is considered in the light of its biochemical and structural specificities to elucidate potential intrinsic regulatory mechanisms that could contribute to dysregulation of ATP synthesis which leads to perturbation of the cellular ATP homeostasis and elimination of both life forms in the human host. However, to this day, there is a growing body of evidence describing the structural determinants of the subunits comprising the mycobacterial ATP synthase, and to understand these evolutionarily conserved molecular machines, the look is taken through the kingdom of prokaryotes concluding with the mycobacteria specific structural and functional features. 


\subsubsection{F-ATP synthases}

$\mathrm{F}_{1} \mathrm{~F}_{\mathrm{O}}$-ATP synthases are versatile, multi-subunit, molecular motors that generate the currency of life, ATP. Across the various species, genera and even kingdoms, the general architecture of F-ATP synthase is conserved, but interestingly species-specific adaptations emerged to accommodate the energy requirements of a particular ecological niche. Numerous studies of the composition of F-ATP synthase (unc) operons as well as structural studies have provided unprecedented insight into the adaptations that provide the upper hand in energy generation and regulation of these molecular machineries.

Often, the studies of an uncharacterized protein complex start with a comparison of it operon with operons of known organisms whose homologous protein complexes had been well characterized. One of the most extensively studied bacterial F-ATP synthases are Escherichia coli and Geobacillus stearothermophilus enzymes (former Bacillus PS3) (131-136) due to relatively easiness of isolation in their pure form that allows biochemical and structural characterization. A typical operon of prokaryotic F-ATP synthases is comprised of multiple genes encoding individual subunits that co/post-translationally assemble into a functional $\mathrm{F}_{1} \mathrm{~F}_{\mathrm{O}}-\mathrm{ATP}$ synthase (137-139). Across the genera, there are operonic differences that support a variety of adaptations to accommodate the energy generation requirements reflected in various subunit composition.

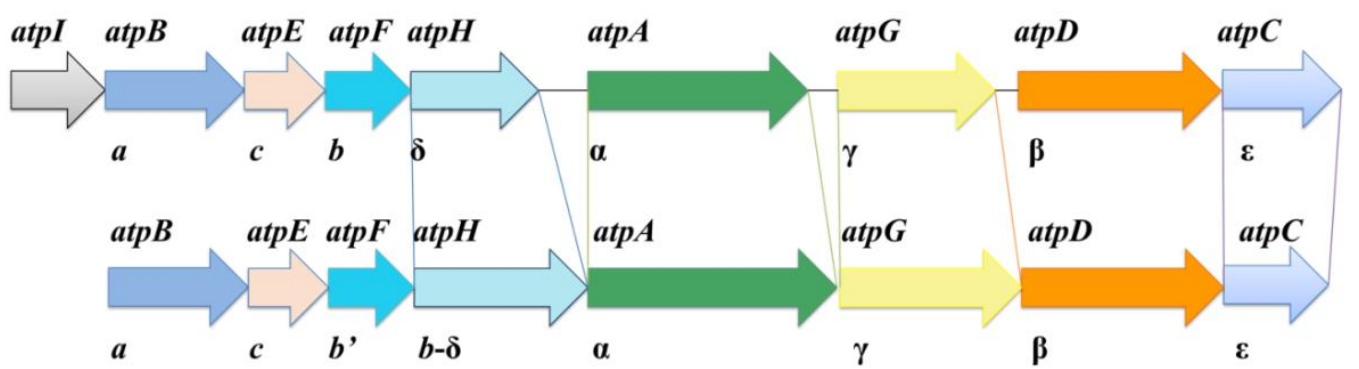

Figure 1.5: F-ATP synthase operons of $\boldsymbol{E}$. coli and $\boldsymbol{M} \boldsymbol{t} \boldsymbol{b}$. The operon structure of $E$. coli (top) shows the annotated genes and their relative size in comparison to the genes of the Mtb F-ATP synthase operon. Several differences are notable: atpI does not exist in $M t b, a t p H(F H)$ gene of $M t b$ encodes a fusion protein of $b$ - $\delta$ subunits, while $E$. coli encodes $b$ and $\delta$ separately. Mtb atpF encodes unique $b^{\prime}$ subunit homologous to $b$ in $E$. coli. Mt $\alpha$ subunit is larger than E. coli homolog, while MtE is shorter than its counterpart in E.coli.

A functional bacterial-type F-ATP synthase is comprised of the two parts, a water-soluble $\mathrm{F}_{1}$, and a membrane-spanning part, $\mathrm{F}_{\mathrm{O}}$ part (140-142) (Figure 1.6). 


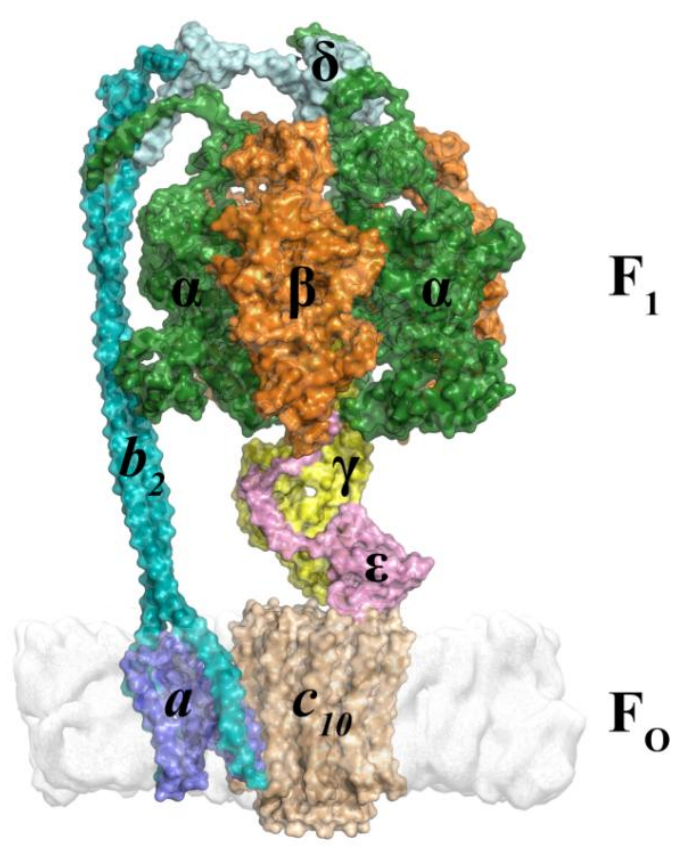

Figure 1.6: Structure of the $\boldsymbol{E}$. coli F-ATP synthase solved using the cryo-EM (143). The surface representation of the $\mathrm{F}_{1} \mathrm{~F}_{\mathrm{O}}$-ATP synthase shows the individual subunits that define two distinct parts, $\mathrm{F}_{1}$ and $\mathrm{F}_{\mathrm{O}}$. The structural coordinates are deposited in the Protein Data Bank under the accession number 5 T4O (144). The $\alpha_{3} \beta_{3} \gamma \varepsilon \delta$ subunits are organized in the $\mathrm{F}_{1}$ region, while $a b_{2} c_{10}$ make up the $\mathrm{F}_{\mathrm{O}}$ part of E. coli F-ATP synthase.

The $\mathrm{F}_{\mathrm{O}}$ domain is comprised of multiple subunits with the stoichiometry of $a b_{2} c_{n}$. The $c$ subunit, encoded by atpE, forms a homo-oligomeric ring containing a variable number of monomers in different species (145). The $c$-ring displays a rotary capacity against a static subunit $a$ and together they form a proton sink. On the opposite side of subunit $a$ (encoded by $a t p B$ ), the N-terminal helices of the $b$ subunit dimer insert into the membrane to form a clamp around subunit $a$. These "clamp" interactions provide rigid support to the rotary $c$-ring, while subunit $b$ C-terminal helices extend out from the membrane to establish contact with $\mathrm{F}_{1}$ subunits $\delta(a t p H)$ and $\alpha(a t p A)$ to form a $b_{2}$ backbone of the $\mathrm{F}_{1} \mathrm{~F}_{\mathrm{O}}$-ATP synthase termed peripheral stalk.

The catalytically active $F_{1}$ part is made up of multiple subunits with the stoichiometry $\alpha_{3} \beta_{3} \gamma \delta \varepsilon$ (atpADGHC). By association in an alternate fashion, subunits $\alpha$ and $\beta$ form a pseudo-hexameric, catalytic head around the central cavity. The pseudohexamer harbors six nucleotide binding pockets $(146,147)$, of which three perform catalysis while the other three play a role in fine-tuning the enzymatic activity. The central cavity fits the asymmetric subunit $\gamma$ which together with subunit $\varepsilon$ forms a central, rotary stalk $(143,148)$. This interaction is established through the contact of the 
N-terminal domain (NTD) of subunit $\varepsilon$ with the paraxial domain of $\gamma$ subunit, forming a contact surface with the $\mathrm{F}_{\mathrm{O}} c$-ring. Peripherally, subunit $\delta$ associates with the catalytic hexamer and acts as a link to connect the peripheral stalk to the $\mathrm{F}_{1}$ part that subsequently interacts with subunit $a$ of $\mathrm{F}_{\mathrm{O}}$.

Functionally, the enzyme can be divided into the stator part comprised of the $\alpha_{3} \beta_{3}$ hexameric head, subunits $b_{2}$ and $a$. Subunit $\gamma$ together with $\varepsilon$ interacts with the $c$ ring and rotate together relative to the stator $(147,149)$. This functional separation provides the basis for the rotary catalysis. F-ATP synthases can function bidirectionally; translocation of the protons through the $c$-ring/subunit $a$ interface provides the torque utilized for ATP synthesis (Figure 1.7) while the energy stored in the $\gamma$-phosphate bond of ATP is utilized to generate torque in the opposite direction and pump the protons against their gradient (140). These two catalytic directions are well regulated by subunit $\varepsilon$ (132) and intrinsic interactions of subunits $\alpha, \beta$, and $\varepsilon$ with $\mathrm{Mg}^{2+}$-ADP and $\mathrm{P}_{\mathrm{i}}(150)$, which will be described in more detail.

(A)

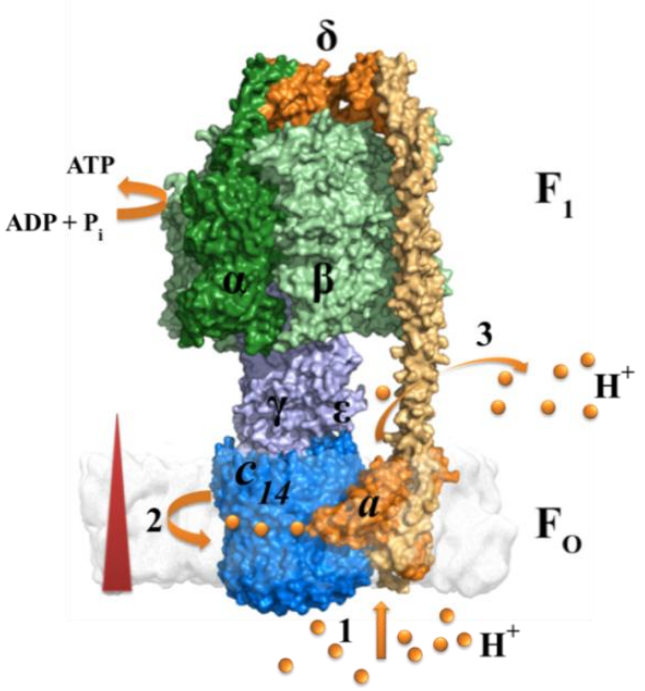

(B)

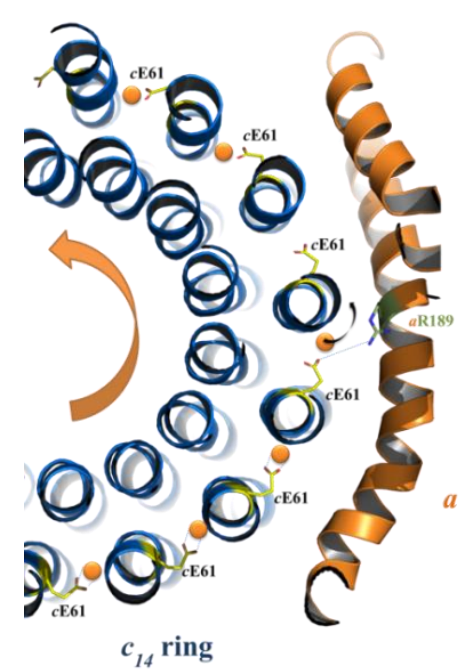

Figure 1.7: Schematic representation of ATP synthesis. (A) A generalized representation of the $S$. oleracea chloroplast $\mathrm{F}_{1} \mathrm{~F}_{\mathrm{O}}$-ATP synthase embedded into the membrane (151). The proton gradient and the direction of the translocation are indicated with the red arrow. The $c$-ring (dark blue) loaded with the protons (the orange spheres) rotates and loads the protons into the subunit $a$ (dark orange). The protons exit subunit $a$ on the other side of the membrane, whereby the energy of this process is utilized to drive the rotation of the central stalk (subunits $\gamma$ and $\varepsilon$, light violet) and cause alternative changes of the conformations of the catalytic hexameric head (subunits $\alpha$ and $\beta$, green shades). The catalytic head synthesizes ATP from ADP and $\mathrm{P}_{\mathrm{i}}$. (B) Detailed schematic representation of the subunit $a / c$-ring loading mechanism. The blue helices represent the transmembrane hairpin organization of the fourteen $c$ monomers. The $c$ E61 sidechains (yellow) interact with a proton (orange spheres) obtained from the extracellular medium, delivering it towards the specific residue $a \mathrm{R} 189$ (green). The interaction of the E61 and R189 allows the unloading of the proton into the channel of the subunit and subsequent expulsion of the proton on the opposite side of the membrane. Color coding is consistent with the adjacent image. 
The proton motive force generated at the membrane provides the proton pool that interacts with the $c$-ring and subunit $a$. This mechanism is studied extensively, and it was recently described to the detail in the structural studies performed on the F-ATP synthase of the spinach chloroplast (151). In the chloroplast F-ATP synthase, a single proton is taken up through the half channel of the subunit $a$ by interaction with the specific residue of the $c$-ring monomer, $c$ E61 (151). The proton loaded $c$-ring, through its rotational movement against the subunit $a$, delivers the proton to the proximity of the residue $a \mathrm{R} 189$. The positively charged side chain of the residue $a \mathrm{R} 189$ interacts with the negatively-charged $c$ E61 sidechain, enabling the release of the bound proton in the hydrophilic half-channel of the $a$ subunit and its subsequent expulsion to the opposite side of the membrane $(151,152)$. Every interface between the two $c$-ring monomers is loaded subsequently with protons creating a linked proton supply and securing the continuous utilization of the proton motive force. Such continuous rotatory motion drives the rotation of the central shaft.

The main component of the shaft is an asymmetrical subunit $\gamma$ that reaches deep into the $\alpha_{3} \beta_{3}$ hexameric head with its coiled-coil domain inducing the change in their conformations. This event is mediated mainly through the globular domain of the subunit $\gamma$ that specifically interacts with the catalytic subunit $\beta$ through $\mathrm{C}$-terminal “DELSEED” region (149,153-155). A similar interaction occurs with the non-catalytic subunit $\alpha$ employing the "DELSEED" homologous region ${ }_{396} \mathrm{AQFGSDLDK}_{404} ; G$. stearothermophilus numbering) (156) to couple the rotation with the catalytic events in the $\alpha_{3} \beta_{3}$ hexamer. The asymmetry observed in the subunit $\gamma$ plays a fundamental role in driving the conformational change and forcing differential occupancy of the nucleotide-binding sites in the non-catalytic and catalytic subunits $\alpha$ and $\beta$. This process is known as rotary catalysis or the Boyer's binding change mechanism (157), and it is being regulated by $\mathrm{Mg}-\mathrm{ADP}(158)$ and subunit $\varepsilon(159,160)$.

\subsubsection{Rotary catalysis and it's regulation}

In order to catalyze the reaction of ATP synthesis or hydrolysis, the subunits $\alpha$ and $\beta$ form a highly dynamic interface. In virtually all known F-ATP synthases, the catalytic subunit $\beta$ harbors a specific short loop in its central domain capable of binding the phosphorylated nucleotides (ADP and ATP) termed phosphate-binding loop (P-loop). P-loop also known as Walker A motif $(161,162)$ represents a highly 
conserved sequence present in other ATP hydrolysing enzymes such is myosin, adenylate kinase, $\operatorname{Rec} A$ with a general sequence $\mathrm{G}-\mathrm{X}-\mathrm{X}-\mathrm{X}-\mathrm{X}-\mathrm{G}-\mathrm{K}(\mathrm{T}) \mathrm{X}-\mathrm{X}-\mathrm{X}-\mathrm{X}-\mathrm{X}-\mathrm{X}-$ $\mathrm{I} / \mathrm{V}$ (163) that enables the binding of the phosphate moiety of the phosphorylated nucleotides (ADP, ATP) and is an active spot of ATP synthesis/hydrolysis (161). In FATP synthases, the selectivity of the P-loop towards ADP/ATP is a function of the position of rotary subunit $\gamma$ that interacts through the "DELSEED" region with $\beta$ altering the intramolecular interactions and affecting the P-loop topology $(164,165)$.

Similarly to the subunit $\beta$, the subunit $\alpha$ contains the P-loop as well, however, its role is not entirely clear. Studies are advocating that $\alpha$ P-loop serves as a noncatalytic site capable of sensing the intracellular ADP/ATP homeostasis and modulating the catalytic events that occur in $\beta$, promoting either ATP synthesis or hydrolysis (166) through a conformational change. Defined by the angle of $\gamma$, it is possible to distinguish three different conformations of subunits $\alpha$ and $\beta$ : $\beta_{\mathrm{E}}$, where the nucleotide site is open and empty, $\beta_{\mathrm{DP}}$, site where ADP and $\mathrm{P}_{\mathrm{i}}$ are bound and $\beta_{\mathrm{TP}}$, site that contains ATP $(167,168)$. All three conformations exist in both catalytic events, synthesis as well as hydrolysis of ATP, but the order of catalytic events differs (169).

Operation of the F-ATP synthase in the synthesis direction requires the purified $\mathrm{F}_{1} \mathrm{~F}_{\mathrm{O}}$ integrated into the membrane system in which PMF is established to drive the catalytic events. Until today, it has been proven to be difficult to establish such an experimental setup and thus, most studies of the catalytic events were carried out on the isolated $F_{1}$ part operating in the ATP hydrolysis direction as it only requires ATP as a substrate. Considering that ATP synthesis and hydrolysis are not just reversed reactions (170), this approach allowed only a partial understanding of ATP synthesis, and thus only ATPase activity will be considered in more details (170-172).

\subsubsection{ATP hydrolysis}

The catalytic events that lead to the enzymatic cleavage of the $\gamma$-phosphate bond of ATP are elucidated to great detail $(167,168,173)$, however here a general overview will be provided.

At any given point of time, three catalytic sites in subunit $\beta$ will be considered. Due to the asymmetry of the $\gamma$ subunit, $\beta$ subunits have different nucleotide occupancies and assume different configurations, namely, $\beta_{\mathrm{E}}, \beta_{\mathrm{TP}}$, and $\beta_{\mathrm{DP}}$ (Figure 1.8) (174). Due to the high cellular concentrations of ATP, $\beta_{E}$, a site without the nucleotide 
is in the open conformation promoting the binding of ATP. Upon the initial interactions between ATP and the P-loop of the $\beta_{\mathrm{E}}$, the binding event induces the conformational change in the $\beta$ subunit that transitions to the closed conformation $\beta_{\mathrm{TP}}$ binding ATP tightly. P-loop amino acid side chains establish a coordination complex through $\mathrm{Mg}^{2+}$ that coordinates the $\beta$ - and $\gamma$-phosphate groups of the ATP. In the next step, due to the local stereo-chemical rearrangements, the $\gamma$-phosphate bond is cleaved, $\mathrm{ADP}$ and $\mathrm{P}_{\mathrm{i}}$ are produced, and the close contact is still maintained in the conformation referred to as $\beta_{\mathrm{DP}}$ which assumes a different topology than the one present in the $\beta_{\mathrm{TP}}$. This catalytic event drives the rotation of the subunit $\gamma$, which in turn affects the other two $\beta$ subunits altering their conformation propagating multi-site catalysis (164). In order for the next catalytic cycle to occur, ADP and $\mathrm{P}_{\mathrm{i}}$ need to be released from the catalytic site. Some targeted molecular dynamic simulation studies emphasize that this release is a two-step process, whereby $\mathrm{P}_{\mathrm{i}}$ is released first, followed by the transition of the $\beta_{\mathrm{DP}}$ to $\beta_{\mathrm{E}}$ and release of $\mathrm{ADP}(175,176)$. One catalytic cycle is complete, and $\beta_{\mathrm{E}}$ is again receptive of ATP.

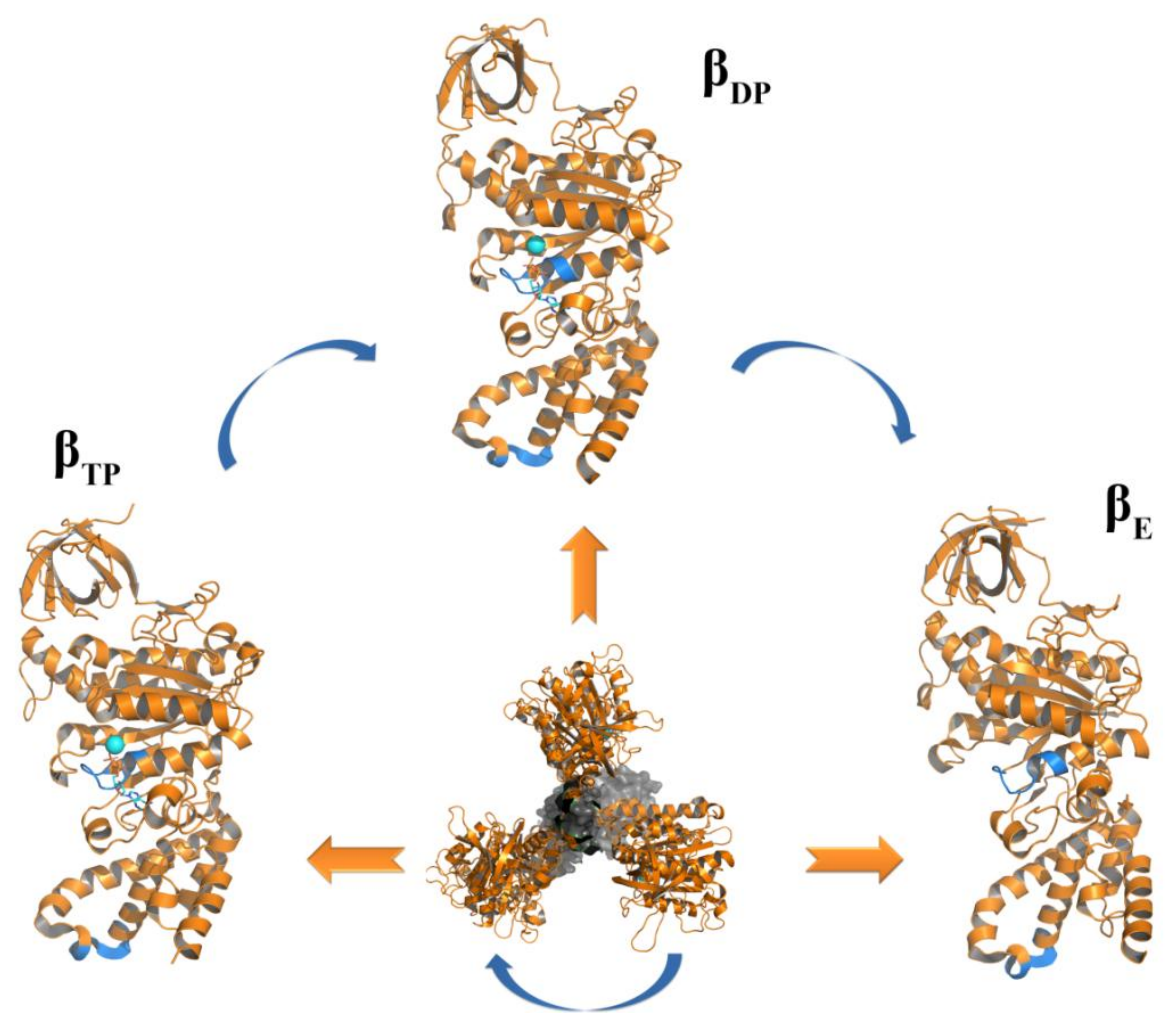

Figure 1.8: The conformations of subunit $\boldsymbol{\beta}$ during catalysis. The cartoon representation of the subunit $E$. coli $\beta$ (PDB ID: 3OAA: D) conformational change during the catalytic cycle. $\beta_{\mathrm{E}}-$ open conformation binds ATP and progresses into the ATP-bound conformation $\beta_{\mathrm{TP}}$. The cleavage of the ATP to ADP and $P_{i}$ occurs and the conformation changes to $\beta_{D P}$ to release $P_{i}$ while ADP is still bound to the catalytic site. Upon further conformational change to $\beta_{\mathrm{E}} \mathrm{ADP}$ dissociates lowing $\beta_{\mathrm{E}}$ to accept next ATP molecule. Phosphate-binding loop (up) and coupling DELSEED region (down) labeled in blue. 
All these conformational alterations are driving the rotation of subunit $\gamma$ that in turn stabilizes the particular $\beta$ conformation during its rotary motion enabling the multi-site catalysis (164). The multi-site catalysis occurs in all three catalytic sites with a "phase" shift, which was initially proposed by Boyer and termed "binding change mechanism" (157).

Such a complex sequence of catalytic events and the transition between synthetic and hydrolytic mode is regulated in response to the energy requirements of the cell. In various organisms such as E. coli and G. stearothermophilus, the regulation is accomplished mostly via two evolutionary conserved mechanisms: a phenomenon known as Mg-ADP inhibition (177) and through conformational changes of the $\mathrm{F}_{1}$ subunit $\varepsilon(132,159,178-182)$.

A question was raised whether such regulation was present in the pathogenic mycobacterial F-ATP synthase considering that current facts indicate its preferred operation in the ATP synthesis direction exhibiting none or low ATPase activity (2). In order to understand the specificities of the mycobacterial enzyme, the attention is primarily focused on the well-characterized enzymes of other bacterial species and considered in the perspective of potential exploitation of mycobacteria-specific structural and functional traits.

\subsubsection{Structural features of bacterial subunit $\varepsilon$ and its regulatory capacity}

High-resolution studies conducted on an E. coli $\varepsilon$ (Ece) and $G$. stearothermophilus $\varepsilon$ (Gse) reveal that the subunit $\varepsilon$ is composed of two structurally distinct domains. The NTD is comprised of the multiple antiparallel $\beta$-sheets forming a compact and rigid $\beta$-barrel, and the C-terminal domain (CTD) composed of two $\alpha$ helices, helix 1 and helix 2, connected by a linker of various lengths specific to the organism (Figure 1.9) $(183,184)$. In the isolated state, the CTD forms a hairpin by stacking the two helices in a parallel manner. In this conformation, further referred to as a "closed" or "compact", the tip of the CTD interacts with the NTD and stabilizes this structural motif (184).

Similarly, there is evidence that the contributing factor to the stabilization of the closed conformation is an ATP molecule, whose binding to subunit $\varepsilon$ was shown in the titration experiments. Namely, the binding constant of ATP to isolated EcE measured at $25{ }^{\circ} \mathrm{C}$ was $22 \mathrm{mM}$ (184), far beyond the physiologically relevant value ( $2 \mathrm{mM}$ ATP in the E. coli cell lysate) (185). In contrast, the isolated Gse binds ATP with $4 \mu \mathrm{M}$, which represents roughly 5,000 times higher affinity, indicating that ATP 
stabilizes the closed conformation and directly contributes to regulation via subunit Gsc. These differences stem from the structural organization of the ATP-binding pocket.

(A)

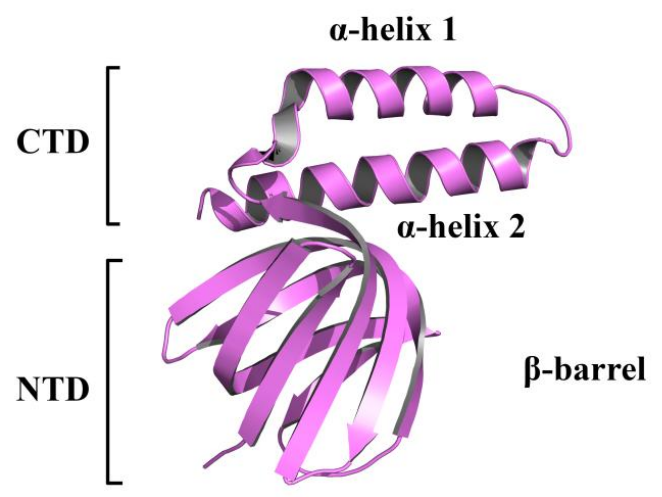

(B)

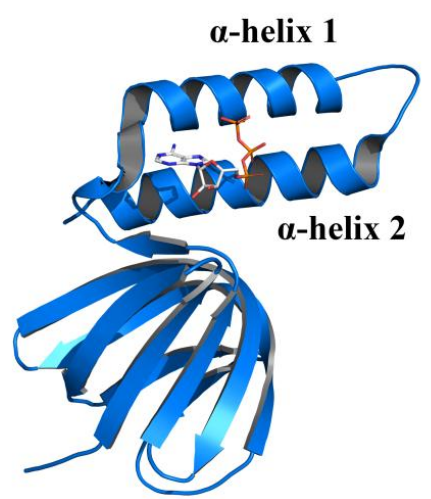

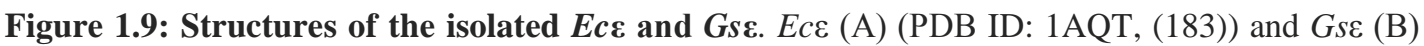
(PDB ID: 2E5Y,(184)) structures solved by the crystallography showing the $\beta$-barrel NTD domains and compacted CTD helices 1 and 2 connected by a linker. CTD tip of the helix 2 is found in the proximity of NTD, partaking in the stabilizing interactions. Isolated Gse is shown in complex with ATP while there is no ATP bound to the EcE.

The NMR titration studies showed significant perturbations of the residues E83, D89, R92, R122 and R126 upon addition of ATP, indicating their contribution to the architecture of the ATP-binding pocket. Complimentary mutational studies confirmed that the binding was abolished when the residues E83, D89, R92, R122, and R126 were substituted with alanine, which effectively disrupted the geometrical requirements of the ATP-binding pocket and rendered the mutants irresponsive to ATP (186).

According to the sequence alignment, EcE harbors alternative residues in the matching positions I84, K99, K123 and Q127 which drastically change the electrostatic network, as well as geometry responsible for ATP binding, thus lowering the affinity. This phenomenon could also have physiological implications and in principle indicates that the ATP is not an essential regulatory component of the armlike motion of the Ec\&CTD (186) (Figure 1.10) but acts as a potent regulator in on the conformational alterations of the Gse.

In order to regulate the catalytic events and to function as an ATPase inhibitor in response to the variable energy homeostasis of the cell, subunit $\varepsilon$ undergoes 
significant conformational change induced by multiple factors such as the reduction of the cellular ATP pools, changes of the PMF and reversal of the rotational direction of the subunit $\gamma(160,179,187,188)$. Due to the thermodynamic instability, there are no reported structures of the isolated extended bacterial $\varepsilon$ to this date, possibly due to the requirements of the sterical input from other subunits of ATP synthase that could induce $\varepsilon$ into a conformational transition and stabilize the extended state.
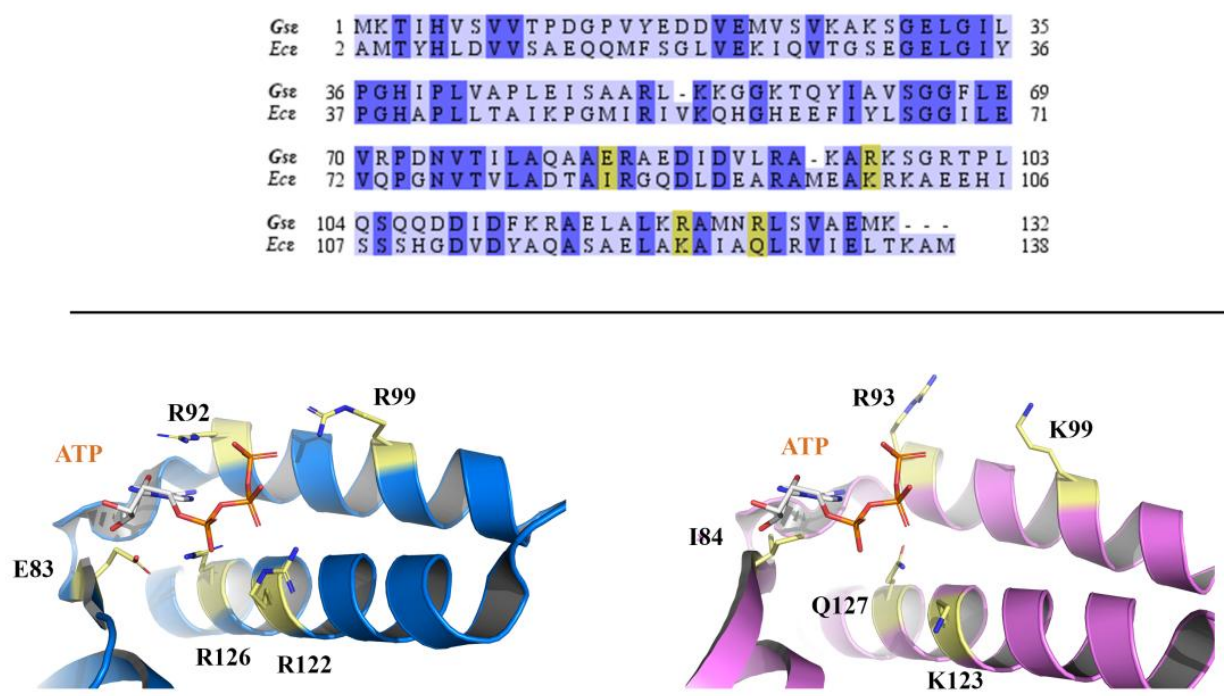

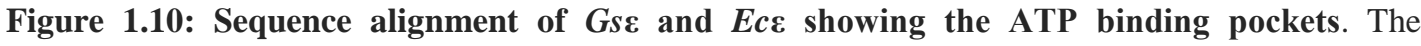
GseCTD binds ATP with $4 \mu \mathrm{M}(186,189)$ affinity into the pocket comprised of highly polar E83, R92, R99, R122, and R126. The EcعCTD (184) harbors the less polar homologous residues I84, R93, K99, K123, and Q127, which bind ATP with the affinity of $22 \mathrm{mM}$.

Indeed, the first evidence came with the isolation of the $E c \gamma-\varepsilon$ complex that displays the subunit $\varepsilon$ in the half-extended state (PDB ID: 1FS0) (190) confirming the necessity of the stabilizing interactions to overcome the thermodynamic equilibrium of the closed conformation (Figure 1.11-B).

Further studies on the bacterial $F_{1}$ complexes offer a different perspective. One of the earliest isolated and described structure by crystallographic methods was an $E$. coli $\mathrm{F}_{1}$ containing the $\alpha_{3} \beta_{3} \gamma \varepsilon$ subunits (PDB ID: 1D8S) (191) (Figure 1.11-A). The hexameric $\alpha_{3} \beta_{3}$ head was well resolved to the resolution of $4.4 \AA$, while subunit $\gamma$ was represented with discontinued $\alpha$-helices enabling the resolution of most of the features of the $\alpha_{3} \beta_{3} \gamma$. However, even though $\varepsilon$ was present in the isolated complex, it was not possible to assign its density with any degree of certainty. Complementary crosslinking studies, however, showed that the residue in the $\varepsilon$ CTD at the position 108 could interact with the $\beta$-DELSEED region indicating that the subunit $\varepsilon$ exists in the 
extended conformation that can reach the catalytic hexamer $\alpha_{3} \beta_{3}(192,193)$. Additional confirmation of this phenomenon came with the further development of crystallography. The structure of isolated $E c \mathrm{~F}_{1}$ was solved with the resolution of 3.26 $\AA$ (PDB ID: 3OAA) (194), enabling placement of the subunit $\varepsilon$ in the context of the $\mathrm{F}_{1}$ (Figure 1.11-C).

(A)

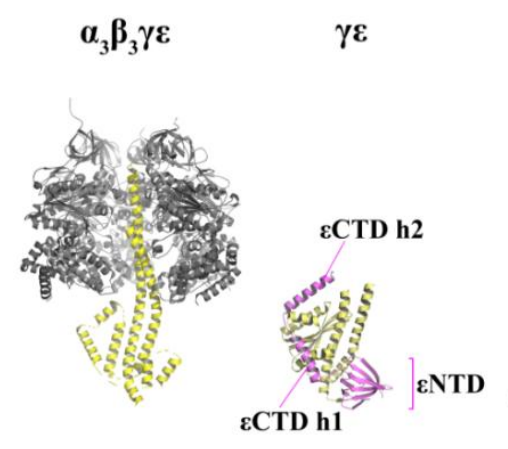

1999

(B)

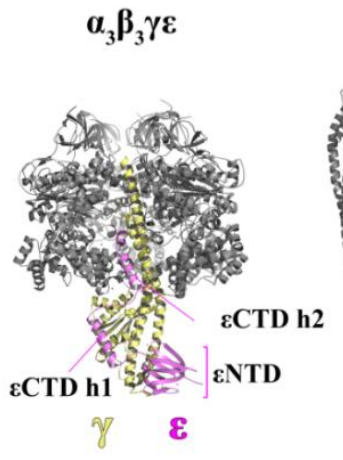

2011
(D)<smiles></smiles>

Figure 1.11: Overview of the $E c \varepsilon$ in the extended conformation. (A) Isolated complex $E c \alpha_{3} \beta_{3} \gamma \varepsilon$ structure (PDB ID: 1D8S) (191) solved by x-ray crystallography to $4.4 \AA$ shows the well resolved $\alpha_{3} \beta_{3}$ (gray) while $\gamma$ subunit (yellow) features discontinued helices. Densities of subunit $\varepsilon$ not visible.

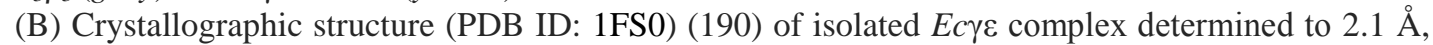
showing the $\varepsilon$ in a half-extended state. (C) Crystallographic structure of $E c \alpha_{3} \beta_{3} \gamma \varepsilon$ (PDB ID: 3OAA) (194) solved to $3.2 \AA$, showing subunit $\varepsilon$ in a fully extended conformation, inserting into the $\alpha_{3} \beta_{3}$ hexamer. (D) Cryo-EM structure $E c \mathrm{~F}_{1} \mathrm{~F}_{\mathrm{O}}$ (PDB ID: 5T4O) (143) solved to $6.9 \AA$, showing the extended $\varepsilon$ state in the context of the entire F-ATP synthase.

It was shown that $\varepsilon N T D$ binds to the foot domain of subunit $\gamma$ while the $\varepsilon$ CTD helices extend and assume the non-linear structural motif helix-linker-helix. While the helix 1 stacks against the globular domain of subunit $\gamma$, helix 2 intercalates the catalytic hexamer $\alpha_{3} \beta_{3}$, interacting with the coiled-coil of $\gamma(132,195)$.

Furthermore, the structural studies employing cryo-EM, for the first time, revealed the structure of the entire $E c \mathrm{~F}_{1} \mathrm{~F}_{\mathrm{O}}$ with the extended conformation of subunit $\varepsilon$ (143). The arrangement of the $\varepsilon$ CTD helices reflects the arrangement observed in the isolated $F_{1}$ indicating that the presence of the other subunits, mainly the entire rotor $\left(c_{10}: \gamma\right)$ does not alter the capacity of $\varepsilon$ to assume the extended conformation with topological arrangement similar to the ones described for the isolated $\mathrm{F}_{1}$ (Figure 1.11- 
D). The shown structures provide snapshots of the two states of $\varepsilon$, but the entire process of the conformational transition is not entirely understood to this day.

Similarly to the E. coli enzyme, other bacterial F-ATP synthases were studied extensively. The recent crystal structure of the isolated $\alpha_{3} \beta_{3} \gamma \varepsilon$ complex of $G$. stearothermophilus revealed that its $\varepsilon$ subunit (Gse) maintained the conserved structural features of a $\beta$-barrel in its NTD, present in EcENTD, while the arrangement of CTD helices is strikingly different (196,197) (Figure 1.12). The extended conformation of $G s$ shows CTD helices arranged in a linear fashion connected by a short unstructured linker, forming together a pike-like helical domain.

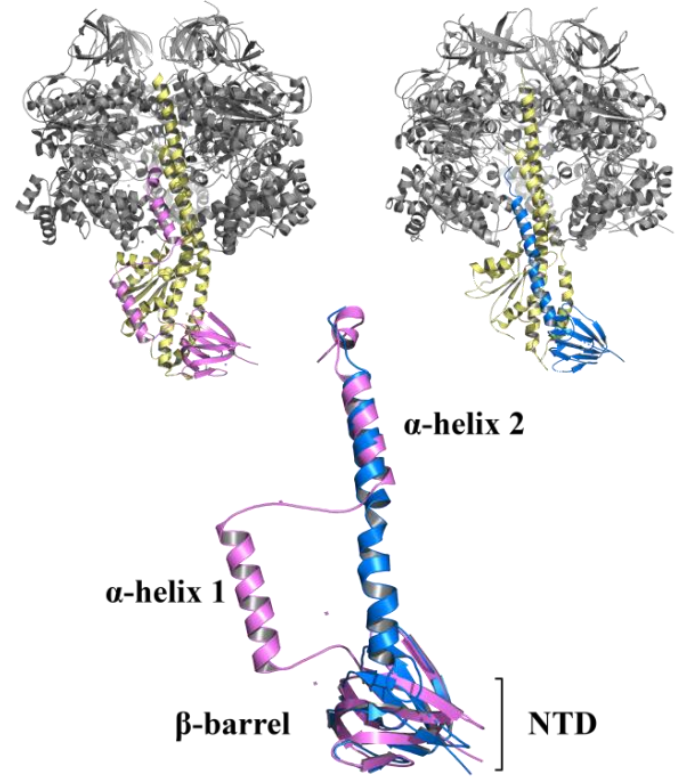

Figure 1.12: Structural specificities of $G s \varepsilon$ and $E c \varepsilon$ in an extended conformation. Cartoon representation of subunit Gse (blue) (PDB ID: 4XD7: H, (196)) aligned with the Ece (magenta) (PDB ID: 3OAA: H, \}(197)) shows the CTD helices 1 and 2 extended to form a linear elongated structure. EcECTD shows lateral displacement of the helix 1 in respect to Gse helix 1. Both structures were obtained in the complexes with $G s \alpha_{3} \beta_{3} \gamma$ (top left) and $E c \alpha_{3} \beta_{3} \gamma$ (top right), respectively, and shown in an isolated state.

The helix 2 assumes the canonical position by intercalating into the catalytic head, interacting directly with the coupling, $\beta$-DELSDED region and coiled-coil domain of $\gamma$ (148). The parallel is drawn with the Ec\& in this case, while Gse helix 1 does not seem to interact with subunit $\gamma$ making it an essential structural feature that contributes to the conformational transitions (Figure 1.12).

From the mechanistic aspect, the inhibition of the wasteful ATP hydrolysis is not entirely understood, and many assumptions still govern the field. However, the sequence of events should take into consideration multiple factors such is ADP/ATP 
homeostasis, PMF, speed of rotation of central stalk and strength of the interactions between $\varepsilon$ CTD-NTD that need to be abolished to accomplish conformational extension. In E. coli and G. stearothermophilus, the F-ATP synthase can be used to generate a proton gradient across the membrane at the expense of cellular ATP (160). In E. coli, upon drop of ATP below the homeostatic level, the inhibition of ATP hydrolysis occurs to prevent the depletion of the cellular pool. It has been suggested that the first in the sequence of events is the dissociation of ATP from subunit $\varepsilon$ found in the closed conformation, enabling weakening of the interactions that maintain the closed conformation $(186,189)$. Due to the hydrodynamic drag occurring during the rotation of the central stalk in ATP hydrolysis direction, ECTD could be dislodged from the closed conformation, undergoing the stochastic movements. The initial step of the extension could involve the interaction of the ECTD helix 1 with globular domain of $\gamma$, partially stabilizing this transitional state and allowing only helix 2 to move stochastically. These events would increase thermodynamic stability of the rotating central stalk forcing even further stabilization by finding the $\alpha_{3} \beta_{3}$ cavity that can accommodate $\varepsilon$ CTD helix 2 and stop the rotation. Once this condition is met, the helix 2 inserts into the $\alpha_{3} \beta_{3}$ interface stacking against the $\gamma$ coiled-coil domain imposing the sterical clashes with the coupling $\beta$-DELSEED region $(132,195)$. Even though this final conformation is supported with numerous interactions found in the interface (197), it cannot be said with certainty whether these interactions occur before binding of $\varepsilon$ CTD helix 1 to $\gamma$.

Similarly to this mode of regulation in E. coli enzyme, it has been reported that these events occur much faster in the enzyme of G. stearothermophilus (184) owing to the increased sensitivity of Gse to ATP and lower flexibility of the extended CTD domain. This phenomenon could find the support considering that the $G$. stearothermophilus thrives at the environmental temperature as high as $65{ }^{\circ} \mathrm{C}$ $(198,199)$, where the enzymes have to impose an adept control of the rate of metabolic reactions.

It was observed that the numerous adaptations in F-ATP synthase, particularly in regulatory subunit $\varepsilon$, enable adaptation of the organism to the environmental conditions and accommodate the energy generation needs. Considering the pathogenic, highly specialized nature of $M t b$ that relies on tight energy coupling mechanisms, high metabolic requests for the ATP and F-ATP synthase essentiality for viability and pathogenicity, the research was directed towards understanding the mechanisms 
employed to establish the close-to impregnable inhibition of wasteful ATP hydrolysis. With this goal, the next chapter focuses on the structural and functional properties of mycobacterial F-ATP synthases reported to the date that constitute such formidable regulation of the ATPase activity in mycobacteria.

\subsubsection{Structural and functional features of mycobacterial F-ATP synthase}

The $c$-ring subunits of mycobacteria are encoded by a single copy of the atpE gene. In other bacterial F-ATP synthases, the stoichiometry of the $c$-ring is in correlation with the energization of the membrane which depends on the environmental conditions. In the F-ATP synthases of the organisms that thrive in environments with a nutritional abundance, the cellular respiration generates a high proton gradient across the membrane. In order to accommodate the high flux of protons through the rotary $c$-ring, organisms evolved the increased stoichiometry of the $c$ monomers $(145,151)$. According to the Boyer binding change mechanism, one complete rotation of subunit $\gamma$ driven by the rotation of the $c$-ring induces conformational changes in three catalytic sites in $\beta$, synthesizing/hydrolyzing three ATP molecules. In the decameric $c$-ring of E coli ATP synthase $\left(c_{10}\right)(194)$, the $\mathrm{H}^{+} /$ATP ratio equals to roughly 3.3 , leading to the requirement of translocation of 3.3 protons to synthesize one ATP molecule.

Interestingly, the chloroplast F-ATP synthase $c$-ring encompasses $14 c$ monomers $\left(c_{14}\right)(151)$, increasing the $\mathrm{H}^{+}$/ATP ratio to $\sim 4.6$. Such a high requirement for protons is rooted in a physiological context of the high energization levels of the chloroplast membrane due to the proton pumping mediated by membrane-bound photo-systems that generate high PMF in light conditions. Such high stoichiometry enables the chloroplast F-ATP synthase to act as a highly effective proton sink that prevents the buildup of the backpressure on the photosystems due to the increased proton concentration in the inter-membrane space of the chloroplast (200).

In mycobacteria, the energy requirements and generally low transmembrane proton gradient were as well the factors that drove the evolution of the $c$-ring stoichiometry (145). The recently solved crystal structure provided unprecedented detail of the overall organization of the $c$-ring of non-pathogenic $M$. phlei showing the assembly of $9 c$ monomers arranged around the central cavity (109). Each monomer is comprised of the typical two $\alpha$-helices connected by a conserved polar loop $\left({ }_{45} \mathrm{RQPE}_{48}\right)$ on the cytoplasmic side of the membrane. The interface between 
monomers has a binding site for protons and partakes in the proton shuttling to the subunit $a$.

It has been proposed that the $c$-ring has such number of $c$ monomers to accommodate the full rotation of the $\gamma$ even in the conditions of the low transmembrane proton gradient since it requires translocation of only 9 protons for the synthesis of 3 ATP molecules. From a physiological perspective, such architecture can maintain the constant ATP synthesis even at the low PMF values, often found in the macrophage environment during infection. Such an adaptation of the $c$-ring provides the mycobacteria the means to thrive at energetically unfavorable conditions, giving rise to the persistence of the TB.

The recent advancements in the drug discovery uncovered a potent antimycobacterial agent, BDQ that drastically reduces the bacterial loads in lungs during the onset of active TB as well as in the latent TB infection (18). The mechanism of BDQ action was long unresolved even though the spontaneous mutations in the $M$. tuberculosis and $M$. smegmatis c-ring that conveyed the resistance revealed it as a BDQ target (18). Recently, by using the crystallographic approach, it has been confirmed that the BDQ targets specifically the mycobacterial $c$-ring binding to the residues D32, E65 of one $c$-monomer while $\mathrm{I70}$ belongs to the adjacent monomer (109) that together form the proton-binding pocket. Interestingly, upon the BDQ binding, the side chain of the residue F69 undergoes a significant conformational alteration to accommodate the bulky BDQ moiety (Figure 1.13), which is not observed in the case of carrying the $\mathrm{H}^{+}$. 


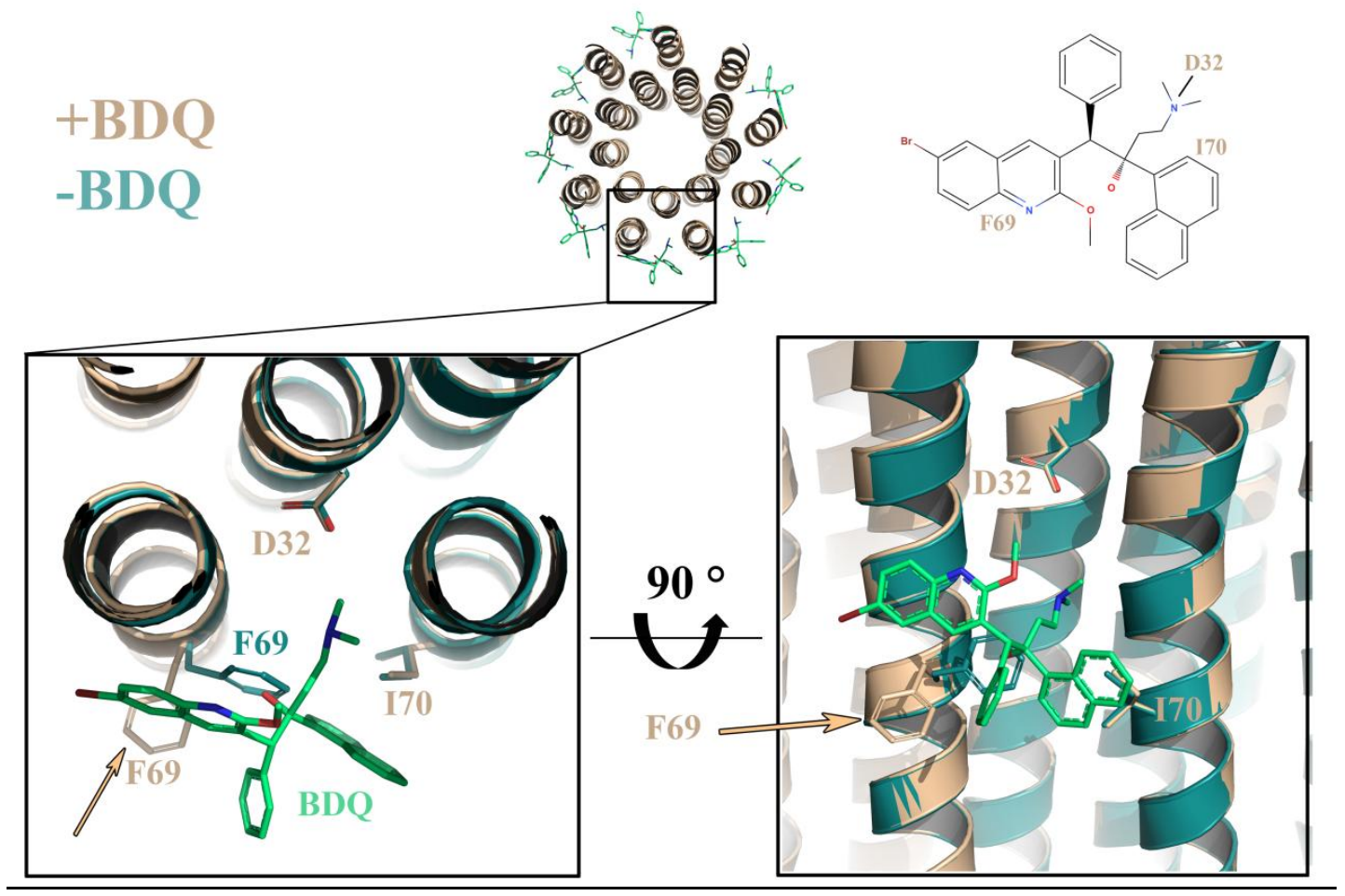

Figure 1.13: The structural alterations of the BDQ binging pocket in the $c$-ring. 3D crystal structure showing the $c_{9}$ ring of non-pathogenic $M$. phlei (PDB ID: 4V1F - wheat and 4V1G - emerald green, (109)) in complex with BDQ (light green) (top). The top view of the entire ring shows multiple binding sites between adjacent $c$ monomers. Enhanced top view (left frame) of the $c$-ring: BDQ (wheat) complex aligned with the structure of the $c$-ring in apo-form (emerald green) indicates the F69 (arrow) in altering conformation upon BDQ binding. The residues D32, I70 are also a part of the binding pocket. A side view (right frame) showing the geometry of the BDQ binding site.

From the functional aspect, the bulky moiety of the BDQ prevents the rotation of the $c$-ring against the membrane-embedded subunit $a$ by introducing the sterical clashes (109). As a result, the proton translocation through the $a$-c-ring interface is halted, and the enzyme arrests in the non-catalytic mode. This example has structurally validated mycobacterial ATP synthase as a clinically relevant drug target. As a prolonged utilization of BDQ has already caused the emergence of the resistant strains of $M t b$ (129), it is critically important to find other mycobacteria-specific drugs that bind exclusively to the epitopes in its F-ATP synthase. Such selective activity could disable the catalytic activity and deplete Mtb ATP pools during the infection of the human host, avoiding the occurrence of the resistance.

Comparative study of the sequences of mycobacterial F-ATP synthase pointed out that $\gamma$ subunit harbors a unique epitope whose presence was not determined in $E$. coli and any other bacterial subunits $\gamma$ (Figure 1.14-A). Subunit $\gamma$ is the main shaft of the central stalk that couples the catalytic events in the headpiece with the proton translocation through the $c$-ring (201). In structurally characterized bacterial enzymes, 
$\gamma$ is comprised of two elongated helices that partially wound around each other, forming the central rotary axle, attached to the globular domain that harbors both, short helices and $\beta$-sheet elements better known as Rossmann fold (202) (Figure 1.14B).

During the $\gamma$ rotation, in all described F-ATP synthases, the Rossmann fold interacts directly with the coupling parts of the subunits $\alpha$-SDLD and $\beta$-DELSEED to convey the conformational change to the catalytic hexamer but interestingly, in mycobacteria, the subunit $\gamma$ harbors an unusual insertion of the 13 highly polar ${ }_{166}$ DNGEDQRSDSGEG $_{178}$ residues in proximity of the Rossmann fold (Figure 1.14B). According to the NMR study, the synthesized peptide $M t \gamma_{166-178}$ assumes a structure of a flexible polar loop (203) whose role has been established in blocking the proton translocation through the $c$-ring. The experiments on the M. smegmatis IMVs with a genetic deletion of the $M t \gamma_{166-178}$ generated mutants whose ATP synthase shows a higher proton conductance, an increased ATP hydrolysis, as well as a reduced ATP synthesis in comparison to the WT (204). These properties actively contribute to the role of $M t \gamma_{166-178}$ in the prevention of the proton backflow through the $c$-ring. The interaction between $\gamma$-loop polar residues $\left(M t \gamma_{166-178}\right)$ and conserved ${ }_{45} \mathrm{RQPE}_{48}$ motif of the $c$-ring monomer are proposed epitopes that contribute to this phenomenon.

(A)

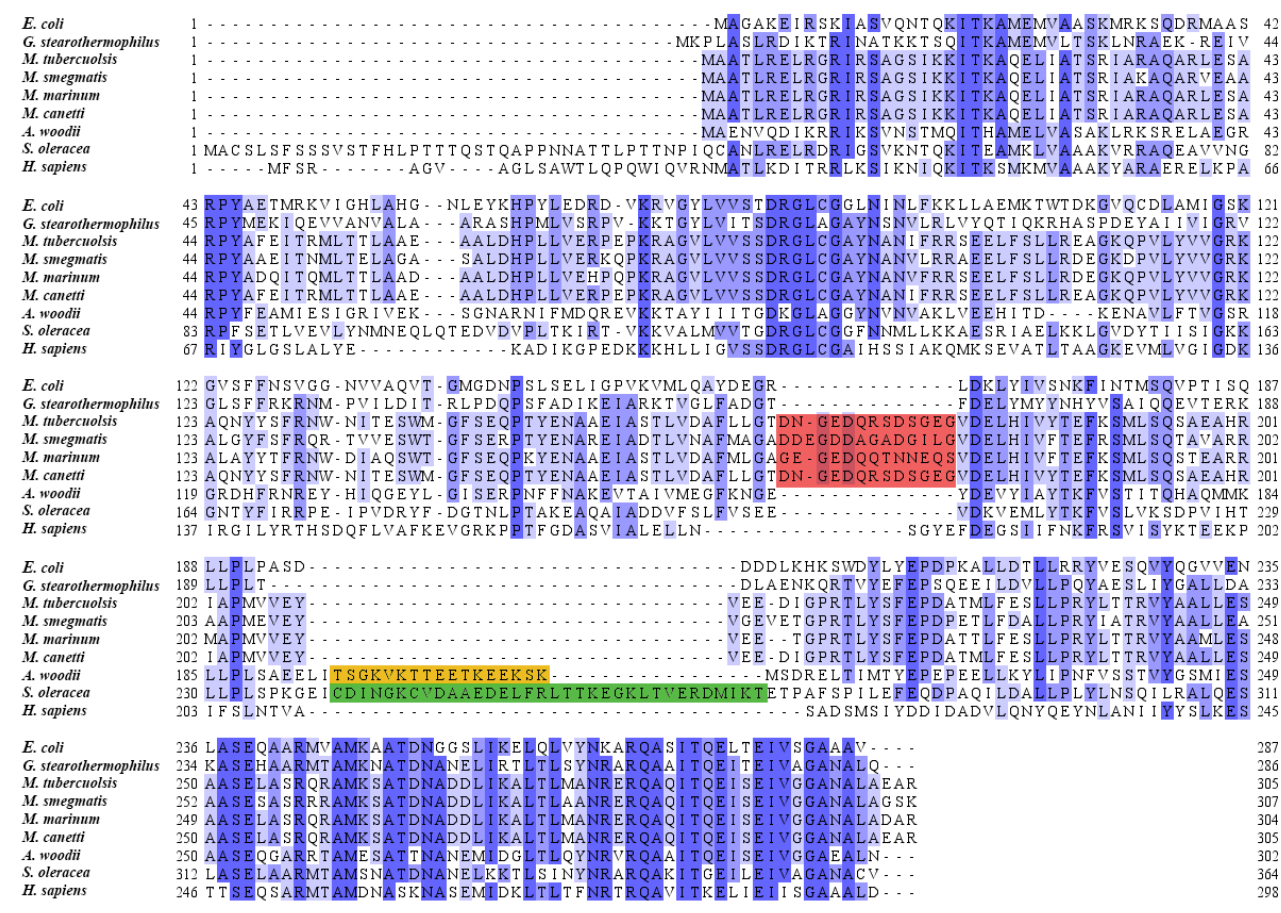


(B)

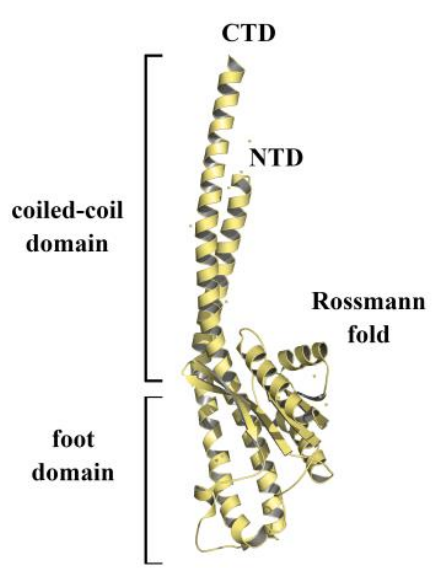

E. coli $\gamma$

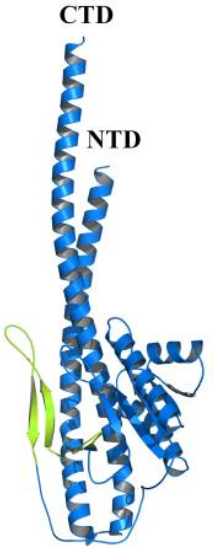

S. oleacea $\gamma_{240-273}$

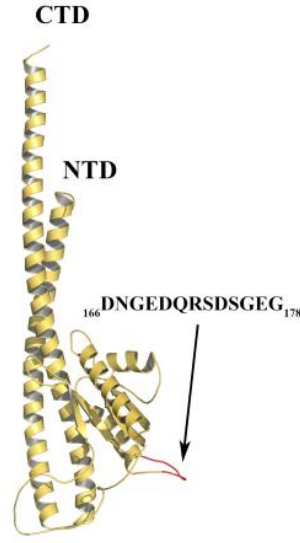

M. tuberculosis $\gamma_{166-178}$

Figure 1.14: Sequence alignment and comparison of $\gamma$ and its unique features. (A) Protein sequences of subunit $\gamma$ of various organisms display the unique stretches. The red square indicates a 14/15 amino acid insertion specific to mycobacteria. The yellow square indicates the unique sequence of 17 residues in subunit $\gamma$ of $A$. woodii with a proposed regulatory role in the catalysis. Green square represents the unique sequence in chloroplast $\gamma$, encoding the hairpin structure with a defined regulatory role in catalysis. (B) Comparison of the structural features of the $\gamma$ subunits shows the evolutionarily conserved 3D structure of $E c \gamma$ (PDB ID: 3OAA, G, (197)) (left) comprised of the extended coiled-coil domain, globular Rossmann fold and a foot domain. The chloroplast So (PDB ID: 6FKF, G, (151)) (center) displays the preserved fold with a unique regulatory hairpin loop (green). The homology model of Mty (right) showing the unique loop of 13 residues in proximity to the Rossmann fold. The figure under (A) is a part of (205).

In summary, the presented data indicate that mycobacteria-specific $\gamma$-loop is a critical epitope for coupling the rotation of central stalk with catalytic events and could be selectively targeted by anti-microbials $(203,206)$.

To further explore mycobacterial epitopes as a drug space, the other specific features of mycobacterial F-ATP synthases were investigated. The functional sequence motifs required for nucleotide binding (P-loop), torque transmission ( $\beta$-DELSEED), and the residues required for ATP hydrolysis ( $\beta E 183$ and $\alpha \mathrm{R} 376)$ are all conserved in mycobacteria (207) and yet, the inability of mycobacterial F-ATP synthase to operate in the hydrolysis direction is prominent in the case of $M$. bovis BCG while in $M$. smegmatis it is measurable but to a lower degree (2). It has been proposed that this phenomenon does not stem from the differences in the central catalytic mechanism; instead, it is proprietary to a different mycobacterial specialization.

Indeed, it is found that Mt $\alpha$ contains a specific 36 amino acid long C-terminal domain (Figure 1.15) in comparison to the F-ATP synthases of bacteria E. coli and $G$. stearothermophilus (3). In order to investigate the role of this epitope in the catalysis, the CTD peptide (514-549) was deleted $\left(\Delta \alpha_{514-549}\right)$ from the $M$. smegmatis genome using the recombineering approach. In the experiments conducted on the $M$. 
smegmatis WT and $\Delta \alpha_{514-549}$ mutant IMVs, deletion of the $\alpha_{514-549}$ peptide increased the ATPase activity by $64 \%$ and caused a $40 \%$ drop in the rate of ATP synthesis, indicating an important regulatory role of this unique Mta epitope (3). The regulation is most likely conveyed through the interactions with other mycobacteria-specific epitopes, however, this claim has not received a reliable experimental validation.

As no high-resolution structural information on the mycobacterial $F_{1}$ complex is available, further efforts were made to isolate and structurally characterize only the $M t \alpha_{514-549}$ peptide. The NMR studies have shown that, in its isolated form, $M t \alpha_{514-549}$ adopts a partial helical conformation in the $M t \alpha_{520-541}$ region flanked by two unstructured regions (514-519 and 542-549) (3) (Figure 1.15). However, during the study, it was not feasible to determine with certainty whether those regions would assume an ordered structure in the structural context of the entire $M t \alpha$.

(A)

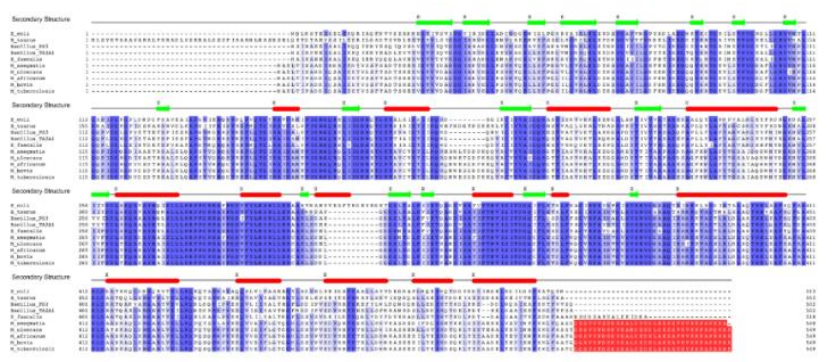

(B)
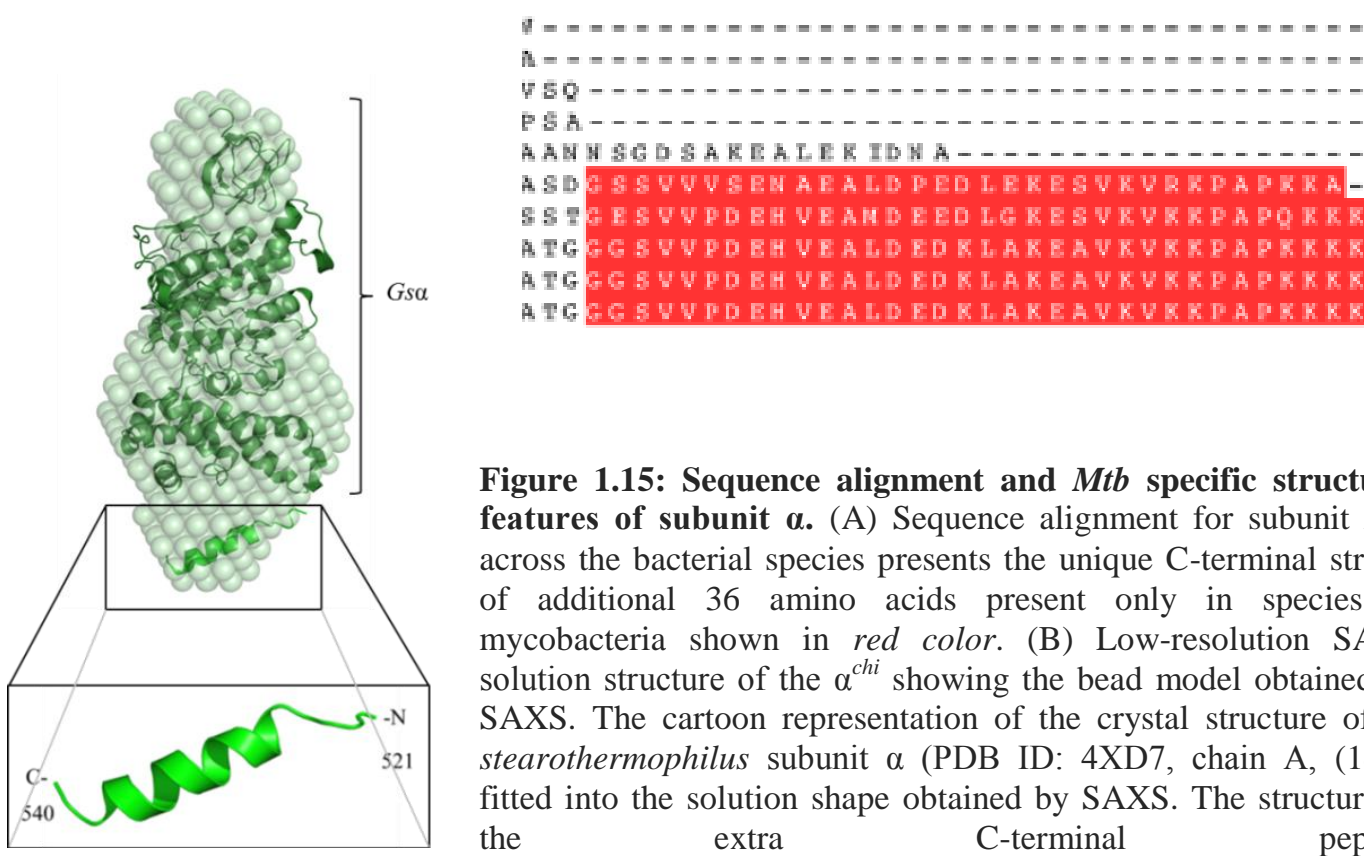

Figure 1.15: Sequence alignment and $M t b$ specific structural features of subunit $\boldsymbol{\alpha}$. (A) Sequence alignment for subunit $M t \alpha$ across the bacterial species presents the unique $\mathrm{C}$-terminal stretch of additional 36 amino acids present only in species of mycobacteria shown in red color. (B) Low-resolution SAXS solution structure of the $\alpha^{\text {chi }}$ showing the bead model obtained by SAXS. The cartoon representation of the crystal structure of $G$. stearothermophilus subunit $\alpha$ (PDB ID: 4XD7, chain A, (148)) fitted into the solution shape obtained by SAXS. The structure of the extra C-terminal peptide $\left({ }_{521}\right.$ PDEHVEALDEDKLAKEAVKV $\left.{ }_{540}\right)$ of $M t \alpha$ solved by NMR (3) is fitted into the solution shape. The figure under (A) credited to (3). 
Furthermore, the genetic manipulation was employed to generate a chimeric protein, hereon termed $\alpha^{c h i}$, consisting of mycobacteria-specific epitope Mt $\alpha_{514-549}$, fused to the C-terminus of $G s \alpha$, in order to observe its overall arrangement. Smallangle X-ray scattering (SAXS) studies (3) performed on $\alpha^{\text {chi }}$ showed the density corresponding to the mycobacteria-specific epitope in the proximity of the GsaCTD. Such placement could bring the $M t \alpha_{514-549}$ in the proximity of the $\gamma$ Rossmann fold and $\varepsilon \mathrm{CTD}$, and modulate the catalysis of the mycobacterial F-ATP synthase. Even though the recent isolated Mta SAXS studies corroborate these findings (4), the exact position of $M t \alpha_{514-549}$ epitope within the complex remains undetermined, and its interaction partners remain unresolved giving rise to one of the research questions addressed in this thesis. However, it is clear that the mycobacterial $\alpha \mathrm{CTD}$ extension $\left(M t \alpha_{514-549}\right)$ plays a vital role as the regulatory epitope and its precise interaction partners, as well as the mechanism of action, need to be further studied.

Considering well-studied role of bacterial subunit $\varepsilon$ in regulation of the catalysis, it was proposed that the $M t \alpha_{514-549}$ interaction partner could be CTD of subunit $\varepsilon$, here on referred to as MtECTD and thus in the next segment, the attention will be focused on the structural and functional aspects of $M t \varepsilon$ that could define it as a regulator of catalysis.

The protein sequence comparison pointed out that MtE consists of 121 amino acid residues and that it is 17 residues shorter than EcE (Figure 1.16) which prompted the structural and functional characterization (208).

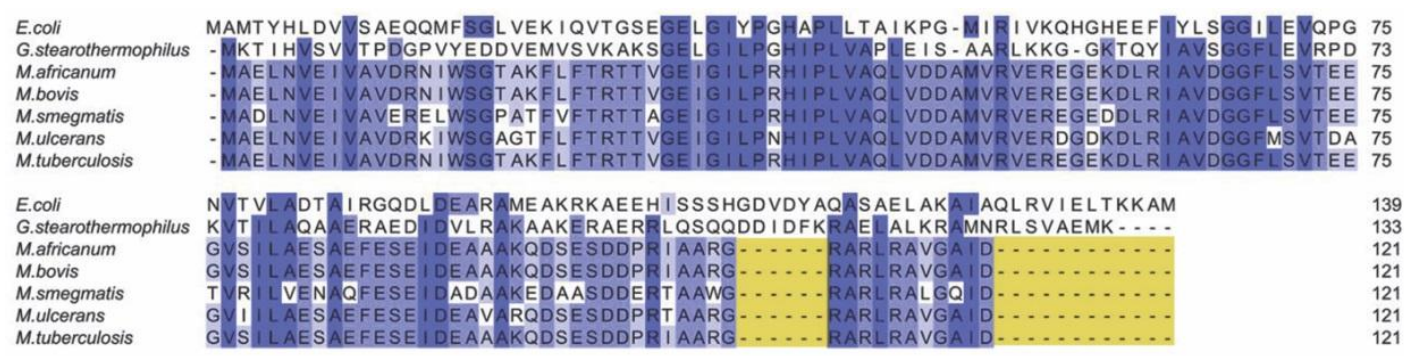

Figure 1.16: Protein sequence alignment of $\boldsymbol{M t \varepsilon}$. Sequence alignment shows shorted $M t \varepsilon C T D$ (yellow) in comparison with bacterial homologs.

The recently published MtE NMR solution structure revealed the overall arrangements of the functional domains, its dynamic properties and intramolecular network that sustain the conformational integrity in the solution (209). The entire protein is organized in two domains, evolutionary conserved $\beta$-barrel NTD and the 
specific CTD domain comprised of two short antiparallel helices (helix 1 and helix 2)

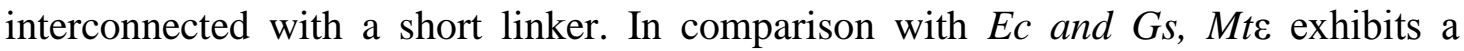
unique organization of its CTD. The two short helices are positioned in the proximity of the NTD, forming an interaction surface that encompasses NTD residues D47, D48, and A49 with CTD residues A108 and A112 NTD (Figure 1.17). The NMR dynamics show that these residues form a tight connection that reduces the flexibility of the CTD with respect to NTD and forces the two domains to act as a single globular protein.

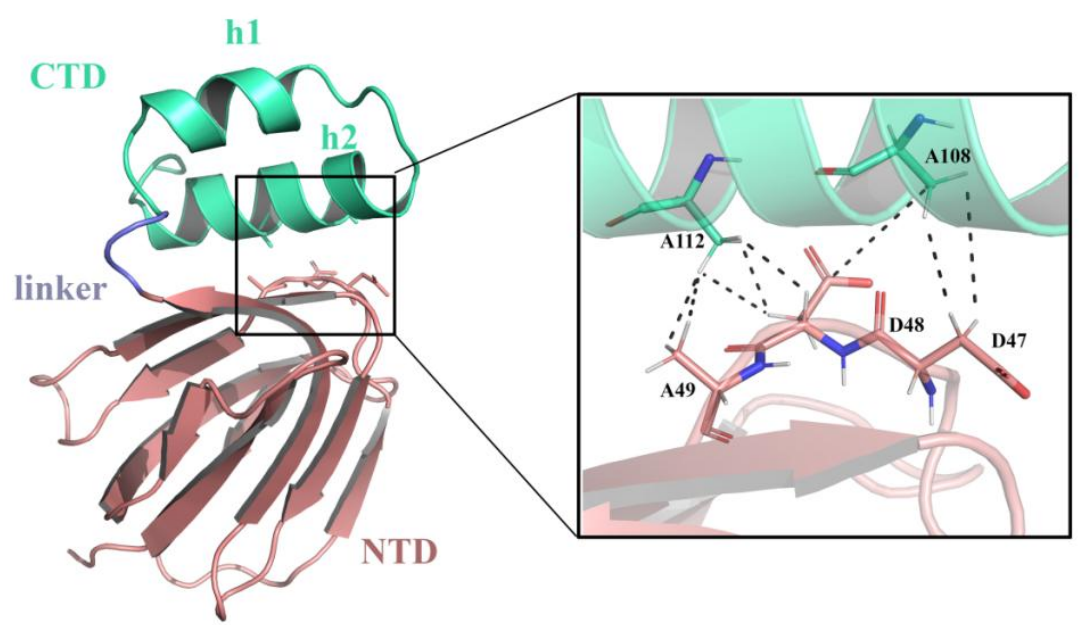

Figure 1.17: MtE inter-domain interactions. $M t \varepsilon$ displays a topological arrangement of its domains. $\beta$-barrel NTD (maroon) connects to the CTD (lime) via linker (violet); $h 1$ and $h 2$, helix 1 and helix 2. The insert shows an enhanced view of the residues forming the interface. The NTD residues D47, D48, and A49 engage in hydrogen bonding and hydrophobic interactions with CTD A108 and A112. Figure adapted from (209).

Furthermore, it has been shown that the NTD residues I15, W16, L61, R62, I63, F86 form a hydrophobic cleft and together with the linker residues E87, S88 and E89 couple to the CTD residues I90, D91, A112, R113, A116 and V117 forming a network of interactions (209) (Figure 1.18).

These interactions paired with the CTD epitope G118-D121 contribute to the maintenance of the closed conformation and tight coupling of the NTD with the CTD. Considering the fact that MtE does not bind the regulatory ATP (208), the authors have proposed that, in contact with a rotary $c$-ring during the catalysis, this network plays a crucial role in the $M t \varepsilon$ conformational transition from closed to open in the intact $\mathrm{F}_{1} \mathrm{~F}_{\mathrm{O}}$, which presents a novel regulatory mechanism involving subunit $\varepsilon$, specific only for the mycobacteria (209). 


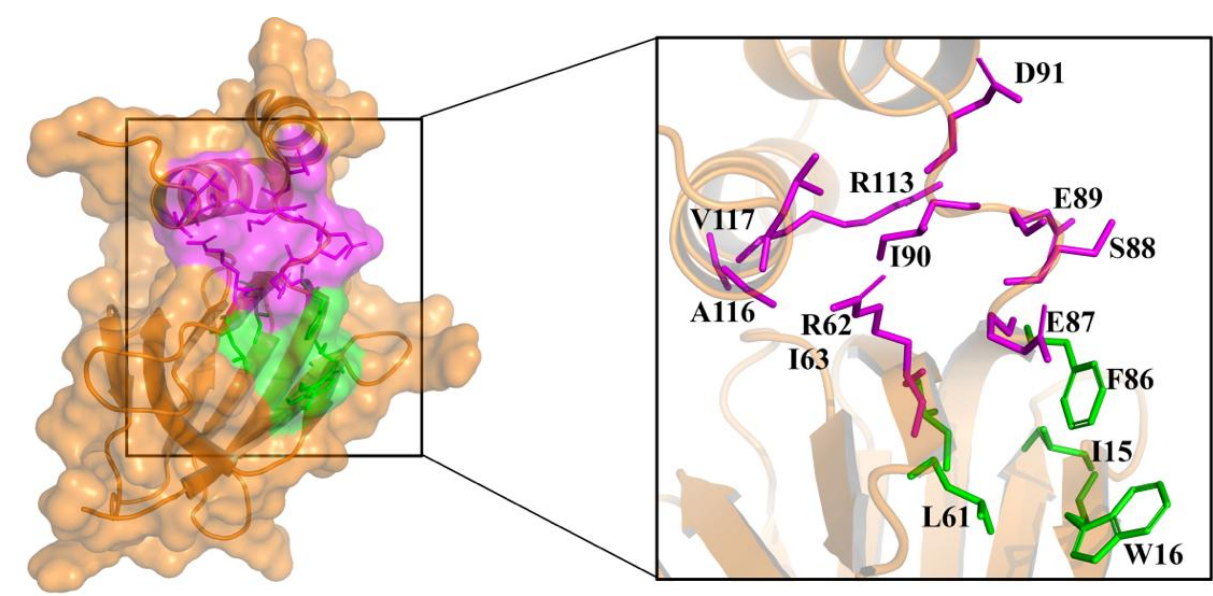

Figure 1.18: Interaction network within the MtE. Surface representation of $M t \varepsilon$ shows the formation of the hydrophobic cleft (green) that couples with residues in CTD. The frame shows the enhanced view of the interacting residues. The interacting NTD residues I15, W16, L61, and F86 are shown as stick representation and couple with CTD residues (magenta). Figure adapted from (209).

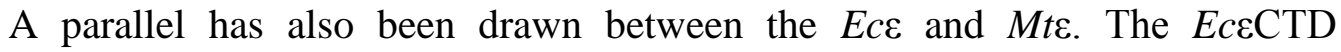
terminal residues ${ }_{136} \mathrm{KAM}_{138}$ are shown to be essential for the stabilization of the ATP hydrolysis inhibitory extended conformation within the $\alpha_{3} \beta_{3}$ hexameric head by forming a hook-like motif (197). Similarly, the effect of MtcCTD terminal residue D121 was investigated. The deletion of the terminal aspartate in the genomic atpC sequence of $M$. smegmatis is carried out via the recombineering to generate the mutant atp $C^{1-120}$. The mutation caused a $13 \%$ increase in the ATP hydrolysis and a $65 \%$ drop in ATP synthetic activity when compared to the WT, as measured in the IMVs. These outcomes indicated that D121 residue interacts with the catalytic head in an undefined manner and contributes significantly to the regulation of the catalytic activity of mycobacterial F-ATP synthase (209).

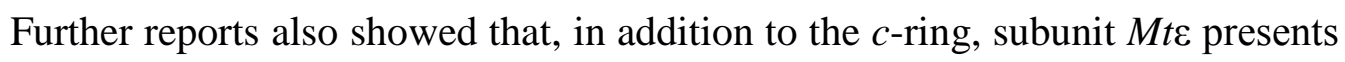
an alternate BDQ binding site (108). The tryptophan fluorescence (208) and NMR experiments (209) presented that, similarly to $c$-ring (F69), the BDQ binding site on the $\varepsilon$ involves the aromatic residues residues $\mathrm{W} 16$ and F86 that together with other residues (R13, N14, I15, S17 and A84), form a pocket of hydrophobic interactions. (Figure 1.19) (209). As for the fact that some of BDQ-binding residues also belong to the epitope that forms the network of interactions between MtECTD and -NTD, BDQ binding event could indeed affect the coupling between those domains.

The additional confirmation of the BDQ action on this epitope was uncovered in the experiments on the M. smegmatis IMVs. Specifically, the bulky side chain of the residue W16 was substituted with an alanine using the genetic recombineering to 
generate a mutant named $a t p C^{\mathrm{W} 16 \mathrm{~A}}$. It has been shown that the mutation causes a $9 \%$ increase in the ATPase activity and strikingly, 83\% decrease of the ATP synthetic capacity in comparison with the WT. Ultimately the in vivo experiments on the $M$. smegmatis atp $C^{\mathrm{W} 16 \mathrm{~A}}$ demonstrated that the mutation causes severe growth impairments in the presence of BDQ (108) emphasizing the role of subunit $\varepsilon$ as a potent inhibitor of ATP hydrolysis in mycobacteria.
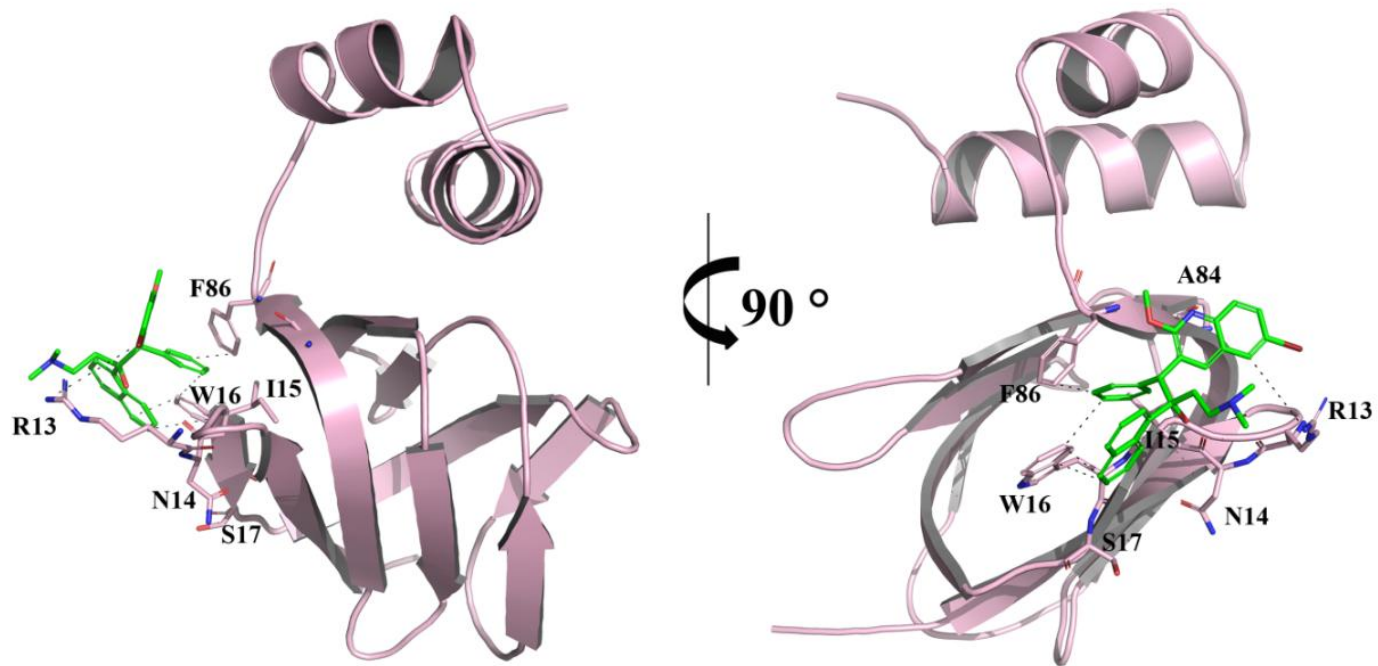

Figure 1.19: MtE in complex with BDQ. The cartoon representation of the docking model of $M t \varepsilon$ showing the residues involved in the geometrical organization of the BDQ binding pocket. The aromatic residues W16 and F86 form $\pi-\pi$ hydrophobic interactions with the BDQ (green) while R13 interacts with it via the $\pi$-cation interactions.

These facts presented previously undoubtedly show that the mycobacterial FATP synthase has many adaptations and specific epitopes that selectively prevent ATP hydrolysis. As described here, the most prominent epitopes are certainly unique Cterminal extension of subunit $M t \alpha\left(M t \alpha_{514-549}\right)$ and $M t \varepsilon$ with its comparatively short Cterminal domain. Here the study on the interactions of these two epitopes needs to take place together with the elucidation of the protein structure. However, obtaining the pure mycobacterial $\mathrm{F}_{1} \mathrm{~F}_{\mathrm{O}}$-ATP synthase proved to be challenging, and thus an alternative approach was taken to investigate the synergistic effect of these two epitopes on the catalytic activity. 


\subsubsection{Catalytic features of $\alpha^{c h i}{ }_{3}: \beta_{3}: \gamma$ complex and modeling of the $\alpha^{c h i}{ }_{-\varepsilon}$ interactions}

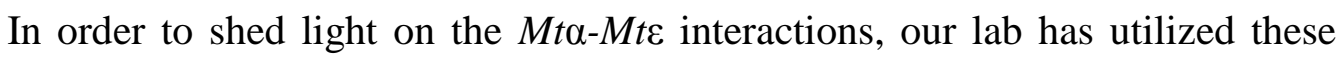
unique epitopes to generate a hybrid $\mathrm{F}_{1}$ complex and study its catalytic traits. This model system was based on well-studied enzymatic sub-complex $\alpha_{3} \beta_{3} \gamma$ from $G$. stearothermophilus whose $G s \alpha_{1-502}$ was genetically fused with the unique $M t \alpha_{514-549}$, to create a chimeric complex termed $\alpha^{\text {chi }}{ }_{3} \beta_{3} \gamma$ (3). The isolation of the highly pure complex enabled its characterization using single-molecule techniques. Namely, it has been proven that the incorporation of this mycobacterial epitope reduces the ATP hydrolytic activity by $13 \%$ in comparison to the $G s \alpha_{3} \beta_{3} \gamma$ which was caused by the reduced angular velocity of the rotating $\alpha^{c h i}{ }_{3} \beta_{3} \gamma$ complex upon binding of ATP to the catalytic site (3). This result goes well in line with the findings on the $M$. smegmatis IMVs that the deletion of the same epitope $\left(M t \alpha_{514-549}\right)$ caused a similar increase in ATPase activity (3), thus validating our model for further experimental use. In addition, the structural data from the SAXS studies conducted on the isolated $\alpha^{c h i}$ and $M t \alpha$ confirmed that their overall elongated shape was a consequence of the placement of the unique $M t \alpha_{514-549}$ epitope in proximity of the GsaCTD.

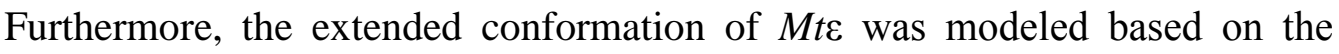
NMR structure of the closed conformation (PDB ID: 5YIO) (209) considering the fact that its proposed extended length is estimated to around $42 \AA$, which is $25 \AA$ shorter than the measured length of EcE and Gse in their extended conformations. Due to this structural trait, it has been postulated that the several CTD residues of the extended Mte model could interact with the coupling regions ( $\beta$-DELSEED and $\alpha$ ${ }_{396} \mathrm{AQFGSDLDK}_{404}$ ) and unique extended mycobacterial epitope $M t \alpha_{514-549}$ (Figure 1.20). The unique evolutionary $M t \alpha$ extension and $M t \varepsilon$ shortening, could potentially suggest co-operativity of these epitopes in terms of ATP hydrolysis functional inhibition in mycobacteria, which represents a theme in the scope of this thesis. 


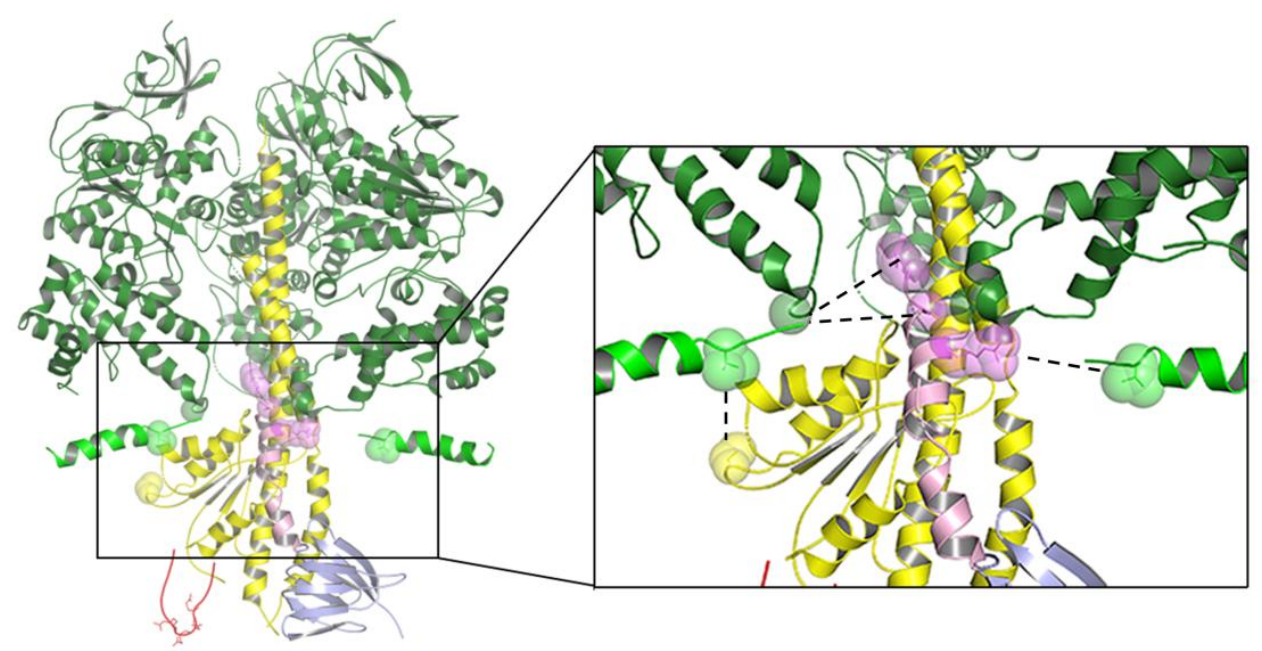

Figure 1.20: Proposed interactions of $\boldsymbol{\alpha}^{c h i}$ and MtECTD. The chimeric subunit $\alpha^{c h i}$ shown in green variants features the mycobacteria-specific CTD peptide (light green) genetically fused to the Gsa (forest green). The subunit Gs $\gamma$, (yellow) features the mycobacteria-specific loop $\gamma_{166-179}$ (red) that has been modeled to reflect its dimensions and hypothetic position within the complex. The extended conformation of subunit $M t \varepsilon$ was modeled to reflect its hypothetic length and emphasize the interacting epitopes within the complex $\alpha^{c h i}{ }_{3}: \beta_{3}: \gamma: M t \varepsilon$ represented as spheres.

This structural assembly model $\alpha^{\text {chi }}{ }_{3} \beta_{3}: \gamma: M t \varepsilon$ serves as a platform to define the interacting partners between $\alpha$ and $\varepsilon$ as well as to identify the crucial residues of Mt $\varepsilon$ that contribute to the activation/inhibition of ATP hydrolysis. Ultimately, with the profound understanding of these interactions, it could be possible to drive the discovery of the specific anti-microbial agent that would interfere with these interactions in order to promote/trap the mycobacterial ATP synthase in the continuous mode of ATP hydrolysis, wasting cellular ATP of $M t b$. As seen from the previous studies, BDQ targets specifically the mycobacterial F-ATP synthase $c$-ring and subunit $\varepsilon$ and stresses the essentiality of the F-ATP synthase in mycobacteria for their survival. Targeting those specific epitopes and causing the activation of the latent ATP hydrolysis would have implications in the treatment of the active TB as well as a latent infection.

\subsection{Overview of Acetobacterium woodii}

\subsubsection{Metabolic pathways of carbon assimilation and energy generation}

Virtually all living organisms must generate energy for their metabolic processes that allow them to assimilate a vast number of different substrates to sustain cell growth, division, and populations. The cyanobacteria, algae and higher plants utilize the photoactive pigments like chlorophyll to convert the energy of the sunlight into the chemical energy that fuels the assimilation of the atmospheric carbon dioxide 
$\left(\mathrm{CO}_{2}\right)$ and produce the organic, multi-carbon compounds in a process known as photosynthesis $(210,211)$. The assimilation can occur following distinct biochemical pathways known as C3, C4 and CAM metabolism $(212,213)$ that include various enzymes, specific to the life form and environment which the organism inhabits (212214), and this relation to the nutrient and energy acquisition is characterized as a photo-autotrophy.

On the other side of evolution, there are organisms classified as Eubacteria which had evolved to obtain the energy and carbon from the organic matter. The cleavage of the covalent bond of the organic substrate molecules provides both, the electrons required for the redox homeostasis of the cell, as well as the metabolic intermediates used as building blocks. The vast majority of these organisms thrive as saprophytic life forms, assimilating the organics from the soil, and generating the reducing equivalents in the form of $\mathrm{NADH}$ in the process. The energy in the form of ATP is generated via complex biochemical pathways involving the multiple enzyme cascades and the subsequent reduction of the molecular oxygen to water. Canonical examples of such organisms are prokaryotes like E. coli, M. smegmatis, $G$. stearothermophilus all characterized as saprophytic/commensalistic chemoheterotrophs with a species-specific modification of biochemical pathways to adapt to a particular niche they occupy.

Apart from saprophytes who obtain their essentials from the environment, there are life forms that competitively utilize the metabolites of other organisms, known as pathogens $(215,216)$. The pathogens reduce the viability/vitality of the host by employing more efficient systems and enzymes that outcompete the host defenses. One such example is $M t b$, an evolutionarily well-equipped pathogenic organism capable of surviving the macrophagal environment by entering into the persistence and resuscitating upon stabilization of the favorable conditions $(217,218)$. They owe this remarkable feat to the vast array of enzymes that are being selectively up/downregulated to enable metabolic shift accommodating the lack of the nutrients and oxygen found in the host $(79,219,220)$.

During the active growth phase, $M t b$ utilizes the carbohydrates as the primary carbon source (221), while the persisters switch the metabolism to utilization of the macrophage-derived cholesterol and other lipids to sustain the persistence (222-224). In the actively growing $M t b$, the assimilated carbohydrates get converted to glucose that is further metabolised through the glycolytic pathway, followed by the Krebs 
cycle and oxidative phosphorylation. These processes result in the generation of the reducing equivalents (NADH) and ATP that serve as the core energy currency of the cell and support its growth.

In an alternate evolutionary branch from Eubacteria (Figure 1.20), the separate, ancient group of organisms has evolved in the primordial atmosphere and the niches devoid of organic material. The Archaea have long avoided identification and placement in the phylogenetic tree of life due to the insufficient characterization of their morphological and metabolic traits as well as a faulty classification system that was based on the dichotomy of life. In the early nineties of the $20^{\text {th }}$ century, the taxonomic classification system was revised, and Archaea have emerged as a separate kingdom of life (Figure 1.21) (225).

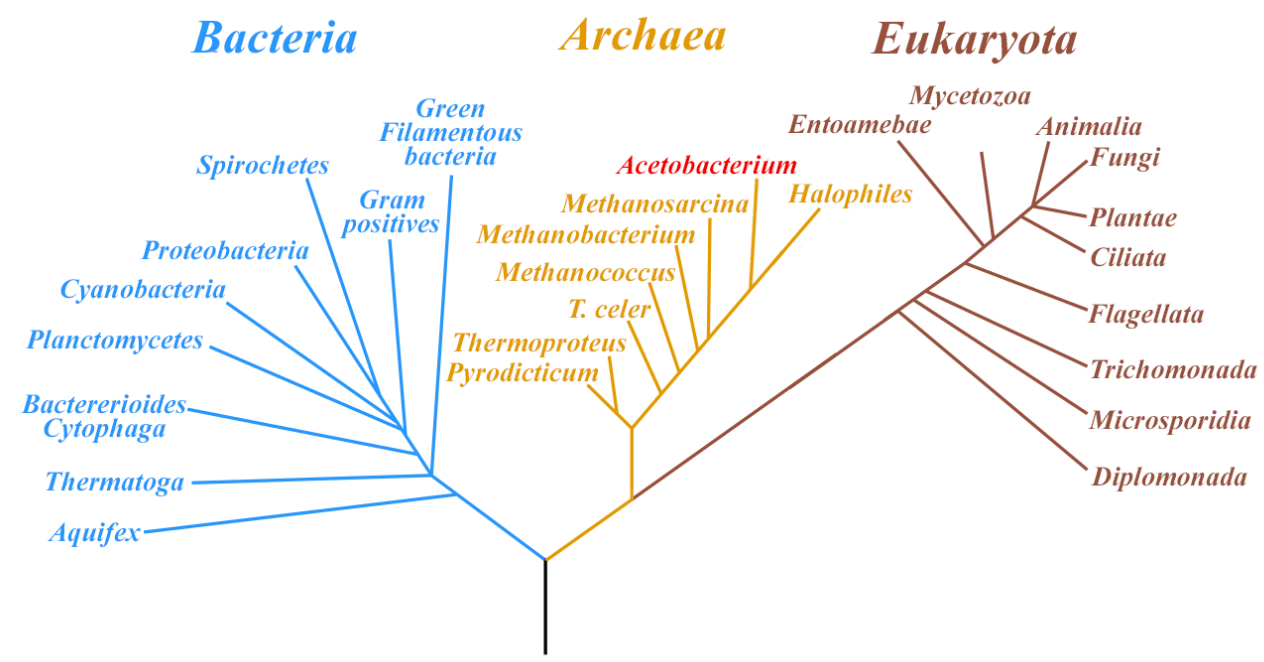

Figure 1.21: The tree of life. The rooted tree represents taxa segregated into the three domains of life: Eubacteria, Archaea, and Eukaryotes according to the revised phylogenetic classification (225). Acetobacterium woodii (red) is classified as an archaeon and its origins are stemming from a methanogenic/halophilic group of Archaea. Figure adapted from (225).

Owing to their evolutionary origins, groups of prokaryotes like methanogenic (226,227) (Metanosarcina mazei Gö1) and acetogenic bacteria (Acetobacterium woodii) $(228,229)$, have developed what is now considered as unconventional biochemical pathways that support the assimilation of atmospheric $\mathrm{CO}_{2}$ and hydrogen $\left(\mathrm{H}_{2}\right)$. The assimilation of $\mathrm{CO}_{2}$ and $\mathrm{H}_{2}$ provides the chemical energy and the substrate for the synthesis of ATP and higher metabolites earning the name chemo-autotrophs $(230,231)$. Most known chemo-autotrophs inhabit and thrive in the environments with extreme environmental conditions such as high pressure, extreme temperatures, 
salinity, and $\mathrm{pH}$, often found in the deep marine sediments, volcanic gutters, glacial ice, and anoxic subterranean niches, earning them the name extremophiles $(232,233)$.

In nature, there are many examples of adaptations arising from environmental pressure, and as a result, the niche emerges as an evolutionary factor that provides both, the nutrients and limitations, and the delicate balance between these factors drives and tunes these adaptations. In this context, the acetogenic bacteria, in particular, A. woodii, has evolved a specific metabolic system for the assimilation of $\mathrm{CO}_{2}$ and $\mathrm{H}_{2}$ yielding acetate as an end product via highly specific Wood-Ljungdahl pathway (234-236), synthesizing ATP at substrate-level phosphorylation. In addition, A. woodii utilizes the unique "hybrid" F-ATP synthase $(6,237-239)$ and a $\mathrm{Na}^{+}$-motive force to generate sufficient ATP for the sustenance of life. How these adaptations arose as well as their origins will be considered in the following chapter.

\subsubsection{Introduction to $A$. woodii}

The early life emerged in a reducing atmosphere saturated with $\mathrm{H}_{2}$ and $\mathrm{CO}_{2}$ where no or extremely low oxygen amount $(0.001 \%)$ was present $(240,241)$. These conditions, along with low concentration of organics, forced newly emerging life to adapt or perish. There are multiple examples of various adaptations of the cell to overcome the limiting factors, thrive and multiply and one remarkable example is $A$. woodii, a prokaryote with metabolic adaptations like no other $(237,238,242-245)$. It has been discovered and isolated from black sediment of Oyster Pond inlet, in Massachusetts, USA which has specific salinity and amount of organics to fuel its growth (246).

A. woodii is described as an anaerobic bacillus without the capability to sporulate (246). Its cell wall structure classifies it in the gram-positive group of rodshaped acetogenic bacteria. The single cells (Figure 1.22) most often tend to adhere to an adjacent cell forming a defined pair with a single, long flagellum utilized for motility. However, eventhough there are particular morphological adaptations, the impact of the evolution in these conditions is best seen at the level of metabolic prowess of $A$. woodii. 


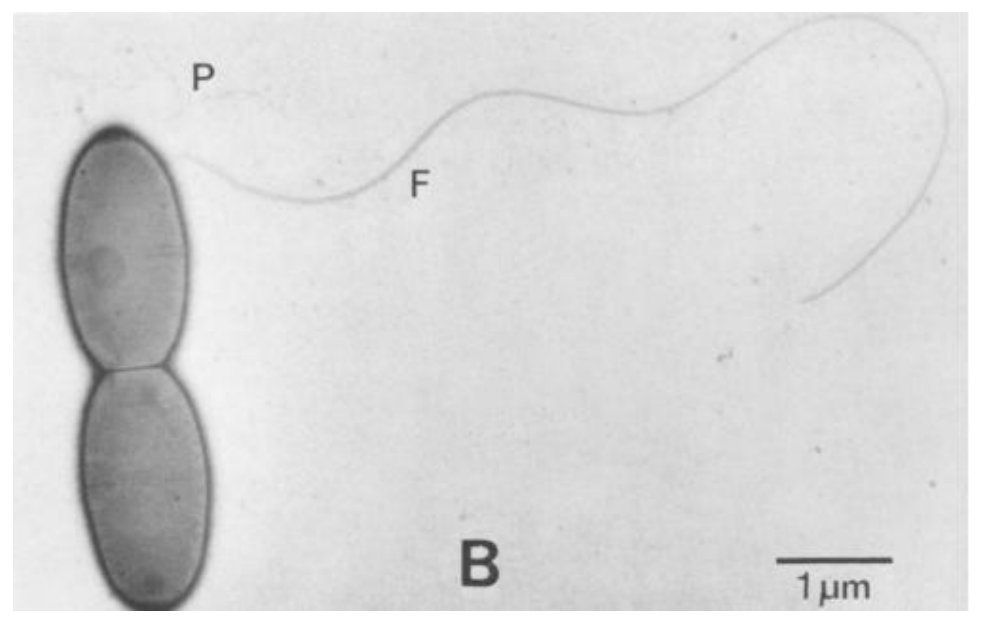

Figure 1.22: Electron micrograph of the $A$. woodii. The cells usually occur in pairs $(\mathrm{P})$ and use a single flagellum $(\mathrm{F})$ for the motility through the environment. (Image credited to (247)).

The anaerobic, hypersaline $\left(\mathrm{Na}^{+}\right)$waters, where $A$. woodii was initially isolated, imposed selective pressure on its energy generation mechanisms, primarily respiratory chain components and F-ATP synthase, as well as flagellar motion and nutrient intake systems (248-250). As a chemo-autotrophic bacterium, A. woodii relies on the reduction of $\mathrm{CO}_{2}$ by $\mathrm{H}_{2}$ for the production of its biomass, which is facilitated by highly specific enzymes of Wood-Ljungdahl (WL) pathway that yield ATP and acetate as the end products of anaerobic metabolism $(236,250)$. The ATP generated in WL pathway does not provide sufficient energy for the sustenance of life, and thus A. woodii has employed other systems that fuel the ATP generation such as specialized electron transport chain (251) and $\mathrm{Na}^{+}$-dependent oxidative phosphorylation carried out by $\mathrm{F}$ ATP synthase $(238,239,252)$.

\subsubsection{Carbon fixation and Wood-Ljungdahl pathway}

The Wood-Ljungdahl pathway combines the fixation of the $\mathrm{CO}_{2}$ and ATP synthesis by using molecular hydrogen as the energy source $\left(\mathrm{H}_{2}\right)$ and, could be considered to have evolved on earth very early on the conditions present in the reductive atmosphere $(253,254)$.

$$
2 \mathrm{CO}_{2}+4 \mathrm{H}_{2} \rightarrow \mathrm{CH}_{3} \mathrm{COOH}+2 \mathrm{H}_{2} \mathrm{O} \Delta \mathrm{G}=-25.6 \mathrm{~J} / \mathrm{mol}
$$

The pathway follows the reduction of carbon dioxide to formate, using the tetrahydrofolate as a cofactor (THF) and subsequent reduction of the formyl group to methyl-tetrahydrofolate. Additional carbon dioxide fixed by reducing it to carbon 
monoxide that stays bound to the enzyme and subsequently condensed with a methyl group and coenzyme A to yield acetyl-CoA (Fig. 1.23). Acetyl-CoA is the precursor in amphibolic reactions, namely molecule for biosynthetic reactions and in the catabolic route the precursor of acetate. The newly formed Acetyl-CoA could be used for the synthesis of ATP via substrate-level phosphorylation which is being canceled out in the formate activation step to formyltetrahydrofolate $(229,234,235,250)$. Therefore, the net production of ATP via this pathway by substrate-level phosphorylation is zero leaving unanswered questions of the origin of metabolic energy. As the end product of this pathway, the acetate is produced and secreted into the medium which lowers the $\mathrm{pH}$.

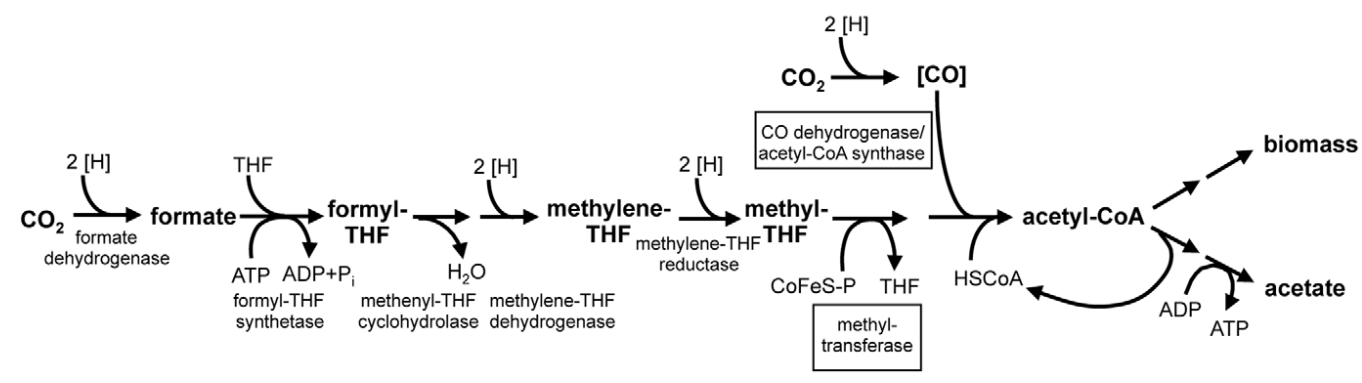

Figure 1.23. Overview of the Wood-Ljungdahl pathway (235). The assimilation of atmospheric $\mathrm{CO}_{2}$ and $\mathrm{H}_{2}$ begins with the reduction of $\mathrm{CO}_{2}$ to formate $(1 \mathrm{C}$ atom) by the formate dehydrogenase. The next step is facilitated by formyl-THF synthetase by utilization of ATP to form formyl-THF (1C atom). The next transformation occurs with the release of $\mathrm{H}_{2} \mathrm{O}$ via methenyl-THF cyclohydrolase and further reduction via methylene-THF dehydrogenase to produce methylene-THF (1C atom). Methylene-THF reductase uses $\mathrm{H}_{2}$ for further reduction to methyl-THF ( $1 \mathrm{C}$ atom) that gets converted into acetyl-CoA in two subsequent steps involving methyl-transferase and reduction of another $\mathrm{CO}_{2}$ molecule via acetyl-CoA synthase. The CoA is recycled while the acetate is produced yielding ATP in the reaction. Figure credited to (235).

In aerobic bacteria, the electron transport chain is utilized to generate protonmotive force that drives the F-ATP synthase to produce ATP in a process called oxidative phosphorylation. The proton gradient across the cell membrane is generated via oxidation of $\mathrm{NADH}$, succinate, and transfer of the electrons to molecular $\mathrm{O}_{2}$ in the ETC of aerobic organisms. However, due to the reliance of $A$. woodii on the anaerobic respiration to generate the ion-motive force, the mechanism of the utilization of the gradient across the membrane might differ significantly and till recently were insufficiently characterized.

The anaerobic respiration occurs in the absence of the molecular $\mathrm{O}_{2}$ that acts as a terminal electron acceptor. The existence of the alternate complexes partaking in the redox reactions has long been proposed, and finally, the respiratory chain of $A$. woodii has been described (251). The bulk of the redox reactions are being carried out by 
ferredoxin:NAD ${ }^{+}$oxidoreductase (Rnf complex), a member of the family of oxidoreductases whose role in electron transfer was initially proposed in anaerobic bacterium Rhodobacter caspulatus (255). The Rnf complex is defined as ferredoxindependent $\mathrm{NAD}^{+}$oxidoreductase, a membrane-integral hexameric protein complex that utilizes multiple iron-sulfur clusters [4Fe-4S], FMN and FAD as cofactors and assumes a role in respiration homologous to the role of ETC complexes found in aerobic bacteria. In $A$. woodii, the Rnf-complex is shown to couple the reversible electron flow with exclusive $\mathrm{Na}^{+}$translocation across the cellular membrane, generating the $\mathrm{Na}^{+}$-motive force that can be utilized by F-ATP synthase to produce ATP.

Most organisms rely on PMF for ATP synthesis, however, A. woodii adaptation to exclusively utilize $\mathrm{Na}^{+}$-motive force might have arisen due to the incompatibility of the WL pathway and PMF utilization. Considering that the acetate generated in the WL pathway, can shuttle protons across the membrane, collapsing the proton gradient in the process, $A$. woodii had adapted its F-ATP synthase to accommodate exclusive and un-promiscuous bi-directional translocation of sodium ions and use this energy for ATP synthesis.

\subsubsection{Structural and functional components of the $\mathrm{Na}^{+}$-translocating $\mathrm{F}$-ATP synthase of $A$. woodii}

The research of the bacterial genomes helped to develop a concept that the prokaryotic genes related to a single function, are often found clustered into operons. The operonic organization of coding and regulatory sequences promotes functional combining of single gene products (peptides) to form multimeric protein complexes whose expression is under the control of a single promoter. The clustering enabled such genes to undergo fast recombineering, and their inheritance/exchange through the microbial community was facilitated.

For virtually all organisms, the genes related to the monitoring of the external nutrients and respiratory substrates, as well as genes encoding the enzymes of the ETC are essential for survival and adaptation to the changing conditions of the environment. Through evolution, one such operon is highly conserved but also highly divergent. The operonic sequence of $A$. woodii F-ATP synthase genes is comprised of single copies of the atpI, atpA, atpG, atpD, atpC, atpB, and atpH genes, while the atpE gene that encodes subunits of the $c$-ring, has multiple copies with various sequences $a t p E_{l}$, 
$\operatorname{atp} E_{2}, a t p E_{3}$ that encode the subunits $c_{1}, c_{2}$, and $c_{3}$, respectively (252) (109). This indicated early on that the adaptive radiation had occurred and that gene duplications/recombinations were stabilized to facilitate the ion/proton translocation through the $\mathrm{F}_{\mathrm{O}} c$-ring, however not much was known about its composition or the stoichiometry of $A$. woodii $\mathrm{F}_{1} \mathrm{~F}_{\mathrm{O}}$-complex.

Detailed analysis of its operon showed that the atpE exists in 3 variants: atp $E_{1}$, $\operatorname{atp} E_{2}, a t p E_{3}$ encode subunits $c_{1}, c_{2}$, and $c_{3}$, respectively (252) (109). The sequence of $a t p E_{1}$ gene is roughly two times longer than that of the $a t p E_{2}$ and $a t p E_{3}$, which have identical sequences. These facts prompted a question of the expression pattern and stoichiometric assembly of $c$-monomers to form a functional $c$-ring.

However, not much structural information was obtained until the first attempt to characterize the $\mathrm{F}_{1} \mathrm{~F}_{\mathrm{O}}$ isolated from membrane vesicles. As initially determined by negative-stain electron microscopy (NS-EM), the ultrastructure of the A. woodii FATP synthase reflects the F-ATP synthase canonical organization into two domains, $\mathrm{F}_{1} \mathrm{~F}_{\mathrm{O}}$ connected by the central stalk (256). The dimensions inferred from the micrographs closely resemble the dimensions of the characterized F-ATP synthases from E. coli (257), bovine mitochondria and chloroplasts (258). The $\mathrm{F}_{1}$ domain measured $92 \times 105 \AA, F_{O} 78 \times 105 \AA$ with a length of central stalk spanning to about $42 \AA$, while the length of the entire complex was $\sim 205 \AA$, underlining that despite its biochemical specialization to operate on $\mathrm{Na}^{+}$, its overall structure does not deviate from the canonical form.

In more recent days, as the previous structural characterization did not answer the question regarding the $\mathrm{Na}^{+}$specificity and assembly of $A$. woodii F-ATP synthase, the structural and biochemical characterization of $\mathrm{Na}^{+}$binding pocket drew more attention.

In other F-ATP synthases, the ion/proton translocation occurs at the interface of two $c$-ring helices with a specific glutamate residue that can be reversibly protonated (Figure 1.7). The ratio of the protons to ATP synthesized is defined by the stoichiometry of the $c$-ring, more precisely the number of protonophoric spots. The $E$. coli $c_{10}$-ring translocates ten protons to synthesize 3 ATP molecules ( 3 catalytic sites in one rotation) contributing to the $\mathrm{H}^{+}$/ATP ratio of 3.3. Another F-ATP synthase, from chloroplast, harbors the $c_{14}$-ring requiring even higher $\mathrm{H}^{+} / \mathrm{ATP}$ ratio of roughly 4.6 while the $c_{9}$-ring of $M t b$ completes the synthesis of ATP even at a low proton motive force due to the $\mathrm{H}^{+} / \mathrm{ATP}$ ratio of 3 . The $c$-ring stoichiometry and number of 
ionophoric spots within seem to be correlated with the energetic state of the environment.

Considering the divergence of $A$. woodii in energy coupling mechanism (Rnfcomplex) (251) and the fact that $A$. woodii $c$-ring translocates $\mathrm{Na}^{+}$ions, it plausible that involved mechanism might differ from the $\mathrm{H}^{+}$translocation mechanism found in other F-ATP synthases. As shown by the extensive structural studies (259-262), this mechanism relies on the interactions between $c$-ring specific protonophoric glutamate and the specific "ratchet" arginine in the subunit $a$ that coordinate proton shuttling and release. In proton translocating F-ATP synthases, the inhibitor dicyclohexacarbodiimide (DCCD) is shown to block this activity by binding to the specific protonophoric $c$-ring glutamate. In analogy, the experiments carried out on A. woodii ATP synthase showed a pronounced ATPase inhibition with DCCD, proposedly via the similar interaction seen in the $\mathrm{H}^{+}$-translocating ATP synthases (239). Indeed, it was shown that $\mathrm{Na}^{+}$ions outcompete the labeled $\left[\mathrm{C}^{14}\right]$-DCCD manifesting as diminished labeling of $A$. woodii F-ATP synthase which undoubtedly confirmed that the binding spot involves the same specific $c$-ring glutamate.

Furthermore, a group of organic compounds with a guanidino functional group, the ameliorides, is shown to inhibit A. woodii F-ATP synthase (263) as well. The $\mathrm{Na}^{+}$ was able to abolish this inhibition, suggesting that amelioride guanidino group and $\mathrm{Na}^{+}$ compete for the same binding site. Considering that the amelioride guanidino group is analogous to the guanidino group found in arginine, it is discerned that the specific arginine is also involved in $\mathrm{Na}^{+}$shuttling in a way analogous to proton shuttling (263). As concluded from these experiments, A. woodii employs mainly a non-divergent mechanism for $\mathrm{Na}^{+}$translocation and the adaptation of its $c$-ring does not involve an alternate ionophoric site. However, the organization of it $c$-ring was still a subject of the scientific debate.

\subsubsection{Structural and functional characterization of the $A$. woodii $c$-ring}

The high-resolution crystal structure of the $A$. woodii $c$-ring was solved recently to $2.1 \AA$ (7). The hybrid $c$-ring is comprised of $c_{1}, c_{2}$, and $c_{3}$ subunits assembly that forms a heterodecameric ring with the stoichiometry $c_{1}: c_{2}: c_{3}$ to $1: 8.3: 0.7$, which presents a unique arrangement found to this day (7). The ring is organized into 22 transmembrane helices forming two concentric circles of eleven transmembrane helices each contributing to the total height of $72 \AA$ and widest diameter of $55 \AA$. 
Similar to the other c-ring structures described for Ilyobacter tataricus (264) the A. woodii c-ring assumes the lateral hourglass shape. The cytoplasmic surface exposes almost uniformly distributed negative charge, while the opposite side of the ring, at the periplasmic space, exposes polar residues and a highly hydrophobic extra NTD helix of the $c_{1}$ subunit that covers the central pore (Figure 1.24).

The hybrid $c$-ring contains the evolutionarily conserved multiple copies of 8 $\mathrm{kDa} c_{2 / 3}$ subunit encoded by atp $E_{2 / 3}$ and the unique subunit $16 \mathrm{kDa} c_{1}$ encoded by $\operatorname{atp} E_{1}$. The $c_{2 / 3}$ subunit is comprised of the two anti-parallel transmembrane membrane helices connected with a short, conserved electronegative ${ }_{42} \mathrm{RQPE}_{45}$ loop (Figure 1.24) on the cytoplasmic side which is shown to be essential for the coupling of the $c$-ring with the rotation of the central stalk. The $c_{2 / 3}$ helices maintain the canonical fold and a $\mathrm{Na}^{+}$binding site wedged between the helices of the two adjacent $c_{2 / 3}$ subunits. The central residue involved in $\mathrm{Na}^{+}$binding is a conserved glutamate E62 that coordinates $\mathrm{Na}^{+}$together with the tyrosine, threonine alanine, valine, and glutamine that from the pocket (Figure 1.24-B).
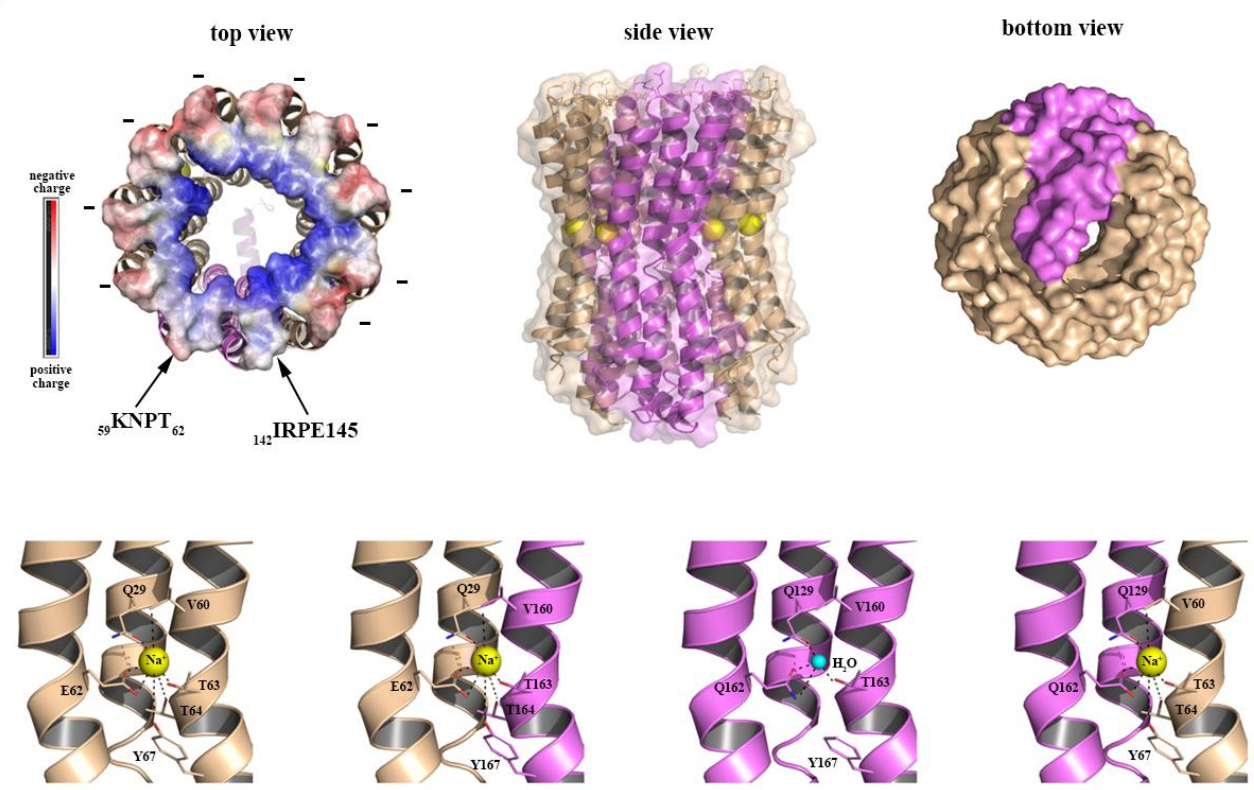

Figure 1.24: The structural properties of $A$. woodii $c$-ring (PDB ID: 4BEM, (109)). The calculated electrostatic potential shows mostly electronegative charge on the cytoplasmic surface of the $c$-ring (top left). The red color indicates electronegative charge; blue indicates electropositive charge located on the inner rim of the $c$-ring. The $c$-ring shown from the lateral perspective assumes the characteristic hourglass shape and reveals the hetero-oligomeric composition. Yellow spheres indicate $\mathrm{Na}^{+}$ion bound to the interface. The bottom view of the $c$-ring reveals the NTD helix of the subunit $c_{l}$ (top right) that covers the central cavity of the $c$-ring. The subunit $c_{I}$ (magenta) does not bind the $\mathrm{Na}^{+}$at its canonical position due to the replacements in the amino acid composition of the binding pocket while $\mathrm{Na}^{+}$is bound to all the $c_{2 / 3}$ subunits. The $\mathrm{Na}^{+}$ions bind to the $c_{2 / 3}-c_{2 / 3}, c_{2 / 3}-c_{1}, c_{1}$ $-c_{2 / 3}$ but not the $c_{1}-c_{1}$ interface as shown in the inserts in the second row. 
The other constituent of the hybrid $c$-ring is a unique $16 \mathrm{kDa} c_{1}$ subunit, comprised of four transmembrane helices connected with two unique cytoplasmic loops ${ }_{59} \mathrm{KNPT}_{62}$ and ${ }_{142} \mathrm{IRPE}_{145}$. The helices 1 and 3 comprise the inner part of the ring while 2 and 4 face the lipid membrane bilayer. The overall structure resembles the duplicated structure of the $c_{2 / 3}$ with the addition of unique $\mathrm{N}$-terminal short helix that projects across the periplasmic pore facing the distal $c_{2 / 3}$ subunit. The $\mathrm{Na}^{+}$binding sites are found to be located only at the helices 2 and 4 that form the binding pocket with adjacent subunits $c_{2 / 3}$, while the interface between helix 2 and 4 contains the nonconserved glutamine Q162 (substitution of glutamate) whose neutral charge does not promote the electrostatic interaction with $\mathrm{Na}^{+}$ion. However, the other residues comprising the pocket, together with Q162 bind the water molecule instead of $\mathrm{Na}^{+}$. As a result, the $c$-ring even though comprised of 10 subunits, was found to translocate only $8 \mathrm{Na}^{+}$ions per cycle generating the efficient ratio of $\mathrm{Na}^{+} / \mathrm{ATP}$ of around 2.7 (239). However, the functional role of this phenomenon is not entirely clear as well as the role of the extra NTD helix of the $c_{l}$ subunit.

In order to establish a role of variants of $c$ subunit in the $\mathrm{Na}^{+}$transport, the experiments on inverted membrane vesicles (IMVs) were carried out (6). The systematic deletion of $c_{3}$ from the operon of overexpressed protein caused very little or no alterations in the capacity of the $\Delta c_{3} \mathrm{~F}_{1} \mathrm{~F}_{\mathrm{O}}$ to catalyze the ATP synthesis/hydrolysis and translocate $\mathrm{Na}^{+}$as $c_{3}$ was replaced with $c_{2}$, deeming the $c_{3}$ dispensable for the assembly and function.

However, when the same approach was taken for the $c_{2}$ subunit, the enzyme assembly was hampered, and the $38 \%$ of ATPase activity was detected in comparison to WT. In addition, the combination of the deletion of both $c_{2}$ and $c_{3}$ subunits $\left(\Delta c_{2 / 3}\right)$ lead to a complete failure of $\mathrm{F}_{1} \mathrm{~F}_{\mathrm{O}}$ to assemble indicating that the $c_{1}$ subunit cannot form the homo-oligomeric $c$-ring (6). It was considered that the failure to assemble the $c_{1}$ ring occurred due to the presence of its N-terminal extra helix that would cause the sterical clashes in the decameric ring supra-structure, however, the helix deletion $\left(c_{142-}\right.$ ${ }_{18}$ ) did not remedy the outcome, and the $c_{1}$ ring did not assemble. Finally, the $c_{1}$ gene was deleted to establish its role. The obtained $\Delta c_{1} \mathrm{~F}_{1} \mathrm{~F}_{\mathrm{O}}$-ATP synthase, even though assembled from eleven $c_{2 / 3}$ subunits and functional, had drastically reduced stoichiometry of subunit $\varepsilon$ in the $\mathrm{F}_{1}$, which manifested as $24 \%$ ATPase activity in respect to the WT, possibly due to the synergistic effect of reduced efficiency in sodium transport and weakened coupling of $c$-ring and central stalk rotation caused by 
partial $\varepsilon$ depletion. These findings summarize particular roles of each $c$ subunit variant on the assembly and catalysis, indicating that the $c_{1}$ and $c_{2}$ are required for the proper assembly and coupling in the stoichiometric ratio present in the ring while $c_{3}$ variant is dispensable for assembly and catalysis (6).

Considering the specificities bound to the existence of the unique $c_{1}$ variant, other potential epitopes that regulate the assembly and catalysis were investigated. Here, in this thesis $\gamma$ emerges as unusual subunit due to the existence of the unique epitope whose role is not yet defined. 


\subsubsection{The unique epitope of $A$. woodii subunit $\gamma$}

From the comparisons of the A. woodii F-ATP synthase genes with other bacterial species whose F-ATP synthase was structurally and functionally characterized, it was spotted that the subunit $\gamma$ harbors an unusual stretch of amino acids absent in any other bacterial genus, the epitope ${ }_{195}$ TSGKVKTTEETKEEKSK $_{211}$ comprised of the 17 amino acids (Figure 1.25). In analogy to the functional role of the unique $\gamma$-loops in chloroplast and $M t \mathrm{~F}_{1} \mathrm{~F}_{\mathrm{O}}$ who mediate the inhibition of ATP hydrolysis and proton translocation, respectively $(151,206)$, the regulatory role of $A$. woodii subunit $\gamma$ is proposed.

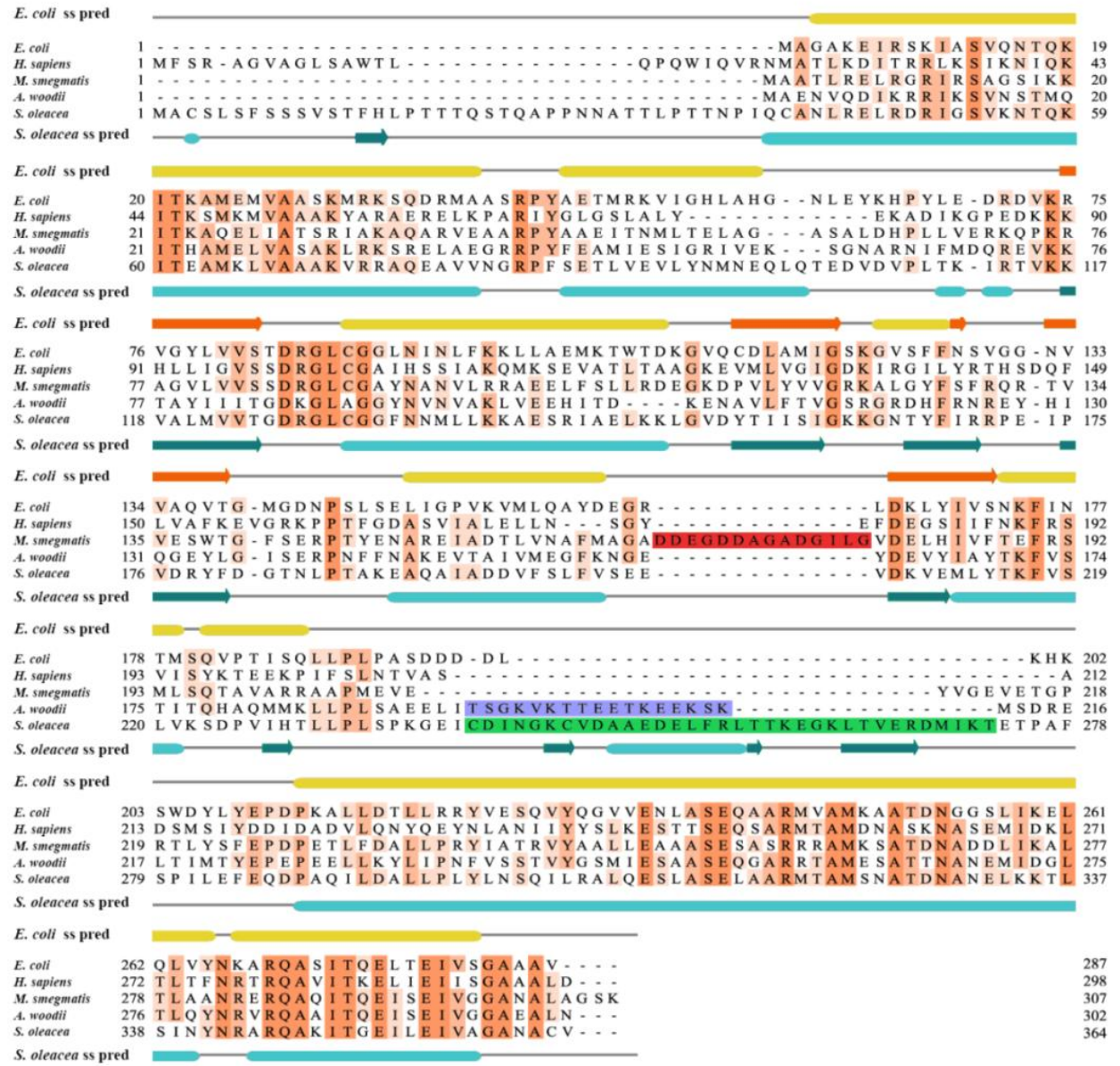

Figure 1.25. Protein sequence alignments of the $\gamma$ subunit of F-ATP synthases. The sequence alignment of subunit $\gamma$ from Escherichia coli, human mitochondria, Mycobacterium smegmatis, Acetobacterium woodii and Spinacea oleracea chloroplast featuring its unique regions. The secondary structural elements (yellow) of the E. coli $\gamma$ subunit, placed above the sequences, are based on the crystal structure PDB ID 3OAA and the cryo-EM structure 5T4O, 5T4P, 5T4Q. The secondary structures of $S$. oleracea, which are based on the cryo-EM structure (PDB ID: 6FKF; (151)), are depicted in light green and shown below the sequence alignment. The residues of the unique $\gamma$-loops of M. smegmatis, A. woodii and S. oleracea are boxed in red, magenta and green, respectively. The alignments were performed in Jalview software using default ClustalOmega algorithm settings (Sievers et al., 2011). The figure is the part of the author's publication (265). 
In order to derive possible effects of $A$. woodii $\gamma$-loop on the $\mathrm{Na}^{+}$translocation and catalysis, it was essential to consider it in the context of the interactions within $\mathrm{F}_{1} \mathrm{~F}_{\mathrm{O}}$. Since there is no high-resolution structural data available, as a part of this thesis, the homology model of $A$. woodii $\mathrm{F}_{1} \mathrm{~F}_{\mathrm{O}}$ was generated to test the importance of the $\gamma_{195}$ TSGKVKTTEETKEEKSK $_{211}$ epitope and propose the interaction partners. None of the subunits excluding $c$-ring crystal structure have been structurally described and thus the assembled structural homology models were derived using the online servers (section 2.2.35). The obtained model of $A$. woodii F-ATP synthase fitted well with the E. coli F-ATP synthase structure determined by cryo-EM. Apart from the structural heterogeneity of the previously solved $c$-ring structure (7), another crucial inferred difference was the existence of the extra $\gamma$-loop (Figure 1.26).
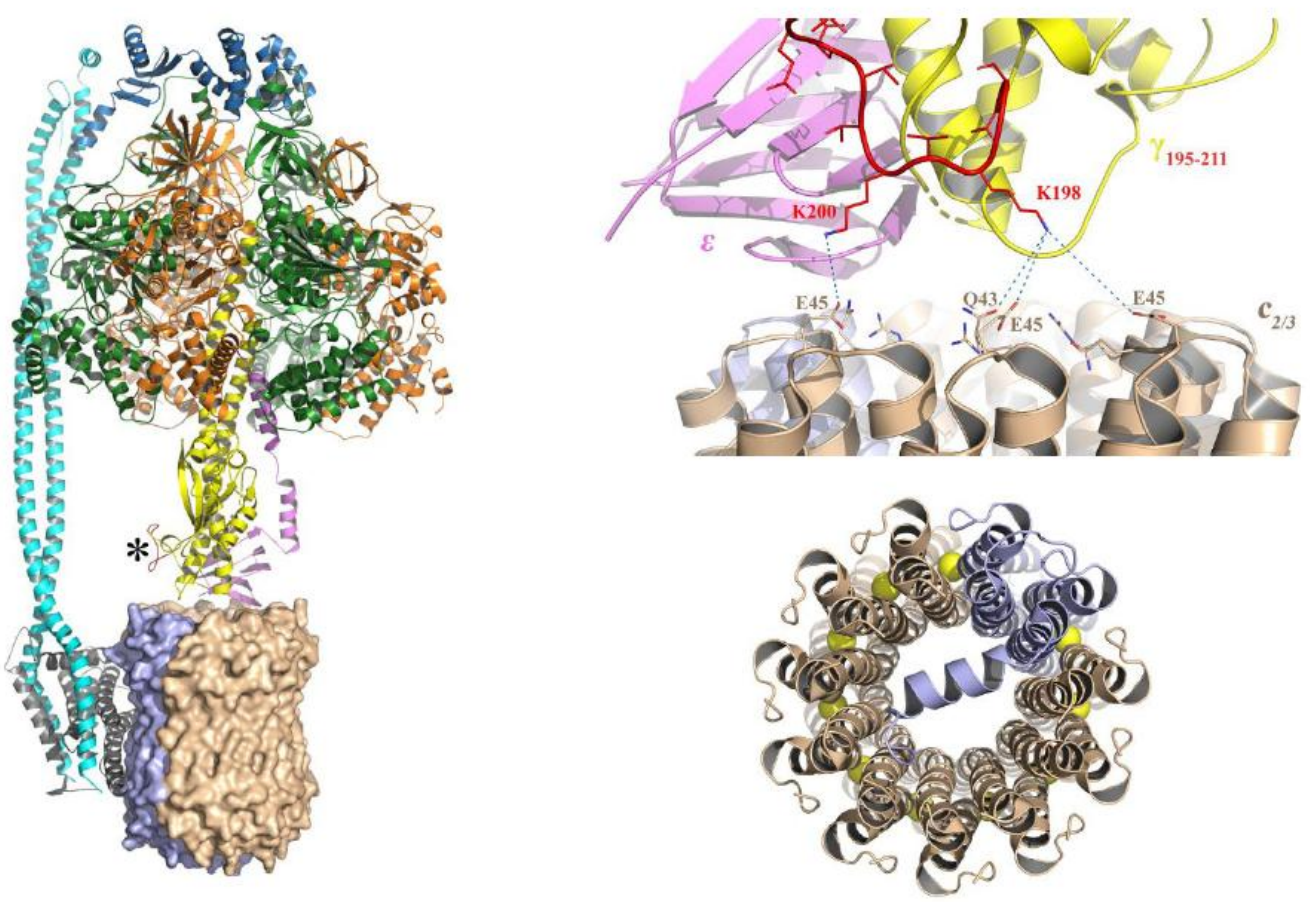

Figure 1.26: The unique A. woodii subunit $\gamma$-loop ${ }_{195}$ TSGKVKITEETKEEKSK $_{211}$. (A) Structural model of the A. woodii F-ATP synthase showing the overall arrangements of its constituents. The subunit composition is $\alpha_{3}$ (green), $\beta_{3}$ (orange), $\gamma$ (yellow) with its unique loop (red and highlighted by a $*$ ), $\delta$ (slate blue), $\varepsilon$ (pink), a (grey), $b_{2}$ (cyan), $c_{1}$ (purple) and $c_{2 / 3}$ (wheat). (right) periplasmic view of the $c$-ring of the $A$. woodii F-ATP synthase (PDB ID: 4BEM) (7) consisting of nine $c_{2 / 3}$ subunits (wheat) and a single $c_{1}$ subunit (light blue). Each subunit binds one $\mathrm{Na}^{+}$-ion (yellow spheres; bottom right). The $\mathrm{N}$-terminal helix in subunit $c_{1}$ lies across the central pore. Enhanced side view (upper right) shows the proposed interactions between the unique loop of subunit $\gamma$ ( ${ }_{195}$ TSGKVKITEETKEEKSK 211 ; red) and subunits $c_{2}$ (wheat). $\gamma \mathrm{K} 198$ is in the vicinity to Q43 and E45 of one subunit $c_{2}$ as well as with an E45 of a neighboring subunit $c_{2}$. Furthermore, $\gamma \mathrm{K} 200$ is in proximity to E45 of a third $c_{2}$-loop. Subunit $\varepsilon$ is presented in magenta. The structural models of the individual subunits were generated by SWISS-PROT server (266), and the best scoring hits were aligned with the E. coli $\mathrm{F}_{1} \mathrm{~F}_{\mathrm{O}}$ structure (PDB ID: 5T4O) using Pymol v1.8 (267). The figure is a part of the (265). 
From the model, it is visualized that the $\gamma_{195}$ TSGKVKTTEETKEEKSK $_{211}$ epitope assumes a disordered topology due to the lack of reference in the database for this structural motif. The $\gamma$-loop is modeled in the proximity of the Rossmann fold of subunit $\gamma$ occupying the space close to the $c$-ring cytoplasmatic epitopes ${ }_{42} \mathrm{RQPE}_{45}$. The distances between the sidechains of $\gamma$-loop and $c_{42} \mathrm{RQPE}_{45}$ were inferred to be between 5 and $7 \AA$, which is slightly higher than the average distances found in an obligate protein-protein interface. Considering potential dynamic properties and flexibility of the loop, it could be possible that the $\gamma$-loop extends towards the $c$-ring and interacts with the ${ }_{42} \mathrm{RQPE}_{45}$ epitope during the regulatory event.

As seen from the comparison of the amino acid sequence of $\gamma$ across the bacterial species (Figure 1.27), the $A$. woodii emerges as a separate branch, indicating that the regulation of its ATP synthase catalytic activity might have taken an evolutionary turn. As a part of this thesis, I am interested in describing the regulatory role of the $\gamma$-loop and proposing structural communication between the epitopes of $c$ ring with the $\gamma$-loop and $\varepsilon$, as well to structurally characterize the entire A. woodii $\mathrm{F}_{1} \mathrm{~F}_{\mathrm{O}}$-ATP synthase complex. To that end, the model was biochemically and structurally investigated and the interactions with $c$-ring were proposed.

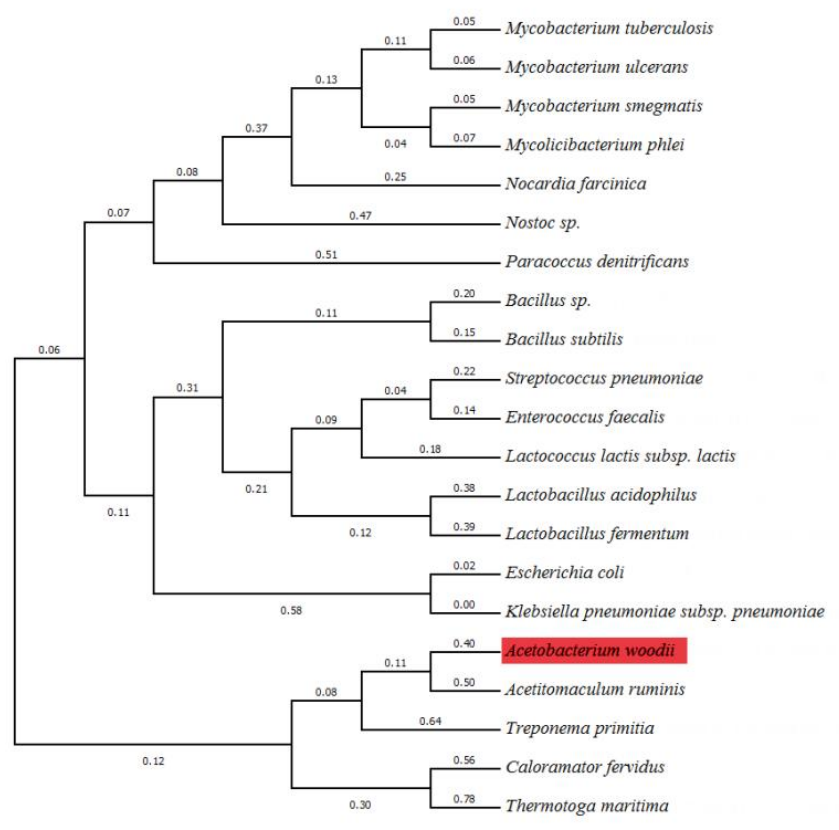

Figure 1.27: The phylogenetic tree of the selected bacterial and archaeal species. The three is formed by comparing the protein sequences of FATP synthase subunit $\gamma$. The $A$. woodii is related closely to the acetogenic archaea Acetitomaculum ruminis residing in the ruminant gut. The second clade shows the relation to Treponema primitia an acetogenic bacterium firstly isolated from the highly-acidic thermite gut. The third clade includes thermophilic bacteria Caloramator fervidus, isolated from the hot springs and Thermatoga maritima found in the hot springs and well as hydrothermal vents growing at staggering $80-90{ }^{\circ} \mathrm{C}$, resembling archaeal ecotypes. The organization of these organisms in the same quarternary clade indicates the evolutionary correlation between the energetic conditions of the environment and the adaptations in FATP synthase. Numbers indicate the genetic distance from the closest ancestral species. The phylogenetic tree is generated using MEGA7 (268). 


\subsection{Goals of the thesis}

The epitopes governing the regulation of the catalytic activity of the mycobacterial F-ATP synthase currently remain structurally and mechanistically uncharacterized. The biochemical and allosteric factors that regulate its activity are mostly undescribed. Mycobacterial F-ATP synthases predominantly carry out ATP synthesis with virtually no or residual ATPase activity, and, in addition, this enzyme was also identified as a drug target of the antibiotics that tackle $M t b$ infections. In order to manipulate its catalytic activity and exploit the potential lethality of ATP depletion, it is of primary importance to understand the mechanisms that regulate it. Precise mapping of the regulatory epitopes and insight in their interaction could hypothetically contribute to the activation of the latent ATP hydrolytic mode of catalysis, forcing the enzyme to hydrolyze ATP against the homeostatic equilibrium. By describing the subunits' $\alpha^{c h i}$ and $M t \varepsilon$ interaction space and uncovering the regulatory epitopes, obtained facts would provide the tools for drug-mediated selective activation of ATP hydrolysis with the ultimate goal to disrupt Mtb cellular ATP homeostasis and eradicate the pathogen. Considering the former, I am interested in:

1. Describing the 3D structure of the model complex $\alpha^{c h i}{ }_{3}: \beta_{3}: \gamma: M t \varepsilon$ using the negative-stain electron microscopy and validating the position of the $M t \alpha_{514-549}$ within the complex by using the crosslinking experiments.

2. Validating the specific residues of $M t \varepsilon$ that interact with $\alpha^{c h i}: \beta_{3}: \gamma$ complex using crosslinking experiments.

3. Describing the kinetic properties of the $\alpha^{c h i}{ }_{3}: \beta_{3}: \gamma: M t \varepsilon$ during the ATP hydrolysis and assigning the contribution of Mt\& to the catalysis.

4. Defining the specific residues and domains of $M t \varepsilon$ that affect the catalysis and/or association of the $M t \varepsilon$ with the $\alpha^{c h i}{ }_{3}: \beta_{3}: \gamma$

5. Investigating the effect of the $\operatorname{atp} C^{\mathrm{E} 87 \mathrm{~A}}$ mutation on the phenotype and BDQ susceptibility of $M$. smegmatis using the recombineering approach for mutant strain generation 
A. woodii has displayed remarkable evolutionary adaptations to the harsh conditions in which it thrives. There are a number of the theories and opinions on how the metabolism of this homoacetogen fuels ATP production and maintains energy homeostasis. We proposed that the specific epitope of subunit $\gamma$ is a critical catalytic factor that plays a role in blocking the utilization of ATP via ATP synthase, by interacting with the sodium translocating $c$-ring. We describe the structural homology model and use it as a tool to validate the role of the $\gamma$-subunit unique epitope ${ }_{195}{ }_{\text {TSGKVKITEETKEEKSK }} 211$ in catalysis and sodium translocation via the $c$-ring. As a part of this work, I am interested in:

1. Describing the $2 \mathrm{D}$ structure of the $A$. woodii $\mathrm{F}_{1} \mathrm{~F}_{\mathrm{O}}$-ATP synthase using the negative stain electron microscopy

2. Describing the role of unique ${ }_{195}$ TSGKVKITEETKEEKSK $_{211}$ of subunit $\gamma$ in ATP synthesis, ATP hydrolysis, and sodium translocation

3. Proposing the interactions between $c$-ring with $\gamma$-loop and $\varepsilon$ subunit

The description of the unique features of $M t b$ and $A$. woodii ATP synthases could contribute to:

- understating of the evolutionary steps taken towards the development of the functional aspects of these enzymes

- designing of the potent activators/inhibitors that can be a factor in the $M t b$-related health and disease

- enhancement of the industrial application of A. woodii in production of acetate on a large-scale 
2. Materials and methods 


\subsection{Materials}

\subsubsection{Chemicals}

All the chemicals used in the current study were of at least analytical grade. Chemicals were purchased from the following companies:

Buffers, Media and Salts

Dyes, ATTO fluorescent

DTT

$\mathrm{Ni}^{2+}$-NTA

Pefabloc $^{\text {SC }}$

PMSF

LB Media

$\mathrm{N}$-Acetyl-L-cysteine

Antibiotics

IPTG

BSA

ATP / ADP / AMPPNP
Sigma (St. Louis, MO, USA)

USB (Swampscott, MA)

Calbiochem (Darmstadt, Germany)

Fluka (Sigma, Buchs Germany)

Roth (Karlsruhe, Germany)

Serva (Heidelberg, Germany)

ATTO-TEC GmbH (Siegen,

Germany)

Hoefer (San Francisco, CA, USA)

QIAGEN (Hilden, Germany)

BIOMOL (Hamburg,

Germany)

Sigma (St. Louis, MO, USA)

BD (Sparks, MD, USA)

Sigma (St. Louis, MO, USA)

Calbiochem, (Darmstadt, Germany),

Sigma and Gibco, (St. Louis, MO, USA)

Fermentas (Vilnius, Lithuania)

GERBU (Heidelberg, Germany)

Sigma (St. Louis, MO, USA) 


\subsubsection{Electrophoresis Chemicals}

Agarose

SDS

Glycine

APS
Bio-Rad (Hercules, CA, USA)

Bio-Rad (Hercules, CA, USA)

Bio-Rad (Hercules, CA, USA)

Bio-Rad (Hercules, CA, USA)

\subsubsection{Molecular biology materials} plasmid DNA

KAPA HiFi polymerase

NcoI, SacI, BamHI, HindIII

T4 DNA Ligase

T4 Quick Ligase

Miniprep Plasmid Kit

Escherichia coli strains

pET-24b(+)

pET-9d(+)

KAPA Biosystems (UK)

Fermentas and New England

Biolabs, (UK)

Fermentas and New England Biolabs, (UK)

Promega, (Wisconsin, United States)

Qiagen (Hilden, Germany)

DH5 $\alpha$, Top10, Bl-21 (DE3), DK8, C41

Novagen, (Schwalbach am Taunus,

Germany

Grüber et al., 2002 


\subsubsection{Chromatography}

\subsubsection{Ion exchange}

RESOURCE $^{\mathrm{TM}} \mathrm{Q}(6 \mathrm{ml})$

Amersham Biosciences (New York

City, USA)

$\mathrm{Ni}^{2+}$-NTA resin

Qiagen (Hilden, Germany)

\subsubsection{Gel filtration}

Superdex 75 HR (10/30)

GE Healthcare (Uppsala, Sweden)

Superdex 300

GE Healthcare (Uppsala, Sweden)

\subsubsection{Instruments and accessories}

Akta FPLC, Akta Prime

Millex Filters $(0.45 \mu \mathrm{M})$

Syringe, needles and accessories

USA)
GE Healthcare (Uppsala, Sweden)

Millipore (Bradford, USA)

BD Biosciences, (San Jose, California,

\subsubsection{Protein concentration devices and estimation}

Centriprep YM10

Amicon ultra (100 kDa)

BCA Assay

BioSpec-nano

\subsubsection{Other instrumentation}

PCR Thermocycler:

Biometra $\mathrm{T}$ personal

Biometra $\mathrm{T}$ gradient

Sonoplus Sonicator

Micropulser Electroporator

Ultraspec 2100 Pro Spectrophotometer

Chalfont, UK)
Millipore (Co-cork, Ireland)

Millipore (Co-cork, Ireland)

Kit Pierce (Rockford, IL, USA)

Shimadzu Scientific, (Kyoto, Japan)

Biometra, (Göttingen, Germany)

Biometra, (Göttingen, Germany)

Bendelin, (Göttingen, Germany)

Bio-Rad, (Göttingen, Germany)

Amersham Biosciences, (Little 


\subsubsection{Computer software}

Vector NTI 10.3.0

PyMOL v1.5

Quantity One

SPARKY

\section{Topspin 1.3}

Jalview 2.10.4b1

UCSF Chimera

Eman2

Genome compiler

Snapgene software

MolProbity
Invitrogen (California, United States)

DeLano Scientific LLC (USA)

Bio-Rad (Hercules, CA, USA)

University of California (USA)

Bruker Biospin (Karlsruhe, Germany)

(269)

(270)

(271)

http://www.genomecompiler.com/aboutgenome-compiler/

GSL Biotech; www.snapgene.com

(272) 


\subsection{Methods}

\subsubsection{Generation of the plasmid pET-24b: $\alpha^{c h i}{ }_{3}: \beta_{3}: \gamma$}

The plasmid with a backbone pET-24b was manipulated and the genes of $\alpha^{\text {chi }}{ }_{3} \beta_{3}: \gamma$ were introduced as described in the Ph.D. Thesis of Dr. Lavanya Sundararaman: "The role of N157 of A-ATP synthase subunit B in nucleotide binding and expression, production and structural and mechanistic features of subunits $\alpha$ and $\varepsilon$ of Mycobacterium tuberculosis F-ATP synthase"

\subsubsection{Site-directed mutagenesis of F-ATP synthase}

In order to incorporate the desired mutations for this study, mutagenic primers have been used to clone the entire plasmid. Mutants have been assigned the following names: $\alpha^{\text {chi }}$ S400C, $\alpha^{\text {chi }}$ D401C, $\alpha^{\text {chi }}$ V525C and $\alpha^{\text {chi }}$-D401C- $\gamma$ R97C and graphically shown in Figure 2.2.

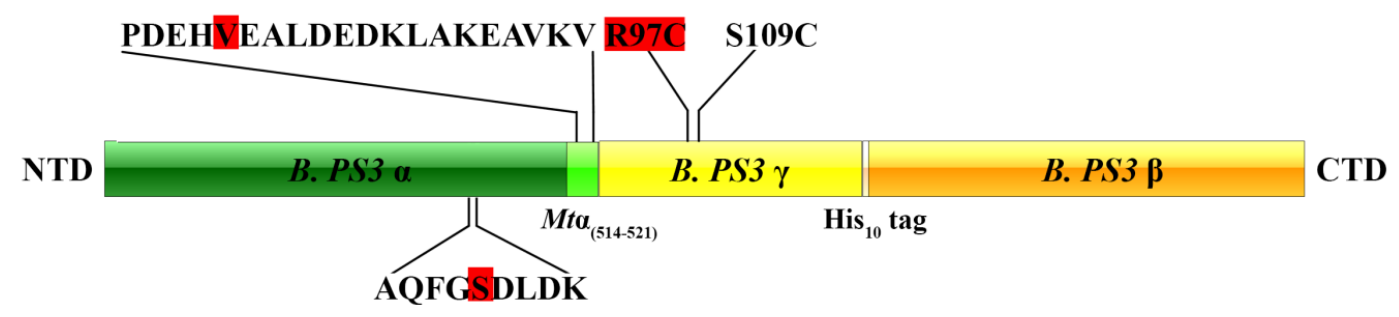

Figure 2.1: Bar diagram mapping the mutations in $\alpha^{c h i}: \beta_{3}: \gamma$ protein complex

\begin{tabular}{|c|c|c|c|}
\hline Construct & & Oligonucleotide sequence 5 '-3' & $\mathbf{T}_{\mathrm{m}}\left({ }^{\circ} \mathrm{C}\right)$ \\
\hline \multirow[t]{2}{*}{$\alpha^{\text {chi }} \mathbf{S 4 0 0 C}$} & forward & CGCCCAATTCGGCTGCGACCTCGATAAA & 65.9 \\
\hline & reverse & TTTATCGAGGTCGCAGCCGAATTGGGCG & 65.9 \\
\hline \multirow[t]{2}{*}{$\alpha^{\text {chi } D 401 C}$} & forward & CCAATTCGGCTCCTGCCTCGATAAAGCGA & 65.2 \\
\hline & reverse & TCGCTTTATCGAGGCAGGAGCCGAATTGG & 65.2 \\
\hline \multirow[t]{2}{*}{$\alpha^{\text {chi }}$ V525C } & forward & CGCCAAGGAAGCCTGCAAGGTCAAAAAGCC & 66.7 \\
\hline & reverse & GGCTTTTTGACCTTGCAGGCTTCCTTGGCG & 66.7 \\
\hline \multirow[t]{2}{*}{$\gamma R 97 C$} & forward & GGCGCGTACAACAGCAACGTGCTGTGCCTCGTG & 70.7 \\
\hline & reverse & $\begin{array}{l}\text { AGCATGGCGTTTTTGGATCGTTTGGTACACGAGGCACA } \\
\text { GCAC }\end{array}$ & 69.7 \\
\hline
\end{tabular}

Table 2.1: Primers for the generation of $\alpha^{c h i}{ }_{3}: \beta_{3}: \gamma$ mutants

In the polymerase chain reaction (PCR), $\sim 25 \mathrm{ng}$ of plasmid DNA encoding $\alpha^{\text {chi }}{ }_{3} \beta_{3} \gamma$ complex has been used as a template. Total reaction volume has been set to 50 $\mu l$ and pipetting of the reaction mixture to PCR tubes has been done on ice for all the 
steps. The reaction has been set following the order and the volumes of the reagents in table 2.2. The mixture has been homogenized by pipetting and centrifugation on a conventional table-top centrifuge (Eppendorf Mini-centrifuge).

\begin{tabular}{ll}
\hline Reagents & Volume $(\boldsymbol{\mu l})$ \\
\hline Template (plasmid DNA) & 1.00 \\
MiliQ water & 35.9 \\
Primers $(100 \mu \mathrm{M})$ & $2 \times 0.30$ \\
dNTP $(2 \mathrm{mM})$ & 1.50 \\
Kappa buffer $(5 \mathrm{x})$ & 10.0 \\
Kappa HiFi DNA polymerase & 1 \\
\hline
\end{tabular}

Table 2.2: Reaction mix for PCR

PCR Thermocycler (Biometra $\mathrm{T}$ personal) was preheated to $99{ }^{\circ} \mathrm{C}$ before placing the PCR tubes on the heating block. The following program has been used for the amplification:

\begin{tabular}{|c|c|c|c|}
\hline Cycle steps & Temperature $\left({ }^{\circ} \mathrm{C}\right)$ & Time & \\
\hline Lid & 99 & & \\
\hline Initial denaturation & 95 & $2 \min$ & \\
\hline Cycle denaturation & 98 & $20 \mathrm{sec}$ & \\
\hline Annealing & $*$ & $\begin{array}{l}15 \mathrm{sec} \\
\text { cycles }\end{array}$ & \} 16 \\
\hline Extension & 72 & $5 \min 30 \mathrm{sec}$ & \\
\hline Final extension & 72 & $7 \mathrm{~min}$ & \\
\hline End & 4 & & \\
\hline
\end{tabular}

Table 2.3: PCR cycling program; * primer-based $\mathrm{T}_{\mathrm{a}}$

Cycle denaturation, annealing and extension steps were repeated for another 15 cycles. Total time taken for the PCR program was $1 \mathrm{~h} 46 \mathrm{~min} 30 \mathrm{~s}$. To check the quality, $5 \mu \mathrm{l}$ of each the PCR product was applied on a $1.2 \%$ agarose gel. The gel was subsequently stained with Ethidium bromide (EtBr) solution for $20 \mathrm{~min}$ and observed under UV light upon de-staining. The size of the products was confirmed by comparing it with the appropriate DNA marker. For each of the constructs, the remaining reaction mixture $(45 \mu 1)$ was used to isolate the amplified plasmid DNA containing the mutation following the next protocol: 


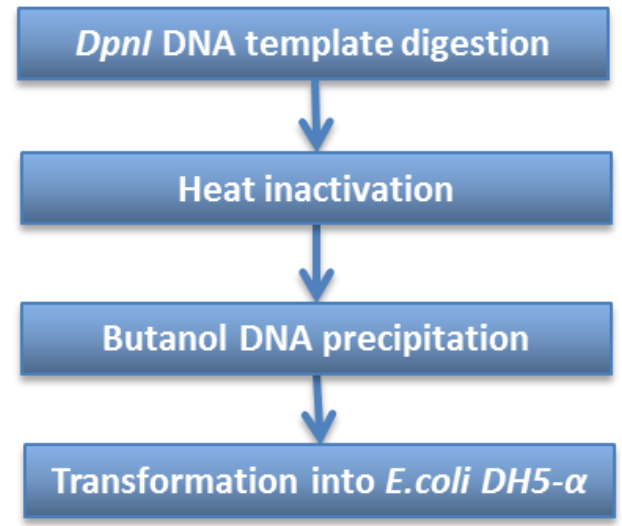

1. DpnI digestion and inactivation:

To digest the methylated plasmid DNA that has been used as the template in PCR, restriction with endonuclease DpnI has been set up. Components listed below have been pipetted into a tube and mixed till homogeneity. The reaction mixture was incubated for $1 \mathrm{~h}$ in a water bath at $37^{\circ} \mathrm{C}$ followed by $20 \mathrm{~min}$ of incubation on $80{ }^{\circ} \mathrm{C}$ to inactivate the DpnI endonuclease.

\begin{tabular}{ll}
\hline Reagents & Volume $(\boldsymbol{\mu l})$ \\
\hline PCR mixture & 30.0 \\
CutSmart® buffer $(10 \mathrm{x})$ & 35.9 \\
DpnI & $2 \times 0.30$ \\
MiliQ water & 1.50 \\
\hline
\end{tabular}

Table 2.4: Reaction mix for $D p n I$ digestion

\section{DNA precipitation}

In order to isolate DNA for subsequent transformation into the electrocompetent cells, $40 \mu \mathrm{l}$ of MiliQ water followed by $900 \mu \mathrm{l}$ of $n$-butanol (HPLC grade) was added to the reaction mixture. After thorough mixing and homogenizing, DNA was precipitated out using the conventional table-top centrifuge (Eppendorf Minicentrifuge) for $10 \mathrm{~min}$ on $13,000 \mathrm{x} \mathrm{g}$. The supernatant was pipetted out and replaced with $500 \mu \mathrm{l}$ of $70 \%$ ethanol (HPLC grade) to remove the residual butanol. Centrifugation was repeated and subsequently, ethanol was removed. The tube containing the pellet with DNA was inverted on a paper inside of the sterile hood in the duration of 10 minutes to allow residual ethanol to evaporate. Dry DNA pellet was re-dissolved in $7 \mu \mathrm{l}$ of MiliQ water on ice in the duration of $30 \mathrm{~min}$. 


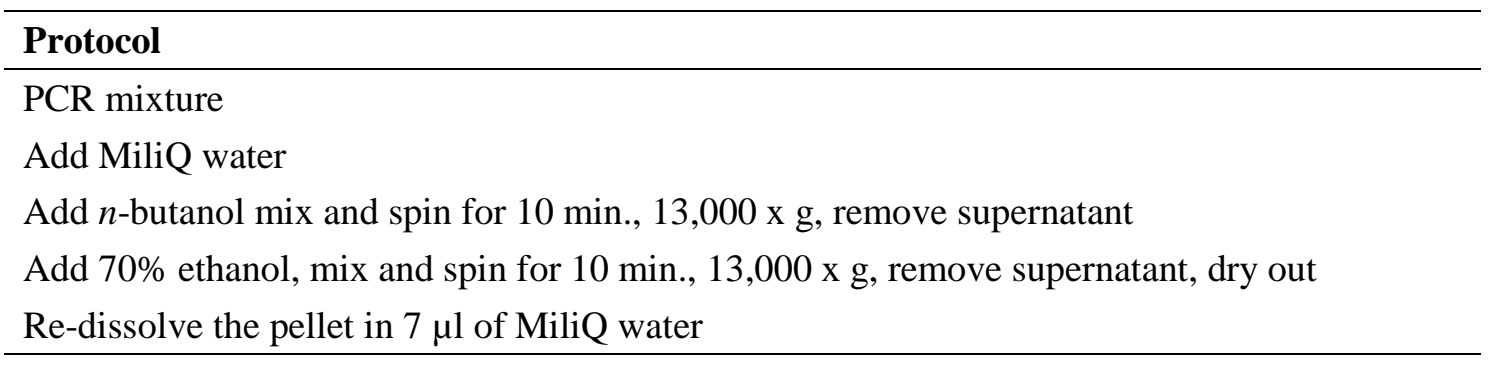

Table 2.5: Protocol for butanol precipitation of DNA

\subsubsection{Transformation by electroporation}

Reagents used: Luria Bertani (LB) media: 10 g/l Tryptone

$5 \mathrm{~g} / \mathrm{l}$ Yeast Extract

$5 \mathrm{~g} / \mathrm{l} \mathrm{NaCl}$

E. coli Top10 electro-competent cells were prepared as per the protocol from "Current Protocols in Molecular Biology" (Wiley InterScience) manual. Approximately $100 \mathrm{ng}$ of plasmid DNA was added to $\sim 100 \mu \mathrm{l}$ of competent cells incubated 1-2 $\min$ on ice and transferred to pre-chilled electroporation cuvette. Electroporation was performed using constant voltage of $2500 \mathrm{~V}$ on Micropulser Electroporator, Bio-Rad. The cuvette was then immediately placed back on the ice in the duration of 1 min., following washing of cells with $900 \mu \mathrm{l}$ of LB media and transferring to an Eppendorf tube. The new culture of freshly transformed cells was incubated at $37{ }^{\circ} \mathrm{C}$ for $1 \mathrm{~h}$ and appropriate dilutions were prepared before plating them on antibiotic selection plates. The plates contained $30 \mu \mathrm{g} / \mathrm{ml}$ of kanamycin and the culture spread was incubated overnight at $37^{\circ} \mathrm{C}$. Single colonies were picked from the plate on the next day and were incubated at $37^{\circ} \mathrm{C}$ in the LB media. The plasmid DNA was isolated using standard protocol (QIAGEN mini-prep kit), subsequently double digested with BamHI and HindIII and applied onto 1.2\% agarose gel, in order to confirm the presence of the insert. Size of the insert and the vector were compared with appropriate controls and markers. Plasmids were sent for sequencing and the verified ones were finally transformed into E. coli DK8 cells, whose entire F-ATP synthase operon was deleted $(\Delta u n c)$ for protein production. The cells were cultivated on $30 \mu \mathrm{g} / \mathrm{ml}$ kanamycin-containing $2 \mathrm{YT}$ agar plates. 


\subsubsection{Cultivation conditions and expression of complex $\alpha^{\text {chi }}{ }_{3}: \beta_{3}: \gamma$ F-ATP synthase}

2YT media provides comparatively more nutrients than $L B$, in order to achieve a healthy log phase.

Composition: $16 \mathrm{~g} / \mathrm{l}$ Tryptone

$10 \mathrm{~g} / \mathrm{l}$ Yeast Extract

$5 \mathrm{~g} / \mathrm{l} \mathrm{NaCl}$

Glycerol stock of $E$. coli DK8 cells containing pET-24(b) + subunits $\alpha^{\text {chi }} \beta_{3} \gamma$ was used for over-night small scale inoculation in $500 \mathrm{ml}$ of kanamycin positive (30 $\mu \mathrm{g} / \mathrm{ml}$ ) 2YT medium. Upscale secondary culture up to 101 distributed equally into $8 \mathrm{x}$ 51 Erlenmeyer flasks were grown to $\mathrm{OD}_{600}$ of $0.6-0.7$ at $37^{\circ} \mathrm{C}$ with shaking at $180 \mathrm{rpm}$ (Infors HT Minitron shaker). Induction was started by addition of $1 \mathrm{mM}$ of isopropyl (thio)- $\beta$-D-galactopyranoside (IPTG) and kept at $37{ }^{\circ} \mathrm{C}$ for $6 \mathrm{~h}$ (as per the established protocol). Cells were harvested at 9,000 x g for $11 \mathrm{~min}, 4{ }^{\circ} \mathrm{C}$, snap-frozen with liquid nitrogen and subsequently stored in $-80^{\circ} \mathrm{C}$.

\subsubsection{Purification of complex $\alpha^{\text {chi }}: \beta_{3}: \gamma$ F-ATP from $E$. coli DK8 cells}

In order to purify the $\mathrm{His}_{10^{-}} \alpha^{c h i}{ }_{3} \beta_{3} \gamma$ F-ATP synthase, roughly $24 \mathrm{~g}$ of cells were re-suspended in buffer 1 (50 mM Tris/ $\mathrm{HCl}, \mathrm{pH}$ 7.5, $150 \mathrm{mM} \mathrm{NaCl}, 2 \mathrm{mM}$ PMSF, 2 $\mathrm{mM}$ Pefabloc ${ }^{\mathrm{SC}}$ (BIOMOL) and $0.8 \mathrm{mM}$ DTT and lysed on ice by sonication for $4 \mathrm{x} 1$ min. Precipitated material was separated by centrifugation at $14,500 \mathrm{x} g$ for $35 \mathrm{~min}$. The supernatant was filtered $(0.45 \mu \mathrm{m}$; Millipore $)$

The filtrate containing the His-tagged protein was incubated with $3 \mathrm{ml} \mathrm{Ni}^{2+}$ NTA resin, which was previously equilibrated with buffer 1 on a rotational wheel in the duration of $1.5 \mathrm{~h}$ at $4{ }^{\circ} \mathrm{C}$. After incubation, the protein was eluted with an imidazole-gradient (0-600 mM) in buffer 1 and applied onto an SDS-PAGE. Fractions containing His-tagged $\alpha^{\text {chi }}{ }_{3} \beta_{3} \gamma$ were pooled and applied onto anion-exchange chromatography column (Resource ${ }^{\mathrm{TM}} \mathrm{Q}$ column $6 \mathrm{ml}$ GE Healthcare). The buffers used were A: $50 \mathrm{mM}$ Tris- $\mathrm{HCl} \mathrm{pH}$ 7.5, $0.8 \mathrm{mM}$ DTT and B: $50 \mathrm{mM}$ Tris/ $\mathrm{HCl} \mathrm{pH}$ 7.5, $1 \mathrm{M} \mathrm{NaCl}$ and $0.8 \mathrm{mM}$ DTT. A step gradient was performed, starting from $50 \mathrm{mM}$ of $\mathrm{NaCl}$ to $1 \mathrm{M}$ of $\mathrm{NaCl}$, collected fractions were applied on an SDS-PAGE. The peak of interest appeared at $250 \mathrm{mM} \mathrm{NaCl}$. The fractions under this peak were pooled and concentrated using a $100 \mathrm{kD}$ centricon ( $100 \mathrm{kDa}$ cut off) to the final volume of $40 \mu \mathrm{l}$ and loaded on an SDS-PAGE for further analysis. 
The purity and the stoichiometry of the complex were observed by staining the gel with Coomassie Brilliant Blue G250. Protein concentrations were determined by Biospec-nano®.

\subsubsection{Optimization of the purification of complex $\alpha^{\text {chi }}{ }_{3}: \beta_{3}: \gamma$ F-ATP}

To increase the protein production, vector pET-24(b) containing the insert $\alpha$ chi3 $\beta 3 \gamma$ was transformed by electroporation into the E. coli OverExpress C41(DE3): F - ompT hsdSB (rB- mB-) gal dcm (DE3) using the protocol described in section 2.2.3. Protein production upon $1 \mathrm{mM}$ of IPTG addition was verified using the standardized induction test as described in section 2.2.10. Upon successful induction, cultivation conditions were maintained as done for the purification from DK8 cells. The protein was purified according to the protocol mentioned in section 2.2.5.

\subsubsection{Cloning of various constructs of subunit $\varepsilon$ of F-ATP synthase from Mycobacterium tuberculosis}

Plasmid DNA containing the construct has previously been generated in our lab by Dr. Lavanya Sundararaman. The construct contains subunits $\varepsilon$ cloned between NcoI and SacI (restriction sites) in vector pET-9d(+)(273), as shown in (Figure 2.2). His $_{6}$-tag was introduced at the $\mathrm{N}$-terminal, to enable the purification using $\mathrm{Ni}^{2+}$-NTA affinity chromatography. The series of mutations have been introduced using two different approaches; conventional cloning as well as site-directed mutagenesis.

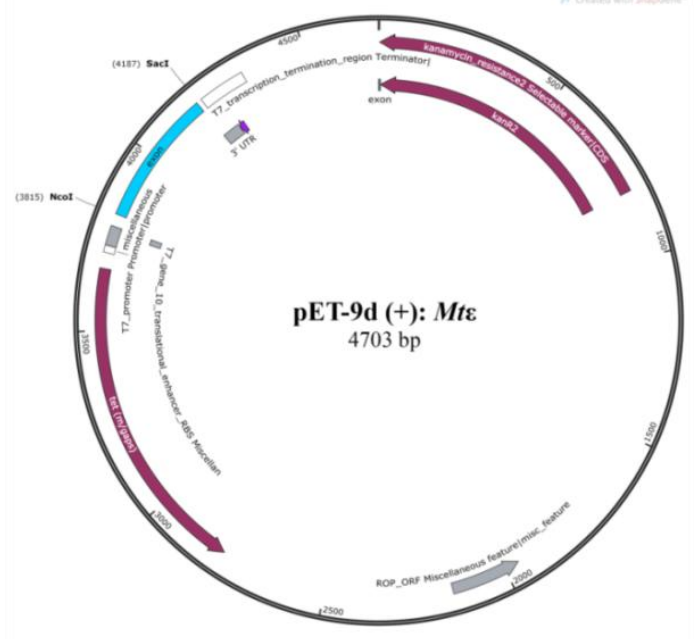

Figure 2.2: Map of the pET-9d(+):Mte vector. The atpC gene encodes mycobacterial F-ATP synthase, subunit $\varepsilon$ inserted into the vector pET-9d (+), between the restriction sites $N c o I$ and SacI . 


\subsubsection{Conventional cloning method}

To generate mutants annotated as V11C, R113C, G118C, DNA fragment coding for the subunit $\varepsilon$ was amplified from the template DNA (vector+insert) by using the mutagenic primers listed below:

\begin{tabular}{|c|c|c|c|}
\hline Construct & & Oligonucleotide sequence 5 '-3' & $\mathbf{T}_{\mathrm{m}}\left({ }^{\circ} \mathrm{C}\right)$ \\
\hline \multirow[t]{2}{*}{$M t \varepsilon V 11 C$} & fwd & $\begin{array}{l}\text { CACCCCATGGCCGAATTGAACGTTGAGATCGTCGCC } \\
\text { TGCGACCGGAA }\end{array}$ & 73.0 \\
\hline & rev & $\begin{array}{l}\text { ATCCGAGCTCTTAGTCGATCGCGCCGACGGCGCGCA } \\
\text { АTCT }\end{array}$ & 73.1 \\
\hline \multirow[t]{2}{*}{ MtER113C } & fwd & $\begin{array}{l}\text { CACCCCATGGCCGAATTGAACGTTGAGATCGTCGCC } \\
\text { GTCGACCGGAAC }\end{array}$ & 72.6 \\
\hline & rev & $\begin{array}{l}\text { ATCCGAGCTCTTAGTCGATCGCGCCGACGGCGCGCA } \\
\text { A } \underline{\text { ACAGGCGCGGCC }}\end{array}$ & 77.1 \\
\hline \multirow[t]{2}{*}{ MtEG118C } & fwd & $\begin{array}{l}\text { CACCCCATGGCCGAATTGAACGTTGAGATCGTCGCC } \\
\text { GTCGACCGGAAC }\end{array}$ & 72.6 \\
\hline & rev & $\begin{array}{l}\text { ATCCGAGCTCTTAGTCGATCGCGCAGACGGCGCGCA } \\
\text { АTCTG }\end{array}$ & 71.9 \\
\hline
\end{tabular}

Table 2.6: Primers used for generation of $M t \varepsilon$ mutants

In the PCR, 70 ng of plasmid DNA encoding subunit $\varepsilon(\operatorname{atp} C)$ has been used as a template. A total reaction volume was set to $50 \mu \mathrm{l}$ and the PCR was done on ice for all the steps. The reaction was set following the order and the volumes of the reagents in table 2.7 provided below. The mixture was homogenized by pipetting and centrifugation using an Eppendorf Mini-centrifuge.

\begin{tabular}{ll}
\hline Reagents & Volume $(\boldsymbol{\mu l})$ \\
\hline Template (plasmid DNA) & 1.00 \\
MiliQ water & 40.5 \\
Primers $(100 \mu \mathrm{M})$ & $2 \times 0.50$ \\
dNTP $(2 \mathrm{mM})$ & 1.50 \\
$P f u$ buffer $(10 \mathrm{x})$ & 5.00 \\
Pfu DNA polymerase & 1.00 \\
\hline
\end{tabular}

Table 2.7: Reaction mix for PCR for the generation of Mtع mutants 
PCR Thermocycler (Biometra T personal) was preheated to $99{ }^{\circ} \mathrm{C}$ before placing the PCR tubes on the heating block. The following program has been used for the amplification:

\begin{tabular}{lll}
\hline Cycle steps & Temperature $\left({ }^{\circ} \mathbf{C}\right)$ & Time \\
\hline Lid & 99 & \\
Initial denaturation & 96 & $3 \mathrm{~min}$ \\
Cycle denaturation & 96 & $30 \mathrm{sec}$ \\
Annealing & $67^{*}$ & $45 \mathrm{sec}$ \\
Extension & 72 & $1 \mathrm{~min}$ \\
Final extension & 72 & $5 \mathrm{~min}$ \\
End & 4 & \\
\hline
\end{tabular}

Table 2.8: PCR cycling program; * primer-based $\mathrm{T}_{\mathrm{a}}$

Cycle denaturation, annealing and extension steps were repeated for another 29 cycles. Total time taken for the PCR program was $1 \mathrm{~h} 11 \mathrm{~min} 30 \mathrm{~s}$. To check the quality, $5 \mu \mathrm{l}$ of each the PCR product was applied on a $1.2 \%$ agarose gel. The gel was then placed in Ethidium bromide (EtBr) solution for $20 \mathrm{~min}$ and observed under UV light on de-staining. The sizes of the products were confirmed by comparing it with the appropriate DNA marker. For each of the constructs, the remaining reaction mixture $(45 \mu \mathrm{l})$ was applied on a $1.2 \%$ preparative agarose gel and the portions corresponding to the correct PCR product were cut out immediately and purified by gel extraction kit (QIAGEN) as per the manufacturer's protocol. The product was finally eluted in $50 \mu \mathrm{l}$ volume of Tris- $\mathrm{HCl}$ buffer of $\mathrm{pH}$ 8.0. $1 \mu \mathrm{l}$ of gel extracted PCR product was applied onto a $1.2 \%$ analytical agarose gel for estimating the purity of the sample. Concentrations of the PCR products were noted down and used to calculate the appropriate ratio for the ligation reaction.

To obtain the insert fragments that can be ligated, overnight double digestion using NcoI and SacI was setup (shown in the table below) while the reaction mixture was purified by an enzyme reaction purification kit from QIAGEN. The ligation reaction was subsequently performed in order to fuse the insert with pre-digested vector; pET 9-d(+) vector used for ligation was also double digested with NcoI and SacI restriction enzymes and treated with CIAP to prevent self-ligation. 


\begin{tabular}{ll}
\hline Reagents & Volume $(\boldsymbol{\mu l})$ \\
\hline Insert DNA & 30.0 \\
NEBuffer 1.1 $(10 \mathrm{x})$ & 6.00 \\
NcoI & 1.50 \\
SacI & 1.50 \\
BSA $(100 \mathrm{x})$ & 0.60 \\
MiliQ water & 20.4 \\
\hline
\end{tabular}

Table 2.9: Reaction mix insert double digestion

The molar ratio of vector and insert was calculated using the formula:

$$
\text { insert mass }[\mathrm{ng}]=\text { volume } * 3 * \frac{\text { insert lenght }[\mathrm{bp}]}{\text { vector lenght }[\mathrm{bp}]} * \text { vector mass }[\mathrm{ng}]
$$

The ligation reaction was set up as follows:

\begin{tabular}{ll}
\hline Reagents & Amount \\
\hline Ligase buffer $(10 \mathrm{x})$ & $1 \mu \mathrm{l}$ \\
Dephosphorylated vector & $100 \mathrm{ng}$ \\
Insert & variable (3 or 5 molar excess of a vector) \\
T4 Ligase $(40,000 \mathrm{U} / \mathrm{ml})$ & $0.35 \mu \mathrm{l}$ \\
MiliQ water & up to $10 \mu \mathrm{l}$ \\
\hline
\end{tabular}

Table 2.10: Reaction mix for ligation of DNA fragments

The ligation mixture was kept at room temperature for $2 \mathrm{~h}$ and the reaction was terminated by adding $90 \mu \mathrm{l}$ of MilliQ water to make a final volume of $100 \mu \mathrm{l}$. Desired DNA was precipitated out using $n$-butanol as previously described in section 2.2.2.

$7 \mu \mathrm{l}$ of re-dissolved DNA was used to transform the DH5- $\alpha$ as described in section 2.2.3. Control restriction digestion was performed using NcoI and SacI restriction endonucleases and relevant clones were confirmed by sequencing. Plasmids carrying the desired mutations were finally transformed into E. coli BL21 (DE3) cells for protein production followed by $1 \mathrm{~h}$ reviving of culture on $37{ }^{\circ} \mathrm{C}$ and plating on 30 $\mu \mathrm{g} / \mathrm{ml}$ kanamycin-containing LB agar plates. Before the large scale protein production, induction and solubility tests were performed as described in section 2.2.9. E. coli BL21 expression strains were purchased from Novagene (Darmstadt, Germany). 


\subsubsection{Site-directed mutagenesis of MtE mutants}

Site-directed mutagenesis is a method for amplification of the entire plasmid carrying the gene of interest by the usage of mutagenic primers (274). To substitute the desired residues and/or delete the segment, sets of mutagenic primers were designed according to the table below and plasmid DNA used in these procedures was described in the previous section 2.3.1 and Figure 2.2. For the construct D121C, the whole plasmid amplification is performed using phosphorylated "tail-to-tail" primers to facilitate the subsequent ligation step. The constructs $M t \varepsilon_{1-101}$ and $M t \varepsilon_{1-119}$ featuring the C-terminal 20 and 2 amino acid residues deletion, respectively, as well as substitution mutants R62L, E87A and E100C are amplified using regular, non-phosphorylated primers.

\begin{tabular}{|c|c|c|c|}
\hline Construct & & Oligonucleotide sequence 5'-3' & $\mathbf{T}_{\mathrm{m}}\left({ }^{\circ} \mathrm{C}\right)$ \\
\hline \multirow[t]{2}{*}{$M t \varepsilon D 121 C$} & fwd & $\begin{array}{l}\text { P-TGCTAAGAGCTCGGATCCGGCTGCTAACAAAGCCCG- } \\
\text { P }\end{array}$ & 69.6 \\
\hline & rev & P-GATCGCGCCGACGGCGCGCAATC-P & 69.5 \\
\hline \multirow[t]{2}{*}{$M t \varepsilon_{6-121}$} & fwd & GTTGAGATCGTCGCCGTCGACCGGAAC & 66.0 \\
\hline & rev & GGCGACGATCTCAACGGGGTGATGGTGATG & 66.7 \\
\hline \multirow[t]{2}{*}{ MtEE87A } & fwd & AGTTCGCGTCGGAGATCGACGAGGCCG & 68.6 \\
\hline & rev & TCCGACGGCGAACTCGGCAGATTCGGC & 68.1 \\
\hline \multirow[t]{2}{*}{ MtEE100C } & fwd & CAGGATTCCTGTTCCGACGATCCCCGCATC & 66.5 \\
\hline & rev & TCGGAACAGGAATCCTGCTTGGCGGC & 66.7 \\
\hline \multirow[t]{2}{*}{$M t \varepsilon R 62 L$} & fwd & GACCTGCTCATCGCGGTCGACGGCG & \\
\hline & rev & GCGATGAGCAGGTCCTTTTCTCCCTCCCGC & \\
\hline
\end{tabular}

Table 2.11: Primers for the amplification of $M t \varepsilon$ mutant constructs

In the polymerase chain reaction, $\sim 25 \mathrm{ng}$ of plasmid DNA was used as the template and the reaction was set up by mixing all the reagents in a manner specified in table 2.12 .

\begin{tabular}{ll}
\hline Reagents & Volume $(\boldsymbol{\mu l})$ \\
\hline Template (plasmid DNA) & 1.00 \\
MiliQ water & 35.9 \\
Primers $(100 \mu \mathrm{M})$ & $2 \times 0.30$ \\
dNTP $(2 \mathrm{mM})$ & 1.50 \\
Kappa buffer $(5 x)$ & 10.0 \\
Kappa HiFi DNA polymerase & 1.00 \\
\hline
\end{tabular}

Table 2.12: Reaction mix for PCR for the generation of the Mt\& mutants 
To start the reaction, PCR tubes containing the mixture are placed in a preheated block of the PCR Thermocycler (Biometra $\mathrm{T}$ personal) to $99{ }^{\circ} \mathrm{C}$. The program resulted in amplification of the entire plasmid is shown below:

\begin{tabular}{|c|c|c|c|}
\hline Cycle steps & Temperature $\left({ }^{\circ} \mathrm{C}\right)$ & Time & \\
\hline Lid & 99 & & \\
\hline Initial denaturation & 95 & $2 \mathrm{~min}$ & \\
\hline Cycle denaturation & 98 & $20 \mathrm{sec}$ & \\
\hline Annealing & $*$ & $\begin{array}{l}15 \mathrm{sec} \\
\text { cycles }\end{array}$ & 20 \\
\hline Extension & 72 & $3 \mathrm{~min} 30 \mathrm{sec}$ & \\
\hline Final extension & 72 & $5 \mathrm{~min}$ & \\
\hline End & 4 & & \\
\hline
\end{tabular}

Table 2.13: PCR cycling program; * primer-based $\mathrm{T}_{\mathrm{a}}$

Cycle denaturation, annealing and extension steps were repeated for another 19 cycles. Total time taken for the PCR program was $1 \mathrm{~h} 22 \mathrm{~min}$.

Protocol for a quality check was performed identically to that described in section 2.2.7. The isolation of the was performed as described for the complex $\alpha_{3}^{\text {chi }}{ }_{3} \beta_{3}: \gamma$ in section 2.2.2, with an additional step that included the ligation of the amplified plasmid. Ligation was performed between the heat inactivation and butanol precipitation steps by incubation of the ligation mixture for $3 \mathrm{~h}$ at room temperature. The following table 2.14 describes the ligation:

\begin{tabular}{ll}
\hline Reagents & Volume $(\boldsymbol{\mu l})$ \\
\hline DpnI digested mix & 57.4 \\
ATP $(100 \mathrm{mM})$ & 0.60 \\
T4 DNA ligase & 2.00 \\
\hline
\end{tabular}

Table 2.14: Reaction mix for ligation of the $M t \varepsilon D 121 \mathrm{C}$ mutant pDNA

Transformation of the precipitated plasmid DNA was performed according to the procedure described in section 2.2.3 with a few changes as listed below. E. coli DH5- $\alpha$ was used for plasmid propagation. Control restriction digestion is performed using NcoI and SacI restriction endonucleases and relevant clones were confirmed by sequencing. Plasmids carrying the desired mutations were finally transformed into $E$. coli BL21 (DE3) cells for protein production. Before the large scale protein 
production, the induction and the solubility test were performed as described in section 2.9 .

\subsubsection{Induction test for MtE mutants}

Composition of 4 x Lysis buffer: 250 mM Tris-HCl, pH 6.8

$$
\begin{aligned}
& 9.2 \% \text { SDS } \\
& 40 \% \text { Glycerol } \\
& 0.2 \% \text { Bromophenol Blue }
\end{aligned}
$$

At least 3-4 single colonies were randomly picked for each construct and were grown to $\mathrm{OD}_{600}$ of $0.6-0.7$ at $37^{\circ} \mathrm{C}$ with shaking of $180 \mathrm{rpm}$ (Infors HT Minitron shaker). The final concentration of $1 \mathrm{mM}$ IPTG was then added to start the induction. The mixture was kept at $37^{\circ} \mathrm{C}$ for $2.5 \mathrm{~h}$. Respective controls were left un-induced under identical conditions. The cells were pelleted down and re-suspended with $30 \mu 1$ of $2 \mathrm{x}$ lysis buffer in presence of $1 \mathrm{mM}$ DTT. Re-suspended cell mass was heated up at $95^{\circ} \mathrm{C}$ for $5 \mathrm{~min}$. and $15-20 \mu \mathrm{l}$ of each sample was loaded onto a 17\% SDS-PAGE. The expression level was higher in the induced cultures $((+)$ supplemented with IPTG) than un-induced cultures ((-) not supplemented with IPTG).

\subsubsection{Solubility test for $M t \varepsilon$ mutants}

To check for the optimal solubility of the protein, culture was grown and induced overnight with $1 \mathrm{mM}$ IPTG at $15{ }^{\circ} \mathrm{C}$. Following the successful induction of proteins, solubility of the produced proteins was tested in various buffers as shown below.

$50 \mathrm{mM}$ Tris-HCl, $\mathrm{pH} 8.5,200 \mathrm{mM} \mathrm{NaCl}$

$50 \mathrm{mM}$ Tris- $\mathrm{HCl}, \mathrm{pH} 7.5,200 \mathrm{mM} \mathrm{NaCl}$

$50 \mathrm{mM}$ Hepes, pH 7.5, $200 \mathrm{mM} \mathrm{NaCl}$

50 mM MES, pH $7.0200 \mathrm{mM} \mathrm{NaCl}$

$50 \mathrm{mM}$ Phosphate, pH $6.8200 \mathrm{mM} \mathrm{NaCl}$

$50 \mathrm{ml}$ of culture used to pellet the cells into five equal fractions by centrifuging at $10,000 \times \mathrm{g}$ for $7 \mathrm{~min}$. Freshly prepared protease inhibitor Pefabloc ${ }^{\mathrm{SC}}$ in water was added to a final concentration $(8 \mathrm{mM})$. Reducing agent such as DTT (1 mM) was used in the preparation. Resuspension was sonicated at $10 \%$ power with KE 76 tip of sonicator (Bandelin Sonoplus) three times for one min each, with a cooling interval of 
two min between each cycle. Supernatant was then separated from the pellet by centrifugation at $10,000 \mathrm{x} \mathrm{g}$ and transferred to a new tube. Pellets were resuspended in the respective buffers. All the steps were carried out on ice. $15 \mu 1$ of pellet and supernatant were loaded onto a $17 \%$ SDS-gel.

\subsubsection{Purification of MtE mutant constructs}

After successful solubility test, proteins were then purified in large scale. To produce $\mathrm{His}_{6}-\mathrm{Mt \varepsilon}$ various constructs, liquid cultures were incubated in kanamycinpositive $(30 \mathrm{mg} / \mathrm{ml}) \mathrm{LB}$ medium at $37{ }^{\circ} \mathrm{C}$ with shaking of $180 \mathrm{rpm}$, until an optical density $\mathrm{OD}_{600}$ of 0.6-0.7 was achieved. Expression of $\mathrm{His}_{6}-\mathrm{Mt \varepsilon}$ was obtained by adding IPTG to a final concentration of $1 \mathrm{mM}$. Following the overnight incubation at $15{ }^{\circ} \mathrm{C}$ for 16 hours, the cells were harvested at $9,000 \mathrm{x} \mathrm{g}$ for $11 \mathrm{~min}, 4{ }^{\circ} \mathrm{C}$. Subsequently, they were lysed on ice by sonication for 3 x 1 min in buffer A (50 mM Tris-HCl, pH 7.5, $200 \mathrm{mM} \mathrm{NaCl}, 2 \mathrm{mM}$ PMSF and $2 \mathrm{mM}$ Pefabloc $^{\mathrm{SC}}$ (BIOMOL) and $10 \%$ glycerol. For cysteine mutants, $0.8 \mathrm{mM}$ DTT was also included in the buffer. The precipitated material was separated by centrifugation at $10000 \times \mathrm{g}$ for $35 \mathrm{~min}$, and the supernatant was filtered $\left(0.45 \mu \mathrm{m}\right.$; Millipore). This was passed over a $1 \mathrm{ml} \mathrm{Ni}^{2+}$-NTA resin column to isolate subunit $\varepsilon$. His-tagged protein was allowed to bind to the matrix for $1.5 \mathrm{~h}$ at $4{ }^{\circ} \mathrm{C}$ and eluted with an imidazole-gradient $(0-400 \mathrm{mM})$ in buffer $\mathrm{A}$. Fractions containing His-tagged $M t \varepsilon$ and its mutant forms were pooled and concentrated using Centricon YM-3 (3 kDa molecular mass cut off) spin concentrators (Millipore). The imidazole was removed by gel filtration chromatography using a Superdex 75 HR 10/30 column (GE Healthcare) and a buffer of $50 \mathrm{mM}$ Tris/HCl (pH 7.5), $200 \mathrm{mM} \mathrm{NaCl}, 10 \%$ glycerol. After the gel filtration, samples are concentrated and used for experiments such as crosslinking and ATP hydrolysis assay described in section 2.2.15.

The mutant Mt\&E87A was also cultivated in ${ }^{15} \mathrm{~N}$-enriched minimal media whose composition is listed in the section 2.2.13. To obtain proteins of very high homogeneity and purity for NMR studies, Mtc-E87A was subjected to a three-step purification protocol including $\mathrm{Ni}^{2+}$-NTA affinity chromatography, ion exchange, and gel filtration chromatography. The pooled fractions from the imidazole gradient were applied on an ion-exchange column, Resource ${ }^{\mathrm{TM}} \mathrm{Q}$ (GE Healthcare). The buffers used were A: $50 \mathrm{mM}$ Tris- $\mathrm{HCl}(\mathrm{pH} 7.5)$ and $10 \%$ glycerol and B: $50 \mathrm{mM}$ Tris/ $\mathrm{HCl}(\mathrm{pH}$ 
7.5), $1 \mathrm{M} \mathrm{NaCl}$ and $10 \%$ glycerol. The flow-through collected was concentrated as required using Centricon YM-3 (3 kDa molecular mass cut off) spin concentrators (Millipore) and then applied on gel filtration chromatography column using a Superdex 75 HR 10/30 column (GE Healthcare) using buffer of $50 \mathrm{mM}$ Tris-HCl (pH 7.5), $200 \mathrm{mM} \mathrm{NaCl}$ and $10 \%$ glycerol. Protein concentrations were determined by BioSpec-nano®.

\subsubsection{Isotopic labeling $\left({ }^{15} \mathrm{~N}\right)$ of proteins}

Reagents for Minimal media (M9): $42 \mathrm{mM} \mathrm{Na}_{2} \mathrm{HPO}_{4}$

$$
\begin{aligned}
& 22 \mathrm{mM} \mathrm{KH}_{2} \mathrm{PO}_{4} \\
& 8.5 \mathrm{mM} \mathrm{NaCl} \\
& 1 \mathrm{~g} / \mathrm{I}^{15} \mathrm{NH}_{4} \mathrm{Cl} \\
& 0.1 \mathrm{mM} \mathrm{CaCl}_{2} \\
& 2 \mathrm{mM} \mathrm{MgSO}_{4} \\
& 10 \mathrm{~g} \mathrm{glucose} \\
& 30 \mu \mathrm{M} \mathrm{FeCl} \\
& 5 \mathrm{ng} / 1 \text { thiamine } \\
& \text { Antibiotic } \\
& \text { LB medium }
\end{aligned}
$$

For the production of uniformly labeled ${ }^{15} \mathrm{~N}$ recombinant proteins in E. coli C41 and E. coli BL21 (DE3), M9 minimal medium was used. All unlabelled reagents were of standard laboratory reagents grade. All solutions were prepared using MilliQ water (Millipore, Billerica, MA). $50 \mathrm{ml}$ of the overnight culture was grown. The cells were pelleted down by centrifugation at 5,000 $\mathrm{g}$ at room temperature. The supernatant LB was decanted and the pellet was re-suspended and washed in M9 media. An appropriate volume of this was used to inoculate $500 \mathrm{ml}$ of minimal medium to 0.1 OD. Cells were shaken at $37^{\circ} \mathrm{C}$ till an $\mathrm{OD}_{600}$ of 0.6 was reached. The culture was then induced with $1 \mathrm{mM}$ IPTG at $15^{\circ} \mathrm{C}$ overnight. The cells were harvested and frozen in liquid nitrogen and stored at $-80{ }^{\circ} \mathrm{C}$ until purification. 


\subsubsection{4 ${ }^{15} \mathrm{~N}$-labeled MtEE87A mutant spectrum assignment}

To understand the role that the substituted residue play in the maintenance of the protein fold/structure, a $10 \% \mathrm{D}_{2} \mathrm{O}$ was added to the ${ }^{15} \mathrm{~N}$-labelled MtcE87A that was purified in the same buffer as the Mte and it was subjected to the HSQC analysis. The data were collected with the help of Dr. Shin Joon from our lab.

In order to investigate possible structural changes between recombinant $M t \varepsilon$ and substitution mutant $M t \varepsilon E 87 \mathrm{~A}$, the NMR sample was uniformly ${ }^{15} \mathrm{~N}-{ }^{1} \mathrm{HN}$-labeled and supplied in $50 \mathrm{mM}$ Tris/ $\mathrm{HCl}, \mathrm{pH} 7.5,200 \mathrm{mM} \mathrm{NaCl}$, and $10 \% \mathrm{D}_{2} \mathrm{O}$. The ${ }^{15} \mathrm{~N}-{ }^{1} \mathrm{H}-$ HSQC experiment was performed on a Bruker Avance 700 spectrometer at $293 \mathrm{~K}$ by Dr. Shin Joon from our lab. The conventional 2D heteronuclear NMR data were collected using a uniformly ${ }^{15} \mathrm{~N}$-labeled sample. The NMR spectrum was processed with NMRPipe (275) and analyzed using SPARKY (276). Chemical shifts were monitored for backbone ${ }^{15} \mathrm{~N}$ and ${ }^{1} \mathrm{HN}$ and were calculated by the formula $\delta=\left[(\Delta \mathrm{N} / 5)^{2}\right.$ -

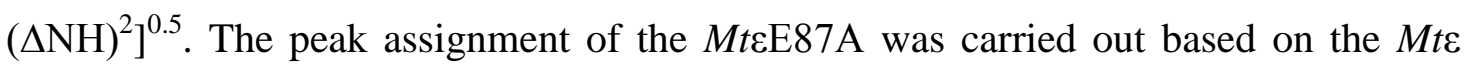
spectrum obtained and processed by Dr. Shin Joon (209) and the weighted CSPs between $M t \varepsilon$ and mutant $M t \varepsilon E 87$ A were inferred from the overlaid spectra. Residue mapping on the surface of the protein structure was performed in Pymol (267) according to the calculated CSPs.

\subsubsection{NADH coupled continuous assay for ATP hydrolytic activity measurements}

ATPase activity of the complex $\alpha_{3}^{c h i}: \beta_{3}: \gamma(: M t \varepsilon)$ and IMVs was measured using the NADH coupled continuous assay. This ATP regenerating system is driven by pyruvate kinase $(\mathrm{PK})$ which catalyzes the conversion of phospho-enol pyruvate (PEP) to pyruvate, by transferring the phosphate to an ADP to generate an ATP molecule. In the process the resulting pyruvate is converted to lactate by the lactate dehydrogenase $(\mathrm{LDH})$ at the expense of $\mathrm{NADH}$, which gets oxidized to $\mathrm{NAD}^{+}$. NADH shows an additional absorbance peak at $340 \mathrm{~nm}$ owing to the presence of the adenine moiety which is absent in the oxidized form. Spectrophotometric measurement of the reducing population of NADH at $340 \mathrm{~nm}$, allows monitoring the rate of steady-state hydrolysis of ATP in real time. The measurements were done over a period of $300 \mathrm{~s}$ with an interval of 2 seconds on addition of the ATPase to the enzymatic solution containing 2 $\mathrm{mM}$ ATP in $25 \mathrm{mM}$ HEPES, $25 \mathrm{mM} \mathrm{KCl}, 5 \mathrm{mM} \mathrm{MgCl} 2,5 \mathrm{mM} \mathrm{KCN}, 2 \mathrm{mM}$ PEP, 0.5 
mM $\beta$-NADH, 30 units each of LDH and PK enzymes. The activity of the enzyme was measured by accounting for the slope of the linear part of the curve.

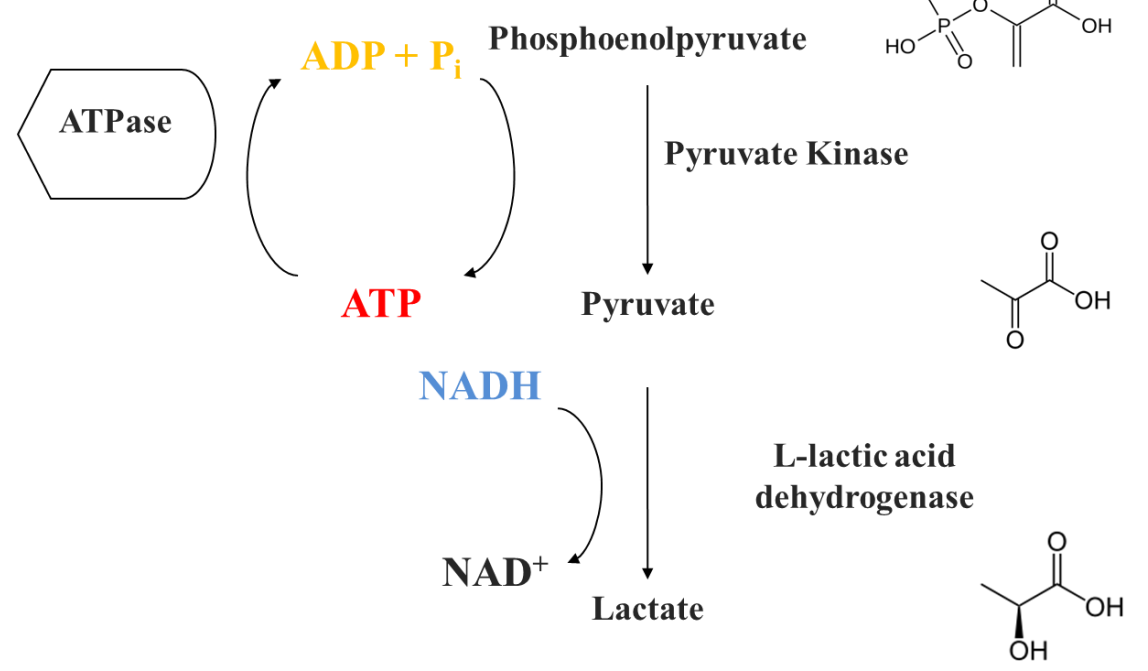

Figure 2.3: Scheme for the reaction of NADH coupled continuous assay. To measure ATP hydrolysis, NADH coupled continuous assay showing the conversion of PEP to lactate through pyruvate with the generation of ATP and the oxidation of NADH.

For the measurements of the enzyme kinetics, the Michaelis-Menten constant $\left(K_{m}\right)$ and the maximum rate $\left(V_{\max }\right)$ of $\alpha^{c h i}: \beta_{3}: \gamma$ and $\alpha^{c h i}{ }_{3}: \beta_{3}: \gamma: M t \varepsilon$ were determined by substrate-dependent ATP-hydrolysis experiments with varying concentrations of 0.02 $2 \mathrm{mM}$ ATP. $200 \mathrm{mM} \mathrm{MgCl} 2$ was pre-incubated with $200 \mathrm{mM}$ ATP (pH adjusted to 7.5) for $30 \mathrm{~min}$ on ice. Necessary pre-dilutions were made and Mg-ATP was added to each assay cuvette before the measurement.

\subsubsection{Reconstitution of the $\alpha^{c h i}{ }_{3:}: \beta_{3}: \gamma$ complex with MtE mutants}

The purified protein complex $\alpha^{c h i}: \beta_{3}: \gamma$ was incubated in the duration of $1 \mathrm{~h}, 4$

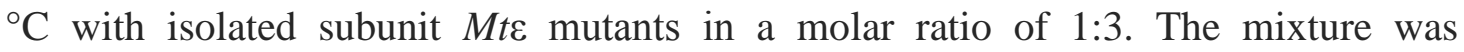
subsequently subjected to the size-exclusion S300 column to remove the excess of unbound $M t \varepsilon$ and utilized for downstream experiments.

\subsubsection{Negative stain electron microscopy (NS-EM) and 2D image analysis of the} $\alpha^{c h i}{ }_{3}: \beta_{3}: \gamma-$ and $\alpha^{c h i}{ }_{3}: \beta_{3}: \gamma: M t \varepsilon$ complexes of $F_{1}$-ATP synthase

The $\alpha^{\text {chi }}{ }_{3}: \beta_{3}: \gamma-$ and $\alpha^{\text {chi }}{ }_{3}: \beta_{3}: \gamma: M t \varepsilon$ complexes were diluted to a final concentration of $40 \mu \mathrm{g} / \mathrm{ml}$ in buffer B. A volume of $4 \mu \mathrm{l}$ of protein sample was applied 
to a glow-discharged carbon-coated copper TEM grid and stained with $2 \%(\mathrm{v} / \mathrm{v})$ uranyl acetate. Electron micrographs were recorded on a Tecnai spirit T12 transmission electron microscope (FEI) equipped with a $4 \mathrm{~K}$ CCD camera (FEI) operated at a voltage of $120 \mathrm{kV}$ at a calibrated magnification of $66,350 \mathrm{x}$ under low dose conditions. Sixty to eighty micrographs were recorded at $0^{\circ}$ angle for both $\alpha^{\text {chi }}{ }_{3}: \beta_{3}: \gamma-$ and $\alpha^{\text {chi }}{ }_{3}: \beta_{3}: \gamma: M t \varepsilon$ complexes, respectively. A total of $\sim 8,000$ particles for recombinant $\alpha^{c h i}: \beta_{3}: \gamma$ and 28,349 particles for $\alpha^{c h i}: \beta_{3}: \gamma: M t \varepsilon$ were selected. The selection criteria were clear visibility of single molecules and separation from neighboring particles. The particle selection and exporting was performed using EMAN2 (277). For the $\alpha^{c h i}: \beta_{3}: \gamma: M t \varepsilon$ complex, two rounds of $2 \mathrm{D}$ classification were executed whereby first round had the input of the whole data set of 28,349 particles, which were further refined to 12,860 particles that were finally segregated in 50 classes. The 3D model generation used experimentally obtained and selected particles from 2D classification and a surface density map generated based on the PDB structure 4XD7: A, B, C, D, E, F, G. Density map was filtered to $30 \AA$ and used as a reference map to generate the 3D density map based on the experimentally selected particles. The resulting map was refined and the resolution was estimated by Fourier shell correlation (FSC) at 1.43.

Following the same workflow as for the $\alpha^{\text {chi }}{ }_{3}: \beta_{3}: \gamma: M t \varepsilon$ complex, $\sim 8,000$ particles of $\alpha^{\text {chi }}{ }_{3}: \beta_{3}: \gamma$ were classified in fifteen $2 \mathrm{D}$ classes. $2 \mathrm{D}$ and $3 \mathrm{D}$ classification and modeling was done using RELION v1.3 (278) and USCD Chimera (279-281) for density map generation and visualization.

\subsubsection{Crosslinking experiment and 2D gel preparation}

In order to map the positions of putative critical residues in the subunits $\alpha^{\text {chi }}, \gamma$ and $\varepsilon$, those residues have been substituted with cysteine by mutagenesis. Formation of the disulfide bridge can be promoted with oxidative agents such as $\mathrm{CuCl}_{2}$ provided that the residues are in proximity of each other. For establishing a crosslink, $\alpha^{c h i}{ }_{3}: \beta_{3}: \gamma$ was reconstituted with $M t \varepsilon$ in a molar ratio 1:3 and incubated on a shaker for 1-4 h. The reconstituted complex was subsequently treated with $2 \mathrm{mM}$ final concentrations of $\mathrm{MgCl}_{2}$ and nucleotides (AMP-PnP, ADP, ATP) in the duration of 5 min followed by 15 min incubation with $0.1 \mathrm{mM} \mathrm{CuCl}_{2}$ to enhance the cross-link formation. The reaction was stopped by adding $0.1 \mathrm{mM}$ EDTA to the mix. All the steps were performed on ice while incubation was performed at $4{ }^{\circ} \mathrm{C}$. Loading dye (Laemli, $5 \mathrm{x}$, 
no DTT) was added to the samples before loading on a 9-17\% SDS-gel. Gels were stained with Coomassie Brilliant Blue G250.

For the second dimension, bands containing the possible crosslinking products were cut out and incubated in a buffer $(50 \mathrm{mM}$ Tris/ $\mathrm{HCl}, 250 \mathrm{mM} \mathrm{NaCl}, \mathrm{pH}=7.5 ; 20$ mM DTT) for $20 \mathrm{~min}$. After incubation, gel pieces containing the proteins were loaded on the $1 \mathrm{~mm}$ thick, 9-17\% SDS-gel, whereby stacking gel was cast with modified combs to create wider wells in order to accommodate the incised bands. On top of the loaded pieces, $10 \mu \mathrm{l}$ of Laemli buffer $(5 \mathrm{x})$ with $0.8 \mathrm{mM}$ DTT was added. The gel was run on the constant current of $10 \mathrm{~mA}$ for approx. $3 \mathrm{~h}$, and gels were stained with Coomassie Brilliant Blue G250 for band visualization.

\subsubsection{Densitometric analysis of reconstituted $\alpha^{c h i}{ }_{3}: \beta_{3}: \gamma: M t \varepsilon$ complexes}

The reconstitution efficiency of $\alpha^{c h i}{ }_{3}: \beta_{3}: \gamma$ complex with $M t \varepsilon$ and its mutant forms $M t \varepsilon$ R62L, $M t \varepsilon E 87 \mathrm{~A}, M t \varepsilon E 100 \mathrm{C}, M t \varepsilon_{1-120}$ and $M t \varepsilon_{6-121}$ was analyzed by measuring the intensities of the bands of the corresponding subunits applied on the $12 \%$ SDS gel. The intensities were converted into two-dimensional peaks whose integrals were calculated using ImageJ software (282). The analysis yielded values for the intensities of subunit $\gamma$ and $M t \varepsilon$ which were normalized to $\gamma$. The typical intensity ratio of $\gamma: M t \varepsilon$ detected for the $\alpha^{c h i}{ }_{3}: \beta_{3}: \gamma: M t \varepsilon$ complex is $1: 0.5$.

\subsubsection{Models of the interaction of $\alpha^{\text {chi }}$ with subunits $\gamma$ and MtE}

The structure of Gs $\alpha$ (PDB ID: 4XD7: A, B, C) was superimposed on to the $\alpha$ subunits of the E. coli $\mathrm{F}_{1} \mathrm{~F}_{\mathrm{O}}$-ATP synthase structure (PDB ID: 5T4O) (143) in all the three states, with r.m.s. deviation being $2.195 \AA$ for $\alpha_{E}, 2.144 \AA$ for $\alpha_{D P}$ and $2.25 \AA$ for $\alpha_{\text {TP. The }} \gamma$ subunit of Gs (PDB ID: 4XD7) was superimposed on to the $\gamma$ subunit of the E. coli with an r.m.s.d. of $2.146 \AA$. The solution shape of the $\alpha^{\text {chi }}$ was superimposed on to the $G s \alpha$ structure in all sites with non-spatial discrepancy (NSD) (283) of 1.56. The NMR solution structure of the C-terminal peptide $M t \alpha_{521-540}$ (3) was placed in the extra density of the SAXS-shape. Similar orientations were maintained for all the three sites

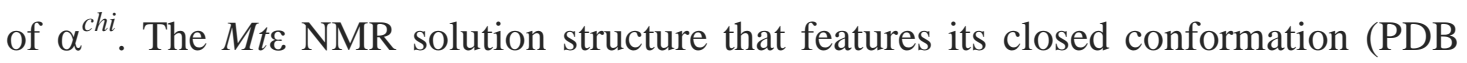
ID: 5YIO) (209) was superimposed with Gse (PDBID: 4XD7: H, (148) with r.m.s.d. of $3.123 \AA$. The extended conformation was modeled to feature the CTD helices arranged in a similar fashion as seen in the example of Gse (PDB ID: 4XD7: H). The total 


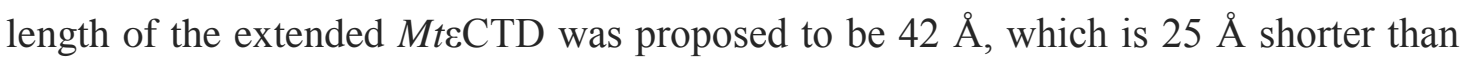

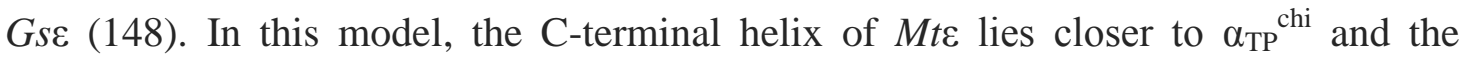
central globular domain of subunit $\gamma$ lies in between $\alpha_{\mathrm{DP}}{ }^{c h i}$ and $\alpha_{\mathrm{TP}}{ }^{c h i}$.

\subsubsection{Preparation of the labeled proteins $\alpha^{c h i}{ }_{3}: \beta_{3}: \gamma$ with $M t \varepsilon E 100 C$ for smFRET}

To label, the proteins for the smFRET experiment, an isolated and purified mutant of subunit $M t \varepsilon E 100 \mathrm{C}$ and $\alpha^{c h i}{ }_{3} \beta_{3} \gamma$ complex were individually labeled. The $M t \varepsilon E 100 \mathrm{C}$ was selectively labeled with Atto647N-maleimide while subunit $\gamma$ of the $\alpha^{\text {chi }}{ }_{3} \beta_{3} \gamma$ complex was labeled with Atto488-maleimide.

In brief, proteins were separately incubated with the respective dyes on ice in the duration of $1 \mathrm{~min}$, followed by the inactivation of the excess dye with $1 \mathrm{mM}$ DTT. Subsequently, the free dye was initially separated from the labeled proteins by washing 3 times with buffer containing $1 \mathrm{mM}$ DTT, in the centricons of a corresponding cut-off (MtcE100C - 3k; $\left.\alpha^{c h i}{ }_{3} \beta_{3} \gamma-100 \mathrm{k}\right)$. Buffers used for the washing are $50 \mathrm{mM}$ Tris/ $\mathrm{HCl} 200 \mathrm{mM} \mathrm{NaCl}, 10 \%$ glycerol, $\mathrm{pH} 7.5$ for the subunit $M t \varepsilon E 100 \mathrm{C}$ and $50 \mathrm{mM}$ Tris/ $\mathrm{HCl} 200 \mathrm{mM} \mathrm{NaCl}, \mathrm{pH} 7.5$ for the $\alpha^{\text {chi }}{ }_{3}: \beta_{3}: \gamma$ complex. After the washing, the reconstitution was performed for $1 \mathrm{~h}$ at $4{ }^{\circ} \mathrm{C}$ by incubating the proteins in 1:3 molar ratio, having subunit $M t \varepsilon$ in excess. The unbound $M t \varepsilon$ was subsequently removed by size exclusion chromatography (Superdex 300 HR 10/30, GE Healthcare). Specific labeling was confirmed using the Typhoon scanner (GE Healthcare) with the following settings: ATTO488-maleimide bound to subunit $\gamma$ was excited using $501 \mathrm{~nm}$ wavelength while ATTO647N-maleimide bound to subunit Mt\&E100C was excited using $629 \mathrm{~nm}$ wavelength. Fluorescence was registered at the $523 \mathrm{~nm}$ and $657 \mathrm{~nm}$, respectively. For both scans, the PMT value was set to 500.

\subsubsection{Preparation of electrocompetent cells of $M$. smegmatis $\mathrm{mc}^{155^{2}}$}

The electro-competent cells of $M$. smegmatis were made by inoculation of the overnight $(18 \mathrm{~h})$ primary culture in $200 \mathrm{ml}$ of in a 1:150 to 1:200 ratio of inoculum to media volume $(284,285)$. Cells were harvested upon reaching of $\mathrm{OD}_{600}=0.5$, transferred to a $50 \mathrm{ml}$ falcon tube and placed on ice for $1.5 \mathrm{~h}$. Subsequently, they were centrifuged at 3,500 $\mathrm{rpm}$ for $10 \mathrm{~min}$ at $4{ }^{\circ} \mathrm{C}$, the supernatant was discarded, and the pellets were washed with one volume $(200 \mathrm{ml})$ of ice-cold $10 \%$ glycerol. For the following two washes, one half and one-fourth of the original volume were used, 
respectively. Finally, the cell pellet was re-suspended in $4 \mathrm{ml}$ of sterile, ice-cold $10 \%$ glycerol. Cells were then aliquoted in $120 \mu \mathrm{l}$ aliquots and stored at $-80{ }^{\circ} \mathrm{C}$ until use. The entire workflow was guided by Dr. Carolyn Mulu Wu and conducted in the lab of Prof, Thomas Dick, NUS, Singapore.

\subsubsection{Transformation and preparation of the $M$. smegmatis mc155 ${ }^{2}$ pJV62 recombineering strain}

To prepare the strain expressing the protein gp61, necessary for recombineering and introduction of point mutations, M. smegmatis mc $155^{2}$ electrocompetent cells were transformed with the plasmid pJV62 as described here.

In brief, 50-100 ng of pJV62 plasmid DNA and $100 \mu \mathrm{l}$ of M. smegmatis electrocompetent (EC), both provided kindly by Prof. Thomas Dick, were added to a pre-chilled $0.2 \mathrm{~cm}$ electroporation cuvette and incubated on ice in the duration of 10 min. By using the specific electroporation conditions $(2.5 \mathrm{kV}, 25 \mu \mathrm{F}, 1000 \Omega$ (Tc: 20 $25 \mathrm{~ms})$ ), the plasmid DNA was introduced into the cells which were recovered immediately with $1 \mathrm{ml}$ of pre-warmed $7 \mathrm{H} 9$ media. Cells were subsequently revived by shaking at $160 \mathrm{rpm}$ for $4 \mathrm{~h}$ at $37{ }^{\circ} \mathrm{C}$ and plated onto $7 \mathrm{H} 10$ agar plate containing 25 $\mu \mathrm{g} / \mathrm{ml}$ of kanamycin. Plates were incubated on $37{ }^{\circ} \mathrm{C}$ in the duration of two days and observed colonies were picked and re-streaked on a new plate to confirm the plasmid presence. A single colony was inoculated for the overnight culture and upon reaching $\mathrm{OD}_{600}=0.8-1$, equi-volumetric amount of $50 \%$ sterile glycerol was added to the culture. Upon mixing, $1 \mathrm{ml}$ aliquots are taken and frozen in cryo-vials on $-80{ }^{\circ} \mathrm{C}$ for further expansion. The entire workflow was guided by Dr. Carolyn Mulu Wu and conducted in the lab of Prof, Thomas Dick, NUS, Singapore.

\subsubsection{Preparation of $M$. smegmatis $\operatorname{mc}^{2} 5^{2}$ pJV62 EC}

To prepare the electro-competent cells that express the gp61 protein required

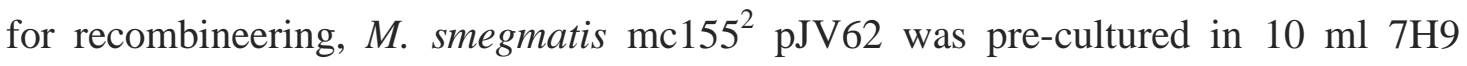
media containing $25 \mu \mathrm{g} / \mathrm{ml}$ of kanamycin to $\mathrm{OD}_{600}$ of $0.4-0.6$. One $\mathrm{ml}$ of the preculture was inoculated into the induction media overnight and upon reaching the $\mathrm{OD}_{600}$ of $0.4-0.6,2 \mathrm{ml}$ of $20 \%$ sterile acetamide was added into the cultures to a final conc. $0.2 \%$ and incubated for $3 \mathrm{~h}$ and kept on ice in the duration of $1.5 \mathrm{~h}$. The EC cells were then prepared using the same procedure as described above, for the 
Mycobacterium smegmatis $m c 155^{2}$ EC. The entire workflow was guided by Dr. Carolyn Mulu Wu and conducted in the lab of Prof, Thomas Dick, NUS, Singapore.

\subsubsection{Design, transformation of the ssDNA $M$. smegmatis atp $C^{\mathrm{E} 87 \mathrm{~A}}$ oligo into the recombineering strain $M$. smegmatis $\mathrm{mc155}^{2}$ pJV62}

The ssDNA oligo, complementary to the lagging strand, was designed to contain the mutated nucleotide pair E87A in the middle of the sequence whose length is 75 nucleotides as shown in table 2.15 below.

\begin{tabular}{ll}
\hline Construct & Oligonucleotide sequence 5'-3' \\
\hline E87A oligo fwd & CTG CGT CTT CCT TGG CAG CAT CCG CGT CGA TCT CGG \\
& TCGA ATT GTG CGT TCT CCA CGA GGA TCC GGA CGG \\
\hline
\end{tabular}

Table 2.15: Single strand DNA E87A oligonucleotide for recombineering

The $250 \mathrm{ng}$ ssDNA (in $5 \mu \mathrm{l}$ ) was used together with $1 \mu \mathrm{l}(50 \mathrm{ng}$ ) of integrative plasmid pSJ25 to transform $100 \mu$ l of $M$. smegmatis mc $155^{2}$ pJV62 EC as described in section 2.2.22. Therefore, DNA was added to the EC and incubated on ice for $10 \mathrm{~min}$, followed by rapid electroporation and immediate recovery with $1 \mathrm{ml}$ of pre-warmed $7 \mathrm{H} 9$ media. The newly transformed cells were revived for $4 \mathrm{~h}$ incubated at $37{ }^{\circ} \mathrm{C}$ with shaking (200 rpm). Subsequently, cells were plated onto 7H10 agar plate containing $25 \mu \mathrm{g} / \mathrm{ml}$ of kanamycin and $50 \mu \mathrm{g} / \mathrm{ml}$ of hygromycin to recover successful transformants. Recovered colonies isolated on the grid plate and screened for the desired mutation using the PCR screening method. The entire workflow was guided by Dr. Carolyn Mulu Wu and conducted in the lab of Prof, Thomas Dick, NUS, Singapore.

\subsubsection{PCR screening of E87A mutation in M. smegmatis $\mathrm{mc}^{2} 5^{2}$ pJV62}

The transformants' colonies plated on a 7H10 agar plate containing $25 \mu \mathrm{g} / \mathrm{ml}$ of kanamycin and $50 \mu \mathrm{g} / \mathrm{ml}$ of hygromycin were analyzed by colony PCR for the presence of E87A mutation in Msme gene. The 3' end of the forward primer was designed to end at the nucleotide sequence specific for the mutation as shown in table 2.16. 


\begin{tabular}{llll}
\hline Construct & & Oligonucleotide sequence 5' -3' $^{\prime}$ & $\mathbf{T}_{\mathbf{m}}\left({ }^{\circ} \mathrm{C}\right)$ \\
\hline E87A & fwd & 5'- TCC GCG TCG ATC TCG GAC G -3' & $61.9^{\circ} \mathrm{C}$ \\
& rev & 5'- GAC AAG CTC ACC AAG GGC G -3 & $59.0^{\circ} \mathrm{C}$
\end{tabular}

Table 2.16: Primers for the colony PCR screening of atp $C^{\mathrm{E} 87 \mathrm{~A}} M$. smegmatis mutation

In the polymerase chain reaction, the entire colony was used as the template and the reaction was set up by mixing all the reagents in a manner specified in table 2.17 below.

\begin{tabular}{ll}
\hline Reagents & Volume $(\boldsymbol{\mu l})$ \\
\hline Entire colony & \\
$\mathrm{ddH}_{2} \mathrm{O}$ & 13.8 \\
$\left(\mathrm{NH}_{4}\right)_{2} \mathrm{SO}_{4} \mathrm{TAQ}$ buffer & 2.00 \\
$\mathrm{MgCl}_{2}(25 \mathrm{mM})$ & 1.60 \\
$\mathrm{dNTP}(10 \mathrm{mM})$ & 1.50 \\
primer E87A screening_FWD & 1.00 \\
primer PCR control_REV & 1.00 \\
Taq polymerase & 0.20 \\
\hline
\end{tabular}

Table 2.17: Reaction mix for screening PCR of $a t p C^{\mathrm{E} 87 \mathrm{~A}}$ mutants

To start the reaction, colonies were scraped using the sterile yellow tip into the PCR tubes containing the mixture. The tubes were subsequently placed in a preheated block of the PCR Thermocycler (Biometra T personal) to $95{ }^{\circ} \mathrm{C}$. The program shown in Table 2.18 resulted in amplification of the sequence-specific only to the desired mutant. The entire workflow was guided by Dr. Carolyn Mulu Wu and conducted in the lab of Prof, Thomas Dick, NUS, Singapore.

\begin{tabular}{lll}
\hline Cycle steps & Temperature $\left({ }^{\circ} \mathbf{C}\right)$ & Time \\
\hline Lid & 95 & \\
Initial denaturation & 95 & $5 \mathrm{~min}$ \\
Cycle denaturation & 95 & $30 \mathrm{sec}$ \\
Annealing & 62 & $30 \mathrm{sec}$ \\
cycles \\
Extension & 72 & $50 \mathrm{sec}$ \\
Final extension & 72 & $10 \mathrm{~min}$ \\
End & 4 & \\
\hline
\end{tabular}

Table 2.18: PCR program for screening PCR of $a t p C^{\mathrm{E} 87 \mathrm{~A}}$ mutants; 


\subsubsection{Isolation of inverted membrane vesicle (IMV) isolation from $M$. smegmatis $m c 155^{2}(\mathrm{WT})$ and mutant $a t p C^{\mathrm{E} 87 \mathrm{~A}}$}

In order to purify membrane fractions (inverted vesicles) of M. smegmatis for ATP synthesis, hydrolysis, and proton pumping assays, cells were cultivated overnight at $37^{\circ} \mathrm{C}$ in $7 \mathrm{H} 9$ medium supplemented with $10 \%$ albumin/glucose/catalase enrichment (ADC), $0.5 \%$ glycerol and $0.05 \%$ Tween-80 until they reached an attenuance at 600 $\mathrm{nm}$ of 0.6-0.7. The culture was expanded in $200 \mathrm{~mL}$ supplemented 7H9 medium, and grown in 11 shake flasks $(180 \mathrm{rpm})$ until it reached an attenuance at $600 \mathrm{~nm}$ of 0.6 0.7. This culture was used to inoculate a $500 \mathrm{~mL}$ culture that was then grown overnight in 21 shake flasks (180 rpm) until it reached an attenuance at $600 \mathrm{~nm}$ of 0.60.7. Approximately $5 \mathrm{~g}$ (wet weight) of WT $M$. smegmatis and the $\operatorname{atp} C^{\mathrm{E} 87 \mathrm{~A}}$ mutant were re-suspended in $20 \mathrm{~mL}$ membrane preparation buffer $(50 \mathrm{mM}$ MOPS, $2 \mathrm{mM}$ $\mathrm{MgCl} 2, \mathrm{pH} 7.5$ ) containing EDTA-free protease inhibitor cocktail (one tablet per 20 $\mathrm{ml}$ buffer, Roche) and $1.2 \mathrm{mg} / \mathrm{ml}$ lysozyme. The suspension was stirred at room temperature for $45 \mathrm{~min}$. and additionally supplemented with $300 \mu 1$ of $1 \mathrm{M} \mathrm{MgCl}_{2}$ and $50 \mu \mathrm{l}$ DNase I and stirring was continued for another $15 \mathrm{~min}$ at room temperature. All subsequent steps were performed on ice. Cells were lysed by three passages through an ice-cooled microfluidizer (model M-110L, Microfluidics, Westwood, MA, USA) at $18,000 \mathrm{psi}$. The suspension containing lysed cells was centrifuged at $4,200 \mathrm{~g}$ at $4{ }^{\circ} \mathrm{C}$ for $20 \mathrm{~min}$. The supernatant containing the membrane fraction was further subjected to ultracentrifugation at $45,000 \mathrm{~g}$ at $4{ }^{\circ} \mathrm{C}$ for $1 \mathrm{~h}$. The supernatant was discarded, and the precipitated membrane fraction was re-suspended in membrane preparation buffer containing $15 \%$ glycerol, separated into aliquots, snap-frozen and stored at $-80{ }^{\circ} \mathrm{C}$. The concentrations of the proteins in the vesicles were determined by the bicinchoninic acid assay (BCA; Pierce, Rockford, IL, USA). IMVs were stored at $-80{ }^{\circ} \mathrm{C}$.

\subsubsection{ATP synthesis assay for screening the activities of $M$. smegmatis mc155 $^{2}$ (WT), mutant atp $C^{\mathrm{E} 87 \mathrm{~A}}$ and mutant $a t p C^{\mathrm{E} 87 \mathrm{~A}}$ complemented strains IMVs}

ATP synthesis was measured in a flat bottom white micro-titer 96 well plates. The reaction mix $(50 \mu \mathrm{l})$, made in assay buffer (50 mM MOPS, pH 7.5, $10 \mathrm{mM}$ $\mathrm{MgCl}_{2}$ ) containing $10 \mu \mathrm{M}$ ADP, $250 \mu \mathrm{M} \mathrm{P} i$ and $1 \mathrm{mM}$ NADH. The concentration of $\mathrm{P} i$ was adjusted by the addition of $100 \mathrm{mM} \mathrm{KH}_{2} \mathrm{PO}_{4}$ salt dissolved in the assay buffer. ATP synthesis was initiated by adding IMVs of WT M. smegmatis and its mutants to a 
final concentration of $5 \mu \mathrm{g} / \mathrm{ml}$. The reaction mix was incubated at room temperature and aliquots of $50 \mu \mathrm{l}$ were transferred to a 96 well plate at $\mathrm{t}=0,2,5,10,15,20,15$, and $30 \mathrm{~min}$, followed by the addition of $50 \mu \mathrm{l}$ of the luciferin-luciferase assay. The mixture was incubated for another $10 \mathrm{~min}$ in dark at room temperature. Produced luminescence, which was correlated with the synthesized ATP, was measured by a Tecan plate reader Infinite 200 Pro, using the following parameters: luminescence, integration time $500 \mathrm{~ms}$, attenuation none.

\subsubsection{ATP hydrolysis assay for $M$. smegmatis $m c 155^{2}$ (WT), mutant atp $C^{\mathrm{E} 87 \mathrm{~A}}$ and mutant atp $^{\mathrm{E} 87 \mathrm{~A}}$ complemented strains IMVs}

A continuous ATP hydrolysis assay was applied to measure the ATPase activity of IMVs according to section 2.2.15. To prevent the NADH consumption by the type II NADH dehydrogenase (NDH-2) found in the IMVs, the assay was supplemented with the inhibitor, thioridazine to the final concentration of $80 \mu \mathrm{M}$. In addition, ATPase activity of IMVs was also measured in the absence of ATP and presence of thioridazine to identify any slight background oxidation of NADH and to determine the amount of $\mathrm{Mg}$-ATP hydrolyzed per minute and total protein.

\subsubsection{ATP-driven proton translocation}

ATP-driven proton translocation into IMVs of $M$. smegmatis mc1552 was measured by a decrease of 9-amino-6-chloro-2-methoxy acridine (ACMA) fluorescence using a Cary Eclipse Fluorescence spectrophotometer (Varian Inc.,Palo Alto). IMVs $(0.18 \mathrm{mg} / \mathrm{ml})$ were pre-incubated at $37^{\circ} \mathrm{C}$ in $10 \mathrm{mM}$ HEPES-KOH $(\mathrm{pH}$ 7.5), $100 \mathrm{mM} \mathrm{KCl}, 5 \mathrm{mM} \mathrm{MgCl}_{2}$ containing $2 \mathrm{mM}$ ACMA and a baseline was monitored for $5 \mathrm{~min}$. The reaction was then started by adding $2 \mathrm{mM}$ ATP or $2 \mathrm{mM}$ $\mathrm{NADH}$ (control). After $20 \mathrm{~min}$, any proton gradient was collapsed by the addition of 1 $\mathrm{mM}$ SF6847. The excitation and emission wavelengths were 410 and $480 \mathrm{~nm}$, respectively.

\subsubsection{Determination of the intracellular ATP level of $M$. smegmatis $m c 155^{2}$ and mutant $\operatorname{atp} C^{\mathrm{E} 87 \mathrm{~A}}$}

To measure the intercellular content of ATP in cultures, upon reaching the $\mathrm{OD}_{600}$ of $1,25 \mu \mathrm{l}$ aliquots were mixed $25 \mu \mathrm{l}$ of freshly prepared BacTiter-Glo 
Microbial Cell Viability Assay kit from Promega according to the manufacturer's instructions. The intracellular ATP was quantified in a 96-well white opaque Nunc plates and incubated for $5 \mathrm{~min}$ in the dark. The emitted luminescence was detected using a Tecan Infinite M200 Pro plate reader (Tecan instruments) and displayed as relative light units (r.l.u.); the readings were directly correlated with the ATP content of the respective sample. All experiments were standardized using ATP solutions ranging from 0.1 to $100 \mathrm{nM}$ as an internal control. The absolute amount of ATP per bacterium was calculated by dividing mol ATP $\mathrm{ml}^{-1}$ by c.f.u. $\mathrm{ml}^{-1}$.

\subsubsection{BDQ susceptibility test}

Cultures of $M$. smegmatis $\mathrm{mc} 155^{2}$ and $a t p C^{\mathrm{E} 87 \mathrm{~A}}$ mutant were cultivated in an overnight culture and seeded into a secondary culture at the starting $\mathrm{OD}_{600} 0.1$ until it reached 1. Serial dilutions of the drug BDQ, dissolved in 95\% DMSO, were prepared in a flat-bottom 96-well transparent plate to the following concentrations: $0,4,8,16$, 32, 64, 128, 256, 512, 1024, $2048 \mathrm{nM}$. Wells contained $50 \mu \mathrm{l}$ of BDQ solution which was mixed with the equi-volumetric amount of bacterial culture to the final BDQ concentrations reaching half the value mentioned above $(0,2,4,8,16,32,64,128$, $256,512,1024)$ and $\mathrm{OD}_{600}=0.5$. Cultures were then incubated on $37{ }^{\circ} \mathrm{C}$ with shaking, and the $\mathrm{OD}_{600}$ values were read using the Tecan plate reader Infinite 200 Pro.

\subsubsection{Acid-Fast Staining and Light Microscopy}

The acid-fast staining was carried out using a TB stain kit (BD, 212520) according to the manufacturer's instructions. An aliquot of the culture in log phase $\left(\mathrm{OD}_{600}=0.6\right)$ was pipetted onto a microscope glass slide and dyed as described further. The microscope slides were placed onto the beaker rim and were flooded with TB Carbolfuchsin ZN. The excess of the dye was blotted away with filter paper. The gentle heating was applied using the hotplate until steaming occurred (5 min). The microscope slide was washed gently in running water. The de-colorization with TB Decolorizer was done in two subsequent steps, each 1-2 min until no more red color appeared in washing. Subsequently, the slides were washed gently in running water. After the drying, the cover glass was placed on top of the sample smear and sealed with the nail polish. The samples were observed under the white-field microscope (Olympus BX60, bright field). 


\subsubsection{Quantification of proteins using bicinchoninic acid (BCA) assay and nanodrop}

BSA is used as a standard for protein quantification by BCA method. BCA reagent available in kit form from Pierce (Rockford, IL, USA.) was used for measuring protein concentration. In general, two dilutions of protein samples were taken and each of them was measured in triplicates. Optical density was measured at $562 \mathrm{~nm}$ against a blank. A standard curve with $50 \mu \mathrm{g} / \mathrm{ml}, 125 \mu \mathrm{g} / \mathrm{ml}, 200 \mu \mathrm{g} / \mathrm{ml}$ and $250 \mu \mathrm{g} / \mathrm{ml}$ of BSA was then drawn from the $\mathrm{OD}_{562}$ and the fitting equation was derived. The same equation was used to calculate the protein concentration. The protein concentration was also measured with the help of a nanodrop (BioSpec-Nano, Shimadzu) by measuring the absorption of aromatic amino acids at $280 \mathrm{~nm}$. Molar extinction coefficients were determined as $147,620 \mathrm{M}^{-1} \mathrm{~cm}^{-1}$ for the $\alpha^{\text {chi }}{ }_{3}: \beta_{3}: \gamma$ and $153,120 \mathrm{M}^{-1} \mathrm{~cm}^{-}$

${ }^{1}$ for the $\alpha^{c h i}{ }_{3}: \beta_{3}: \gamma: M t \varepsilon$, using ExPASy ProtParam (286). The values obtained were reliable and reproducible, so this technique was accepted for its feasibility.

\subsubsection{Structural model of the $A$. woodii F-ATP synthase and interactions of subunits $\gamma$ with $c_{1}$ and $c_{2 / 3}$}

A homology model of the $A$. woodii $\mathrm{F}_{1} \mathrm{~F}_{\mathrm{O}}$ ATP synthase was built by combining the structural information of the $c$-ring (PBDID: 4BEM) (7) and 3D models obtained by using the 3D structure prediction server, SWISS-MODEL (287). In brief, to model the $\mathrm{F}_{1}$ subunits $\alpha, \beta, \gamma, \delta, \varepsilon$ and $b$ and subunit $a$ of the $\mathrm{F}_{\mathrm{O}}$ part, their protein sequence information was obtained from the UniProt server (288) and used as the input for modeling. The default server settings were used, without imposing any constraints on the model calculations. As the output, single/several models of each subunit were generated and the files of the highest-scoring hits in PDB format were used for further processing. To frame the 3D models of individual subunits in the distance constraints present in the determined F-ATP synthase structure, the E. coli FATP synthase cryo-EM structure (PBD ID: 5T4O) (143) was used as a template. The structural alignment of $\alpha, \beta, \gamma, \delta, \varepsilon, b$ and subunit $a$ with their counterparts was performed in Pymol (289). The A. woodii c-ring structure was also superimposed on the E. coli $c$-ring. This workflow generated a model that enabled distance approximations between the unique loop of subunit $\gamma$ and subunits of the $c$-ring, without introducing steric clashes. 


\subsubsection{Cloning of the $A$. woodii $\mathbf{F}_{1} \mathbf{F}_{0}$-ATP synthase deletion mutant $\gamma_{\Delta 195-211}$}

The $\gamma$-loop deletion mutant $\gamma_{\Delta 195-211}$ has been constructed by site-directed mutagenesis using the vector pKB3-His (Brandt et al., 2013), as a template. The forward primer 5'Phospho-GCA AAT GGG TCG GGA TCC GAT CCA TGC ATC ACC ATC ACC ATC ACC ATC AC -3' binds to the downstream region of the DNA sequence that codes for the peptide ${ }_{195}$ TSGKVKTTEETKEEKSK 211 of the $\gamma$ subunit. The reverse primer 5'- TAT CAA CTC TTC GGC CGA AAG CGG CAA CAA TTT CAT CAT TTG AGC -3' binds to the sequence upstream of the peptide designated for the deletion. The amplification of the entire plasmid was carried out using the Kappa $\mathrm{HiFi}$ polymerase. The PCR product was purified and circularized using T4 DNA ligase (New England Biolabs). Precipitated pDNA was transformed into plasmid amplification strain, E. coli DH5- $\alpha$. After mutant verification by sequencing, the selected pDNA carrying the $\gamma_{\Delta 195-211}$ mutation was transformed into E. coli DK8 for protein expression.

\subsubsection{Purification of the $A$. woodii $\mathrm{F}_{1} \mathrm{~F}_{\mathrm{O}}$-ATP synthase WT and mutant $\gamma_{\Delta 195-211}$}

The WT recombinant $A$. woodii $\mathrm{F}_{1} \mathrm{~F}_{\mathrm{O}}$ ATP synthase and its mutant $\gamma_{\Delta 195-211}$ were purified according to the protocol in Brandt et al. (2013) with the following modifications. E. coli DK8 cells containing the plasmid encoding WT or the mutant $\gamma_{\Delta 195-211}$ was cultivated in 101 of $2 x Y T$-medium at $37{ }^{\circ} \mathrm{C}$ under aeration. Gene expression was induced at $\mathrm{OD}_{600}$ of 1.0-1.5 by addition of $0.5 \mathrm{mM}$ IPTG. After 1.5-2 $\mathrm{h}$ of growth, cells were harvested and frozen in liquid nitrogen for storage.

The recombinant proteins were purified at $4{ }^{\circ} \mathrm{C}$ unless otherwise indicated. Cells were re-suspended in $25 \mathrm{ml}$ buffer A $(50 \mathrm{mM}$ Tris/ $\mathrm{HCl}, 10 \mathrm{mM} \mathrm{MgCl} 2,10 \%$ [v/v] glycerol, pH 7.5) per $10 \mathrm{~g}$ of wet weight. Cells were disrupted by ultrasonication (3x1 min, 25\%; Bandelin, Sonoplus HD2200 with cone tip KE76). After removal of cell debris $(14,000 \mathrm{xg}, 30 \mathrm{~min})$, membranes were prepared by ultracentrifugation (180,000xg, $45 \mathrm{~min})$. To solubilize the ATP synthase the membrane pellet was dissolved in buffer B containing $1 \%[\mathrm{w} / \mathrm{v}] \mathrm{N}$-dodecyl- $\beta$-maltoside (DDM; Glycon, Germany) and incubated for $1 \mathrm{~h}$. Remaining membranes were removed by ultracentrifugation $(180,000 \mathrm{xg}, 45 \mathrm{~min})$ and the solubilisate was applied to a $\mathrm{Ni}^{2+}$-NTA-chromatography. $\mathrm{Ni}^{2+}$-NTA beads were equilibrated with buffer B $(50 \mathrm{mM}$ 
Tris/HCl, $300 \mathrm{mM} \mathrm{NaCl}, 5 \mathrm{mM} \mathrm{MgCl}_{2}, 20 \mathrm{mM}$ imidazole, $10 \%$ [v/v] glycerol, $0.1 \%$ [w/v] DDM, 0.8 mM DTT, pH 7.5) prior to incubation with the solubilisate for $1 \mathrm{~h}$.

The material was transferred to a column and after washing the column with 15 column volumes (cv) of buffer B, the F-ATP synthase was eluted with a stepwise (2 cv) imidazole gradient in buffer B. The final imidazole concentrations were $40 \mathrm{mM}$, $60 \mathrm{mM}, 80 \mathrm{mM}, 100 \mathrm{mM}$, and $150 \mathrm{mM}$. Fractions containing the F-ATP synthase $(60 \mathrm{mM}, 80 \mathrm{mM}, 100 \mathrm{mM})$ were pooled and concentrated to a volume of $1 \mathrm{ml}$ (Vivaspin 20 columns, $100 \mathrm{kDa}$ MWCO, Sartorius, Germany). To further purify the sample an additional size exclusion chromatography was performed at room temperature. $1 \mathrm{ml}$ of sample was applied onto a Superdex HR 200 column (GE Healthcare) that was equilibrated with buffer $\mathrm{C}(50 \mathrm{mM}$ Tris/ $\mathrm{HCl}, 150 \mathrm{mM} \mathrm{NaCl}, 5$ $\mathrm{mM} \mathrm{MgCl}_{2}, 10 \%$ [v/v] glycerol, $0.05 \%$ [w/v] DDM, pH 7.5). The protein was eluted with buffer $\mathrm{C}$ after around $11.5 \mathrm{ml}$ (Peak 1) with a flow rate of $0.5 \mathrm{ml} / \mathrm{min}$. Only the major fractions from the peak I were pooled and applied onto a 12\% SDS polyacrylamide gel (290). ATPase activity of the enzymes was done as described in section 2.2.15.

\subsubsection{Electron microscopy and $2 \mathrm{D}$ image analysis of $A$. woodii F-ATP synthase}

A. woodii F-ATP synthase was diluted in buffer B $30 \mu \mathrm{g} / \mathrm{ml}$ and a volume of 4 $\mu \mathrm{l}$ of protein sample was applied to a glow-discharged carbon coated copper transmission electron microscopy grid and stained with $2 \%(\mathrm{v} / \mathrm{v})$ uranyl acetate. Electron micrographs were recorded on a Tecnai spirit T12 transmission electron microscope (FEI) equipped with a 4K CCD camera (FEI) operated at a voltage of 120 $\mathrm{kV}$ at a calibrated magnification of $66,350 \mathrm{x}$ under low dose conditions. 49 micrographs were recorded at $0^{\circ}$ angle for the enzyme complex. A total of 1,674 particles for A. woodii F-ATP synthase were selected for 2D analysis. The selection criteria were clear visibility of single molecules and separation from neighboring particles. Particles were picked using EMAN2 (271) and processed using RELION 1.3 (278). 


\section{Results}


3.1 Characterization of the $\alpha^{c h i}{ }_{3}: \beta_{3}: \gamma: M t \varepsilon$ complex 


\subsubsection{Optimized production, purification of the $\alpha^{c h i}{ }_{3}: \beta_{3}: \gamma$ sub-complex and reconstitution with $M t \varepsilon$}

Plasmid pET-24b containing the genes $\alpha$ (modified to $\alpha^{\text {chi }}$ as described previously (209)), $\gamma$ and $\beta$ (4,012 bp) of the F-ATP synthase from Geobacillus stearothermophilus was generated as described in the Dr. Lavanya Sundararaman Ph.D. thesis (291). The plasmid DNA (pDNA) was transformed into E. coli C41 cells (292) for the production of the recombinant $\alpha^{c h i}{ }_{3}: \beta_{3}: \gamma$ as described in section 2.2.3. Purification of $\alpha^{\text {chi }}{ }_{3}: \beta_{3}: \gamma$ was performed as per the established two-step purification protocol (3). The complex eluting from the $\mathrm{Ni}^{2+}$-NTA affinity column was analyzed on a $12 \%$ SDS-gel, showing that subunits $\alpha^{c h i}, \beta$, and $\gamma$ were obtained (3.1A). Fractions containing 100-400 mM of imidazole were pooled and applied on an ion-exchange column (Resource Q, $6 \mathrm{ml}$ ) which resulted in the elution of homogeneous $\alpha^{c h i}: \beta_{3} \gamma$ (3.1B). The desired protein was obtained at $200 \mathrm{mM}$ of $\mathrm{NaCl}$.

Subsequently, the protein was used for reconstitution with subunit Mte purified separately according to an established protocol, section 2.2.10 and (291);. Freshly purified sub-complex $\alpha^{\text {chi }}{ }_{3}: \beta_{3}: \gamma$ was incubated with a 3-fold molar excess of $M t \varepsilon$ at 4 ${ }^{\circ} \mathrm{C}$ for $1 \mathrm{~h}$ in $50 \mathrm{mM}$ Tris/ $\mathrm{HCl}, \mathrm{pH} 7.5,200 \mathrm{mM} \mathrm{NaCl}$. The reconstituted mixture was injected into the gel filtration column (Superdex 300, GE-Healthcare) to remove the excess of unbound $M t \varepsilon$ (3.1C). The protein complex eluted at $11.6 \mathrm{ml}$ and was concentrated using a $100 \mathrm{kDa}$ centricon. The concentrated sample, when analyzed on an SDS-gel, confirmed that individual subunit $M t \varepsilon$ has bound to the $\alpha^{\text {chi }}{ }_{3}: \beta_{3}: \gamma$ complex (3.1D).

Furthermore, to quantify the efficiency of $M t \varepsilon$ binding to the complex, the reconstituted sample was applied on $12 \%$ SDS gel and subjected to densitometric analysis using ImageJ software (Figure 3.2; (282). The band intensities belonging to subunit $\gamma$ and $M t \varepsilon$ were converted into the 2D peaks whose integrals were calculated. The value obtained for subunit $\gamma$ was normalized to $1(100 \%)$ while the $M t \varepsilon$ peak was shown as relative to $\gamma$. In the case of the $\alpha^{c h i}: \beta_{3}: \gamma: M t \varepsilon$ complex, the $\gamma: M t \varepsilon$ ratio was calculated to be 1:0.5 as shown in Figure 3.2.

The $\alpha^{c h i}: \beta_{3}: \gamma:$ Mt $\varepsilon$ complex showed an ATP hydrolysis activity of 8.4 $\mu \mathrm{mol} / \mathrm{min} / \mathrm{mg}$ protein, which is roughly 1.8 times higher than the activity of $\alpha^{\text {chi }}{ }_{3}: \beta_{3}: \gamma$ ( $4.7 \mu \mathrm{mol} / \mathrm{min} / \mathrm{mg}$ of protein). These results indicate that the $M t \varepsilon$ is a pre-requisite for the maximal activity of the complex. 
(A)

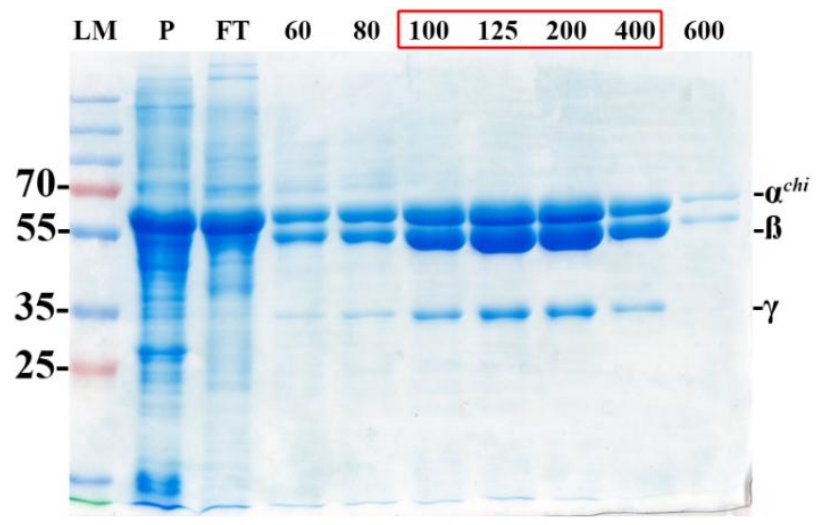

(C)

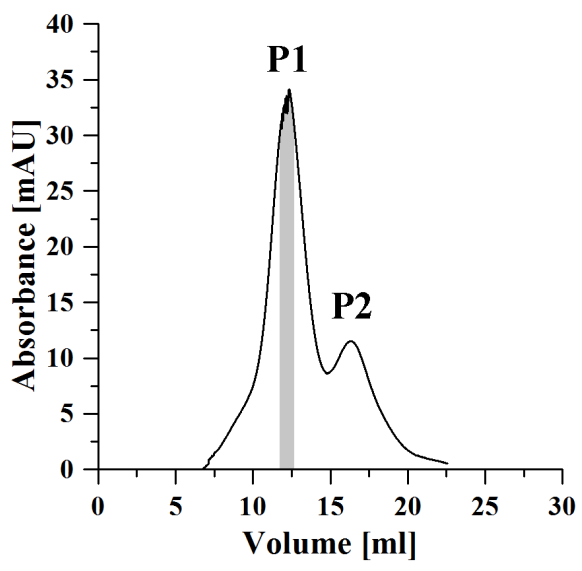

(B)

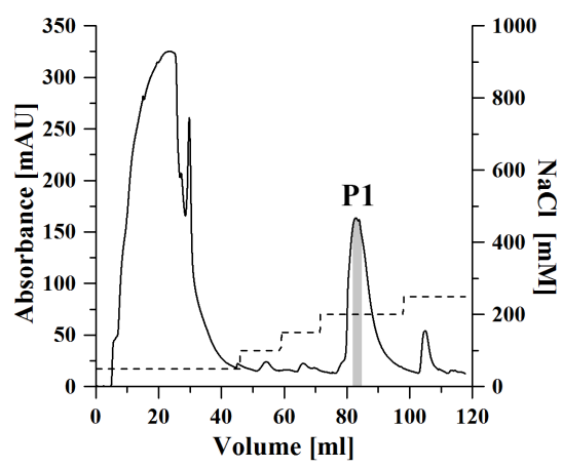

(D)

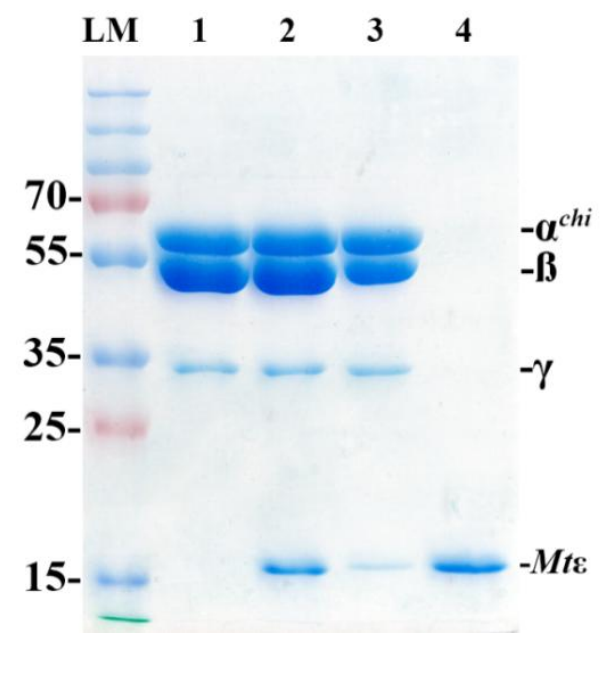

Figure 3.1: Purification of $\boldsymbol{\alpha}^{c h i}: \beta_{3}: \gamma$ and reconstitution with $\boldsymbol{M t} \boldsymbol{\varepsilon}(\mathrm{A}) \mathrm{Ni}^{2+}{ }_{-} \mathrm{NTA}$ purification profile showing the desired bands of subunits $\alpha^{c h i}, \beta$, and $\gamma$. Fractions from $100 \mathrm{mM}$ to $400 \mathrm{mM}$ imidazole were pooled and applied onto an (B) anion exchange column (Resource Q, $6 \mathrm{ml}$ ) at a constant flow rate of $1 \mathrm{ml} / \mathrm{min}$ with a $\mathrm{NaCl}$ step gradient. The grey area of the peak indicates the pooled fractions. (C) Protein obtained after the anion exchanger was reconstituted with a 3-fold molar excess of MtE and applied onto a gel filtration column (Superdex 300) and eluted at $11.6 \mathrm{ml}$ column volume; greyed area indicates the fractions pooled for the SDS-PAGE analysis and negative stain electron microscopy. Purity of $\alpha^{c h i}{ }_{3}: \beta_{3}: \gamma: M t \varepsilon$ was confirmed by a $12 \%$ SDS-PAGE gel (D); Lane 1 shows the $\alpha^{\text {chi }}{ }_{3}: \beta_{3}: \gamma$ sub-complex loaded after anion exchange column; Reconstituted sub-complex $\alpha^{c h i}: \beta_{3}: \gamma: M t \varepsilon$ was loaded in lane 2 (before the size-exclusion column) and -3 (after the size-exclusion column). Lane 4 represents purified recombinant $M t \varepsilon$. 
(A)

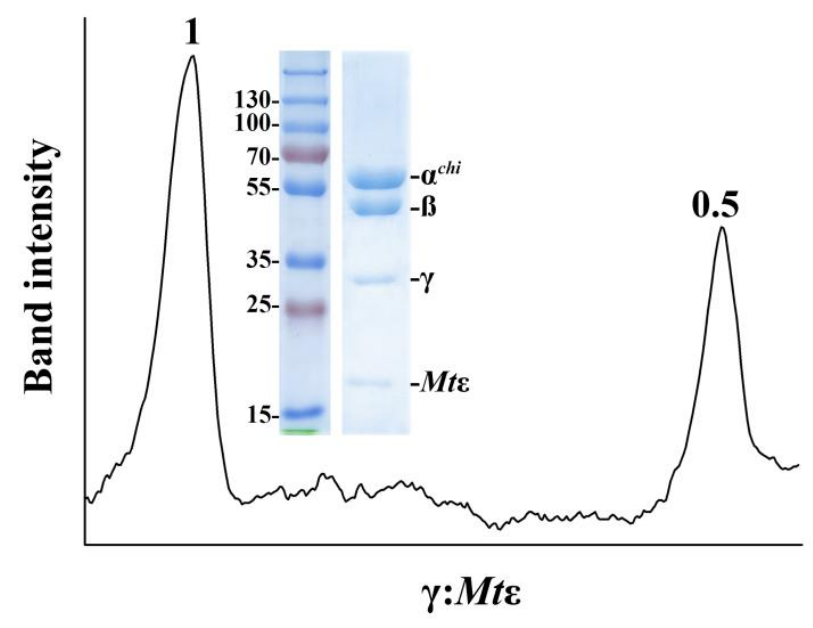

(B)

(C)
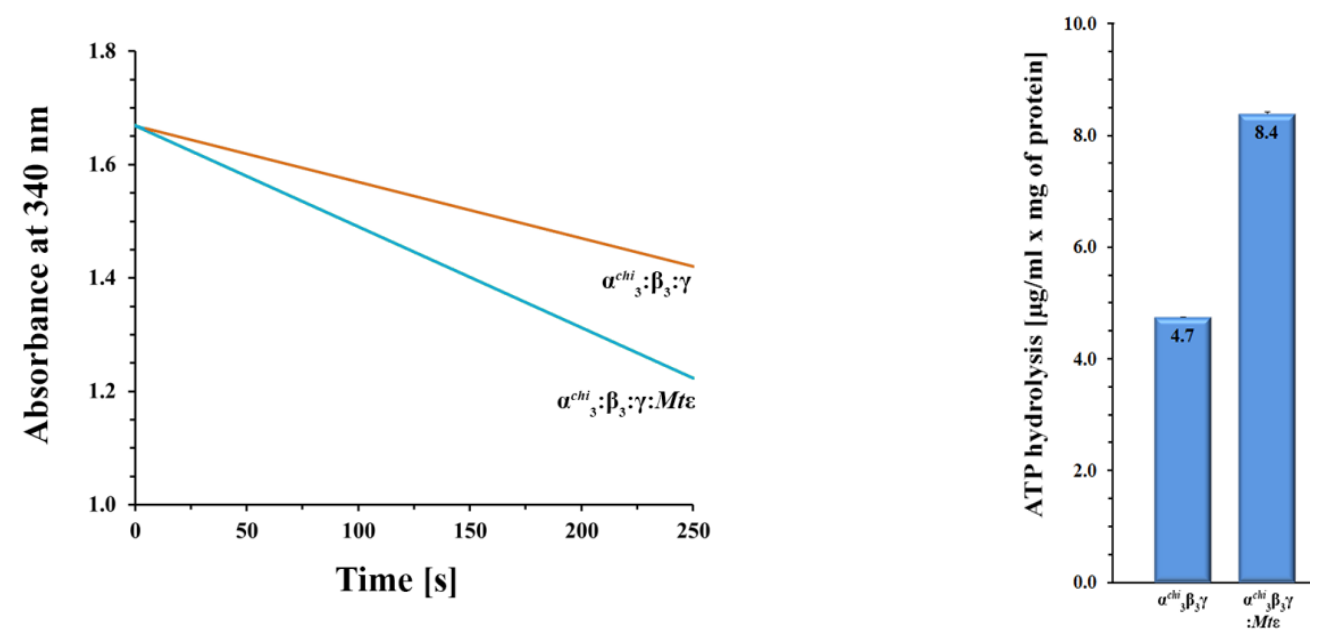

Figure 3.2: Densitogram and ATP hydrolysis of $\alpha^{c h i}{ }_{3}: \beta_{3}: \gamma$ and $\alpha^{c h i}{ }_{3}: \beta_{3}: \gamma: M t \varepsilon$ complexes. (A) Densitogram revealing the ratio of subunits $\gamma: M t \varepsilon$ of 1:0.5 within the reconstituted $\alpha^{\text {chi }}{ }_{3}: \beta_{3}: \gamma: M t \varepsilon$ complex. (B) Continuous ATPase activity is shown as the orange and turquoise line representing the profiles of $\alpha^{c h i}{ }_{3}: \beta_{3}: \gamma$ and $\alpha^{c h i}{ }_{3}: \beta_{3}: \gamma: M t \varepsilon$, respectively. (C) Bar diagram representing the specific ATPase activities of $\alpha^{c h i}{ }_{3}: \beta_{3}: \gamma$ and $\alpha^{c h i}{ }_{3}: \beta_{3}: \gamma: M t \varepsilon$ in the presence of $2 \mathrm{mM} \mathrm{Mg-ATP}$. Figures credited to (205).

\subsubsection{Electron microscopy, $2 \mathrm{D}$ and 3D reconstruction of $\alpha^{c h i}{ }_{3}: \beta_{3}: \gamma: M t \varepsilon$}

To analyze the complex formation and association of subunit $M t \varepsilon$ with $\alpha^{c h i}{ }_{3} \beta_{3} \gamma$, reconstituted sub-complex sample was subjected to single particle analysis (SPA) in negative stain electron microscopy. The protein sample was diluted with $50 \mathrm{mM}$ Tris/ $\mathrm{HCl}, \mathrm{pH} 7.5$, and $200 \mathrm{mM} \mathrm{NaCl}$ to an optimal concentration of $40 \mu \mathrm{g} / \mathrm{ml}$, and stained with uranyl acetate (negative stain) to improve the visibility of the molecules by providing better contrast. A total of 80 images were collected, and 28,349 particles were picked and extracted by the software EMAN 2 (271). The first round of $2 \mathrm{D}$ class averaging had an input of total 28,349 particles which were refined and segregated in 
better-defined classes using software RELION 1.3 (278). Subsequently, the second run of $2 \mathrm{D}$ classification contained 12,860 selected particles that were finally segregated in 50 classes (Figure 3.3).

As observed in the most populated class 1, the sub-complex viewed from the side, reveals the distinct hexameric catalytic headpiece and the position and shape of the central stalk proposed to contain subunits $\gamma$ and Mte (Figure 3.3 and class 1 focused as image 1). The second class represents a sub-complex viewed from the top, revealing the pseudo-hexagonal arrangement of the densities belonging to the nucleotide-binding subunits $\alpha^{\text {chi }}$ and $\beta$ that form a "crown" with the central cavity that is delimited by $\alpha^{\text {chi }}$ and $\beta$ N-terminal domains (NTDs) (Figure 3.3 and class 2 focused as image 2). Additionally, the third class shows the bottom side of the sub-complex where a seventh, central density represents the central stalk subunits $\gamma$ and $M t \varepsilon$, thus confirming the complex formation (Figure 3.3 and class 3 focused as image 3 ).

To produce a 3D model based on the experimental data, two conditions needed to be met: a) generation of the reference map for the software fitting and b) providing the pre-selected particles. For the generation of the reference map, the crystal structure of $\mathrm{F}_{1}$-ATP synthase from G. stearothermophilus was used with modification. Namely, chain $H$ that is assigned to subunit $\varepsilon$ was removed from the structure. The map was filtered to $30 \AA$, and the data were fitted to generate the output of five $3 \mathrm{D}$ classes. Finally, the refinement yielded a 3D model which was used to validate the position of the chimeric tail of $\alpha^{\text {chi }}$ and the presence of $M t \varepsilon$ in the reconstituted sub-complex. As seen in Figure 3.4, the experimental density map shows distinct features of the subunits $\alpha^{c h i}$ in the form of additional density and increased size when compared to subunit $\beta$. The density corresponds to the extra $\mathrm{C}$-terminal helix which could be seen when the modeled $\alpha^{c h i}: \beta_{3}: \gamma: M t \varepsilon$ cartoon representation is fitted into the density map (Figure 3.4). Additionally, it is possible to observe the increase in the size of the density map corresponding to the base of subunit $\gamma$ which could be caused by the association of NTD of MtE with $\gamma$, while the position of its' C-terminal helices is not yet revealed.

Due to the limited resolution of the density map, calculated from the gold standard Fourier shell correlation to be $18 \AA$ (Figure 3.5), it was not possible to reveal the exact orientation of the chimeric helix of $\alpha^{\text {chi }} \mathrm{CTD}$ and the position of subunit $M t \varepsilon C T D$ in respect to the catalytic subunits. 
(A)

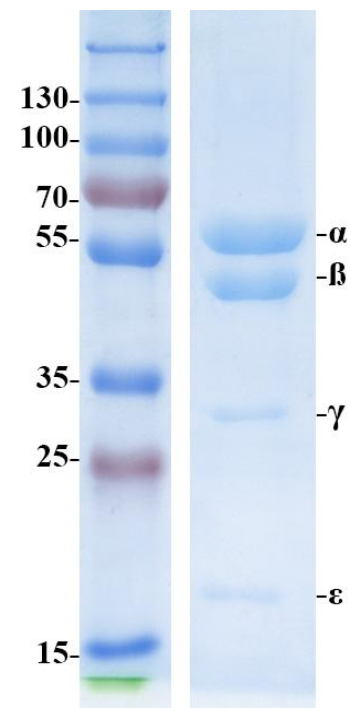

(B)
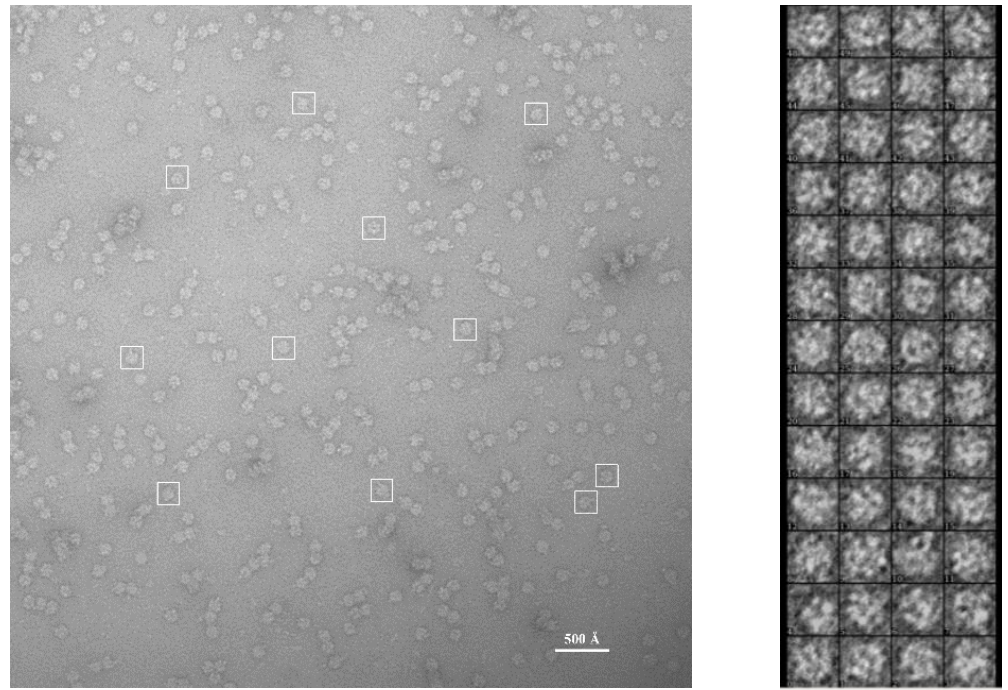

(D)

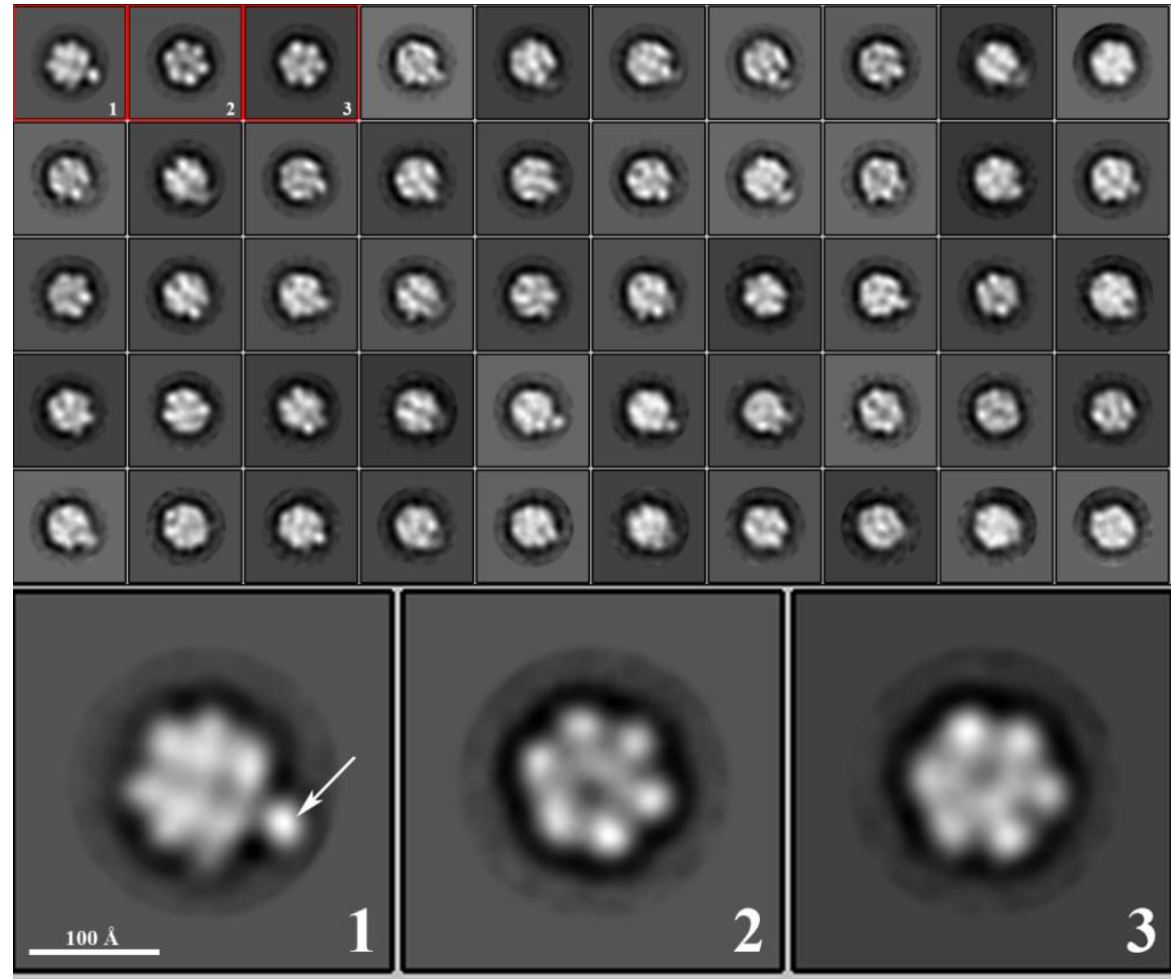

Figure 3.3: Negative stain electron microscopy: (A) SDS gel shows the reconstituted complex used for the negative staining EM. (B) Representative micrograph of negatively-stained reconstituted complex $\alpha^{c h i} \beta_{3} \gamma-M t \varepsilon$ displays well-dispersed particles in various orientations. (C) The panel contains particles selected for the 2D classification using EMAN2. (D) Upper panel shows $\alpha$ chi3 $33 \gamma-\mathrm{Mt} \varepsilon$ particles sorted into fifty $2 \mathrm{D}$ classes using RELION 1.3. The classification was performed on 28,349 particles in two rounds resulting in 12,860 particles used to obtain the classes. Lower panel shows classes with prominent features: 1 . side view showing the central stalk (arrow); 2 . top view shows the pseudo-hexameric arrangement of alternating subunits $\alpha$ chi and $\beta$ with the central cavity; 3 . bottom view showing additional density that possibly corresponds to the central stalk subunits ( $\gamma$ and Mte). Figures credited for (205). 
1

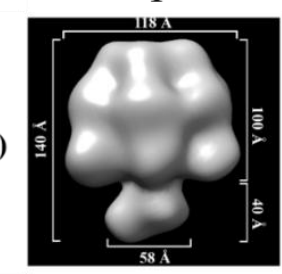

B)

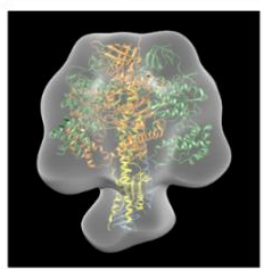

2
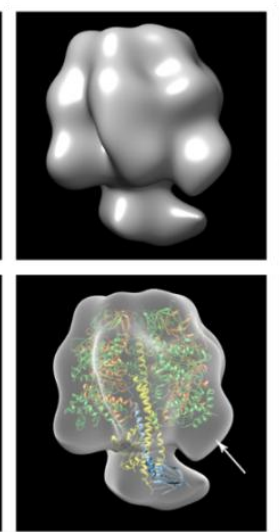

3
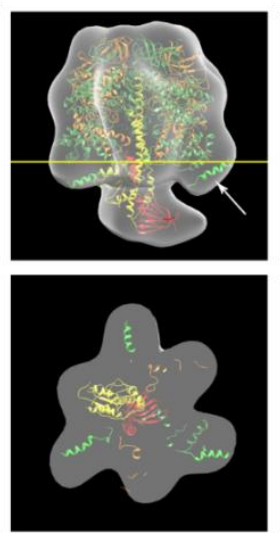

4
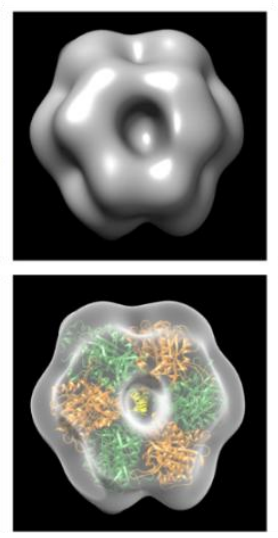

5
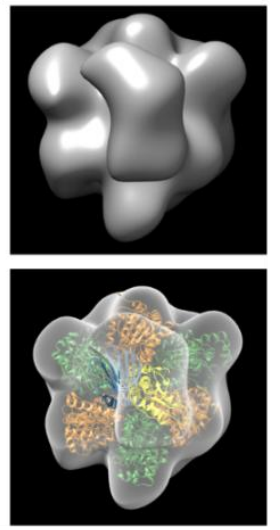

Figure 3.4: Surface representation of 3D reconstruction of $\alpha^{c h i}{ }_{3}: \beta_{3}: \gamma: M t \varepsilon$ complex. (A) Surface 3D map refined to $\sim 18 \AA$ using RELION 1.3. 12,860 particles selected through the 2D classification were used for the reconstruction. (A1, A2) Side views show asymmetric subunits $\alpha^{\text {chi }}$ and $\beta$ and a central stalk $\gamma$ and Mte.; (A3) Side view sectioned at the position of extra C-terminal densities of $\alpha^{\text {chi }}$ subunits; yellow line indicates the level of the section, arrow indicates the C-terminal helix fitted into the density; (A4) Top view is represented with the "crown" of alternating subunits $\alpha^{\text {chi }}$ and $\beta$ arranged to form a central cavity; (A5) The bottom view of the complex represents subunits $\alpha^{\text {chi }}$ and $\beta$ arranged in pseudo-hexagonal manner and the additional density corresponding to the central stalk subunits $\gamma$ and $M t \varepsilon ;(B)$ Panel contains the cartoon representations of $G$. stearothermophilus $\alpha_{3}: \beta_{3}: \gamma: \varepsilon$ and the $\alpha^{c h i}: \beta_{3}: \gamma: M t \varepsilon$ complex model fitted into the surface density map obtained from the NS-EM of the reconstituted complex $\left(\alpha_{3}^{\text {chi }}{ }_{3}: \beta_{3}: \gamma: M t \varepsilon\right)$. Colour code: $\alpha$-forest green, extended $C$-terminus shown in light green; $\beta$-orange, $\gamma$-yellow, $\varepsilon$-blue and $M t \varepsilon$-red. (B1) Side view of the complex reveals the unoccupied densities of the subunits $\alpha$ that correspond to the extra C-terminal peptide of $\alpha^{\text {chi }}$; (B2) shows the model of the extended state of subunit $\varepsilon$, subunit $\beta$ hidden for viewing convenience, arrow indicates unoccupied densities of the subunit $\alpha$; (B3) Cross section shows extra C-terminal helices of $\alpha^{\text {chi }}$ nested in the density map in an alternating fashion; (B4) Top view of the hexamer and central shaft; (B5) Bottom view of the complex emphasizes the central stalk subunits $\gamma$ and $\varepsilon$. Graphics are visualized using UCSF CHIMERA. Figures credited to (205).

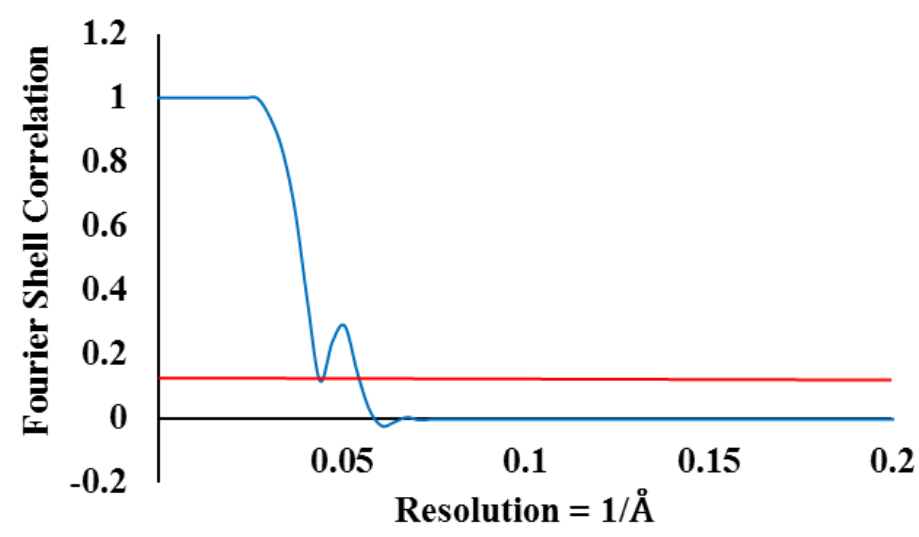

Figure 3.5: FSC graph for negatively-stained reconstituted $\alpha^{c h i}{ }_{3} \beta_{3} \gamma: M t \varepsilon$ complex. Density map resolution calculated for the FSC value of approx. $0.143(0.146)$. Global resolution based on FSC is $1 / 0.55=\sim 18 \AA$ A. Figures credited to (205). 


\subsubsection{Kinetic properties of the $\alpha^{c h i}{ }_{3}: \beta_{3}: \gamma-$ and $\alpha^{c h i}{ }_{3}: \beta_{3}: \gamma: M t \varepsilon$ complexes}

To understand the enzymatic activity of the $\alpha^{c h i}{ }_{3}: \beta_{3}: \gamma$ and $\alpha^{c h i}{ }_{3}: \beta_{3}: \gamma: M t \varepsilon$, an NADH-coupled ATPase assay was performed using $2 \mathrm{mM}$ ATP as the substrate. The assay revealed an ATPase activity of around $4.7 \mu \mathrm{mol} / \mathrm{min} / \mathrm{mg}$ protein for $\alpha^{\text {chi }}{ }_{3} \beta_{3} \gamma$ and $8.4 \mu \mathrm{mol} / \mathrm{min} / \mathrm{mg}$ protein for $\alpha_{3}^{c h i}: \beta_{3}: \gamma: M t \varepsilon$ (Figure 3.6). The higher activity of the $\alpha^{c h i}: \beta_{3}: \gamma: M t \varepsilon$ complex does signify the importance of subunit $M t \varepsilon$ as an activator for ATP hydrolysis. To determine the role of subunit $M t \varepsilon$, substrate-dependent ATPhydrolysis experiments with varying concentrations of ATP $(200 \mu \mathrm{M}, 60 \mu \mathrm{M}, 20 \mu \mathrm{M}$, and $6 \mu \mathrm{M} \mathrm{MgATP}$ ) were performed (Figure 3.6). The Michaelis-Menten constant $\left(K_{m}\right)$ and the maximum rate $\left(V_{\max }\right)$ of $\alpha^{c h i}: \beta_{3}: \gamma$ and $\alpha^{c h i}{ }_{3}: \beta_{3}: \gamma: M t \varepsilon$ were determined (Figure 3.7).

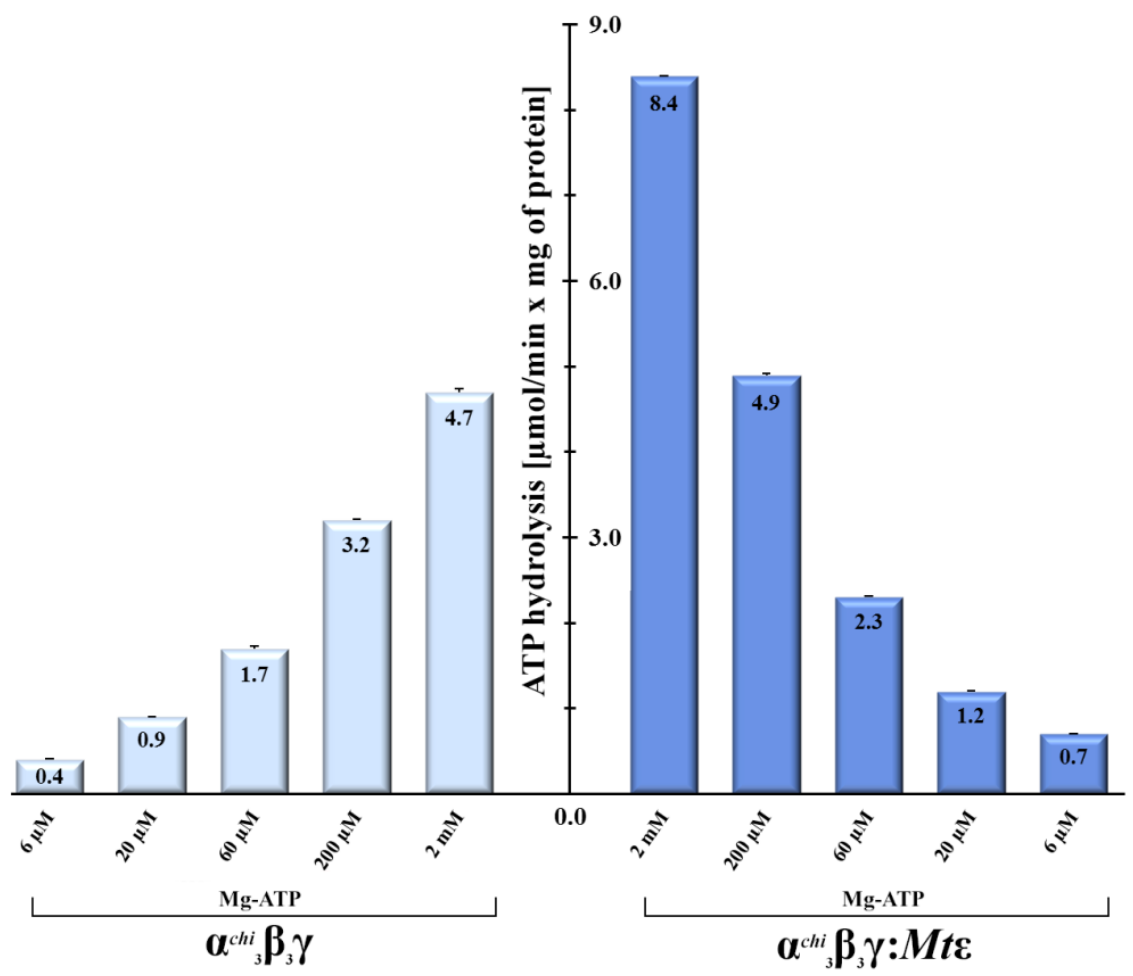

Figure 3.6: ATPase activity of $\alpha^{c h i}{ }_{3}: \beta_{3}: \gamma \alpha^{c h i}{ }_{3}: \beta_{3}: \gamma: M t \varepsilon$ at variable Mg-ATP. Continuous ATPase activity of $\alpha^{c h i}: \beta_{3}: \gamma$ (left) and $\alpha^{c h i}: \beta_{3}: \gamma: M t \varepsilon$ (right) measured at different concentrations of Mg-ATP ranging from 0.006 to $2 \mathrm{mM}$. The decrease in NADH absorption at $340 \mathrm{~nm}$ is plotted against the progressing time in seconds, and the resulting values are shown as a bar diagram. Figures credited to (205).

The calculated $V_{\max }$ values of $\alpha^{\text {chi }}{ }_{3}: \beta_{3}: \gamma$ and $\alpha^{c h i}{ }_{3}: \beta_{3}: \gamma: M t \varepsilon$ was $4.97 \pm 0.12 \mathrm{~s}^{-1}$ and $8.63 \pm 0.25 \mathrm{~s}^{-1}$, respectively, while the $K_{m}$ values were close for both $(0.11 \pm 0.01$ $\mathrm{mM}$ and $0.164 \pm 0.02 \mathrm{mM}$, respectively) (Figure 3.7). The second order binding rate constant $V_{\max } / K_{m}$ was calculated for both complexes for and it was found that 
$\alpha^{c h i}: \beta_{3}: \gamma: M t \varepsilon \quad\left(0.52 \cdot 10^{5} \mathrm{M}^{-1} \cdot \mathrm{s}^{-1}\right)$ is catalytically more effective than $\alpha^{c h i}{ }_{3}: \beta_{3}: \gamma$ $\left(0.46 \cdot 10^{5} \mathrm{M}^{-1} \cdot \mathrm{s}^{-1}\right)$. The kinetic data unequivocally show that the presence of subunit $M t \varepsilon$ in the $\alpha_{3}^{c h i}: \beta_{3}: \gamma$ complex does not significantly alter the substrate binding affinity $\left(K_{m}\right)$. However, $V_{m}$ is 1.8 times lower in the complex lacking subunit $M t \varepsilon$, indicating its- role in another step of the ATP hydrolysis reaction, possibly relieving of Mg-ADP inhibition, cleavage of the ATP to ADP and $\mathrm{P}_{\mathrm{i}}$ and/or release of the products.

\begin{tabular}{lccc}
\hline Enzyme & $\boldsymbol{V}_{\max }\left[\mathrm{s}^{-1}\right]$ & $\boldsymbol{K}_{\boldsymbol{m}}$ & $V_{\max } / K_{m}\left[\mathrm{M}^{-1} \cdot \mathrm{s}^{-1}\right]$ \\
\hline$\alpha^{c h i}{ }_{3}: \gamma: \beta_{3}$ & $4.97 \pm 0.12$ & $0.11 \pm 0.01$ & $0.46 \times 10^{5}$ \\
$\alpha^{c h i}{ }_{3}: \gamma: \beta_{3}: M t \varepsilon$ & $8.63 \pm 0.25$ & $0.164 \pm 0.02$ & $0.52 \times 10^{5}$ \\
\hline
\end{tabular}

Table 3.1: Enzymatic activities of the $\alpha^{c h i}{ }_{3}: \beta_{3}: \gamma$ and $\alpha^{c h i}: \beta_{3}: \gamma: M t \varepsilon$ complexes

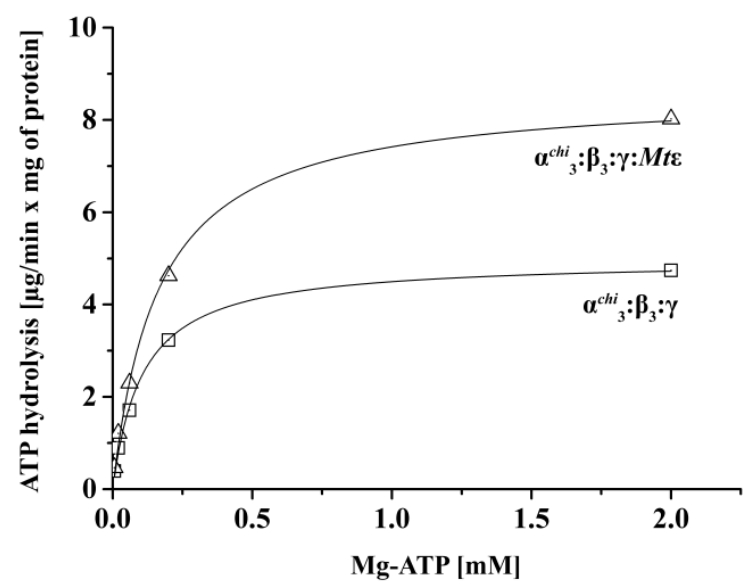

Figure 3.7: Michaelis-Menten plot of $\boldsymbol{\alpha}^{\text {chi }}{ }_{3}: \boldsymbol{\beta}_{3}: \gamma$ and $\boldsymbol{\alpha}^{\text {chi }}{ }_{3}: \boldsymbol{\beta}_{3}: \gamma:$ Mte. The measurements for $\alpha^{\text {chi }}{ }_{3}: \beta_{3}: \gamma$ (口) and $\alpha_{3}^{\text {chi }}: \beta_{3}: \gamma: M t \varepsilon(\Delta)$ were performed at varying concentrations of MgATP $(200 \mu \mathrm{M}, 60 \mu \mathrm{M}, 20$ $\mu \mathrm{M}$, and $6 \mu \mathrm{M})$ performed at $37^{\circ} \mathrm{C}$. Figures credited to (205).

In the related F-ATP synthases the cleavage of ATP in the nucleotide-binding site is independent of rotation, while the release of phosphate from the active site is coupled to the rotation of the central stalk, which is necessary for the overall hydrolysis reaction to continue with the binding of ATP to an empty site. A strong coupling between the reaction site and the central rotating stalk is needed for the enzyme to be effective (293). As subunit Mte can interact with both subunit $\gamma$ and subunit $\alpha^{c h i}$ (294-296), it seems to act as a mediator element for rotation. Increasing the tightness of the coupling between rotor and stator subunits might increase the maximal velocity of the reaction (293). 


\subsubsection{Role of subunit MtE in Mg-ADP-mediated inhibition of the $\alpha^{\text {chi }}{ }_{3}: \beta_{3}: \gamma: M t \varepsilon$}

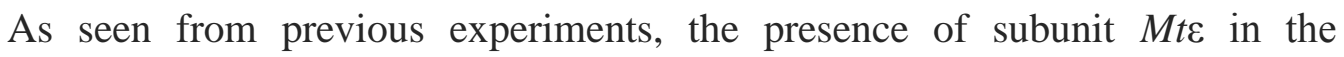
$\alpha_{3}^{c h i}: \beta_{3}: \gamma$ complex does not significantly alter the substrate binding affinity $(\mathrm{Km})$. However, the complex reconstituted with subunit Mte displays 1.8-times faster consumption rate $\left(V_{m}\right)$ of Mg-ATP, indicating its' role in another step of the ATP hydrolysis reaction, possibly relieving of $\mathrm{Mg}$-ADP inhibition, cleavage of the ATP to $\mathrm{ADP}$ and $\mathrm{Pi}$ and/or release of these products. In order to test whether $\mathrm{Mg}$-ADP binding may in part cause this difference, $\alpha^{c h i}{ }_{3}: \beta_{3}: \gamma$ - and $\alpha^{c h i}{ }_{3}: \beta_{3}: \gamma: M t \varepsilon$ were pre-incubated with varying $\mathrm{Mg}$-ADP concentrations. As shown in Figure 3.8 the presence of $10 \mu \mathrm{M}$ and $100 \mu \mathrm{M}$ of $\mathrm{Mg}$-ADP reduced the ATPase activity of $\alpha^{\text {chi }}: \beta_{3}: \gamma \quad(4.7 \pm 0.01$ $\mu \mathrm{mol} / \mathrm{min} / \mathrm{mg}$ protein) slightly to $4.3 \pm 0.05 \mu \mathrm{mol} / \mathrm{min} / \mathrm{mg}$ protein and $4.0 \pm 0.02$ $\mu \mathrm{mol} / \mathrm{min} / \mathrm{mg}$ protein, respectively.

(A)

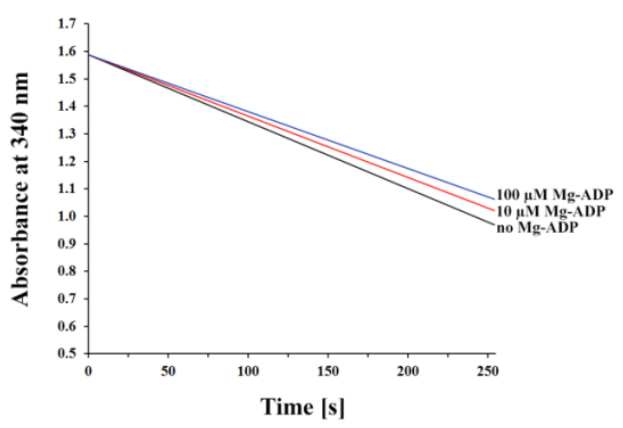

(B)

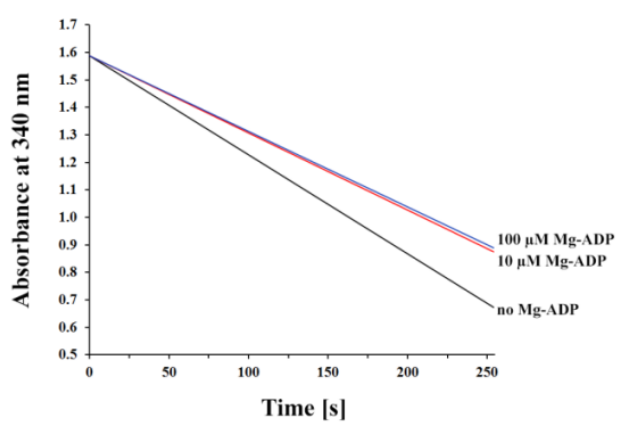

(C)

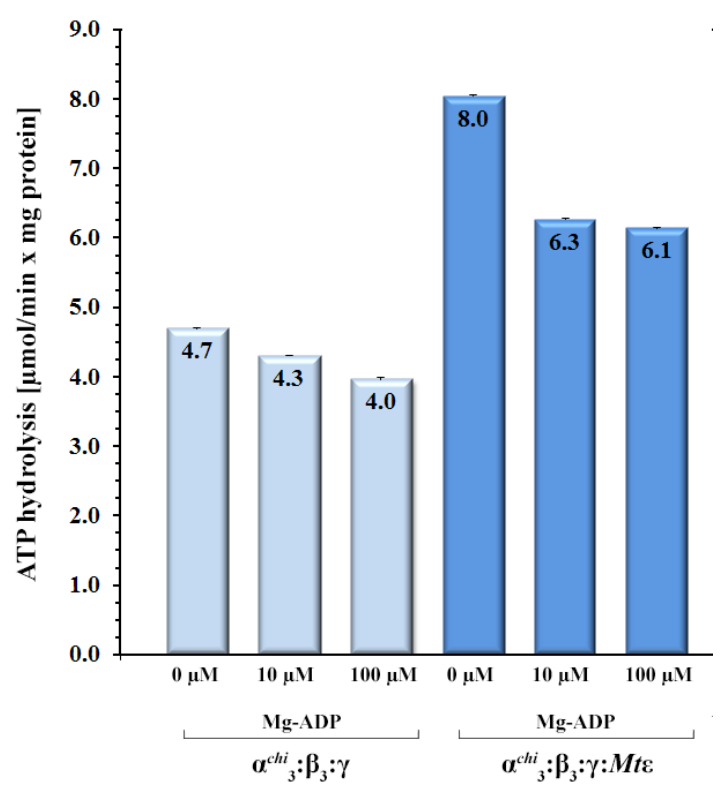

Figure 3.8: Effect of Mg-ADP on the inhibition of ATPase activity. Profiles of the ATPase activity of $\alpha^{\text {chi }}{ }_{3}: \beta_{3}: \gamma(A)$ and $\alpha^{c h i}: \beta_{3}: \gamma: M t \varepsilon(B)$ measured in the presence of $2 \mathrm{mM} \mathrm{Mg-ATP}$ and varying Mg-ADP concentrations; Complexes incubated without $\mathrm{Mg}$-ADP (black), $10 \mu \mathrm{M} \mathrm{Mg-ADP}$ (red) and $100 \mu \mathrm{M} \mathrm{Mg-ADP} \mathrm{(blue).} \mathrm{(C)} \mathrm{Bar} \mathrm{diagrams} \mathrm{showing} \mathrm{the} \mathrm{specific} \mathrm{ATPase} \mathrm{activity} \mathrm{of}$ $\alpha^{c h i}: \beta_{3}: \gamma$ and $\alpha^{c h i}{ }_{3}: \beta_{3}: \gamma: M t \varepsilon$ in the presence of $2 \mathrm{mM} \mathrm{Mg-ATP}$ and varying Mg-ADP concentrations. All values displayed are a mean value of three measurements. Figures credited to (205). 
In comparison, a significant decrease in ATPase activity was observed in the $\alpha_{3}^{\text {chi }} \beta_{3}: \gamma: M t \varepsilon$ complex $(8.0 \pm 0.02 \mu \mathrm{mol} / \mathrm{min} / \mathrm{mg}$ protein $)$ after pre-incubation of 10 $\mu \mathrm{M}$ Mg-ADP (Fig 3.16), causing a reduction of $21 \%$ in ATPase activity (6.3 \pm 0.02 $\mu \mathrm{mol} / \mathrm{min} / \mathrm{mg}$ protein), which was comparable to the value determined in the presence of $100 \mu \mathrm{M}$ of $\mathrm{Mg}$-ADP $(6.1 \pm 0.01 \mu \mathrm{mol} / \mathrm{min} / \mathrm{mg}$ protein $)$.

Considering that $10 \mu \mathrm{M}$ Mg-ADP causes a drop in ATPase activity of only $8 \%$ versus $21 \%$ seen for the $M t \varepsilon$-bound complex, these data suggest that the inhibitory Mg-ADP gets partially trapped by the reconstituted Mt $\varepsilon$ within the $\alpha^{\text {chi }}{ }_{3}: \beta_{3}: \gamma: M t \varepsilon$ complex and that $M t \varepsilon$ in part, enhances the sensitivity of the complex towards $\mathrm{Mg}$ ADP inhibition.

\subsubsection{Epitope important for Mg-ADP inhibition of $\alpha^{c h i}{ }_{3: \beta_{3}: \gamma: M t \varepsilon}$}

As recently described, the mycobacterial subunit $\varepsilon$ exhibits a peculiar effect on the F-ATP synthase catalytic activity in the IMVs of the M. smegmatis. The deletion of the C-terminal domain last residue D121 of subunit $\varepsilon$ leads to the increase of ATPase activity by $13 \%$ and a drastic drop of ATP synthesis by $65 \%$ (209).

Here the recombinant mutant $M t \varepsilon_{1-120}$ was purified (Fig. 3.17) and reconstituted

with $\alpha^{\text {chi }}{ }_{3}: \beta_{3}: \gamma$ complex, its' ATP hydrolytic activity and the effect of the mutation on the $\mathrm{Mg}$-ADP inhibition were determined.

In brief, the protein was purified, as described in section 2.2.10. The $\mathrm{Ni}^{2+}$-NTA fractions (Figure 3.9-A) containing the protein (75-125 $\mathrm{mM}$ imidazole) were injected into the size exclusion column (S75) (Figure 3.9-B) to obtain a highly pure monomeric fraction of the protein for reconstitution experiments

The isolated $M t \varepsilon_{1-120}$ was combined with purified $\alpha^{c h i}{ }_{3}: \beta_{3}: \gamma$ in a $3: 1$ molar ratio and the mixture was subjected to the size exclusion column (GE Healthcare, S300) to remove unbound $M t \varepsilon_{1-120}$ (Figure 3.18). The purity was ascertained on the $12 \%$ SDSPAGE. As described in section 2.2.35, the stoichiometric ratio was determined from the densitometric analysis of 2D peaks representing the intensities of the subunits $\gamma$ and $M t \varepsilon$ to be typical 1:0.5. Figure 3.18-B shows that the mutation does not affect the binding of the subunit $M t \varepsilon_{1-120}$ to the $\alpha^{c h i}: \beta_{3}: \gamma$ complex. 
(A)

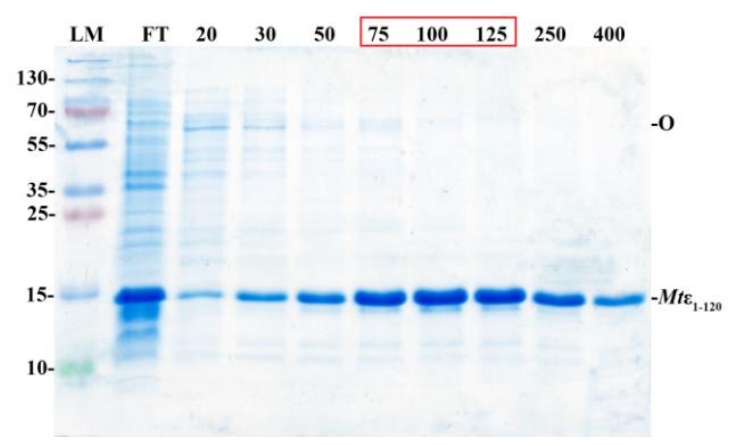

(B)

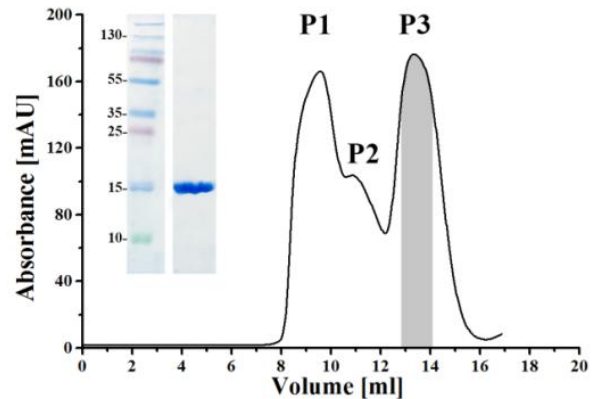

Figure 3.9: Purification of $\boldsymbol{M} t \varepsilon_{1-120}$ by gel filtration. Protein obtained after $\mathrm{Ni}^{2+}-\mathrm{NTA}$ chromatography (A) was applied onto a gel filtration column (Superdex 75) and eluted at $13.5 \mathrm{ml}$ volume, as seen in the chromatogram (B). P1 peak contains the protein in an unspecific oligomeric state, $\mathrm{P} 2$, contains the protein in a dimeric state, $\mathrm{P} 3$ represents the monomeric peak. The purity was confirmed by running a $17 \%$ SDS-PAGE (C). P1, P2, and P3 represent the two main fractions of each peak; Purified protein loaded without DTT is labeled "apo" while the label "+DTT" indicates the presence of the reducing agent.

(A)

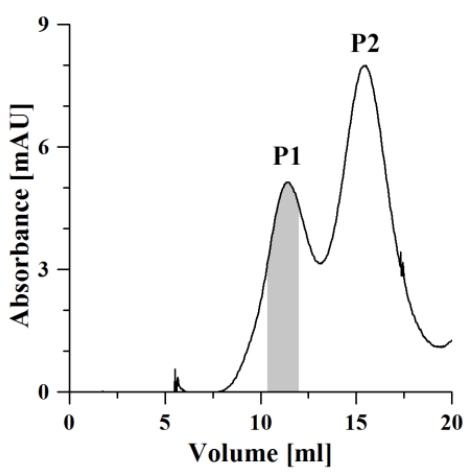

(B)

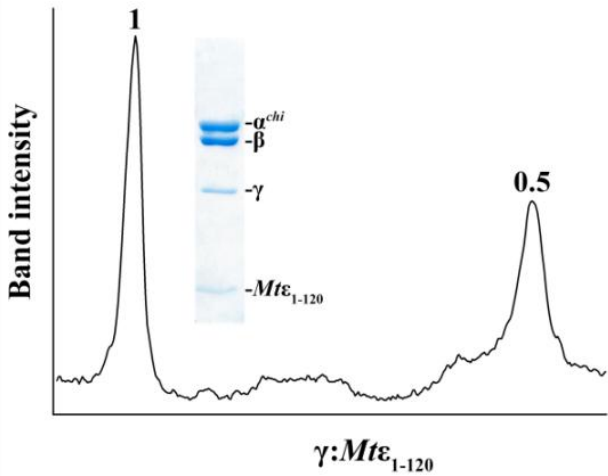

Figure 3.10: Reconstitution and densitometry of $\boldsymbol{\alpha}^{c h i}{ }_{3}: \beta_{3}: \gamma: M t \varepsilon_{1-120}$ complex. (A) $\alpha^{c h i}: \beta_{3}: \gamma$ complex was reconstituted with a 3 -fold molar excess of $M t \varepsilon_{1-120}$, applied onto a gel filtration column (Superdex 300) and eluted at $11.6 \mathrm{ml}$ column volume; greyed area indicates the fractions pooled for the SDS-PAGE. (B) Purity (inset) and stoichiometry were confirmed on the densitogram revealing the ratio of subunits $\gamma: M t \varepsilon_{1-120}$ of 1:0.5 within the reconstituted $\alpha^{c h i}: \beta_{3}: \gamma: M t \varepsilon_{1-120}$ complex. Figure 3.10-B credited to (205).

By using the continuous ATPase assay, it was confirmed that the deletion of D121 from the Mtc caused the increase of the specific ATPase activity to $9.5 \pm 0.04$ $\mu \mathrm{mol} / \mathrm{min} / \mathrm{mg}$ protein, which is $13 \%$ higher than the activity measured for the $\alpha^{\text {chi }}{ }_{3} \beta_{3}: \gamma: M t \varepsilon$ complex $(8.4 \pm 0.04 \mu \mathrm{mol} / \mathrm{min} / \mathrm{mg}$ protein) (Figure 3.11). These values pointed out that the increase in activity was caused by the mutation and not due to altered binding of $M t \varepsilon_{1-120}$ to the complex, further corroborating the findings that $M$. smegmatis IMVs, designed to harbor the same mutation $\left(\varepsilon_{1-120}\right)$, displayed $13 \%$ higher ATPase activity than wild-type M. smegmatis IMVs (209). 
Considering this phenomenon and having in mind that the presence of $M t \varepsilon$ affects the Mg-ADP release from the active site of the $\alpha^{c h i}{ }_{3}: \beta_{3}: \gamma: M t \varepsilon$ complex it was hypothesized, that the residue D121 takes part in the mechanism involving Mg-ADP inhibition.

(A)

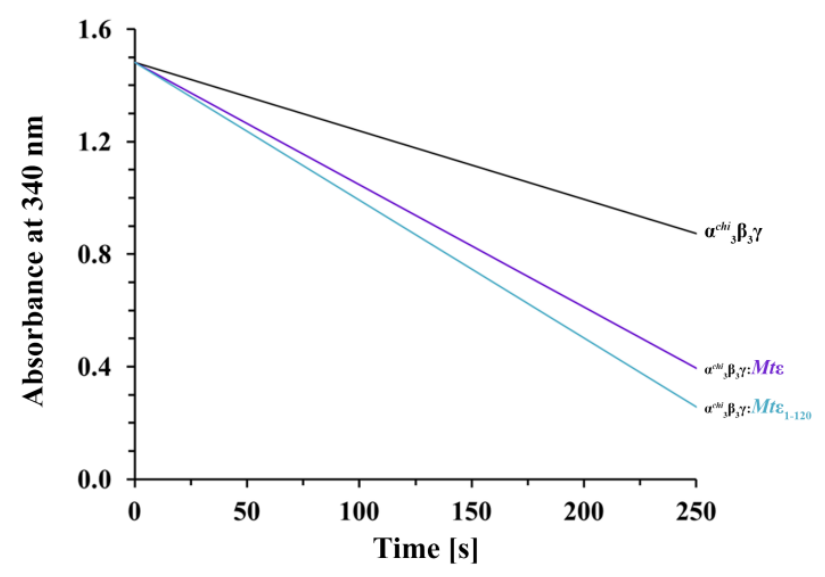

(B)

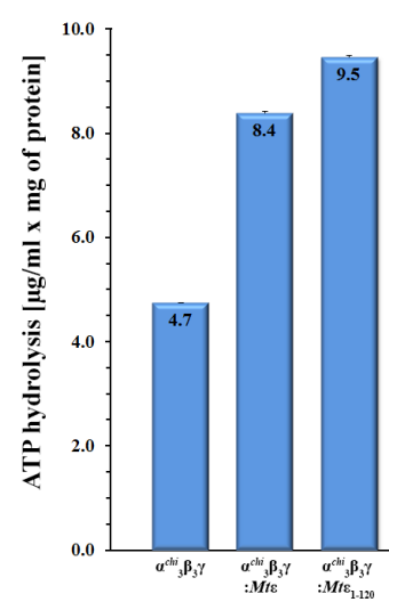

Figure 3.11: ATPase activity of the $\boldsymbol{\alpha}^{c h i}: \beta_{3}: \gamma: M t \varepsilon_{1-120}$ complex. ATPase activity profiles (A) and bar diagrams (B) revealing the activities of the complex $\alpha^{c h i}: \beta_{3}: \gamma$ and reconstituted complexes $\alpha^{c h i}: \beta_{3}: \gamma: M t \varepsilon, \alpha^{c h i}: \beta_{3}: \gamma: M t \varepsilon_{1-120}$, respectively, in the presence of $2 \mathrm{mM}$ ATP. Figures adapted from (205).

The reconstituted protein complex $\alpha^{c h i}{ }_{3}: \beta_{3}: \gamma: M t \varepsilon_{1-120}$ was pre-incubated with 10 $\mu \mathrm{M}$ Mg-ADP in duration of 10 minutes prior to ATP hydrolysis measurements which revealed, that $\mathrm{Mg}$-ADP caused an activity drop of around $10 \%(8.7 \pm 0.01$ $\mu \mathrm{mol} / \mathrm{min} / \mathrm{mg}$ protein) in comparison to the non-treated form of the $\alpha^{\text {chi }}{ }_{3: \beta_{3}: \gamma: M t \varepsilon_{1-120}}$ complex $(9.7 \pm 0.03 \mu \mathrm{mol} / \mathrm{min} / \mathrm{mg}$ protein). When compared with the Mg-ADP inhibitory effect of around $21 \%$ of the $\alpha^{c h i}: \beta_{3}: \gamma: M t \varepsilon$ complex described in section 3.6.4 above, the results indicate that residue D121 of $M t \varepsilon$ is in part involved in trapping the Mg-ADP and increasing the sensitivity to this inhibitory nucleotide (Fig $3.20)$. 
(A)

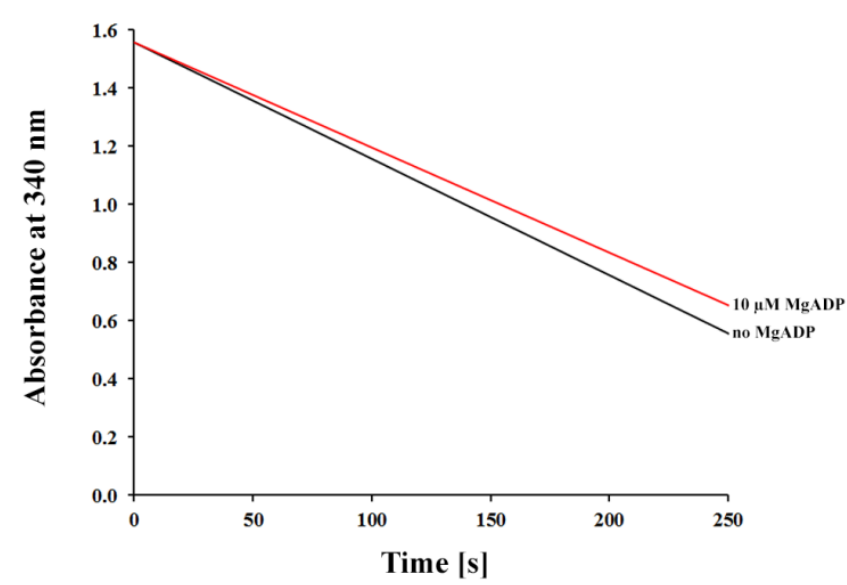

(B)

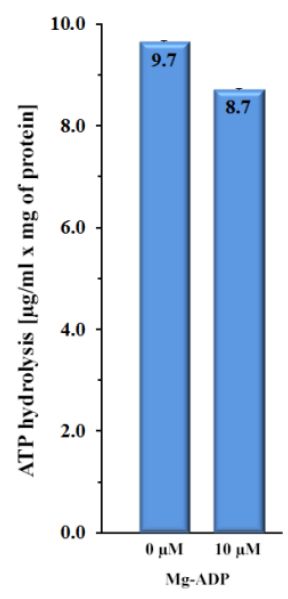

Figure 3.12: Inhibitory of Mg-ADP on the ATPase activity of the $\alpha^{c h i}{ }_{3}: \beta_{3}: \gamma: M t \varepsilon_{1-120}$ complex. Profiles (A) show the ATPase activity of the $\alpha^{\text {chi }}{ }_{3}: \beta_{3}: \gamma: M t \varepsilon_{1-120}$ without Mg-ADP (black) and preincubated with $10 \mu \mathrm{M} \mathrm{Mg}$-ADP (red), as reflected in the bar diagrams (B). Figures credited to (205). 
3.2 Characterization of epitopes of MtE important for catalytic activity and assembly 


\subsubsection{Characterization of epitopes of MtE important for catalytic activity and assembly}

Analyzing the solution NMR structure of MtE (209) the network of interactions between the NTD and CTD was defined (Figure 3.13). The residues involved in the transmission of the conformational alterations from NTD to CTD include the interactions between NTD epitopes A10-W16 and L61-A64 in the NTD that propagates further to the epitope A81-I90 found in the region connection NTD to CTD.

The side chain of the residue E87, positioned in the connecting region, makes the polar contacts with the positively-charged side chain of R62 and interacts through van der Waals forces with sidechains of L61 and neighboring F86 (209). In order to understand whether E87 plays an essential role in the maintenance of the network of interactions between NTD and CTD, it was substituted with alanine and effectively removing its- negatively-charged side chain. The plausible structural alterations resulting from the mutation could affect the binding properties of the $M t \mathrm{E} 87 \mathrm{~A}$ mutant protein to the $\alpha^{\text {chi }}{ }_{3}: \beta_{3}: \gamma$ complex and the catalytic activity within the complex. Thus, the mutant $M t$ E87A was generated using site-directed mutagenesis, purified and reconstituted with $\alpha^{\text {chi }}{ }_{3}: \beta_{3}: \gamma$ complex to perform ATP hydrolysis assay and assess the binding using densitometry. A ${ }^{15} \mathrm{~N}-\mathrm{HSQC}$ spectrum of MteE87A was assigned, and chemical shift perturbations were analyzed to provide insight into possible topological rearrangements.

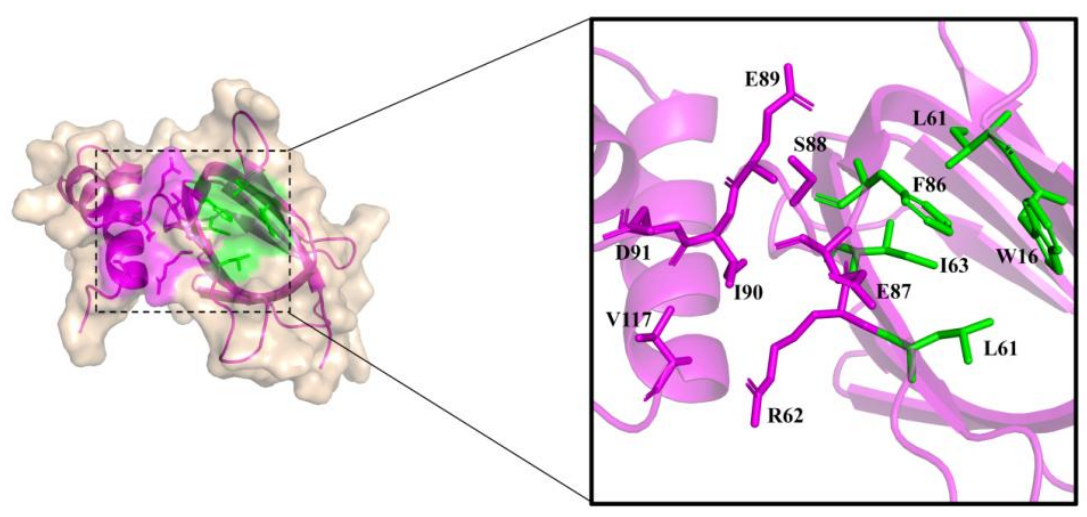

Figure 3.13: The MtE intramolecular network of interactions around residue E87. Surface representation of $M t \varepsilon$ showing the formation of a network of residues connecting the NTD to the CTD. The residues partaking in the network formation are shown as sticks and are represented in green and magenta color for the NTD and the CTD, respectively. The enlarged panel shows the detailed view of the amino acid residues in the network connecting NTD to CTD, represented as sticks. The residue E87 is wedged between the NTD residues (green) and the CTD residues (magenta). The critical residues comprising the network are labeled with one-letter amino acid codes. Figure adapted from (205). 


\subsubsection{Generating the mutant MtEE87A and its reconstitution with $\alpha^{c h i}{ }_{3}: \beta_{3}: \gamma$ complex}

In order to replace the E87 residue with alanine, a site-directed mutagenesis approach was employed (274). The plasmid pET-9d(+) containing the recombinant

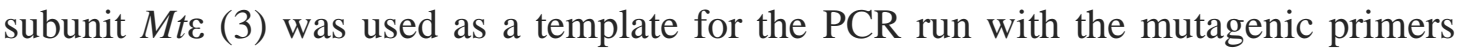
described in the section 2.2.11. The entire amplified plasmid of 4,703 bp was isolated (Figure 3.14), and the E87A mutation in the Mtc gene was confirmed by sequencing. Subsequently, the verified clone pDNA was transformed into E. coli OverExpress C41(DE3): F - ompT hsdSB ( $r B$ - mB-) gal dcm (DE3) (292) for protein production and the protein was purified using the two-step protocol established in (3) and section 2.2.11.

To purify the mutant protein, cell lysate in buffer $50 \mathrm{mM}$ Tris/HCl $200 \mathrm{mM}$ $\mathrm{NaCl}, 10 \%$ glycerol, $\mathrm{pH} 7.5$ was loaded onto pre-equilibrated $\mathrm{Ni}^{2+}$-NTA column and eluted with imidazole $(0-400 \mathrm{mM})$. The fractions 75-125 mM imidazole were pooled, concentrated to $2 \mathrm{ml}$ and loaded onto the size exclusion column Superdex 75 HR 10/30 column (GE Healthcare). The protein was eluted at, and its homogeneity was verified by $17 \%$ SDS - PAGE (Figure $3.15)$.

Freshly purified and homogenous

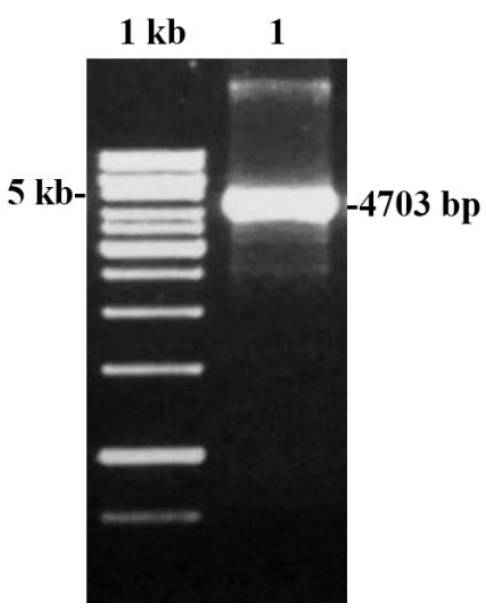

Figure 3.14: Cloning of the substitution mutants MtEE87A. (A) Amplification by site-directed mutagenesis of the entire plasmid DNA containing the $M t \varepsilon$ sequence $(\mathrm{pET} 9 \mathrm{~d}(+): M t \varepsilon)$ yielded the product shown in lane $1 ; 1 \mathrm{~kb}$ represents the DNA marker.

mutant protein was reconstituted with the purified $\alpha^{c h i}: \beta_{3}: \gamma$ complex and assayed for ATPase activity. The reconstitution was performed by incubating the $\alpha^{c h i}{ }_{3}: \beta_{3}: \gamma$ with the 3-fold molar excess of $M t \varepsilon E 87 \mathrm{~A}$ at $+4{ }^{\circ} \mathrm{C}$ for $1 \mathrm{~h}$ in $50 \mathrm{mM}$ Tris/ $\mathrm{HCl}, 200 \mathrm{mM}$ $\mathrm{NaCl}, \mathrm{pH}$ 7.5. Unbound subunit Mt\&E87A was removed by passing the mixture through the size-exclusion column (S300 10/30, GE-Healthcare) (Figure 3.16). The protein complex eluted at $11.6 \mathrm{ml}$ and was further concentrated using a $100 \mathrm{kDa}$ centricon. The concentrated sample was analyzed on the 12\% SDS gel and the presence of the subunit MtEE87A bound to the complex was confirmed (Figure 3.16). 
(A)

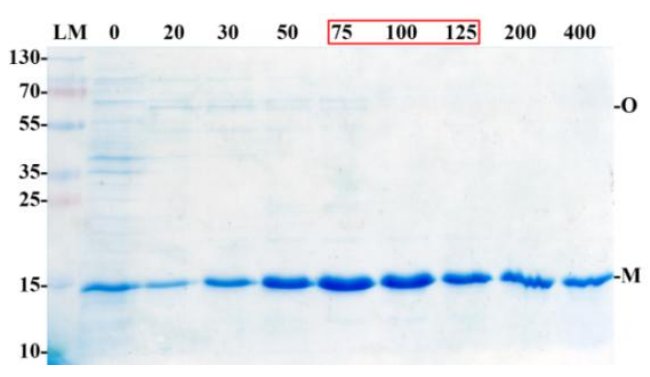

(B)

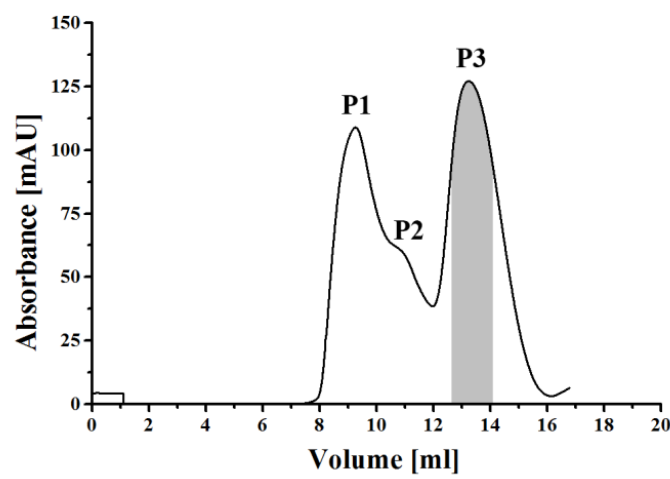

(C)

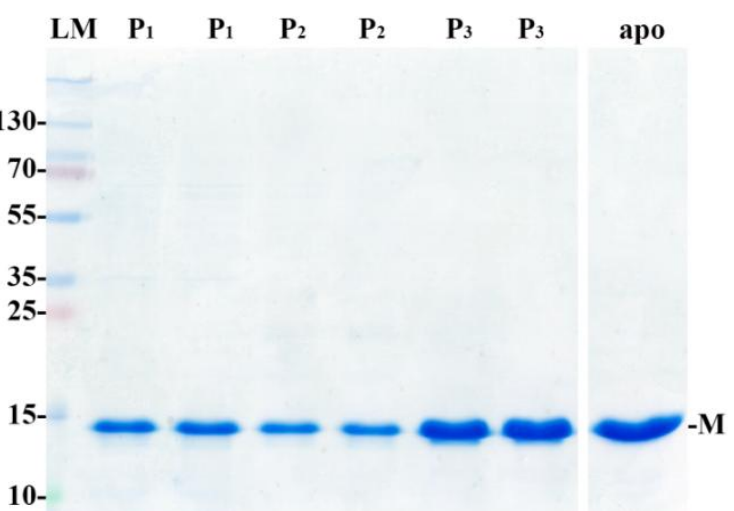

Figure 3.15: Purification of $M t \varepsilon E 87 \mathrm{~A}$ by gel filtration. Protein obtained after $\mathrm{Ni}^{2+}-\mathrm{NTA}$ chromatography (A) was applied onto a gel filtration column (Superdex 75) and eluted at $13 \mathrm{ml}$ volume, as seen in the chromatogram (B). P1 peak contains the protein in an unspecific oligomeric state, P2, contains the protein in a dimeric state, $\mathrm{P} 3$ represents the monomeric peak. The purity was confirmed by running a 17\% SDS-PAGE (C). P1, P2, and P3 represent the two main fractions of each peak; Purified protein was labeled as "apo" and shown in the righter most lane. Figure 3.15C adapted from (205).

ATPase activity of this complex was lower $(78 \%$ or $6.6 \mu \mathrm{mol} / \mathrm{min} \mathrm{x} \mathrm{mg}$ of protein) in comparison to the $\alpha^{c h i}{ }_{3}: \beta_{3}: \gamma: M t \varepsilon$ complex $(8.4 \mu \mathrm{mol} / \mathrm{min} \mathrm{x} \mathrm{mg}$ of protein). To verify that the resulting activity is not a consequence of the reduced binding affinity caused by mutation, the formed complex, collected after the size exclusion column was analyzed by densitometry. The ratio of $\gamma: M t \varepsilon$ was quantified from the $12 \%$ gels using ImageJ software (282). After the conversion of the bands to 2D peaks and calculation of their integrals, the ratio of $\gamma: M t \varepsilon$ was determined to be $1: 0.5$, as shown for the $\alpha^{c h i}{ }_{3}: \beta_{3}: \gamma: M t \varepsilon$ complex. This finding pointed out that the lower activity 
of the $\alpha^{c h i}: \beta_{3}: \gamma: M t \varepsilon E 87 A$ complex indeed was a result of the introduced mutation in the subunit $M t \varepsilon$ and not the artifact of the sub-stoichiometric complex formation.

(A)

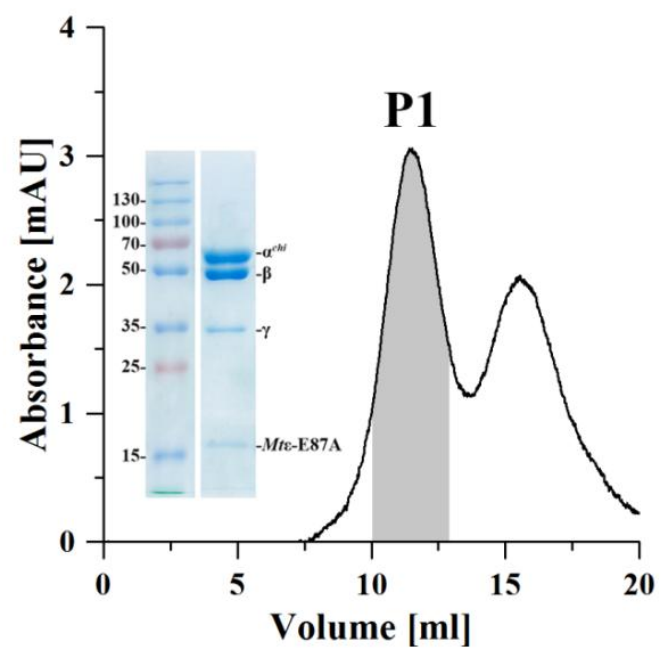

(C)

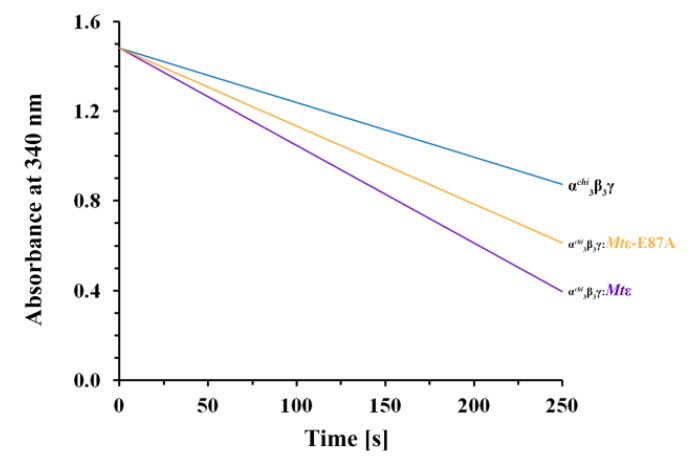

(B)

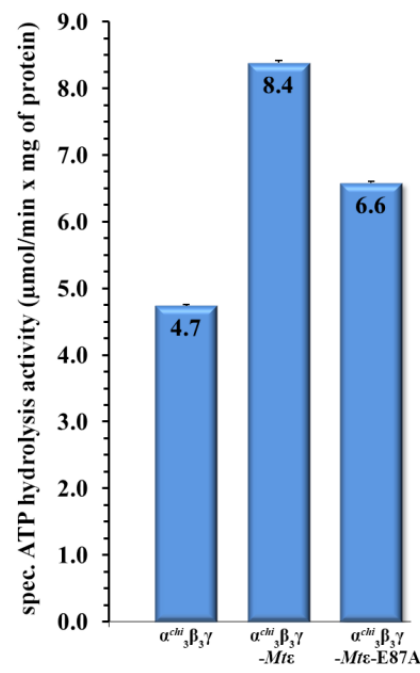

(D)

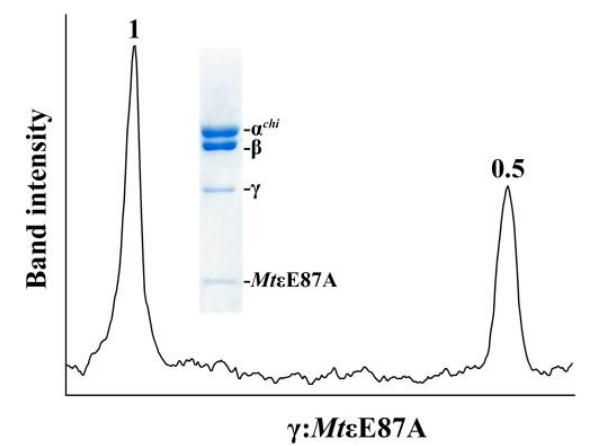

Figure 3.16: Reconstitution of $\boldsymbol{\alpha}^{\text {chi }}{ }_{3}: \beta_{3}: \gamma$ and subunit MtEE87A mutant. (A) Purified $\alpha^{\text {chi }}{ }_{3}: \beta_{3}: \gamma$ and $M t \varepsilon E 87 \mathrm{~A}$ were reconstituted and passed through a Superdex S300 column (A) and the peak containing reconstituted $\alpha^{c h i}: \beta_{3}: \gamma: M t \varepsilon E 87 \mathrm{~A}$ was run on a $12 \%$ SDS-gel (insert). (B) Bar diagram and activity profiles (C) showing the specific activities of $\alpha^{c h i}{ }_{3}: \beta_{3}: \gamma, \alpha^{c h i}: \beta_{3}: \gamma: M t \varepsilon$ and $\alpha^{c h i}{ }_{3}: \beta_{3}: \gamma: M t \varepsilon \mathrm{E} 87 \mathrm{~A}$, indicating the stimulatory effect of $M t \varepsilon$ on the $\alpha^{c h i}{ }_{3}: \beta_{3}: \gamma$ complex, as well as the effect of mutant protein form, MtcE87A. (D) Densitogram showing the band intensities of subunits $\gamma$ (left peak) and MtEE87A (right peak) in the $\alpha^{\text {chi }}{ }_{3}: \beta_{3}: \gamma: M t \varepsilon E 87 \mathrm{~A}$ complex derived from the $12 \%$ SDS-gel (inset). Integrals of the peaks were used to determine the ratio of $\gamma: M t \varepsilon E 87 \mathrm{~A}$ as $1: 0.5$. Figures credited to (205). 


\subsection{3 $\quad{ }^{15} \mathrm{~N}$ isotopic labeling and purification of labeled $M t \varepsilon E 87 \mathrm{~A}$}

Considering that the binding of the MtcE87A mutant to the complex is not altered and the fact that the drop in ATPase activity for the $\alpha^{c h i}{ }_{3}: \beta_{3}: \gamma: M t \varepsilon E 87 \mathrm{~A}$ complex was measured to be $13 \%$, it was hypothesized that the observed phenomenon occurred due to the altered MtE intramolecular interactions. As the N-terminal and Cterminal domain are tightly coupled via the short inter-domain loop, the mutation E87A could possibly disrupt the interaction network and affect the conformational arrangements of the NTD- and CTD in respect to each other. In order to understand the extent of the E87A effect, the protein was labeled with ${ }^{15} \mathrm{~N}$-ammonium sulfate $\left({ }^{15} \mathrm{NH}_{4} \mathrm{SO}_{4}\right)$, and the 2D-NMR experiment was conducted to assign ${ }^{15} \mathrm{~N}-\mathrm{HSQC}$ and identify residues affected by the mutation.

Isotopic protein labeling required the cultivation of the E. coli $\mathrm{C} 41$ cells harboring the plasmid pET-9d(+):MteE87A in minimum media with ${ }^{15} \mathrm{NH}_{4} \mathrm{SO}_{4}$ as the nitrogen source (section 2.2.13). The protein isolation and purification were carried out in three steps. To isolate the His $_{6}$-tagged $M t \varepsilon E 87 \mathrm{~A}$ from the other cellular proteins, the cells lysed in buffer containing $50 \mathrm{mM}$ Tris/ $\mathrm{HCl}, 200 \mathrm{mM} \mathrm{NaCl}, 10 \%$ glycerol, $\mathrm{pH} 7.5$ supplemented with Pefabloc ${ }^{\mathrm{SC}}$, PMSF and DTT. After lysis, cell debris was separated from the soluble fraction by centrifugation which was subsequently incubated with $\mathrm{Ni}^{2+}$-NTA affinity beads in the duration of $1.5 \mathrm{~h}$. The $M t \varepsilon E 87 \mathrm{~A}$ protein was eluted with the imidazole gradient $(0-400 \mathrm{mM})$, and sufficient amounts of protein were found in fractions $75 \mathrm{mM}-125 \mathrm{mM}$ (Figure 3.17-A), which were pooled together and diluted down two times with buffer $50 \mathrm{mM}$ Tris/ $\mathrm{HCl}, 10 \%$ glycerol, $\mathrm{pH}$ 7.5. The sample was applied on the anion exchange Resource Q column (Figure 3.17-B), pre-equilibrated with $50 \mathrm{mM}$ Tris/ $\mathrm{HCl}, 100 \mathrm{mM} \mathrm{NaCl}, 10 \%$ glycerol, $\mathrm{pH} 7.5$ and the flow-through was collected while the undesired proteins were bound to the matrix. The flow-through was further concentrated using a $3 \mathrm{~K}$ cut-off centricon and subjected to the size exclusion S75 column using $50 \mathrm{mM}$ Tris/ $\mathrm{HCl}, 200 \mathrm{mM} \mathrm{NaCl}, 10 \%$ glycerol, $\mathrm{pH} 7.5$ buffer. The monomeric protein was eluted at $\sim 13 \mathrm{ml}$ volume fraction from the $\mathrm{S} 75$ size exclusion column (Figure 3.17-C), concentrated until the concentration of $0.2 \mathrm{mM}$ was achieved and directly utilized to obtain the ${ }^{15} \mathrm{~N}-\mathrm{HSQC}$ spectrum.

The three-step purification described, resulted in a highly homogenous and pure, monomeric form of $M t \varepsilon E 87 \mathrm{~A}$ used for the ${ }^{15} \mathrm{~N}-\mathrm{HSQC}$ spectrum assignment. The NMR spectra were collected with the support of Dr. Shin Joon from our group. 
(A)

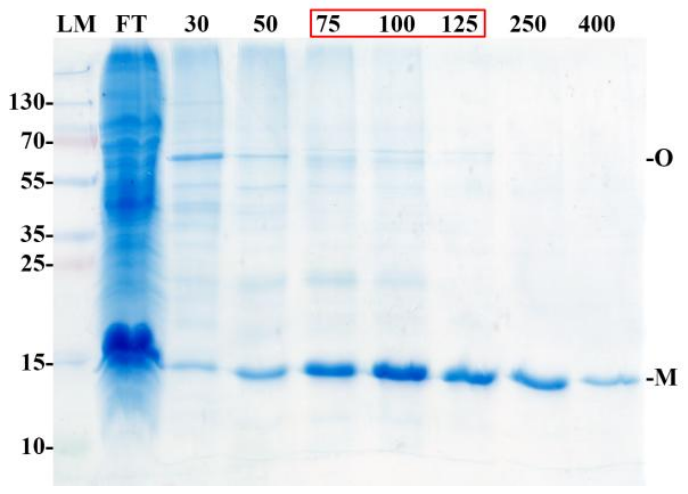

(B)

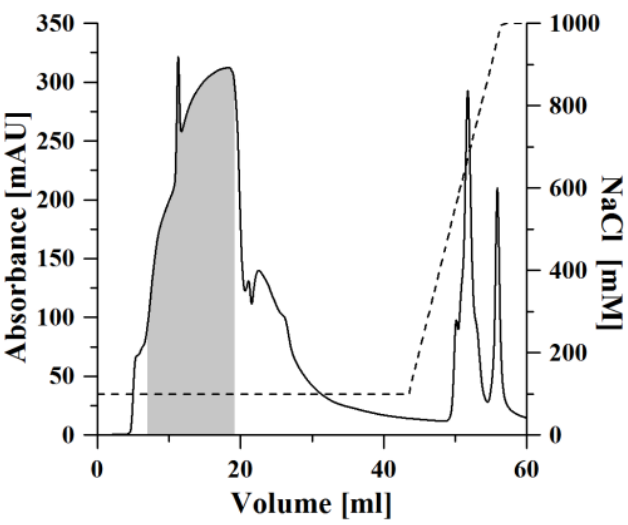

(C)

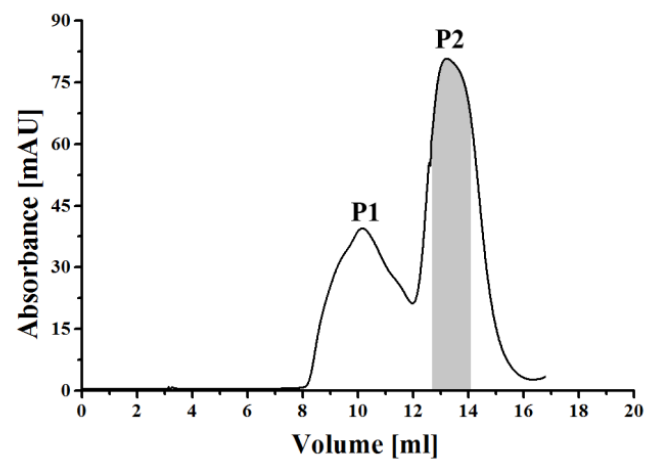

Figure 3.17: Purification of ${ }^{15} \mathrm{~N}$-labelled MtEE87A. (A) Affinity purification with $\mathrm{Ni}^{2+}$-NTA shows the profile of the fractions eluted by imidazole gradient $(0-400 \mathrm{mM})$, loaded on the $17 \%$ SDS gel. (B) Fractions framed in red in (A) are pooled and injected into the ResourceQ anion exchange column which resulted in the shown chromatogram. The gray area under the peak indicates the collected volume. (C) The protein isolated in the previous step was injected in the size-exclusion S75 column resulting in the shown chromatogram. P1 peak contains the protein in an oligomeric state, P2, eluted at about $13.5 \mathrm{ml}$, contains the protein in monomeric form. The gray area indicates the volumetric fractions collected and used to obtain the ${ }^{15} \mathrm{~N}-\mathrm{HSQC}$ spectrum.

The recombinant mutant $M t \varepsilon E 87 \mathrm{~A}$ spectrum was overlaid with a previously

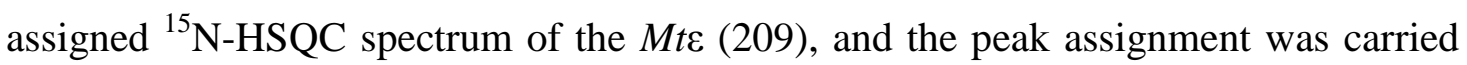
out by comparing the two spectra (Figure 3.18-A). A total of 107 peaks were identified excluding the 14 residues (L35, P36, R37, H38, I39, P40, M50, D60, R62, A81, P104, A112 and V117, and the mutated A87) whose peaks were not apparent or could be ambiguously interpreted. Out of the assigned peaks, the residues undergoing significant chemical shift perturbation (CSP) included W16, V51, V53, I63, E85 of the NTD, the linker residue S88 as well as the C-terminal residues R113, R115, A116, G118, I120 of the CTD (Fig. 3.26-B). These residues were mapped onto the NMR solution structure of $M t \varepsilon$ (Fig. 3.26-C) and are shown to belong to the amino acid 
epitope that provides the interaction of the NTD and CTD (209). It is, however, possible that in the mutant MteE87A, the lack of polar interactions/salt bridges that E87 was forming with adjacent residues, caused a disruption in the organization of the network and thus the topology/dynamics of the mutant protein have altered. This, in turn, could possibly affect the dynamics of conformational alterations and the arrangement of the protein domains which manifests as a lower ATPase activity of the $\alpha^{c h i}{ }_{3}: \beta_{3}: \gamma: M t \varepsilon E 87 \mathrm{~A}$ when compared to the activity of the $\alpha^{c h i}: \beta_{3}: \gamma: M t \varepsilon$.

(A)

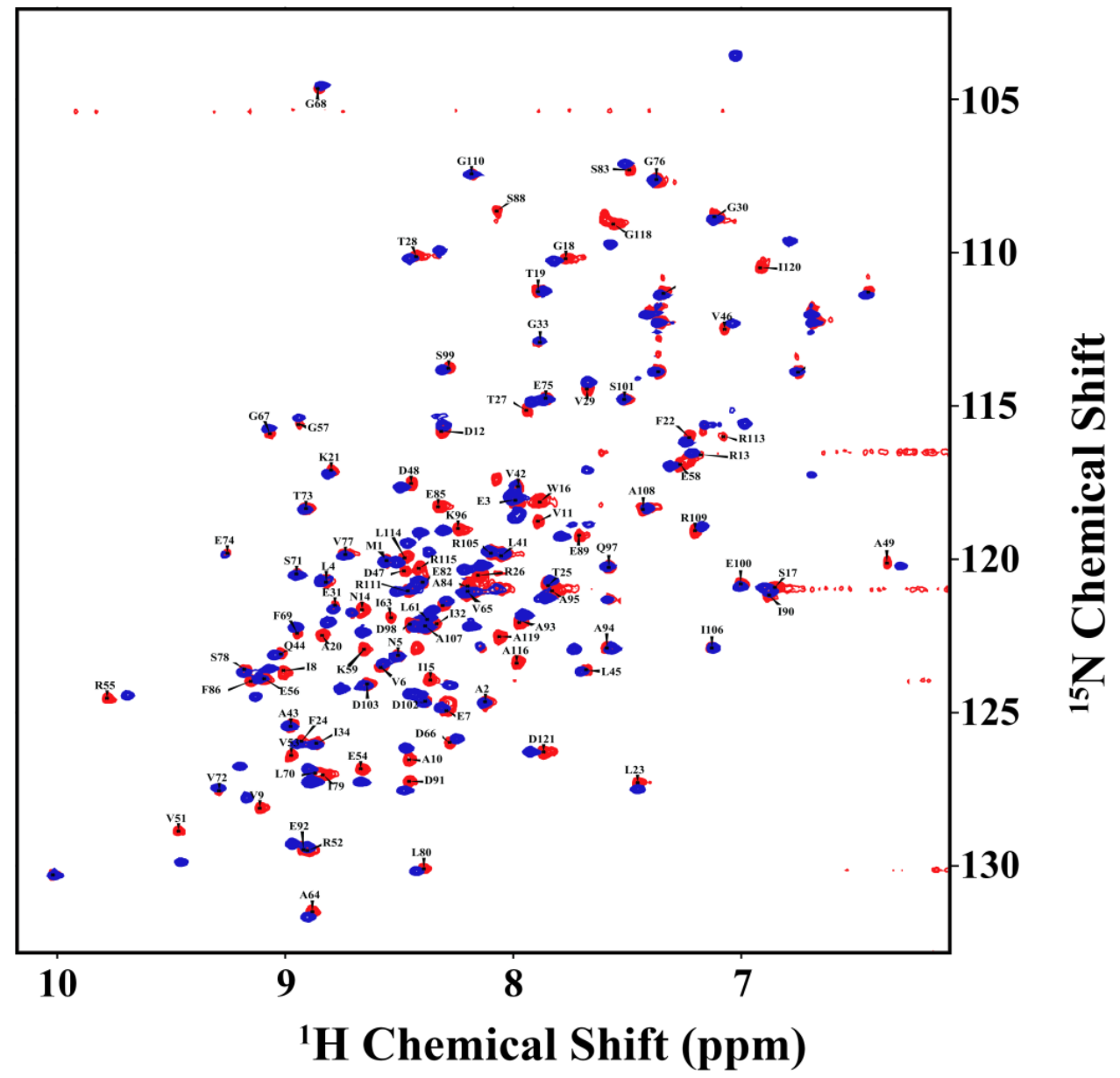


(B)

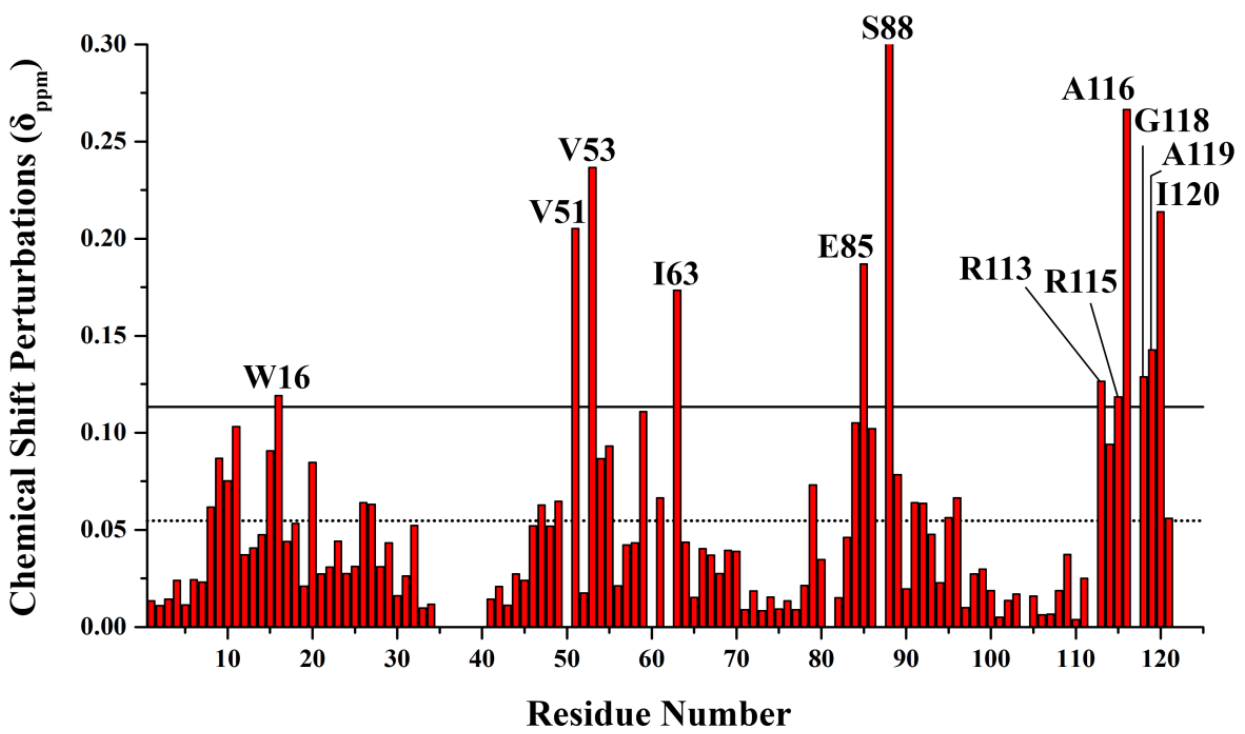

(C)
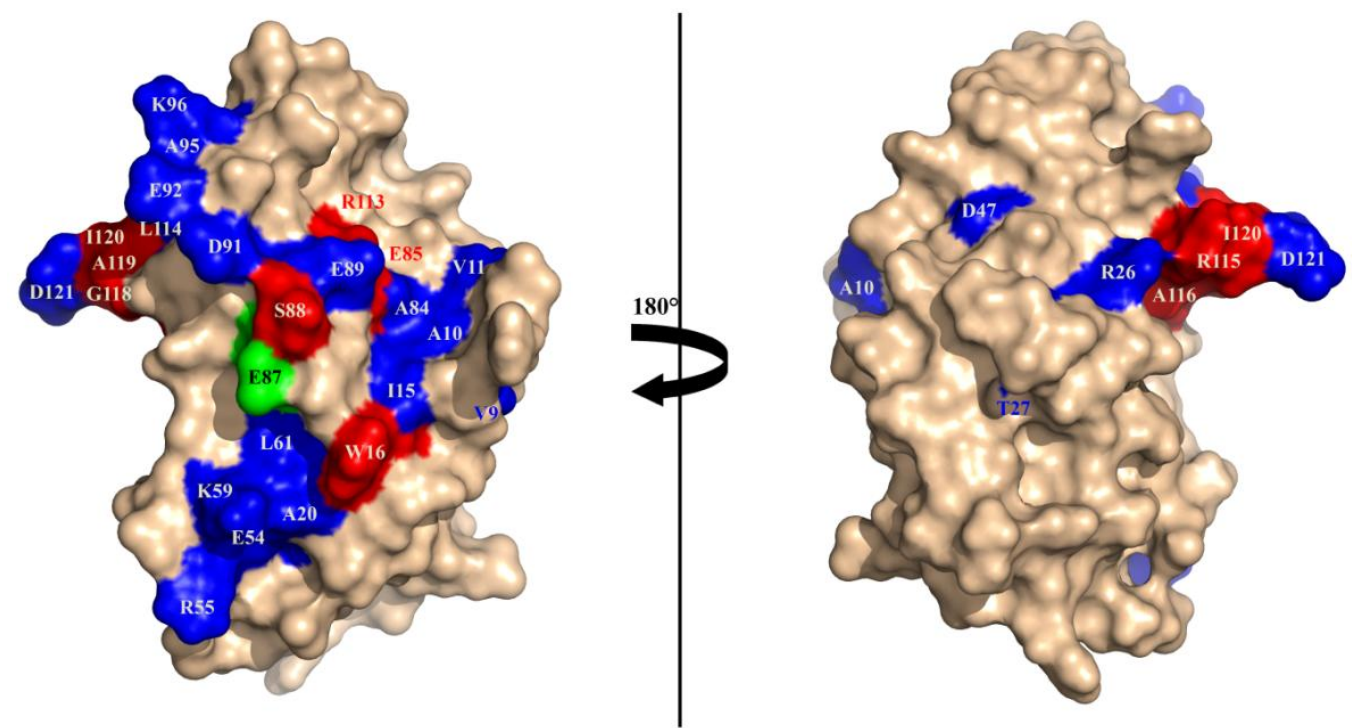

Figure 3.18. Effect of the E87A mutation Mte revealed by NMR. (A) The overlaid ${ }^{15} \mathrm{~N}-\mathrm{HSQC}$ spectra of MtE (blue) and the mutant E87A (red). (B) Weighted CSPs were derived for the ${ }^{15} \mathrm{~N}$ and ${ }^{1} \mathrm{H}$ resonance of $M t \varepsilon$ and mutant $M t \varepsilon E 87 \mathrm{~A}$ from formula $\Delta \delta=\left[(\Delta \mathrm{N} / 5)^{2}+(\Delta \mathrm{HN})^{2}\right]^{0.5}$. The residues with significant CSP (CSP > $0.1 \mathrm{ppm}$ ) are marked with one-letter code. (C) Perturbed residues were mapped on the surface of $M t \varepsilon$; residues with CSP $>0.1 \mathrm{ppm}$ ( red), residues with CSP values between 0.05 and 0.1 (blue). The substituted residue, E87 (fluorescent green). Figures credited to (205). 


\subsubsection{Effects of he atp $C^{\mathrm{E} 87 \mathrm{~A}}$ on $M$. smegmatis bioenergetics}

Mycobacterial subunit $\varepsilon$ partakes in the regulation of catalytic activity in the $\alpha_{3}^{c h i}: \beta_{3}: \gamma$ : Mte complex (179-182,297,298). Mutation altering the intramolecular interactions of $M t \varepsilon$ and altering its topological arrangement could potentially affect certain aspects of the mycobacterial energy homeostasis. To investigate this, the mutant $a t p C^{\mathrm{E} 87 \mathrm{~A}}$ was engineered using the single-strand mutagenic oligonucleotide in the recombineering protocol (299) and the E87A mutation was introduced into the genomic sequence of the atpC gene of the saprophytic model organism, Mycobacterium smegmatis under the guidance of Dr. Carolyn Mulu Wu, National University of Singapore,(NUS). It was hypothesized that the $a t p C^{\mathrm{E} 87 \mathrm{~A}}$ mutation could affect the ATP synthesis, hydrolysis and ATP-dependant proton pumping which could, in turn, alter the ATP homeostasis and contribute to a phenotype different than that of WT.

To generate the strain, $\operatorname{atp} C^{\mathrm{E} 87 \mathrm{~A}}$, the single-stranded oligonucleotide $(73 \mathrm{nt})$ that carries the mutagenic codon $(\mathrm{GAG} \rightarrow \mathrm{GCG})$ in the middle of the sequence was designed to bind to the negative DNA strand (Figure 3.19) and recombine into the chromosome $(206,300)$. One out of two primer pairs, necessary for the PCR mutant screening, displayed no binding to the WT M. smegmatis DNA, producing no bands on the agarose gel, indicating its specificity only to the mutant sequence, while the second pair amplified a 209 bp fragment from the WT DNA and was not utilized for the mutant screening due to the lack of specificity. Additional primer pair, designed to amplify the entire $a t p C$ fragment with its 5' and 3' flanking regions produced the 593 bp band used for the sequencing purposes (Figure 3.19).

After establishing the suitable primer pair for screening, the transformation of the mutagenic atp $C^{\mathrm{E} 87 \mathrm{~A}}$ ss-oligo DNA along with $\mathrm{pSJ} 25$ co-transformation plasmid conferring hygromycin resistance yielded a high number of M. smegmatis mc $^{2} 55^{2}$ : pJV62 transformants. The initial PCR screening for the positive transformants with the incorporated mutation was conducted using the primer pair that specifically binds to the mutated DNA code of the recombinants while it does not bind the WT sequence (Figure 3.19, E87A_FWD and Ctrl_REV). This approach yielded a single band of 425 bp in two out of thirty-two screened colonies, indicating a recombination successes rate of about $6.25 \%$ (Figure 3.20). Subsequently, the colonies identified by PCR were re-streaked, and the grid plate was made to select the suitable colonies further. The second round of the PCR screening yielded eleven positive reactions out of 18 
screened colonies as seen from the apparent single band of 425 base pair, which comprised the $\sim 61 \%$.

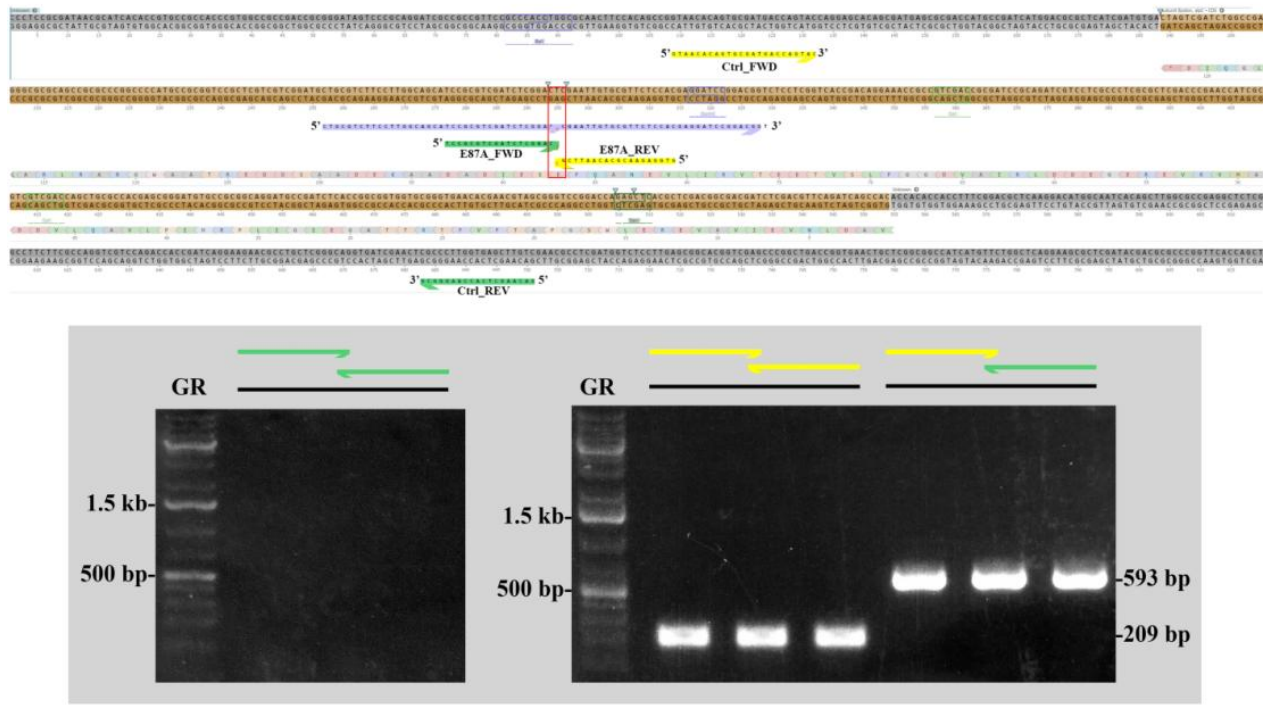

Figure 3.19: Generation of the $M$. smegmatis $\operatorname{atp}^{\mathrm{E} 87 \mathrm{~A}}$ using recombineering. Design of the $\operatorname{atp} C^{\mathrm{E} 87 \mathrm{~A}}$ mutagenic oligonucleotide and mutant screening primers for in vivo recombineering in $M$. smegmatis; The top panel shows the sequence of the entire atpC gene (shades of brown) in a reversed manner reflecting its orientation in the M. smegmatis genome (negative DNA strand). The one-letter codes, positioned below the nucleotide sequence represent the protein sequence. Greyed-out nucleotide sequence represents up/downstream unannotated regions flanking the atpC gene. Singlestranded oligonucleotide (violet, $73 \mathrm{nt}$ ) is complementary to the negative strand (dark brown) and harbors the anti-codon CGC in the middle, whereby G is the only mutagenic nucleotide. Agarose gel on the left shows mutant-specific primer pair (E87A_FWD + Ctrl_REV, green arrows) yielding no amplified product in reaction with $M$. smegmatis WT colonies. Agarose gel on the right shows the mutant-specific alternate primer pair (Ctrl_FWD + E87A_REV, yellow arrows) that amplified the $M$. smegmatis WT DNA fragment, resulting in the 209 bp band. The control primer pair (Ctrl_FWD + Ctrl_REV, yellow rightward arrow, green leftward arrow, respectively) was utilized to amplify the $593 \mathrm{bp}$ fragment containing the entire atp $C$ region (366 nt) for sequencing.

Two PCR-positive colonies were picked randomly for the subsequent colony PCR amplification using the non-mutagenic, flanking primers, yielded product visualized as a single band of 593 base pair. The incorporation of the E87A mutation into the atpC gene was confirmed by sequencing the 593 base pair amplified fragment (Figure 3.20). The strain M. smegmatis $m c 155^{2}$ : atp $C^{\mathrm{E} 87 \mathrm{~A}}$ : $\mathrm{pJV62:} \mathrm{pJS} 25$ verified by colony screening PCR and sequencing, was stabilized, the pSJ25 vector removed by several rounds of passaging and further utilized for the studies.

In order to generate a strain with the inducible expression of the WT form of subunit $\varepsilon$, the newly-engineered $a t p C^{\mathrm{E} 87 \mathrm{~A}}$ strain was further manipulated. A vector harboring the WT copy of the atpC gene, pMV262:atp $C^{\mathrm{WT}}$ was transformed into the M. smegmatis $m c 155^{2}$ : atp $C^{\mathrm{E} 87 \mathrm{~A}}: \mathrm{pJV} 62$ and the protein expression was under control 
of the $h s p 60$ promoter. All the plasmids and a M. smegmatis $m c 155^{2}$ : pJV62 strain were generated and supplied kindly by Prof. Thomas Dick's lab at NUS, Singapore.

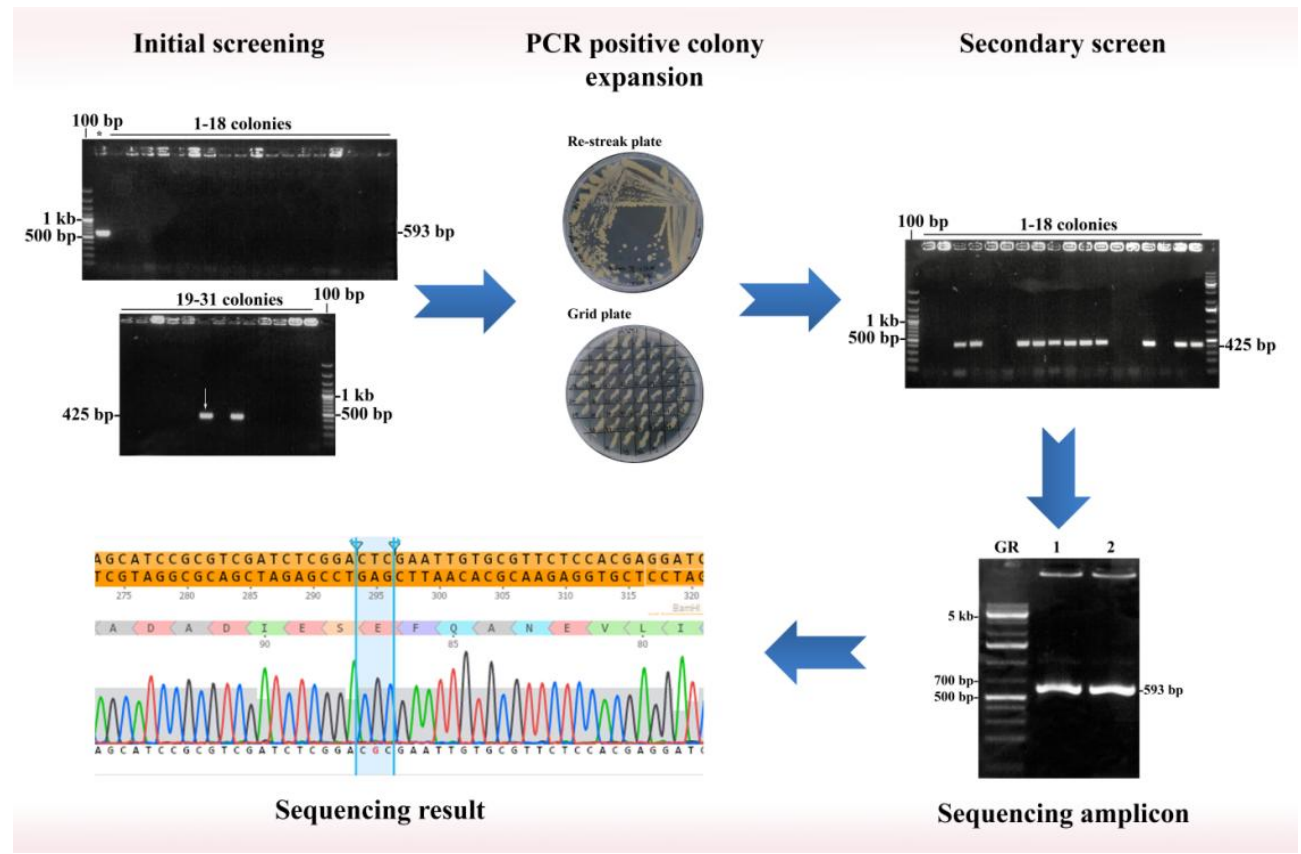

Figure 3.20: Screening for the $M$. smegmatis atp $C^{\mathrm{E} 87 \mathrm{~A}}$ mutant strain. Initial screening performed with the E87A mutant-specific primer pair (E87A_FWD and Ctrl_REV) that amplifies the 425 base pair sequence. A number of 31 screened colonies yielded 2 colonies positive for the E87A mutation; "*" in the top left gel indicates the control reaction (593 bp) obtained using the Ctrl_FWD and Ctrl_REV primer pair. The PCR-positive colonies were re-streaked on a 7H10/hyg plate and randomly-selected for expansion on a grid plate. The secondary screen was conducted with the identical PCR to observe the clonal expansion; 11/18 colonies showed mutant-positive PCR (425 bp band). Two positive colonies were selected for colony PCR with the flanking primer pair (Ctrl_FWD and Crtl_REV), and the sequencing amplicon of 593 base pairs was amplified. Lane 1 and lane 2 (the gel in the lower-right corner) represent the amplified fragments from two different colonies for sequencing. Sequencing of the fragment confirmed the successful substitution of E87 with alanine; CGC anti-code shown above represents a complement sequence of the GCG, a code for alanine.

M. smegmatis atp $C^{\mathrm{E} 87 \mathrm{~A}}$ mutant displayed a growth behavior, cell and colony morphologies that were indistinguishable from that of the parental WT strain when cultivated at $37^{\circ} \mathrm{C}$ in the aerobic conditions using Middlebrook $7 \mathrm{H} 9$ media and $7 \mathrm{H} 10$ agar plates (Figure 3.21A-B). Preliminary screening of the cell viability in the presence of the Bedaquiline showed slight hyposensitivity of the $\operatorname{atp} C^{\mathrm{E} 87 \mathrm{~A}}$ mutant, whose minimum inhibitory concentration $\left(\mathrm{MIC}_{50}\right)$ was 1.6 times higher of the $\mathrm{MIC}_{50}$ specific for the WT.

To isolate IMVs, the WT, atp $C^{\mathrm{E} 87 \mathrm{~A},}$ and $\mathrm{pMV} 262: a t p C^{\mathrm{WT}}$ mutant strains were cultivated in a conventional, Middlebrook 7H9 media and were harvested after reaching the log phase characterized by the optimal optical density of $\sim 0.6$ at $\mathrm{OD}_{600}$. IMVs containing complexes of the respiratory chain, including the $\mathrm{F}_{1} \mathrm{~F}_{\mathrm{O}}$-ATP synthase 
were isolated using the established protocol (3) and used for the ATPase/-synthase activity measurements and proton pumping activity screening, as described earlier.

(A)

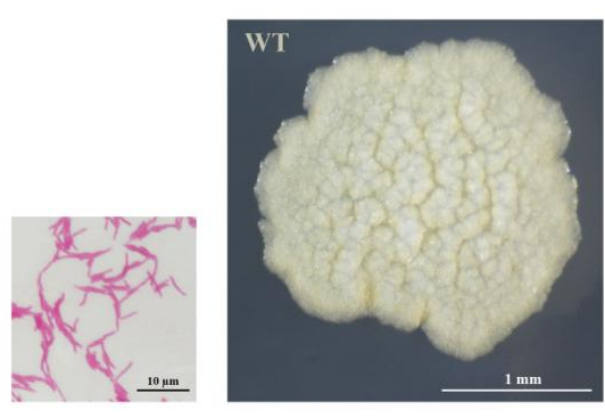

(C)

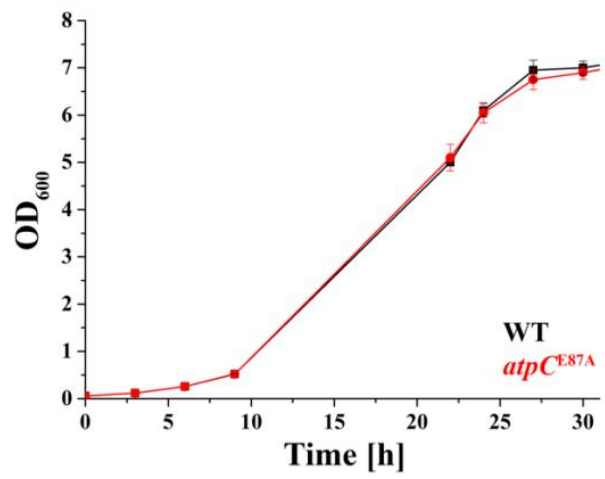

(B)

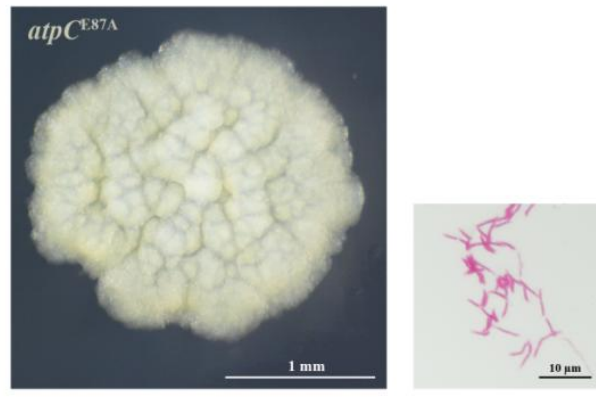

(D)

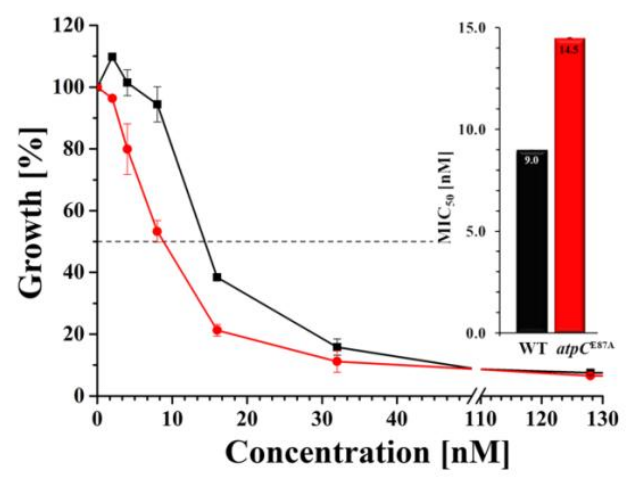

Figure 3.21: Growth of $M$. smegmatis $m c 155^{2}$ and $a t p C^{\mathrm{E} 87 \mathrm{~A}}$ and its susceptibility to BDQ. (A) Colonies of both strains were cultivated on 7H10 medium and are showing the typical morphology after 4 days of incubation. Cells were screened for variable morphology using hematoxylin-eosin (HE) staining. (B) The strain $a t p C^{\mathrm{E} 87 \mathrm{~A}}$ displays the growth behavior similar to that of the WT in the liquid, 7H9 media. (C) Bedaquiline growth inhibition dose-response curve shows the alteration of the minimal inhibitory concentration $\left(\mathrm{MIC}_{50}\right)$. The $\operatorname{atp} C^{\mathrm{E} 87 \mathrm{~A}}$ mutant displayed increased tolerance to bedaquiline.

The IMVs of the WT, atp $C^{\mathrm{E} 87 \mathrm{~A},}$ and $\mathrm{pMV} 262: \operatorname{atp} C^{\mathrm{WT}}$ were used to determine the ATPase activities. Upon addition of $2 \mathrm{mM}$ ATP as a substrate, the IMVs of the $a t p C^{\mathrm{E} 87 \mathrm{~A}}$ mutant strain exhibited a reduced ATP hydrolysis rate of $\sim 11 \%$, when compared to the one of the WT, while the complementation of the mutant with the constitutively expressed WT $\varepsilon$, partially restored the hydrolysis rate to about $93 \%$ (Figure 3.22-A). To determine whether the ATP hydrolysis events are coupled to proton pumping, the IMVs of all three strains were tested in the presence of the $\mathrm{pH}$ sensitive, hydrophobic, fluorescent fluorophore, 9-amino-6-chloro-2-methoxy-acridine (ACMA) $(3,206)$. In its deprotonated form, ACMA molecules diffuse freely through the IMV membrane. Upon addition of the $2 \mathrm{mM}$ ATP, the ATP synthase utilizes the 
chemical energy stored in the $\gamma$-phosphate bond to translocate protons inside the IMVs. The resulting decrease in $\mathrm{pH}$ within the IMVs could protonate ACMA molecules, decreasing its hydrophobicity and, in turn, trapping it within the membrane compartment. The protonation also reduces ACMA fluorescence which could be observed as a fluorescent emission drop in the spectrum. The addition of the uncoupler SF6847 (301) to the IMVs, caused the leakiness of the IMV membrane for protons, triggering the collapse of the $\Delta \mathrm{pH}$, equalizing the $\mathrm{pH}$ on both sides of the IMV membrane. This promoted the deprotonation of the ACMA molecules trapped within the IMVs which in turn caused its free diffusion through the membrane and the increase in the fluorescence.

To verify that the IMVs obtained for this study were proton-tight (Figure 3.22B) and that there is no unspecific proton translocation, the NADH was used as a substrate for the proton-translocating respiratory complex I (NDH-I). The addition of the NADH to the WT IMVs, caused a significant fluorescence drop which was restored after the addition of the un-coupler SF6847, indicating that the IMVs were not leaky.

As shown in the Figure 3.22-B, when $2 \mathrm{mM}$ ATP was used as a substrate, both WT and atp $C^{\mathrm{E} 87 \mathrm{~A}}$ strains' IMVs revealed an initial fluorescence quenching, showing no significant differences of the ATPase-driven proton pumping between the two strains. The addition of the SF6847 un-coupler to the WT and atp $C^{\text {E87A }}$ strain IMVs caused the restoration of the fluorescent signal, indicating that the vesicles were proton-tight and that observed initial fluorescence quenching was a result of the ATP synthase-mediated acidification of the IMV compartment. As no differences were observed in ATPase activities of WT and $a t p C^{\mathrm{E} 87 \mathrm{~A}}$, the measurements did not include the vesicles of the $a t p C^{\mathrm{E} 87 \mathrm{Ac}}$. 
(A)

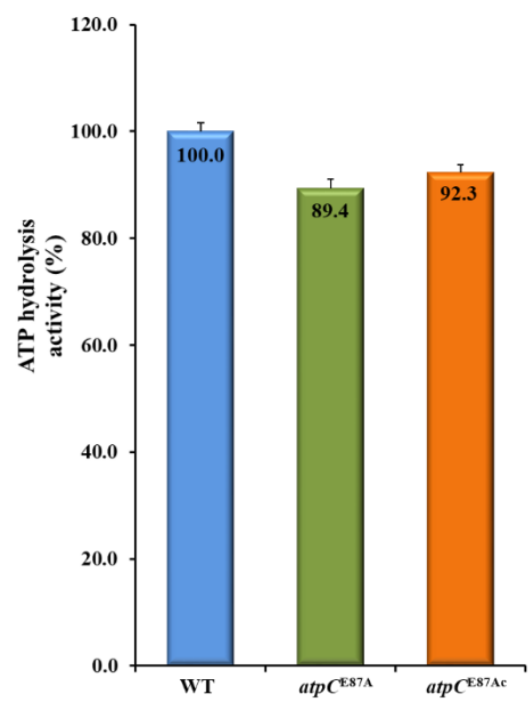

(B)

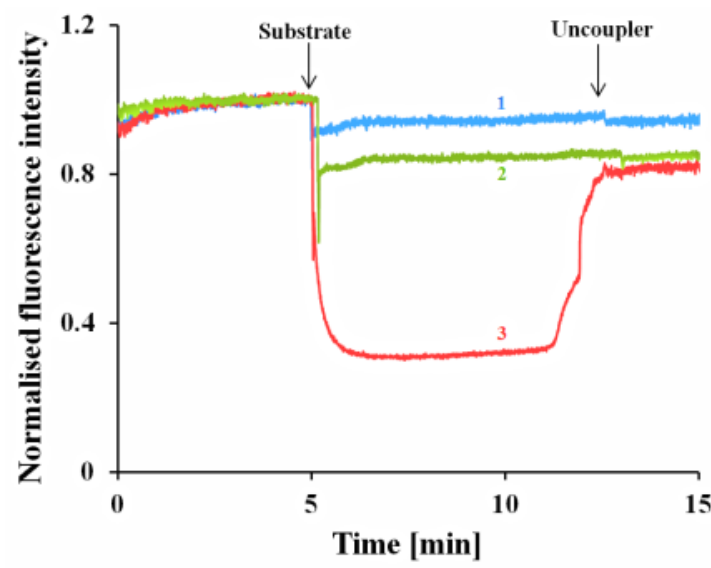

Figure 3.22: ATPase activity and proton pumping of the $M$. smegmatis IMVs. (A) The bar diagram represents the ATP hydrolysis measured in the IMVs of the M. smegmatis WT, atpCE87A mutant, and the atpCE87A complement strain converted into the percentage of the respective absolute values. Standard deviation was calculated based on (at least three) individual measurements for each strains' IMVs and is represented as the error bars converted into the respective percentages. (B) The proton pumping of the M. smegmatis WT (blue), $a t p C^{\mathrm{E} 87 \mathrm{~A}}$ strain (green) vesicles in the presence of the $2 \mathrm{mM}$ ATP. The red profile represents the control, M. smegmatis WT IMVs proton pumping in the presence of the $2 \mathrm{mM}$ NADH.

Furthermore, the capacity of IMVs to synthesize ATP was investigated. As shown in Figure 3.23, the ATP synthesis of WT IMVs was $1.65 \mathrm{nmol} / \mathrm{min} / \mathrm{mg}$ of protein while the mutant $a t p C^{\mathrm{E} 87 \mathrm{~A}} \mathrm{IMVs}$ displayed the increased ATP synthesis rate $(2.53 \mathrm{nmol} / \mathrm{min} / \mathrm{mg}$ of protein) which is around $53 \%$ higher than the rate determined for the WT IMVs.

The ATP synthesis rate was calculated for the complemented strain atp $C^{\mathrm{E} 87 \mathrm{Ac}}$ to be $1.93 \mathrm{nmol} / \mathrm{min} / \mathrm{mg}$ of protein, which is an increase of $17 \%$ in comparison to the WT and a $34 \%$ decrease in respect to the rate of the $a t p C^{\mathrm{E} 87 \mathrm{~A}}$ mutant. This indicated that the E87A mutation indeed caused the increase of ATP synthesis, probably due to the altered intramolecular interactions between NTD and CTD, as revealed in the NMR experiment in the section 3.23, emphasizing the importance of the tight coupling on the ATP synthetic activity. 
(A)

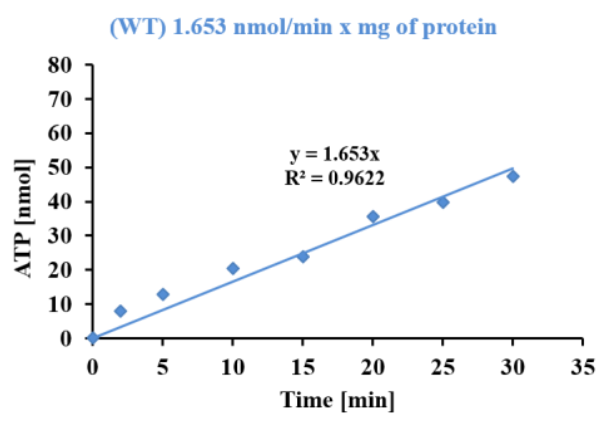

(C)

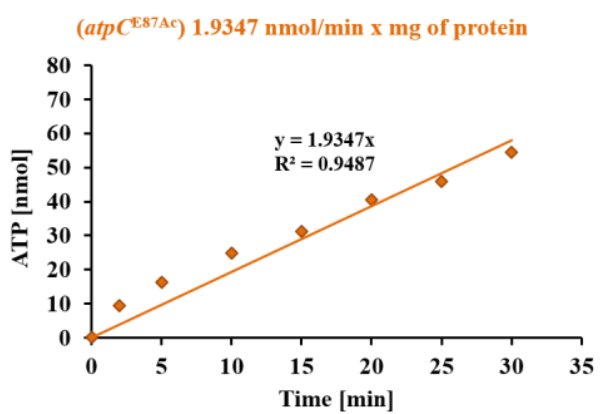

(B)

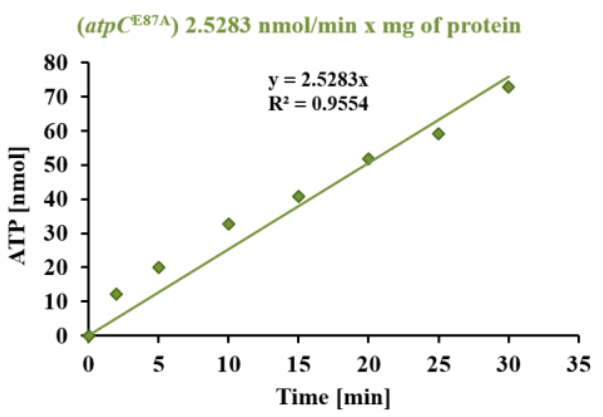

(D)

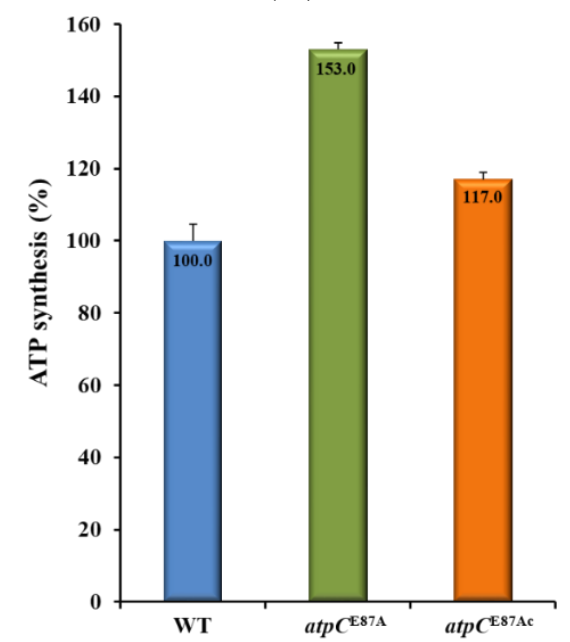

Figure 3.23: ATP synthesis of the M. smegmatis IMVs. The graphs represent the rate of ATP synthesis; (A) WT, (B) atpC ${ }^{\mathrm{E} 87 \mathrm{~A}}$, and (C) atpCE87Ac. (D) The bar diagram represents the ATP synthesis shown as the percentage (\%) of the absolute activity determined for individual IMVs of the M. smegmatis WT, atp $C^{\mathrm{E} 87 \mathrm{~A}}$ mutant, and the atp $C^{\mathrm{E} 87 \mathrm{Ac}}$ complement strain. 


\subsubsection{Purification of and the characterization of the mutant MtER62L}

As shown in section 3.23, the substitution of MtcE87 to A87 caused the removal of the negatively charged carboxylic group from the network of interactions described in section 1.1.11 (Figure 1.17). This altered the intramolecular interactions in turn affecting the enzymatic activity of the $\alpha^{c h i}{ }_{3}: \beta_{3}: \gamma: M t \varepsilon$. The residue R62 belongs to the same network and could possibly contribute to the coupling in a similar way as E87.

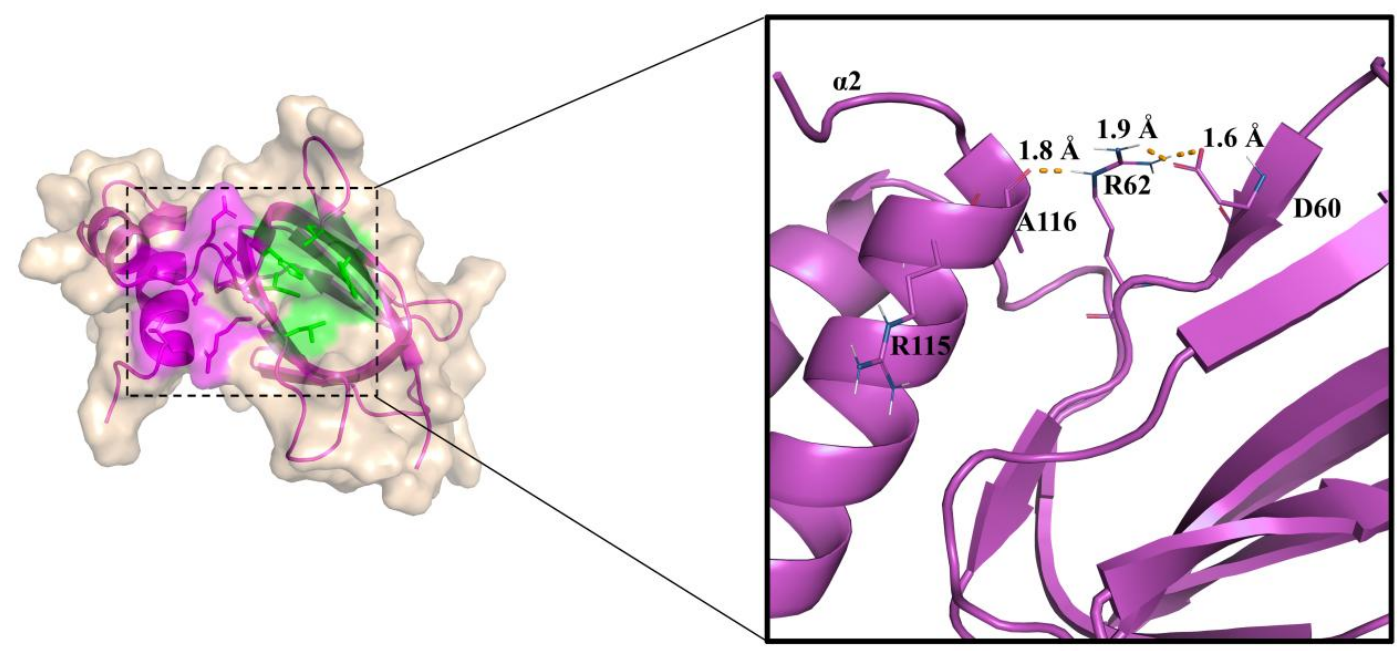

Figure 3.24: The MtE intramolecular network of interactions around residue R62. Surface representation of $M t \varepsilon$ showing the formation of a network of residues connecting the NTD to the CTD. The residues partaking in the network formation are shown as sticks and are represented in green and magenta color for the NTD and the CTD, respectively. The enlarged panel shows the detailed view of the amino acid residues in the network connecting NTD to CTD, represented as sticks. The residue R62 is wedged between the NTD residues (green) and the CTD residues (magenta). The important residues are labeled with one-letter amino acid codes and inter-proton distances less than $5 \AA$ are marked as dashed lines. Figure adapted from (205).

It was investigated here whether the positively-charged guanidino group or the long aliphatic chain of the R62 plays a role in the maintenance of that network (Figure 3.24). Here the mutant MtcR62L that omits the charge of the arginine side chain by replacing it with the long aliphatic side chain of lysine was generated.

Site-directed mutagenesis was used to generate the MtcR62L mutant, using the plasmid containing the $M t \varepsilon$ as a template. The substitution of arginine to lysine was carried out using the mutagenic primers described in section 2.2.9. The PCR resulted in an amplified plasmid (Figure 3.25) and the mutation was confirmed by DNAsequencing. The mutant was generated using the same workflow described for the Mt\&E87A mutant including the purification steps (sections 2.2.12 and 3.2.2). 
To isolate the mutant protein, the cells were lysed in buffer $50 \mathrm{mM}$ Tris/HCl $200 \quad \mathrm{mM} \quad \mathrm{NaCl}, \quad 10 \%$ glycerol, $\mathrm{pH} 7.5$ and the lysate was loaded onto a pre-equilibrated $\mathrm{Ni}^{2+}$ NTA column. The elution was performed with imidazole (0-400 $\mathrm{mM}$ ) and the fractions $75-125 \mathrm{mM}$ imidazole were pooled, concentrated to $2 \mathrm{ml}$ and loaded onto the size exclusion column Superdex 75 HR 10/30 column (GE Healthcare). The homogeneity of the eluted protein was verified by a $17 \%$ SDS - PAGE (Figure 3.26).

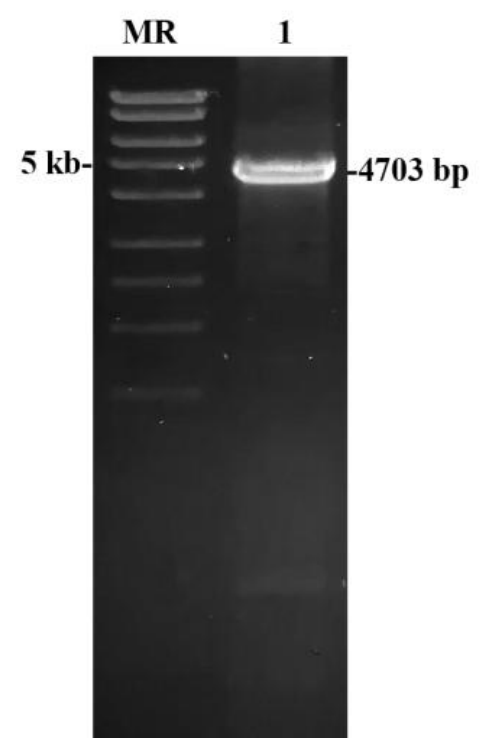

Figure 3.25: Cloning of the substitution mutant Mt\&R62L. Amplification by site-directed mutagenesis of the entire plasmid DNA containing the $M t \varepsilon$ sequence $(\mathrm{pET} 9 \mathrm{~d}(+): M t \varepsilon)$ yielded the product shown in the lane $1 ; 1 \mathrm{~kb}$ represents the DNA marker.

(A)

(B)
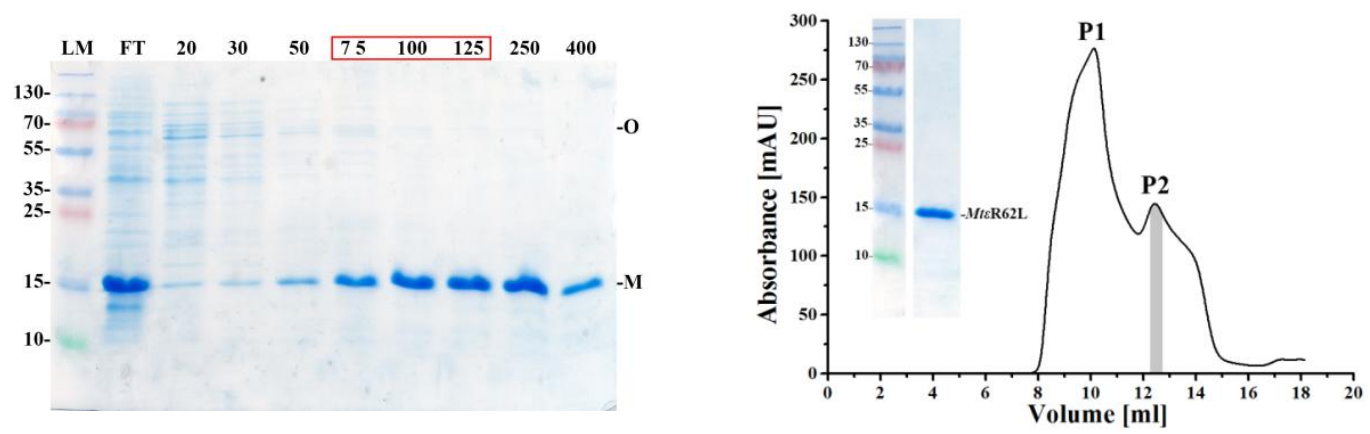

Figure 3.26: Purification of Mt\&R62L by gel filtration. Protein obtained after $\mathrm{Ni}^{2+}-\mathrm{NTA}^{-}$ chromatography (A) was applied onto a gel filtration column (Superdex 75) and eluted at $13 \mathrm{ml}$ volume, as seen in the chromatogram (B). P1 peak contains the protein in an unspecific oligomeric state, $\mathrm{P} 2$, contains the protein in the dimeric state, $\mathrm{P} 3$ represents the monomeric peak. The purity was confirmed by running a $17 \%$ SDS-PAGE (C). P1, P2, and P3 represent the two main fractions of each peak; Purified protein is labeled as "apo" and shown in the righter-most lane. 
Reconstitution with the $\alpha^{c h i}: \beta_{3}: \gamma$ complex was achieved using the standardized protocol, described in section 2.2.16 and shown in Figure 3.27. The stoichiometry of the reconstituted complex was verified with the densitometric analysis, while ATP hydrolysis measurements were conducted using the described continuous ATPase assay.

(A)

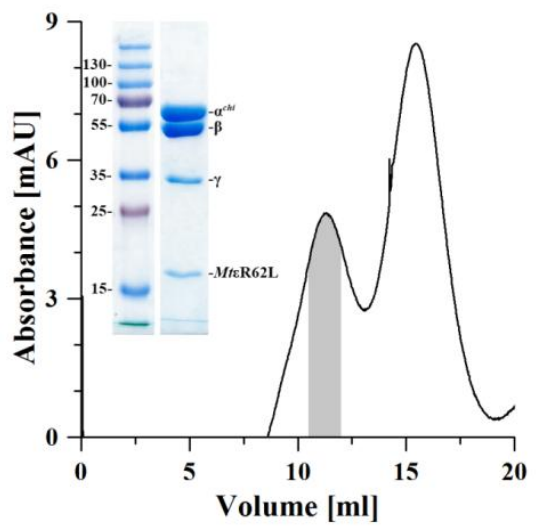

(C)

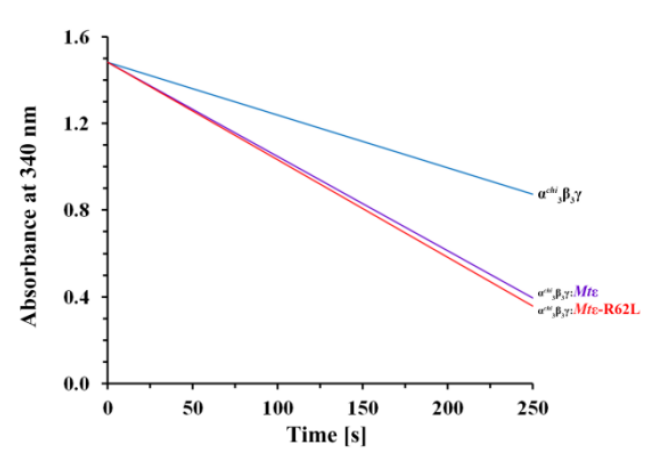

(B)

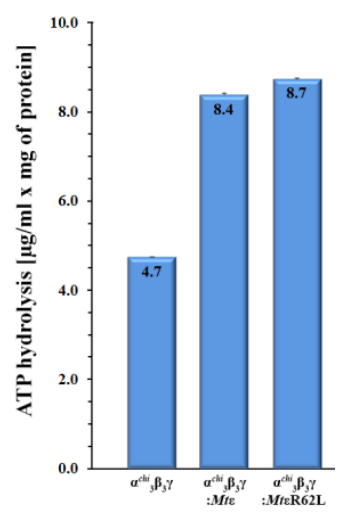

(D)

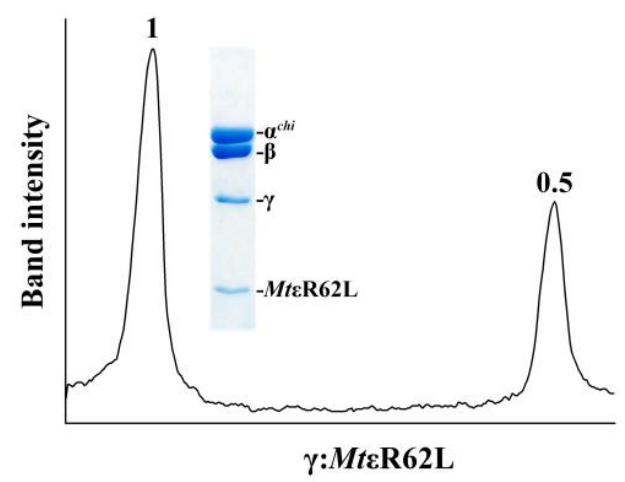

Figure 3.27: Reconstitution of $\alpha^{c h i}{ }_{3}: \beta_{3}: \gamma$ and subunit Mt\&R62L mutant. Purified $\alpha^{c h i}{ }_{3}: \beta_{3}: \gamma$ and MtcR62L were reconstituted and passed through a Superdex S300 column (A) and the peak containing reconstituted $\alpha^{c h i}: \beta_{3}: \gamma: M t \varepsilon R 62 \mathrm{~L}$ was run on a $12 \%$ SDS-gel (inset). (B) Bar diagram and activity profiles (C) showing the specific activities of $\alpha^{c h i}{ }_{3}: \beta_{3}: \gamma, \alpha^{c h i}: \beta_{3}: \gamma: M t \varepsilon$ and $\alpha^{c h i}{ }_{3}: \beta_{3}: \gamma: M t \varepsilon R 62 \mathrm{~L}$, indicating that the mutation R62L does not alter significantly the ATPase activity of the $\alpha^{c h i}{ }_{3}: \beta_{3}: \gamma: M t \varepsilon$ R62L complex. (D) Densitogram showing the band intensities of subunits $\gamma$ (left peak) and MtER62L (right peak) in the $\alpha^{c h i}: \beta_{3}: \gamma: M t \varepsilon R 62 \mathrm{~L}$ complex derived from the 12\% SDS-gel (inset). Integrals of the peaks were used to determine the ratio of $\gamma: M t \varepsilon R 62 \mathrm{~L}$ as 1:0.5. Figures credited to (205).

The results show, that the R62L mutation in subunit Mte does not alter the binding to the $\alpha^{c h i}: \beta_{3}: \gamma$, allowing the stoichiometric reconstitution of subunits $\gamma: \varepsilon$ in ratio 1:0.5, observed for the $\alpha^{c h i}{ }_{3}: \beta_{3}: \gamma: M t \varepsilon$ complex. Additionally, the ATPase activity 
of this new complex was not altered to a great extent $(8.7 \pm 0.01 \mu \mathrm{mol} / \mathrm{min} / \mathrm{mg}$ protein), when compared to the value obtained $\alpha_{3}^{\text {chi }}: \beta_{3}: \gamma: M t \varepsilon(8.4 \pm 0.04 \mu \mathrm{mol} / \mathrm{min} / \mathrm{mg}$ protein). These results point out that the guanidino group of R62 is not crucial for the coupling efficiency within subunit $M t \varepsilon$ and that its substitution does not affect the enzymatic activity of the $\alpha^{c h i}: \beta_{3}: \gamma: M t \varepsilon R 62 \mathrm{~L}$ complex.

\subsubsection{Importance of the N-terminal domain for the stability of MtE}

Early studies on the E. coli $\mathrm{F}_{1} \mathrm{~F}_{\mathrm{O}}$-ATP synthase emphasized the importance of the NTD of subunit $\varepsilon$ to provide a coupling capacity in association with subunit $\gamma(5)$. Systematic deletions of the $\varepsilon$ NTD residues showed that the first 15 amino acids had no significant influence on binding to $\mathrm{F}_{\mathrm{O}}$ in a functional manner and did not affect the enzymatic activity. However, the mutant $\varepsilon$ subunit which harbored an NTD truncation of 16 amino acids suffered a reduced binding capacity to central stalk and perturbed catalytic activity. Considering the high degree of structural homology, but significant degree of sequence uniqueness of $\varepsilon N T D$ across bacterial species, the epitope that could provide critical interactions for the stability of the mycobacterial subunit $\varepsilon$ and in theory affect the binding capacity to the subunit $\gamma(5)$ was investigated.

The assembled model contained structural coordinates of recently solved subunit Mtc (PDB ID: 5YIO) (209) and mycobacterial $c_{9}$-ring (PDB ID: 4V1G) and a model of mycobacterial subunit $\gamma$, generated as described in the section 1.11 (302). The residues ${ }_{1} \mathrm{MAELN}_{5}$ are prone to interact with loop residues Q46, E48, and A49 of two adjacent $c$-ring monomers, transferring the rotational energy of the $c$-ring. In

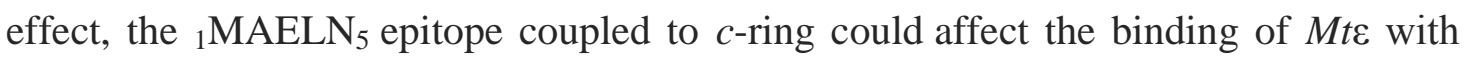
subunit $\gamma$ via their respective epitopes ${ }_{10} \mathrm{AVDRN}_{14}$ of and residues ${ }_{40} \mathrm{KSFV}_{43}$ as mentioned in section 1.11 .

To generate the truncation mutant subunit $M t \varepsilon_{6-121}$, by deleting the NTD epitope ${ }_{1} \mathrm{MAELN}_{5}$, the entire plasmid containing $M t \varepsilon$ gene was amplified by sitedirected mutagenesis according to the established protocol in the section 2.2.9. The amplicon size of 4,688 bp was verified on the 1\% agarose gel (Figure 3.28) and was transformed into the E. coli $\mathrm{C} 41$ cells for protein production and purification two-step protocol as described in section 2.2.11. 
To purify the mutant protein, the cells were lysed in buffer $50 \mathrm{mM}$ Tris/HCl $200 \quad \mathrm{mM} \quad \mathrm{NaCl}, \quad 10 \%$ glycerol, $\mathrm{pH} 7.5$ and the lysate was loaded onto a pre-equilibrated $\mathrm{Ni}^{2+}$ NTA column. The elution was performed with imidazole (0-400 mM) and the fractions 75-125 $\mathrm{mM}$ imidazole were pooled, concentrated to $2 \mathrm{ml}$ and loaded onto the size exclusion column Superdex 75 HR

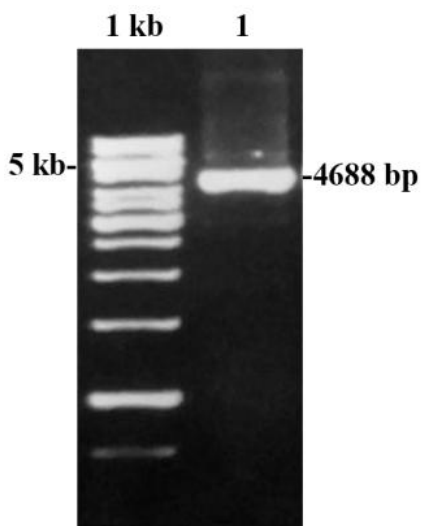

Figure 3.28: Cloning of the truncation mutant $\boldsymbol{M t \varepsilon}_{6-121}$. Amplification by site-directed mutagenesis

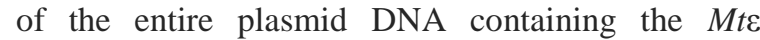
sequence $(\mathrm{pET} 9 \mathrm{~d}(+): M t \varepsilon)$ yielded the product shown in lane $1 ; 1 \mathrm{~kb}$ represents the DNA marker. 10/30 column (GE Healthcare).

From the $\mathrm{Ni}^{2+}$-NTA profile, it was apparent that the imidazole fractions $75-125$

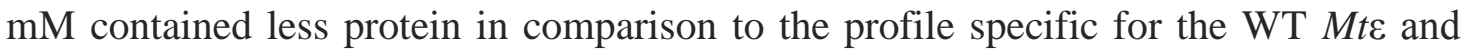
that the entire profile was shifted towards elution with higher molarity of imidazole (Figure 3.29). This indicated the presence of higher oligomers which was further confirmed from the size-exclusion diagram. It was apparent that the major part of the typical, well-resolved peak of the monomeric fraction that elutes at around $13 \mathrm{ml}$ in case of the WT MtE was now shifted towards the peak that represented oligomeric fractions, eluting at around $8 \mathrm{ml}$. To verify the purity and homogeneity of the eluted protein after the size exclusion column, the samples were applied on a 17\% SDSPAGE (Figure 3.29). The monomeric fraction was poorly resolved after the sizeexclusion column, indicating that the equilibrium was shifted towards the oligomeric state (Figure 3.29-B) due to the truncation of the ${ }_{1} \mathrm{MAELN}_{5}$ peptide. To understand whether the structural alterations resulting from ${ }_{1} \mathrm{MAELN}_{5}$ truncation affect the binding to the central stalk subunit $\gamma$, reconstitution of $M t \varepsilon_{6-121}$ with $\alpha^{\text {chi }}{ }_{3}: \beta_{3}: \gamma$ was carried out (section 2.2.31) and the catalytic activity of the newly formed complex $\alpha^{c h i}: \beta_{3}: \gamma: M t \varepsilon_{6-121}$ was measured. 
(A)

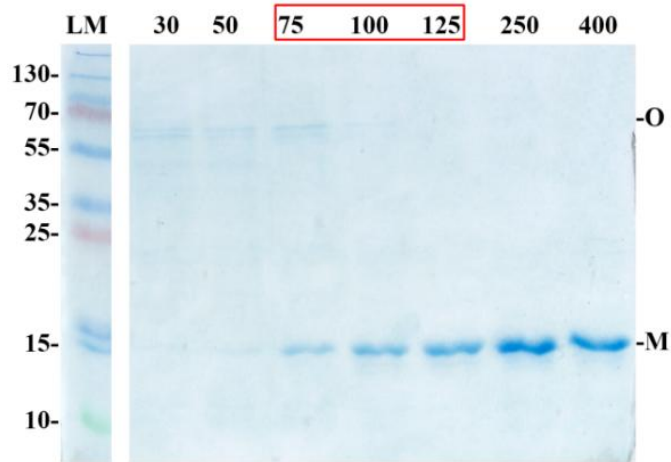

(B)

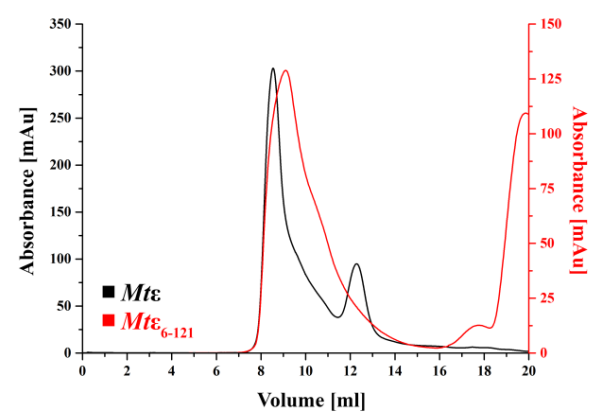

(C)

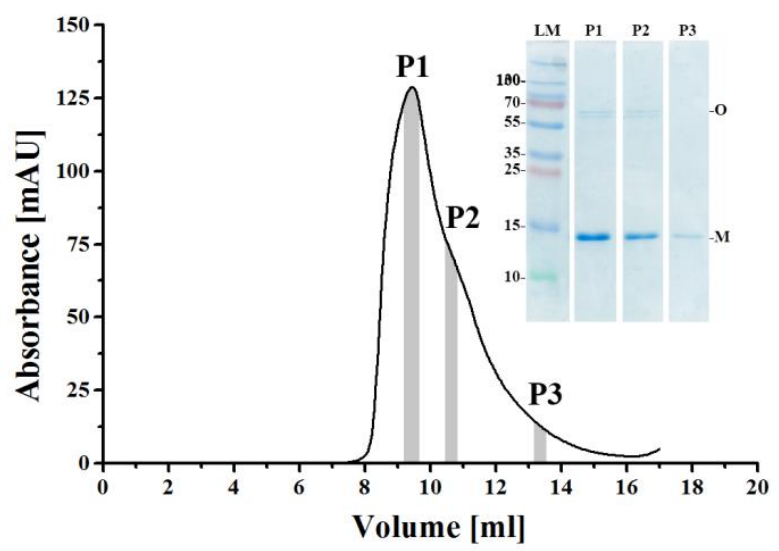

Figure 3.29: Purification of $\boldsymbol{M} \boldsymbol{\varepsilon}_{6-121}$ by gel filtration. Protein obtained after $\mathrm{Ni}^{2+}-\mathrm{NTA}$ chromatography (A) was applied onto a gel filtration column (Superdex 75). (B) The comparison of elution profiles of $M t \varepsilon$ (black) and $M t \varepsilon_{6-121}$ (red) (C) Size exclusion diagram reveals the oligomerization of the $M t \varepsilon_{6-121}$ protein; Peak fractions indicated in gray were analyzed for the purity and homogeneity on 17\% SDS-PAGE. P1 and P2 peaks contain the protein in an unspecific oligomeric state, $\mathrm{P} 3$ represents the monomeric peak as shown in the insets. $M$-monomer, $O$-oligomer. Figures credited to (205).

The complex $\alpha^{\text {chi }}{ }_{3}: \beta_{3}: \gamma: M t \varepsilon_{6-121}$ was reconstituted by mixing the $\alpha^{c h i}: \beta_{3}: \gamma$ and the monomeric fraction (peak 1) of $M t \varepsilon_{6-121}$ in a molar ratio of 1:3, respectively. When applied on the $12 \%$ SDS-gel, it was revealed by densitometric analysis that the substoichiometric complex $\alpha^{c h i}: \beta_{3}: \gamma: M t \varepsilon_{6-121}$ was formed and that the ratio of $\gamma: M t \varepsilon_{6-121}$ was 1:0.14 (Figure 3.30-A), which is significantly lower than the ratio established for the $\alpha^{c h i}{ }_{3}: \beta_{3}: \gamma: M t \varepsilon$ complex $(\gamma: M t \varepsilon=1: 0.5)$. The capacity of the newly formed complex to hydrolyze ATP was measured to be $5.7 \pm 0.01 \mu \mathrm{mol} / \mathrm{min} / \mathrm{mg}$ protein. Even though the sub-stoichiometric complex was formed, the mutant $M t \varepsilon_{6-121}$ was able to bind to 
the central stalk subunit $\gamma$ and increased the catalytic rate when compared to $\alpha^{\text {chi }}{ }_{3}: \beta_{3}: \gamma$ $(4.7 \pm 0.01 \mu \mathrm{mol} / \mathrm{min} / \mathrm{mg}$ ) (Figure 3.30-B, C).

\section{(A)}

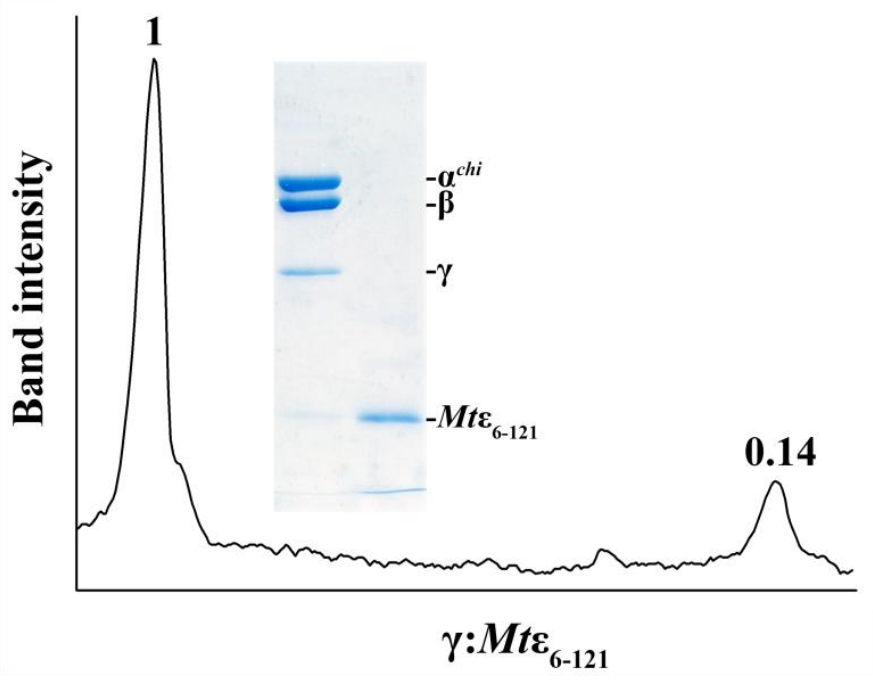

(B)

(C)
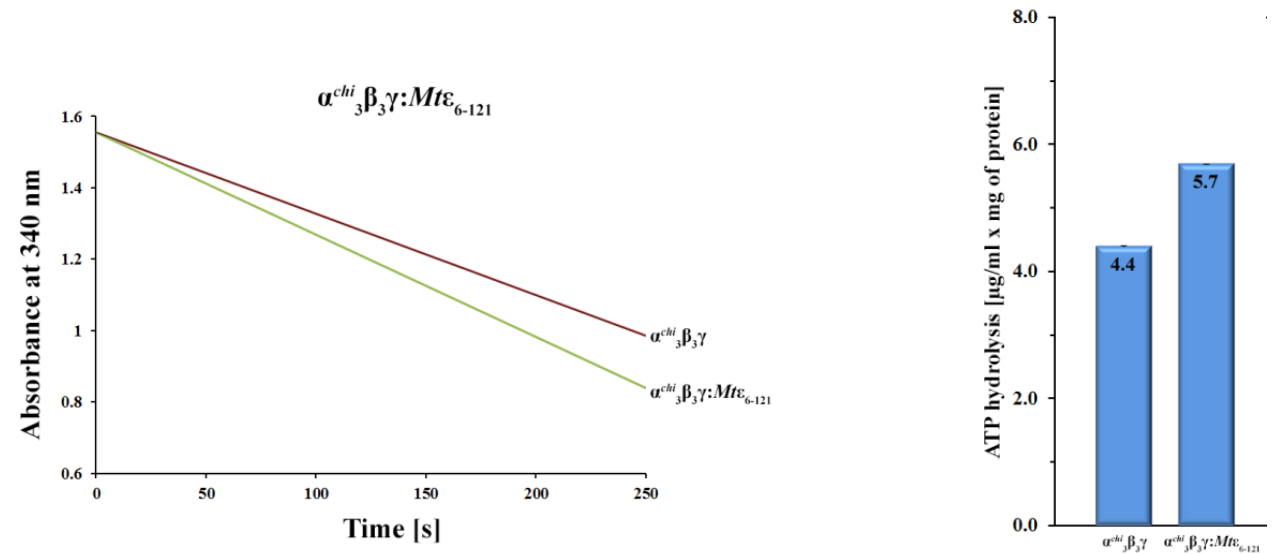

Figure 3.30: Reconstitution of $\alpha c^{h i}{ }_{3}: \beta_{3}: \gamma$ and subunit $M t \varepsilon_{6-121}$ mutant. (A) Purified $\alpha^{\text {chi }}{ }_{3}: \beta_{3}: \gamma$ and $M t \varepsilon_{6-121}$ were reconstituted and passed through a Superdex S300 column; Densitometric analysis of the $12 \%$ SDS gel (inset) shows the ratio of $\gamma: M t \varepsilon_{6-121}$ is 1:0.14 in lane 1; Lane 2 contains the monomeric fraction of $M t \varepsilon_{6-121}$ used for the reconstitution experiment. Activity profiles (B) and bar diagram (C) indicate the specific activities of $\alpha^{c h i}: \beta_{3}: \gamma$ and $\alpha^{c h i}: \beta_{3}: \gamma: M t_{6-121}$. Figures credited to (205).

These findings indicate that the first five residues of $M t \varepsilon$ are essential for the formation of proper structural traits and capability of the protein to remain in a monomeric form that enables it to successfully associate to the $\alpha^{c h i}: \beta_{3}: \gamma$ complex. 
3.3 Interactions of mycobacteria-specific epitopes within mutant $\alpha^{c h i}{ }_{3}: \beta_{3}: \gamma: M t \varepsilon$ complexes 
3.3.1 Cloning, production, and purification of $\left(\alpha^{c h i}-\mathrm{S} 400 \mathrm{C}\right)-$ and $\left(\alpha^{c h i}\right.$ V525C) $)_{3}: \beta_{3}: \gamma$ complexes

Numerous studies have reported subunit $\varepsilon$ as a key regulator of the catalytic capacity of bacterial $\mathrm{F}_{1} \mathrm{~F}_{\mathrm{O}}$-ATP synthases via its conformational alterations from compact to extended state (180-182,184,303,304). Considering the shorter length of mycobacterial subunit $\varepsilon$ (209), in comparison to its bacterial counterparts, the question was raised whether the $M t \varepsilon$ could undergo similar transitions and interact with the subunit $\alpha$ in the catalytic head. As the complex $\alpha^{\text {chi }}{ }_{3}: \beta_{3}: \gamma$ has been previously shown to be an important model for studying the epitope-specific properties of mycobacterial FATP synthases (3), Dr. Sony Manimakalai from our team built the model of the $\alpha^{c h i}{ }_{3}: \beta_{3}: \gamma: M t \varepsilon$ as described in section 2.2.20. As the NMR solution structure of $M t \varepsilon$ reported the structural coordinates of the subunit in the closed conformation, the extended conformation was modeled to reflect the extension of the two Mt\&CTD helices into the cavity formed by the subunits $\alpha^{c h i}$ and $\beta$ (Figure 3.31).

From the model, it was inferred that the residue S400 in the coupling motif $\left({ }_{396} \mathrm{AQFGSDLDK}_{404}\right)$ of $\alpha^{\text {chi }}$ as well as V525 of the mycobacteria-specific CTD epitope $\left(M t \alpha_{514-549}\right)$ of $\alpha^{c h i}$, could possibly interact with the $M t \varepsilon$ residues R113, G118

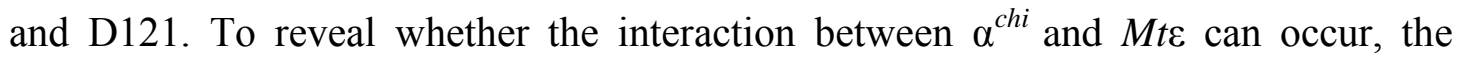
mutations $\left(\alpha^{c h i}-\mathrm{S} 400 \mathrm{C}\right)_{3}: \beta_{3}: \gamma$ and $\left(\alpha^{c h i}-\mathrm{V} 525 \mathrm{C}\right){ }_{3}: \beta_{3}: \gamma$ were designed and the crosslinking experiments were carried out with the $M t \varepsilon$ mutants R113C, G118C, and D121C.

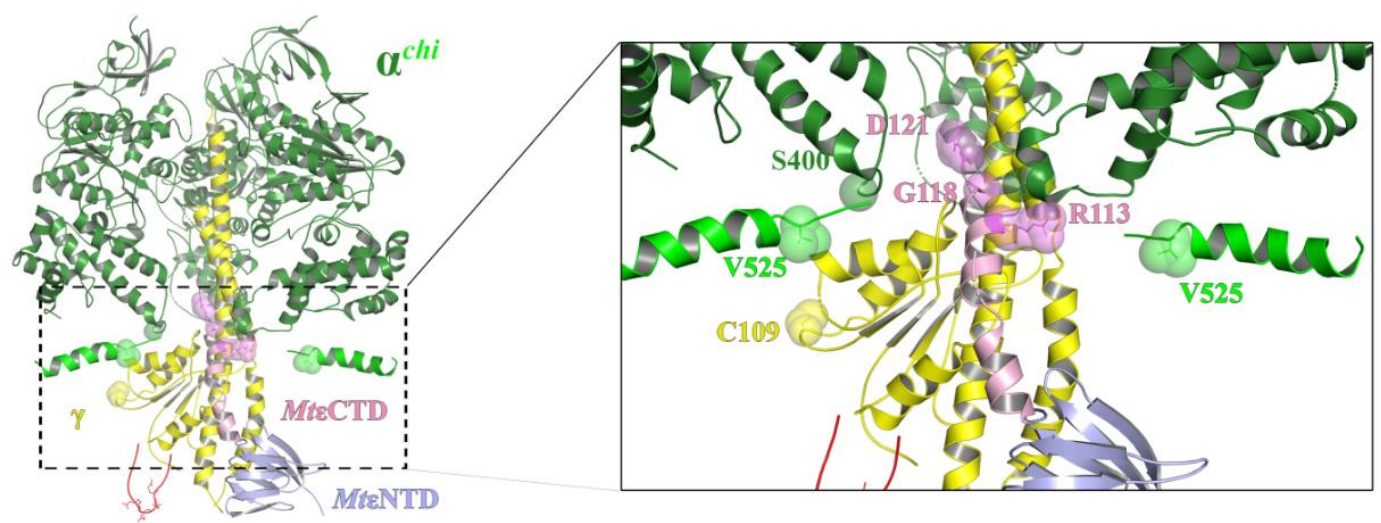

Figure 3.31: The model of the interacting residues in $\alpha^{\text {chi }}{ }_{3}: \boldsymbol{\beta}_{3}: \gamma: M t \varepsilon$ complex. The model shows the subunits $\alpha^{\text {chi }}$ (shades of green), $\gamma$ (yellow) and MtE (shades of purple), revealing the hypothesized residues labeled with a one-letter code and shaded by a chain. All shown residues were mutated to cysteines for the crosslinking experiments. 
To engineer the S400C and V525C mutations in the subunit $\alpha^{c h i}$, the plasmid harboring genes that code $\alpha^{c h i}, \gamma$ and $\beta$ subunits, generated and described in the thesis of Dr. Lavanya Sundararaman (291) was used as a template for the site-directed mutagenesis. The PCR amplification of the entire plasmid was carried out as described in the section 2.2.2 together with Dr. Dhirendra Singh from our group, yielding a single band of 9,316 base pairs, which was visualized on the $1 \%$ agarose gel (Figure 3.32). The plasmid containing the correct sequence (verified by the sequencing) was transformed into $E$. coli DK8 cells, as described in section 2.2.5. The proteins were expressed in the E. coli DK8 cell strain and isolated using the two-step purification protocol as described in section 2.2.6.

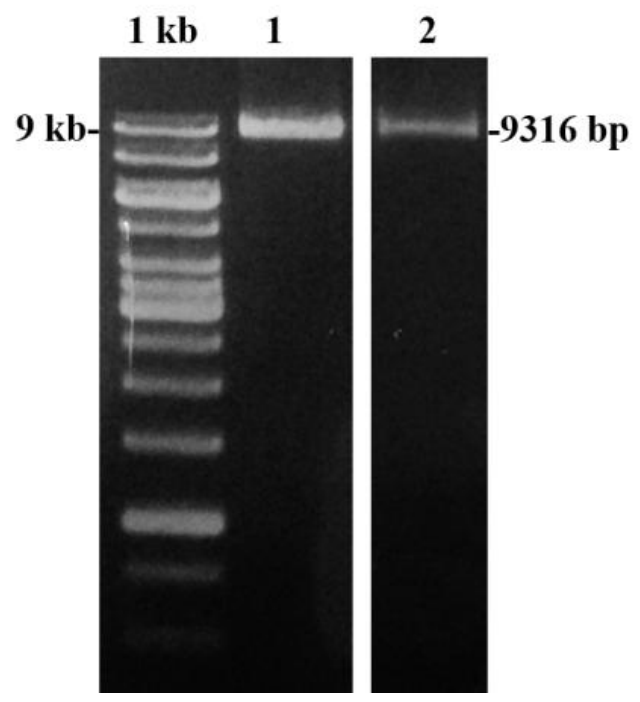

Figure 3.32: Cloning of the cysteine mutant complexes $\alpha^{c h i}: \beta_{3}: \gamma$. Site-directed mutagenesis generated the PCR products loaded on the agarose gel. The products represent complexes $\left(\alpha^{c h i}-\right.$ $\mathrm{S} 400 \mathrm{C})_{3}: \beta_{3}: \gamma($ lane 1$)$ and $\left(\alpha^{\text {chi }}-\mathrm{V} 525 \mathrm{C}\right)_{3}: \beta_{3}: \gamma$ (lane 2$) .1 \mathrm{~kb}$ represents the DNA marker;

By using the His 10 -tag on the subunit $\beta$ of the complex $\left(\alpha^{\text {chi }}-\mathrm{S} 400 \mathrm{C}\right)_{3}: \beta_{3}: \gamma$ and $\left(\alpha^{c h i}-\mathrm{V} 525 \mathrm{C}\right)_{3}: \beta_{3}: \gamma$, the enzyme was separated from the bulk of cellular proteins. The cell lysate was allowed to bind to the $\mathrm{Ni}^{2+}$-NTA matrix and was eluted with an imidazole gradient (Figure 3.33-A, C). To further homogenize the complex, the fractions containing the highest yield of protein (125-400 $\mathrm{mM}$ imidazole) were applied on a ResourceQ column $(6 \mathrm{ml})$. The protein was eluted at $250 \mathrm{mM} \mathrm{NaCl}$ in buffer containing $50 \mathrm{mM}$ Tris/ $\mathrm{HCl}, \mathrm{pH} 7.5$, and applied on the 9\% SDS-PAGE (Figure 3.33B, D). 
(A)

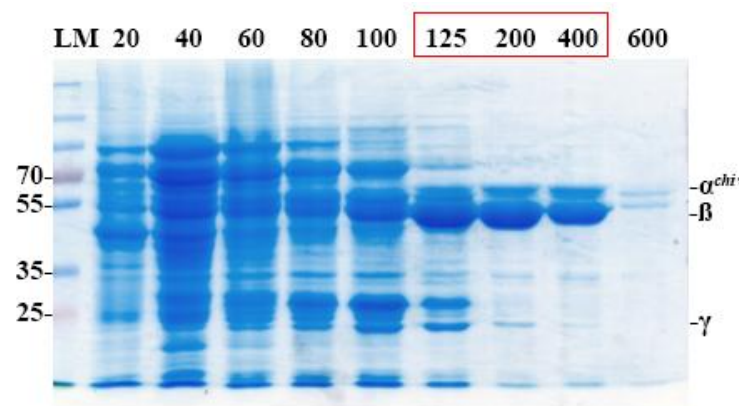

(C)

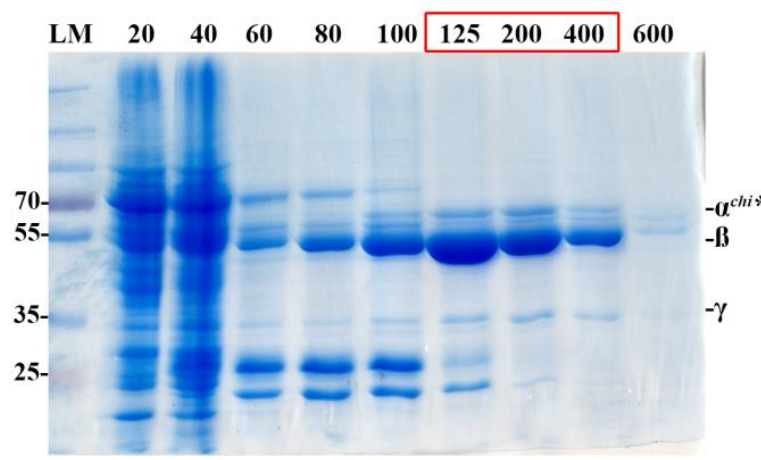

(B)

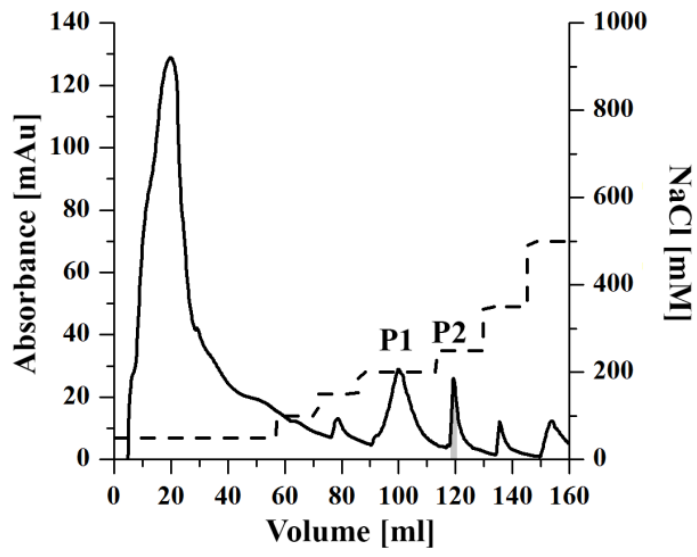

(D)

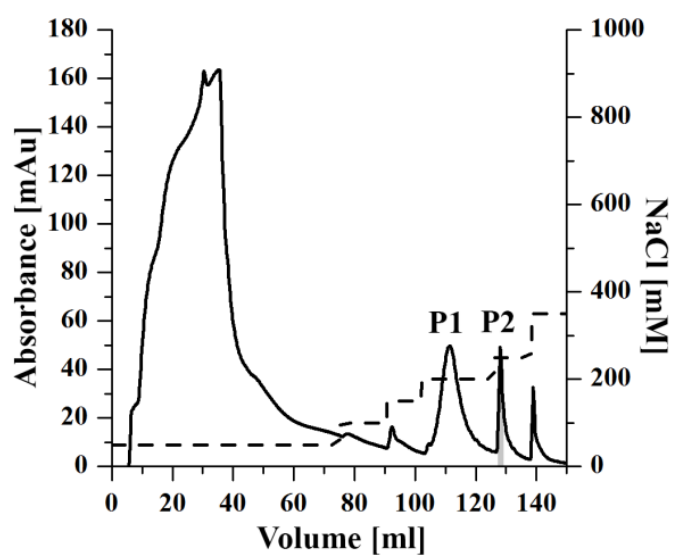

Figure 3.33: Purification of $\left(\boldsymbol{\alpha}^{\text {chi }}-\mathrm{S400C}\right)_{3}: \beta_{3}: \gamma$ and $\left(\boldsymbol{\alpha}^{\text {chi }}-\mathrm{V} 525 \mathrm{C}\right)_{3}: \boldsymbol{\beta}_{3}: \gamma$. (A, C) $\mathrm{Ni}^{2+}-\mathrm{NTA}$ purification profiles of $\left(\alpha^{c h i}-\mathrm{S} 400 \mathrm{C}\right)_{3}: \beta_{3}: \gamma$ and $\left(\alpha^{c h i}-\mathrm{V} 525 \mathrm{C}\right)_{3}: \beta_{3}: \gamma$, respectively, showing the bands of subunits $\alpha^{\text {chi }}$, $\beta$, and $\gamma$. Asterisk $(*)$ replaces the mutation name for brevity. Fractions from $125 \mathrm{mM}$ to $400 \mathrm{mM}$ imidazole of the respective complexes were pooled and applied onto an (B, D) anion exchange column (Resource Q, $6 \mathrm{ml}$ ) at a constant flow rate of $1 \mathrm{ml} / \mathrm{min}$ with a $\mathrm{NaCl}$ step gradient. Peak 1 (P1), eluting at $200 \mathrm{mM} \mathrm{NaCl}$, contains subunit $\beta$ (data not shown), while the grey area of peak 2 (P2) indicates the collected fractions containing the complex.

The mutant complexes displayed the ratio of subunits $\alpha^{c h i}: \beta: \gamma$ to be $3: 3: 1$, a typical stoichiometry found in ATP synthases, and confirming that S400C and V525C mutations do not affect the complex stability. As expected, the crosslinking product was not formed between $\alpha^{\text {chi }}-$ S400C and cysteine residue, C109 present in subunit $\gamma$ when incubated with $100 \mu \mathrm{M} \mathrm{CuCl}_{2}$. In comparison, the mutant $\left(\alpha^{\text {chi }}-\mathrm{V} 525 \mathrm{C}\right)_{3}: \beta_{3}: \gamma$ produced two distinct higher molecular weight bands upon $\mathrm{CuCl}_{2}$ incubation (Figure 3.34). To determine the constituent proteins, the 100- (I) and $120 \mathrm{kDa}$ (II) bands were cut out, incubated in buffer Tris/ $\mathrm{HCl}, \mathrm{pH} 7.5,250 \mathrm{mM} \mathrm{NaCl}, 0.8 \mathrm{mM}$ DTT and embedded in second SDS-gel in presence of $20 \mathrm{mM}$ DTT to ensure the reduction of 
disulfides. A $100 \mathrm{kDa}$ band (I) resolved to subunits $\alpha^{\text {chi }}$-V525C (59 kDa) and $\gamma(33$ $\mathrm{kDa}$ ), $120 \mathrm{kDa}$ band (II) resolved to subunit $\alpha^{\text {chi }}$-V525C (Figure 3.34-C).

(A)

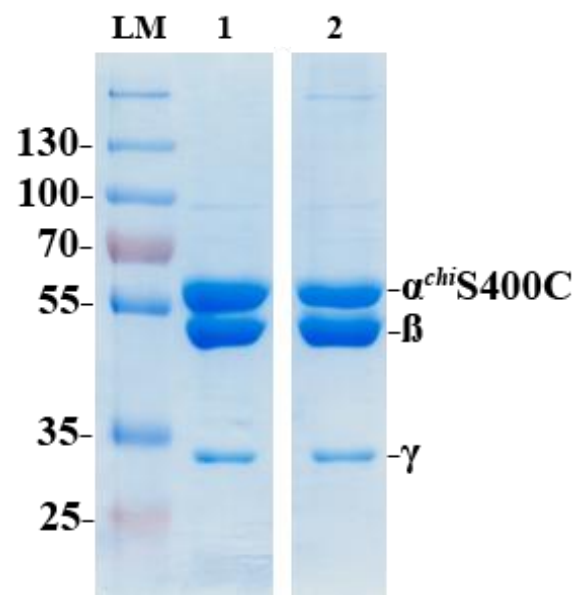

(B)

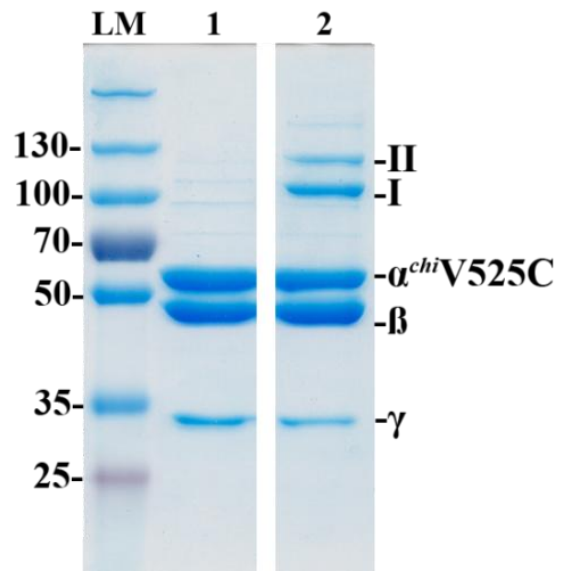

(C)

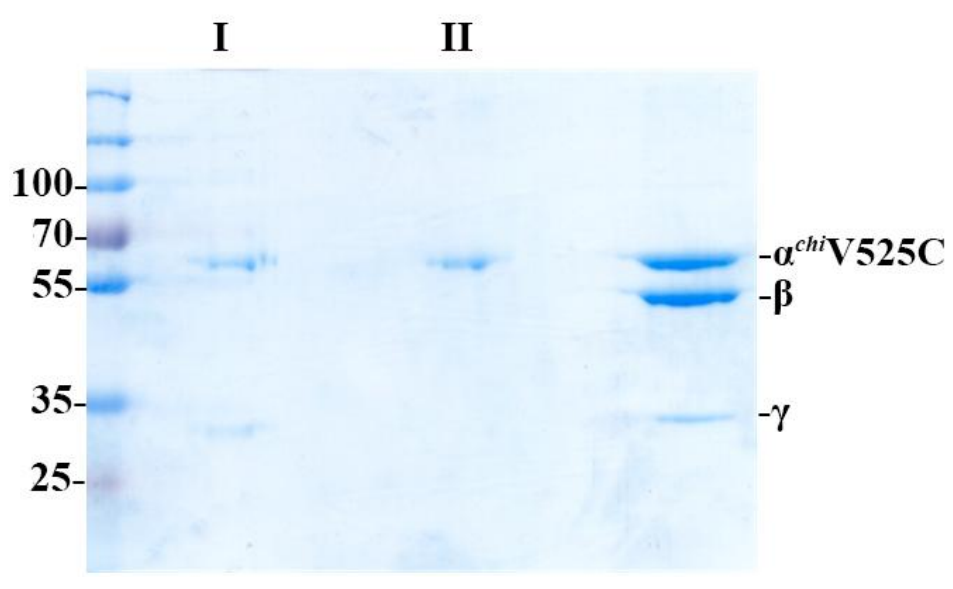

Figure 3.34: Purification of $\left(\boldsymbol{\alpha}^{c h i}-\mathrm{S400C}\right)_{3}: \boldsymbol{\beta}_{3}: \gamma$ and $\left(\boldsymbol{\alpha}^{c h i}-\mathrm{V} 525 \mathrm{C}\right)_{3}: \boldsymbol{\beta}_{3}: \gamma$. The protein complexes purified using a two-step protocol, as described above, were applied on a 9\% SDS-gel. (A) Shows that $\left(\alpha^{\text {chi }} \text {-S400C) }\right)_{3}: \beta_{3}: \gamma$ treated with $0.8 \mathrm{mM}$ DTT (lane 1) and $100 \mu \mathrm{M} \mathrm{CuCl}_{2}$ (lane 2) does not feature crosslink formation. (B) Complex $\left(\alpha^{\text {chi }}-\mathrm{V} 525 \mathrm{C}\right)_{3}: \beta_{3}: \gamma$ treated with $100 \mu \mathrm{M} \mathrm{CuCl}_{2}$ (lane 2) forms two higher molecular weight bands of estimated to be $\sim 100 \mathrm{kDa}(I)$ and $120 \mathrm{kDa}$ (II), which were not apparent in the DTT-treated sample. (C) A 2D 9\% SDS-PAGE showing the crosslinking products of $\left(\alpha^{\text {chi }}-\mathrm{V} 525 \mathrm{C}\right)$ and $\gamma \mathrm{S} 109 \mathrm{C}$ within the complex $\left(\alpha^{\text {chi }}-\mathrm{V} 525 \mathrm{C}\right)_{3}: \beta_{3}: \gamma$ resolved in the second dimension. The band labeled (I) in under (B), was cut out, incubated in $20 \mathrm{mM}$ DTT and embedded into the lane I which shows two bands corresponding to subunits $\alpha^{\text {chi }}$ and $\gamma$. The band labeled (II) in the gel under (B) was loaded in the lane II and resolved to subunit $\alpha^{\text {chi }}$. Righter-most lane contains the complex $\left(\alpha^{\text {chi }}-\mathrm{V} 525 \mathrm{C}\right)_{3}: \beta_{3}: \gamma$ to serve as a control. Figure adapted from $(3,209)$.

This revealed that the mycobacteria-specific epitope $\left(M t \alpha_{514-549}\right)$ could be found in the close proximity of $\gamma \mathrm{C} 109$ and supported the notion that this epitope interacts with the Rossmann fold of subunit $\gamma$, during rotation events. The purified protein was further used for the reconstitution with cysteine substitution mutant of subunit $M t \varepsilon \mathrm{D} 121 \mathrm{C}$ as described in the sections below. 


\subsubsection{Cloning, production, and purification of Mte mutants R113C, G118C, and D121C}

For the purpose of the crosslinking experiments, the mutants MtcR $113 \mathrm{C}$ and $M t \varepsilon G 118 \mathrm{C}$ were cloned by a conventional method. The $M t \varepsilon$ gene was amplified using the mutagenic primers and the products are shown as the $382 \mathrm{bp}$ single bands on $1.2 \%$ agarose gel (Figure 3.35-A). These products were purified, digested with the restriction enzymes and ligated with modified pET9d(+) vector. The ligation product was subsequently transformed into E. coli $\mathrm{DH} 5 \alpha$ cells for plasmid selection and amplification. The isolated plasmid was subjected to the control restriction digestion and it showed two distinct bands on $1 \%$ agarose gel (Figure 3.35-B). The upper bands $(4,377 \mathrm{bp})$ correspond to the vector backbone while the lower bands (366 bp) correspond to the insert (gene). The mutation was verified by sequencing and the plasmid DNA isolated from the confirmed clones was further transformed into the $E$. coli $\mathrm{B} 121$ cells for the protein production.

(A)

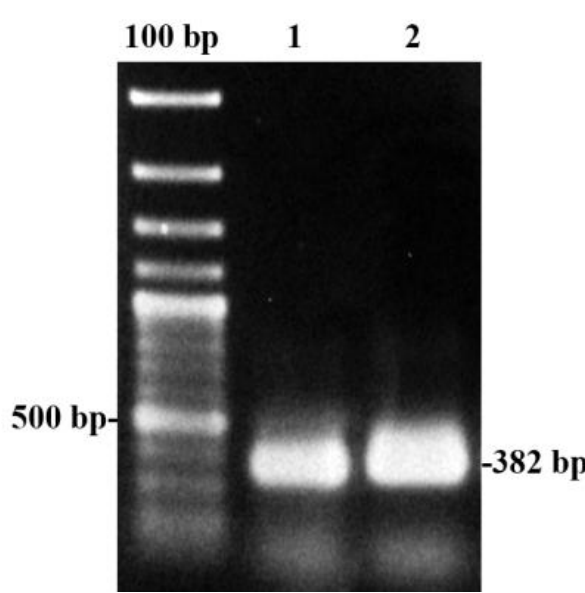

(B)

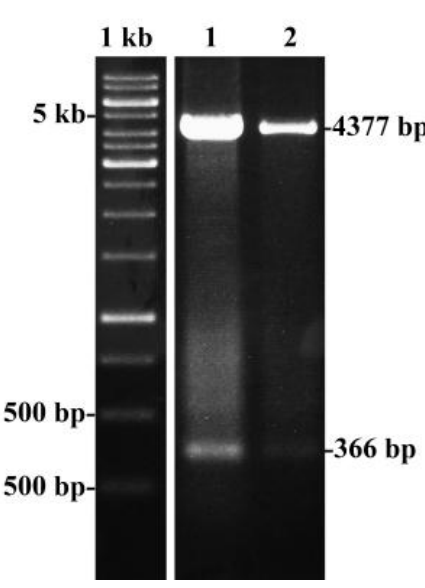

(C)

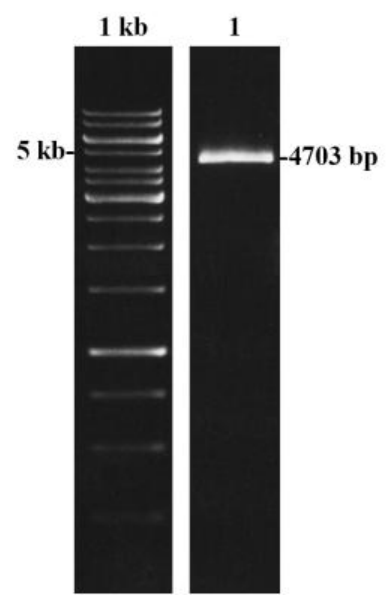

Figure 3.35: Cloning of the cysteine substitution mutants of Mtc. (A) PCR amplification of the $M t \varepsilon$ DNA fragment yields the DNA products corresponding to the Mt\&R113C mutant (lane 1) and $M t \varepsilon G 118 \mathrm{C}$ (lane 2), $100 \mathrm{bp}$ represents the DNA marker. (B) Control restriction digestion of the plasmids harboring extracted from E. coli DH5- $\alpha$ Mt\&R113 (lane 1) and -G118C (lane 2) the mutations. The band of 4,377 bp corresponds to the vector backbone, while $366 \mathrm{bp}$ correspond to the inserted sequence $(M t \varepsilon)$; (C) The amplification of the entire plasmid DNA containing the $M t \varepsilon$ sequence $(\mathrm{pET} 9 \mathrm{~d}(+): M t \varepsilon)$ by site-directed mutagenesis using the mutagenic primer pair (D121C) yielded the product shown in the lane $1(4,703 \mathrm{bp}) ; 1 \mathrm{~kb}$ represents the DNA marker. 
To produce $M t \varepsilon \mathrm{D} 121 \mathrm{C}$ for the crosslinking studies, the plasmid encoding the mycobacterial gene $a t p C$, designed and described earlier (section 2.2.7) was used as a template to introduce a substitution of terminal aspartate residue, D121 with redoxactive cysteine by using the site-directed mutagenesis as previously explained (section 2.2.7). The PCR amplification with mutagenic primers yielded the entire plasmid of 4,703 bp resolved as a single band on a 1\% agarose gel (Figure 3.35-C). The linearized PCR product was 5' phosphorylated during the amplification and subsequently was ligated (circularized). The isolated plasmid was transformed into the E.coli B121 for protein production.

The purifications of the three mutant forms were conducted in following the two-step protocol that includes $\mathrm{Ni}^{2+}$-NTA affinity step that separates the $\mathrm{His}_{6}-\mathrm{Mt \varepsilon}$ mutants from the bulk of cellular proteins, followed by the size exclusion column. Imidazole fractions containing the protein $(75-250 \mathrm{mM})$ were pooled together, concentrated (spin concentrator, molecular cut-off: $3 \mathrm{kDa}$, Millipore) and applied to a size exclusion column (Superdex 200 HR 10/30, GE Healthcare). The protein was eluted with a buffer containing $50 \mathrm{mM}$ Tris/ $\mathrm{HCl}, \mathrm{pH} 7.5$, and $200 \mathrm{mM} \mathrm{NaCl}, 10 \%$ glycerol, $80 \mathrm{mM}$ DTT (Figure 3.36), followed by the buffer exchange to remove the excess of DTT and allow disulfide formation. The purity was assessed on a 17\% SDSPAGE. To check for the spontaneous dimer formation via the disulfide bridge, the proteins were subjected to the buffer exchange followed by 15 mins incubation at $4{ }^{\circ} \mathrm{C}$ to allow for the re-oxidation of cysteines.

The $17 \%$ SDS-PAGE underlines that purification yields highly homogenous MtcD121C in its reduced monomeric form, while the cysteines re-oxidize to form a small population of dimers after the non-reducing conditions were introduced upon buffer exchange (Figure 3.4-A). Oppositely to this observation, the mutants MteR113C and $M t \varepsilon G 118 \mathrm{C}$ do not form disulfide-mediated dimers spontaneously (Figure 3.4-B) under the same conditions, possibly due to the lowered/restricted accessibility of the residues $\mathrm{C} 113$ and $\mathrm{C} 118$. 
(A)

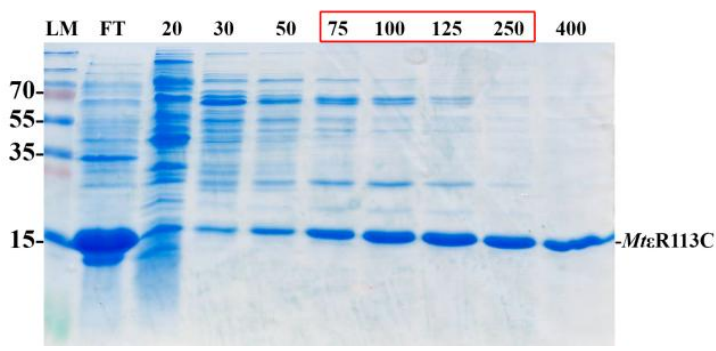

(C)

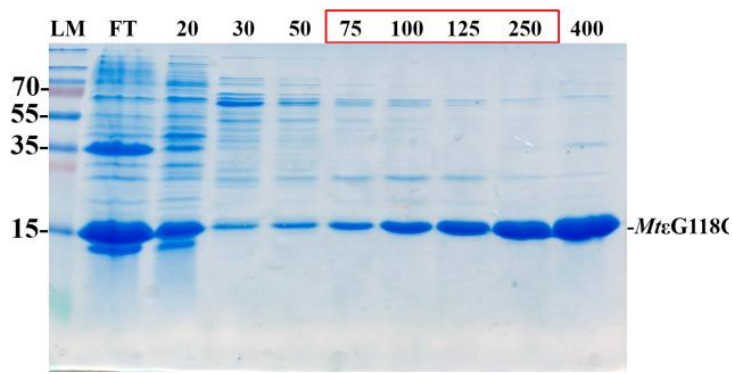

(E)

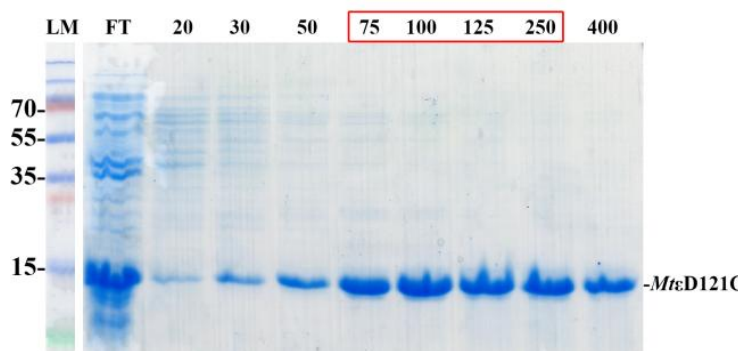

(B)

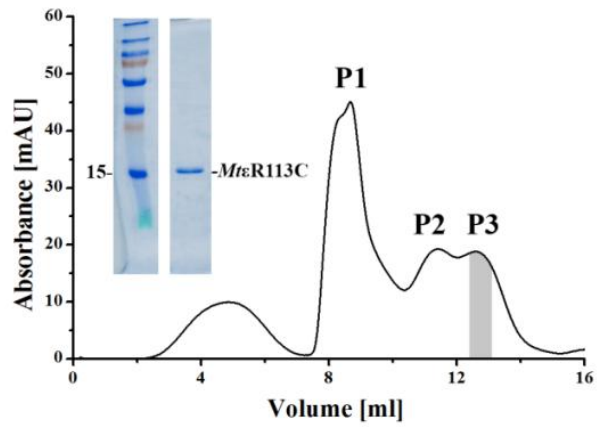

(D)

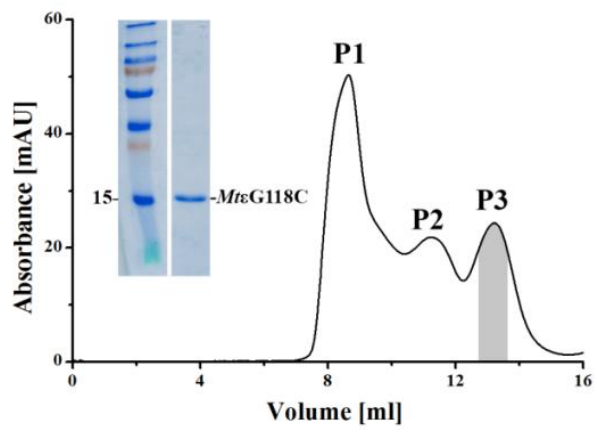

(F)

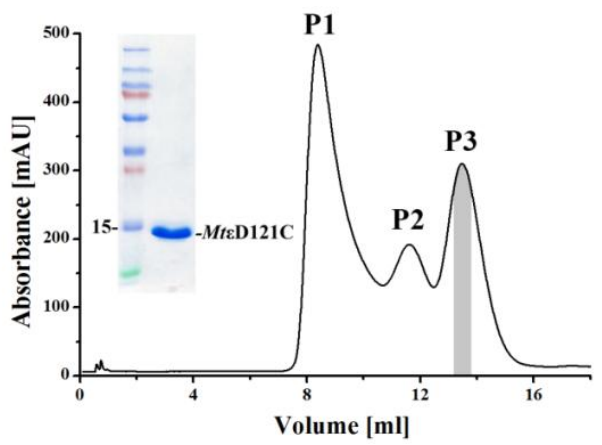

Figure 3.36: Purification of $M t \varepsilon$ mutants R113C, G118C, and D121C by gel filtration. Individually expressed protein obtained after $\mathrm{Ni}^{2+} \mathrm{NTA}$ chromatography (17\% SDS-gel; 75-250 mM imidazole fractions in red) (A) was applied onto a gel filtration column (Superdex 75) and eluted at around $13.5 \mathrm{ml}$ volume, as seen in the chromatograms (B, D, and E). Peaks P1 (eluted at $\sim 8 \mathrm{ml}$ ) and P2 (eluted at $\sim 11 \mathrm{ml}$ ) represent the oligomeric fractions of proteins, the greyed-out area under P3 represents the monomeric fraction shown on a 17\% SDS-gel, inset. 
(A)

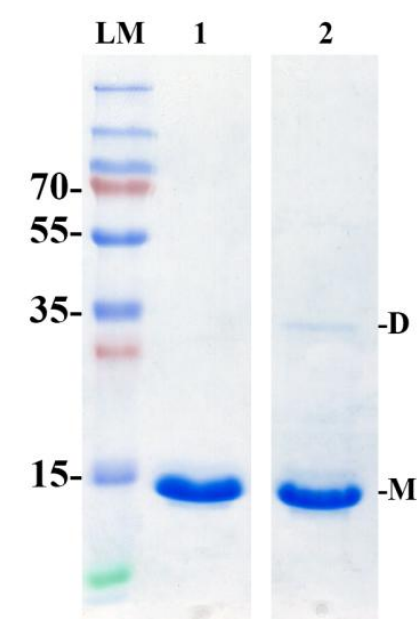

(B)

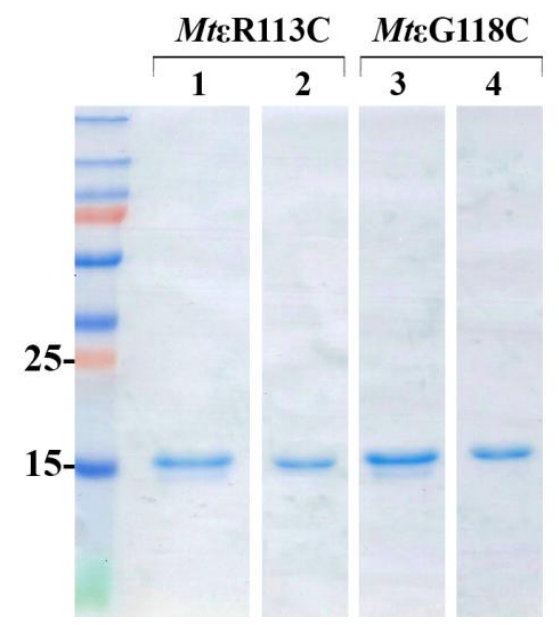

Figure 3.37: Redox-mediated formation of intramolecular disulfide bridges in Mt\& mutants. The freshly purified proteins were subjected to the 3 rounds of buffer exchange to remove the reducing agent, DTT used in the purification. (A) The mutant $M t \varepsilon D 121 \mathrm{C}$ displays the capacity to form disulfides in the absence of DTT (lane 2) while only a monomeric band was observed in reducing conditions (lane 1). $M$-monomer, $D$-dimer. (B) The Mutants $M t \varepsilon R 113 \mathrm{C}$ and $M t \varepsilon G 118 \mathrm{C}$ do not show spontaneous dimer formation in non-reducing conditions obtained after the buffer exchange.

\subsubsection{Reconstitution of $\left(\alpha^{c h i}-\mathrm{S} 400 \mathrm{C}\right)_{3}: \beta_{3}: \gamma$ and $M t \varepsilon D 121 C$}

For reconstitution of the $M t \varepsilon D 121 \mathrm{C}$ with the $\left(\alpha^{\text {chi }}-\mathrm{S} 400 \mathrm{C}\right)_{3}: \beta_{3}: \gamma$ complex, freshly purified $\left(\alpha^{\text {chi }}-\mathrm{S} 400 \mathrm{C}\right)_{3}: \beta_{3}: \gamma$ was incubated with a 3 -fold molar excess of subunit $M t \varepsilon \mathrm{D} 121 \mathrm{C}$ at $4{ }^{\circ} \mathrm{C}$ during $1 \mathrm{~h}$ in $50 \mathrm{mM}$ Tris/ $\mathrm{HCl}, \mathrm{pH} 7.5,250 \mathrm{mM} \mathrm{NaCl}$. The reconstituted mixture was aliquoted, and the protein was treated either with $0.8 \mathrm{mM}$ DTT to reduce disulfides or incubated for 15 minutes with $100 \mu \mathrm{M} \mathrm{CuCl}_{2}$ to promote crosslinkformation. The samples were applied on the 12\% SDS-PAGE, as shown in Figure 3.38-A. Apart from the bands of the individual subunits $\alpha^{\text {chi }}-\mathrm{S} 400 \mathrm{C}, \beta, \gamma$ and MtED121C, major and two minor cross-linked products were observed (lane 2) which were not present in the DTT-treated sample (lane 1). In order to reveal the constituent proteins, the bands of the crosslinking product were cut out, incubated it in the buffer containing $20 \mathrm{mM}$ DTT, embedded in a $12 \%$ SDS-PAGE to reduce the potential disulfides of the product and reveal its constituent proteins. As shown in the Fig 3.46B, the major crosslinking product

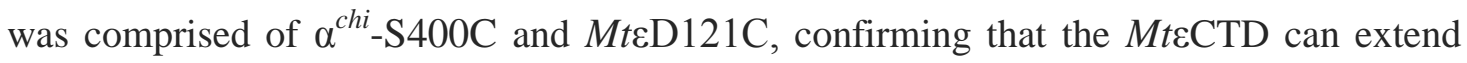
towards and interact with ${ }_{396} \mathrm{AQFGSDLDK} 404$ region. 
(A)

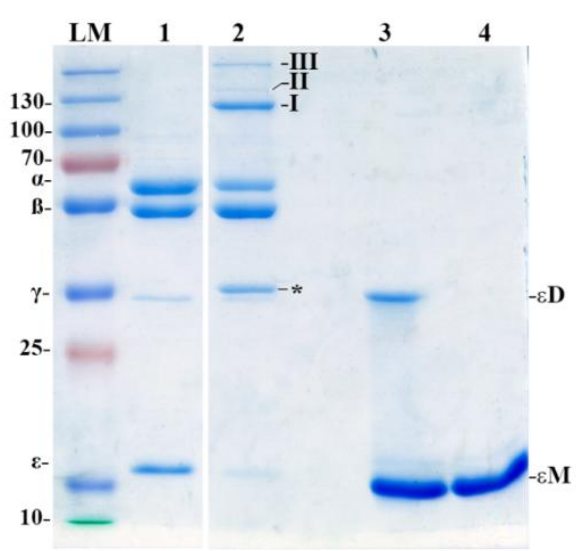

(B)

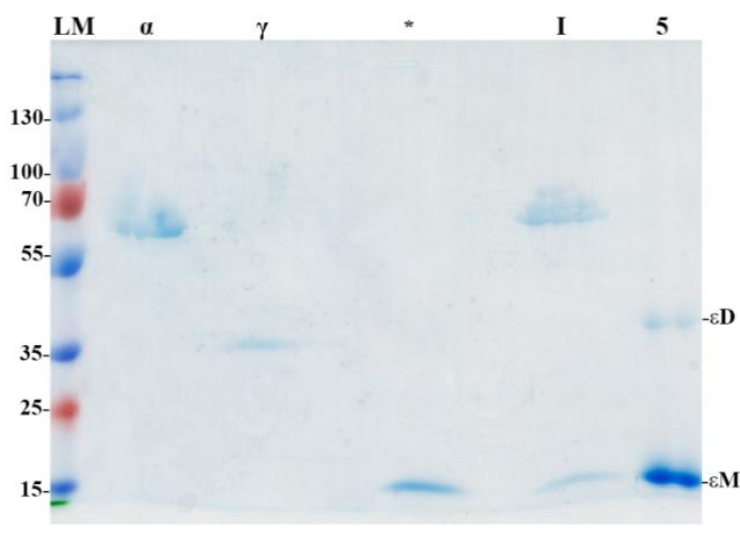

Figure 3.38: Crosslinking of the $\left(\alpha^{c h i}-\mathrm{S400C}\right)_{3}: \boldsymbol{\beta}_{3}: \gamma$ and $M t \varepsilon D 121 C$. The reconstituted complex was treated with $0.8 \mathrm{mM}$ DTT to obtain the resolved bands of individual subunits (lane 1 ) as indicated on the left side of the gel. Treatment of the complex (lane 2) with $100 \mu \mathrm{M} \mathrm{CuCl}_{2}$ promoted disulfide bridge formation resulting in products marked with roman numbers I-III and the asterisk (*). Lanes 3 and 4 reveal $M t \varepsilon D 121 \mathrm{C}$ without and with $20 \mathrm{mM}$ DTT, respectively. $\varepsilon M$ indicates the monomeric form of $M t \varepsilon D 121 \mathrm{C}$ and $\varepsilon D$ the dimeric one in the absence of DTT (lane 3). All lanes displayed are a part of a single gel. $L M$, represents molecular weight standard proteins. (B) The bands of the product I, * and constituent proteins $\alpha^{\text {chi }}-\mathrm{S} 400 \mathrm{C}$, and $\gamma$ of the gel displayed under (A) were cut out, treated with $20 \mathrm{mM}$ DTT and embedded in the second 12\% SDS-gel. Constituent proteins are mentioned above the gel. $L M$, represents molecular weight standard proteins. The lane of $*$ reveals that the band of $*$ in the left gel is formed by an $M t \varepsilon D 121 \mathrm{C}$ dimer, which gets resolved after DTTtreatment as indicated by the single band marked as $\varepsilon M$. Lane 5, $8 \mu \mathrm{g}$ of $M t \varepsilon \mathrm{D} 121 \mathrm{C}$ in the absence of DTT revealing the monomeric form $(\varepsilon M)$ of $M t \varepsilon D 121 \mathrm{C}$ and the dimeric one $(\varepsilon D)$ again running slightly above subunit $\gamma$. Figure adapted from (209).

\subsubsection{Reconstitution of $\left(\alpha^{c h i}-\mathrm{V} 525 \mathrm{C}\right)_{3}: \beta_{3}: \gamma$ and $M t \varepsilon$ mutants D121C, R113C and G118C}

Observations that the MtcCTD tip residue D121 could reach the $\alpha^{\text {chi }}$ coupling epitope ${ }_{396} \mathrm{AQFGSDLDK}_{404}$ in what is proposed to be the extended conformation prompted to investigate whether the Mt\&CTD could reach the mycobacteria-specific $M t \alpha_{514-549}$ epitope. To that end the $\left(\alpha^{\text {chi }}-\mathrm{V} 525 \mathrm{C}\right)_{3}: \beta_{3}: \gamma$ and the MtE mutants D121C, $\mathrm{R} 113 \mathrm{C}$ and promoted formation of potential disulfide bridges using $\mathrm{CuCl}_{2}$ as an oxidative agent.

The reconstitution of $\left(\alpha^{\text {chi }}-\mathrm{V} 525 \mathrm{C}\right)_{3}: \beta_{3}: \gamma$ with the single $M t \varepsilon$ mutants was performed as previously described in section 3.1.3. Complex and MtE mutants were allowed to bind in buffer Tris/ $\mathrm{HCl}, \mathrm{pH} 7.5,250 \mathrm{mM} \mathrm{NaCl}$ at $4^{\circ} \mathrm{C}$ in a molar ratio $1: 3$, respectively. The incubation proceeded during $1 \mathrm{~h}$, followed by 15 min incubation with $\mathrm{CuCl}_{2}$.

The reconstituted $\left(\alpha^{\text {chi }}-\mathrm{V} 525 \mathrm{C}\right)_{3}: \beta_{3}: \gamma: M t \varepsilon D 121 \mathrm{C},\left(\alpha^{\text {chi }}-\mathrm{V} 525 \mathrm{C}\right)_{3}: \beta_{3}: \gamma: M t \varepsilon \mathrm{R} 113 \mathrm{C}$ and $\left(\alpha^{\text {chi }}-\mathrm{V} 525 \mathrm{C}\right)_{3}: \beta_{3}: \gamma: M t \varepsilon G 118 \mathrm{C}$ complexes displayed three higher molecular weight 
bands around $90 \mathrm{kDa}, 100 \mathrm{kDa}$ and $120 \mathrm{kDa}$, labeled III, I and II, respectively. The 100 - and $120 \mathrm{kDa}$ bands display the same intensity in all three complexes, while the band III with a molecular weight around $90 \mathrm{kDa}$ is more prominent in the $\left(\alpha^{\text {chi }}\right.$ V525C) $)_{3}: \beta_{3}: \gamma: M t \varepsilon D 121 \mathrm{C}$ complex (Figure 3.39). The bands of 100- and $120 \mathrm{kDa}$ were cut out and described earlier (Figure 3.34-C). The $90 \mathrm{kDa}$ band (III) was cut out, incubated in the $50 \mathrm{mM}$ Tris/ $\mathrm{HCl}, \mathrm{pH} 7.5,250 \mathrm{mM} \mathrm{NaCl}, 0.8 \mathrm{mM}$ DTT and embedded into the second dimension of a 12\% SDS gel in the presence of $20 \mathrm{mM}$ DTT, to reduce the disulfide bonds. The band III resolved to subunits $\alpha^{c h i}-\mathrm{V} 525 \mathrm{C}$ and MtcD121C (Figure 3.40). Similarly to this observation, MtcC113 and -C118 residues could also interact with the C525 of the mycobacterial epitope in subunit $\alpha^{c h i}$. This points out that the proximity of the MteCTD residues R113, G118, and D121 to $\alpha^{\text {chi }}$-V525 allows the interaction to occur and that these two epitopes play a role in the regulation of the catalytic activity of mycobacterial $\mathrm{F}_{1} \mathrm{~F}_{\mathrm{O}}$-ATP synthase.

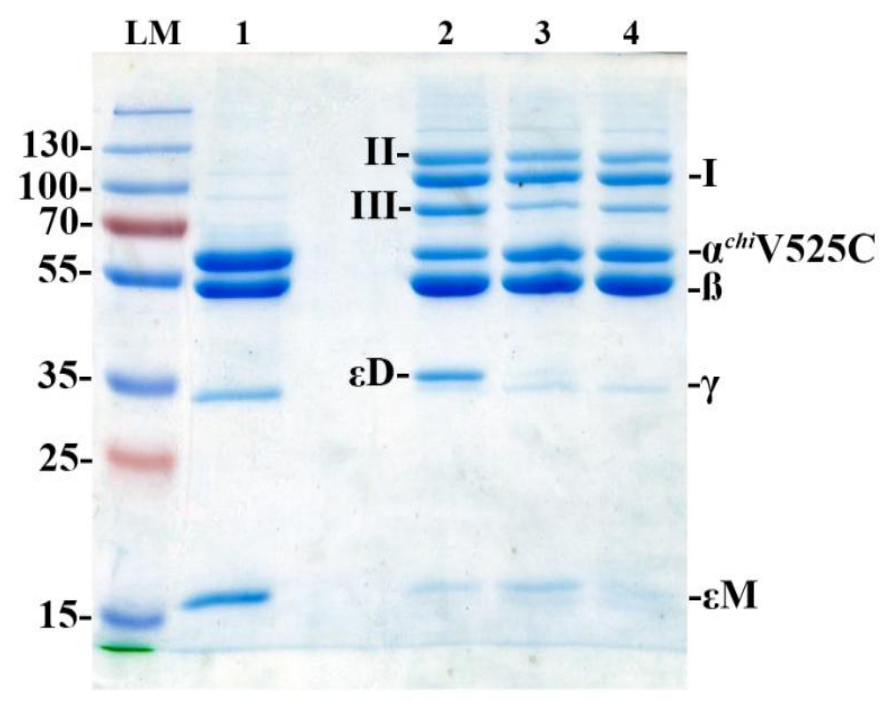

Figure 3.39: Crosslinking of the $\left(\alpha^{c h i}-\mathrm{V} 525 \mathrm{C}\right)_{3}: \beta_{3}: \gamma$ with MtE mutants D121C, G118C and R113C. The reconstituted complex $\left(\alpha^{\text {chi }}-\mathrm{V} 525 \mathrm{C}\right)_{3}: \beta_{3}: \gamma:$ MtcD121C treated with $0.8 \mathrm{mM}$ DTT (lane 1) and $100 \mu \mathrm{M} \mathrm{CuCl}$ (lane 2). Lanes 3 and 4 reveal the reconstituted complexes $\left(\alpha^{\text {chi }}\right.$ V525C $)_{3}: \beta_{3}: \gamma: M t \varepsilon G 118 \mathrm{C}$ and $\left(\alpha^{\text {chi }}-\mathrm{V} 525 \mathrm{C}\right)_{3}: \beta_{3}: \gamma: M t \varepsilon R 113 \mathrm{C}$, respectively, treated with $100 \mu \mathrm{M}$ $\mathrm{CuCl}_{2}$. $\mathrm{CuCl}_{2}$ treatment promoted the crosslink formation in lanes 2-4, resulting in products marked with roman numbers I-III. $L M$ represents molecular weight standard proteins, $\varepsilon M$ indicates the monomeric form of $M t \varepsilon$ mutants and $\varepsilon D$ the dimeric one seen only in the mutant MtcD121C (lane 2). 


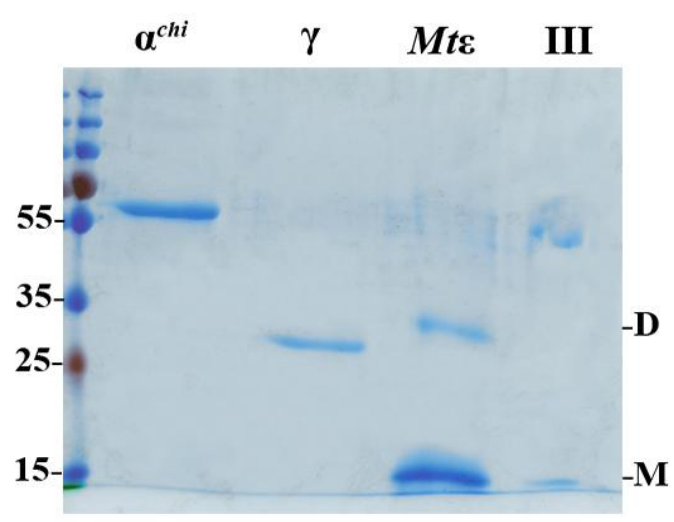

Figure 3.40: Determination of the constituent proteins of the crosslinking products. The band labeled (III) in Fig. 3.7 was cut out, incubated in $20 \mathrm{mM}$ DTT and embedded into the lane III of the $12 \%$ SDS-gel which shows two bands corresponding to subunits $\alpha^{\text {chi }}$ and Mte. Similarly, bands $\alpha^{c h i}$ and $\gamma$ were cut out from the gel in Fig. 3.7 and embedded in the respective lanes to serve as a control. Non-reduced $M t \varepsilon D 121 \mathrm{C}$ protein loaded in the respective lane $(M t \varepsilon)$. $M$-monomer, $D$-dimer.

\subsubsection{Cloning, production, and purification of the recombinant MtEE100C mutant for SmFRET}

Data suggests that the mycobacterial subunit $\varepsilon$ stimulates the ATP hydrolysis in the reconstituted $\alpha^{c h i}: \beta_{3}: \gamma: M t \varepsilon$ complex by a factor of 1.8 when compared to the $\alpha^{c h i}: \beta_{3}: \gamma$ complex. Based on structural and functional information available for the subunit $\varepsilon$ on the F-ATP synthases of E. coli $(143,194)$, B. PS3 (148) and B. subtilis (179) it has been previously reported that the regulation of the catalytic activity is mediated by the subunit $E$ CTD that undergoes the transition between two conformational states; compact whereby it sits in proximity of its NTD and extended state that can reach the catalytic interfaces of the subunits $\alpha$ and $\beta$. In the crosslinking experiments performed on the cysteine mutants of $\alpha^{c h i}{ }_{3}: \beta_{3}: \gamma: M t \varepsilon$ complex, it was shown that the MtECTD was capable of interacting with epitopes in subunit $\alpha^{c h i}$ which, together with kinetic studies, confirmed Mte involvement in the regulation of the catalytic events.

To study the conformational dynamics of Mte within the $\alpha^{\text {chi }}: \beta_{3}: \gamma: M t \varepsilon$ complex, namely the MtcCTD transition between two states, compact and extended, the single-molecule Förster resonance energy transfer (smFRET) was utilized (305). The FRET technique employs a confocal microscope setup to register the changes in locally-emitted fluorescence originating from the interaction of two specific fluorescent donor and acceptor dyes. To achieve FRET, it is a requirement that the emission spectrum of the donor overlaps with the absorption spectrum of the acceptor. The photochemical reaction is initiated when the system is excited with 
monochromatic laser light of a wavelength that matches the absorbance maximum of the donor dye. The photon excites the donor to a higher energy state. Upon this event, the spontaneous vibrational (non-radiative) process causes relaxation of the electrons to the lowest energy level of the excited state. Subsequently, the electrons can undergo further relaxation and return to their ground state, causing the emission of a photon in the process called fluorescence.

In another radiationless process, the energy of the donor electron is transferred to the acceptor dye via dipole-dipole coupling without the release of a photon. This phenomenon causes quenching of the donor fluorescence, as well as the subsequent emission of a photon from the acceptor. For FRET, certain conditions must be met. First, the dipoles of the donor and acceptor fluorophores must be oriented parallel towards each other. Second, the absorption coefficients and the quantum yields of the donor and the acceptor should be within a similar range. Otherwise, this has to be corrected when calculating the FRET efficiency.

In our setup, there are several undefined parameters such as the refractive index of the sample, the orientation of the dipoles, and the instrumental cross-talk, all preventing a quantitative analysis of the distances between fluorophores. Instead of this approach, a qualitative method that does not take all these parameters into account was employed. Namely, this method allowed the detection of the relative changes in FRET levels by calculating the proximity factor $\mathrm{p}$, which is defined as:

$$
\mathrm{p}=\mathrm{I}_{\mathrm{A}} /\left(\mathrm{I}_{\mathrm{A}}+\mathrm{I}_{\mathrm{D}}\right)
$$

where $I_{A}$ and $I_{D}$ are the intensities of the acceptor and donor fluorophores, as detected by the instruments.

Another aspect to be considered is the choice of the covalent modification of the fluorophores that serve as a link to the protein hotspots under investigation. Several modifications are available, such as carboxyl ester, streptavidin, biotin, phalloidin, amines, azides, iodoacetamide, hydrazine, and maleimide. To incorporate the selected dye pair into the protein epitopes whose interactions are under investigation, the dye was conjugated to engineered cysteine residues via cysteinereactive maleimides.

It was shown earlier that the subunit F of archaeal A-ATP synthase, (homolog of subunit $\varepsilon$ of F-ATP synthase) undergoes conformational alterations measurable by smFRET (306). The donor dye was conjugated to the subunit D (homolog to $\gamma$ ) while 
the acceptor dye was introduced to the various positions in subunit $\mathrm{F}$, revealing the conformational dynamics on a time nanoscale.

In order to measure the conformational dynamics of the Mtc within the $\alpha^{\text {chi }}{ }_{3} \beta_{3}: \gamma: M t \varepsilon$ complex, the $M t \varepsilon$ was modeled in the extended state and the suitable residues for smFRET were identified. As seen from the model (Figure 3.41), the cysteine residue $\gamma \mathrm{C} 109$ and MtEE100 fall within the distance of 45-52 $\AA$ and the solvent exposure/dye accessibility requirements were fulfilled. The mutant $M t \varepsilon E 100 \mathrm{C}$ was generated, purified, labeled with an acceptor dye and reconstituted with the donor dye-labeled $\alpha^{c h i}: \beta_{3}: \gamma$.
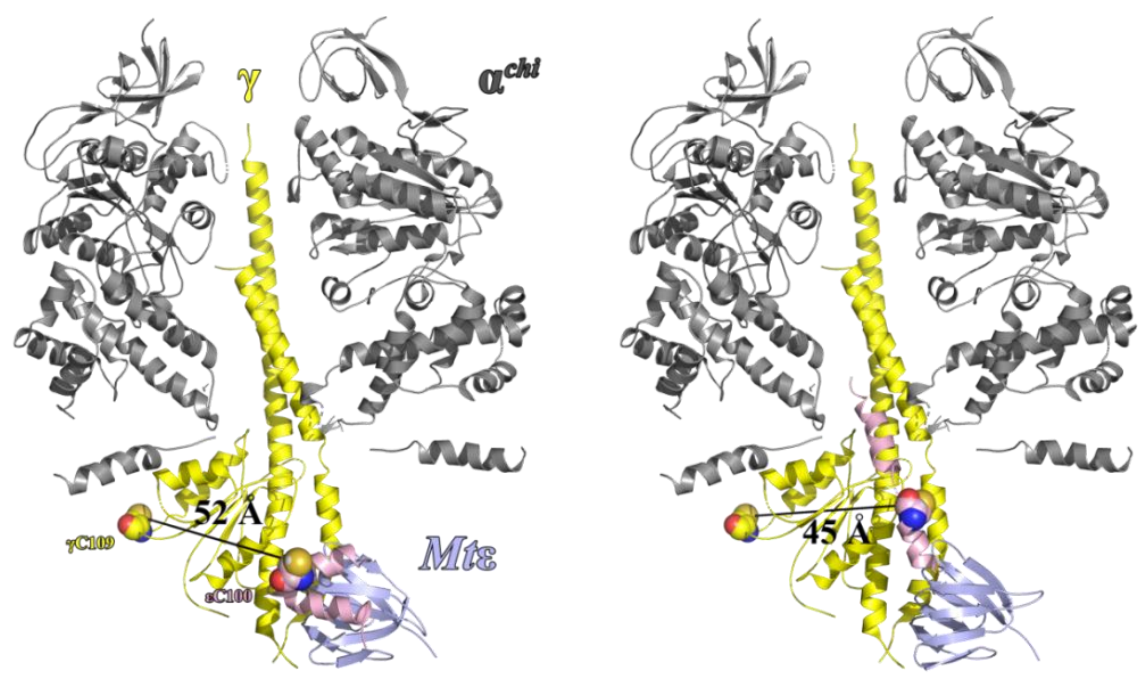

Figure 3.41. Model of the $\alpha^{\text {chi }}{ }_{3}: \beta_{3}: \gamma: M t \varepsilon E 100 C$ complex used in SmFRET. The cartoon representation highlights the subunits $\alpha^{c h i}, \gamma$, and MtcE100C in grey, yellow and shades of magenta respectively. The cysteines that allow the disulfide bridge formation with maleimide moiety of the ATTO dyes were introduced by site-directed mutagenesis at positions $\gamma \mathrm{S} 109 \mathrm{C}$ and $M t \varepsilon \mathrm{E} 100 \mathrm{C}$ are labeled and shown as spheres. The left figure shows the structure of the compact form of $M t \varepsilon$ displaying the distance between the reactive cysteines of $52 \AA$. The right figure shows the modeled extended conformation of $M t \varepsilon$ whereby the distance between reactive cysteines is reduced to $45 \AA$. Light pink and light violet represent the Mt\&CTD and -NTD, respectively. 
The mutant protein $M t \varepsilon E 100 \mathrm{C}$ was generated using the site-directed mutagenesis (Figure 3.42) described in section 2.2.9 and purified according to the established protocol described in section 2.2.10 and 2.2.11. The cells were resuspended and lysed in buffer containing $50 \mathrm{mM}$ Tris/ $\mathrm{HCl}, 200 \mathrm{mM} \mathrm{NaCl}$, $10 \%$ glycerol, $80 \mathrm{mM}$ DTT, $\mathrm{pH} 7.5$ and incubated with the $\mathrm{Ni}^{2+}$-NTA beads in the duration of $1.5 \mathrm{~h}$. Subsequently, the protein was eluted using the imidazole gradient $(0$ $400 \mathrm{mM}$ ) and the fractions $75-125 \mathrm{mM}$ imidazole were pooled and applied onto the size exclusion column (Superdex S75). The protein fractions were collected at around

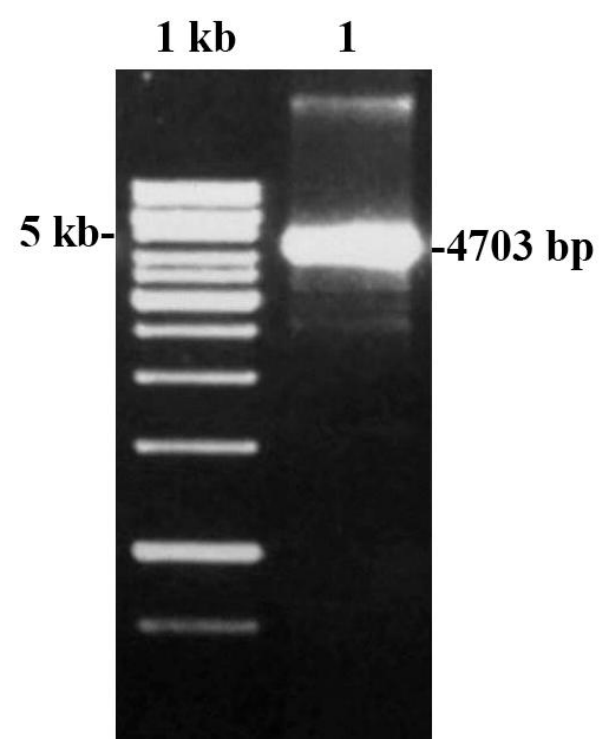

Figure 3.42: Cloning of the MtEE100C. Amplification by site-directed mutagenesis of the entire plasmid DNA containing the $M t \varepsilon$ sequence $(\mathrm{pET} 9 \mathrm{~d}(+): M t \varepsilon)$ yielded the product shown in the lane $1 ; 1 \mathrm{~kb}$ represents the DNA marker

$14.5 \mathrm{ml}$, concentrated using the centricon with $3 \mathrm{k}$ cut-off filter and applied on $17 \%$ SDS gel to check the purity, dimerization, and homogeneity of the protein (Figure 3.43).

To carry out a successful smFRET experiment, it was necessary to confirm that the mutation E100C in subunit Mte does not significantly alter the catalytic activity of the $\alpha^{c h i}{ }_{3}: \beta_{3}: \gamma: M t \varepsilon E 100 \mathrm{C}$ complex by forming a crosslink with the cysteine residue in subunit $\gamma$. Thus, the reconstitution is performed as previously described (section 2.2.16) and the crosslink formation, as well as ATPase activity, were tested. 
(A)

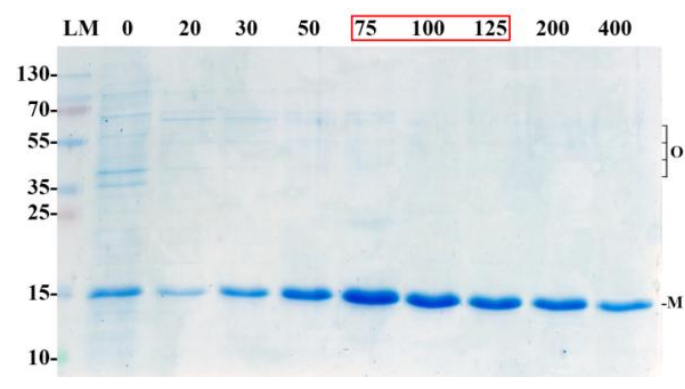

(B)

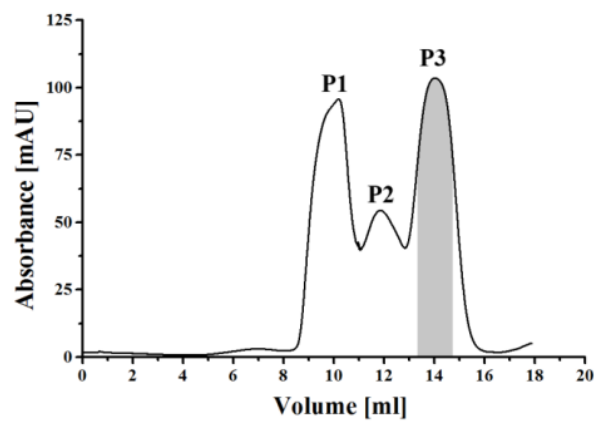

(C)
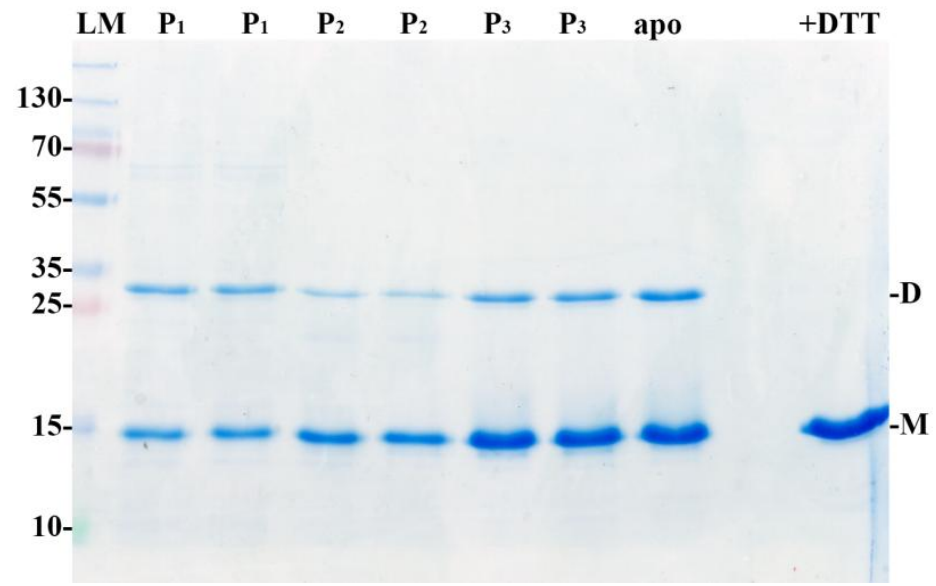

Figure 3.43: Purification of Mt\&E100C by gel filtration. Protein obtained after $\mathrm{Ni}^{2+-} \mathrm{NTA}$ chromatography (A) was applied onto a gel filtration column (Superdex 75) and eluted at $14.5 \mathrm{ml}$ volume, as seen in the chromatogram (B). P1 peak contains the protein in an unspecific oligomeric state, P2, contains the protein in a dimeric state, $\mathrm{P} 3$ represents the monomeric peak. The purity was confirmed by running a 17\% SDS-PAGE (C). P1, P2, and P3 represent the two main fractions of each peak; Purified protein loaded without DTT is labeled "apo" while the label "+DTT" indicates the presence of the reducing agent; $M$ - monomer, $D$ - dimer.

The purified MtcE100C was mixed in 3-fold molar ratio with the $\alpha^{\text {chi }}{ }_{3}: \beta_{3}: \gamma$ complex and incubated in the duration of $1 \mathrm{~h}$ at $4{ }^{\circ} \mathrm{C}$. The reconstitution mixture was subsequently applied onto the size-exclusion column (Superdex S75). The fractions containing the reconstituted $\alpha^{c h i}{ }_{3}: \beta_{3}: \gamma: M t \varepsilon E 100 C$ complex, that eluted at the approximately $11.5 \mathrm{ml}$, were concentrated using $100 \mathrm{~K}$ centricon and the binding of the MtcE100C to the complex was observed (Figure 3.44, inset). Additionally, the ATPase activity of the complex $\alpha^{c h i}{ }_{3}: \beta_{3}: \gamma: M t \varepsilon E 100 \mathrm{C}(8.1 \pm 0.03 \mu \mathrm{g} / \mathrm{ml} / \mathrm{mg}$ of protein $)$ was not significantly altered in respect to the activity measured for the $\alpha^{\text {chi }}{ }_{3}: \beta_{3}: \gamma: M t \varepsilon$ complex $(8.4 \pm 0.05 \mu \mathrm{g} / \mathrm{ml} / \mathrm{mg}$ of protein), indicating that the mutation does not affect the regulatory epitope in $M t \varepsilon$. 
According to the model (Figure 3.41), the two cysteines in the $\alpha^{c h i}{ }_{3}: \beta_{3}: \gamma: M t \varepsilon E 100 C$ complex were found within the radius of 45-52 $\AA$ from each other. Even though the distance for the crosslink formation was unfavorable, the complex was tested for the possible interaction of the $\gamma \mathrm{C} 109$ and $M t \varepsilon \mathrm{C} 100$ which would render the cysteines inaccessible for the FRET dyes. Both, untreated and DTTtreated samples were loaded on the $12 \%$ SDS-gel in which showed that the crosslink formation does not occur in either of the conditions (Figure 3.44-C).

(A)

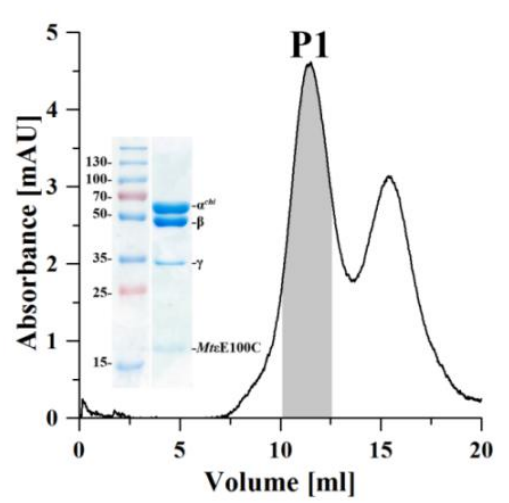

(B)

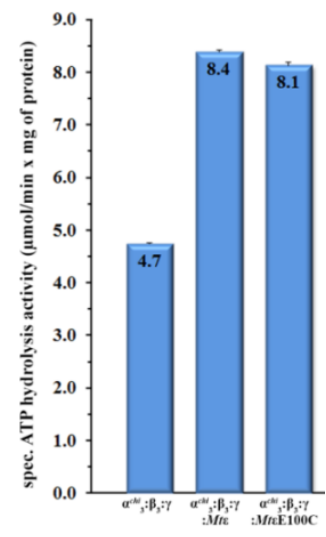

(C)

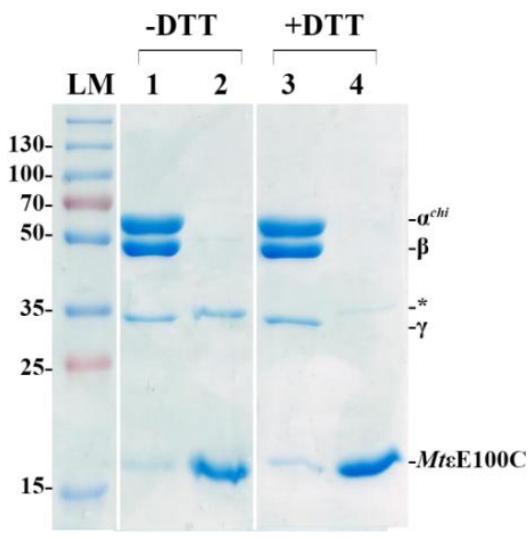

Figure 3.44: Reconstitution of $\alpha^{c h i}{ }_{3}: \beta_{3}: \gamma: M t \varepsilon E 100 C$, ATPase activity and crosslink formation. (A) Purified $\alpha^{c h i}: \beta_{3}: \gamma$ and MtEE100C were reconstituted and applied onto a Superdex S300 column (A) and the peak containing reconstituted $\alpha_{3}^{\text {chi }}: \beta_{3}: \gamma: M t \varepsilon E 100 \mathrm{C}$ was loaded on a $12 \%$ SDS-gel (insert). (B) Bar diagram showing the specific activities of $\alpha^{c h i}{ }_{3}: \beta_{3}: \gamma, \alpha^{c h i}{ }_{3}: \beta_{3}: \gamma: M t \varepsilon$ and $\alpha^{\text {chi }}{ }_{3}: \beta_{3}: \gamma: M t \varepsilon E 100 \mathrm{C}$ indicating the increase in activity of $\alpha^{c h i}: \beta_{3}: \gamma$ after addition of subunit MtE as well as the effect of mutation E100C on the complex activity. (C) $12 \%$ SDS gel showing the $\alpha^{\text {chi }}{ }_{3}: \beta_{3}: \gamma: M t \varepsilon E 100 \mathrm{C}$ (untreated, lane 1 and DTT-treated, lane 3) forming no higher molecular weight bands. Lanes 2 and 3 showing the $M t \varepsilon E 100 \mathrm{C}$, untreated and DTT-treated, respectively.

Taken the facts that the catalytic activity is not altered and that the residues $\gamma \mathrm{C} 109$ and $M t \varepsilon C 100$ remain accessible for the fluorescent dyes, the $\alpha^{\text {chi }}{ }_{3}: \beta_{3}: \gamma: M t \varepsilon E 100 \mathrm{C}$ complex was selectively labeled, as described in details in the following section, in order to study the conformational transition of Mte by smFRET. 


\subsubsection{Fluorescent labeling of the $\alpha^{c h i}{ }_{3}: \beta_{3}: y: M t \varepsilon E 100 C$ complex for the SmFRET experiment}

As mentioned earlier, in smFRET experiments it is possible to infer the distance and the dynamics of the movement between a donor and an acceptor fluorescent dye attached to the moving domains within a protein complex. The donor, ATTO-488 chromophore absorbs the light in $501 \mathrm{~nm}$ range, while the emission occurs at $523 \mathrm{~nm}$. The acceptor dye, ATTO-647N absorbs at the $644 \mathrm{~nm}$, emitting at $669 \mathrm{~nm}$. The non-overlapping spectra allow the selective excitation of the dyes in the two-dye system and thus this pair was used to selectively label the protein complex $\alpha^{\text {chi }}{ }_{3} \beta_{3}: \gamma: M t \varepsilon E 100 \mathrm{C}$. The cysteine residue $\gamma \mathrm{C} 109$ was modified with a donor dye ATTO-488-maleimide, while acceptor ATTO-647N-maleimide was conjugated to the $M t \varepsilon \mathrm{C} 100$.

In more detail, purified $\alpha^{c h i}: \beta_{3}: \gamma$ complex was incubated with an equimolar amount of the donor dye ATTO-488-maleimide in the duration of 1 min on ice and the reaction was stopped with the addition of the DTT to the mixture to the final concentration of $1 \mathrm{mM}$. The excess of the dye was removed by washing the sample with 3-fold buffer (50 mM Tris/HCl, $200 \mathrm{mM} \mathrm{NaCl,} 1 \mathrm{mM}$ DTT, pH 7.5) volumes in $100 \mathrm{k}$ centricon. Similarly, the individual mutant protein $M t \varepsilon E 100 \mathrm{C}$ was labeled with an acceptor dye ATTO-647N-maleimide. The washing step was performed using the centricon with a $3 \mathrm{k}$ cutoff membrane and the buffer containing $50 \mathrm{mM}$ Tris/ $\mathrm{HCl}, 200$ $\mathrm{mM} \mathrm{NaCl}, 10 \%$ glycerol, $1 \mathrm{mM}$ DTT, $\mathrm{pH}$ 7.5. Subsequently, individually labeled proteins were mixed for the reconstitution in 3-fold molar excess of $M t \varepsilon E 100 \mathrm{C}$ in the duration of $1 \mathrm{~h}$ at $4^{\circ} \mathrm{C}$, followed by size-exclusion column (S300, GE Healthcare) to remove the unbound $M t \varepsilon E 100 \mathrm{C}$ (Figure 3.45-A). The labeled complex was successfully reconstituted with the correct stoichiometry and the labeling is confirmed with the multi-wavelength scanner (Typhoon, GE Healthcare). 
(A)

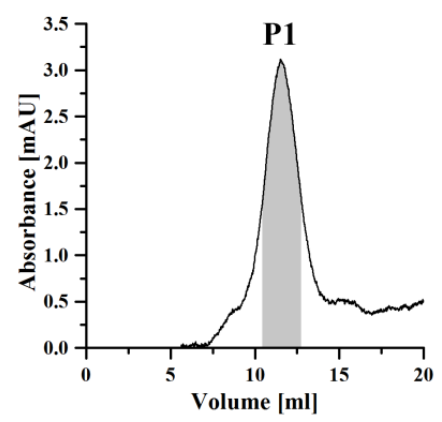

(B)

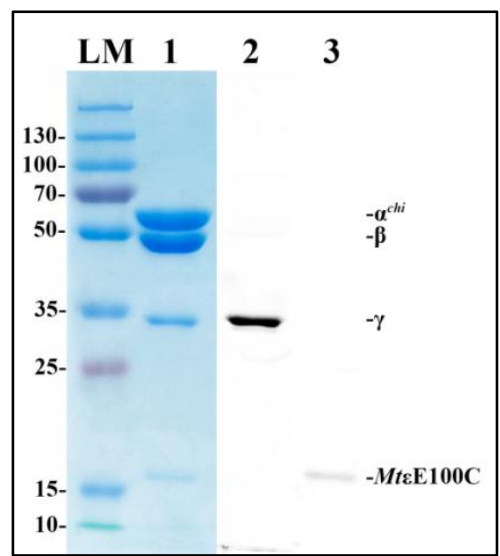

(C)

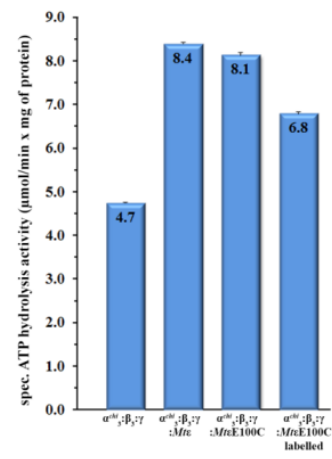

Figure 3.45: Reconstitution of the labeled $\alpha^{c h i}{ }_{3}: \beta_{3}: \gamma$ and subunit $M t \varepsilon E 100 C$ mutant for SmFRET. The separately purified, labelled and reconstituted complex $\alpha^{\text {chi }}{ }_{3:} \beta_{3:} \gamma: M t \varepsilon E 100 \mathrm{C}$ was passed over the size-exclusion column that eliminated the excess of subunit $M t \varepsilon E 100 \mathrm{C}$ and dyes, as shown in the elution diagram (A); The sample was analyzed on the Coomassie blue-stained, 12\% SDS gel, lane 1, where bands of all subunits are clearly resolved (B). The labelling efficiency of subunits $\gamma$ and $M t \varepsilon E 100 \mathrm{C}$ was estimated by using the Typhoon scanner on the wavelengths specific for each dye; $\gamma$-ATTO-488 maleimide in lane 2, MtcE100C-ATTO-647N maleimide, lane 3. (C) Bar diagram showing the specific activities of $\alpha^{\text {chi }}{ }_{3}: \beta_{3}: \gamma, \alpha^{\text {chi }}{ }_{3}: \beta_{3}: \gamma: M t \varepsilon$ and $\alpha^{\text {chi }}{ }_{3}: \beta_{3}: \gamma: M t \varepsilon \mathrm{E} 100 \mathrm{C}$ labeled $\alpha^{c h i}: \beta_{3}: \gamma: M t \varepsilon E 100 \mathrm{C}$ indicating that the labeled complex retained the $83 \%$ of its activity.

As seen in a Figure 3.45-B, there was no cross-reactivity between the dyes and the degree of labeling reflects the amount of the subunit present on the 12\% SDS-gel. Additionally, the ATP hydrolysis of the labeled $\alpha^{c h i}{ }_{3}: \beta_{3}: \gamma: M t \varepsilon E 100 \mathrm{C}$ complex was assayed in presence of $2 \mathrm{mM} \mathrm{Mg-ATP}$ and its activity was comparable to that one of the non-labeled complex, reaching $84 \%$ or $6.8 \mu \mathrm{mol} / \mathrm{min} / \mathrm{mg}$ of protein (Figure 3.45C).

The following smFRET experiment, in which the $\alpha^{c h i}{ }_{3:} \beta_{3: \gamma}$ was labeled with Atto488-maleimide (donor) and MtEE100C with Atto647N-maleimide (acceptor) as described above, was performed by Prof. Dr. Michael Börsch at the Jena University Hospital, Germany. The reconstituted, double-fluorescently labeled complex $\alpha^{\text {chi }}{ }_{3}: \beta_{3}: \gamma: M t \varepsilon E 100 \mathrm{C}$ was diluted to achieve a concentration below $1 \mathrm{nM}$, which is optimal for performing smFRET. The FRET efficiency was measured and compared in the absence and presence of Mg-ATP.

The preliminary smFRET measurements of the complex $\alpha^{c h i}: \beta_{3}: \gamma: M t \varepsilon E 100 \mathrm{C}$ with Mg-ATP showed a slight shift in the histogram profile towards higher count numbers in domain of the lower FRET efficiency when compared to the histogram of the apo-form, which displayed higher counts in higher FRET efficiencies (Figure 3.46). 
Nevertheless, it is difficult to reliably interpret the data due to the several unknown parameters such as the orientation of the dyes, differences in their quantum yields and conformation and transition states of Mte. The fitting of the data could not be performed due to the lack of the underlying model that would describe the movement of subunit MtcCTD. However, to precisely dissect this mechanism, further experiments including various nucleotides (ADP, AMP-PnP, ATP- $\gamma \mathrm{S}$ ) need to be conducted.

(A)

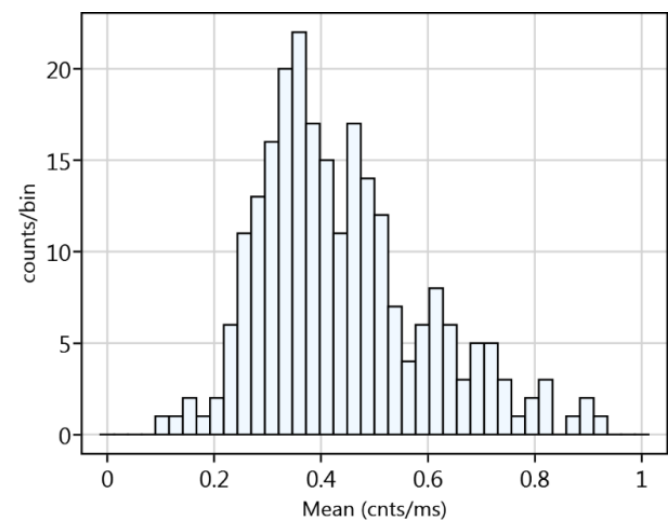

(B)

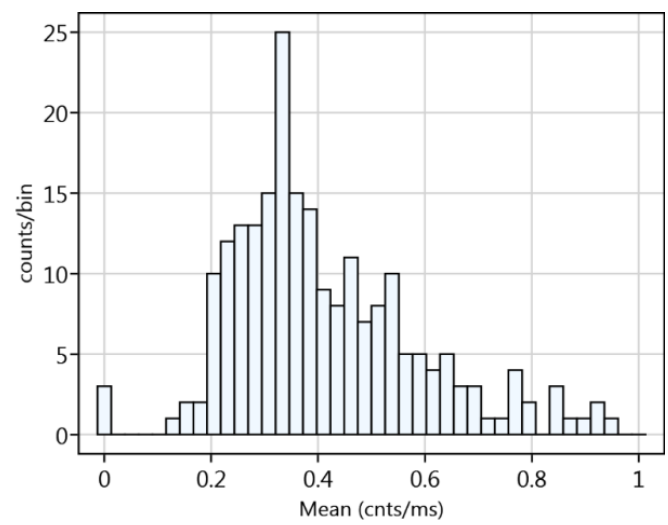

Figure 3.46: FRET efficiency histogram of the $\alpha^{c h i}{ }_{3}: \beta_{3}: \gamma: M t \varepsilon E 100 C$ complex. Histograms of proximity factors of FRET labeled protein complex in the absence (A) and presence (B) of Mg-ATP as an enzyme substrate. 
3.4 Purification and characterization of $A$. woodii $\mathbf{F}_{1} \mathbf{F}_{O}$-ATP synthase using NS-EM and establishing a role of the unique subunit $\gamma$ unique loop 


\subsubsection{Purification and ATPase activity of $A$. woodii $\mathbf{F}_{\mathbf{1}} \mathbf{F}_{\mathrm{O}}$-ATP synthase}

The plasmid pKB3-His obtained from Prof. Volker Müller (Johann-Wolfgang Goethe University, Frankfurt/M., Germany), including the entire F-ATP synthase operon was transformed into E. coli DK8 cells and purified according to the modified protocol described in the section 2.2.36.

The cells were cultivated in 2YT media till an optical density reached $\sim 1$ and the protein production, followed by pelleting of the cells and storing in liquid $\mathrm{N}_{2}$. Roughly $10 \mathrm{~g}$ of cells were homogenized in lysis buffer $(50 \mathrm{mM}$ Tris/HCl, $10 \mathrm{mM}$ $\mathrm{MgCl}_{2}, 10 \%$ [v/v] glycerol, $\left.\mathrm{pH} 7.5\right)$ followed by centrifugation to remove the cell debris. Subsequently, the materials were subjected to three rounds of ultracentrifugation to isolate the membranes containing the embedded proteins (see details in section 2.2.37).

(A)

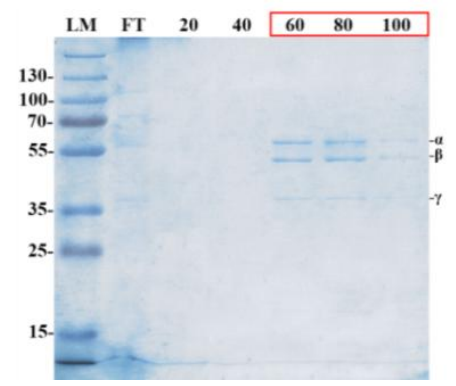

(C)

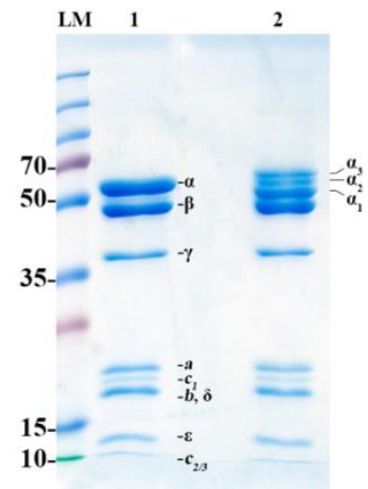

(B)

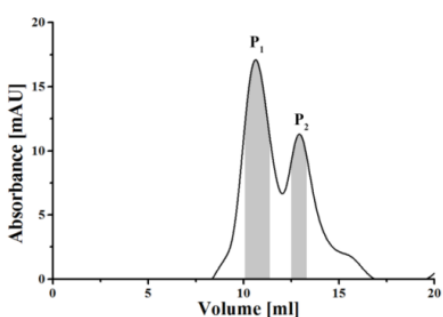

(D)

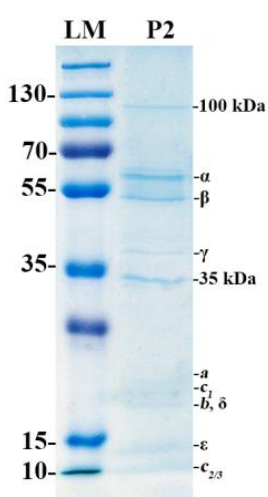

Figure 3.47: Purification of recombinant $A$. woodii $\mathbf{F}_{1} \mathbf{F}_{\mathrm{O}^{-}}$-ATP synthase. (A) $\mathrm{Ni}^{2+}-\mathrm{NTA}$ profile shown on the $12 \%$ SDS-gel; the pooled fractions are squared in red. (B) The size-exclusion profile (Superdex HR 200 column (GE Healthcare)) shows that the enzyme elutes in two peaks at about 11.5 $\mathrm{ml}(\mathrm{P} 1)$ and $13.0 \mathrm{ml}(\mathrm{P} 2)$. (C) The $12 \%$ SDS gel reveals that the highly pure and complete A. woodii $\mathrm{F}_{1} \mathrm{~F}_{\mathrm{O}}$ ATP synthase elutes in peak 1 . Lane 1 represents the protein pre-incubated in $20 \mu \mathrm{M}$ of DTT or non-treated by the reducing agent (lane 2). The oxidized subunit $\alpha$ migrates in three bands $\alpha 1, \alpha 2$, and $\alpha 3$ as verified by MALDI-TOF (Supplement Tables S2A-C). LM represents molecular weight protein marker. (D) Peak 2 of the elution profile was applied on a 12\% SDS gel demonstrating that the A. woodii F-ATP synthase eluted with two E. coli host protein bands of about $35 \mathrm{kDa}$ and 100 kDa (Supplement. Table S3A-C). Figure created for (265). 
To solubilize the F-ATP synthase, membrane fraction was incubated with buffer containing DDM and cell debris were removed once more by another UC step. The isolate was allowed to bind for $1 \mathrm{~h}$ to the pre-equilibrated $\mathrm{Ni}^{2+-}$ column $(50 \mathrm{mM}$ Tris/HCl, $10 \mathrm{mM} \mathrm{MgCl} 2,10 \%$ [v/v] glycerol, $\mathrm{pH}$ 7.5). The elution was initiated by the washing of the matrix with $20 \mathrm{mM}$ imidazole (15 column volume) followed by the step gradient 40-1000 mM. The fractions were applied onto a 12\% SDS gel (Figure. 3.56-A) and the protein was eluted in $60,80,100 \mathrm{mM}$ imidazole. Those fractions were subsequently applied onto the size-exclusion column (define the column) to remove any impurities (Figure 3.47-B) and both peaks observed in the chromatogram were concentrated in $100 \mathrm{k}$ centricon.

The sample isolated under peak 1 was treated with DTT and loaded in lane 1 while the non-treated sample was applied to lane 2 (Figure 3.47-C). Lane 1 shows distinct bands corresponding to the $\mathrm{F}_{1}$ subunits $\alpha, \beta, \gamma, \delta$, and $\varepsilon, \mathrm{F}_{\mathrm{O}}$ subunits $a$ and $c$ ring monomers $c_{1}$ and $c_{2 / 3}$ as well as subunit $b$ of the peripheral stalk, indicating that the high purity and homogeneity of the isolated $\mathrm{F}_{1} \mathrm{~F}_{\mathrm{O}}$ complex was achieved. The sample was further used for the negative stain electron microscopy (NS-EM) as described in the following section 3.5.2. The non-treated sample displayed the three distinct bands above $50 \mathrm{kDa}$, labeled as $\alpha_{1}, \alpha_{2}$, and $\alpha_{3}$ which were shown to be various oxidation adducts of subunit $\alpha$, as shown by mass-spec analysis (Suppl. Table S2A-C). The sample isolated under the peak 2 showed most of the subunits described, represented in the sub-stoichiometric ratio (Figure 3.47-D) with the addition of two $E$. coli host protein contaminants seen at around $110 \mathrm{kDa}$ and $35 \mathrm{kDa}$ (Suppl. Table S3AC).

(A)

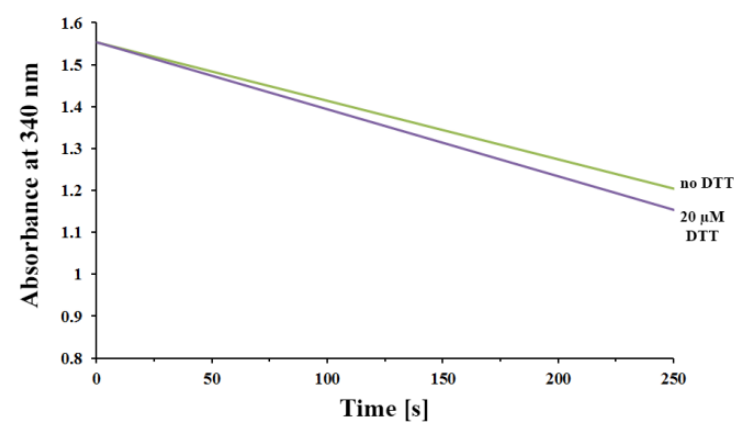

(B)

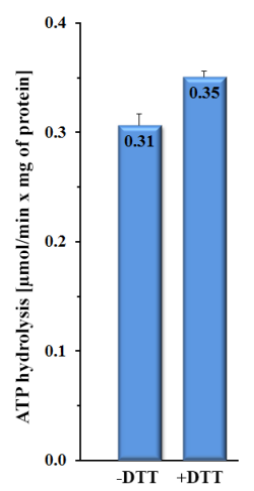

Figure 3.48: ATPase activity of the A. woodii F-ATP synthase (A) ATPase activity profile and (B) bar diagram of WT A. woodii F-ATP synthase in the presence and absence of DTT. Figure created for (265). 
Considering that the non-catalytic subunit $\alpha$ has four cysteine residues and displays the signs of oxidation, the ATPase activities of the reduced (DTT treated sample) and its non-reduced variant was measured, using the coupled ATP hydrolysis assay described in section 2.2.15. The reduced sample displayed an activity of $0.35 \pm$ $0.01 \mu \mathrm{mol} / \mathrm{min} / \mathrm{mg}$ of protein, which is roughly $11 \%$ higher than the activity of the non-treated sample $(0.31 \pm 0.02 \mu \mathrm{mol} / \mathrm{min} / \mathrm{mg}$ of protein). This could be indicative of its role as a redox sensor that, in part, can tune the catalytic activity of the $\mathrm{F}_{1} \mathrm{~F}_{\mathrm{O}}$ ATP synthase.

\subsubsection{Negative stain electron microscopy of $A$. woodii $\mathrm{F}_{1} \mathrm{~F}_{\mathrm{O}}$-ATP synthase}

To visualize the purified protein, the sample was diluted to $30 \mu \mathrm{g} / \mathrm{ml}$. A volume of $4 \mu \mathrm{l}$ of protein sample was applied to a glow discharge carbon coated copper transmission electron microscopy grid and stained with $2 \%$ uranyl acetate. The EM-images were taken by Assistant Professor Shashi Bhushan, NTU. The negatively stained enzyme yielded monodisperse particles shown in Figure 3.49-A. Discrimination criterion was a clear view of the lateral side of the complex, visualizing $\mathrm{F}_{1}$ and $\mathrm{F}_{\mathrm{O}}$ domains. For image processing, I used a total of 1,674 such particles. After multiple rounds of 2D class averaging, the projections of A. woodii F-ATP synthase were generated. As seen in Figure 3.49-B, the averaging resulted in the sorting of the particles into eight classes revealing distinct structural features.

The entire enzyme is comprised of $F_{1}$ and $F_{O}$ parts clearly linked with the central stalk Figure 3.49-C. The main density of $\mathrm{F}_{1}$ displays heterogeneity correlating with the pseudo-hexagonal organization of the subunits $\alpha$ and $\beta$. The density corresponding to subunit $\delta$ is observed best in class 2 (Figure 3.49-C). Subunit $\gamma$ and $\varepsilon$ assume the central position with the highest density possibly correlating with the Rossmann fold domain of subunit $\gamma$ and/or anti-parallel helices of subunit $\varepsilon$ in its compact conformation. The peripheral stalk is visible in several classes and connects to the subunit $\delta$ on the top of the $\mathrm{F}_{1}$ and to the $\mathrm{F}_{\mathrm{O}}$ via subunit $a$, whose density is present but hardly distinguishable from the density of the $c$-ring. The averaged classes were separated based on the orientation of the asymmetrical central stalk, asymmetrical $\mathrm{F}_{\mathrm{O}}$ domain (subunits $a$ and $c$-ring) and/or visibility of the peripheral stalk connecting $\mathrm{F}_{1}$ and $\mathrm{F}_{\mathrm{O}}$ domains. The entire $A$. woodii F-ATP synthase extends to length of around $204 \AA$ with the estimated dimensions of the $F_{1}$ headpiece of $101 \times 101 \AA$, 
the central stalk domain 40 x $38 \AA$, and the membrane part, $\mathrm{F}_{\mathrm{O}}, 63$ x $89 \AA$, confirming the integrity and conformational diversity of the A. woodii $\mathrm{F}_{1} \mathrm{~F}_{\mathrm{O}}$ ATP synthase.

(A)

(B)

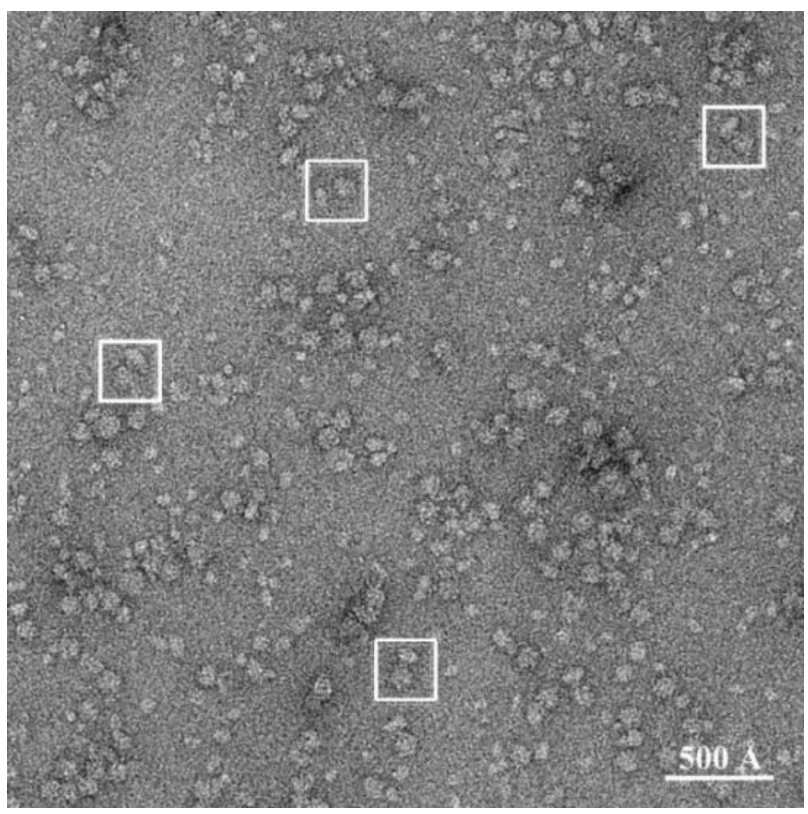
1
2
3
4
5

A

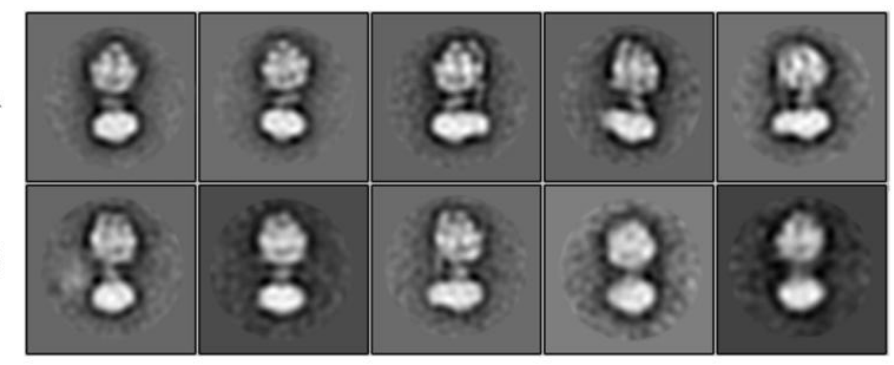

(C)

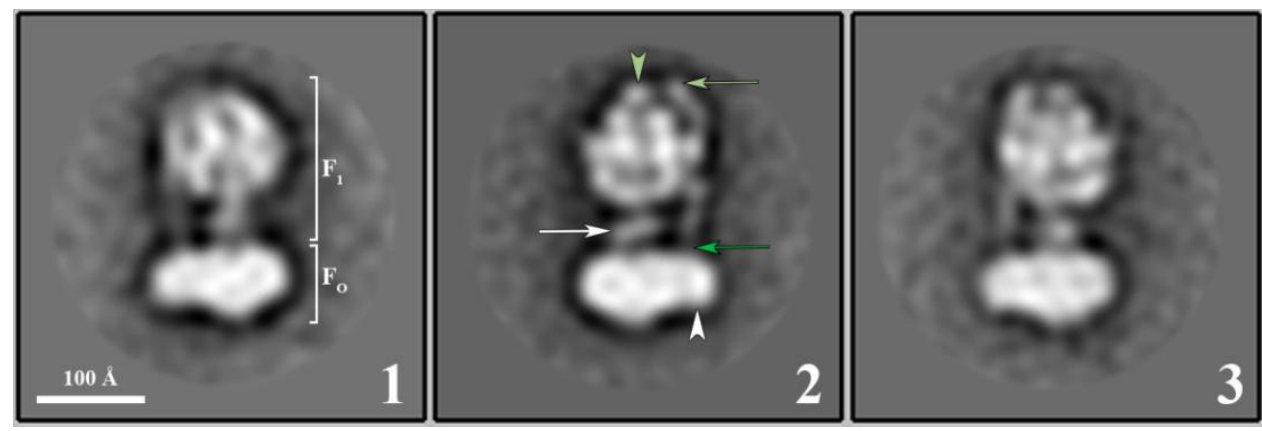

Figure 3.49: Negative stained EM of the recombinant $A$. woodii $\mathbf{F}_{1} \mathbf{F}_{0^{-}}-\mathbf{A T P}$ synthase. (A) Electron micrograph showing the selected particles consisting of two visibly distinguished domains. (B) Gallery shows ten 2D class averages resulting from two rounds of 2D classifications. Classes showing the prominent structural features of the complex are magnified in (C). Panel one shows the lateral view of the complex where the $\mathrm{F}_{1}$ and $\mathrm{F}_{\mathrm{O}}$ parts are clearly distinguished. Green arrows indicate the mass of the peripheral stalk connecting to $\mathrm{F}_{1}$ (light green; top) and $\mathrm{F}_{\mathrm{O}}$ (green; bottom) part, light green arrowhead points to the mass of the $\delta$ subunit. The white arrow indicates the mass of the asymmetrical central stalk. White arrowhead reveals the membrane-embedded subunit $a$ associated with the $c$-ring and a peripheral stalk. Panels 2 and 3 show the side-views of the complex with different rotational positions, as discerned from the orientation of the mass of the central stalk. Figure created for (265). 


\subsubsection{Purification and ATPase activity of $A$. woodii $\gamma_{\Delta 195-211}$ mutant $F_{1} F_{O}$-ATP synthase}

To introduce the deletion of the unique loop of subunit $\gamma$ (195TSGKVKITEETKEEKSK 211 ), the vector pKB3-His obtained from Prof. Volker Müller (Johann-Wolfgang Goethe University, Frankfurt/M., Germany) was used. The deletion was introduced into the gene by using single step site-directed mutagenesis with phosphorylated forward and non-phosphorylated reverse primers described in section 2.2.36.

The WT pDNA ( $p$ TRC99A: $\mathrm{F}_{1} \mathrm{~F}_{\mathrm{O}}$ ) was used as a template in PCR carried out with the mutagenic primers. The product was a single band of the estimated size of 12 $\mathrm{kB}$. The purified PCR product was ligated with the T4 DNA ligase as described in section 2.2.8 and transformed in E. coli DH5 $\alpha$ for plasmid propagation. The extracted plasmid was verified via the double restriction digestion with single-cutter endonucleases BamHI and XbaI. The resulting bands are visualized on the $1 \%$ agarose gel (Figure 3.50-A). The plasmids of the correct size were sent for sequence verification (Figure 3.50-B) and subsequently, plasmids carrying the $\gamma_{\Delta 195-211}$ mutation were transformed into the protein production strain E. Coli DK8.

(A)

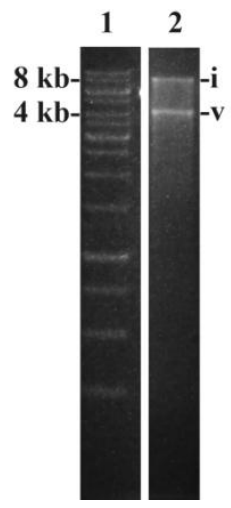

(B)

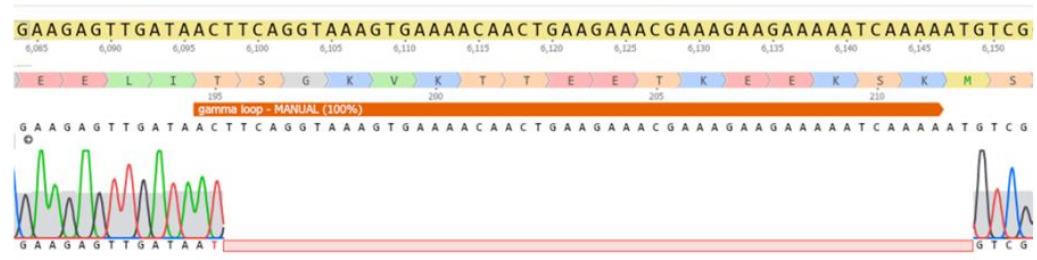

ATATGAACCGGAACCGGAAGAACTTTTAAAGTATCTGATTCCGAATTTTGTCAGTAGTACCGTATATGG

Figure 3.50: Restriction digestion and sequence verification of $\boldsymbol{A}$. woodii $\gamma_{\Delta 195-211}$ mutant. (A) $1 \%$ agarose gel showing the fragments of the double digested plasmid pTRC99A: $\mathrm{F}_{1} \mathrm{~F}_{\mathrm{O}}$ (lane 2). Lane 1 contains the $1 \mathrm{~kb}$ Gene Ruler. (B) The insert shows the deleted nucleotide sequence to obtain the mutant protein. Chromatographic peaks identify the nucleotides at the corresponding position.

The cultivation was carried out in a same way as described for the WT enzyme, followed by the purification using the same protocol (section 2.2.37 and 3.4.1). 
(A)

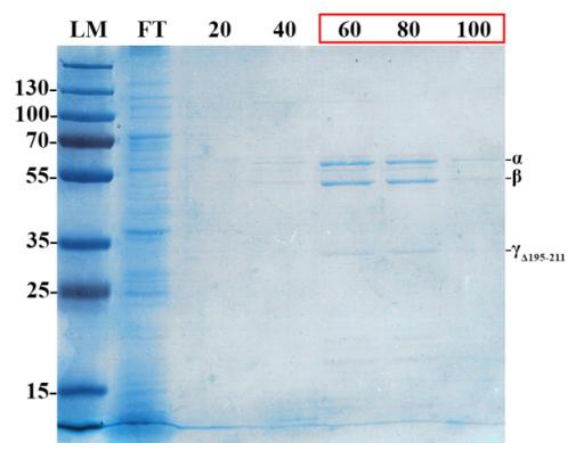

(C)

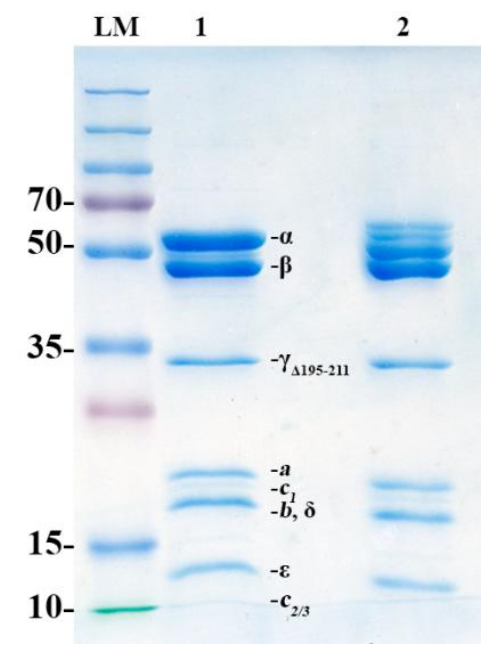

(B)

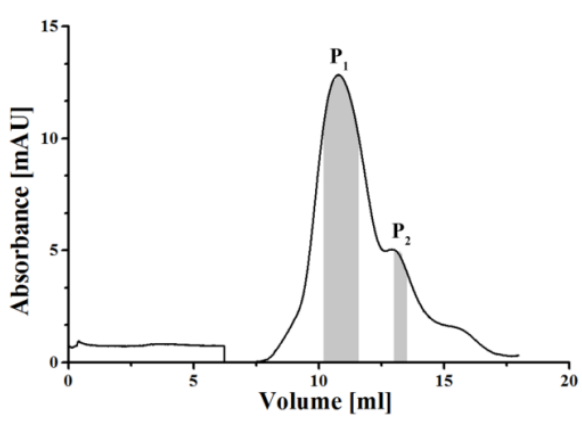

(D)

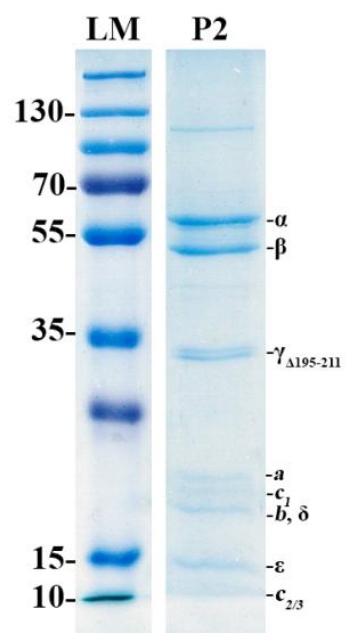

Figure 3.51: Purification of recombinant $A$. woodii $\gamma_{\Delta 195-211}$ mutant $\mathrm{F}_{1} \mathbf{F}_{\mathrm{O}}$-ATP synthase. (A) $\mathrm{Ni}^{2+}$ NTA profile shown on the $12 \%$ SDS-gel; the pooled fractions are squared in red. (B) The sizeexclusion profile (Superdex HR 200 column (GE Healthcare)) shows that the enzyme elutes in two peaks at about $11.5 \mathrm{ml}(\mathrm{P} 1)$ and $13.0 \mathrm{ml}(\mathrm{P} 2)$. (C) The $12 \%$ SDS gel reveals that the highly pure and complete $A$. woodii $\mathrm{F}_{1} \mathrm{~F}_{\mathrm{O}}$ ATP synthase elutes in peak 1. Lane 1 represents the protein pre-incubated in $20 \mu \mathrm{M}$ of DTT or non-treated by the reducing agent (lane 2). LM represents molecular weight protein marker. (D) Peak 2 of the elution profile was applied on a $12 \%$ SDS gel demonstrating that the $A$. woodii F-ATP synthase eluted with two contaminants of about $35 \mathrm{kDa}$ and $100 \mathrm{kDa}$. Figure created for (265).

The protein was eluted from the matrix using the imidazole gradient $(20,40$, $60,80,100,150 \mathrm{mM})$ and subsequently injected into the size-exclusion column (S300, GE Healthcare) (Figure 3.51-A, B). The sample was separated into two distinct peaks. The sample collected from the peak 1 was treated with DTT and analyzed on the $12 \%$ SDS-PAGE (lane 1) which showed the presence of all the subunits; peripheral stalk subunit(s) $b$ and $\alpha, \beta, \gamma, \delta$, and $\varepsilon$ of $\mathrm{F}_{1}$ part as well as $a$ and $c$-ring constituents, $c_{1}$ and $c_{2 / 3}$ of $\mathrm{F}_{\mathrm{O}}$ part, while lane 2 contains non-treated protein showing three distinct bands, apart from the mentioned ones, corresponding to spontaneous oxidation products of 
subunit $\alpha$, as defined with the mass-spectrometry (section 3.4.1, Suppl. Table S2). The sample eluted under peak 2 was showing a sub-stoichiometric complex containing additional bands at around 35 and $100 \mathrm{kDa}$.

The ATPase activity of the $\gamma_{\Delta 195-211}$ mutant complex (Figure 3.52) was determined to be $0.38 \pm 0.04 \mu \mathrm{g} / \mathrm{min} / \mathrm{mg}$ of protein, which is $16 \%$ lower than the ATP hydrolysis rate of the WT enzyme $(0.32 \pm 0.04 \mu \mathrm{g} / \mathrm{min} / \mathrm{mg}$ of protein $)$.

(A)

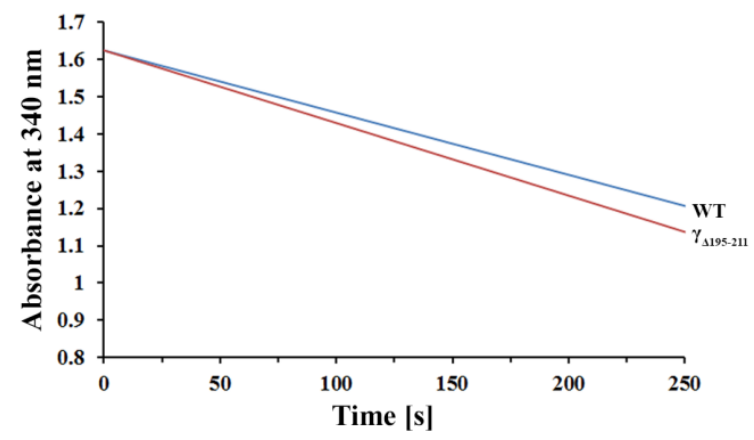

(B)

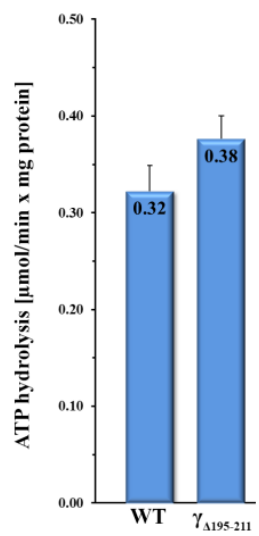

Figure 3.52: ATPase activity of the $A$. woodii $\mathbf{F}_{1} \mathbf{F}_{O}$-ATP synthase (A) ATPase activity profile and (B) bar diagram of WT A. woodii F-ATP synthase in the presence and absence of DTT. Figure created for (265).

In order to measure the ATP synthesis, it was a requirement to provide a gradient of the sodium ions across the membrane and establish the sodium-motive force. Mr. Dragan Trifunović from Prof. Volker Müller's lab isolated and reconstituted the WT and $\gamma_{\Delta 195-211}$ mutant complexes into the proteoliposomes.

To analyze the contribution of the $\gamma_{\Delta 195-211}$ loop in the regulation of the ATP synthesis, the proteoliposomes of the WT and the mutant enzyme were subjected a luciferase/luciferin assay. The WT proteoliposomes revealed the synthesis activity of $32 \pm 5 \mathrm{nmol} / \mathrm{min} / \mathrm{mg}$ of protein in the presence of ADP, while the synthesis of the proteoliposomes harboring the $\gamma_{\Delta 195-211}$ mutation in the same condition, was entirely abolished (Figure 3.53-A). As a control, the WT proteoliposomes do not synthesize ATP in the absence of the substrate, ADP.

As the ATP synthesis is coupled to $\mathrm{Na}^{+}$translocation, it was tested whether the mutation had a similar effect it did on the ATP synthesis. Namely, the sodium translocation was tested using the ${ }^{22} \mathrm{Na}^{+}$radioactive isotope. After the incubation in the buffer containing $100 \mathrm{mM}$ Tris/ $\mathrm{HCl}, 100 \mathrm{mM}$ maleic acid, $5 \mathrm{mM} \mathrm{MgCl}_{2}, \mathrm{pH} 7.4$ 
supplemented with ${ }^{22} \mathrm{NaCl}$, the proteoliposomes were passed over a column (Dowex 50-WX8 (100-200-mesh) to remove the excess of the unused ${ }^{22} \mathrm{Na}$. The proteoliposomes were washed out using the $420 \mathrm{mM}$ sucrose, and the scintillating counter was used to determine the remaining radiation.

The WT proteoliposomes translocate $\mathrm{Na}^{+}$at a rate of $4.5 \pm 1 \mathrm{nmol} \mathrm{Na}{ }^{+} / \mathrm{min} / \mathrm{mg}$ of protein in the presence of ATP, while ADP did not induce the translocation (Figure 3.62-B, C). In the case of the $\gamma \Delta_{195-211}$ mutant, both ATP and ADP did not induce $\mathrm{Na}^{+}$ translocation, pointing out the significance of the $\gamma$-loop in the regulation of the ATP synthesis.

(A)

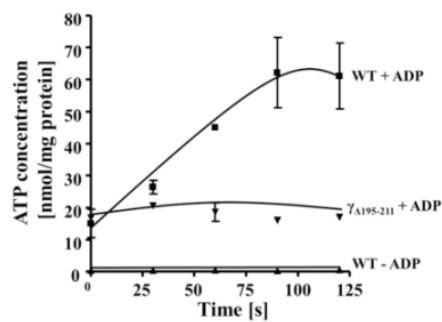

(B)

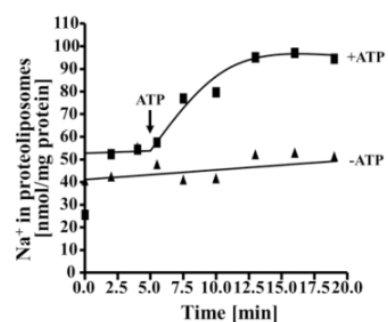

(C)

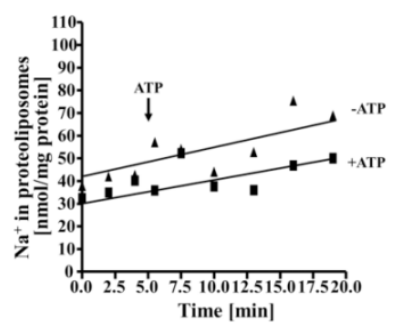

Figure 3.53: Effect of the $\gamma \Delta \mathbf{1 9 5 - 2 1 1}$ deletion on ATP synthesis and Na+ transport. (A) ATP synthesis driven by $\mathrm{Na}^{+}$in the presence and absence of ADP. WT (匹) F-ATP synthase-containing proteoliposomes; the F-ATP synthase $\gamma \Delta 195-211$ mutant $(\boldsymbol{\nabla}) \cdot \mathrm{Na}^{+}$transport measurements of WT (B) and $\gamma \Delta_{195-211}$ deletion mutant (C) F-ATP synthase-containing proteoliposomes. *The experiments performed and figures provided kindly by Mr. Dragan Trifunović, Prof. Volker Müller lab. Figure created for (265). 


\section{Discussion}


F-ATP synthases are one of the most studied molecular machines present in nearly all domains of life. Their primary physiological role is to generate metabolic energy in the form of ATP and take part in its turnover by utilizing the electrochemical gradient established across the cell membrane. Different ecological niches have forced organisms to adapt to conditions therein and thus, their F-ATP synthases reflect those adaptations in order to support the energy requirements of the cellular metabolism. In this thesis, F-ATP synthases of two evolutionarily distant organisms have been examined in order to describe their specific structural adaptations and correlate them with their functional traits. The first part of the work focuses on the protontranslocating F-ATP synthases of pathogenic mycobacteria, primarily $M t b$, while the second part describes an archaeal F-ATP synthase driven by $\mathrm{Na}^{+}$-motive force.

In pathogenic mycobacteria such as Mtb, M. bovis BCG, M. leprae and $M$. avium, F-ATP synthases are characterized as essential for survival and growth due to the unusually high requirements of their cells for metabolic energy. These enzymes have evolved to harbor specific regulatory mechanisms that internally inhibit detrimental ATP hydrolysis in order to conserve cellular ATP (2). The essentiality of mycobacterial F-ATP synthase was exploited in recent works when a potent antimicrobial agent, bedaquiline (BDQ), was shown to specifically inhibit the proton translocation through the $c$-ring (109) and cause a significant bactericidal effect $(307,308)$. These discoveries led to clinical usage of BDQ as the newest antibiotic for the treatment of $\mathrm{TB}$, however, due to multiple human factors, the emergence of resistance to BDQ has been recorded (130). This fact alone created a need to seek out an alternative mechanism to disable the functionality of mycobacterial F-ATP synthase, and thus, the regulatory epitopes that prevent detrimental ATP hydrolysis became significant potential targets. Chapters 4.1-4.6 focus on the structural and mechanistic aspects of specific mycobacterial epitopes $\alpha^{c h i}$ and $M t \varepsilon$ and their synergistic contribution to the regulation of the catalytic activity of the chimeric model complex $\alpha^{c h i}{ }_{3}: \beta_{3}: \gamma: M t \varepsilon$.

In chapter 4.7 , the structural and functional features of archaeal F-ATP synthase from Acetobacterium woodii were investigated. Considering the fact that $A$. woodii thrives in anoxic waters facing high salinity and acidic $\mathrm{pH}$ (309), it was established that these factors were leading contributors to the diversification of its respiratory complexes and its F-ATP synthase. 
Previous studies on F-ATP synthase showed that its specific structural adaptations include the organization of the unique $c$-ring and functional utilization of transmembrane $\mathrm{Na}^{+}$gradient for ATP synthesis (256). However, not much was known about the structural traits that take part in the coupling and regulation of $\mathrm{Na}^{+}$ translocation and ATP synthesis.

Towards that end, in this work, the NS-EM was utilized to visualize the entire $\mathrm{F}_{1} \mathrm{~F}_{\mathrm{O}}$ complex. The mutational studies and biochemical assays uncovered a novel regulatory epitope of subunit $\gamma$ and described its function, while generated structural model provided a basis to understand the coordination of bi-directional $\mathrm{Na}^{+}$ translocation with ATP synthesis/hydrolysis.

\subsection{Structural and functional characterization of complexes $\alpha^{\text {chi }}{ }_{3}: \beta_{3}: \gamma$ and $\alpha^{c h i}: \beta_{3}: \gamma: M t \varepsilon$}

The newly utilized host E. coli OverExpress ${ }^{\circledR}$ C41(292) that tolerates the expression of toxic proteins proved to be receptive to the expression of the chimeric $\alpha^{c h i}: \beta_{3}: \gamma$ complex in high yields. Since this strain harbors its $\mathrm{F}_{1} \mathrm{~F}_{\mathrm{O}}$-ATP synthase and the oxidative phosphorylation is not affected like in E. coli DK8 strain previously used, the question was raised whether the E. coli ATP synthase subunits will associate and co-purify with the His ${ }_{10}$-tagged $\alpha^{c h i}: \beta_{3}: \gamma$ complex. The optimized 3-step purification and reconstitution protocol (section 2.2.6) allowed the isolation of high yields of homogenous complexes $\alpha^{\text {chi }}{ }_{3}: \beta_{3}: \gamma$ and $\alpha^{\text {chi }}{ }_{3}: \beta_{3}: \gamma: M t \varepsilon$ that do not contain any subunits of the host F-ATP synthase, as verified by MALDI-TOF spectrometry (310).

Pure $\alpha^{c h i}: \beta_{3}: \gamma: M t \varepsilon$, exhibiting the typical stoichiometry $\left(\alpha^{c h i}: \beta: \gamma: M t \varepsilon=3: 3: 1: 1\right)$, was analyzed with negative-stain electron microscopy (NS-EM) that yielded a 3D structure $18 \AA$ resolution. Observed from the top, it is apparent that the subunits $\alpha^{c h i}$ and $\beta$ assume the hexagonal hexameric shape. Their $\mathrm{N}$-terminal domains associate with each other to form a coherent ring with a visible cavity in its center (Figure 4.1). Such an arrangement is typical and observed in all solved structures of $F_{1} / F_{1} F_{O}$ to this date. Side projection(s) feature all the present subunits of which subunits $\alpha^{\text {chi }}$ and $\beta$ display distinct differences in their size; Apparently, the density that corresponds to subunit $\alpha^{c h i}$ is more prominent due to the presence of the $M t \alpha_{514-549}$ epitope at its CTD domain, as proposed and later corroborated with crosslinking (section 3.1.3 and 3.1.4) and SAXS studies (3). In addition, the central stalk density allows fitting of the subunits $\gamma$ and $M t \varepsilon N T D$ confirming that heterologous $M t \varepsilon$ is assembling with $\alpha^{c h i}{ }_{3}: \beta_{3}: \gamma$ 
complex at its canonical position (Figure 4.1), disregarding the genetic distance between the G. stearothermophilus and $M t b$, and overcoming the sequence heterogeneity. Similarly, the bottom view of the $\alpha_{3}^{c h i}: \beta_{3}: \gamma: M t \varepsilon$ provides yet another

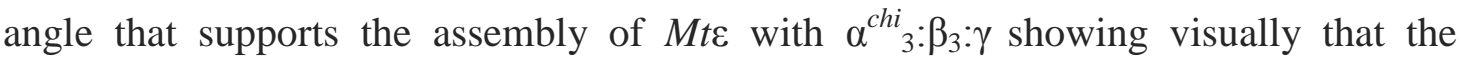
formed complex is stable and gives a solid basis to study the activity alterations introduced by mycobacterial epitopes (Figure 4.1).

1

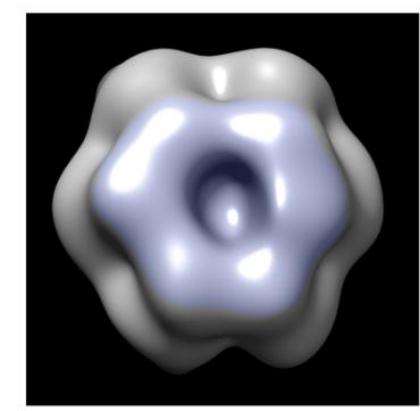

A)

B)

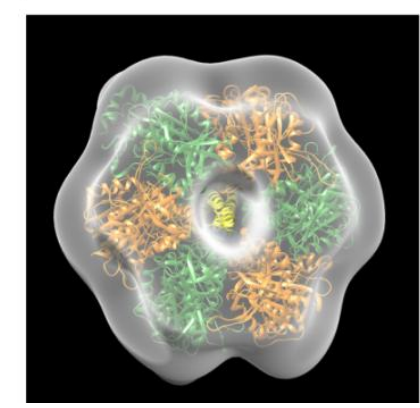

2
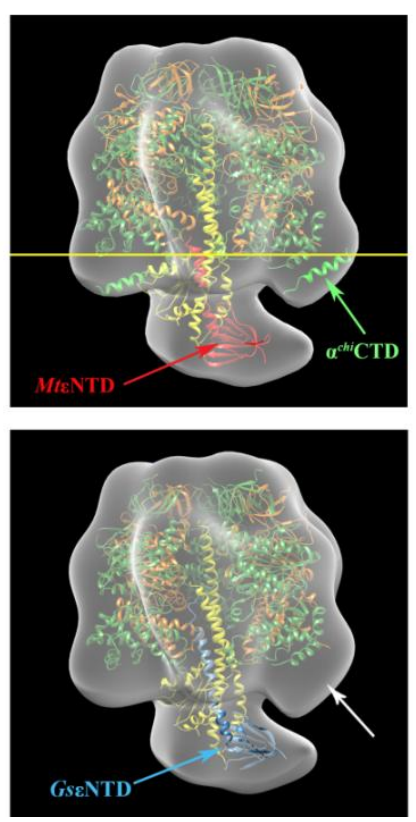

3
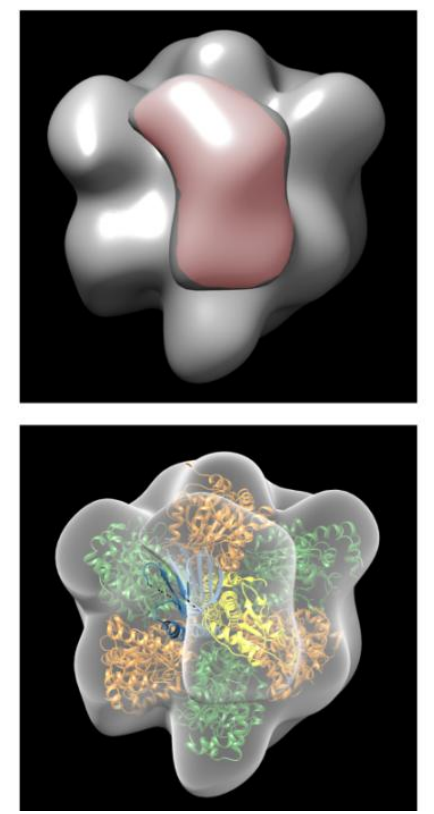

Figure 4.1: Surface representation of 3D reconstruction of $\boldsymbol{\alpha}^{\text {chi }}{ }_{3}: \beta_{3}: \gamma: M t \varepsilon$ complex. (A1) Top surface 3D map refined to $\sim 18 \AA$ using RELION 1.3 reveals the crown like arrangement (violet shade) of $\mathrm{N}$-terminal domains of subunits $\alpha^{\text {chi }}$ (forest green) and $\beta$ (orange) as reflected in panel $B 1$. Panel A2 reveals the side view of the complex reflecting the position of the $\alpha^{\text {chi }}$ CTD helix $M t \alpha_{521-540}$ (light green arrow) and MtENTD (red arrow) associated with the $\gamma$ subunit (yellow). The yellow line indicates section at the position of extra C-terminal densities of $\alpha^{\text {chi }}$ subunits. In comparison, panel B2 shows the structure of $G s \alpha_{3}: \beta_{3}: \gamma: \varepsilon$ (PDB ID: 4XD7, (196)) fitted into the experimental map obtained for the complex $\alpha^{\text {chi }}{ }_{3}: \beta_{3}: \gamma: M t \varepsilon$. The white arrow indicates the unoccupied density of subunit $G s \alpha$, the blue arrow indicates Gse associated with subunit $\gamma$. (A3) The bottom view of the complex represents subunits $\alpha^{\text {chi }}$ and $\beta$ arranged in a pseudo-hexagonal manner and the additional density corresponding to the central stalk subunits $\gamma$ and $M t \varepsilon$, shown in red shade; For comparison, the panel B3 contains the cartoon representations of $G$. stearothermophilus $\alpha_{3}: \beta_{3}: \gamma: \varepsilon$ complex are reflecting the canonical position of central stalk (Gs $\gamma$, yellow and Gse, blue) fitted into the surface density map that was obtained from the NS-EM of the reconstituted complex $\alpha^{c h i}{ }_{3}: \beta_{3}: \gamma: M t \varepsilon$. Color code: $\alpha$-forest green, extended C-terminus shown in light green; $\beta$-orange, $\gamma$-yellow, $\varepsilon$-blue, and Mte-red. Graphics are visualized using UCSF CHIMERA (270). The figure was adapted from (205).

Special attention is dedicated to the role of $M t \varepsilon$ in the regulation of the catalytic activity, and thus the ATP hydrolysis was measured in the presence of the various 
substrate (Mg-ATP) concentrations to determine the kinetic properties (section 3.2.3). At the physiological concentration of $\mathrm{Mg}$-ATP of $2 \mathrm{mM}$, the $\alpha^{\text {chi }}: \beta_{3}: \gamma$ complex displayed ATP hydrolysis rate of $4.7 \pm 0.01 \mu \mathrm{mol} / \mathrm{min} / \mathrm{mg}$ protein, while $8.4 \pm 0.04$ $\mu \mathrm{mol} / \mathrm{min} / \mathrm{mg}$ protein was the value determined for the $\alpha^{c h i}: \beta_{3}: \gamma: M t \varepsilon$ complex. Similarly, both complexes

were subjected to the ATP hydrolysis measurements in the presence of the various Mg-ATP concentrations ranging from $6 \mu \mathrm{M}$ to $2 \mathrm{mM}$. While maximum velocity (Vmax) of $\alpha^{\text {chi }}{ }_{3}: \beta_{3}: \gamma:$ Mt is determined to be 1.8 times higher than the value measured for $\alpha^{c h i}: \beta_{3}: \gamma$ complex, $K_{m}$ values were virtually in the same range. These parameters reflected that $M t \varepsilon$ did not significantly alter the substrate binding affinity, but it played a part in another step of the ATP hydrolysis reaction, possibly relieving of Mg-ADP inhibition, cleavage of the ATP to ADP and $\mathrm{P}_{\mathrm{i}}$ and/or release of the products from the active site. However, the specific epitope and its interactions remain unclear.

Furthermore, it has been observed that the inhibition of Mg-ATP dependent ATPase activity is more pronounced in the $\alpha^{c h i}: \beta_{3}: \gamma: M t \varepsilon$ than in complex lacking MtE (Figure 4.2).

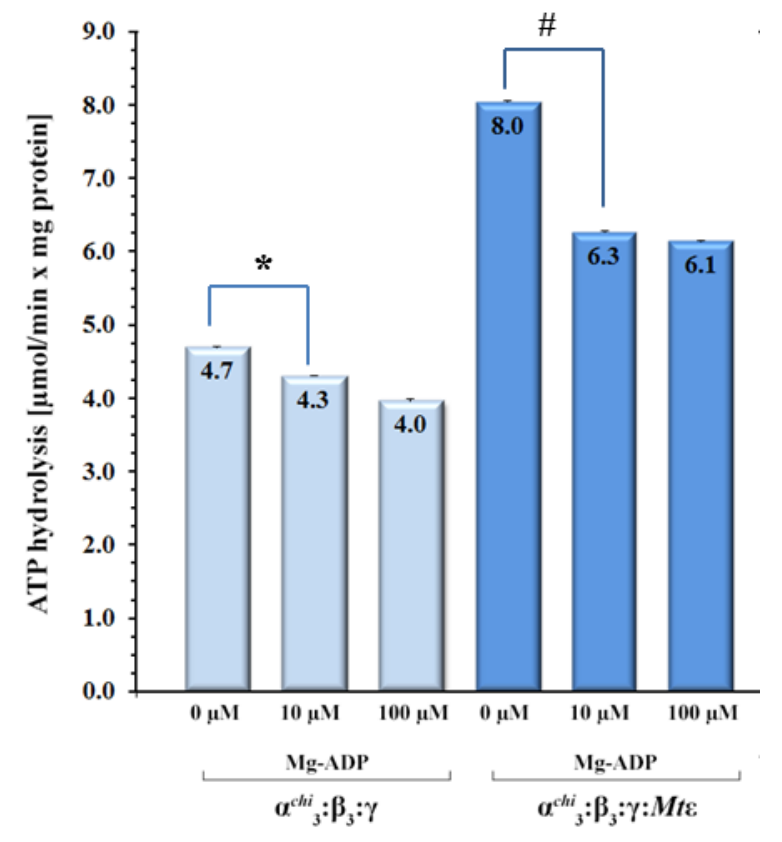

Figure 4.2: Synergistic effect of Mg-ADP and subunit MtE on ATP hydrolysis. The incubation of the complex $\alpha^{\text {chi }}{ }_{3}: \beta_{3}: \gamma$ with $10 \mu \mathrm{M} \mathrm{Mg-}$ ADP showed a reduction in ATPase activity by $8.5 \%$ in comparison to the complex in apo-form (*). The incubation of the complex $\alpha_{3}^{c h i}: \beta_{3}: \gamma: M t \varepsilon$ ATPase with $10 \mu \mathrm{M}$ $\mathrm{Mg}$-ADP reduced its ATPase activity by $\sim 22 \%$ in comparison to the apoform (\#), indicating the $M t \varepsilon$ mediated increase in sensitivity towards inhibitory Mg-ADP. Figure adapted from (205).

These observations are well in line with the finding for Gse which inhibits the hydrolysis under the Mg-ATP concentrations below $1 \mathrm{mM}$ ATP, while the complex is considered to be actively hydrolyzing ATP when its concentrations supersede $1 \mathrm{mM}$ 
(311). It is also shown that Gse has a binding site for ATP $(186,188,312)$, which can be wedged between two CTD helices when Gse is in the compact form. These interactions, in turn, stabilize compact conformation and allow the hydrolysis reaction to proceed unobstructed. Upon depletion of cellular ATP below the homeostatic equilibrium, the ATP bound to Gse dissociates as well, permitting the conformational transition from closed (ATPase non-inhibitory) to extended (ATPase inhibitory) state to occur. In comparison, the mycobacterial subunit $\varepsilon$ lacks the ATP binding motif (208) in its CTD, which makes it highly unlikely that Mte acts as an ATP sensor. In order to establish the inhibition of ATP hydrolysis in low ATP concentrations, the alternative mechanism must be present, and thus the synergy between Mg-ADP and $M t \varepsilon$ was investigated.

\subsection{Epitopes of mycobacterial subunit $\varepsilon$ involved in the regulation of ATPase activity}

Mg-ADP inhibition is a well-documented phenomenon (196,313-316), involving a release of $\mathrm{P}_{\mathrm{i}}$ after ATP cleavage, leaving Mg-ADP bound in the catalytic site. The event causes subsequent rotational shifts of subunit $\gamma$ that propagates further conformational changes in the active spot, trapping Mg-ADP and in turn, further ATP hydrolysis is inhibited (182). In some bacterial F-ATP synthases, it was proposed that the inhibitory effect of subunit $\varepsilon$ is achieved by enhancing the inhibition caused by Mg-ADP (313,317,318). This phenomenon is well known for Gse whose two CTD helices assume extended conformation in a pike-like manner of the total length of $67 \AA$ (PDB ID: 4XD7) (148) (Figure 4.3-B).

These structural traits allow it to extend deep into the $\alpha_{3}: \beta_{3}$ hexamer reaching the P-loop. Similarly, E. coli $\varepsilon$ CTD is slightly shorter than Gse and organized in a helix-linker-helix-hook motif $(143,194)$ (Figure 4.3-A) in its extended conformation which allows the terminal ${ }_{135} \mathrm{KAM}_{138}$ residues to interact with P-loop (PDB ID: 3OAA), (197) to communicate the structural alterations and trap the Mg-ADP. Considering the length requirements and the fact that Mte extended CTD has a proposed length of $42 \AA$, it was not clear whether the entrapment of Mg-ADP would be mediated by this subunit (Figure 4.3). 

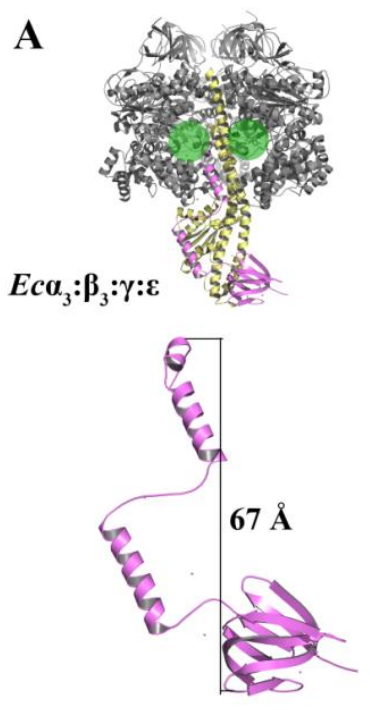

EcE
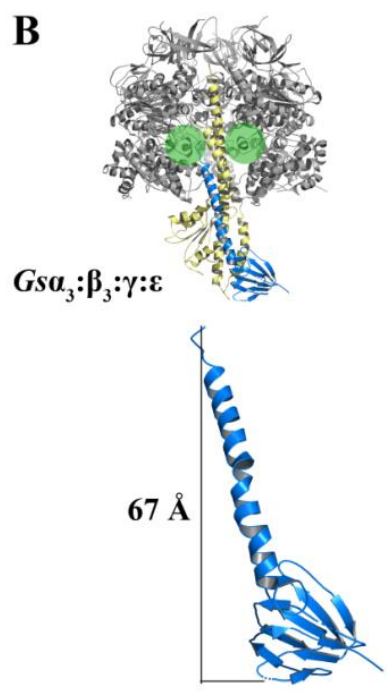

GsE
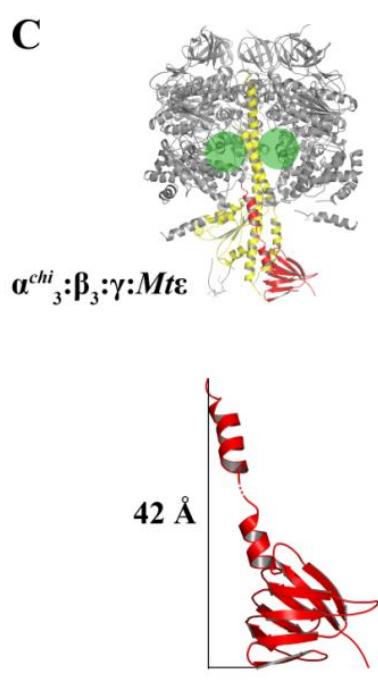

Mts

Figure 4.3: Regulatory epitopes of bacterial F-ATP synthases. The cartoon representation of the subunit $\varepsilon$ in the extended conformation from E. coli (magenta), G. stearothermophilus (blue) and $M t b$ (red). (A) The EcE (PDB ID: 3OAA: chain H, (197)) spans the length of $67 \AA$ and is comprised of $\beta$-barrel NTD and CTD domain featuring two $\alpha$-helices forming a "helix-linker-helix-linker-hook" motif. The $E c \alpha_{3}: \beta_{3}: \gamma: \varepsilon$ complex structure (PDB ID: 3OAA) reflects that position of the very Cterminal "hook" motif of EcECTD in respect to the nucleotide-binding motif in subunits $\alpha$ and $\beta$ (Ploop, green circles). (B) The Gse (PDB ID: 4XD7: chain H, (148)) spans the length of $67 \AA$, similar to EcE and is comprised of $\beta$-barrel NTD and CTD domain featuring two $\alpha$-helices organized in a linear pike-like motif. The $G s \alpha_{3}: \beta_{3}: \gamma: \varepsilon$ complex structure (PDB ID: 4XD7) reflects that position of the very $\mathrm{C}$-terminal of GseCTD in respect to the nucleotide-binding motif in subunits $\alpha$ and $\beta$ (Ploop, green circles). (C) The cartoon representation of subunit MtE in the modeled extended conformation derived from the NMR solution structure (209) spanning $42 \AA$. In comparison to EcE and $G s \varepsilon, M t \varepsilon$ is $25 \AA$ shorter, and it cannot directly reach the nucleotide-binding site (P-loop, green circles) with its CTD.

In the study reported in section 3.2.4, the complexes $\alpha^{\text {chi }}{ }_{3}: \beta_{3}: \gamma$ and $\alpha^{\text {chi }}{ }_{3}: \beta_{3}: \gamma: M t \varepsilon$ were incubated with various concentrations of Mg-ADP. In the presence of $10 \mu \mathrm{M} \mathrm{Mg}$-ADP the complex without subunit $M t \varepsilon$ displayed a moderate inhibition of $8 \%$ while a $100 \mu \mathrm{M}$ concentration decreased its activity by $15 \%$ (Figure 4.2 ). The subunit $M t \varepsilon$ enhanced the inhibition further by lowering the ATPase activities by $21 \%$ and $23 \%$ in the presence of $10 \mu \mathrm{M}$ and $100 \mu \mathrm{M} \mathrm{Mg}$-ADP, respectively. The data confirmed that entrapment of the inhibitory Mg-ADP is indeed enhanced by subunit $M t \varepsilon$ which sensitizes the complex towards Mg-ADP invoking tighter regulation. Apart from the residue $M t \varepsilon D 121$ that seems to enhance the Mg-ADP dependent inhibition, it was not clear, however, which epitope was involved considering that $M t \varepsilon$ is shorter that $G s \varepsilon$ and $E c \varepsilon$ (Figure 4.3-C).

From most recent studies on M. smegmatis IMVs (209), it was observed that the subunit $\varepsilon$ CTD D121 deletion increased ATP hydrolysis by $13 \%$ indicating that 
this particular residue assumes, at least in part, a regulatory role and is vital for the inhibition of ATPase activity. Similarly, $M t \varepsilon_{1-120}$ mutant featuring the same deletion was generated, and the reconstitution was performed to measure its effects on the rate of ATP hydrolysis. After confirming that the binding of the mutant is not affected by this CTD mutation, it was shown that the ATPase activity was indeed increased in the similar range, by $12 \%$ as measured in the M. smegmatis IMVs. The Mg-ADP treated complex $\alpha^{c h i}{ }_{3}: \beta_{3}: \gamma: M t \varepsilon_{1-120}$ exhibited a moderate (10\%) inhibition in the presence of 10 $\mu \mathrm{M}$ Mg-ADP in comparison to the value obtained for the $\alpha^{\text {chi }}{ }_{3}: \beta_{3}: \gamma: M t \varepsilon$ complex $(21 \%$ inhibition) treated in the same way. The $10 \%$ inhibition of the complex $\alpha^{\text {chi }}{ }_{3}: \beta_{3}: \gamma: M t \varepsilon_{1-}$ ${ }_{120}$ resembles the inhibition rate of $\alpha_{3}^{c h i}: \beta_{3}: \gamma$ indicating that $M t \varepsilon D 121$ residue modulates the sensitivity towards Mg-ADP. This phenomenon could be attributed to the $M t \varepsilon D 121$ interactions with an epitope in the $\alpha_{3}: \beta_{3}$ catalytic hexamer that prevents the release of Mg-ADP after the ATP hydrolysis. Since subunit Mte is shorter by $\sim 25 \AA$ than its homologs $G s \varepsilon$ and $E c \varepsilon$, the additional epitope involved in this mode of regulation can indeed be $M t \alpha_{514-549}$, whose interactions will be discussed in section 5.6.

The results describe the structural and functional characterization of Mte that has been performed in the context of the model complex $\alpha^{c h i}{ }_{3}: \beta_{3}: \gamma$. However, very little is known about its dynamics, time-resolved, substrate-dependent structural alterations that provide the regulatory link between $F_{1}$ and $F_{O}$ functional domains. To fully understand the Mt\&CTD conformational transition between its conformations states and correlate it with the proton-motive force that drives the synthesis, or availability of ATP for hydrolysis, it is a pre-requisite to obtain the purified mycobacterial $F_{1} F_{O}$ embedded into lipid nano-discs and perform the smFRET measurements. However, these experiments have not been carried out as a part of this thesis.

\subsection{Critical epitopes of subunit Mte for coupling activity}

The coupling of the c-ring rotation with catalytic activities of the $\alpha_{3}: \beta_{3}$ hexamer is conducted via the central stalk subunits $\gamma$ and $\varepsilon$ in virtually all studied ATP synthases. In E. coli and G. stearothermophilus, \&CTD transits between compact and extended states to allow the tuning of the catalytic activity by inhibiting ATP hydrolysis $(132,303,319)$. In $\alpha$-proteobacteria, subunit $\varepsilon$ has a strictly coupling role, enhancing the association of the central stalk to the $\mathrm{F}_{\mathrm{O}} c$-ring while its role in the regulation of ATP hydrolysis is overtaken by a novel zeta subunit (320). In mycobacteria, this phenomenon is poorly understood due to the issues in isolation and 
downstream characterization of mycobacterial $\mathrm{F}_{1} \mathrm{~F}_{\mathrm{O}}$ ATP synthase, including the lack of a high-resolution structure of the entire complex. Thus we used the $\alpha^{c h i}{ }_{3}: \beta_{3}: \gamma: M t \varepsilon$ complex to investigate the interactions pertaining the coupling and regulation.

The recent NMR structure of isolated MtE (209) was solved in its closed conformation showing two CTD helices compacted in an anti-parallel manner, wherein the second helix folds towards the NTD. The network of hydrophobic interactions and hydrogen bonds was well defined. It included contacts of NTD residues A10-W16 with the epitopes L61-A64 and A81-I90, which together could convey the structural changes from NTD to CTD (209). We proposed a mechanistic model of mycobacterial $\mathrm{F}_{1} \mathrm{~F}_{\mathrm{O}}$ that included the structural coordinates of M. phlei $c_{9^{-}}$ ring (PDB ID: 4V1G) (109), Mte and a homology model of $\gamma$ subunit.

As seen from the structure of the $c_{9}$-ring (109), the monomer consists of two parallel helices inserted in the membrane connected with a short loop on the cytoplasmic side. The loop residues R45, Q46, and E48, could be found in the proximity of the amino acids F24 and K21 of the MtENTD, respectively, and could potentially transfer the rotational energy adopted from the ion transport through the $c$ ring (Figure 4.4). The conformational change would then be communicated to the epitopes V51-V53, L61-A64, I8-A10, and D12-N14 in a sequential manner which would propagate further to the A81-I90 motif found in the proximity of the MtECTD. The residues F86 as well as E87-I90 that belong to the region linking the NTD to the CTD, have a propensity to form a hinge and propel the conformational transition of CTD from compacted to the extended state.

By adhering to this model, the mutant Mt\&E87A was generated and reconstituted to form a $\alpha^{c h i}: \beta_{3}: \gamma: M t \varepsilon E 87 \mathrm{~A}$ complex which showed a $21 \%$ decrease in the ATPase activity in comparison to the $\alpha^{c h i}: \beta_{3}: \gamma: M t \varepsilon$ complex. The residues with the most significant chemical shift perturbations include the NTD residues V51, V53, I63, E85, residue S88 belonging to the linker region and residues R113, R115, A116, G118, I120 found in the second helix of the CTD (section 3.2.3, Figure 3.18). The drop of the ATPase activity associated with E87A mutation could arise due to the stabilization of the $M t \varepsilon$ extended state, which enhances the entrapment of Mg-ADP increasing the inhibition of ATP hydrolysis. 


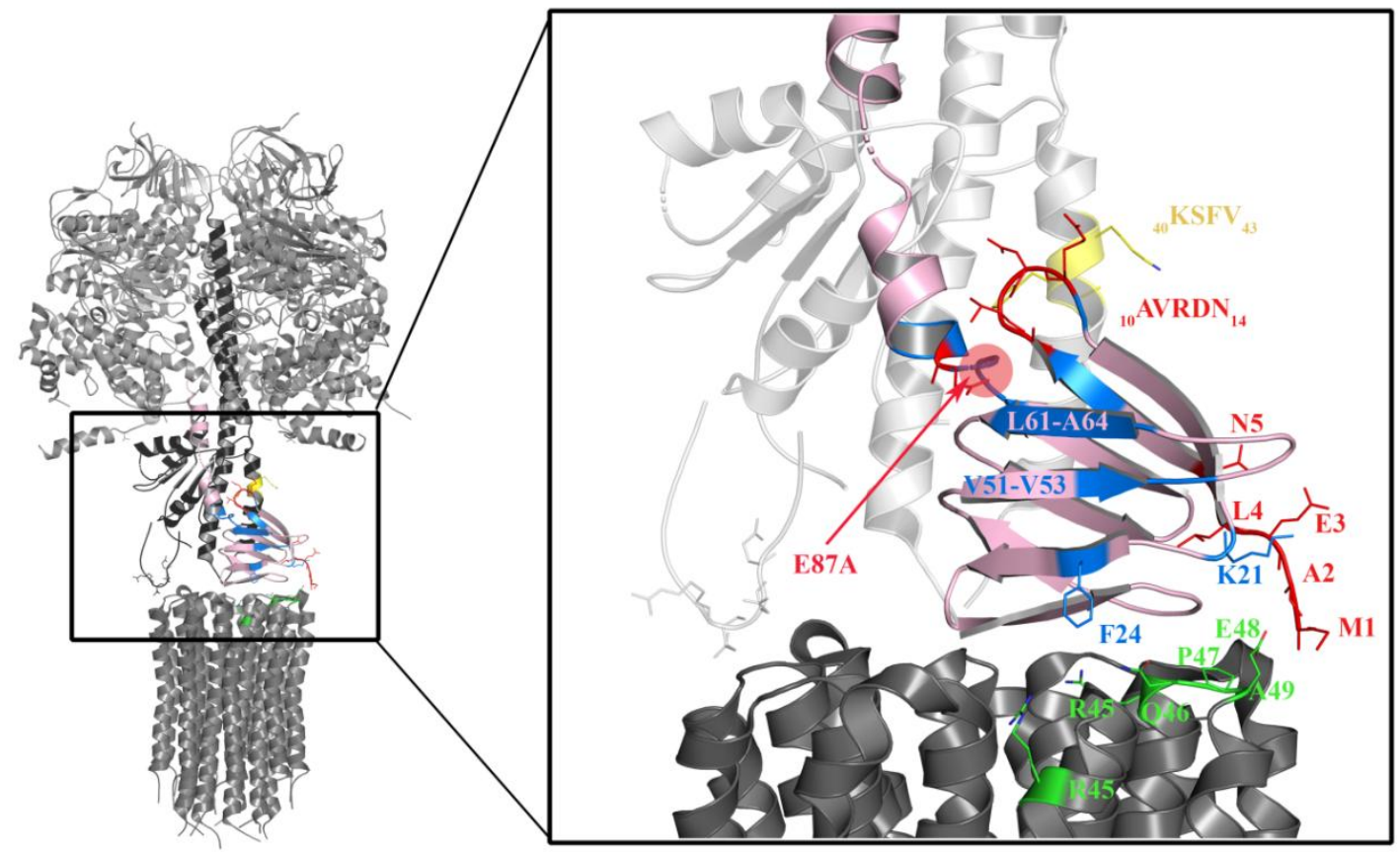

Figure 4.4: Communication of the rotational movement of $\boldsymbol{c}$-ring through $\boldsymbol{M t \varepsilon}$. The enhanced view reveals the proposed residues for the coupling of the $c$-ring rotation with the conformational transition of $M t \varepsilon$ from compact to extended conformation. The $c$-ring residues ${ }_{45} \mathrm{RQPEA}_{49}$ proposed to interact with the $M t \varepsilon N T D$ epitope ${ }_{1}$ MAELN $_{5}$ and residues F24 and/or K21 to transfer the rotational force. The sterical alterations of their side-chains further transfer the force through the epitopesV51-V53, L61A64 towards ${ }_{10} \mathrm{AVRDN}_{14}$ that conveys the structural change to the $\gamma$ epitope ${ }_{40} \mathrm{KSFV}_{43}$. The same structural alterations are also conferred towards the $M t \varepsilon$ hinge region A81-I90 that in turn could affect the extension of $M t \varepsilon C T D$ from compact state and confer the structural alterations to the $\alpha_{3}: \beta_{3}$ hexamer. The mutation in MtcE87A, in the hinge region, is marked with the red circle.

Alternatively, the lack of polar interactions caused by the substitution of the side chain E87A mutant could cause the delay/failure of the Mt\&CTD helices to retract from the central cavity formed by the catalytic subunits $\alpha$ and $\beta$. This could, in turn, cause a sterical hindrance within the catalytic hexamer, stabilizing the unfavorable angular position of subunit $\gamma$ that prevents the binding of the ATP to the empty site in subunit $\beta$. In any case, these alteration(s) could potentially also affect the rate of ATP synthesis, which will be addressed in section 4.6.

Apart from the "hinge" region including the epitope A81-I90, the NTD R62 residue was shown to interact with D60 in NTD and A116 in CTD, forming a link between those two domains. To characterize the type of interaction maintaining this contact, I substituted R62 with leucine and generated the reconstituted complex $\alpha_{3}^{c h i}: \beta_{3}: \gamma: M t \varepsilon R 62 L$. Its ATPase activity was not significantly different from the value obtained for the $\alpha^{c h i}{ }_{3}: \beta_{3}: \gamma: M t \varepsilon$ indicating that the R62L substitution did not alter the interactions within $M t \varepsilon$ that could affect the catalytic activity of the complex. The lack 
of the effect of R62L mutation on the complex activity could indicate that the guanidino group of R62 is not involved in the maintenance of the protein intramolecular interactions and/or topology and that the aliphatic side-chain could play a more significant role. In addition, this finding further establishes the importance of the "hinge" region residue E87 that was shown to reduce the ATPase activity.

\subsection{Importance of the NTD for the Mte stability}

The role of the $\mathrm{N}$-terminal terminal domain of subunit $\varepsilon$ is well-documented (5). As it has been shown for the enzymes of G. stearothermophilus and E. coli, the association of subunit $\varepsilon$ to the $\gamma$ is a critical requirement for the proper assembly of $F_{1}$ to $F_{O}(321)$. The NTD is conserved amongst the wide variety of bacterial species, and it is the primary epitope of subunit $\varepsilon$ that associates with the Rossmann fold motif of subunit $\gamma$ to form a stable, fully functional central stalk. Its association increases the contact surface adding to the interactions of the foot of subunit $\gamma$ and the $c$-ring surface, facilitating the assembly and propagation of the rotational forces between the domains, which was confirmed with the studies that report no membrane-associated ATP hydrolysis activity upon the deletion of subunit $\varepsilon$ in E. coli (321-323). However, when the chloroplast ATP synthase was depleted of the subunit $\varepsilon$, the assembly was not affected, but the interactions that facilitated the coupling of ATP hydrolysis with proton translocation were abolished (324). Moreover, the systematic deletion of the $E$. coli $\varepsilon$ NTD residues showed that the initial 15 residues-deletion did not affect the assembly and catalysis to a great extent, while the truncation of the initial 16 residues leads to a defective enzymatic activity, proton translocation as well as partial assembly (5).

The phenomenon described for $E$. coli $\varepsilon$ could possibly have had a similar manifestation in the mycobacterial enzyme. As seen from the assembly model (Figure 4.4) that includes the structural coordinates of the $M$. phlei $c$-ring (PDB ID: 4V1H) and the recently solved NMR structure of MtE (PDB ID: 5YIO, (209)), the residues of the loop of $c$-ring monomers Q46, E48, and A49 were in the proximity of the ${ }_{1} \mathrm{MAELN}_{5}$ motif of $M t \varepsilon N T D$. Thus the mutant $M t \varepsilon_{6-121}$ was generated by truncating the ${ }_{1}$ MAELN $_{5}$ peptide to form the reconstituted $\alpha^{c h i}{ }_{3}: \beta_{3}: \gamma: M t \varepsilon_{6-121}$ complex (205). From the $M t \varepsilon_{6-121}$ isolation, it was apparent that the truncation caused destabilization of the protein fold, which manifested as oligomerization (Figure 4.5-A). The majority of the 
protein molecules was indeed in the oligomeric fraction, with a small population of molecules found to elute at the monomer-specific volumetric range of 13-14 ml.

The reconstitution efficiency of $\gamma: \varepsilon$ was measured by densitometry to be lowered to 1:0.14, a value far below the one measured for the $\alpha^{c h i}{ }_{3}: \beta_{3}: \gamma: M t \varepsilon(1: 0.5)$, indicating that the ${ }_{1}$ MAELN $_{5}$ epitope is required for proper binding to subunit $\gamma$ and presumably coupling of the catalytic activity with proton translocation. Indeed, when measured, the ATP hydrolysis of the $\alpha^{c h i}: \beta_{3}: \gamma: M t \varepsilon_{6-121}$ complex was $33 \%$ lower than the value measured for the $\alpha^{c h i}{ }_{3}: \beta_{3}: \gamma: M t \varepsilon$ but still higher than the value obtained for the complex lacking MtE $\left(\alpha^{c h i}: \beta_{3}: \gamma\right)$ (Figure 4.5-B). This finding indicated that even

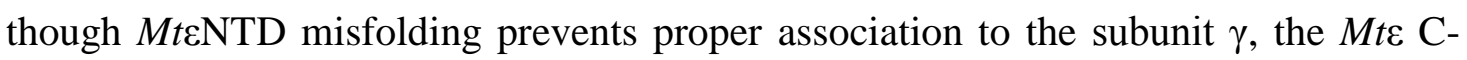
terminal domain was able to convey the alterations to the catalytic hexamer.

As seen (Figure 4.4), the intramolecular interactions derived from the NMR structure (209), define the $M t \varepsilon{ }_{10} \mathrm{AVDRN}_{14}$ epitope as a linking motif that propagates the structural alterations towards the $M t \varepsilon C T D$. The $M t \varepsilon$ epitope ${ }_{1} \mathrm{MAELN}_{5}$ could possibly interact with it via the MtENTD $\beta 1$-sheet and circumstantially influence, at least in part, the binding to the $\gamma_{40} \mathrm{KSFV}_{43}$ motif.

\subsection{Residue E87 of MtE is important for M. smegmatis bioenergetics}

To investigate how such hypothetical conformational alteration would affect mycobacterial energetics and possibly phenotype, a M. smegmatis mutant atp $C^{\mathrm{E} 87 \mathrm{~A}}$ which features the same mutation in subunit $\varepsilon$ was engineered (section 3.24).

The screening of isolated IMVs for their ATP synthetic/hydrolytic activities and the ATP driven proton pumping across the membrane showed alterations of the catalytic activity caused by the mutation. The $M$. smegmatis strain with $\operatorname{atp}^{\mathrm{E} 87 \mathrm{~A}}$ mutation displayed a reduced ATPase activity by $11 \%$ (Figure 4.5), which corroborated the in vitro ATPase activity data obtained for the complex $\alpha^{c h i}: \beta_{3}: \gamma: M t \varepsilon \mathrm{E} 87 \mathrm{~A}$. The verification of the $a t p C^{\mathrm{E} 87 \mathrm{~A}}$ mutation effect also came from the WT complemented strain that showed partial recovery of the ATPase activity up to 93\%. These facts pointed out that the E87 contributed to a strong coupling of NTD to CTD. The substitution E87A has removed the negatively charged side chain of the glutamate from the interaction network described in section 1.1.10 (Figure 1.17) which could cause an unspecific/uncoupled conformational transition of Mt\&CTD between the closed and extended conformations during the catalysis in ATP hydrolysis direction. 

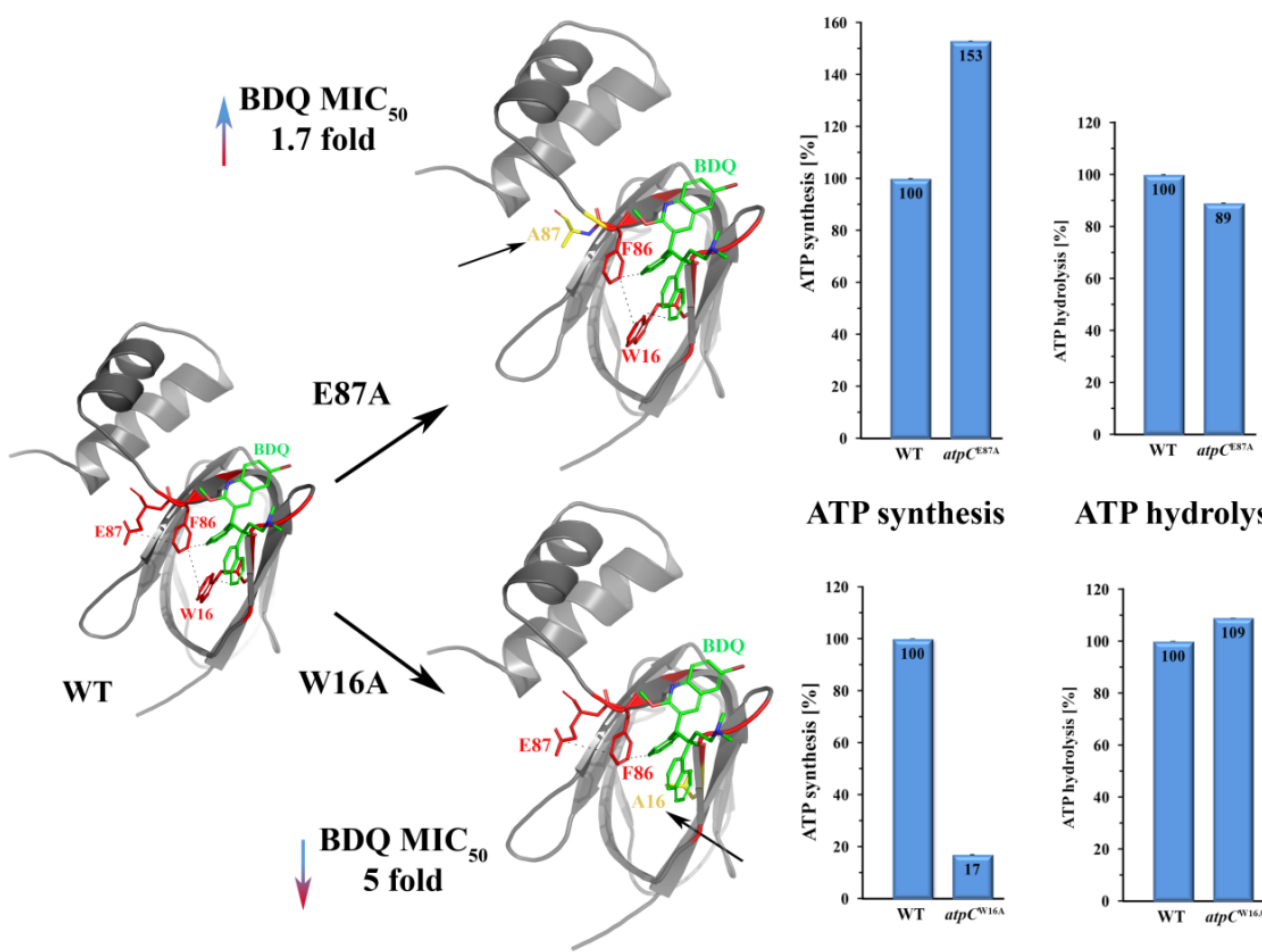

\section{ATP hydrolysis}
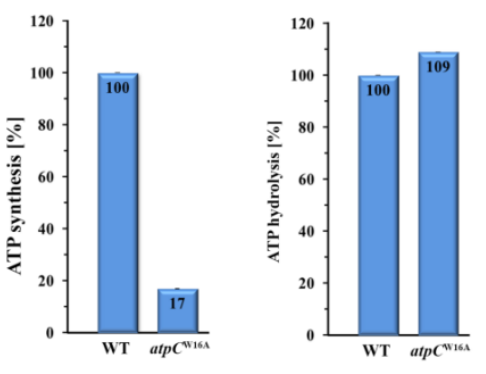

Figure 4.5: Effect of W16A and E87A mutations on BDQ affinity and enzymatic traits in IMVs. The cartoon representation of the $M t \varepsilon$ showing the residues involved in the BDQ binding pocket represented as red sticks. The mutation of the residue E87A (alanine showed in yellow, marked with the arrow, top cartoon) in the hinge region between NTD and CTD lead to the increase in BDQ tolerance (BDQ $\mathrm{MIC}_{50}$ increased 1.7-fold), increase in ATP synthesis (53\%) and decrease in ATP hydrolysis (11\%). The mutation of the residue W16A (alanine shown in yellow, marked with the arrow, bottom cartoon) in the NTD region directly involved in BDQ binding lead to the 5-fold decrease in BDQ tolerance (BDQ $\mathrm{MIC}_{50}$ decreased 5-fold), drop in ATP synthesis (83\%) and mild increase in ATP hydrolysis (9\%). BDQ shown as green sticks.

As ATP hydrolysis is coupled with proton-translocation via the central stalk $\gamma$ and $\varepsilon$, it was expected that the drop of $11 \%$ of mutant ATPase activity would also reduce proton pumping. When the activity was measured in the presence of $2 \mathrm{mM}$ ATP, no difference was observed indicating that the proton pumping activity was not affected by the E87A mutation. These findings were considered in the light of the fact that the mycobacterial F-ATP synthase does not show ATP-driven pumping activity $(2,325)$ and thus the fine differences that could have arisen, could avoid detection due to their low magnitude. To affirm that the vesicles are capable of proton pumping and that the detection of fluorescence was possible, the M. smegmatis WT IMVs were treated with NADH, that successfully activated the proton translocation, but via the NADH dehydrogenase. Upon application of the un-coupler SF6847, the protons leaked outside the IMVs, and the measured ACMA fluorescence returned to the initial 
value. This observation validated that the IMVs are intact and are a reliable tool to measure these parameters.

Considering the bi-directional nature of the F-ATP synthase-mediated catalysis, the effects observed on the hydrolysis could also indicate that the ATP synthesis could be affected. Thus ATP synthesis in the IMVs was measured and interestingly it was shown that the $a t p C^{\mathrm{E} 87 \mathrm{~A}}$ IMVs displayed $53 \%$ higher rate of ATP synthesis than the WT (Figure 4.7), while the $\varepsilon \mathrm{WT}$-complemented atp $C^{\mathrm{E} 87 \mathrm{~A}}$ strain reduced the ATP synthesis rate close to the $117 \%$ of the value measured for the WT. The complemented strain expresses both variants of the subunit $\varepsilon$, WT, and E87A mutant, however, the levels of their expression as well as the efficiency of the assembly into the $\mathrm{F}_{1} \mathrm{~F}_{\mathrm{O}}$ ATP synthase are not yet well documented which obstructed our understanding of the complementation phenomenon.

In contrast, as shown previously (209) the mutation in $\varepsilon \mathrm{NTD}$, atp $C^{\mathrm{W} 16}$ caused the reduction of $83 \%$ of ATP synthetic capacity (Figure 4.7) due to the sterical alterations in the network of interactions significant for conformational transition (section 1.1.10, Figures 1.17 and 1.18). This mutation altered the coupling of proton translocation through the $c$-ring with the catalytic hexamer $\alpha_{3} \beta_{3}$ manifesting as a lower catalytic rate of ATP synthesis. Taken these facts together, it is hypothesized that the mutation E87A could cause such a dramatic increase (53\%) in a similar fashion; the substitution of the negatively charged glutamate side chain with alanine could trigger alteration of intra-molecular network of $\varepsilon$ (section 3.4.3) that could force the topological rearrangement of its closed conformation abolishing the interactions of عCTD with $\alpha_{3}: \beta_{3}$ hexamer. These events could, in turn, increase the speed of rotation by inducing the conformational change in active sites in a faster manner.

Considering that the mutation caused the increased ATP synthesis, we run preliminary phenotypic characterization. While there were no differences in the colony and single cell morphology, growth on solid and liquid media (7H10 and 7H9, respectively), the E87A mutation caused a 1.75 fold decrease in the sensitivity to BDQ (Figure 4.7). The opposite effect was observed in the mutant $a t p C^{\mathrm{W} 16 \mathrm{~A}}$, whereby the mutation W16A caused a 5-fold increase in BDQ sensitivity (WT, $10 \mathrm{nM}$; atp $C^{\mathrm{W} 16 \mathrm{~A}}, 2$ $\mathrm{nM}$ ) (108) which has been caused by geometrical alterations in the BDQ binding pocket (Figure 4.7). As revealed by the NMR experiments, the substitution of the bulky W16 chain with alanine, enhanced the BDQ binding affinity by improving the spatial coordination of the BDQ binding pocket (209). In light of these facts, the 
$\mathrm{MIC}_{50}$ shift from $\sim 8 \mathrm{nM}$ in WT to $\sim 14 \mathrm{nM}$ in $\operatorname{atp} C^{\mathrm{E} 87 \mathrm{~A}}$ could be explained by the decrease in the BDQ affinity of subunit $\varepsilon$, caused by the E87A mutation that could indirectly alter the geometry of its binding pocket through the coupling network (section 1.1.10, Figures 1.17 and 1.18). Alternatively, the reduced BDQ susceptibility of the $a t p C^{\mathrm{E} 87 \mathrm{~A}}$ mutant could have arisen simply due to the mutation-induced increased ATP production via F-ATP synthase, which counteracts the effect of BDQ on the whole cell level.

Taken together, these facts indicated that, in the abundance of the glycolytic metabolites, like the one found in $7 \mathrm{H} 9$ media, the mutant $a t p C^{\mathrm{E} 87 \mathrm{~A}}$ strain does not exhibit any phenotypic effect different than the WT. In contrast, in a more restrictive environment, like the one triggered by BDQ treatment, E87A mutation might prove to be beneficial for the survival of the mycobacterial cell. This illustrates the importance of the subunit $\varepsilon$, in particular, its connecting region, in the coupling, regulation of the catalysis and resistance to antibiotics, similar to what has earlier been described for the $\operatorname{atp} C^{\mathrm{W} 16 \mathrm{~A}}$ mutant (326).

\subsection{Spatial orientation of epitopes $M t \alpha_{514-549}$ and Mt\&CTD within the $\alpha^{c h i}{ }_{3}: \beta_{3}: \gamma: M t \varepsilon$ mutant complexes}

As described in section 1.1.10 (Figure 1.16) and discussed in section 4.2 (Figure 4.3), mycobacterial $\varepsilon$ has a significantly shorter CTD that spans the modeled length of $42 \AA$ while $E c \varepsilon$ and $G s \varepsilon$ extend up to $67 \AA$. Considering that EcF-ATP synthase operates in both ATP hydrolytic/synthetic mode and can utilize ATP to generate PMF, $\varepsilon$ is known to transit from closed to open conformation in a bimodal manner with a slow dynamics due to the organization of its CTD (helix 1-linker-helix 2-linker-hook) (197). It has been shown that helix 2 interacts with the coupling regions of subunit $\alpha$ and $\beta$ while the CTD "hook", comprised of residues ${ }_{136} \mathrm{KAM}_{138}$ extends to the proximity of the P-loop in both $\alpha$ and $\beta$, interacts with specific $\beta Y 331$ affecting the nucleotide binding and stopping the rotation as a consequence (197). Similarly, Gse modulates the P-loop binding affinity as well as the direction of rotation but with faster conformational dynamics than $E c \varepsilon$ (184). These modalities of operation seem to

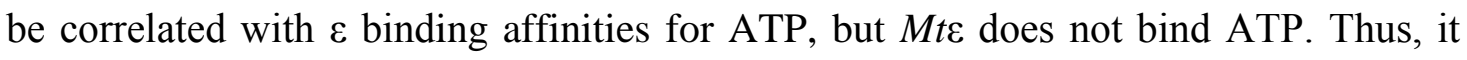
was hypothesized that due to the lack of ATP binding site and a shorter Mt\&CTD, another mycobacteria-specific epitope could interact with Mte to establish the regulation (3). 
The cysteine substitution mutants of $\alpha^{\text {chi }}$ in the chimeric complex $\alpha^{\text {chi }}{ }_{3}: \beta_{3}: \gamma$ : together with $M t \varepsilon$ showed that the epitopes $\alpha^{c h i}$ and $\varepsilon$ CTD helix 2 could interact with each other. Namely, the crosslinks formed between $\alpha^{c h i}-\mathrm{S} 400 \mathrm{C}$ and MtED121C within the $\left(\alpha^{\text {chi }}-\mathrm{S} 400 \mathrm{C}\right)_{3}: \beta_{3}: \gamma: M t \varepsilon \mathrm{D} 121 \mathrm{C}$ complex, revealed that the very C-terminus of $M t \varepsilon$ could extend into the catalytic hexamer (in a homologous way to the "hook" mechanism of EcE) (Figure 4.6) with a difference of reaching only the coupling regions in $\alpha$ and $\beta$ subunits $\left(\alpha-394\right.$ AFAQFGSDLD 403 and $\beta-{ }_{390}$ DELSDED $\left._{396}\right)$ and not the P-loop.

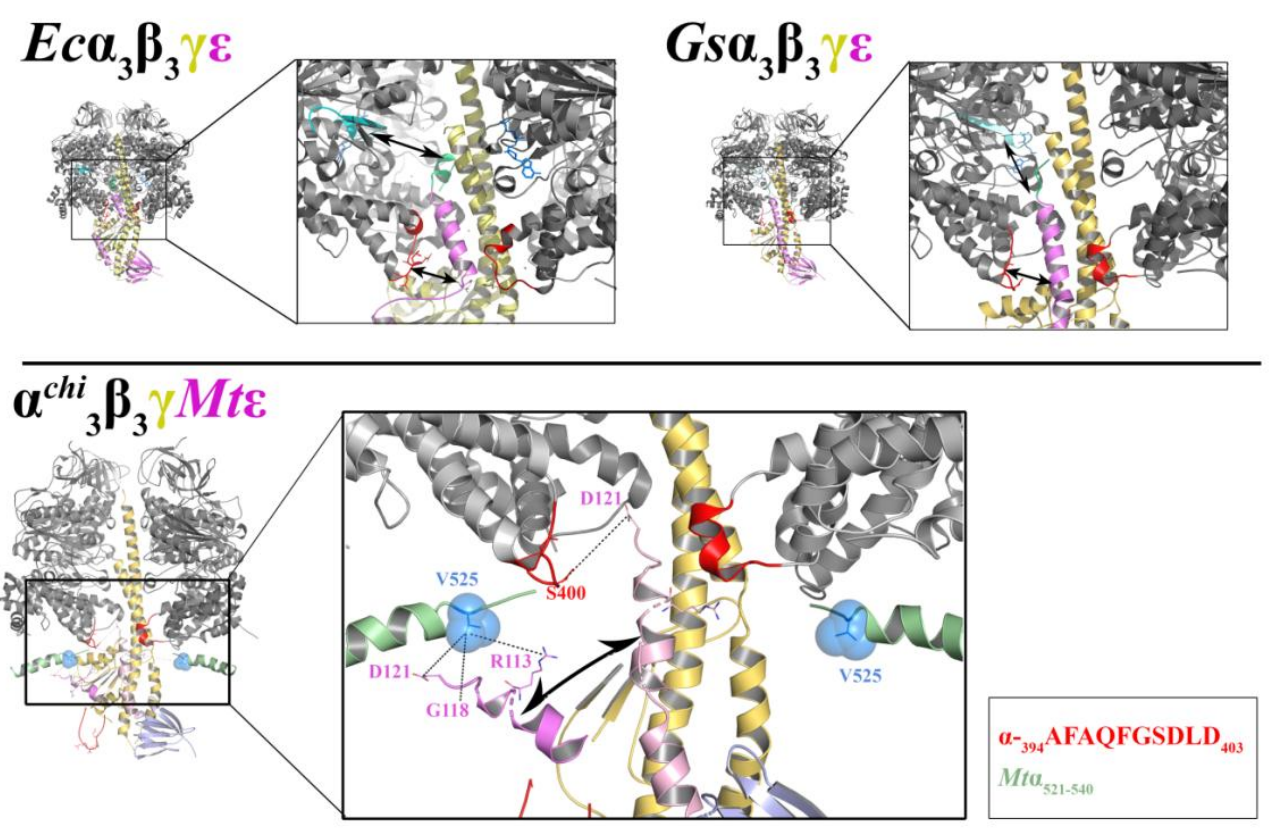

Figure 4.6: The interactions of the subunits $E c \varepsilon, G s \varepsilon$, and MtE within $\mathbf{F}_{1}$ domain. Top panel shows overall and enhanced views of $E c \alpha_{3}: \beta_{3}: \gamma: \varepsilon$ and $G s \alpha_{3}: \beta_{3}: \gamma: \varepsilon$ displaying the length and insertion of subunit $\varepsilon$ (magenta). Lime green indicates $\varepsilon$ CTD tip residues that interact with epitopes in the proximity of the P-loop (sky blue). The second helix of $\varepsilon$ CTD interacts with both $\alpha$ and $\beta$ coupling epitopes (red). Bottom panel shows the overall and enhanced view of the complex $\alpha^{\text {chi }}: \beta_{3}: \gamma: M t \varepsilon$. The interactions between MtcCTD (magenta) and two epitopes in $\alpha^{\text {chi }}$ are shown as the dashed lines. Enhanced view in the frame reveals the crosslink of Mt\&CTD with the coupling region of $\alpha^{\text {chi }}$ ${ }_{394} \mathrm{AFAQFGSDLD}_{403}$, red) that was found between residues $\alpha^{\text {chi }} \mathrm{S} 400$ and $M t \varepsilon \mathrm{D} 121$. The other

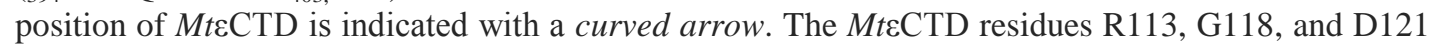
form a crosslink with residue V525 (blue spheres) found in the mycobacteria-specific epitope $M t \alpha_{514}$ 549. The segment of this epitope whose structure is determined to form a helix $\left(M t \alpha_{521-540}\right)$ is labeled in grass green.

In addition, the crosslinking experiments on the mutant complex $\left(\alpha^{\text {chi }}\right.$ -

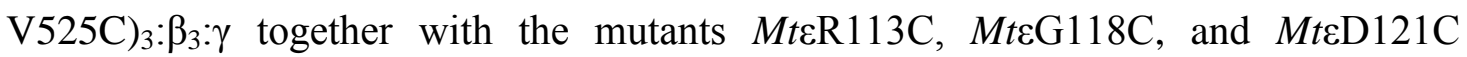
showed the existence of the unique interaction of the mycobacterial $\alpha$ epitope $\left(M t \alpha_{514-}\right.$ 549) with $\gamma$ and MtECTD. 
Firstly, the crosslink formation between cysteines of $\alpha^{\text {chi }}$-V525C and $\gamma \mathrm{C} 109$ in the complex $\left(\alpha^{c h i}-\mathrm{V} 525 \mathrm{C}\right)_{3}: \beta_{3}: \gamma$ placed the $M t \alpha_{514-549}$ in the proximity of the $\gamma$ Rossmann fold residues Q104, R106 and C109, a domain directly involved in the transmission of the rotational energy from $c$-ring to catalytic $\alpha$ and $\beta$ subunits and vice versa. This finding corroborates the previous observations that the $\alpha^{c h i}{ }_{3}: \beta_{3}: \gamma$ complex exhibits lowered ATPase activity and angular velocity of $\gamma$ in comparison to the same parameters of the homologous complex from G. stearothermophilus $\alpha^{c h i}: \beta_{3}: \gamma$ (3).

Furthermore, the crosslinks formed between $\alpha^{c h i}-\mathrm{V} 525 \mathrm{C}$ and $M t \varepsilon \mathrm{R} 113 \mathrm{C}$, $M t \varepsilon \mathrm{G} 118 \mathrm{C}$ and $M t \varepsilon \mathrm{D} 121 \mathrm{C}$ within the reconstituted complexes advocated strongly the fact that the helix 2 of $\varepsilon$ CTD can interact with the mycobacterial epitope $\left(M t \alpha_{514-549}\right)$.

These interactions, taken together with the interaction of $\alpha^{\text {chi }}$ and Rossmann fold, comprise a unique mycobacterial regulatory interface. The "longer" MtaCTD could be a compensatory mechanism for the "shorter" MteCTD, and together with subunit $\gamma$, they suppress the ATP hydrolysis in $M t \mathrm{~F}_{1} \mathrm{~F}_{\mathrm{O}}$ which presents a remarkable evolutionary adaptation in the regulation of mycobacterial F-ATP synthases.

These epitopes can, however, be utilized to disrupt the inhibition of ATPase activity by designing new molecules that could interfere with their interactions. One of the possible ways would include the obstruction of the up-down motion of MtECTD by preventing its contact with partner regulatory epitopes in subunit $\alpha^{\text {chi }}$ (396 $\mathrm{AQFGSDLDK} 404$ and a mycobacteria-specific $M t \alpha_{514-549}$ ). Due to the distribution of the predominantly polar residues in this space, the active molecule would have to exhibit complementary topological charge distribution in order to bind successfully.

As seen in the NMR experiments, the position and the importance of the MtE residue E87 and its adjacent epitopes would make this surface a promising candidate for drug design. In this scenario, the conformational transition of MtECTD from closed to extended could hypothetically be prevented by using a selected molecule with desired properties to clamp on Mt\&CTD anti-parallel helixes and restrict their motion. However, the in silico compound library screening has not been performed until the conclusion of this thesis, and thus, no potential molecules were identified. Nevertheless, the specific binding of the novel compounds would ideally trigger the activation of continuous ATP utilization, which would eventually lead to the depletion of $M t b$ cell ATP pools holding potential for clinical applications. 


\subsection{Special features of $A$. woodii $\mathbf{F}_{1} \mathbf{F}_{\mathrm{O}}$-ATP synthase}

A. woodii has been isolated from a marine estuary and described as homoacetogenic $(234,237)$ bacterium that can synthesize acetate by anaerobic fermentation from $\mathrm{H}_{2}: \mathrm{CO}_{2}$ mixture. The acetate is excreted out of the cell, lowering the $\mathrm{pH}$ of the surrounding media, which makes the utilization of the proton-motive force to synthesize ATP a futile task. However, A. woodii makes use of a different mechanism; it instead utilizes the $\mathrm{Na}^{+}$gradient to drive the ATP synthesis. To enable a function such is sodium conduction, $\mathrm{F}_{1} \mathrm{~F}_{\mathrm{O}}$-ATP synthase has undergone an evolutionary leap and adaptation of its $c$-ring took place (7).

Virtually all the bacterial F-ATP synthases possess the $\mathrm{F}_{\mathrm{O}}$ homo-oligomeric $c$ ring comprised of the $c$ subunit monomers coded by a single gene, atpE (in bacteria), while the $c$-ring of $A$. woodii displayed a unique specialization. From the analysis of its genome, it was determined that the $A$. woodii carries a specific F-ATP synthase operon (238); it harbors the genes $a t p E_{1}, a t p E_{2}$, and $a t p E_{3}$ that code for three subunits $c, c_{1}, c_{2}$, and $c_{3}$. The structural analysis has shown that $c$-ring is comprised of ten subunits (7), the $c_{1}, c_{2}$, and $c_{3}$ in the determined stoichiometry 1:8.3:0.7 (6). The $c_{2}$ and $c_{3}$ possess two identical transmembrane helices connected via a short loop ${ }_{42} \mathrm{RQPE}_{45}$ on the cytoplasmic side while the unique subunit $c_{1}$ is comprised of a short helix on the extracellular side as well as four trans-membrane helices connected via two loops, ${ }_{59} \mathrm{KNPT}_{62}$ and ${ }_{142} \mathrm{IRPE}_{145}$ on the cytoplasmic side (7). The recent studies show that the deletion of the $c_{1}$ subunit causes a defective $\mathrm{Na}^{+}$and a $70-80 \%$ reduction in ATPase activity (6) demonstrating that this subunit plays a significant role in structure and catalysis.

Apart from the chimeric $c$-ring, another unique feature was identified in this thesis to be positioned in subunit $\gamma$, namely a unique 17-amino acid epitope ${ }_{195}$ TSGKV-KITEETKEEKSK 211 whose current structure and function are unknown. Sequence alignment revealed that the peptide is positioned similarly to the ${ }_{240}$ CDINGKCVDAAEDELFRL- TTKEGKLTVERDMIKT $_{273}$ peptide sequence of the spinach $\gamma$, whose regulatory role via redox mechanism is established and well described $(151,327)$

In order to get insight into the overall complex assembly, its activity, and the function of its unique $\gamma$ loop, the WT enzyme was investigated using negative-stain electron microscopy (NS-EM). Obtained images of the pure complex with a defined stoichiometry, yielded ten 2D classes with clearly distinguishable side-views features. 
As seen from the prominent NS-EM projections (Figure 4.7), the fully assembled complex, comprised of $\mathrm{F}_{1}$ and $\mathrm{F}_{\mathrm{O}}$ domains, was obtained. The connections of the two domains were established via the central stalk in the middle and the peripheral stalk on the side. The entire complex spans to around $204 \AA$, a characteristic length observed for most ATP synthases $(1,143,328)$.

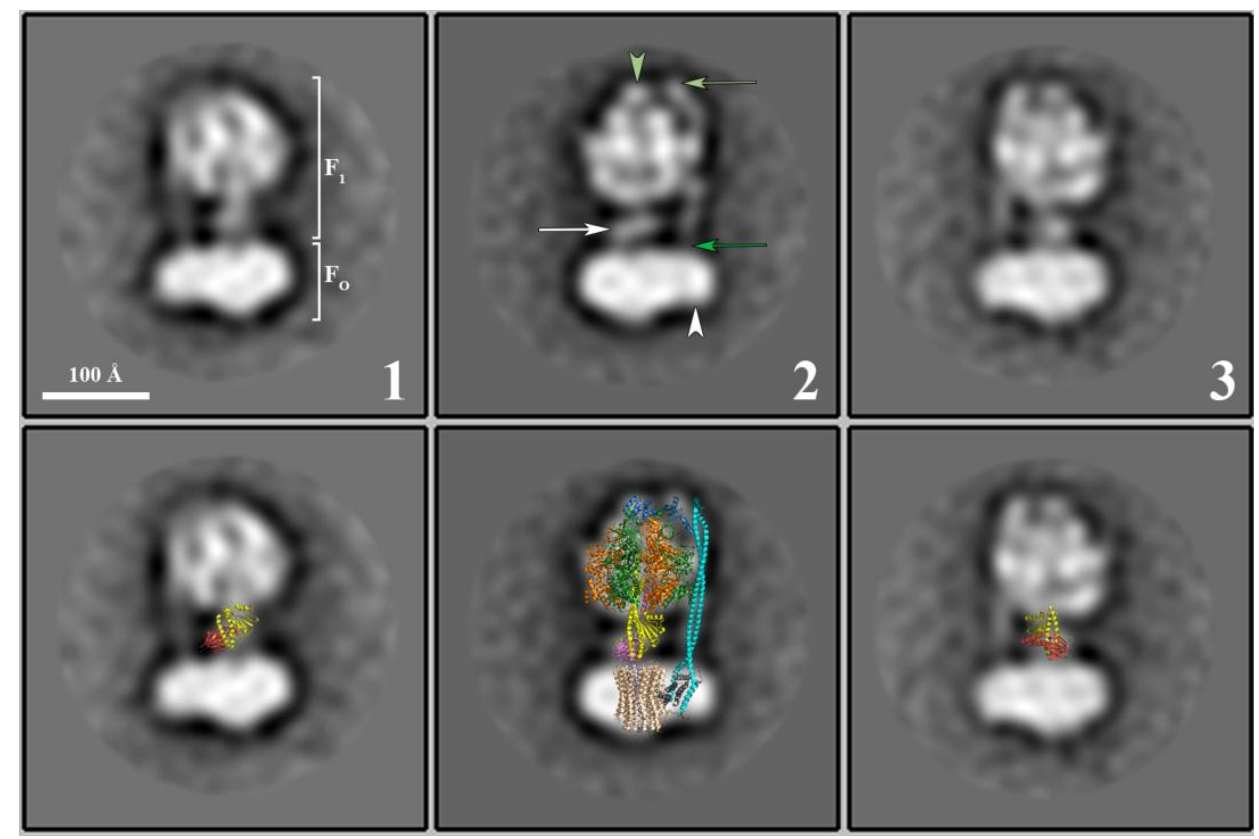

Figure 4.7: 3D homology model fitting the 2D projections of NS A. woodii F-ATP synthase. Panel one shows the lateral view of the complex where the $\mathrm{F}_{1}$ and $\mathrm{F}_{\mathrm{O}}$ parts are distinguished. Green arrows indicate the mass of the peripheral stalk connecting to $\mathrm{F}_{1}$ (light green; top) and $\mathrm{F}_{\mathrm{O}}$ (green; bottom) part, light green arrowhead points to the mass of the $\delta$ subunit, The white arrow indicates the mass of the asymmetrical central stalk. White arrowhead reveals the membrane-embedded subunit $a$ associated with the $c$-ring and a peripheral stalk. Panels 2 and 3 show the side-views of the complex with different rotational positions, as discerned from the orientation of the mass of the central stalk. The lower row reveals the superimposition of the structural homology model of the $A$. woodii F-ATP synthase on its projections acquired with NS-EM. The superimpositions reflect the good fits of the $\alpha_{3}: \beta_{3}$ domain, peripheral- and central stalk subunits as well as the $F_{O}-V_{O}$ domain. The orientations of subunits $\gamma$ (yellow) $\varepsilon$ (red) in the three projections indicate the different orientations of these rotary elements. Additional density in the membrane-part is apparent due to the presence of the detergent micelle around the $\mathrm{F}_{\mathrm{O}}$ part. Figure prepared for (265).

The $\mathrm{F}_{1}$ domain that canonically contains subunits $\alpha, \beta, \gamma \delta$, and $\varepsilon$ is visible as anisotropic globular density, which could indicate the pseudo-hexameric arrangement of subunits $\alpha$ and $\beta$ (Figure 4.9). Furthermore, in comparison to the bacterial VATPases (329) and archaeal Methanosacrina mazei Gö A-ATP synthase (328) that feature two and three peripheral stalks, respectively, A. woodii F-ATP synthase was shown to have only one peripheral stalk, first time visualized in this $2 \mathrm{D}$ reconstruction. As seen in class 2 (Figure 4.7), the A. woodii F-ATP synthase harbors a single 
peripheral stalk comprised of subunits $b_{2}$ connected to subunit $\delta$ on top of the $\alpha_{3} \beta_{3}$ catalytic hexamer and to the $\mathrm{F}_{\mathrm{O}}$ subunit $a$ on the opposite side of its contact to $\mathrm{F}_{1}$. The density corresponding to the peripheral stalk appears to feature an unusual "indent" (Fig 4.7, class 2, peach arrowhead) close to the subunits $\alpha / \beta$ that might interact with their central domain or their interface to stabilize and/or in part contribute to the regulation of the activity.

The density corresponding to $\gamma-\varepsilon$ central stalk is visible in multiple orientations with respect to $\alpha_{3} \beta_{3}$ in the represented class averages. As seen from the fitted structural model (Fig 4.7, lower row), the orientation of central stalk indicates that the isolation of the enzyme in different rotational states was achieved. By comparing the classes 1 and 3 , it is apparent that the central stalk $\gamma-\varepsilon$ assumes different orientation with respect to the rest of the $\mathrm{F}_{1} \mathrm{~F}_{\mathrm{O}}$. In class 1 the highest density, possibly corresponding to the Rossmann fold of the subunit $\gamma$ (330), is facing the peripheral stalk subunits, while in class $3, \gamma-\varepsilon$ faces away from the peripheral stalk.

The $\mathrm{F}_{\mathrm{O}}$ domain features subunit $a$ and $c$-ring whose densities are not clearly resolved but their positions can be inferred from the fitted structural model in the lower row. The additional unoccupied density is proprietary to the associated detergent as the $\mathrm{F}_{\mathrm{O}}$ is highly hydrophobic, membrane-embedded domain. As described above, the homology modeling of the subunits allowed a structural alignment with the individual subunit coordinates of the cryo-EM 3D reconstruction of the E. coli $\mathrm{F}_{1} \mathrm{~F}_{\mathrm{O}^{-}}$ ATP synthase (143). The alignment brought A. woodii subunits $\gamma$ and $\varepsilon$ in the proximity of the $c$-ring loops that connect transmembrane helices of $c$-monomers.

From the model (Fig. 4.8), the interactions of subunit $\varepsilon$ and $c_{1}$ and $c_{2 / 3}$ monomers were predicted. The $\varepsilon$ epitope ${ }_{27} \mathrm{RGIEGELAILAEHTP}_{41}$ is in the proximity of the specific loops that connect the transmembrane helices ${ }_{59} \mathrm{KNPT}_{62}$ and ${ }_{142} \mathrm{IRPE}_{145}$ of the $c_{1}$ subunit on the $\varepsilon N T D$ side, and ${ }_{45} \mathrm{RQPE}_{48}$ of $c_{2 / 3}$ on $\varepsilon \mathrm{CTD}$ side of the peptide. These loops may play a role in limiting the lateral "sliding" motion of the subunit $\varepsilon$ with respect to $\gamma$ during the change of the direction of rotation. The side chains of E32, L36, and E38 of $\varepsilon$ subunit project towards the outer side of the $c$-ring. The clockwise motion of residue $\varepsilon \mathrm{E} 32$ could be limited by the $c_{1}$ residues R143 and E145 due to their electrostatic interactions, while $\varepsilon$ L36 could be wedged between $c_{1}$ P61 and T62 side chains. Similarly, the residue $\varepsilon$ E38 would clash into E45 of the adjacent $c_{2 / 3}$ subunit due to the hydrodynamic drag developed upon rotation arrest/reversal. 


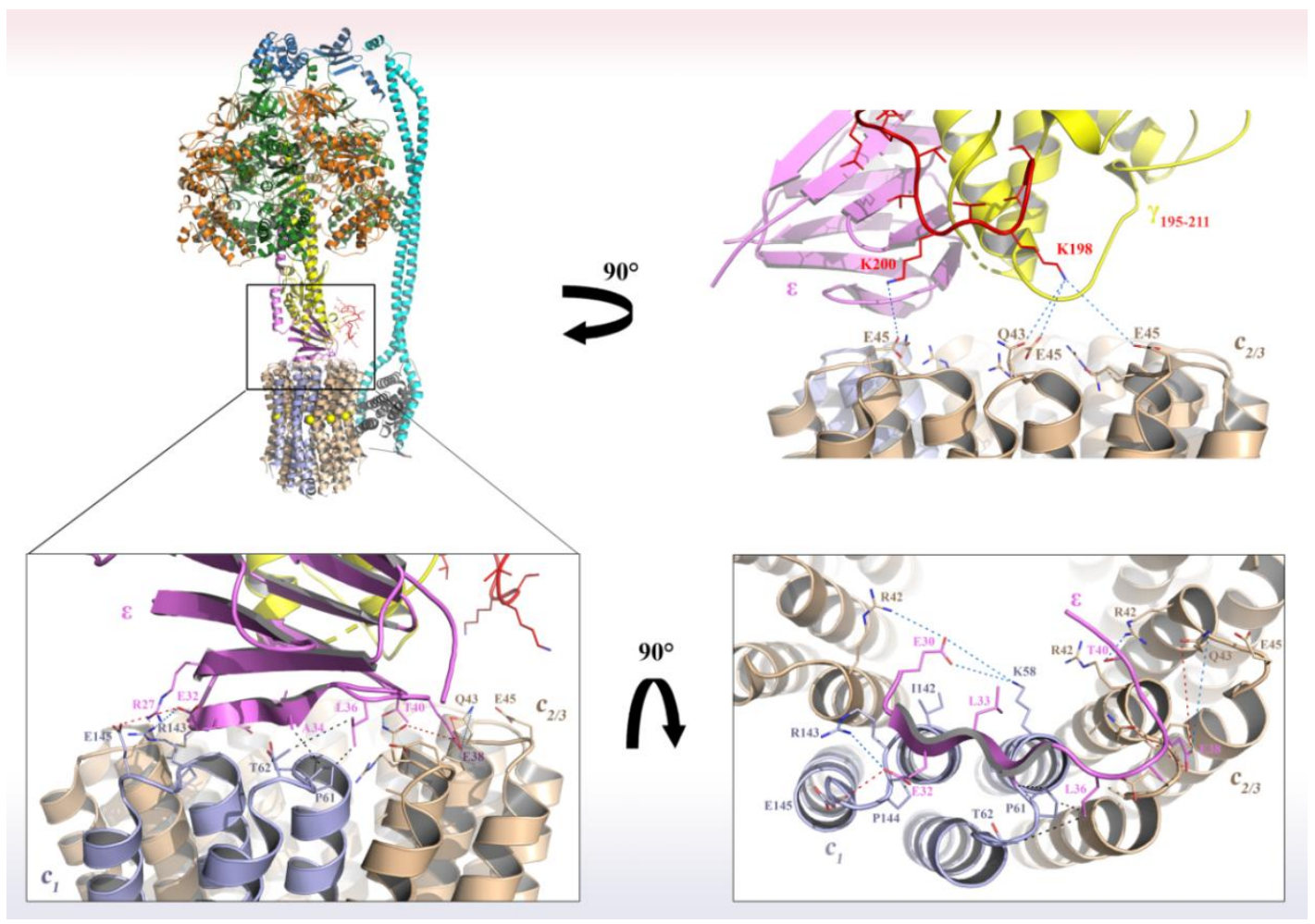

Figure 4.8: Assembly model of $A$. woodii $\mathbf{F}_{1} \mathbf{F}_{\mathbf{O}}$-ATP synthase showing the interactions. The lower left panel shows the side-view of the interactions between subunit $\varepsilon$ (magenta), $c_{1}$ (light blue) and $c_{2 / 3}$ (wheat) subunits. The lower right panel represents a top view of those interactions whereby $\varepsilon$ is represented by the ${ }_{30}$ EGELAILAEHTP $_{41}$ epitope for clarity. The right panel shows the side view of the interactions of subunit $\gamma$ (yellow, loop dyed in red) with the ${ }_{42} \mathrm{RQPE}_{45}$ loops of adjacent $c_{2 / 3}$ subunits. Parts of the figure prepared for (265).

In addition to these interactions, a $\varepsilon E 30$ sidechain projects towards the inner space of the $c$-ring, positioning itself directly above the $c_{l}$ I142 side chain, of the motif ${ }_{142} \mathrm{IRPE}_{145}$, which could be further stabilized via interactions with $c_{1} \mathrm{~K} 59$ of ${ }_{59} \mathrm{KNPT}_{62}$ and R42 of the adjacent $c_{2 / 3}$ monomer loop ${ }_{42} \mathrm{RQPE}_{45}$. To the contrary, if the subunit $\varepsilon$ would associate with the $c_{2 / 3}$ monomer loops ${ }_{42} \mathrm{RQPE}_{45}$ instead of the $c_{1}$, the R42 side chain would invoke a severe sterical clash with $\varepsilon E 30$ residue (Fig. 4.8) reducing the likelihood of such assembly. The outcome of those sterical clashes (Fig 4.9) would be the destabilization of the $\varepsilon$-c-ring interface, which could lead to the defective assembly/function manifesting as disrupted stoichiometry. Indeed, in the previous study, it has been shown that the $c_{1}$ deletion ( $\Delta c_{1}$ mutant) causes defective $\mathrm{Na}^{+}$ translocation, ATPase activity and partial depletion of subunit $\varepsilon$ from the complex (6), which could be explained by the model described here. 

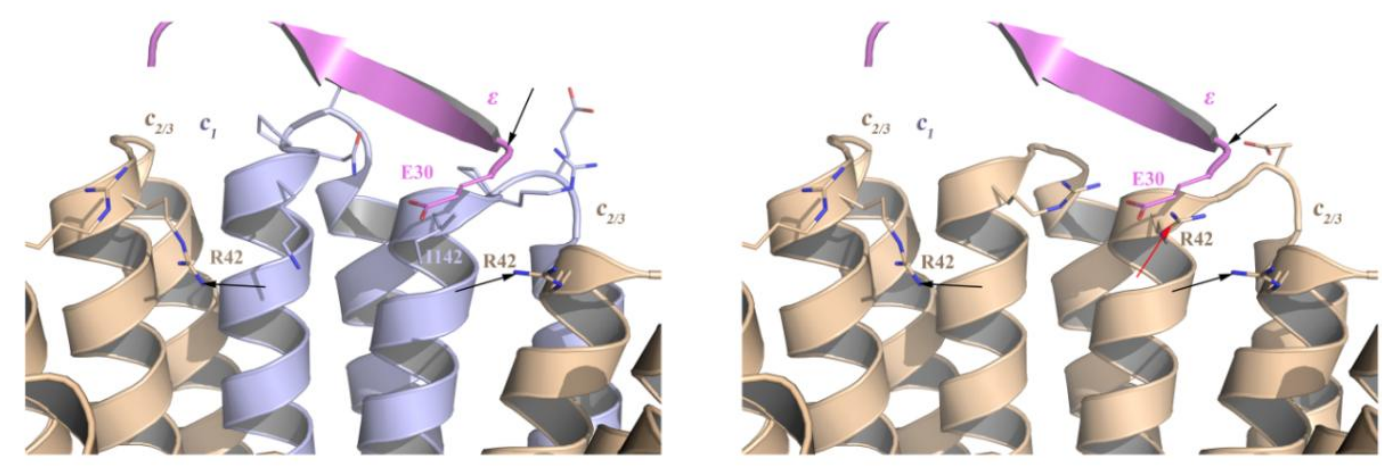

Figure 4.9: Interactions of the inner side of the $c$-ring in $A$. woodii F-ATP synthase. The left panel shows the $\varepsilon E 30$ side chain wedged between R42 of the $c_{2 / 3}$ and K59 of $c_{1}$ in the space provided by $c_{1}$ I142 residue. The right panel shows the arrangement of two subunits $c_{2 / 3}$ in the position of $c_{l}$, which introduces the sterical clash of the $\varepsilon \mathrm{E} 30$ and R42 of $c_{2 / 3}$ marked with the red arrow. Figure prepared for (265).

Furthermore, subunit $\gamma$ harbors a unique 17-amino acid stretch which by its position in the sequence could be a homolog to the specific regulatory loop found in spinach chloroplast $(151,327,331)$. In addition to the interaction interface of $\varepsilon-c_{1}$, described above, the interactions of the $\gamma$ loop and subunit $c_{2 / 3}$ monomers assembled in the $\mathrm{F}_{1} \mathrm{~F}_{\mathrm{O}}$ synthase were modeled. The long side chains of the residue K198, belonging to ${ }_{195}$ TSGKVITEETKEEKSK $_{211}$, come into proximity of the negatively-charged residues, Q43 and E45 of one subunit $c_{2 / 3}$ as well as with a residue E45 of the adjacent subunit $c_{2 / 3}$. Additionally, the side chain of K200 could interact with residue E45 of the third $c_{2 / 3}$ loop, and together these interactions with the interactions of epsilon with $c_{1}$ could strengthen the coupling that enables translocation of $\mathrm{Na}^{+}$and functional catalysis.

Upon deletion of the $\gamma$-loop in the mutant $\gamma_{\Delta 195-211}$, the enzyme reconstituted into proteoliposomes displayed reduced ATPase activity as well as the complete arrest of the ATP synthesis and sodium translocation. Such effects of the $\gamma$-loop deletion hinted that this epitope provides communication of the rotating $c$-ring with the catalytic hexamer. It could be the case that the rotational force of the $c$-ring generated by $\mathrm{Na}^{+}$translocation could be effectively transmitted only if the sufficient interaction surface with both $\gamma$ and $\varepsilon$ subunits was established. This would, in turn, provide enough drag to rotate the entire assembly and drive the ATP synthesis in the catalytic hexamer $\alpha_{3} \beta_{3}$.

In analogy to the regulatory role of $\gamma$-loop in A. woodii, described here, it was reported that the chloroplast $\gamma$-loop acts as an inhibitor of ATP hydrolysis. The 
inhibition occurs upon oxidation of the regulatory cysteine pair, which in turn, causes the conformational alteration of the unique loop and its insertion into the $\alpha-\beta$ interface (151). In addition, mycobacterial F-ATP synthase is characterized by low capacity for ATP hydrolysis, owing to this trait to several adaptations present exclusively in this enzyme (2). One such adaptation is a loop comprised of 14 amino acids of $\gamma_{166-179}$ epitope whose deletion from the genome of $M$. smegmatis caused an increase in ATPase activity, ATP-driven proton translocation and a significant reduction of ATP synthesis (206). These experiments marked the $\gamma_{166-179}$ loop as an inhibitor of ATP consumption coupled to the generation of the proton-motive force and described it as a conditionally unidirectional "proton-tight" seal, present only in mycobacterial F-ATP synthases.

(A)

S. oleracea (chloroplast)

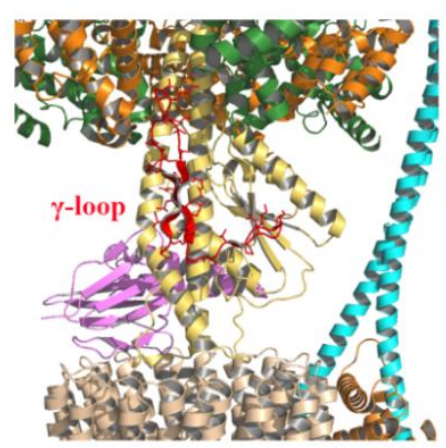

(B)

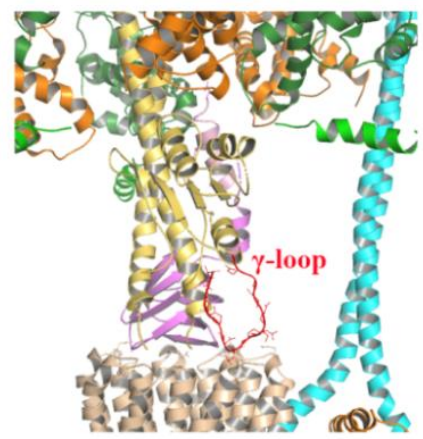

(C) A. woodii

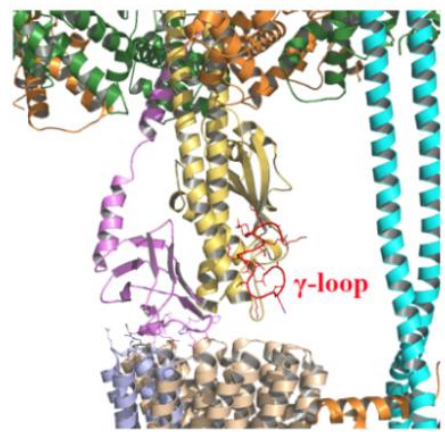

Figure 4.10: The position of the unique regulatory loops in subunit $\gamma$. (A) Cryo-EM reconstruction of $S$. oleracea F-ATP synthase from chloroplast (left panel) shows unique regulatory epitope of subunit $\gamma{ }_{240}$ CDINGKCVDAAEDELFRLTTKEGKLTVERDMIKT $_{273}$ (labeled in red color) (332). The regulation is mediated via oxidation of cysteine pair, conformational alteration of the loop and its insertion into the $\alpha_{3}: \beta_{3}$ catalytic hexamer. (B) A homology model of $M$. tuberculosis F-ATP synthase (central panel) revealing the position of the subunit $\gamma$ unique regulatory loop $\left({ }_{166}\right.$ DNGEDQRSDSGEG $_{178}$ ) (labeled in red color). The regulation is accomplished via interaction with the $c$-ring to establish a unidirectional proton conductance in the ATP synthesis direction. (C) A homology model of $A$. woodii F-ATP synthase revealing the unique regulatory epitope of subunit $\gamma$ ( ${ }_{195}$ TSGKVITEETKEEKSK ${ }_{211}$, colored in red). Color coding: $\alpha$-forest green; $\beta$ - orange; $\gamma-y e l l o w ; \gamma$ loop - red; $b_{2}$ - cyan; $\varepsilon$ - magenta; $c$-ring - wheat; $c_{1}$ - violet. Figure prepared for (265).

As seen from the examples above and Figure 4.10, it is clear that these mechanisms contribute to the energy homeostasis in the conditions optimal for these life forms. In analogy, in order to utilize the sodium-gradient for ATP synthesis, $A$. woodii has developed specific adaptations such as hetero-oligomeric $c$-ring and $\gamma_{195-211}$ 
epitope. The emergence of these mechanisms appears to have occurred as a response to the high salinity and low $\mathrm{pH}$ that resulted from the excretion of acetate during the fermentative growth, which prevented the utilization of the PMF to generate the metabolic energy (250).

Furthermore, it was hypothesized that the $\gamma$-loop could undergo phosphorylation at the specific predicted sites (Table 4.1) as a functional adaptation to regulate the catalysis. Indeed, the preliminary data show the binding of the radioactive $\left[\mathrm{P}^{32}\right]$-phosphate isotopes to the loop residues (unpublished data), however, full characterization of these novel traits requires additional experimental validation.

\begin{tabular}{cccc} 
Residue & Motif & CS & Enzyme \\
\hline $195 \mathrm{~T}$ & EELITSGKV & 0.591 & PKC \\
$196 \mathrm{~S}$ & ELITSGKVK & 0.793 & PKC \\
$201 \mathrm{~T}$ & GKVKTTEET & 0.908 & unsp \\
$201 \mathrm{~T}$ & GKVKTTEET & 0.554 & CKII \\
$202 \mathrm{~T}$ & KVKTTEETK & 0.612 & CKII \\
$205 \mathrm{~T}$ & TTEETKEEK & 0.900 & unsp \\
$205 \mathrm{~T}$ & TTEETKEEK & 0.574 & CKI \\
$205 \mathrm{~T}$ & TTEETKEEK & 0.565 & CKII \\
$210 \mathrm{~S}$ & KEEKSKMSD & 0.992 & unsp \\
\hline
\end{tabular}

Table 4.1: The prediction of the phosphorylation sites in subunit $\gamma$. The residues predicted for phosphorylation are shown in red. CS, confidence score ( 0.5 no phosph.; $0.5<\mathrm{x}<1$ phosphorylation probable). PKC, protein kinase C; unsp, unspecified; CKII, casein kinase 2; CKI, casein kinase 1. Predictions carried out by NetPhos 3.1 server $(333,334)$.

The results presented in this study provided functional and, in part, structural insight for the role of the A. woodii specific $\gamma$-loop as well as the overall enzyme assembly and architecture. The interaction models contribute to the understanding of the underlying structural mechanisms that can explain the phenomena of the loss of ATP synthetic activity, increased ATPase- and sodium translocation activity in the $\gamma_{\Delta 195-211}$ mutant. By using the model, interactions responsible for partial disassembly and depletion of subunit $\varepsilon$ caused by the deletion of $c_{1}$ subunit (6), can now be inferred. Besides these findings, the capacity of the A. woodii F-ATP synthase $\gamma$-loop to bind phosphate reveals the novel regulatory modality in comparison to functions of specific $\gamma$-loops in chloroplast and Mtb F-ATP synthases, which requires further characterization. 
5. Conclusions 
TB remains a disease with the highest mortality and infectiveness despite our efforts to designs targeted therapy. With the discovery of BDQ, a novel target space was identified, and the F-ATP synthase emerged as a potent candidate for drug design. However, resistance has made a single-drug approach almost obsolete, and thus, the need is present to find a more elaborate mechanism of targeting the F-ATP synthase. A strategy paving the way included assigning the 3D structure of F-ATP synthase to derive relevant epitopes in regulation and catalysis.

By using negative stain electron microscopy in this work, the chimeric complex $\alpha^{c h i}{ }_{3}: \beta_{3}: \gamma: M t \varepsilon$ mimicking crucial mycobacterial F-ATP synthase features, was described as a model system to study the catalysis and interactions. The 2D- and 3D reconstruction allowed the assignment of the subunits into the experimental density

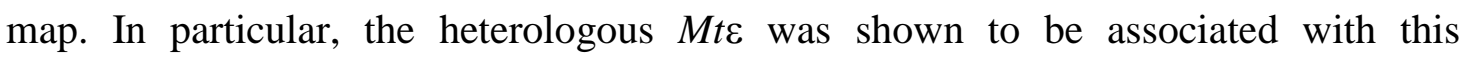
$\alpha^{c h i}{ }_{3}: \beta_{3}: \gamma$ in its canonical position, disregarding the genetic distance between $M t b$ and G. stearothermophilus, an original donor of the catalytic mechanism (complex $\left.\alpha_{3}: \beta_{3}: \gamma\right)$. This association with the heterologous complex emphasized that $M t \varepsilon$ harbors a preserved canonical fold of its NTD, whose critical binding epitopes were investigated further. The deletion of the initial five $M t \varepsilon N T D$ residues, led to a severe structural instability causing a shift of the isolated mutant protein $M t \varepsilon_{6-121}$ to higher oligomeric states, which in turn disrupted functional binding to the $\alpha^{\text {chi }}{ }_{3}: \beta_{3}: \gamma$ : complex. As a result of sub-stoichiometric mutant complex formation, its ATPase activity was impaired in comparison to its non-truncated variant.

Furthermore, Mt\&CTD had been described as unique, due to its shorter length in comparison with its bacterial homologs. Considering that $M t \varepsilon$ is described as a regulatory subunit, the ATPase activity of complexes $\alpha^{\text {chi }}{ }_{3}: \beta_{3}: \gamma$ and $\alpha^{\text {chi }}{ }_{3}: \beta_{3}: \gamma: M t \varepsilon$ was assayed. The kinetic profile of ATP hydrolysis revealed that the complex with MtE had 1.8 times higher $V_{\max }$ while $k_{m}$ of both complexes were in the same range emphasizing that MtE does not affect the cleavage of ATP, but it affects the binding/release of nucleotides from the catalytic centers.

The inhibitory Mg-ADP had a drastically different effect on complex $\alpha^{c h i}{ }_{3}: \beta_{3}: \gamma: M t \varepsilon$ in respect to $\alpha^{c h i}{ }_{3}: \beta_{3}: \gamma$; inhibition of $\alpha^{c h i}{ }_{3}: \beta_{3}: \gamma: M t \varepsilon$ in presence of $10 \mu \mathrm{M}$ Mg-ADP lowered its ATPase activity by $21 \%$, while the same nucleotide concentration inhibited $\alpha^{c h i}: \beta_{3}: \gamma$ by $9 \%$, thus revealing that the subunit MtE rendered the complex more sensitive to this Mg-ADP inhibition. The epitopes mediating this 
sensitivity include the CTD terminal residue D121 whose deletion desensitized the complex to almost the same level as seen for the complex without Mte hinting its essentiality.

The previous NMR studies conducted on isolated $M t \varepsilon$ brought residue E87 in focus as a part of the MtE epitope that maintained the closed conformation. Its systematic replacement with alanine $(M t \varepsilon E 87 \mathrm{~A})$ and subsequent reconstitution of this mutant with a complex $\alpha^{c h i}{ }_{3}: \beta_{3}: \gamma$ revealed a reduced ATP hydrolysis capacity. This indicated that E87A mutation could have triggered dis-regulation and de-coupling that together affected the catalysis. A similar effect on the ATPase activity was also established in the in vivo study on $M$. smegmatis, whereby the introduction of the matching mutation its genomic sequence of $a t p C$ gene similarly impeded the ATPase activity. In addition to these observations, even though the mutant strain displayed unaltered growth behavior, its sensitivity to BDQ was reduced, hinting that subunit $\varepsilon$ could indeed be a target epitope of BDQ. These findings substantiated the fact that novel antibiotics could target the mechanism of regulation, and that this trait could be exploited in clinical settings. Selective activation of ATPase activity and subsequent depletion of $M t b$ cellular ATP would have had a bactericidal effect on activelydividing and dormant bacteria, as hinted in one of the earlier in vitro studies.

Further mutational and structural experiments confirmed that $M t \varepsilon$ can alter its conformation from closed to extended, analogous to the phenomenon observed with $E$.

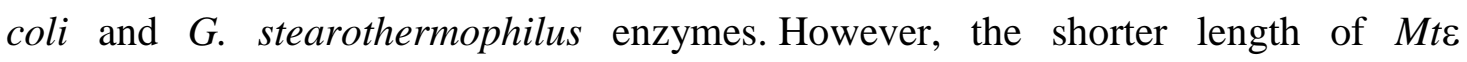
suggested involvement of the alternate epitopes that synergistically mediated the

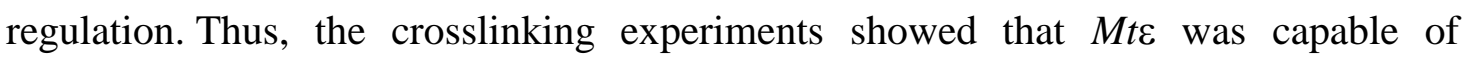
interacting with two epitopes in subunit $\alpha^{\text {chi}}$; a conserved coupling epitope ${ }_{396} \mathrm{AQFGSDLDK}_{404}$ and a mycobacteria-specific $M t \alpha_{514-549}$ epitope, possibly allowing multiple regulatory alterations that lead to the fine-tuning of the catalytic activity.

In summary, the work regarding provides new structural and mechanistic insights in the functionality of mycobacterial F-ATP synthases obtained through the investigations of heterologous $\alpha^{c h i}{ }_{3}: \beta_{3}: \gamma: M t \varepsilon$ complex. Main findings presented here are:

- Heterologous $\alpha^{c h i}: \beta_{3}: \gamma: M t \varepsilon$ complex that features mycobacterial regulatory epitopes was visualized using NS-EM 
- MtE functionally binds to $\alpha^{c h i}: \beta_{3}: \gamma$ disregarding the genetic distance between these proteins

- MtENTD is conserved and vital for functional binding to $\alpha^{c h i}{ }_{3}: \beta_{3}: \gamma ; M t \varepsilon$ residues 1-5 are crucial for its structural integrity and stability

- MtE residue E87 connects its NTD and CTD, maintains the protein topology and its substitution with alanine (E87A) in the genome of $M$. smegmatis leads to slight hyposensitivity of the strain to BDQ

- MtcCTD acts as a regulator of ATPase activity and increases the sensitivity of $\alpha^{c h i}: \beta_{3}: \gamma$ complex to Mg-ADP inhibition via the residue D121 in $M t \varepsilon C T D$

- MtcCTD establishes the regulation through interaction with coupling region of the subunit $\alpha^{c h i}$ and the $M t \alpha$ specific epitope

In reflection of the evidence above, as part of the future work, it would be imperative to isolate the entire mycobacterial $\mathrm{F}_{1} \mathrm{~F}_{\mathrm{O}}$-ATP synthase and conduct the structural characterization in its membrane context. High-resolution cryo-EM studies of the purified complex embedded into the lipid nano-discs would provide unprecedented structural details, while solid-state NMR with selectively labeled MtE would propel the understanding of the dynamics of its inhibitory conformational transitions that affect the ATPase activity. Subsequent smFRET experiments carried out with various relevant nucleotides such as AMP-PNP, ADP, ATP- $\gamma$ S, and ATP would complete the understanding of the spatiotemporal dimension of the conformational changes. The rotational experiments with a conjugated golden nanorod would precisely describe the catalytic cycle, rotation speed and nucleotide dwell in both, ATP synthesis and ATP hydrolysis direction.

Having such a deep understanding would permit manipulation of the catalytic events in order to internally promote ATP hydrolysis. The approach to achieve this would include designing more efficient antimicrobials that could disrupt the interactions between $M t \varepsilon C T D$ and $M t \alpha_{514-549}$ in order to dysregulate the F-ATP synthase. The resulting perpetual ATP hydrolysis could cause the depletion of microbial ATP pools which holds a potential to affect the actively-replicating, as well as dormant bacilli. The novel structure-guided antibiotic with higher bactericidal properties would hold a promise for clinical applications in order to eradicate TB. 
Like $M t b, A$. woodii has undergone adaptations as uniquely reflected by its $\mathrm{F}_{\mathrm{O}} / \mathrm{V}_{\mathrm{O}}$ hybrid F-ATP synthase. A hybrid between two kingdoms of life, A. woodii $\mathrm{F}-$ ATP synthase has displayed a combination of structural and biochemical features found nowhere else. Due to the lack of the complete high-resolution structural coordinates, most of the epitopes within this motor remain uncharacterized. Here, as a part of this thesis, A. woodii F-ATP synthase was isolated and visualized for the first time by using the negative stain electron microscopy. The 2D projections enabled arbitral assignment of the canonical F-ATP synthase subunits into the experimentally obtained data. The entire enzyme retained in the typical $\mathrm{F}_{1} \mathrm{~F}_{\mathrm{O}}$ organization whose linkage is established through the central stalk. The $c$-ring was visualized in the neighborhood of subunit $a$, whose position was inferred from the connection of the single peripheral stalk. The peripheral stalk is also seen to connect to the $\mathrm{F}_{1}$ subunit $\delta$ positioned apically of the $F_{1}$ catalytic hexamer.

The protein sequence comparison of the individual subunits of A. woodii FATP synthase with other bacterial and eukaryal homologs highlighted the presence of a unique segment found in the subunit $\gamma$. In order to establish its role in catalysis and structure, the epitope $\gamma_{195}$ TSGKVKTTEETKEEKSK 211 that was comprised of the 17 amino acids was deleted from the operon to generate and isolate the mutant $\gamma_{\Delta 195-211}$ $\mathrm{F}_{1} \mathrm{~F}_{\mathrm{O}}$-ATP synthase. The biochemical characterization of the mutant revealed that the epitope took part in the coordination of the $\mathrm{Na}^{+}$translocation and ATP synthesis since its deletion abolished these processes. These facts pointed out that the $\gamma_{195}$ TSGKVKTTEETKEEKSK $_{211}$ loop was indeed essential for maintaining the physiological role of the A. woodii F-ATP synthase. Moreover, its deletion from the operon did not cause disassembly of $F_{1}$ from $F_{O}$, which excluded the possibility that the observed effects were an artifact of the compromised structural integrity of the complex.

The assembled homology model of $\mathrm{F}_{1} \mathrm{~F}_{\mathrm{O}}$ provided the insight into the interactions of the $\gamma_{195}$ TSGKVKTTEETKEEKSK ${ }_{211}$ epitope with subunit $\varepsilon$ and $c$-ring. The proposed $\gamma_{195-211} c_{1}$ interactions were marked as indispensable for the coupling of $\mathrm{Na}^{+}$and ATP synthesis, while the $\varepsilon-c_{1}$ interactions were essential for the stability of the central stalk. The structural model build in this work corroborated the findings of an independent study that showed that the $c_{l}$ deletion destabilized the central stalk by triggering the depletion of $\varepsilon$ from the complex. Moreover, the interactions described 
by the model provided a structural basis for the effect exerted by the $\gamma_{\Delta 195-211}$ mutation onto $\mathrm{Na}^{+}$transport and ATP synthesis.

In summary, the work on A. woodii F-ATP synthase provides an insight into the functionality of the hybrid enzyme by contributing with main findings presented here:

- The enzyme retained in the canonical $\mathrm{F}_{1} \mathrm{~F}_{\mathrm{O}}$ organization as visualized by NS-EM

- The $\gamma_{195}$ TSGKVKTTEETKEEKSK 211 peptide is unique amongst bacterial and eukaryal homologs

- Deletion of the $\gamma_{195-211}$ epitope does not cause disassembly of $F_{1}$ from $F_{O}$ as confirmed with the isolation of $\gamma_{\Delta 195^{-211}}$ mutant

- $\gamma_{195-211}$ acts as a coupling epitope of $\mathrm{Na}^{+}$translocation and ATP synthesis since its deletion abolished these processes

- A preliminary study showed phosphorylation of the $\gamma_{195^{-211}}$ that could be essential in the regulation of the catalytic events

- $\mathrm{F}_{1} \mathrm{~F}_{\mathrm{O}}$ structural model describes the proposed $\gamma_{195-211^{-}} c_{1}$ interactions as essential for the coupling of $\mathrm{Na}^{+}$and ATP synthesis, and the $\varepsilon-c_{1}$ interactions as crucial for the stability of the central stalk

There are many unknowns revolving around the hybrid A. woodii F-ATP synthase. The discoveries made as part of this thesis give an insight into its structural and biochemical traits. The future characterizations would include a determination of the $\mathrm{F}_{1} \mathrm{~F}_{\mathrm{O}}$ high-resolution structure by employing cryo-EM which would bring about unprecedented structural details. Apart from seeing the position of the $\gamma$-loop with respect to coupling epitopes, it would be possible to uncover the structural basis of phosphorylation and describe its significance in catalysis. A phosphorylated regulatory epitope could also provide a direct link to the other metabolic processes such as signal transduction cascades and could infer a higher-order communication of the cellular requirements with its energy generation machinery. The understanding gained from such link would benefit metabolic manipulation of $A$. woodii in order to increase its commercial value by producing higher amounts of acetate through fixation of $\mathrm{CO}_{2}$ and $\mathrm{H}_{2}$. 
As seen from all accumulated evidence, F-ATP synthases remain to be an inexhaustive source of solutions fascinating for structural biology, even though their overall architecture is conserved across the domains of life. Uncovering the patterns of their evolution and linking them to the native environmental factors of host organisms holds a great potential to modify and adapt those organisms to the needs of human society. While the complete understanding of the structural traits of Mtb F-ATP synthase could lead to the development of the new antibiotics and a breakthrough in TB treatment, the manipulation of the $A$. woodii hybrid enzyme could hold a promise of more efficient industrial application of this acetogen archaeal species. 


\section{References}


1. Guo, H., Suzuki, T., and Rubinstein, J. L. (2018) Structure of a bacterial ATP synthase. bioRxiv

2. Haagsma, A. C., Driessen, N. N., Hahn, M. M., Lill, H., and Bald, D. (2010) ATP synthase in slow- and fast-growing mycobacteria is active in ATP synthesis and blocked in ATP hydrolysis direction. FEMS microbiology letters 313, 68-74

3. Ragunathan, P., Sielaff, H., Sundararaman, L., Biuković, G., Subramanian Manimekalai, M. S., Singh, D., Kundu, S., Wohland, T., Frasch, W., Dick, T., and Grüber, G. (2017) The uniqueness of subunit alpha of mycobacterial F-ATP synthases: An evolutionary variant for niche adaptation. J Biol Chem 292, 11262-11279

4. Bogdanović, N., Sundararaman, L., Kamariah, N., Tyagi, A., Bhushan, S., Ragunathan, P., Shin, J., Dick, T., and Grüber, G. (2018) Structure and function of Mycobacteriumspecific components of F-ATP synthase subunits alpha and epsilon. Journal of structural biology 204, 420-434

5. Jounouchi, M., Takeyama, M., Noumi, T., Moriyama, Y., Maeda, M., and Futai, M. (1992) Role of the amino terminal region of the epsilon subunit of Escherichia coli H(+)-ATPase (F0F1). Arch Biochem Biophys 292, 87-94

6. Brandt, K., Muller, D. B., Hoffmann, J., Langer, J. D., Brutschy, B., Morgner, N., and Muller, V. (2016) Stoichiometry and deletion analyses of subunits in the heterotrimeric F-ATP synthase c ring from the acetogenic bacterium Acetobacterium woodii. The FEBS journal 283, 510-520

7. Matthies, D., Zhou, W., Klyszejko, A. L., Anselmi, C., Yildiz, O., Brandt, K., Muller, V., Faraldo-Gomez, J. D., and Meier, T. (2014) High-resolution structure and mechanism of an F/V-hybrid rotor ring in a $\mathrm{Na}(+)$-coupled ATP synthase. Nature communications $\mathbf{5}, 5286$

8. Glynn, J. R., Whiteley, J., Bifani, P. J., Kremer, K., and van Soolingen, D. (2002) Worldwide occurrence of Beijing/W strains of Mycobacterium tuberculosis: a systematic review. Emerg Infect Dis 8, 843-849

9. Yew, W. W., and Leung, C. C. (2008) Update in tuberculosis 2007. American journal of respiratory and critical care medicine 177, 479-485

10. Dye, C., and Williams, B. G. (2010) The population dynamics and control of tuberculosis. Science 328, 856-861

11. Dye, C., Scheele, S., Dolin, P., Pathania, V., and Raviglione, M. C. (1999) Consensus statement. Global burden of tuberculosis: estimated incidence, prevalence, and mortality by country. WHO Global Surveillance and Monitoring Project. Jama 282, 677-686

12. Saravanan, M., Niguse, S., Abdulkader, M., Tsegay, E., Hailekiros, H., Gebrekidan, A., Araya, T., and Pugazhendhi, A. (2018) Review on emergence of drug-resistant tuberculosis (MDR \& XDR-TB) and its molecular diagnosis in Ethiopia. Microb Pathog 117, 237-242 
13. Asgedom, S. W., Teweldemedhin, M., and Gebreyesus, H. (2018) Prevalence of Multidrug-Resistant Tuberculosis and Associated Factors in Ethiopia: A Systematic Review. J Pathog 2018, 7104921

14. Gallo, J. F., Pinhata, J. M. W., Simonsen, V., Galesi, V. M. N., Ferrazoli, L., and Oliveira, R. S. (2018) Prevalence, associated factors, outcomes and transmission of extensively drug-resistant tuberculosis among multidrug-resistant tuberculosis patients in Sao Paulo, Brazil: a cross-sectional study. Clinical microbiology and infection : the official publication of the European Society of Clinical Microbiology and Infectious Diseases 24, 889-895

15. Dookie, N., Rambaran, S., Padayatchi, N., Mahomed, S., and Naidoo, K. (2018) Evolution of drug resistance in Mycobacterium tuberculosis: a review on the molecular determinants of resistance and implications for personalized care. $J$ Antimicrob Chemother 73, 1138-1151

16. Wood, T. K., Knabel, S. J., and Kwan, B. W. (2013) Bacterial persister cell formation and dormancy. Appl Environ Microbiol 79, 7116-7121

17. Cohen, N. R., Lobritz, M. A., and Collins, J. J. (2013) Microbial persistence and the road to drug resistance. Cell Host Microbe 13, 632-642

18. Andries, K., Verhasselt, P., Guillemont, J., Gohlmann, H. W., Neefs, J. M., Winkler, H., Van Gestel, J., Timmerman, P., Zhu, M., Lee, E., Williams, P., de Chaffoy, D., Huitric, E., Hoffner, S., Cambau, E., Truffot-Pernot, C., Lounis, N., and Jarlier, V. (2005) A diarylquinoline drug active on the ATP synthase of Mycobacterium tuberculosis. Science 307, 223-227

19. Huitric, E., Verhasselt, P., Andries, K., and Hoffner, S. E. (2007) In vitro antimycobacterial spectrum of a diarylquinoline ATP synthase inhibitor. Antimicrob Agents Chemother 51, 4202-4204

20. Koul, A., Vranckx, L., Dendouga, N., Balemans, W., Van den Wyngaert, I., Vergauwen, K., Gohlmann, H. W., Willebrords, R., Poncelet, A., Guillemont, J., Bald, D., and Andries, K. (2008) Diarylquinolines are bactericidal for dormant mycobacteria as a result of disturbed ATP homeostasis. J Biol Chem 283, 25273-25280

21. Li, S.-Y., Tasneen, R., Tyagi, S., Soni, H., Converse, P. J., Mdluli, K., and Nuermberger, E. L. (2017) Bactericidal and Sterilizing Activity of a Novel Regimen with Bedaquiline, Pretomanid, Moxifloxacin, and Pyrazinamide in a Murine Model of Tuberculosis. Antimicrobial Agents and Chemotherapy 61, e00913-00917

22. Nguyen, T. V. A., Anthony, R. M., Bañuls, A.-L., Nguyen, T. V. A., Vu, D. H., and Alffenaar, J.-W. C. (2018) Bedaquiline Resistance: Its Emergence, Mechanism, and Prevention. Clinical Infectious Diseases 66, 1625-1630

23. Hards, K., and Cook, G. M. (2018) Targeting bacterial energetics to produce new antimicrobials. Drug Resistance Updates 36, 1-12 
24. Sapp, J. (2005) The prokaryote-eukaryote dichotomy: meanings and mythology. Microbiol Mol Biol Rev 69, 292-305

25. Collins, C. H., Grange, J. M., and Yates, M. D. (1984) Mycobacteria in water. J Appl Bacteriol 57, 193-211

26. Waters, W. R., and Palmer, M. V. (2015) Mycobacterium bovis Infection of Cattle and White-Tailed Deer: Translational Research of Relevance to Human Tuberculosis. ILAR J 56, 26-43

27. Grange, J. M. (2001) Mycobacterium bovis infection in human beings. Tuberculosis 81, 71-77

28. Benjak, A., Avanzi, C., Singh, P., Loiseau, C., Girma, S., Busso, P., Fontes, A. N. B., Miyamoto, Y., Namisato, M., Bobosha, K., Salgado, C. G., da Silva, M. B., Bouth, R. C., Frade, M. A. C., Filho, F. B., Barreto, J. G., Nery, J. A. C., Buhrer-Sekula, S., Lupien, A., Al-Samie, A. R., Al-Qubati, Y., Alkubati, A. S., Bretzel, G., Vera-Cabrera, L., Sakho, F., Johnson, C. R., Kodio, M., Fomba, A., Sow, S. O., Gado, M., Konate, O., Stefani, M. M. A., Penna, G. O., Suffys, P. N., Sarno, E. N., Moraes, M. O., Rosa, P. S., Baptista, I., Spencer, J. S., Aseffa, A., Matsuoka, M., Kai, M., and Cole, S. T. (2018) Phylogenomics and antimicrobial resistance of the leprosy bacillus Mycobacterium leprae. Nature communications 9, 352

29. Scherr, N., Bieri, R., Thomas, S. S., Chauffour, A., Kalia, N. P., Schneide, P., Ruf, M. T., Lamelas, A., Manimekalai, M. S. S., Gruber, G., Ishii, N., Suzuki, K., Tanner, M., Moraski, G. C., Miller, M. J., Witschel, M., Jarlier, V., Pluschke, G., and Pethe, K. (2018) Targeting the Mycobacterium ulcerans cytochrome bc1:aa3 for the treatment of Buruli ulcer. Nature communications 9, 5370

30. Garchitorena, A., Ngonghala, C. N., Texier, G., Landier, J., Eyangoh, S., Bonds, M. H., Guegan, J. F., and Roche, B. (2015) Environmental transmission of Mycobacterium ulcerans drives dynamics of Buruli ulcer in endemic regions of Cameroon. Sci Rep 5, 18055

31. Oliveira, M. S., Fraga, A. G., Torrado, E., Castro, A. G., Pereira, J. P., Filho, A. L., Milanezi, F., Schmitt, F. C., Meyers, W. M., Portaels, F., Silva, M. T., and Pedrosa, J. (2005) Infection with Mycobacterium ulcerans induces persistent inflammatory responses in mice. Infection and immunity 73, 6299-6310

32. Shiloh, M. U., and Nathan, C. F. (2000) Reactive nitrogen intermediates and the pathogenesis of Salmonella and mycobacteria. Current opinion in microbiology $\mathbf{3}, 35$ 42

33. Deretic, V., Delgado, M., Vergne, I., Master, S., De Haro, S., Ponpuak, M., and Singh, S. (2009) Autophagy in immunity against mycobacterium tuberculosis: a model system to dissect immunological roles of autophagy. Current topics in microbiology and immunology 335, 169-188 
34. Guilliams, M., Bruhns, P., Saeys, Y., Hammad, H., and Lambrecht, B. N. (2014) The function of $\mathrm{Fc} \gamma$ receptors in dendritic cells and macrophages. Nature Reviews Immunology 14, 94

35. Kambayashi, T., and Laufer, T. M. (2014) Atypical MHC class II-expressing antigenpresenting cells: can anything replace a dendritic cell? Nature Reviews Immunology 14, 719

36. Roche, P. A., and Furuta, K. (2015) The ins and outs of MHC class II-mediated antigen processing and presentation. Nature Reviews Immunology 15, 203

37. Weinstein, E. A., Yano, T., Li, L. S., Avarbock, D., Avarbock, A., Helm, D., McColm, A. A., Duncan, K., Lonsdale, J. T., and Rubin, H. (2005) Inhibitors of type II NADH:menaquinone oxidoreductase represent a class of antitubercular drugs. Proc Natl Acad Sci U S A 102, 4548-4553

38. Baughn, A. D., and Rhee, K. Y. (2014) Metabolomics of Central Carbon Metabolism in Mycobacterium tuberculosis. Microbiology Spectrum 2

39. Podinovskaia, M., Lee, W., Caldwell, S., and Russell, D. G. (2013) Infection of macrophages with Mycobacterium tuberculosis induces global modifications to phagosomal function. Cellular microbiology 15, 843-859

40. Xia, Y., and Zweier, J. L. (1997) Superoxide and peroxynitrite generation from inducible nitric oxide synthase in macrophages. Proceedings of the National Academy of Sciences of the United States of America 94, 6954-6958

41. Linares, E., Giorgio, S., Mortara, R. A., Santos, C. X., Yamada, A. T., and Augusto, O. (2001) Role of peroxynitrite in macrophage microbicidal mechanisms in vivo revealed by protein nitration and hydroxylation. Free radical biology \& medicine 30, 12341242

42. Prolo, C., Alvarez, M. N., and Radi, R. (2014) Peroxynitrite, a potent macrophagederived oxidizing cytotoxin to combat invading pathogens. BioFactors (Oxford, England) 40, 215-225

43. Teixeira, M. M., Williams, T. J., and Hellewell, P. G. (1993) Role of prostaglandins and nitric oxide in acute inflammatory reactions in guinea-pig skin. Br J Pharmacol 110, $1515-1521$

44. Kuo, P. C., and Schroeder, R. A. (1995) The emerging multifaceted roles of nitric oxide. Annals of surgery 221, 220-235

45. Rosselli, M., Keller, P. J., and Dubey, R. K. (1998) Role of nitric oxide in the biology, physiology and pathophysiology of reproduction. Hum Reprod Update 4, 3-24

46. Tripathi, P., Tripathi, P., Kashyap, L., and Singh, V. (2007) The role of nitric oxide in inflammatory reactions. FEMS Immunol Med Microbiol 51, 443-452 
47. (2012) Retraction. The role of nitric oxide in inflammatory reactions. FEMS Immunol Med Microbiol 66, 449

48. Levine, A. B., Punihaole, D., and Levine, T. B. (2012) Characterization of the role of nitric oxide and its clinical applications. Cardiology 122, 55-68

49. Guirado, E., Schlesinger, L. S., and Kaplan, G. (2013) Macrophages in tuberculosis: friend or foe. Seminars in immunopathology 35, 563-583

50. Lamichhane, G. (2011) Mycobacterium tuberculosis response to stress from reactive oxygen and nitrogen species. Front Microbiol 2, 176

51. Voskuil, M. I., Bartek, I. L., Visconti, K., and Schoolnik, G. K. (2011) The response of mycobacterium tuberculosis to reactive oxygen and nitrogen species. Front Microbiol 2, 105

52. Boon, C., and Dick, T. (2002) Mycobacterium bovis BCG response regulator essential for hypoxic dormancy. J Bacteriol 184, 6760-6767

53. Voskuil, M. I., Schnappinger, D., Visconti, K. C., Harrell, M. I., Dolganov, G. M., Sherman, D. R., and Schoolnik, G. K. (2003) Inhibition of respiration by nitric oxide induces a Mycobacterium tuberculosis dormancy program. The Journal of experimental medicine 198, 705-713

54. Gengenbacher, M., and Kaufmann, S. H. (2012) Mycobacterium tuberculosis: success through dormancy. FEMS microbiology reviews 36, 514-532

55. Flynn, J. L., Chan, J., and Lin, P. L. (2011) Macrophages and control of granulomatous inflammation in tuberculosis. Mucosal Immunology 4, 271-278

56. Russell, D. G., Cardona, P.-J., Kim, M.-J., Allain, S., and Altare, F. (2009) Foamy macrophages and the progression of the human TB granuloma. Nature immunology $\mathbf{1 0}$, 943-948

57. Jay, S. M., Skokos, E. A., Zeng, J., Knox, K., and Kyriakides, T. R. (2010) Macrophage fusion leading to foreign body giant cell formation persists under phagocytic stimulation by microspheres in vitro and in vivo in mouse models. Journal of biomedical materials research. Part A 93, 189-199

58. Russell, D. G., Cardona, P.-J., Kim, M.-J., Allain, S., and Altare, F. (2009) Foamy macrophages and the progression of the human tuberculosis granuloma. Nature immunology 10, 943-948

59. Daniel, J., Maamar, H., Deb, C., Sirakova, T. D., and Kolattukudy, P. E. (2011) Mycobacterium tuberculosis Uses Host Triacylglycerol to Accumulate Lipid Droplets and Acquires a Dormancy-Like Phenotype in Lipid-Loaded Macrophages. PLOS Pathogens 7, e1002093 
60. Genoula, M., Marín Franco, J. L., Dupont, M., Kviatcovsky, D., Milillo, A., Schierloh, P., Moraña, E. J., Poggi, S., Palmero, D., Mata-Espinosa, D., González-Domínguez, E., León Contreras, J. C., Barrionuevo, P., Rearte, B., Córdoba Moreno, M. O., Fontanals, A., Crotta Asis, A., Gago, G., Cougoule, C., Neyrolles, O., Maridonneau-Parini, I., Sánchez-Torres, C., Hernández-Pando, R., Vérollet, C., Lugo-Villarino, G., Sasiain, M. d. C., and Balboa, L. (2018) Formation of Foamy Macrophages by Tuberculous Pleural Effusions Is Triggered by the Interleukin-10/Signal Transducer and Activator of Transcription 3 Axis through ACAT Upregulation. Frontiers in Immunology 9

61. Baldán, Á., Gomes, A. V., Ping, P., and Edwards, P. A. (2008) Loss of ABCG1 Results in Chronic Pulmonary Inflammation. The Journal of Immunology 180, 35603568

62. D'Avila, H., Maya-Monteiro, C. M., and Bozza, P. T. (2008) Lipid bodies in innate immune response to bacterial and parasite infections. Int Immunopharmacol 8, 13081315

63. D'Avila, H., Melo, R. C. N., Parreira, G. G., Werneck-Barroso, E., Castro-Faria-Neto, H. C., and Bozza, P. T. (2006) Mycobacterium bovis Bacillus Calmette-Guerin Induces TLR2-Mediated Formation of Lipid Bodies: Intracellular Domains for Eicosanoid Synthesis In Vivo. The Journal of Immunology 176, 3087-3097

64. Silva, A. R., Pacheco, P., Vieira-de-Abreu, A., Maya-Monteiro, C. M., D'Alegria, B., Magalhaes, K. G., de Assis, E. F., Bandeira-Melo, C., Castro-Faria-Neto, H. C., and Bozza, P. T. (2009) Lipid bodies in oxidized LDL-induced foam cells are leukotrienesynthesizing organelles: a MCP-1/CCL2 regulated phenomenon. Biochimica et biophysica acta 1791, 1066-1075

65. Wu, M.-L., Gengenbacher, M., and Dick, T. (2016) Mild Nutrient Starvation Triggers the Development of a Small-Cell Survival Morphotype in Mycobacteria. Frontiers in Microbiology 7

66. McClean, C. M., and Tobin, D. M. (2016) Macrophage form, function, and phenotype in mycobacterial infection: lessons from tuberculosis and other diseases. Pathog Dis 74

67. Cunningham, A. F., and Spreadbury, C. L. (1998) Mycobacterial stationary phase induced by low oxygen tension: cell wall thickening and localization of the 16kilodalton alpha-crystallin homolog. J Bacteriol 180, 801-808

68. Mahamed, D., Boulle, M., Ganga, Y., Mc Arthur, C., Skroch, S., Oom, L., Catinas, O., Pillay, K., Naicker, M., Rampersad, S., Mathonsi, C., Hunter, J., Wong, E. B., Suleman, M., Sreejit, G., Pym, A. S., Lustig, G., and Sigal, A. (2017) Correction: Intracellular growth of Mycobacterium tuberculosis after macrophage cell death leads to serial killing of host cells. Elife 6

69. Cook, G. M., Hards, K., Vilcheze, C., Hartman, T., and Berney, M. (2014) Energetics of Respiration and Oxidative Phosphorylation in Mycobacteria. Microbiology spectrum 2 
70. Rao, S. P., Alonso, S., Rand, L., Dick, T., and Pethe, K. (2008) The protonmotive force is required for maintaining ATP homeostasis and viability of hypoxic, nonreplicating Mycobacterium tuberculosis. Proc Natl Acad Sci U S A 105, 1194511950

71. Searle, A., Wetherell, M. A., Campbell, R., Dayan, C., Weinman, J., and Vedhara, K. (2008) Do patients' beliefs about type 2 diabetes differ in accordance with complications: an investigation into diabetic foot ulceration and retinopathy. International journal of behavioral medicine 15, 173-179

72. Del Vecchio, I., Zuccotti, A., Pisano, F., Canneva, F., Lenzken, S. C., Rousset, F., Corsini, E., Govoni, S., and Racchi, M. (2009) Functional mapping of the promoter region of the GNB2L1 human gene coding for RACK1 scaffold protein. Gene 430, $17-29$

73. Silva Miranda, M., Breiman, A., Allain, S., Deknuydt, F., and Altare, F. (2012) The tuberculous granuloma: an unsuccessful host defence mechanism providing a safety shelter for the bacteria? Clin Dev Immunol 2012, 139127

74. Hunter, R. L. (2016) Tuberculosis as a three-act play: A new paradigm for the pathogenesis of pulmonary tuberculosis. Tuberculosis $\mathbf{9 7 , 8 - 1 7}$

75. Worley, M. V., and Estrada, S. J. (2014) Bedaquiline: a novel antitubercular agent for the treatment of multidrug-resistant tuberculosis. Pharmacotherapy 34, 1187-1197

76. Haagsma, A. C., Abdillahi-Ibrahim, R., Wagner, M. J., Krab, K., Vergauwen, K., Guillemont, J., Andries, K., Lill, H., Koul, A., and Bald, D. (2009) Selectivity of TMC207 towards mycobacterial ATP synthase compared with that towards the eukaryotic homologue. Antimicrob Agents Chemother 53, 1290-1292

77. Weinstein, E. A., Yano, T., Li, L.-S., Avarbock, D., Avarbock, A., Helm, D., McColm, A. A., Duncan, K., Lonsdale, J. T., and Rubin, H. (2005) Inhibitors of type II NADH:menaquinone oxidoreductase represent a class of antitubercular drugs. Proceedings of the National Academy of Sciences of the United States of America 102, 4548-4553

78. Miesel, L., Weisbrod, T. R., Marcinkeviciene, J. A., Bittman, R., and Jacobs, W. R. (1998) NADH Dehydrogenase Defects Confer Isoniazid Resistance and Conditional Lethality in Mycobacterium smegmatis. Journal of Bacteriology 180, 2459-2467

79. Cook, G. M., Berney, M., Gebhard, S., Heinemann, M., Cox, R. A., Danilchanka, O., and Niederweis, M. (2009) Physiology of mycobacteria. Adv Microb Physiol 55, 81$182,318-189$

80. Sassetti, C. M., Boyd, D. H., and Rubin, E. J. (2003) Genes required for mycobacterial growth defined by high density mutagenesis. Molecular microbiology 48, 77-84

81. Schnappinger, D., Ehrt, S., Voskuil, M. I., Liu, Y., Mangan, J. A., Monahan, I. M., Dolganov, G., Efron, B., Butcher, P. D., Nathan, C., and Schoolnik, G. K. (2003) Transcriptional Adaptation of Mycobacterium tuberculosis within Macrophages: 
Insights into the Phagosomal Environment. The Journal of experimental medicine 198, 693-704

82. Hagerhall, C. (1997) Succinate: quinone oxidoreductases. Variations on a conserved theme. Biochimica et biophysica acta 1320, 107-141

83. Pecsi, I., Hards, K., Ekanayaka, N., Berney, M., Hartman, T., Jacobs, W. R., Jr., and Cook, G. M. (2014) Essentiality of succinate dehydrogenase in Mycobacterium smegmatis and its role in the generation of the membrane potential under hypoxia. mBio 5, e01093-01014

84. Watanabe, S., Zimmermann, M., Goodwin, M. B., Sauer, U., Barry, C. E., 3rd, and Boshoff, H. I. (2011) Fumarate reductase activity maintains an energized membrane in anaerobic Mycobacterium tuberculosis. PLoS Pathog 7, e1002287

85. Berney, M., and Cook, G. M. (2010) Unique Flexibility in Energy Metabolism Allows Mycobacteria to Combat Starvation and Hypoxia. PLOS ONE 5, e8614

86. Matsoso, L. G., Kana, B. D., Crellin, P. K., Lea-Smith, D. J., Pelosi, A., Powell, D., Dawes, S. S., Rubin, H., Coppel, R. L., and Mizrahi, V. (2005) Function of the cytochrome bc1-aa3 branch of the respiratory network in mycobacteria and network adaptation occurring in response to its disruption. J Bacteriol 187, 6300-6308

87. Wiseman, B., Nitharwal, R. G., Fedotovskaya, O., Schäfer, J., Guo, H., Kuang, Q., Benlekbir, S., Sjöstrand, D., Ädelroth, P., Rubinstein, J. L., Brzezinski, P., and Högbom, M. (2018) Structure of a functional obligate complex III2IV2 respiratory supercomplex from Mycobacterium smegmatis. Nature structural \& molecular biology 25, 1128-1136

88. Wiseman, B., Nitharwal, R. G., Fedotovskaya, O., Schafer, J., Guo, H., Kuang, Q., Benlekbir, S., Sjostrand, D., Adelroth, P., Rubinstein, J. L., Brzezinski, P., and Hogbom, M. (2018) Structure of a functional obligate complex III2IV2 respiratory supercomplex from Mycobacterium smegmatis. Nature structural \& molecular biology 25, 1128-1136

89. Shi, L., Sohaskey, C. D., Kana, B. D., Dawes, S., North, R. J., Mizrahi, V., and Gennaro, M. L. (2005) Changes in energy metabolism of Mycobacterium tuberculosis in mouse lung and under in vitro conditions affecting aerobic respiration. Proc Natl Acad Sci U S A 102, 15629-15634

90. Borisov, V. B., Murali, R., Verkhovskaya, M. L., Bloch, D. A., Han, H., Gennis, R. B., and Verkhovsky, M. I. (2011) Aerobic respiratory chain of Escherichia coli is not allowed to work in fully uncoupled mode. Proc Natl Acad Sci U S A 108, 1732017324

91. Kana, B. D., Weinstein, E. A., Avarbock, D., Dawes, S. S., Rubin, H., and Mizrahi, V. (2001) Characterization of the cydAB-encoded cytochrome bd oxidase from Mycobacterium smegmatis. J Bacteriol 183, 7076-7086 
92. Poole, R. K., and Cook, G. M. (2000) Redundancy of aerobic respiratory chains in bacteria? Routes, reasons and regulation. Adv Microb Physiol 43, 165-224

93. Cook, G. M., Cruz-Ramos, H., Moir, A. J., and Poole, R. K. (2002) A novel haem compound accumulated in Escherichia coli overexpressing the cydDC operon, encoding an ABC-type transporter required for cytochrome assembly. Archives of microbiology 178, 358-369

94. Green, G. N., Fang, H., Lin, R. J., Newton, G., Mather, M., Georgiou, C. D., and Gennis, R. B. (1988) The nucleotide sequence of the cyd locus encoding the two subunits of the cytochrome d terminal oxidase complex of Escherichia coli. J Biol Chem 263, 13138-13143

95. Poole, R. K., Williams, H. D., Downie, J. A., and Gibson, F. (1989) Mutations affecting the cytochrome d-containing oxidase complex of Escherichia coli K12: identification and mapping of a fourth locus, cydD. J Gen Microbiol 135, 1865-1874

96. Poole, R. K., Hatch, L., Cleeter, M. W., Gibson, F., Cox, G. B., and Wu, G. (1993) Cytochrome bd biosynthesis in Escherichia coli: the sequences of the cydC and cydD genes suggest that they encode the components of an $\mathrm{ABC}$ membrane transporter. Molecular microbiology 10, 421-430

97. Shepherd, M. (2015) The CydDC ABC transporter of Escherichia coli: new roles for a reductant efflux pump. Biochemical Society transactions 43, 908-912

98. Aung, H. L., Berney, M., and Cook, G. M. (2014) Hypoxia-activated cytochrome bd expression in Mycobacterium smegmatis is cyclic AMP receptor protein dependent. $J$ Bacteriol 196, 3091-3097

99. Pethe, K., Bifani, P., Jang, J., Kang, S., Park, S., Ahn, S., Jiricek, J., Jung, J., Jeon, H. K., Cechetto, J., Christophe, T., Lee, H., Kempf, M., Jackson, M., Lenaerts, A. J., Pham, H., Jones, V., Seo, M. J., Kim, Y. M., Seo, M., Seo, J. J., Park, D., Ko, Y., Choi, I., Kim, R., Kim, S. Y., Lim, S., Yim, S. A., Nam, J., Kang, H., Kwon, H., Oh, C. T., Cho, Y., Jang, Y., Kim, J., Chua, A., Tan, B. H., Nanjundappa, M. B., Rao, S. P., Barnes, W. S., Wintjens, R., Walker, J. R., Alonso, S., Lee, S., Kim, J., Oh, S., Oh, T., Nehrbass, U., Han, S. J., No, Z., Lee, J., Brodin, P., Cho, S. N., Nam, K., and Kim, J. (2013) Discovery of Q203, a potent clinical candidate for the treatment of tuberculosis. Nature medicine 19, 1157-1160

100. Dhar, N., and McKinney, J. D. (2010) Mycobacterium tuberculosis persistence mutants identified by screening in isoniazid-treated mice. Proc Natl Acad Sci U S A 107, 12275-12280

101. Lu, P., Heineke, M. H., Koul, A., Andries, K., Cook, G. M., Lill, H., van Spanning, R., and Bald, D. (2015) The cytochrome bd-type quinol oxidase is important for survival of Mycobacterium smegmatis under peroxide and antibiotic-induced stress. Sci Rep 5, 10333

102. Prolo, C., Alvarez, M. N., and Radi, R. (2014) Peroxynitrite, a potent macrophagederived oxidizing cytotoxin to combat invading pathogens. Biofactors 40, 215-225 
103. Jung, J.-Y., Madan-Lala, R., Georgieva, M., Rengarajan, J., Sohaskey, C. D., Bange, F.-C., and Robinson, C. M. (2013) The Intracellular Environment of Human Macrophages That Produce Nitric Oxide Promotes Growth of Mycobacteria. Infection and immunity 81, 3198

104. Arya, S., Sethi, D., Singh, S., Hade, M. D., Singh, V., Raju, P., Chodisetti, S. B., Verma, D., Varshney, G. C., Agrewala, J. N., and Dikshit, K. L. (2013) Truncated hemoglobin, $\mathrm{HbN}$, is post-translationally modified in Mycobacterium tuberculosis and modulates host-pathogen interactions during intracellular infection. J Biol Chem $\mathbf{2 8 8 ,}$ 29987-29999

105. Sohaskey, C. D., and Wayne, L. G. (2003) Role of narK2X and narGHJI in hypoxic upregulation of nitrate reduction by Mycobacterium tuberculosis. J Bacteriol 185, 7247-7256

106. Sohaskey, C. D. (2008) Nitrate enhances the survival of Mycobacterium tuberculosis during inhibition of respiration. J Bacteriol 190, 2981-2986

107. Sohaskey, C. D. (2005) Regulation of nitrate reductase activity in Mycobacterium tuberculosis by oxygen and nitric oxide. Microbiology 151, 3803-3810

108. Kundu, S., Biuković, G., Grüber, G., and Dick, T. (2016) Bedaquiline Targets the epsilon Subunit of Mycobacterial F-ATP Synthase. Antimicrob Agents Chemother 60, 6977-6979

109. Preiss, L., Langer, J. D., Yildiz, O., Eckhardt-Strelau, L., Guillemont, J. E., Koul, A., and Meier, T. (2015) Structure of the mycobacterial ATP synthase Fo rotor ring in complex with the anti-TB drug bedaquiline. Sci Adv 1, e1500106

110. Nyberg, G., Axelsson, R., and Martensson, E. (1981) Cerebrospinal fluid concentrations of thioridazine and its main metabolites in psychiatric patients. European journal of clinical pharmacology 19, 139-148

111. Wen, B., and Zhou, M. (2009) Metabolic activation of the phenothiazine antipsychotics chlorpromazine and thioridazine to electrophilic iminoquinone species in human liver microsomes and recombinant P450s. Chemico-biological interactions 181, 220-226

112. Yano, T., Li, L. S., Weinstein, E., Teh, J. S., and Rubin, H. (2006) Steady-state kinetics and inhibitory action of antitubercular phenothiazines on mycobacterium tuberculosis type-II NADH-menaquinone oxidoreductase (NDH-2). J Biol Chem 281, $11456-11463$

113. Balganesh, M., Dinesh, N., Sharma, S., Kuruppath, S., Nair, A. V., and Sharma, U. (2012) Efflux pumps of Mycobacterium tuberculosis play a significant role in antituberculosis activity of potential drug candidates. Antimicrob Agents Chemother 56, 2643-2651

114. Coelho, T., Machado, D., Couto, I., Maschmann, R., Ramos, D., von Groll, A., Rossetti, M. L., Silva, P. A., and Viveiros, M. (2015) Enhancement of antibiotic 
activity by efflux inhibitors against multidrug resistant Mycobacterium tuberculosis clinical isolates from Brazil. Front Microbiol 6, 330

115. Daniel, W. A., Syrek, M., Haduch, A., and Wojcikowski, J. (2000) Pharmacokinetics and metabolism of thioridazine during co-administration of tricyclic antidepressants. Br J Pharmacol 131, 287-295

116. Sharma, S., and Singh, A. (2011) Phenothiazines as anti-tubercular agents: mechanistic insights and clinical implications. Expert Opin Investig Drugs 20, 16651676

117. Kristiansen, J. E., Dastidar, S. G., Palchoudhuri, S., Roy, D. S., Das, S., Hendricks, O., and Christensen, J. B. (2015) Phenothiazines as a solution for multidrug resistant tuberculosis: From the origin to present. Int Microbiol 18, 1-12

118. Ordway, D., Viveiros, M., Leandro, C., Bettencourt, R., Almeida, J., Martins, M., Kristiansen, J. E., Molnar, J., and Amaral, L. (2003) Clinical Concentrations of Thioridazine Kill Intracellular Multidrug-Resistant \&lt;em\&gt;Mycobacterium tuberculosis\&lt;/em\&gt. Antimicrobial Agents and Chemotherapy 47, 917

119. Yano, T., Kassovska-Bratinova, S., Teh, J. S., Winkler, J., Sullivan, K., Isaacs, A., Schechter, N. M., and Rubin, H. (2011) Reduction of clofazimine by mycobacterial type $2 \mathrm{NADH}$ :quinone oxidoreductase: a pathway for the generation of bactericidal levels of reactive oxygen species. J Biol Chem 286, 10276-10287

120. Grosset, J. H., Tyagi, S., Almeida, D. V., Converse, P. J., Li, S. Y., Ammerman, N. C., Bishai, W. R., Enarson, D., and Trebucq, A. (2013) Assessment of clofazimine activity in a second-line regimen for tuberculosis in mice. American journal of respiratory and critical care medicine 188, 608-612

121. Yoon, H. Y., Jo, K. W., Nam, G. B., and Shim, T. S. (2017) Clinical significance of QT-prolonging drug use in patients with MDR-TB or NTM disease. The International Journal of Tuberculosis and Lung Disease 21, 996-1001

122. Hartkoorn, R. C., Uplekar, S., and Cole, S. T. (2014) Cross-resistance between clofazimine and bedaquiline through upregulation of MmpL5 in Mycobacterium tuberculosis. Antimicrob Agents Chemother 58, 2979-2981

123. Somoskovi, A., Bruderer, V., Homke, R., Bloemberg, G. V., and Bottger, E. C. (2015) A mutation associated with clofazimine and bedaquiline cross-resistance in MDR-TB following bedaquiline treatment. The European respiratory journal 45, 554-557

124. Kamikawa, R., Azuma, T., Ishii, K. I., Matsuno, Y., and Miyashita, H. (2018) Diversity of Organellar Genomes in Non-photosynthetic Diatoms. Protist 169, 351361

125. Lamprecht, D. A., Finin, P. M., Rahman, M. A., Cumming, B. M., Russell, S. L., Jonnala, S. R., Adamson, J. H., and Steyn, A. J. (2016) Turning the respiratory flexibility of Mycobacterium tuberculosis against itself. Nature communications $\mathbf{7}$, 12393 
126. Kalia, N. P., Hasenoehrl, E. J., Ab Rahman, N. B., Koh, V. H., Ang, M. L. T., Sajorda, D. R., Hards, K., Grüber, G., Alonso, S., Cook, G. M., Berney, M., and Pethe, K. (2017) Exploiting the synthetic lethality between terminal respiratory oxidases to kill Mycobacterium tuberculosis and clear host infection. Proceedings of the National Academy of Sciences 114, 7426-7431

127. Mahajan, R. (2013) Bedaquiline: First FDA-approved tuberculosis drug in 40 years. Int J Appl Basic Med Res 3, 1-2

128. Petrella, S., Cambau, E., Chauffour, A., Andries, K., Jarlier, V., and Sougakoff, W. (2006) Genetic basis for natural and acquired resistance to the diarylquinoline R207910 in mycobacteria. Antimicrob Agents Chemother 50, 2853-2856

129. Andries, K., Villellas, C., Coeck, N., Thys, K., Gevers, T., Vranckx, L., Lounis, N., de Jong, B. C., and Koul, A. (2014) Acquired resistance of Mycobacterium tuberculosis to bedaquiline. PLoS One $\mathbf{9}$, e102135

130. Ismail, N. A., Omar, S. V., Joseph, L., Govender, N., Blows, L., Ismail, F., Koornhof, H., Dreyer, A. W., Kaniga, K., and Ndjeka, N. (2018) Defining Bedaquiline Susceptibility, Resistance, Cross-Resistance and Associated Genetic Determinants: A Retrospective Cohort Study. EBioMedicine 28, 136-142

131. Bandyopadhyay, S., and Allison, W. S. (2004) The ionic track in the F1-ATPase from the thermophilic Bacillus PS3. Biochemistry 43, 2533-2540

132. Feniouk, B. A., Kato-Yamada, Y., Yoshida, M., and Suzuki, T. (2010) Conformational transitions of subunit epsilon in ATP synthase from thermophilic Bacillus PS3. Biophys J 98, 434-442

133. Ishii, N., Yoshimura, H., Nagayama, K., Kagawa, Y., and Yoshida, M. (1993) Threedimensional structure of F1-ATPase of thermophilic bacterium PS3 obtained by electron crystallography. Journal of biochemistry 113, 245-250

134. Jault, J. M., Dou, C., Grodsky, N. B., Matsui, T., Yoshida, M., and Allison, W. S. (1996) The $\alpha 3 \beta 3 \gamma$ subcomplex of the F1-ATPase from the thermophilic Bacillus PS3 with the $\beta$ T165S substitution does not entrap inhibitory MgADP in a catalytic site during turnover. Journal of Biological Chemistry 271, 28818-28824

135. Jault, J. M., Kaibara, C., Yoshida, M., Garrod, S., and Allison, W. S. (1994) Probing the specificity of nucleotide binding to the F1-ATPase from thermophilic Bacillus PS3 and its isolated alpha and beta subunits with 2-N3-[beta, gamma-32P]ATP. Arch Biochem Biophys 310, 282-288

136. Richard, P., Pitard, B., and Rigaud, J. L. (1995) ATP synthesis by the F0F1-ATPase from the thermophilic Bacillus PS3 co-reconstituted with bacteriorhodopsin into liposomes. Evidence for stimulation of ATP synthesis by ATP bound to a noncatalytic binding site. J Biol Chem 270, 21571-21578 
137. He, J., Ford, H. C., Carroll, J., Douglas, C., Gonzales, E., Ding, S., Fearnley, I. M., and Walker, J. E. (2018) Assembly of the membrane domain of ATP synthase in human mitochondria. Proc Natl Acad Sci U S A 115, 2988-2993

138. Klionsky, D. J., Brusilow, W. S., and Simoni, R. D. (1983) Assembly of a functional F0 of the proton-translocating ATPase of Escherichia coli. J Biol Chem 258, 1013610143

139. Ruhle, T., and Leister, D. (2015) Assembly of F1F0-ATP synthases. Biochimica et biophysica acta 1847, 849-860

140. Boyer, P. D. (1997) The ATP synthase--a splendid molecular machine. Annual review of biochemistry 66, 717-749

141. Capaldi, R. A., and Aggeler, R. (2002) Mechanism of the F(1)F(0)-type ATP synthase, a biological rotary motor. Trends in biochemical sciences $27,154-160$

142. Dimroth, P., von Ballmoos, C., Meier, T., and Kaim, G. (2003) Electrical power fuels rotary ATP synthase. Structure 11, 1469-1473

143. Sobti, M., Smits, C., Wong, A. S. W., Ishmukhametov, R., Stock, D., Sandin, S., and Stewart, A. G. (2016) Cryo-EM structures of the autoinhibited E. coli ATP synthase in three rotational states. eLife 5, e21598

144. Berman, H. M., Westbrook, J., Feng, Z., Gilliland, G., Bhat, T. N., Weissig, H., Shindyalov, I. N., and Bourne, P. E. (2000) The Protein Data Bank. Nucleic Acids Res 28, 235-242

145. Preiss, L., Klyszejko, A. L., Hicks, D. B., Liu, J., Fackelmayer, O. J., Yildiz, O., Krulwich, T. A., and Meier, T. (2013) The c-ring stoichiometry of ATP synthase is adapted to cell physiological requirements of alkaliphilic Bacillus pseudofirmus OF4. Proc Natl Acad Sci U S A 110, 7874-7879

146. Ahmad, Z., Okafor, F., and Laughlin, T. F. (2011) Role of Charged Residues in the Catalytic Sites of Escherichia coli ATP Synthase. J Amino Acids 2011, 785741

147. Senior, A. E., Nadanaciva, S., and Weber, J. (2002) The molecular mechanism of ATP synthesis by F1F0-ATP synthase. Biochimica et Biophysica Acta (BBA) Bioenergetics 1553, 188-211

148. Shirakihara, Y., Shiratori, A., Tanikawa, H., Nakasako, M., Yoshida, M., and Suzuki, T. (2015) Structure of a thermophilic F1-ATPase inhibited by an epsilon-subunit: deeper insight into the epsilon-inhibition mechanism. The FEBS journal 282, 28952913

149. Watanabe, R., Koyasu, K., You, H., Tanigawara, M., and Noji, H. (2015) Torque transmission mechanism via DELSEED loop of F1-ATPase. Biophys $J$ 108, 11441152 
150. D'Alessandro, M., and Melandri, B. A. (2010) ATP hydrolysis in ATP synthases can be differently coupled to proton transport and modulated by ADP and phosphate: a structure based model of the mechanism. Biochimica et biophysica acta 1797, 755762

151. Hahn, A., Vonck, J., Mills, D. J., Meier, T., and Kühlbrandt, W. (2018) Structure, mechanism, and regulation of the chloroplast ATP synthase. Science $\mathbf{3 6 0}$

152. Dimroth, P. (1990) Mechanisms of sodium transport in bacteria. Philos Trans $R$ Soc Lond B Biol Sci 326, 465-477

153. Azim, S., and Ahmad, Z. (2018) Glu residues of betaDELSEED-motif are essential for peptide binding in Escherichia coli ATP synthase. International journal of biological macromolecules 116, 977-982

154. Hara, K. Y., Noji, H., Bald, D., Yasuda, R., Kinosita, K., Jr., and Yoshida, M. (2000) The role of the DELSEED motif of the beta subunit in rotation of F1-ATPase. J Biol Chem 275, 14260-14263

155. Tanigawara, M., Tabata, K. V., Ito, Y., Ito, J., Watanabe, R., Ueno, H., Ikeguchi, M., and Noji, H. (2012) Role of the DELSEED loop in torque transmission of F1-ATPase. Biophys J 103, 970-978

156. Bald, D., Muneyuki, E., Amano, T., Kruip, J., Hisabori, T., and Yoshida, M. (1999) The noncatalytic site-deficient alpha3beta3gamma subcomplex and FoF1-ATP synthase can continuously catalyse ATP hydrolysis when $\mathrm{Pi}$ is present. European journal of biochemistry / FEBS 262, 563-568

157. Boyer, P. D. (1989) A perspective of the binding change mechanism for ATP synthesis. FASEB journal : official publication of the Federation of American Societies for Experimental Biology 3, 2164-2178

158. Hyndman, D. J., Milgrom, Y. M., Bramhall, E. A., and Cross, R. L. (1994) Nucleotide-binding sites on Escherichia coli F1-ATPase. Specificity of noncatalytic sites and inhibition at catalytic sites by MgADP. The Journal of biological chemistry 269, 28871-28877

159. Taniguchi, N., Suzuki, T., Berney, M., Yoshida, M., and Cook, G. M. (2011) The regulatory C-terminal domain of subunit epsilon of $\mathrm{F}(0) \mathrm{F}(1)$ ATP synthase is dispensable for growth and survival of Escherichia coli. J Bacteriol 193, 2046-2052

160. Feniouk, B. A., Suzuki, T., and Yoshida, M. (2007) Regulatory interplay between proton motive force, ADP, phosphate, and subunit epsilon in bacterial ATP synthase. $J$ Biol Chem 282, 764-772

161. Walker, J. E., Saraste, M., Runswick, M. J., and Gay, N. J. (1982) Distantly related sequences in the alpha- and beta-subunits of ATP synthase, myosin, kinases and other ATP-requiring enzymes and a common nucleotide binding fold. The EMBO journal $\mathbf{1}$, 945-951 
162. Walker, J. E. (2013) The ATP synthase: the understood, the uncertain and the unknown. Biochemical Society transactions 41, 1-16

163. Walker, J. E., Saraste, M., Runswick, M. J., and Gay, N. J. (1982) Distantly related sequences in the alpha- and beta-subunits of ATP synthase, myosin, kinases and other ATP-requiring enzymes and a common nucleotide binding fold. The EMBO journal 1, 945-951

164. Nakamoto, R. K., Baylis Scanlon, J. A., and Al-Shawi, M. K. (2008) The rotary mechanism of the ATP synthase. Arch Biochem Biophys 476, 43-50

165. Nakanishi-Matsui, M., Sekiya, M., Nakamoto, R. K., and Futai, M. (2010) The mechanism of rotating proton pumping ATPases. Biochimica et biophysica acta 1797, 1343-1352

166. Salcedo, G., Cano-Sanchez, P., de Gomez-Puyou, M. T., Velazquez-Campoy, A., and Garcia-Hernandez, E. (2014) Isolated noncatalytic and catalytic subunits of F1ATPase exhibit similar, albeit not identical, energetic strategies for recognizing adenosine nucleotides. Biochim Biophys Acta 1837, 44-50

167. Ito, Y., and Ikeguchi, M. (2015) Mechanism of the alphabeta conformational change in F1-ATPase after ATP hydrolysis: free-energy simulations. Biophys J 108, 85-97

168. Akutsu, H. (2017) Dynamic mechanisms driving conformational conversions of the beta and epsilon subunits involved in rotational catalysis of F1-ATPase. Proc Jpn Acad Ser B Phys Biol Sci 93, 630-647

169. Weber, J., Nadanaciva, S., and Senior, A. E. (2000) ATP-driven rotation of the gamma subunit in $\mathrm{F}(1)$-ATPase. FEBS Lett $\mathbf{4 8 3}, 1-5$

170. Weber, J., and Senior, A. E. (2000) ATP synthase: what we know about ATP hydrolysis and what we do not know about ATP synthesis. Biochim Biophys Acta 1458, 300-309

171. Turina, P. (2000) Structural changes during ATP hydrolysis activity of the ATP synthase from Escherichia coli as revealed by fluorescent probes. Journal of bioenergetics and biomembranes 32, 373-381

172. Suzuki, T., Suzuki, J., Mitome, N., Ueno, H., and Yoshida, M. (2000) Second stalk of ATP synthase. Cross-linking of gamma subunit in F1 to truncated Fob subunit prevents ATP hydrolysis. J Biol Chem 275, 37902-37906

173. Takahashi, H., Umino, S., Miki, Y., Ishizuka, R., Maeda, S., Morita, A., Suzuki, M., and Matubayasi, N. (2017) Drastic Compensation of Electronic and Solvation Effects on ATP Hydrolysis Revealed through Large-Scale QM/MM Simulations Combined with a Theory of Solutions. The journal of physical chemistry. B 121, 2279-2287

174. Yasuda, R., Noji, H., Yoshida, M., Kinosita, K., and Itoh, H. (2001) Resolution of distinct rotational substeps by submillisecond kinetic analysis of F1-ATPase. Nature 410, 898-904 
175. Bockmann, R. A., and Grubmuller, H. (2002) Nanoseconds molecular dynamics simulation of primary mechanical energy transfer steps in F1-ATP synthase. Nat Struct Biol 9, 198-202

176. Ma, J., Flynn, T. C., Cui, Q., Leslie, A. G. W., Walker, J. E., and Karplus, M. (2002) A Dynamic Analysis of the Rotation Mechanism for Conformational Change in F1ATPase. Structure 10, 921-931

177. Ahmad, Z., and Senior, A. E. (2006) Inhibition of the ATPase activity of Escherichia coli ATP synthase by magnesium fluoride. FEBS Lett 580, 517-520

178. Keis, S., Stocker, A., Dimroth, P., and Cook, G. M. (2006) Inhibition of ATP hydrolysis by thermoalkaliphilic F1Fo-ATP synthase is controlled by the C terminus of the epsilon subunit. Journal of bacteriology 188, 3796-3804

179. Mizumoto, J., Kikuchi, Y., Nakanishi, Y.-H., Mouri, N., Cai, A., Ohta, T., Haruyama, T., and Kato-Yamada, Y. (2013) $\varepsilon$ Subunit of Bacillus subtilis F1-ATPase Relieves MgADP Inhibition. PLOS ONE 8, e73888

180. Ferguson, S. A., Cook, G. M., Montgomery, M. G., Leslie, A. G., and Walker, J. E. (2016) Regulation of the thermoalkaliphilic F1-ATPase from Caldalkalibacillus thermarum. Proc Natl Acad Sci U S A 113, 10860-10865

181. Nakanishi-Matsui, M., Sekiya, M., and Futai, M. (2016) ATP synthase from Escherichia coli: Mechanism of rotational catalysis, and inhibition with the epsilon subunit and phytopolyphenols. Biochim Biophys Acta 1857, 129-140

182. D'Alessandro, M., Turina, P., Melandri, B. A., and Dunn, S. D. (2017) Modulation of coupling in the Escherichia coli ATP synthase by ADP and Pi: Role of the epsilon subunit C-terminal domain. Biochim Biophys Acta 1858, 34-44

183. Uhlin, U., Cox, G. B., and Guss, J. M. (1997) Crystal structure of the epsilon subunit of the proton-translocating ATP synthase from Escherichia coli. Structure 5, 12191230

184. Yagi, H., Kajiwara, N., Tanaka, H., Tsukihara, T., Kato-Yamada, Y., Yoshida, M., and Akutsu, H. (2007) Structures of the thermophilic F1-ATPase epsilon subunit suggesting ATP-regulated arm motion of its C-terminal domain in F1. Proc Natl Acad Sci U S A 104, 11233-11238

185. Yaginuma, H., Kawai, S., Tabata, K. V., Tomiyama, K., Kakizuka, A., Komatsuzaki, T., Noji, H., and Imamura, H. (2014) Diversity in ATP concentrations in a single bacterial cell population revealed by quantitative single-cell imaging. Scientific Reports 4

186. Kato, S., Yoshida, M., and Kato-Yamada, Y. (2007) Role of the $\varepsilon$ subunit of thermophilic F1-ATPase as a sensor for ATP. Journal of Biological Chemistry 282, 37618-37623 
187. Bulygin, V. V., Duncan, T. M., and Cross, R. L. (2004) Rotor/Stator interactions of the epsilon subunit in Escherichia coli ATP synthase and implications for enzyme regulation. J Biol Chem 279, 35616-35621

188. Krah, A., Kato-Yamada, Y., and Takada, S. (2017) The structural basis of a high affinity ATP binding epsilon subunit from a bacterial ATP synthase. PLoS One 12, e0177907

189. Kato-Yamada, Y., Bald, D., Koike, M., Motohashi, K., Hisabori, T., and Yoshida, M. (1999) Epsilon subunit, an endogenous inhibitor of bacterial $\mathrm{F}(1)$-ATPase, also inhibits $\mathrm{F}(0) \mathrm{F}(1)$-ATPase. J Biol Chem 274, 33991-33994

190. Rodgers, A. J., and Wilce, M. C. (2000) Structure of the gamma-epsilon complex of ATP synthase. Nat Struct Biol 7, 1051-1054

191. Hausrath, A. C., Gruber, G., Matthews, B. W., and Capaldi, R. A. (1999) Structural features of the gamma subunit of the Escherichia coli F(1) ATPase revealed by a 4.4A resolution map obtained by x-ray crystallography. Proc Natl Acad Sci U S A 96, $13697-13702$

192. Aggeler, R., Weinreich, F., and Capaldi, R. A. (1995) Arrangement of the epsilon subunit in the Escherichia coli ATP synthase from the reactivity of cysteine residues introduced at different positions in this subunit. Biochimica et biophysica acta 1230, $62-68$

193. Aggeler, R., Haughton, M. A., and Capaldi, R. A. (1995) Disulfide bond formation between the $\mathrm{COOH}$-terminal domain of the beta subunits and the gamma and epsilon subunits of the Escherichia coli F1-ATPase. Structural implications and functional consequences. J Biol Chem 270, 9185-9191

194. Cingolani, G., and Duncan, T. M. (2011) Structure of the ATP synthase catalytic complex $(\mathrm{F}(1))$ from Escherichia coli in an autoinhibited conformation. Nature structural \& molecular biology 18, 701-707

195. Yamazaki, A., Bartucca, F., Ting, A., and Bitensky, M. W. (1982) Reciprocal effects of an inhibitory factor on catalytic activity and noncatalytic cGMP binding sites of rod phosphodiesterase. Proceedings of the National Academy of Sciences of the United States of America 79, 3702-3706

196. Shirakihara, Y., Shiratori, A., Tanikawa, H., Nakasako, M., Yoshida, M., and Suzuki, T. (2015) Structure of a thermophilic F<inf $>1</$ inf $>$-ATPase inhibited by an $\varepsilon$-subunit: Deeper insight into the $\varepsilon$-inhibition mechanism. FEBS Journal 282, 2895-2913

197. Cingolani, G., and Duncan, T. M. (2011) Structure of the ATP synthase catalytic complex (F1) from Escherichia coli in an autoinhibited conformation. Nature structural \& molecular biology 18, 701-707

198. Nazina, T. N., Tourova, T. P., Poltaraus, A. B., Novikova, E. V., Grigoryan, A. A., Ivanova, A. E., Lysenko, A. M., Petrunyaka, V. V., Osipov, G. A., Belyaev, S. S., and Ivanov, M. V. (2001) Taxonomic study of aerobic thermophilic bacilli: descriptions of 
Geobacillus subterraneus gen. nov., sp. nov. and Geobacillus uzenensis sp. nov. from petroleum reservoirs and transfer of Bacillus stearothermophilus, Bacillus thermocatenulatus, Bacillus thermoleovorans, Bacillus kaustophilus, Bacillus thermodenitrificans to Geobacillus as the new combinations G. stearothermophilus, G. th. Int J Syst Evol Microbiol 51, 433-446

199. Coorevits, A., Dinsdale, A. E., Halket, G., Lebbe, L., De Vos, P., Van Landschoot, A., and Logan, N. A. (2012) Taxonomic revision of the genus Geobacillus: emendation of Geobacillus, G. stearothermophilus, G. jurassicus, G. toebii, G. thermodenitrificans and G. thermoglucosidans (nom. corrig., formerly 'thermoglucosidasius'); transfer of Bacillus thermantarcticus to the genus as G. thermantarcticus comb. nov.; proposal of Caldibacillus debilis gen. nov., comb. nov.; transfer of G. tepidamans to Anoxybacillus as A. tepidamans comb. nov.; and proposal of Anoxybacillus caldiproteolyticus sp. nov. Int J Syst Evol Microbiol 62, 1470-1485

200. Yang, Y. J., Zhang, S. B., and Huang, W. (2018) Chloroplastic ATP Synthase Alleviates Photoinhibition of Photosystem I in Tobacco Illuminated at Chilling Temperature. Frontiers in plant science 9, 1648

201. Fillingame, R. H. (1997) Coupling H+ transport and ATP synthesis in F1F0-ATP synthases: glimpses of interacting parts in a dynamic molecular machine. $J$ Exp Biol 200, $217-224$

202. Hanukoglu, I. (2015) Proteopedia: Rossmann fold: A beta-alpha-beta fold at dinucleotide binding sites. Biochem Mol Biol Educ 43, 206-209

203. Priya, R., Biuković, G., Manimekalai, M. S. S., Lim, J., Rao, S. P. S., and Grüber, G. (2013) Solution structure of subunit $\gamma(\gamma 1-204)$ of the Mycobacterium tuberculosis FATP synthase and the unique loop of $\gamma 165-178$, representing a novel TB drug target. Journal of bioenergetics and biomembranes 45, 121-129

204. Hotra, A., Suter, M., Biuković, G., Ragunathan, P., Kundu, S., Dick, T., and Grüber, G. (2016) Deletion of a unique loop in the mycobacterial F-ATP synthase gamma subunit sheds light on its inhibitory role in ATP hydrolysis-driven $\mathrm{H}(+)$ pumping. The FEBS journal 283, 1947-1961

205. Bogdanović, N., Sundararaman, L., Kamariah, N., Tyagi, A., Bhushan, S., Ragunathan, P., Shin, J., Dick, T., and Grüber, G. (2018) Structure and function of Mycobacteriumspecific components of F-ATP synthase subunits alpha and epsilon. Journal of structural biology

206. Hotra, A., Suter, M., Biuković, G., Ragunathan, P., Kundu, S., Dick, T., and Grüber, G. (2016) Deletion of a unique loop in the mycobacterial F-ATP synthase gamma subunit sheds light in its inhibitory role in ATP hydrolysis driven $\mathrm{H}$-pumping. The FEBS journal

207. Lu, P., Lill, H., and Bald, D. (2014) ATP synthase in mycobacteria: special features and implications for a function as drug target. Biochimica et biophysica acta 1837, $1208-1218$ 
208. Biuković, G., Basak, S., Manimekalai, M. S., Rishikesan, S., Roessle, M., Dick, T., Rao, S. P., Hunke, C., and Grüber, G. (2013) Variations of subunit epsilon of the Mycobacterium tuberculosis F1FO ATP synthase and a novel model for mechanism of action of the tuberculosis drug TMC207. Antimicrobial agents and chemotherapy 57, $168-176$

209. Joon, S., Ragunathan, P., Sundararaman, L., Nartey, W., Kundu, S., Manimekalai, M. S. S., Bogdanović, N., Dick, T., and Grüber, G. (2018) The NMR solution structure of Mycobacterium tuberculosis F-ATP synthase subunit epsilon provides new insight into energy coupling inside the rotary engine. The FEBS journal 285, 1111-1128

210. Bryant, D. A., and Frigaard, N. U. (2006) Prokaryotic photosynthesis and phototrophy illuminated. Trends Microbiol 14, 488-496

211. Olson, J. M. (2006) Photosynthesis in the Archean era. Photosynthesis research 88, $109-117$

212. Wang, C., Guo, L., Li, Y., and Wang, Z. (2012) Systematic comparison of C3 and C4 plants based on metabolic network analysis. BMC Syst Biol 6 Suppl 2, S9

213. Weise, S. E., van Wijk, K. J., and Sharkey, T. D. (2011) The role of transitory starch in C(3), CAM, and C(4) metabolism and opportunities for engineering leaf starch accumulation. Journal of experimental botany 62, 3109-3118

214. Angelo, C. L., and Daehler, C. C. (2014) Temperature is the major driver of distribution patterns for $\mathrm{C} 4$ and $\mathrm{C} 3 \mathrm{BEP}$ grasses along tropical elevation gradients in Hawai' $i$, and comparison with worldwide patterns. Botany 93, 9-22

215. Jansen, G., Crummenerl, L. L., Gilbert, F., Mohr, T., Pfefferkorn, R., Thanert, R., Rosenstiel, P., and Schulenburg, H. (2015) Evolutionary Transition from Pathogenicity to Commensalism: Global Regulator Mutations Mediate Fitness Gains through Virulence Attenuation. Mol Biol Evol 32, 2883-2896

216. Wilson, J. W., Schurr, M. J., LeBlanc, C. L., Ramamurthy, R., Buchanan, K. L., and Nickerson, C. A. (2002) Mechanisms of bacterial pathogenicity. Postgraduate medical journal 78, 216-224

217. Tufariello, J. M., Jacobs, W. R., Jr., and Chan, J. (2004) Individual Mycobacterium tuberculosis resuscitation-promoting factor homologues are dispensable for growth in vitro and in vivo. Infection and immunity 72, 515-526

218. Rosser, A., Stover, C., Pareek, M., and Mukamolova, G. V. (2017) Resuscitationpromoting factors are important determinants of the pathophysiology in Mycobacterium tuberculosis infection. Crit Rev Microbiol 43, 621-630

219. Wayne, L. G., and Hayes, L. G. (1996) An in vitro model for sequential study of shiftdown of Mycobacterium tuberculosis through two stages of nonreplicating persistence. Infection and immunity 64, 2062-2069 
220. Alnimr, A. M. (2015) Dormancy models for Mycobacterium tuberculosis: A minireview. Brazilian journal of microbiology : [publication of the Brazilian Society for Microbiology] 46, 641-647

221. Gleeson, L. E., Sheedy, F. J., Palsson-McDermott, E. M., Triglia, D., O'Leary, S. M., O'Sullivan, M. P., O'Neill, L. A., and Keane, J. (2016) Cutting Edge: Mycobacterium tuberculosis Induces Aerobic Glycolysis in Human Alveolar Macrophages That Is Required for Control of Intracellular Bacillary Replication. Journal of immunology 196, 2444-2449

222. McKinney, J. D., Honer zu Bentrup, K., Munoz-Elias, E. J., Miczak, A., Chen, B., Chan, W. T., Swenson, D., Sacchettini, J. C., Jacobs, W. R., Jr., and Russell, D. G. (2000) Persistence of Mycobacterium tuberculosis in macrophages and mice requires the glyoxylate shunt enzyme isocitrate lyase. Nature 406, 735-738

223. Movahedzadeh, F., Smith, D. A., Norman, R. A., Dinadayala, P., Murray-Rust, J., Russell, D. G., Kendall, S. L., Rison, S. C., McAlister, M. S., Bancroft, G. J., McDonald, N. Q., Daffe, M., Av-Gay, Y., and Stoker, N. G. (2004) The Mycobacterium tuberculosis ino1 gene is essential for growth and virulence. Molecular microbiology 51, 1003-1014

224. Nesbitt, N. M., Yang, X., Fontan, P., Kolesnikova, I., Smith, I., Sampson, N. S., and Dubnau, E. (2010) A thiolase of Mycobacterium tuberculosis is required for virulence and production of androstenedione and androstadienedione from cholesterol. Infection and immunity 78, 275-282

225. Woese, C. R., Kandler, O., and Wheelis, M. L. (1990) Towards a natural system of organisms: proposal for the domains Archaea, Bacteria, and Eucarya. Proc Natl Acad Sci U S A 87, 4576-4579

226. Balch, W. E., Fox, G. E., Magrum, L. J., Woese, C. R., and Wolfe, R. S. (1979) Methanogens: reevaluation of a unique biological group. Microbiological reviews $\mathbf{4 3}$, 260-296

227. Balch, W. E., Magrum, L. J., Fox, G. E., Wolfe, R. S., and Woese, C. R. (1977) An ancient divergence among the bacteria. Journal of molecular evolution 9, 305-311

228. Fuchs, G. (1986) CO2 fixation in acetogenic bacteria: Variations on a theme. FEMS microbiology letters 39, 181-213

229. Schuchmann, K., and Muller, V. (2014) Autotrophy at the thermodynamic limit of life: a model for energy conservation in acetogenic bacteria. Nature reviews. Microbiology 12, 809-821

230. Cavanaugh, C. M. (1983) Symbiotic chemoautotrophic bacteria in marine invertebrates from sulphide-rich habitats. Nature 302, 58

231. Boschker, H. T., Vasquez-Cardenas, D., Bolhuis, H., Moerdijk-Poortvliet, T. W., and Moodley, L. (2014) Chemoautotrophic carbon fixation rates and active bacterial communities in intertidal marine sediments. PLoS One 9, e101443 
232. Bonch-Osmolovskaya, E., and Atomi, H. (2015) Editorial overview: Extremophiles: From extreme environments to highly stable biocatalysts. Current opinion in microbiology 25, vii-viii

233. Rampelotto, P. H. (2013) Extremophiles and extreme environments. Life (Basel) 3, $482-485$

234. Ljungdahl, L. G. (1986) The autotrophic pathway of acetate synthesis in acetogenic bacteria. Annual review of microbiology 40, 415-450

235. Poehlein, A., Schmidt, S., Kaster, A. K., Goenrich, M., Vollmers, J., Thurmer, A., Bertsch, J., Schuchmann, K., Voigt, B., Hecker, M., Daniel, R., Thauer, R. K., Gottschalk, G., and Muller, V. (2012) An ancient pathway combining carbon dioxide fixation with the generation and utilization of a sodium ion gradient for ATP synthesis. PLoS One 7, e33439

236. Bertsch, J., and Müller, V. (2015) CO Metabolism in the Acetogen Acetobacterium woodii. Applied and Environmental Microbiology 81, 5949-5956

237. Heise, R., Reidlinger, J., Muller, V., and Gottschalk, G. (1991) A sodium-stimulated ATP synthase in the acetogenic bacterium Acetobacterium woodii. FEBS Lett 295, $119-122$

238. Rahlfs, S., Aufurth, S., and Muller, V. (1999) The Na(+)-F(1)F(0)-ATPase operon from Acetobacterium woodii. Operon structure and presence of multiple copies of atpE which encode proteolipids of 8- and 18-kda. J Biol Chem 274, 33999-34004

239. Müller, V., Aufurth, S., and Rahlfs, S. (2001) The Na+ cycle in Acetobacterium woodii: identification and characterization of a Na+ translocating F1F0-ATPase with a mixed oligomer of 8 and $16 \mathrm{kDa}$ proteolipids. Biochimica et Biophysica Acta (BBA) Bioenergetics 1505, 108-120

240. Lyons, T. W., Reinhard, C. T., and Planavsky, N. J. (2014) The rise of oxygen in Earth's early ocean and atmosphere. Nature 506, 307-315

241. Pavlov, A. A., and Kasting, J. F. (2002) Mass-independent fractionation of sulfur isotopes in Archean sediments: strong evidence for an anoxic Archean atmosphere. Astrobiology 2, 27-41

242. Mayer, F., Lurz, R., and Schoberth, S. (1977) Electron microscopic investigation of the hydrogen-oxidizing acetate-forming anaerobic bacterium Acetobacterium woodii. Archives of microbiology 115, 207-213

243. Braun, K., and Gottschalk, G. (1981) Effect of molecular hydrogen and carbon dioxide on chemo-organotrophic growth of Acetobacterium woodii and Clostridium aceticum. Archives of microbiology 128, 294-298

244. Shanmugasundaram, T., Ragsdale, S. W., and Wood, H. G. (1988) Role of carbon monoxide dehydrogenase in acetate synthesis by the acetogenic bacterium, Acetobacterium woodii. Biofactors 1, 147-152 
245. Aufurth, S., Schagger, H., and Müller, V. (2000) Identification of subunits a, b, and c1 from Acetobacterium woodii Na+-F1F0-ATPase. Subunits c1, c2, AND c3 constitute a mixed c-oligomer. The Journal of biological chemistry 275, 33297-33301

246. Balch, W. E., Schoberth, S., Tanner, R. S., and Wolfe, R. S. (1977) Acetobacterium, a New Genus of Hydrogen-Oxidizing, Carbon Dioxide-Reducing, Anaerobic Bacteria. International Journal of Systematic Bacteriology 27, 355-361

247. BALCH, W. E., SCHOBERTH, S., TANNER, R. S., and WOLFE, R. S. (1977) Acetobacterium, a New Genus of Hydrogen-Oxidizing, Carbon Dioxide-Reducing, Anaerobic Bacteria. International Journal of Systematic and Evolutionary Microbiology 27, 355-361

248. Heise, R., Muller, V., and Gottschalk, G. (1992) Presence of a sodium-translocating ATPase in membrane vesicles of the homoacetogenic bacterium Acetobacterium woodii. European journal of biochemistry / FEBS 206, 553-557

249. Müller, V., and Bowien, S. (1995) Differential effects of sodium ions on motility in the homoacetogenic bacteriaAcetobacterium woodii andSporomusa sphaeroides. Archives of microbiology 164, 363-369

250. Muller, V. (2003) Energy conservation in acetogenic bacteria. Appl Environ Microbiol 69, 6345-6353

251. Hess, V., Schuchmann, K., and Müller, V. (2013) The ferredoxin:NAD+ oxidoreductase (Rnf) from the acetogen Acetobacterium woodii requires $\mathrm{Na}+$ and is reversibly coupled to the membrane potential. The Journal of biological chemistry $\mathbf{2 8 8}$, 31496-31502

252. Brandt, K., Müller, D. B., Hoffmann, J., Hübert, C., Brutschy, B., Deckers-Hebestreit, G., and Müller, V. (2013) Functional production of the Na+ F1FO ATP synthase from Acetobacterium woodii in Escherichia coli requires the native AtpI. Journal of bioenergetics and biomembranes 45, 15-23

253. Martin, W. F. (2012) Hydrogen, metals, bifurcating electrons, and proton gradients: the early evolution of biological energy conservation. FEBS Lett 586, 485-493

254. Takami, H., Noguchi, H., Takaki, Y., Uchiyama, I., Toyoda, A., Nishi, S., Chee, G. J., Arai, W., Nunoura, T., Itoh, T., Hattori, M., and Takai, K. (2012) A deeply branching thermophilic bacterium with an ancient acetyl-CoA pathway dominates a subsurface ecosystem. PLoS One 7, e30559

255. Schmehl, M., Jahn, A., Meyer zu Vilsendorf, A., Hennecke, S., Masepohl, B., Schuppler, M., Marxer, M., Oelze, J. r., and Klipp, W. (1993) Identification of a new class of nitrogen fixation genes in Rhodobacter capsalatus: a putative membrane complex involved in electron transport to nitrogenase. MGG Molecular \& General Genetics 241-241, 602-615 
256. Reidlinger, J., Mayer, F., and Muller, V. (1994) The molecular structure of the $\mathrm{Na}(+)-$ translocating F1F0-ATPase of Acetobacterium woodii, as revealed by electron microscopy, resembles that of $\mathrm{H}(+)$-translocating ATPases. FEBS Lett 356, 17-20

257. Akey, C. W., Crepeau, R. H., Dunn, S. D., McCarty, R. E., and Edelstein, S. J. (1983) Electron microscopy and single molecule averaging of subunit-deficient F1-ATPases from Escherichia coli and spinach chloroplasts. The EMBO journal 2, 1409-1415

258. Gräber, P., Fromme, P., Schmidt, G., and Boekema, E. (1988) Structure of the ATPsynthase from chloroplasts as revealed from biochemical studies and electron microscopy. Progress in clinical and biological research 273, 67-74

259. Vorburger, T., Ebneter, J. Z., Wiedenmann, A., Morger, D., Weber, G., Diederichs, K., Dimroth, P., and von Ballmoos, C. (2008) Arginine-induced conformational change in the c-ring/a-subunit interface of ATP synthase. The FEBS journal 275, 2137-2150

260. Segala, E., Sougakoff, W., Nevejans-Chauffour, A., Jarlier, V., and Petrella, S. (2012) New mutations in the mycobacterial ATP synthase: new insights into the binding of the diarylquinoline TMC207 to the ATP synthase C-ring structure. Antimicrob Agents Chemother 56, 2326-2334

261. Balakrishna, A. M., Seelert, H., Marx, S. H., Dencher, N. A., and Gruber, G. (2014) Crystallographic structure of the turbine c-ring from spinach chloroplast F-ATP synthase. Bioscience reports

262. Amodeo, G. F., Solesio, M. E., and Pavlov, E. V. (2017) From ATP synthase dimers to C-ring conformational changes: unified model of the mitochondrial permeability transition pore. Cell death \& disease $\mathbf{8}, 1$

263. Spruth, M., Reidlinger, J., and Müller, V. (1995) Sodium ion dependence of inhibition of the Na+-translocating F1F0-ATPase from Acetobacterium woodii. Probing the site(s) involved in ion transport. Biochimica et Biophysica Acta (BBA) - Bioenergetics 1229, 96-102

264. Meier, T., Polzer, P., Diederichs, K., Welte, W., and Dimroth, P. (2005) Structure of the Rotor Ring of F-Type Na+-ATPase from Ilyobacter tartaricus. Science 308, 659662

265. Bogdanović, N., Trifunović, D., Sielaff, H., Westphal, L., Bhushan, S., Müller, V., and Grüber, G. (2019) Structural features of the Acetobacterium woodii F-ATP synthase and the importance of the unique subunit $\gamma$-loop in Na+ translocation and ATP synthesis. The FEBS journal 286, 1894-1907

266. Waterhouse, A., Bertoni, M., Bienert, S., Studer, G., Tauriello, G., Gumienny, R., Heer, F. T., de Beer, T. A. P., Rempfer, C., Bordoli, L., Lepore, R., and Schwede, T. (2018) SWISS-MODEL: homology modelling of protein structures and complexes. Nucleic Acids Res 46, W296-W303

267. Schrodinger, LLC. (2015) The PyMOL Molecular Graphics System, Version 1.8. 
268. Kumar, S., Stecher, G., and Tamura, K. (2016) MEGA7: Molecular Evolutionary Genetics Analysis Version 7.0 for Bigger Datasets. Molecular biology and evolution 33, $1870-1874$

269. Waterhouse, A. M., Procter, J. B., Martin, D. M. A., Clamp, M., and Barton, G. J. (2009) Jalview Version 2-a multiple sequence alignment editor and analysis workbench. Bioinformatics 25, 1189-1191

270. Pettersen, E. F., Goddard, T. D., Huang, C. C., Couch, G. S., Greenblatt, D. M., Meng, E. C., and Ferrin, T. E. (2004) UCSF Chimera--a visualization system for exploratory research and analysis. J Comput Chem 25, 1605-1612

271. Tang, G., Peng, L., Baldwin, P. R., Mann, D. S., Jiang, W., Rees, I., and Ludtke, S. J. (2007) EMAN2: an extensible image processing suite for electron microscopy. Journal of structural biology 157, 38-46

272. Davis, I. W., Murray, L. W., Richardson, J. S., and Richardson, D. C. (2004) MOLPROBITY: structure validation and all-atom contact analysis for nucleic acids and their complexes. Nucleic Acids Res 32, W615-619

273. Li, J. M., Li, N., Zhu, D. Y., Wan, L. G., He, Y. L., and Yang, C. (2008) Isocitrate lyase from Mycobacterium tuberculosis promotes survival of Mycobacterium smegmatis within macrophage by suppressing cell apoptosis. Chinese medical journal 121, 1114-1119

274. Ho, S. N., Hunt, H. D., Horton, R. M., Pullen, J. K., and Pease, L. R. (1989) Sitedirected mutagenesis by overlap extension using the polymerase chain reaction. Gene 77, 51-59

275. Delaglio, F., Grzesiek, S., Vuister, G. W., Zhu, G., Pfeifer, J., and Bax, A. (1995) NMRPipe: a multidimensional spectral processing system based on UNIX pipes. $J$ Biomol NMR 6, 277-293

276. Small, J. (1983) Sparky. Geriatr Nurs 4, 166

277. Tang, G., Peng, L., Baldwin, P. R., Mann, D. S., Jiang, W., Rees, I., and Ludtke, S. J. (2007) EMAN2: an extensible image processing suite for electron microscopy. $J$. Struct. Biol. 157, 38-46

278. Scheres, S. H. (2012) RELION: implementation of a Bayesian approach to cryo-EM structure determination. Journal of structural biology 180, 519-530

279. Yang, Z., Lasker, K., Schneidman-Duhovny, D., Webb, B., Huang, C. C., Pettersen, E. F., Goddard, T. D., Meng, E. C., Sali, A., and Ferrin, T. E. (2012) UCSF Chimera, MODELLER, and IMP: an integrated modeling system. Journal of structural biology 179, 269-278

280. Huang, C. C., Meng, E. C., Morris, J. H., Pettersen, E. F., and Ferrin, T. E. (2014) Enhancing UCSF Chimera through web services. Nucleic Acids Res 42, W478-484 
281. Rodriguez-Guerra Pedregal, J., and Marechal, J. D. (2018) PyChimera: use UCSF Chimera modules in any Python 2.7 project. Bioinformatics 34, 1784-1785

282. Schneider, C. A., Rasband, W. S., and Eliceiri, K. W. (2012) NIH Image to ImageJ: 25 years of image analysis. Nat Methods 9, 671-675

283. Kozin, M. B., and Svergun, D. I. (2001) Automated matching of high- and lowresolution structural models. J Appl Crystallogr 34, 33-41

284. Gopalaswamy, R., Narayanan, S., Jacobs, W. R., Jr., and Av-Gay, Y. (2008) Mycobacterium smegmatis biofilm formation and sliding motility are affected by the serine/threonine protein kinase PknF. FEMS microbiology letters 278, 121-127

285. Wu, M. L., Gengenbacher, M., and Dick, T. (2016) Mild Nutrient Starvation Triggers the Development of a Small-Cell Survival Morphotype in Mycobacteria. Front Microbiol 7, 947

286. Wilkins, M. R., Gasteiger, E., Bairoch, A., Sanchez, J. C., Williams, K. L., Appel, R. D., and Hochstrasser, D. F. (1999) Protein identification and analysis tools in the ExPASy server. Methods. Mol. Biol. 112, 531-552

287. Waterhouse, A., Bertoni, M., Bienert, S., Studer, G., Tauriello, G., Gumienny, R., Heer, F. T., de Beer, T. A P., Rempfer, C., Bordoli, L., Lepore, R., and Schwede, T. (2018) SWISS-MODEL: homology modelling of protein structures and complexes. Nucleic Acids Research 46, W296-W303

288. The UniProt Consortium. (2017) UniProt: the universal protein knowledgebase. Nucleic Acids Research 45, D158-D169

289. Rigsby, R. E., and Parker, A. B. (2016) Using the PyMOL application to reinforce visual understanding of protein structure. Biochem Mol Biol Educ

290. Laemmli, U. K. (1970) Cleavage of structural proteins during the assembly of the head of bacteriophage T4. Nature 227, 680-685

291. Sundararaman, L. (2016) The role of N157 of A-ATP synthase subunit B in nucleotide binding and expression, production and structural and mechanistic features of subunits $\alpha$ and $\varepsilon$ of Mycobacterium tuberculosis F-ATP synthase. DR-NTU Institutional Repository

292. Dumon-Seignovert, L., Cariot, G., and Vuillard, L. (2004) The toxicity of recombinant proteins in Escherichia coli: a comparison of overexpression in BL21(DE3), C41(DE3), and C43(DE3). Protein Expr Purif 37, 203-206

293. Singh, D., Sielaff, H., Sundararaman, L., Bhushan, S., and Grüber, G. (2016) The stimulating role of subunit $\mathrm{F}$ in ATPase activity inside the $\mathrm{A}_{1}$-complex of the Methanosarcina mazei Gö1 $\mathrm{A}_{1} \mathrm{~A}_{\mathrm{O}}$ ATP synthase. Biochim. Biophys. Acta. (BBA) Bioenergetics 1857, 177-187 
294. Schäfer, I., Rössle, M., Biuković, G., Müller, V., and Grüber, G. (2006) Structural and functional analysis of the coupling subunit $\mathrm{F}$ in solution and topological arrangement of the stalk domains of the methanogenic $\mathrm{A}_{1} \mathrm{~A}_{\mathrm{O}}$ ATP synthase. J. Bioenerg. Biomembr. 38, $83-92$

295. Raghunathan, D., Gayen, S., Grüber, G., and Verma, C. S. (2010) Crosstalk along the Stalk: Dynamics of the Interaction of Subunits $B$ and $F$ in the $A_{1} A_{O}$ ATP Synthase of Methanosarcina mazei Gö1. Biochemistry 49, 4181-4190

296. Raghunathan, D., Gayen, S., Kumar, A., Hunke, C., Grüber, G., and Verma, C. S. (2012) Subunit F modulates ATP binding and migration in the nucleotide-binding subunit $\mathrm{B}$ of the $\mathrm{A}_{1} \mathrm{~A}_{\mathrm{O}} \mathrm{ATP}$ synthase of Methanosarcina mazei Gö1. J. Bioenerg. Biomembr. 44, 213-224

297. Muhleip, A. W., Dewar, C. E., Schnaufer, A., Kuhlbrandt, W., and Davies, K. M. (2017) In situ structure of trypanosomal ATP synthase dimer reveals a unique arrangement of catalytic subunits. Proc Natl Acad Sci U S A 114, 992-997

298. Krah, A., Zarco-Zavala, M., and McMillan, D. G. G. (2018) Insights into the regulatory function of the varepsilon subunit from bacterial F-type ATP synthases: a comparison of structural, biochemical and biophysical data. Open Biol $\mathbf{8}$

299. van Kessel, J. C., and Hatfull, G. F. (2008) Efficient point mutagenesis in mycobacteria using single-stranded DNA recombineering: characterization of antimycobacterial drug targets. Molecular microbiology 67, 1094-1107

300. van Kessel, J. C., and Hatfull, G. F. (2007) Recombineering in Mycobacterium tuberculosis. Nat Methods 4

301. Yoshikawa, K., Kumazawa, N., Terada, H., and Akagi, K. (1980) Physicochemical properties of SF 6847, a potent uncoupler of oxidative phosphorylation in mitochondria in relation to its activity. International Journal of Quantum Chemistry 18, 539-544

302. Kelley, L. A., Mezulis, S., Yates, C. M., Wass, M. N., and Sternberg, M. J. E. (2015) The Phyre2 web portal for protein modeling, prediction and analysis. Nature protocols 10, 845

303. Iino, R., Hasegawa, R., Tabata, K. V., and Noji, H. (2009) Mechanism of inhibition by C-terminal alpha-helices of the epsilon subunit of Escherichia coli FoF1-ATP synthase. J Biol Chem 284, 17457-17464

304. Oka, H., Hosokawa, H., Nakanishi-Matsui, M., Dunn, S. D., Futai, M., and IwamotoKihara, A. (2014) Elastic rotation of Escherichia coli $\mathrm{F}(\mathrm{O}) \mathrm{F}(1)$ having epsilon subunit fused with cytochrome $\mathrm{b}(562)$ or flavodoxin reductase. Biochem Biophys Res Commun 446, 889-893

305. Kruger, A. C., and Birkedal, V. (2013) Single molecule FRET data analysis procedures for FRET efficiency determination: probing the conformations of nucleic acid structures. Methods 64, 36-42 
306. Singh, D., Sielaff, H., Borsch, M., and Gruber, G. (2017) Conformational dynamics of the rotary subunit F in the A3 B3 DF complex of Methanosarcina mazei Go1 A-ATP synthase monitored by single-molecule FRET. FEBS Lett 591, 854-862

307. Hards, K., Robson, J. R., Berney, M., Shaw, L., Bald, D., Koul, A., Andries, K., and Cook, G. M. (2015) Bactericidal mode of action of bedaquiline. J Antimicrob Chemother 70, 2028-2037

308. Hards, K., McMillan, D. G. G., Schurig-Briccio, L. A., Gennis, R. B., Lill, H., Bald, D., and Cook, G. M. (2018) Ionophoric effects of the antitubercular drug bedaquiline. Proc Natl Acad Sci U S A 115, 7326-7331

309. Lowe, S. E., Jain, M. K., and Zeikus, J. G. (1993) Biology, ecology, and biotechnological applications of anaerobic bacteria adapted to environmental stresses in temperature, $\mathrm{pH}$, salinity, or substrates. Microbiological reviews 57, 451-509

310. Meng, W., Zhang, H., Guo, T., Pandey, C., Zhu, Y., Kon, O. L., and Sze, S. K. (2008) One-step procedure for peptide extraction from in-gel digestion sample for mass spectrometric analysis. Anal Chem 80, 9797-9805

311. Kato, Y., Matsui, T., Tanaka, N., Muneyuki, E., Hisabori, T., and Yoshida, M. (1997) Thermophilic F1-ATPase is activated without dissociation of an endogenous inhibitor, epsilon subunit. J Biol Chem 272, 24906-24912

312. Krah, A., and Takada, S. (2016) On the ATP binding site of the epsilon subunit from bacterial F-type ATP synthases. Biochimica et biophysica acta 1857, 332-340

313. Feniouk, B. A., Suzuki, T., and Yoshida, M. (2007) Regulatory interplay between proton motive force, ADP, phosphate, and subunit $\varepsilon$ in bacterial ATP synthase. Journal of Biological Chemistry 282, 764-772

314. Mizumoto, J., Kikuchi, Y., Nakanishi, Y. H., Mouri, N., Cai, A., Ohta, T., Haruyama, T., and Kato-Yamada, Y. (2013) $\varepsilon$ Subunit of Bacillus subtilis F1-ATPase Relieves MgADP Inhibition. PLoS ONE 8

315. Sekiya, M., Hosokawa, H., Nakanishi-Matsui, M., Al-Shawi, M. K., Nakamoto, R. K., and Futai, M. (2010) Single molecule behavior of inhibited and active states of escherichia coli ATP synthase F1 rotation. Journal of Biological Chemistry 285, 42058-42067

316. Shah, N. B., Hutcheon, M. L., Haarer, B. K., and Duncan, T. M. (2013) F1-ATPase of Escherichia coli: The $\varepsilon$-inhibited state forms after ATP hydrolysis, is distinct from the ADP-inhibited state, and responds dynamically to catalytic site ligands. Journal of Biological Chemistry 288, 9383-9395

317. Konno, H., Murakami-Fuse, T., Fujii, F., Koyama, F., Ueoka-Nakanishi, H., Pack, C. G., Kinjo, M., and Hisabori, T. (2006) The regulator of the F1 motor: Inhibition of rotation of cyanobacterial F1-ATPase by the $\varepsilon$ subunit. EMBO Journal 25, 4596-4604 
318. Tsumuraya, M., Furuike, S., Adachi, K., Kinosita Jr, K., and Yoshida, M. (2009) Effect of $\varepsilon$ subunit on the rotation of thermophilic Bacillus F1-ATPase. FEBS Letters 583, $1121-1126$

319. Feniouk, B. A., Suzuki, T., and Yoshida, M. (2006) The role of subunit epsilon in the catalysis and regulation of FOF1-ATP synthase. Biochim Biophys Acta 1757, 326-338

320. Garcia-Trejo, J. J., Zarco-Zavala, M., Mendoza-Hoffmann, F., Hernandez-Luna, E., Ortega, R., and Mendoza-Hernandez, G. (2016) The Inhibitory Mechanism of the zeta Subunit of the F1FO-ATPase Nanomotor of Paracoccus denitrificans and Related alpha-Proteobacteria. J Biol Chem 291, 538-546

321. Yoshida, M., Okamoto, H., Sone, N., Hirata, H., and Kagawa, Y. (1977) Reconstitution of thermostable ATPase capable of energy coupling from its purified subunits. Proc Natl Acad Sci U S A 74, 936-940

322. Klionsky, D. J., Brusilow, W. S., and Simoni, R. D. (1984) In vivo evidence for the role of the epsilon subunit as an inhibitor of the proton-translocating ATPase of Escherichia coli. J Bacteriol 160, 1055-1060

323. Kuki, M., Noumi, T., Maeda, M., Amemura, A., and Futai, M. (1988) Functional domains of epsilon subunit of Escherichia coli H+-ATPase (F0F1). J Biol Chem 263, 17437-17442

324. Patrie, W. J., and McCarty, R. E. (1984) Specific binding of coupling factor 1 lacking the delta and epsilon subunits to thylakoids. J Biol Chem 259, 11121-11128

325. Higashi, T., Kalra, V. K., Lee, S. H., Bogin, E., and Brodie, A. F. (1975) Energytransducing membrane-bound coupling factor-ATPase from Mycobacterium phlei. I. Purification, homogeneity, and properties. J Biol Chem 250, 6541-6548

326. Kundu, S., Biukovic, G., Gruber, G., and Dick, T. (2016) Bedaquiline targets the subunit of mycobacterial F-ATP synthase. Antimicrob Agents Chemother

327. Samra, H. S., Gao, F., He, F., Hoang, E., Chen, Z., Gegenheimer, P. A., Berrie, C. L., and Richter, M. L. (2006) Structural analysis of the regulatory dithiol-containing domain of the chloroplast ATP synthase gamma subunit. The Journal of biological chemistry 281, 31041-31049

328. Coskun, U., Chaban, Y. L., Lingl, A., Muller, V., Keegstra, W., Boekema, E. J., and Gruber, G. (2004) Structure and subunit arrangement of the A-type ATP synthase complex from the archaeon Methanococcus jannaschii visualized by electron microscopy. J Biol Chem 279, 38644-38648

329. Lolkema, J. S., Chaban, Y., and Boekema, E. J. (2003) Subunit composition, structure, and distribution of bacterial V-type ATPases. Journal of bioenergetics and biomembranes 35, 323-335

330. Rao, S. T., and Rossmann, M. G. (1973) Comparison of super-secondary structures in proteins. J Mol Biol 76, 241-256 
331. Sunamura, E., Konno, H., Imashimizu, M., Mochimaru, M., and Hisabori, T. (2012) A conformational change of the gamma subunit indirectly regulates the activity of cyanobacterial F1-ATPase. The Journal of biological chemistry 287, 38695-38704

332. Hahn, A., Parey, K., Bublitz, M., Mills, D. J., Zickermann, V., Vonck, J., Kuhlbrandt, W., and Meier, T. (2016) Structure of a Complete ATP Synthase Dimer Reveals the Molecular Basis of Inner Mitochondrial Membrane Morphology. Molecular cell 63, $445-456$

333. Blom, N., Gammeltoft, S., and Brunak, S. (1999) Sequence and structure-based prediction of eukaryotic protein phosphorylation sites. J Mol Biol 294, 1351-1362

334. Blom, N., Sicheritz-Ponten, T., Gupta, R., Gammeltoft, S., and Brunak, S. (2004) Prediction of post-translational glycosylation and phosphorylation of proteins from the amino acid sequence. Proteomics 4, 1633-1649 


\section{List of Author's publications:}

- Bogdanović, N., Sundararaman, L., Kamariah, N., Tyagi, A., Bhushan, S., Ragunathan, P., Shin, J., Dick, T., and Grüber, G. (2018) Structure and function of Mycobacterium-specific components of F-ATP synthase subunits alpha and epsilon. Journal of structural biology 204, 420-434

- Joon, S., Ragunathan, P., Sundararaman, L., Nartey, W., Kundu, S., Manimekalai, M. S. S., Bogdanović, N., Dick, T., and Grüber, G. (2018) The NMR solution structure of Mycobacterium tuberculosis F-ATP synthase subunit epsilon provides new insight into energy coupling inside the rotary engine. The FEBS journal 285, 1111-1128

- Bogdanović, N., Trifunović, D., Sielaff, H., Westphal, L., Bhushan, S., Müller, V., and Grüber, G. (2019) The structural features of Acetobacterium woodii F-ATP synthase reveal the importance of the unique subunit gamma-loop in $\mathrm{Na}(+)$ translocation and ATP synthesis. The FEBS journal 286, 1894-1907

\section{Conference attendance, speaker invitation, and posters:}

- $\quad$ "1st Annual symposium, From crisis to cures: New approaches to TB Drug discovery, Singapore held from 19th - 20th November 2018, organized by TOPNet. Title: "Mechanistic features of subunit $\varepsilon$ of the Mycobacterial F-ATP synthase" (Poster presentation)

- 20th European Bioenergetics Conference (EBEC), Budapest, Hungary held from 25th - 30th August 2018. Title: "Mechanistic features of subunit $\varepsilon$ of the Mycobacterial FATP synthase" (Poster presentation)

- "2nd TOPNet meeting, Singapore held on 18th June 2018. Title: "Mycobacterial subunit $\varepsilon$ within the catalytic complex; a structural and mechanistic view" (Oral presentation)

\section{Awards:}

- Nanyang Technological University Research Scholarship (2015-2019). 


\section{Appendix}


$\begin{array}{lll}\text { A. woodii } & \text { E. coli }\end{array}$

\begin{tabular}{cccccccc}
$\begin{array}{c}\text { subunit } \\
\text { name }\end{array}$ & gene & length & MW (kDa) & length & MW (kDa) & length & MW (kDa) \\
\hline$\alpha$ & atpA & 502 & 55,0 & 513 & 55,2 & 549 & 59,3 \\
$\beta$ & atpD & 466 & 50,7 & 460 & 50,3 & 486 & 53,0 \\
$\gamma$ & atpG & 302 & 34,0 & 287 & 31,6 & 305 & 33,9 \\
$\varepsilon$ & atpC & 133 & 14,8 & 139 & 15,1 & 121 & 13,1 \\
$\delta$ & atpH & 180 & 20,7 & 177 & 19,3 & & \\
$b-\delta$ & atpFH & & & & & 446 & 48,8 \\
$b$ & atpF & 169 & 19,2 & 156 & 17,3 & 171 & 18,3 \\
$a$ & atpB & 221 & 24,4 & 271 & 30,3 & 250 & 27,5 \\
$c 1$ & atpE1 & 182 & 18,4 & & & & \\
$c 2$ & atpE2 & 82 & 8,2 & & & & \\
$c 3$ & atpE3 & 82 & 8,2 & & & & \\
$c$ & atpE & & & 79 & 8,3 & 81 & 8,1 \\
\hline
\end{tabular}

Supp. Table S1: The composition of the subunits in F-ATP synthases 
Supplementary Table S2A: MALDI-TOF data of the $\alpha 1$ band of lane 2 in Figure 3.47C

\begin{tabular}{|c|c|c|c|c|c|c|c|c|c|c|}
\hline \multicolumn{6}{|c|}{ Protein name } & \multicolumn{2}{|l|}{ Accession No. } & \multicolumn{3}{|l|}{ Protein MW } \\
\hline \multicolumn{6}{|c|}{$\begin{array}{l}\text { ATP synthase subunit } \alpha \text {, sodium ion specific } \\
\text { OS=Acetobacterium woodii (strain ATCC } 29683 \text { / DSM }\end{array}$} & \multicolumn{2}{|l|}{ sp|P50000|ATPA_ACEWD } & \multicolumn{3}{|l|}{55212.7} \\
\hline Calc. Mass & Obsrv. Mass & \pm da & $\pm \mathrm{ppm}$ & $\begin{array}{c}\text { Start } \\
\text { seq. }\end{array}$ & $\begin{array}{l}\text { End } \\
\text { seq. }\end{array}$ & Sequence & ION score & C. I. $\%$ & Modification & $\begin{array}{l}\text { Rank Result } \\
\text { Type }\end{array}$ \\
\hline 803.4807 & 803.4785 & -0.0022 & -3 & 273 & 279 & AMSLILR & 24 & 0 & & Mascot \\
\hline 815.4621 & 815.4594 & -0.0027 & -3 & 165 & 171 & ELIIGDR & & & & Mascot \\
\hline 819.4756 & 819.4702 & -0.0054 & -7 & 273 & 279 & AMSLILR & & & Oxidation $(\mathrm{M})[2]$ & Mascot \\
\hline 819.4756 & 819.4702 & -0.0054 & -7 & 273 & 279 & AMSLILR & & & Oxidation $(\mathrm{M})[2]$ & Mascot \\
\hline 936.4785 & 936.4823 & 0.0038 & 4 & 450 & 456 & DIREFEK & & & & Mascot \\
\hline 936.4785 & 936.4823 & 0.0038 & 4 & 450 & 456 & DIREFEK & & & & Mascot \\
\hline 942.4679 & 942.4653 & -0.0026 & -3 & 382 & 388 & IEYAQYR & & & & Mascot \\
\hline 1072.5819 & 1072.5790 & -0.0029 & -3 & 152 & 161 & AIDSMIPIGR & 54 & 95.659 & & Mascot \\
\hline 1072.5819 & 1072.5790 & -0.0029 & -3 & 152 & 161 & AIDSMIPIGR & & & & Mascot \\
\hline 1088.5769 & 1088.5741 & -0.0028 & -3 & 152 & 161 & AIDSMIPIGR & 14 & 0 & Oxidation $(\mathrm{M})[5]$ & Mascot \\
\hline 1088.5769 & 1088.5741 & -0.0028 & -3 & 152 & 161 & AIDSMIPIGR & & & Oxidation $(\mathrm{M})[5]$ & Mascot \\
\hline 1215.6732 & 1215.6704 & -0.0028 & -2 & 107 & 118 & VVNALGFPVDGK & 63 & 99.451 & & Mascot \\
\hline 1215.6732 & 1215.6704 & -0.0028 & -2 & 107 & 118 & VVNALGFPVDGK & & & & Mascot \\
\hline 1293.6937 & 1293.6938 & 0.0001 & 0 & 256 & 266 & DVLIIYDDLSK & 70 & 99.894 & & Mascot \\
\hline 1293.6937 & 1293.6938 & 0.0001 & 0 & 256 & 266 & DVLIIYDDLSK & & & & Mascot \\
\hline
\end{tabular}




\begin{tabular}{|c|c|c|c|c|c|c|c|c|c|c|}
\hline 1300.7471 & 1300.7438 & -0.0033 & -3 & 176 & 187 & TALAIDTIINQK & 10 & 0 & & Mascot \\
\hline 1300.7471 & 1300.7438 & -0.0033 & -3 & 176 & 187 & TALAIDTIINQK & & & & Mascot \\
\hline 1363.6852 & 1363.681 & -0.0042 & -3 & 140 & 151 & ESVNQPIQTGYK & & & & Mascot \\
\hline 1369.7507 & 1369.7467 & -0.004 & -3 & 94 & 106 & IVEVPVGEAMIGR & 88 & 99.998 & & Mascot \\
\hline 1369.7507 & 1369.7467 & -0.004 & -3 & 94 & 106 & IVEVPVGEAMIGR & & & & Mascot \\
\hline 1385.7457 & 1385.7395 & -0.0062 & -4 & 94 & 106 & IVEVPVGEAMIGR & 39 & 0 & Oxidation $(\mathrm{M})[10]$ & Mascot \\
\hline 1385.7457 & 1385.7395 & -0.0062 & -4 & 94 & 106 & IVEVPVGEAMIGR & & & Oxidation (M)[10] & Mascot \\
\hline 1509.8496 & 1509.8427 & -0.0069 & -5 & 349 & 363 & SGIRPAVNPGISVSR & 27 & 0 & & Mascot \\
\hline 1509.8496 & 1509.8427 & -0.0069 & -5 & 349 & 363 & SGIRPAVNPGISVSR & & & & Mascot \\
\hline 1535.8329 & 1535.8243 & -0.0086 & -6 & 376 & 388 & VAGPLRIEYAQYR & 31 & 0 & & Mascot \\
\hline 1535.8329 & 1535.8243 & -0.0086 & -6 & 376 & 388 & VAGPLRIEYAQYR & & & & Mascot \\
\hline 1553.7383 & 1553.7311 & -0.0072 & -5 & 285 & 297 & EAYPGDVFYLHSR & 106 & 100 & & Mascot \\
\hline 1553.7383 & 1553.7311 & -0.0072 & -5 & 285 & 297 & EAYPGDVFYLHSR & & & & Mascot \\
\hline 1570.8622 & 1570.8296 & -0.0326 & -21 & 1 & 13 & MNLRPEEISQIIK & & & & Mascot \\
\hline 1570.8622 & 1570.8296 & -0.0326 & -21 & 1 & 13 & MNLRPEEISQIIK & & & & Mascot \\
\hline 1586.8571 & 1586.8517 & -0.0054 & -3 & 1 & 13 & MNLRPEEISQIIK & & & Oxidation (M)[1] & Mascot \\
\hline 1586.8571 & 1586.8517 & -0.0054 & -3 & 1 & 13 & MNLRPEEISQIIK & & & Oxidation (M)[1] & Mascot \\
\hline 1872.8497 & 1872.8429 & -0.0068 & -4 & 389 & 405 & ELASFAQFGSDLDDETK & 95 & 100 & & Mascot \\
\hline 1872.8497 & 1872.8429 & -0.0068 & -4 & 389 & 405 & ELASFAQFGSDLDDETK & & & & Mascot \\
\hline 1991.0597 & 1991.0559 & -0.0038 & -2 & 256 & 272 & DVLIIYDDLSKHAVAYR & 47 & 77.376 & & Mascot \\
\hline 1991.0597 & 1991.0559 & -0.0038 & -2 & 256 & 272 & DVLIIYDDLSKHAVAYR & & & & Mascot \\
\hline 2060.0771 & 2060.0693 & -0.0078 & -4 & 133 & 151 & AAGVIERESVNQPIQTGY K & 124 & 100 & & Mascot \\
\hline 2060.0771 & 2060.0693 & -0.0078 & -4 & 133 & 151 & AAGVIERESVNQPIQTGY K & & & & Mascot \\
\hline
\end{tabular}




\begin{tabular}{|c|c|c|c|c|c|c|c|c|c|}
\hline 2091.1345 & 2091.1243 & -0.0102 & -5 & 107 & 126 & $\begin{array}{l}\text { VVNALGFPVDGKGPIVTD } \\
\text { HR }\end{array}$ & 122 & 100 & Mascot \\
\hline 2091.1345 & 2091.1243 & -0.0102 & -5 & 107 & 126 & $\begin{array}{l}\text { VVNALGFPVDGKGPIVTD } \\
\text { HR }\end{array}$ & & & Mascot \\
\hline 2361.2295 & 2361.2231 & -0.0064 & -3 & 19 & 40 & $\begin{array}{l}\text { YEDKLEVVDVGTVIQVGD } \\
\text { GVAR }\end{array}$ & 83 & 99.995 & Mascot \\
\hline 2361.2295 & 2361.2231 & -0.0064 & -3 & 19 & 40 & $\begin{array}{l}\text { YEDKLEVVDVGTVIQVGD } \\
\text { GVAR }\end{array}$ & & & Mascot \\
\hline 2384.1616 & 2384.146 & -0.0156 & -7 & 389 & 410 & $\begin{array}{l}\text { ELASFAQFGSDLDDETKA } \\
\text { QLAK }\end{array}$ & & & Mascot \\
\hline 2726.3267 & 2726.3198 & -0.0069 & -3 & 389 & 413 & $\begin{array}{l}\text { ELASFAQFGSDLDDETKA } \\
\text { QLAKGER }\end{array}$ & 192 & 100 & Mascot \\
\hline 2726.3267 & 2726.3198 & -0.0069 & -3 & 389 & 413 & $\begin{array}{l}\text { ELASFAQFGSDLDDETKA } \\
\text { QLAKGER }\end{array}$ & & & Mascot \\
\hline
\end{tabular}

The MALDI TOF/TOF experiments were carried out on an ABI 4800 Proteomics Analyzer MALDI TOF/TOF mass spectrometer (Applied Biosystems) using the established protocols of the Proteomics Department, School of Biological Sciences, Nanyang Technological University Singapore (310). 
Supplementary Table S2B: MALDI TOF-data of the $\alpha 2$ band of lane 2 in Figure 3.47C

\begin{tabular}{|c|c|c|c|c|c|c|c|c|c|c|}
\hline \multicolumn{6}{|c|}{ Protein name } & \multicolumn{2}{|l|}{ Accession No. } & \multicolumn{3}{|c|}{ Protein MW } \\
\hline \multicolumn{6}{|c|}{$\begin{array}{l}\text { ATP synthase subunit } \alpha \text {, sodium ion specific } \\
\text { OS=Acetobacterium woodii (strain ATCC } 29683 \text { / DSM }\end{array}$} & \multicolumn{2}{|l|}{ sp|P50000|ATPA_ACEWD } & \multicolumn{2}{|l|}{55212.7} & \multirow[b]{2}{*}{$\begin{array}{l}\text { Rank Result } \\
\text { Type }\end{array}$} \\
\hline Calc. Mass & Obsrv. Mass & \pm da & $\pm \mathbf{p p m}$ & $\begin{array}{l}\text { Start } \\
\text { seq. }\end{array}$ & $\begin{array}{l}\text { End } \\
\text { seq. }\end{array}$ & Sequence & ION score & C. I. $\%$ & Modification & \\
\hline 803.4807 & 803.4734 & -0.0073 & -9 & 273 & 279 & AMSLILR & & & & Mascot \\
\hline 803.4807 & 803.4734 & -0.0073 & -9 & 273 & 279 & AMSLILR & & & & Mascot \\
\hline 815.4621 & 815.4536 & -0.0085 & -10 & 165 & 171 & ELIIGDR & & & & Mascot \\
\hline 819.4756 & 819.4673 & -0.0083 & -10 & 273 & 279 & AMSLILR & & & Oxidation (M)[2] & Mascot \\
\hline 819.4756 & 819.4673 & -0.0083 & -10 & 273 & 279 & AMSLILR & & & Oxidation (M)[2] & Mascot \\
\hline 894.4791 & 894.4694 & -0.0097 & -11 & 119 & 126 & GPIVTDHR & & & & Mascot \\
\hline 936.4785 & 936.4728 & -0.0057 & -6 & 450 & 456 & DIREFEK & & & & Mascot \\
\hline 942.4679 & 942.4625 & -0.0054 & -6 & 382 & 388 & IEYAQYR & 31 & 0 & & Mascot \\
\hline 942.4679 & 942.4625 & -0.0054 & -6 & 382 & 388 & IEYAQYR & & & & Mascot \\
\hline 1072.5819 & 1072.5762 & -0.0057 & -5 & 152 & 161 & AIDSMIPIGR & 60 & 99.04 & & Mascot \\
\hline 1072.5819 & 1072.5762 & -0.0057 & -5 & 152 & 161 & AIDSMIPIGR & & & & Mascot \\
\hline 1088.5769 & 1088.5701 & -0.0068 & -6 & 152 & 161 & AIDSMIPIGR & 11 & 0 & Oxidation (M)[5] & Mascot \\
\hline 1088.5769 & 1088.5701 & -0.0068 & -6 & 152 & 161 & AIDSMIPIGR & & & Oxidation (M)[5] & Mascot \\
\hline 1156.6433 & 1156.5886 & -0.0547 & -47 & 162 & 171 & GQRELIIGDR & & & & Mascot \\
\hline 1156.6433 & 1156.5886 & -0.0547 & -47 & 162 & 171 & GQRELIIGDR & & & & Mascot \\
\hline 1215.6732 & 1215.6642 & -0.009 & -7 & 107 & 118 & VVNALGFPVDGK & 58 & 98.509 & & Mascot \\
\hline 1215.6732 & 1215.6642 & -0.009 & -7 & 107 & 118 & VVNALGFPVDGK & & & & Mascot \\
\hline 1229.6848 & 1229.6793 & -0.0055 & -4 & 165 & 175 & ELIIGDRQTGK & & & & Mascot \\
\hline
\end{tabular}




\begin{tabular}{|c|c|c|c|c|c|c|c|c|c|c|}
\hline 1293.6937 & 1293.6844 & -0.0093 & -7 & 256 & 266 & DVLIIYDDLSK & 60 & 99.066 & & Mascot \\
\hline 1293.6937 & 1293.6844 & -0.0093 & -7 & 256 & 266 & DVLIIYDDLSK & & & & Mascot \\
\hline 1300.7471 & 1300.7368 & -0.0103 & -8 & 176 & 187 & TALAIDTIINQK & 9 & 0 & & Mascot \\
\hline 1300.7471 & 1300.7368 & -0.0103 & -8 & 176 & 187 & TALAIDTIINQK & & & & Mascot \\
\hline 1369.7507 & 1369.7417 & -0.009 & -7 & 94 & 106 & IVEVPVGEAMIGR & 86 & 99.998 & & Mascot \\
\hline 1369.7507 & 1369.7417 & -0.009 & -7 & 94 & 106 & IVEVPVGEAMIGR & & & & Mascot \\
\hline 1385.7457 & 1385.7335 & -0.0122 & -9 & 94 & 106 & IVEVPVGEAMIGR & 31 & 0 & Oxidation (M)[10] & Mascot \\
\hline 1385.7457 & 1385.7335 & -0.0122 & -9 & 94 & 106 & IVEVPVGEAMIGR & & & Oxidation (M)[10] & Mascot \\
\hline 1509.8496 & 1509.8378 & -0.0118 & -8 & 349 & 363 & SGIRPAVNPGISVSR & 21 & 0 & & Mascot \\
\hline 1509.8496 & 1509.8378 & -0.0118 & -8 & 349 & 363 & SGIRPAVNPGISVSR & & & & Mascot \\
\hline 1535.8329 & 1535.8175 & -0.0154 & -10 & 376 & 388 & VAGPLRIEYAQYR & 19 & 0 & & Mascot \\
\hline 1535.8329 & 1535.8175 & -0.0154 & -10 & 376 & 388 & VAGPLRIEYAQYR & & & & Mascot \\
\hline 1553.7383 & 1553.7251 & -0.0132 & -8 & 285 & 297 & EAYPGDVFYLHSR & 100 & 100 & & Mascot \\
\hline 1553.7383 & 1553.7251 & -0.0132 & -8 & 285 & 297 & EAYPGDVFYLHSR & & & & Mascot \\
\hline 1570.8622 & 1570.8079 & -0.0543 & -35 & 1 & 13 & MNLRPEEISQIIK & & & & Mascot \\
\hline 1570.8622 & 1570.8079 & -0.0543 & -35 & 1 & 13 & MNLRPEEISQIIK & & & & Mascot \\
\hline 1586.8571 & 1586.8423 & -0.0148 & -9 & 1 & 13 & MNLRPEEISQIIK & & & Oxidation (M)[1] & Mascot \\
\hline 1586.8571 & 1586.8423 & -0.0148 & -9 & 1 & 13 & MNLRPEEISQIIK & & & Oxidation (M)[1] & Mascot \\
\hline 1602.9075 & 1602.8854 & -0.0221 & -14 & 119 & 132 & GPIVTDHRRPVEVK & 5 & 0 & & Mascot \\
\hline 1602.9075 & 1602.8854 & -0.0221 & -14 & 119 & 132 & GPIVTDHRRPVEVK & & & & Mascot \\
\hline 1872.8497 & 1872.833 & -0.0167 & -9 & 389 & 405 & ELASFAQFGSDLDDETK & 76 & 99.979 & & Mascot \\
\hline 1872.8497 & 1872.833 & -0.0167 & -9 & 389 & 405 & ELASFAQFGSDLDDETK & & & & Mascot \\
\hline 1991.0597 & 1991.049 & -0.0107 & -5 & 256 & 272 & DVLIIYDDLSKHAVAYR & & & & Mascot \\
\hline
\end{tabular}




\begin{tabular}{|c|c|c|c|c|c|c|c|c|c|}
\hline 2060.0771 & 2060.0603 & -0.0168 & -8 & 133 & 151 & AAGVIERESVNQPIQTGY K & 71 & 99.93 & Mascot \\
\hline 2060.0771 & 2060.0603 & -0.0168 & -8 & 133 & 151 & AAGVIERESVNQPIQTGY K & & & Mascot \\
\hline 2091.1345 & 2091.1125 & -0.022 & -11 & 107 & 126 & $\begin{array}{l}\text { VVNALGFPVDGKGPIVTD } \\
\text { HR }\end{array}$ & 99 & 100 & Mascot \\
\hline 2091.1345 & 2091.1125 & -0.022 & -11 & 107 & 126 & $\begin{array}{l}\text { VVNALGFPVDGKGPIVTD } \\
\text { HR }\end{array}$ & & & Mascot \\
\hline 2361.2295 & 2361.2021 & -0.0274 & -12 & 19 & 40 & $\begin{array}{l}\text { YEDKLEVVDVGTVIQVGD } \\
\text { GVAR }\end{array}$ & 11 & 0 & Mascot \\
\hline 2361.2295 & 2361.2021 & -0.0274 & -12 & 19 & 40 & $\begin{array}{l}\text { YEDKLEVVDVGTVIQVGD } \\
\text { GVAR }\end{array}$ & 11 & 0 & Mascot \\
\hline 2384.1616 & 2384.1384 & -0.0232 & -10 & 389 & 410 & $\begin{array}{l}\text { ELASFAQFGSDLDDETKAQL } \\
\text { AK }\end{array}$ & & & Mascot \\
\hline 2726.3267 & 2726.3042 & -0.0225 & -8 & 389 & 413 & $\begin{array}{l}\text { ELASFAQFGSDLDDETKAQL } \\
\text { AKGER }\end{array}$ & 116 & 100 & Mascot \\
\hline 2726.3267 & 2726.3042 & -0.0225 & -8 & 389 & 413 & $\begin{array}{l}\text { ELASFAQFGSDLDDETKAQL } \\
\text { AKGER }\end{array}$ & & & Mascot \\
\hline 2799.5627 & 2799.5288 & -0.0339 & -12 & 107 & 132 & $\begin{array}{l}\text { VVNALGFPVDGKGPIVTDH } \\
\text { RRPVEVK }\end{array}$ & 31 & 0 & Mascot \\
\hline 2799.5627 & 2799.5288 & -0.0339 & -12 & 107 & 132 & $\begin{array}{l}\text { VVNALGFPVDGKGPIVTDH } \\
\text { RRPVEVK }\end{array}$ & & & Mascot \\
\hline 3002.5427 & 3002.5073 & -0.0354 & -12 & 14 & 40 & $\begin{array}{l}\text { NEIERYEDKLEVVDVGTVIQ } \\
\text { VGDGVAR }\end{array}$ & 10 & 0 & Mascot \\
\hline 3002.5427 & 3002.5073 & -0.0354 & -12 & 14 & 40 & $\begin{array}{l}\text { NEIERYEDKLEVVDVGTVIQ } \\
\text { VGDGVAR }\end{array}$ & & & Mascot \\
\hline
\end{tabular}

The MALDI TOF/TOF experiments were carried out on an ABI 4800 Proteomics Analyzer MALDI TOF/TOF mass spectrometer (Applied Biosystems) using the established protocols of the Proteomics Department, School of Biological Sciences, Nanyang Technological University Singapore (310). 
Supplementary Table S2C: MALDI-TOF data of the $\alpha 3$ band of lane 2 in Figure 3.47C

\begin{tabular}{|c|c|c|c|c|c|c|c|c|c|c|}
\hline \multicolumn{6}{|c|}{ Protein name } & \multicolumn{2}{|l|}{ Accession No. } & \multicolumn{3}{|l|}{ Protein MW } \\
\hline \multicolumn{6}{|c|}{$\begin{array}{l}\text { ATP synthase subunit } \alpha \text {, sodium ion specific } \\
\text { OS=Acetobacterium woodii (strain ATCC } 29683 \text { / DSM }\end{array}$} & \multicolumn{2}{|l|}{ sp|P50000|ATPA_ACEWD } & \multicolumn{2}{|l|}{55212.7} & \multirow[b]{2}{*}{$\begin{array}{l}\text { Rank Result } \\
\text { Type }\end{array}$} \\
\hline Calc. Mass & Obsrv. Mass & \pm da & $\pm \mathbf{p p m}$ & $\begin{array}{l}\text { Start } \\
\text { seq. }\end{array}$ & $\begin{array}{l}\text { End } \\
\text { seq. }\end{array}$ & Sequence & ION score & C. I. \% & Modification & \\
\hline 803.4807 & 803.4676 & -0.0131 & -16 & 273 & 279 & AMSLILR & 23 & 0 & & Mascot \\
\hline 803.4807 & 803.4676 & -0.0131 & -16 & 273 & 279 & AMSLILR & & & & Mascot \\
\hline 815.4621 & 815.4455 & -0.0166 & -20 & 165 & 171 & ELIIGDR & & & & Mascot \\
\hline 819.4756 & 819.4614 & -0.0142 & -17 & 273 & 279 & AMSLILR & & & Oxidation (M)[2] & Mascot \\
\hline 819.4756 & 819.4614 & -0.0142 & -17 & 273 & 279 & AMSLILR & & & Oxidation (M)[2] & Mascot \\
\hline 942.4679 & 942.4541 & -0.0138 & -15 & 382 & 388 & IEYAQYR & 24 & 0 & & Mascot \\
\hline 942.4679 & 942.4541 & -0.0138 & -15 & 382 & 388 & IEYAQYR & & & & Mascot \\
\hline 1072.5819 & 1072.5685 & -0.0134 & -12 & 152 & 161 & AIDSMIPIGR & 36 & 0 & & Mascot \\
\hline 1072.5819 & 1072.5685 & -0.0134 & -12 & 152 & 161 & AIDSMIPIGR & & & & Mascot \\
\hline 1088.5769 & 1088.561 & -0.0159 & -15 & 152 & 161 & AIDSMIPIGR & 6 & 0 & Oxidation (M)[5] & Mascot \\
\hline 1088.5769 & 1088.561 & -0.0159 & -15 & 152 & 161 & AIDSMIPIGR & & & Oxidation (M)[5] & Mascot \\
\hline 1156.6433 & 1156.5796 & -0.0637 & -55 & 162 & 171 & GQRELIIGDR & & & & Mascot \\
\hline 1156.6433 & 1156.5796 & -0.0637 & -55 & 162 & 171 & GQRELIIGDR & & & & Mascot \\
\hline 1215.6732 & 1215.6533 & -0.0199 & -16 & 107 & 118 & VVNALGFPVDGK & 58 & 98.431 & & Mascot \\
\hline 1215.6732 & 1215.6533 & -0.0199 & -16 & 107 & 118 & VVNALGFPVDGK & & & & Mascot \\
\hline 1293.6937 & 1293.6716 & -0.0221 & -17 & 256 & 266 & DVLIIYDDLSK & 65 & 99.641 & & Mascot \\
\hline 1293.6937 & 1293.6716 & -0.0221 & -17 & 256 & 266 & DVLIIYDDLSK & & & & Mascot \\
\hline 1300.7471 & 1300.7271 & -0.02 & -15 & 176 & 187 & TALAIDTIINQK & 9 & 0 & & Mascot \\
\hline
\end{tabular}




\begin{tabular}{|c|c|c|c|c|c|c|c|c|c|c|}
\hline 1300.7471 & 1300.7271 & -0.02 & -15 & 176 & 187 & TALAIDTIINQK & & & & Mascot \\
\hline 1363.6852 & 1363.6608 & -0.0244 & -18 & 140 & 151 & ESVNQPIQTGYK & & & & Mascot \\
\hline 1369.7507 & 1369.7296 & -0.0211 & -15 & 94 & 106 & IVEVPVGEAMIGR & 74 & 99.954 & & Mascot \\
\hline 1369.7507 & 1369.7296 & -0.0211 & -15 & 94 & 106 & IVEVPVGEAMIGR & & & & Mascot \\
\hline 1385.7457 & 1385.7189 & -0.0268 & -19 & 94 & 106 & IVEVPVGEAMIGR & 35 & 0 & Oxidation (M)[10] & Mascot \\
\hline 1385.7457 & 1385.7189 & -0.0268 & -19 & 94 & 106 & IVEVPVGEAMIGR & & & Oxidation (M)[10] & Mascot \\
\hline 1509.8496 & 1509.822 & -0.0276 & -18 & 349 & 363 & SGIRPAVNPGISVSR & 26 & 0 & & Mascot \\
\hline 1509.8496 & 1509.822 & -0.0276 & -18 & 349 & 363 & SGIRPAVNPGISVSR & & & & Mascot \\
\hline 1535.8329 & 1535.8024 & -0.0305 & -20 & 376 & 388 & VAGPLRIEYAQYR & 6 & 0 & & Mascot \\
\hline 1535.8329 & 1535.8024 & -0.0305 & -20 & 376 & 388 & VAGPLRIEYAQYR & & & & Mascot \\
\hline 1553.7383 & 1553.7086 & -0.0297 & -19 & 285 & 297 & EAYPGDVFYLHSR & 91 & 100 & & Mascot \\
\hline 1553.7383 & 1553.7086 & -0.0297 & -19 & 285 & 297 & EAYPGDVFYLHSR & & & & Mascot \\
\hline 1586.8571 & 1586.8201 & -0.037 & -23 & 1 & 13 & MNLRPEEISQIIK & & & Oxidation (M)[1] & Mascot \\
\hline 1586.8571 & 1586.8201 & -0.037 & -23 & 1 & 13 & MNLRPEEISQIIK & & & Oxidation (M)[1] & Mascot \\
\hline 1602.9075 & 1602.8782 & -0.0293 & -18 & 119 & 132 & GPIVTDHRRPVEVK & & & & Mascot \\
\hline 1602.9075 & 1602.8782 & -0.0293 & -18 & 119 & 132 & GPIVTDHRRPVEVK & & & & Mascot \\
\hline 1872.8497 & 1872.816 & -0.0337 & -18 & 389 & 405 & ELASFAQFGSDLDDETK & 83 & 99.995 & & Mascot \\
\hline 1872.8497 & 1872.816 & -0.0337 & -18 & 389 & 405 & ELASFAQFGSDLDDETK & & & & Mascot \\
\hline 2060.0771 & 2060.0391 & -0.038 & -18 & 133 & 151 & AAGVIERESVNQPIQTGYK & 71 & 99.913 & & Mascot \\
\hline 2060.0771 & 2060.0391 & -0.038 & -18 & 133 & 151 & AAGVIERESVNQPIQTGYK & & & & Mascot \\
\hline 2091.1345 & 2091.0979 & -0.0366 & -18 & 107 & 126 & $\begin{array}{l}\text { VVNALGFPVDGKGPIVTD } \\
\text { HR }\end{array}$ & 100 & 100 & & Mascot \\
\hline 2091.1345 & 2091.0979 & -0.0366 & -18 & 107 & 126 & $\begin{array}{l}\text { VVNALGFPVDGKGPIVTD } \\
\text { HR }\end{array}$ & & & & Mascot \\
\hline
\end{tabular}




\begin{tabular}{|c|c|c|c|c|c|c|c|c|c|}
\hline 2361.2295 & 2361.1868 & -0.0427 & -18 & 19 & 40 & $\begin{array}{l}\text { YEDKLEVVDVGTVIQVGD } \\
\text { GVAR }\end{array}$ & 11 & 0 & Mascot \\
\hline 2361.2295 & 2361.1868 & -0.0427 & -18 & 19 & 40 & $\begin{array}{l}\text { YEDKLEVVDVGTVIQVGD } \\
\text { GVAR }\end{array}$ & & & Mascot \\
\hline 2384.1616 & 2384.113 & -0.0486 & -20 & 389 & 410 & $\begin{array}{l}\text { ELASFAQFGSDLDDETKA } \\
\text { QLAK }\end{array}$ & & & Mascot \\
\hline 2726.3267 & 2726.2798 & -0.0469 & -17 & 389 & 413 & $\begin{array}{l}\text { ELASFAQFGSDLDDETKA } \\
\text { QLAKGER }\end{array}$ & 112 & 100 & Mascot \\
\hline 2726.3267 & 2726.2798 & -0.0469 & -17 & 389 & 413 & $\begin{array}{l}\text { ELASFAQFGSDLDDETKA } \\
\text { QLAKGER }\end{array}$ & & & Mascot \\
\hline
\end{tabular}

The MALDI TOF/TOF experiments were carried out on an ABI 4800 Proteomics Analyzer MALDI TOF/TOF mass spectrometer (Applied Biosystems) using the established protocols of the Proteomics Department, School of Biological Sciences, Nanyang Technological University Singapore (310). 
Supplementary Table S3A: MALDI-TOF data of the $110 \mathrm{kDa}$ band in Figure 3.47D

\begin{tabular}{|c|c|c|c|c|c|c|c|c|c|c|}
\hline \multicolumn{6}{|c|}{ Protein name } & \multirow{3}{*}{$\begin{array}{l}\text { Accession No. } \\
\text { sp|P31224|ACRB_ECOLI }\end{array}$} & \multicolumn{4}{|c|}{ Protein MW } \\
\hline \multicolumn{6}{|c|}{$\begin{array}{l}\text { Multidrug efflux pump subunit AcrB OS=Escherichia coli } \\
\text { (strain K12) OX=83333 GN=acrB PE=1 SV=1 }\end{array}$} & & \multicolumn{4}{|l|}{113615} \\
\hline Calc. Mass & Obsrv. Mass & \pm da & $\pm \mathbf{p p m}$ & $\begin{array}{l}\text { Start } \\
\text { seq. }\end{array}$ & $\begin{array}{l}\text { End } \\
\text { seq. }\end{array}$ & & ION score & $\begin{array}{l}\text { C. } \\
\text { I. } \%\end{array}$ & Modification & $\begin{array}{l}\text { Rank } \\
\text { Result Type }\end{array}$ \\
\hline 894.4104 & 894.3942 & -0.0162 & -18 & 809 & 815 & WEYGSPR & 9 & 0 & & Mascot \\
\hline 894.4104 & 894.3942 & -0.0162 & -18 & 809 & 815 & WEYGSPR & & & & Mascot \\
\hline 1152.5758 & 1152.5658 & -0.01 & -9 & 313 & 322 & MEPFFPSGLK & 22 & 0 & & Mascot \\
\hline 1168.5707 & 1168.5647 & -0.006 & -5 & 313 & 322 & MEPFFPSGLK & 18 & 0 & Oxidation (M)[1] & Mascot \\
\hline 1168.5707 & 1168.5647 & -0.006 & -5 & 313 & 322 & MEPFFPSGLK & & & Oxidation (M)[1] & Mascot \\
\hline 1442.7566 & 1442.7378 & -0.0188 & -13 & 323 & 334 & IVYPYDTTPFVK & & & & Mascot \\
\hline 1479.6937 & 1479.6891 & -0.0046 & -3 & 781 & 792 & MLPDDIGDWYVR & 21 & 0 & & Mascot \\
\hline 1479.6937 & 1479.6891 & -0.0046 & -3 & 781 & 792 & MLPDDIGDWYVR & & & & Mascot \\
\hline 1495.6886 & 1495.6708 & -0.0178 & -12 & 781 & 792 & MLPDDIGDWYVR & 14 & 0 & Oxidation (M)[1] & Mascot \\
\hline 1657.7639 & 1657.7477 & -0.0162 & -10 & 793 & 808 & AADGQMVPFSAFSSSR & & & & Mascot \\
\hline 1673.7588 & 1673.7281 & -0.0307 & -18 & 793 & 808 & AADGQMVPFSAFSSSR & & & Oxidation (M)[6] & Mascot \\
\hline 1673.7588 & 1673.7281 & -0.0307 & -18 & 793 & 808 & AADGQMVPFSAFSSSR & & & Oxidation (M)[6] & Mascot \\
\hline
\end{tabular}

The MALDI TOF/TOF experiments were carried out on an ABI 4800 Proteomics Analyzer MALDI TOF/TOF mass spectrometer (Applied

Biosystems) using the established protocols of the Proteomics Department, School of Biological Sciences, Nanyang Technological University

Singapore (310) 
Supplementary Table S3B: MALDI-TOF data of the $35 \mathrm{kDa}$ band in Figure 3.47D

\begin{tabular}{|c|c|c|c|c|c|c|c|c|c|c|}
\hline \multicolumn{6}{|c|}{ Protein name } & \multirow{2}{*}{$\frac{\text { Accession No. }}{\text { sp|P0ABJ1|CYOA_ECOLI }}$} & \multicolumn{4}{|c|}{ Protein MW } \\
\hline \multicolumn{6}{|c|}{$\begin{array}{l}\text { Cytochrome bo(3) ubiquinol oxidase subunit } 2 \text { OS=Escherichia coli } \\
\text { O6:H1 (strain CFT073 / ATCC } 700928\end{array}$} & & \multicolumn{4}{|l|}{34945.6} \\
\hline Calc. Mass & Obsrv. Mass & $\pm \mathrm{da}$ & \pm ppm & $\begin{array}{l}\text { Start } \\
\text { seq. }\end{array}$ & $\begin{array}{l}\text { End } \\
\text { seq. }\end{array}$ & Sequence & ION score & $\begin{array}{l}\text { C. } \\
\text { I. } \%\end{array}$ & Modification & $\begin{array}{l}\text { Rank } \\
\text { Result Type }\end{array}$ \\
\hline 1035.5259 & 1035.5085 & -0.0174 & -17 & 229 & 237 & AAFDQWVAK & 59 & 98.708 & & Mascot \\
\hline 1035.5259 & 1035.5085 & -0.0174 & -17 & 229 & 237 & AAFDQWVAK & & & & Mascot \\
\hline 1542.7404 & 1542.704 & -0.0364 & -24 & 179 & 192 & LGSQIYAMAGMQTR & & & Oxidation (M)[8] & Mascot \\
\hline 1542.7404 & 1542.704 & -0.0364 & -24 & 179 & 192 & LGSQIYAMAGMQTR & & & Oxidation (M)[8] & Mascot \\
\hline 1598.7996 & 1598.765 & -0.0346 & -22 & 165 & 178 & VTSNSVMNSFFIPR & 39 & 0 & & Mascot \\
\hline 1598.7996 & 1598.765 & -0.0346 & -22 & 165 & 178 & VTSNSVMNSFFIPR & & & & Mascot \\
\hline 1614.7944 & 1614.7517 & -0.0427 & -26 & 165 & 178 & VTSNSVMNSFFIPR & 10 & 0 & Oxidation (M)[7] & Mascot \\
\hline 1614.7944 & 1614.7517 & -0.0427 & -26 & 165 & 178 & VTSNSVMNSFFIPR & & & Oxidation (M)[7] & Mascot \\
\hline 1759.9126 & 1759.8702 & -0.0424 & -24 & 222 & 237 & AIATPDRAAFDQWVAK & 40 & 0 & & Mascot \\
\hline 1759.9126 & 1759.8702 & -0.0424 & -24 & 222 & 237 & AIATPDRAAFDQWVAK & & & & Mascot \\
\hline
\end{tabular}

The MALDI TOF/TOF experiments were carried out on an ABI 4800 Proteomics Analyzer MALDI TOF/TOF mass spectrometer (Applied Biosystems) using the established protocols of the Proteomics Department, School of Biological Sciences, Nanyang Technological University Singapore (310). 
Supplementary Table S3C: MALDI-TOF data of the $35 \mathrm{kDa}$ band in Figure 3.47D

\begin{tabular}{|c|c|c|c|c|c|c|c|c|c|c|}
\hline \multicolumn{6}{|c|}{ Protein name } & \multirow{2}{*}{$\begin{array}{l}\text { Accession No. } \\
\text { sp|B5YQR7|SUCC_ECO5E }\end{array}$} & \multicolumn{4}{|c|}{ Protein MW } \\
\hline \multicolumn{6}{|c|}{$\begin{array}{l}\text { Succinate--CoA ligase [ADP-forming] subunit beta } \\
\text { OS=Escherichia coli O157:H7 (strain EC4115 / EHEC) }\end{array}$} & & \multicolumn{4}{|l|}{41651.8} \\
\hline Calc. Mass & Obsrv. Mass & \pm da & \pm ppm & $\begin{array}{c}\text { Start } \\
\text { seq. }\end{array}$ & $\begin{array}{l}\text { End } \\
\text { seq. }\end{array}$ & Sequence & ION score & $\begin{array}{l}\text { C. } \\
\text { I. } \%\end{array}$ & Modification & $\begin{array}{l}\text { Rank } \\
\text { Result Type }\end{array}$ \\
\hline 1035.5258 & 1035.5085 & -0.0173 & -17 & 71 & 79 & AFAENWLGK & 22 & 0 & & Mascot \\
\hline 1035.5258 & 1035.5085 & -0.0173 & -17 & 71 & 79 & AFAENWLGK & & & & Mascot \\
\hline 1059.5793 & 1060.0417 & 0.4624 & 436 & 62 & 70 & VVNSKEDIR & & & & Mascot \\
\hline 1059.5793 & 1060.0417 & 0.4624 & 436 & 62 & 70 & VVNSKEDIR & & & & Mascot \\
\hline 1614.8308 & 1614.7517 & -0.0791 & -49 & 147 & 161 & VALDPLTGPMPYQGR & & & & Mascot \\
\hline 1614.8308 & 1614.7517 & -0.0791 & -49 & 147 & 161 & VALDPLTGPMPYQGR & & & & Mascot \\
\hline 1791.9435 & 1791.8688 & -0.0747 & -42 & 38 & 54 & IGAGPWVVKCQVHAGGR & & & $\begin{array}{l}\text { Carbamidomethyl } \\
\text { (C)[10] }\end{array}$ & Mascot \\
\hline 1791.9435 & 1791.8688 & -0.0747 & -42 & 38 & 54 & IGAGPWVVKCQVHAGGR & & & $\begin{array}{l}\text { Carbamidomethyl } \\
\text { (C) }[10]\end{array}$ & Mascot \\
\hline
\end{tabular}

The MALDI TOF/TOF experiments were carried out on an ABI 4800 Proteomics Analyzer MALDI TOF/TOF mass spectrometer (Applied Biosystems) using the established protocols of the Proteomics Department, School of Biological Sciences, Nanyang Technological University Singapore (310). 
"My brain is only a receiver, in the Universe, there is a core from which we obtain knowledge, strength, and inspiration. I have not penetrated into the secrets of this core, but I know that it exists".

Sikola Tesla 